\title{
LNG Measurement
}

\section{A User's Manual for Custody Transfer}

\author{
Douglas Mann, General Editor \\ Chemical Engineering Science Division \\ Center for Chemical Engineering \\ National Bureau of Standards
}

Boulder, Colorado 80303

\section{Sponsored by}

Groupe International des Importateurs de Gaz Naturel Liquéfié (G.I.I.G.N.L.)
Center for Chemical Engineering National Bureau of Standards

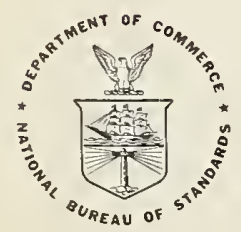

\section{FIRST EDITION}

1985

NBSIR 85-3028

U.S. DEPARTMENT OF COMMERCE, Malcolm Ealdrige, Secretary

National Bureau of Standards, Ernest Ambler, Director 



\section{PREF ACE}

This LNG Measurement Manual in combination with the previously published LNG Materials and Fluids User's Manual [19,50,55 of section 1.] will provide measurement engineers and others with a source of critically evaluated basic physical property data, a description of recent relevant measurement research, and detailed examples of several methods of establishing the quantity and quality of liquefied natural gas(LNG) as a commercial commodity at the custody transfer point of sale. The contents of the manual are edited condensations of published research on properties and measurement processes. Explanations of the several methods of cargo valuation are considered as examples only and are not intended as recommended practice. Other methods of determining LNG cargo value may certainly be used. The procedures examined here utilize a consistent set of basic physical and thermophysical properties data and conversion factors which must be considered when comparing other measurement methods with those considered in this study. The concept of measurement as a process operating on a system is used both to describe the individual measurement elements and to show the integration of these elements into a total measurement process for LNG.

A fundamental requirement in the selection of reference material for this manual was an inclusion in the references of detailed descriptions of all aspects of the particular measurement process including sources and magnitude of error, and the calculation and propagation of measurement error through the entire measurement process. This procedure allows independent assessment of the magnitude of error of an individual measurement process and the intercomparison of several different measurement processes.

For the purposes of this measurement manual, liquefied natural gas(LNG) is considered a mixture of methane, ethane, propane, butane, pentane, hexane and nitrogen. Heavier hydrocarbons, such as the pentanes and hexanes, are generally present in LNG at less than 0.5 mol percent. Properties of mixtures containing these heavier constituents are less well known than mixtures of the lighter fractions, but the greater heating value (mol basis) of the heavier constituents can have a significant effect on the total mixture value. For example; the molar heating value of 3-methylpentane (a hexane isomer) is nearly five times the molar heating value of methane.

The major component of LNG is methane, which is generally present at a concentration of greater than $80 \mathrm{~mol}$ percent. Typical mixture compositions for imported LNG as a function of source are given in table 2.1.1 of the manual section on Elements. Typical mixture compositions of LNG in peak-shaving or satellite facilities are a strong function of the pipeline source and will vary as to location of the natural gas liquefaction plant. Concentrations of ethane and propane in most LNG are generally less than 10 mol percent, and the concentrations of butanes, pentanes, hexanes and nitrogen are generally less than 5 mol percent.

The value of LNG is assessed primarily in respect to its end point use as a heat energy source or as a chemical feedstock. Accurate and precise knowledge of the mol fraction of each component and the total quantity of the component and the mixture are necessary and sufficient measurements for feed stock use. Additional knowledge of heating value per unit of quantity is necessary to establish value as a heat energy fuel. Heating values and methods of gas analysis are presented in this manual as applied to the LNG as a liquid commodity at temperatures of 90 to $150 \mathrm{~K}$. The data and procedures could, of course, be used for other fluid mixtures.

LNG is a commodity in national and international commerce. It is bought and sold worldwide on the basis of values established at the point of sale. It is the intent of NBS to provide a common base for a broad range of calculations which may be necessary to establish commodity value under a wide variety of field conditions. The selection of the SI system for dimensional units with extenslve conversion tables should allow use of the included measurement methods by most workers in the fleld.

Section 1 provides base physical properties data and is intended as a definition of the common base from which most if not all the measurement calculations are made. It is not the purpose to des!gnate this section as a basic reference source for these values, but it is the intent to deflne the number values of the most currently acceptable physical property data, provide the uncertainty for these values, and to reference the source documents. The benefits of this approach should be tworold: calculations will be made on a common base so that different methods may be compared, and the tot 11 estimated uncertainty in the measurement of the value of the commodity should allow mors lntollifrnt economic decisions. 
Once the base physical properties data are defined, it is then possible to describe in detail the individual measurement elements, the summary process for combining these elements, and finally the examination of field applications. At the time the manual was planned, it was anticipated that one or more of the major LNG import terminals would be available to experimentally verify and compare the individual measurement processes. This would include both calculated and measured values off-loaded from the LNG ship, at a measurement station located in the off-loading pipeline and at the shore-based storage tank. As the supply situation within the natural gas industry changed in the early 1980 's from shortage to surplus, only cursory field measurements were possible as the larger LNG import terminals were shut down and new construction was postponed.

The content of the applications section of the manual was most affected by this turn of events. Simple combinations of the individual measurement elements were substituted for actual field measurements under real operating conditions. The result of this modification to the original plan was to give, what will be, an optimistic view of the accuracy and precision of the LNG measurement process. The errors found in practice may be greater than estimated from controlled laboratory-type conditions. The editor regrets this possible limitation, but it is believed to be unavoidable even though care was taken during most of the laboratory experiments to anticipate field-type experimental errors.

\section{ACKNOWLEDGMENT}

The technical program which produced this LNG Measurement Manual has been guided by a Steering Committee appointed by Groupe International des Importateurs de Gaz Naturel Liquéfié (G.I.I.G.N.L.). The members of this committee have contributed freely of their time and talent to make this manual a success. Committee meetings were held at NBS in Boulder, Colorado, on a semiannual basis. The individual steering Comittee members in their capacities as technical reviewers spent many hours with the manuscript and their comments and suggestions are greatly appreciated. The members of the Steering Committee were:

\author{
Mr. Lee Bell (Chairman) \\ Manager of Engineering \\ Western LNG Terminal Associates \\ Mr. Kimio Kurahashi, Manager \\ Production and Engineering Department \\ Tokyo Gas Company
Mr. Bland Osborn (past Chairman)
Chief Engineer
Columbia LNG Corporation

\author{
Mr. Ivan W. Schmitt (past Chairman) \\ Vice President \\ El Paso LNG Company \\ Dr. Klaus Schwier \\ Managing Director \\ Ruhrgas LNG \\ Mr. Hiroaki Tanaka, Manager \\ Control \& Systems Eng. Lab. \\ Tokyo Gas Company
}

The Steering Committee, in outlining the content of the manual, requested that particular attention be directed by NBS to the numeric values of the base physical properties. These values would include molecular and atomic masses of the LNG components and the combustion enthalpies of the pure components and their mixtures. The selected values should reflect the most recent national and international agreement and contain the full support of NBS and other standards groups.

To accomplish this objective, a sub-task was set up by NBS to issue a separate publication containing the required base physical property data but with additional supporting data and references. This program was placed under the direction of Dr. George T. Armstrong a recognized national and international expert in the subject. Dr. Armstrong, with the assistance of Mr. Thomas L. Jobe, Jr., had carried the program to near completion at the time of his death in March of 1982. Dr. David Garvin of the NBS Chemical Thermodynamics Data Center assumed the responsibility for completion and publication of the document (see [12a] of section 1.). The contributions of Dr. Armstrong, Dr. Garvin and the staff of the Data Center, to the original publication and the resulting portions of that study included in this manual are gratefully acknowledged. 



\section{LNG MEASUREMENT}

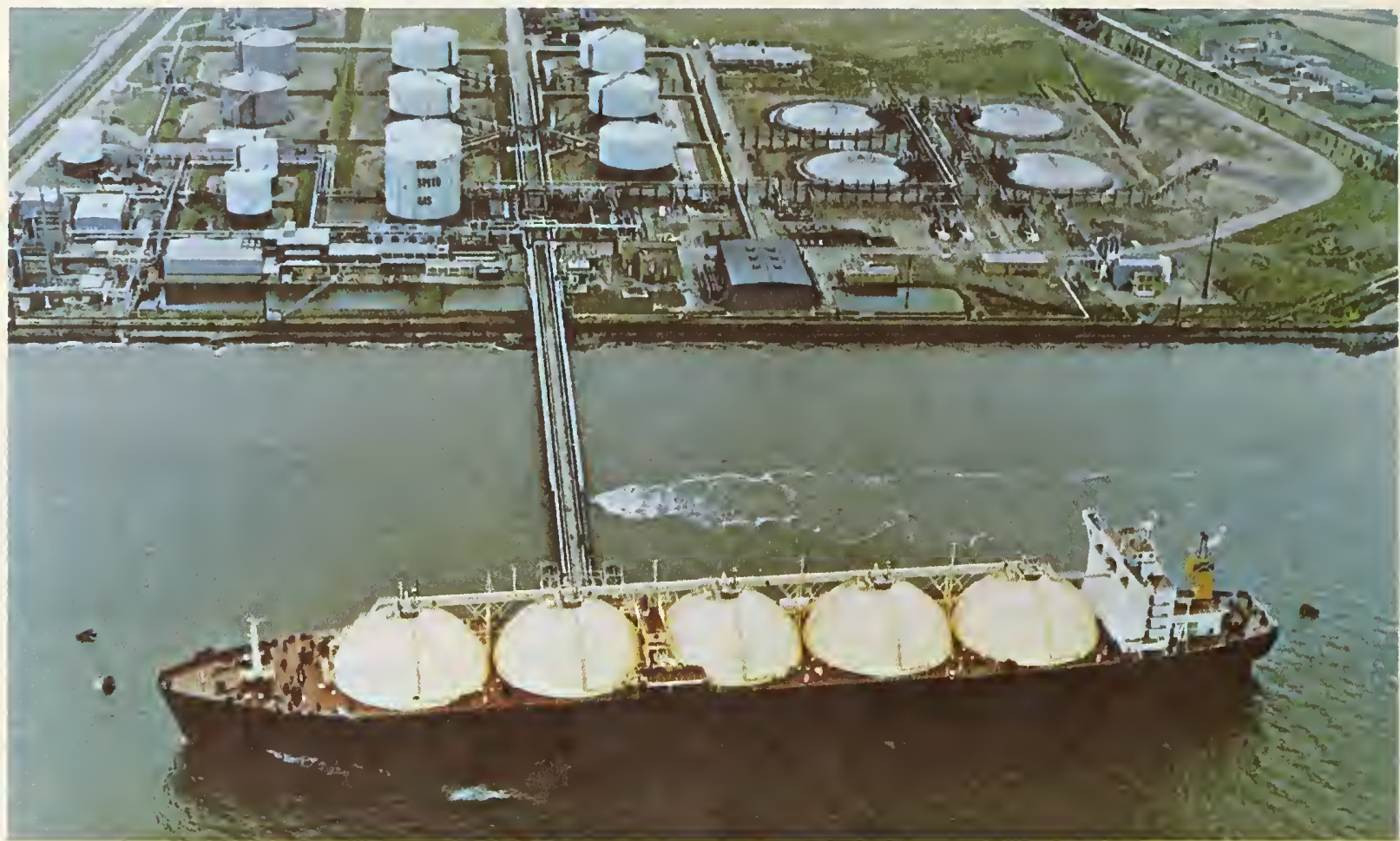

\section{CANVEY TERMINAL}

The Canvey Terminal, operated by the British Gas Corporation (BGC) and constructed on the Canvey Island, located at the mouth of the Thames river in Great Britain, was the world's first LNG receiving terminal which depended on marine LNG transport. The terminal first received LNG in 1958 . The total storage capacity of $265,000 \mathrm{~kL}$ was composed of four inground tanks of 50,000 $k L$, shown in the upper right of the photograph, six 10,000 kL above ground tanks at the upper center of the photograph and two smaller $2,500 \mathrm{~kL}$ aboveground tanks at the upper left of the photograph. Vaporizer units of the open rack type, intermediate fluid type and submerged combustion type have a capacity of $300 \mathrm{t} / \mathrm{h}$ and are located just to the left of the bojl off gas holder (High Speed Gas). The terminal also had a re-liquefaction capacity of $480 \mathrm{t} / \mathrm{d}$.

The size and relative positions of the LNG carrier berth, offloading lines, storage tanks and vaporizers provide a graphic display of the magnitude of the measurements required in establishing the value of imported LNG. (Photograph by courtesy of The Japan Gas Association, Tokyo, Japan, on behalf of the 1981 Japan LNG Congress) 
CONTENTS

1.0 BASE PHYSICAL PROPERTIES PAGE

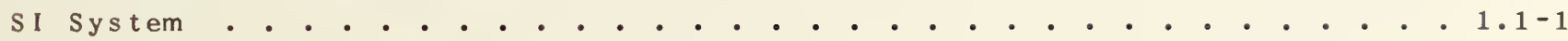

Pure Fluid Properties . . . . . . . . . . . . . . . . . . . . . 1.2-1 Combustion Enthalpies . . . . . . . . . . . . . . . . . . . . . . 1. $3-1$

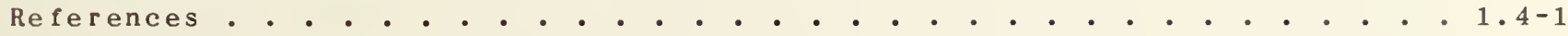

\section{0 MEASUR EMENT ELEMENTS}

The Measurement Process Applied to LNG . . . . . . . . . . . . . . . . . 2.1-1

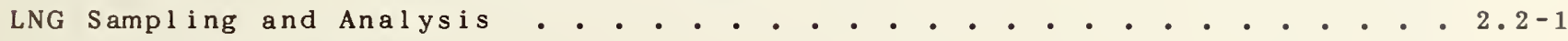

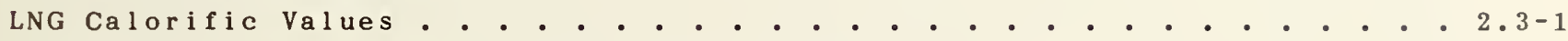

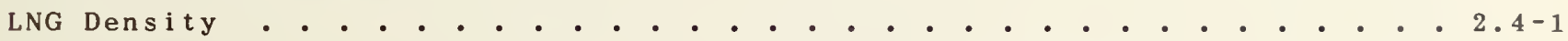

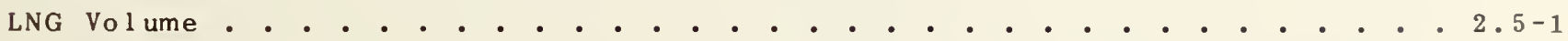

\section{0 MBAS UREMENT APPLICATIONS}

Measurement Uncertainties . . . . . . . . . . . . . . . . . . . . . 3.1

Ship Unloading/Loading . . . . . . . . . . . . . . . . . . . . . . $3.2-1$

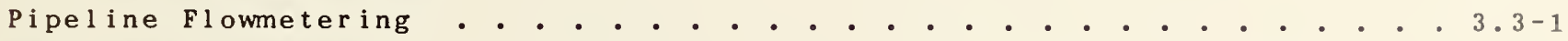

Land Based Storage . . . . . . . . . . . . . . . . . . . . . . . 4 - 1 




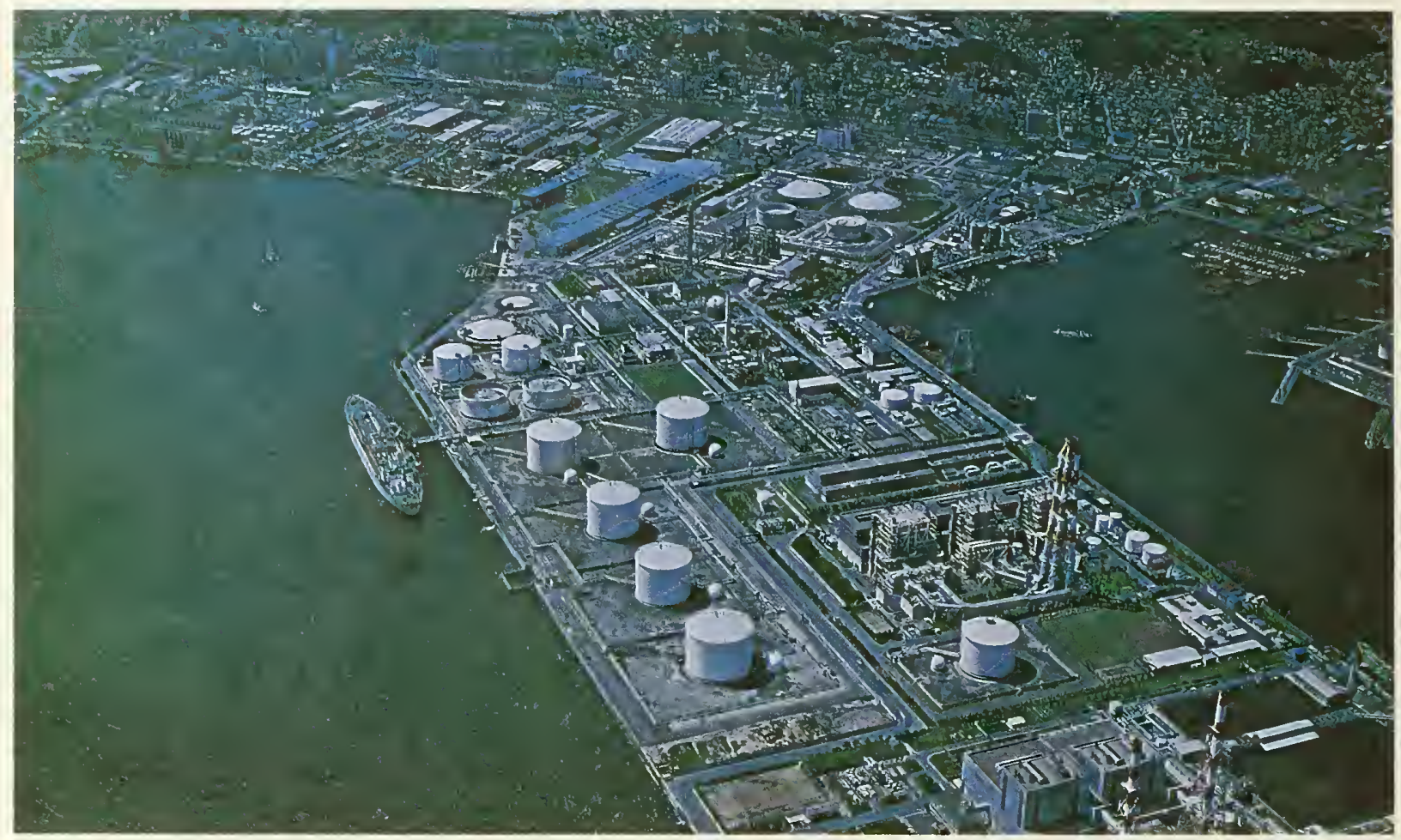

\section{NEGISHI TERMINAL}

The Negishi Terminal is composed of two utility facilities, the Negishi Works of Tokyo Gas and the Minami-Yokohama Thermal Power Station of Tokyo Electric Power. The two installations are situated in a special industrial area facing the Negishi Bay south of Yokohama, Japan. Shared facilities such as berths, storage tanks and vaporizers owned by the two companies result in an efficient operation. The photograph shows six aboveground tanks with a total capacity of $255,000 \mathrm{~kL}$ at the center and five inground tanks (two under construction) of about $260,000 \mathrm{~kL}$ at the top center. 0pen rack (410 t/h) and submerged combustion ( $335 \mathrm{t} / \mathrm{h})$ type vaporizers are located just to the right of the off-loading line from the LNG carrier berth.

LNG from both Alaska and Brunei are brought to this terminal. The wide variation in mixture between the two sources makes accurate property data a necessary requirement in assessing the value of the LNG as an energy source. (Photograph by courtesy of The Japan Gas Association, Tokyo, Japan, on behalf of the 1981 Japan LNG Congress) 
CONTENTS

\section{0 BASE PHYS ICAL PROPERTIES}

SI System

Page

1.1 Th

The International System of Units (SI)

1. $1-1$

1. 1. 1

Units Acceptable for Use with SI.

1. 1.2

Descriptive and Essential Data

Pure Fluid Properties

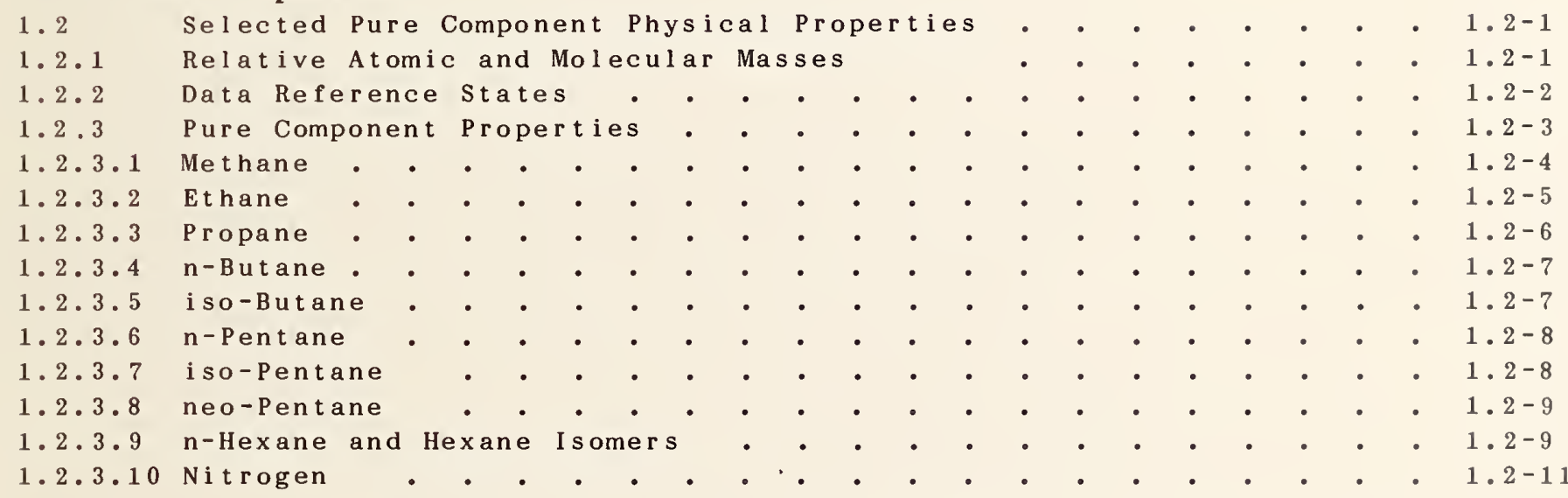

Combustion Enthalpies

1.3

1. $3 \cdot 1$

1. 3.2

1. $3 \cdot 3$

1. $3 \cdot 4$

1. 3.5
Heating Value of Natural Gas Components

Enthalpy of Combustion

St andard Enthalpy of Combustion a

Real Gas Enthalpy of Combustion

Total Uncertainty Estimates for Combustion Enthalpy
1. $1-1$

$1.1-4$ 





\section{LNG MEASUREMENT \\ A User's Manual for Custody Transfer}

\subsection{BASE PHYSICAL PROPERTIES}

\subsection{The International System of Units(SI).}

In 1948 the 9 th General Conference on Weights and Measures (CGPM), by means of Resolution 6 , instructed the International Committee for Weights and Measures (CIPM) "to make recommendations for a single practical system of units of measurement, suitable for adoption by all countries adhering to the Meter Convention". This same CGPM gave general principles for unit symbols and a list of units with special names. Subsequent CGPM of 1954 and 1960 established base, derived and supplementary units for this "practical system" and adopted the name International system of units with the international abbreviation SI.

The United States holds a place on these international bodies by virtue of its adherence to the Treaty of the Meter, slgned in 1875. The National Bureau of Standards (NBS) acts as the official U.S. representative to the various International bodies formed by the treaty. NBS, in light of the Metric Conversion Act of 1975, recommends the use of metric units except in contexts where the exclusive use of metric units would needlessly confuse the intended audience. In these cases, the dual use of metric and customary units will serve the two purposes of communicating the contents and also familiarizing the reader with the SI system.

In the following sections on the SI system, reference is made to the original work published by the International Bureau of Weights and Measures [44], and NBS Special Publication 330 [22], which is the English translation published independently by NBS and Her Majesty's Stationery Office, UK. In addition, a number of sections and tables have been taken verbatim from Guidelines for Use of the Modernized Metric System [72], which are the NBS recommendations on the use of the SI system of units. These tables include entries which may be beyond the immediate scope of this LNG manual but are included for completeness and general interest.

The Metric System: SI The SI is constructed from seven base units for independent quantities plus two supplementary units for plane angle and solid angle. (See table 1.1.1). Units for all other quantities are derived from these nine units. In table 1.1.2 are listed 19 SI derived units with special names. These units are derived from the base and supplementary units in a coherent manner, which means they are expressed as products and quotients of the nine base and supplementary units without numerical factors. All other SI derived units, such as those in tables 1.1.3 and 1.1.4, are similarly derived in a coherent manner from the 28 base, supplementary, and special-name SI units. For use with the SI units, there is a set of 16 prefixes (see table 1.1.5) to form multiples and submultiples of these units. For mass, the prefixes are to be applied to the gram instead of to the SI base unit, the kilogram.

\subsubsection{Units Acceptable for Use with SI}

Certain units which are not part of the SI are used so widely that it is impractical to abandon them. The units that are accepted by NBS for continued used with the International system are 11 sted 1 n table 1.1.6. In those cases where their usage is already well established, the International Commltee for Welghts and Measures (CIPM) also has authorized, for a limited time, the use of the common unlts shown with an asterisk in table 1.1.7.

Table 1.1.1 SI Base and Supplementary Units

\begin{tabular}{|c|l|l|l|}
\hline & Quantity & $\begin{array}{l}\text { Unit } \\
\text { Name }\end{array}$ & $\begin{array}{c}\text { Unit } \\
\text { Symbol }\end{array}$ \\
\hline \hline \multirow{2}{*}{ SI Base Units } & length & meter & $\mathrm{m}$ \\
& mass & kilogram & $\mathrm{kg}$ \\
& time & second & $\mathrm{s}$ \\
& electric current & ampere & $\mathrm{A}$ \\
& thermodynamic temperature & kelvin & $\mathrm{K}$ \\
& amount of substance & mole & mol \\
& luminous intensity & candela & $\mathrm{cd}$ \\
& plane angle & radian & rad \\
& solid angle & steradian & sr \\
\hline
\end{tabular}


Table 1.1.2 SI Derived Units with Special Names

\begin{tabular}{|c|c|c|c|c|}
\hline \multirow[b]{2}{*}{ Quantity } & \multirow[b]{2}{*}{ Name } & \multicolumn{3}{|c|}{ SI Unit } \\
\hline & & Symbol & $\begin{array}{l}\text { Expression } \\
\text { in terms } \\
\text { of other } \\
\text { units }\end{array}$ & $\begin{array}{l}\text { Expression } \\
\text { in terms } \\
\text { of SI base } \\
\text { units }\end{array}$ \\
\hline frequency & hertz & $\mathrm{Hz}$ & $\ldots$ & $s^{-1}$ \\
\hline force & newton & $\mathrm{N}$ & - & $\mathrm{m} \cdot \mathrm{kg} \cdot \mathrm{s}^{-2}$ \\
\hline power, radiant flux & watt & W & $\mathrm{J} / \mathrm{s}$ & $\mathrm{m}^{2} \cdot \mathrm{kg} \cdot \mathrm{s}^{-3}$ \\
\hline $\begin{array}{l}\text { quantity of electricity, electric charge } \\
\text { electrical potential, electromotive force, }\end{array}$ & coulomb & C & $A \cdot S$ & $S \cdot A$ \\
\hline potential difference, & volt & $\mathrm{V}$ & W/A & $\mathrm{m}^{2} \cdot \mathrm{kg} \cdot \mathrm{s}^{-3} \cdot \mathrm{s}^{-1}$ \\
\hline capacitance & farad & $\mathrm{F}$ & $\mathrm{C} / \mathrm{V}$ & $\mathrm{m}^{-2} \cdot \mathrm{kg}^{-1} \mathrm{~s}^{4} \cdot \mathrm{A}^{2}$ \\
\hline electric resistance & ohm & $\Omega$ & $\mathrm{V} / \mathrm{A}$ & $\mathrm{m}^{2} \cdot \mathrm{kg} \cdot \mathrm{s}^{-3} \cdot \mathrm{A}^{-2}$ \\
\hline Celsius temperature & degree Celsius & $\mathrm{C}^{0}$ & -- & $\mathrm{K}$ \\
\hline luminous flux & lumen & $\operatorname{lm}$ & - & $c d \cdot s r$ \\
\hline illuminance & lux & $1 x$ & $1 \mathrm{~m} / \mathrm{m}^{2}$ & $m^{-2} \cdot c d \cdot s r$ \\
\hline
\end{tabular}

Table 1.1.3 Some SI Derived Units in Terms of Base Units

\begin{tabular}{|l|l|l|}
\hline \multicolumn{1}{|c|}{ Quantity } & SI Unit & \multicolumn{1}{c|}{$\begin{array}{l}\text { Unit } \\
\text { Symbol }\end{array}$} \\
\hline \hline area & square meter & $\mathrm{m}^{2}$ \\
volume & cubic meter & $\mathrm{m}^{3}$ \\
speed, velocity & meter per second & $\mathrm{m} / \mathrm{s}$ \\
acceleration & meter per second squared & $\mathrm{m} / \mathrm{s}^{2}$ \\
wave number & per meter & $\mathrm{m}^{-1}$ \\
density, mass density & kilogram per cubic meter & $\mathrm{kg} / \mathrm{m}^{3}$ \\
current density & ampere per square meter & $\mathrm{A} / \mathrm{m}^{2}$ \\
magnetic field strength & ampere per meter & $\mathrm{A} / \mathrm{m}^{3}$ \\
concentration & mol per cubic meter & $\mathrm{mol} / \mathrm{m}^{3}$ \\
specific volume & cubic meter per kilogram & $\mathrm{m} / \mathrm{kg}^{2}$ \\
luminance & candela per square meter & $\mathrm{cd} / \mathrm{m}^{2}$ \\
\hline
\end{tabular}


Table 1.1.4 Some SI Derived Units Expressed by Means of Special Names

\begin{tabular}{|c|c|c|c|}
\hline & \multicolumn{3}{|c|}{ SI Unit } \\
\hline Quantity & Name & Symbol & $\begin{array}{l}\text { Expression } \\
\text { in terms of } \\
\text { SI Base Units }\end{array}$ \\
\hline $\begin{array}{l}\text { dynamic viscosity } \\
\text { moment of force } \\
\text { surface tension } \\
\text { power density, irradiance } \\
\text { heat flux density } \\
\text { heat capacity, entropy } \\
\text { specific heat capacity, } \\
\text { specific entropy } \\
\text { specific energy } \\
\text { thermal conductivity } \\
\text { energy density } \\
\text { electric field strength } \\
\text { electric charge density } \\
\text { electric flux density } \\
\text { permittivity } \\
\text { permeability } \\
\text { molar energy } \\
\text { molar entropy, } \\
\text { molar heat capacity }\end{array}$ & $\begin{array}{l}\text { pascal second } \\
\text { newton meter } \\
\text { newton per meter } \\
\text { watt per square meter } \\
\text { joule per kelvin } \\
\text { joule per kilogram/kelvin } \\
\text { joule per kilogram } \\
\text { watt per meter kelvin } \\
\text { joule per cubic meter } \\
\text { volt per meter } \\
\text { coulomb per cubic meter } \\
\text { coulomb per square meter } \\
\text { farad per meter } \\
\text { henry per meter } \\
\text { joule per mole } \\
\text { joule per mole kelvin }\end{array}$ & $\begin{array}{l}\mathrm{Pa} \cdot \mathrm{s} \\
\mathrm{N} \cdot \mathrm{m} \\
\mathrm{N} / \mathrm{m} \mathrm{kg} \cdot \mathrm{s}^{-2} \\
\mathrm{~W} / \mathrm{m}^{2} \\
\mathrm{~J} / \mathrm{K} \\
\mathrm{J} /(\mathrm{kg} \cdot \mathrm{K}) \\
\mathrm{J} / \mathrm{kg} \\
\mathrm{W} /(\mathrm{m} \cdot \mathrm{K}) \\
\mathrm{J} / \mathrm{m}^{3} \\
\mathrm{~V} / \mathrm{m}^{3} \\
\mathrm{C} / \mathrm{m}^{3} \\
\mathrm{C} / \mathrm{m}^{2} \\
\mathrm{~F} / \mathrm{m} \\
\mathrm{H} / \mathrm{m} \\
\mathrm{J} / \mathrm{mol}^{2} \\
\mathrm{~J} /(\mathrm{mol} \cdot \mathrm{K})\end{array}$ & $\begin{array}{l}\mathrm{m}^{-1} \cdot \mathrm{kg} \cdot \mathrm{s}^{-1} \\
\mathrm{~m}^{2} \cdot \mathrm{kg} \cdot \mathrm{s}^{-2} \\
\mathrm{~kg} \cdot \mathrm{s}^{-3} \\
\mathrm{~m}^{2} \cdot \mathrm{kg} \cdot \mathrm{s}^{-2} \cdot \mathrm{K}^{-1} \\
\mathrm{~m}^{2} \cdot \mathrm{s}^{-2} \cdot \mathrm{K}^{-1} \\
\mathrm{~m}^{2} \cdot \mathrm{s}^{-2} \\
\mathrm{~m} \cdot \mathrm{kg} \cdot \mathrm{s}^{-3} \cdot \mathrm{K}^{-1} \\
\mathrm{~m}^{-1} \cdot \mathrm{kg} \cdot \mathrm{s}^{-2} \\
\mathrm{~m} \cdot \mathrm{kg} \cdot \mathrm{s}^{-3} \cdot \mathrm{A}^{-1} \\
\mathrm{~m}^{-3} \cdot \mathrm{s} \cdot \mathrm{A} \\
\mathrm{m}^{-2} \cdot \mathrm{s} \cdot \mathrm{A} \\
\mathrm{m}^{-3} \cdot \mathrm{kg} \mathrm{g}^{-1} \cdot \mathrm{s}^{4} \cdot \mathrm{A}^{2} \\
\mathrm{~m} \cdot \mathrm{kg} \cdot \mathrm{s}^{-2} \cdot \mathrm{A}^{-2} \\
\mathrm{~m}^{2} \cdot \mathrm{kg} \cdot \mathrm{s}^{-2} \cdot \mathrm{mol}^{-1} \\
\mathrm{~m}^{2} \cdot \mathrm{kg} \cdot \mathrm{s}^{-2} \cdot \mathrm{K}^{-1} \cdot \mathrm{mol}^{-1}\end{array}$ \\
\hline
\end{tabular}

Table 1.1.5 SI Prefixes

\begin{tabular}{|c|l|l|l|l|l|}
\hline factor & prefix & symbol & factor & prefix & symbol \\
\hline \hline $10^{18}$ & exa & $\mathrm{E}$ & $10^{-1}$ & deci & $\mathrm{d}$ \\
$10^{15}$ & peta & $\mathrm{P}$ & $10^{-2}$ & centi & $\mathrm{c}$ \\
$10^{12}$ & tera & $\mathrm{T}$ & $10^{-3}$ & milli & $\mathrm{m}$ \\
$10^{9}$ & giga & $\mathrm{G}$ & $10^{-6}$ & micro & $\mathrm{\mu}$ \\
$10^{6}$ & mega & $\mathrm{M}$ & $10^{-9}$ & nano & $\mathrm{n}$ \\
$10^{3}$ & kilo & $\mathrm{k}$ & $10^{-12}$ & pico & $\mathrm{p}$ \\
$10^{2}$ & hecto & $\mathrm{h}$ & $10^{-15}$ & femto & $\mathrm{f}$ \\
$10^{1}$ & deka & $\mathrm{da}$ & $10^{-18}$ & atto & $\mathrm{a}$ \\
\hline
\end{tabular}

Table 1.1.6 Units in Use with the International System

\begin{tabular}{|c|c|c|}
\hline Name & Symbol & Value in SI Unit \\
\hline minute & $\min$ & $1 \mathrm{~min}=60 \mathrm{~s}$ \\
\hline hour & $\mathrm{h}$ & $1 \mathrm{~h}=60 \mathrm{~min}=3600 \mathrm{~s}$ \\
\hline day & $d$ & $=24 \mathrm{~h}=86400 \mathrm{~s}$ \\
\hline degree & 0 & $=(\pi / 180) \mathrm{rad}$ \\
\hline minute & ' & $=(1 / 60)^{0}=(\pi / 108000) \mathrm{rad}$ \\
\hline second & $"$ & $=(1 / 60)^{\prime}=(\pi / 648000) \mathrm{rad}$ \\
\hline $\begin{array}{l}\text { liter } \\
\text { metric ton }\end{array}$ & $\begin{array}{l}\mathrm{L} \\
\mathrm{t}\end{array}$ & $\begin{array}{l}1 \mathrm{~L}=1 \mathrm{dm}^{3}=10^{-3} \mathrm{~m}^{3} \\
1 \mathrm{t}=10^{3} \mathrm{~kg}\end{array}$ \\
\hline hectare & ha & $=10^{4} \mathrm{~m}^{2}$ \\
\hline
\end{tabular}




\section{Table 1.1.7 Examples of Conversion Factors from Non-SI Units to SI Units}

\begin{tabular}{|c|c|c|c|}
\hline $\begin{array}{l}\text { Physical } \\
\text { Quantity }\end{array}$ & Name of Unit & $\begin{array}{l}\text { Symbol } \\
\text { for } \\
\text { Unit }\end{array}$ & $\begin{array}{l}\text { Definition in } \\
\text { SI Units }\end{array}$ \\
\hline $\begin{array}{l}\text { length } \\
\text { length } \\
\text { length } \\
\text { velocity } \\
\text { cross section } \\
\text { acceleration } \\
\text { mass } \\
\text { force } \\
\text { pressure } \\
\text { pressure } \\
\text { pressure } \\
\text { pressure } \\
\text { stress } \\
\text { energy } \\
\text { energy } \\
\text { energy }\end{array}$ & $\begin{array}{l}\text { inch } \\
\text { nautical mile* } \\
\text { angstrom } \\
\text { knot* }^{*} \\
\text { barn* } \\
\text { gal } \\
\text { pound (avoirdupois) } \\
\text { kilogram-force } \\
\text { conventional millimeter } \\
\text { of mercury } \\
\text { atmosphere } \\
\text { torr } \\
\text { bar } \\
\text { pound-force per sq.in } \\
\text { British thermal unit } \\
\text { (Int. Table) } \\
\text { kilowatt hour } \\
\text { calorie (thermochemical) }\end{array}$ & $\begin{array}{l}\text { in } \\
\mathrm{nmi} \\
\text { A } \\
\mathrm{kn} \\
\mathrm{b} \\
\mathrm{Gal} \\
\mathrm{lb} \\
\mathrm{kgf} \\
\mathrm{mmHg} \\
\text { atm } \\
\text { Torr } \\
\text { bar } \\
\text { lbf / in } \\
\\
\text { Btu } \\
\mathrm{kWh} \\
\mathrm{cal}\end{array}$ & $\begin{array}{l}2.54 \mathrm{x} 10^{2} \\
1852 \mathrm{~m} \\
10^{-10} \mathrm{~m} \\
(1852 / 3600) \mathrm{m} / \mathrm{s} \\
10^{-28} \mathrm{~m}^{2} \\
10^{-2} \mathrm{~m} / \mathrm{s}^{2} \\
0.45359237 \mathrm{~kg} \\
9.80665 \mathrm{~N} \\
13.5951 \mathrm{x} 9.80665 \mathrm{~N} \cdot \mathrm{m}^{-2} \\
101325 \mathrm{~N} \cdot \mathrm{m}^{-2} \\
(101325 / 760) \mathrm{N} \cdot \mathrm{m}^{-2} \\
10^{5} \mathrm{~Pa} \\
6894.757 \mathrm{~Pa} \\
1055.056 \mathrm{~J} \\
3.6 \times 10^{6} \mathrm{~J} \\
4.184 \mathrm{~J}\end{array}$ \\
\hline
\end{tabular}

The short names for compound units (such as "coulomb" for "ampere second" and "pascal" for "newton per square meter") exist for convenience, and either form is correct (see table 1.1.2). For example, communication sometimes is facilitated if the author expresses magnetic flux in the compound term volt seconds (instead of using the synonym, webers) because of the descriptive value implicit in the compound phrase.

Special Considerations The kelvin $(K)$ is the SI base unit of temperature; this unit is properly used for expressing temperature and temperature intervals; however, wide use is also made of the degree Celsius (C) for expressing temperature and temperature intervals. The Celsius scale (formerly called centigrade) is related directly to thermodynamic temperature (kelvins) as follows;

The temperature interval one degree celsius equals one kelvin exactly.

Celsius temperature $(t)$ is related to thermodynamic temperature $(T)$ by the equation:

$$
t=T-T_{0}
$$

where $\mathrm{T}_{0}=273.15 \mathrm{~K}$ by definition.

Over the years the term "weight" has been used to designate two quantities: mass and force. The word weight denotes a quantity of the same nature as a force; the weight of a body is the product of its mass and acceleration caused by gravity; in particular, the standard weight of a body is the product of its mass and standard acceleration caused by gravity. NBS supports the recommendation in the American National Standard for Metric Practice, 2219.1-1976, that the term weight should be avoided in technical publications except under circumstances in which its meaning is completely clear.

It is also recommended that the terms atomic weight and molecular weight be replaced by relative atomic mass and relative molecular mass in accordance with established international practice.

\subsubsection{Descriptive and Essential Data}

Descriptive data describe arrangements, environments, noncritical dimensions and shapes of apparatus, and similar measurements not affecting calculations or results. Such data should be expressed in SI units unless this makes the expression excessively complicated. For example, commercial gage designations, commonly used items identified by nominal dimension, or other commercial nomenclatures (such as drill sizes, or standards for weights and measures) expressed in customary units are acceptable. 
Essential data express or interpret the quantitative results being reported. All such data shall be expressed solely in SI units except in those fields where (a) the sole use of SI units would create a serious impediment to communications, or (b) SI units have not been specified. Exceptions may also occur when dealing with commercial devices, standards, or units having some legal definition, such as commercial weights and measures. Even in such instances, SI units should be used when practical and meaningful; for example, this may be done by adding non-SI units in parentheses after SI units. In tables, SI and customary units may be shown in parallel columns. If coordinate markings in non-SI units are included in graphs, they should be displayed on the top and right-hand sides of the figure. 




\subsection{Selected Pure Component Physical Properties}

Measurements of value for LNG are influenced greatly by three very important factors. These factors relate primarily to physical properties of the commodity. The first factor is the density of the liquid relative to the density of the gas at atmospheric conditions. An increase in density of 600 to 650 times can be realized depending on composition. The second factor is that the temperature of this high density liquid (just less than half the density of water) is cold, a cryogenic fluid at or near $110 \mathrm{~K}$. To store the liquid for transport or peak shaving in a most efficient manner, the insulated container should be quite large with a low surface-to-volume ratio such as a sphere or a right circular cylinder in order to minimize the surface exposed to ambient environment. There is significant economic value in large LNG containers as small containers lose proportionately larger percentages of gas through vaporization, and this gas must be disposed of or reliquefied.

Finally, most LNG is a mixture of relatively light hydrocarbons with nitrogen as an inert impurity. This mixture is non-ideal in the sense that the properties cannot be predicted using the Ideal Gas Law $(P V=n R T)$. The non-ideality can be as large as three percent, which will influence the value calculation of quantity of LNG. Empirical relationships for fixed compositions can serve, for example, for calculation of liquid density as a function of temperature, pressure and composition for a single liquefaction operation, but on an international basis where mixture compositions at liquefaction and during storage can change greatly, broadly based mathematical models with theoretical background are necessary for calculation of accurate physical and derived thermophysical properties of the pure fluids and the mixtures.

Mathematical models use, as a fundamental quantity, the relative molecular mass of both the constituent and the mixture. Values of relative molecular and atomic mass vary somewhat depending on the source of data and other factors $[12,13,37,38,40,41]$ and it was believed necessary for this work to define relative atomic and molecular masses based on the most recent scientific information. This has been done in the following sections and includes references and estimates of error [12a,b]. In addition, sources for calculating pure fluid physical property data at selected reference conditions are given along with estimates of error.

\subsubsection{Relative Atomic and Molecular Masses}

Relative atomic mass is the dimensionless relative mass of an atom or of a mole of atoms of normal isotopic composition based on a scale for which the isotope carbon 12 has a relative mass of 12.0000 exactly. Naturally occurring elements and compounds are composed of mixtures of the isotopes of the individual elements, and this mixture composition may vary with location on the Earth's surface.

The assessment of the relative abundance of isotopic elements is a continuing effort to characterize accurately the relative atomic and molecular masses of such naturally occurring compounds and mixtures as found in LNG. Periodically, the scientific literature is examined and data evaluated to produce a table of recommended isotopic abundances for the elements and atomic masses calculated from these abundances [40]. An estimate of uncertainty for each value is also given. The variation of the values over the years reflects the improvement in knowledge of relative isotopic abundance and the ability of the scientist to make more accurate and precise measurements.

Table 1.2.1 lists the internationally accepted values of the relative atomic mass for the elements of importance to natural gas adopted in this manual (IUPAC Atomic Weights 1981) [12a,b]. In addition. the estimates of uncertainty are presented which alone or in combination can provide error $11 \mathrm{~m}$ its to calculations of quantity and quality.

Table 1.2 .1

Relative Atomic Masses of Elements of LNG [12a,b]

\begin{tabular}{|l|c|c|c|}
\hline Substance & Symbol & Atomic Mass & $\begin{array}{c}\text { Estimated } \\
\text { Uncertainty } \\
\times\end{array}$ \\
\hline \hline Hydrogen & H & 1.00794 & 0.7 \\
Carbon & $\mathrm{C}$ & 12.011 & 10. \\
Nitrogen & $\mathrm{N}$ & 14.0067 & 1. \\
Oxygen & $\mathrm{O}$ & 15.9994 & 3. \\
\hline
\end{tabular}


Calculation of the values of relative molecular masses for pure components of natural gas follow the simple combination of the relative atomic masses. For example; the relative molecular mass of methane would be $12.011+(4 \times 1.00794)=16.0428$ or 16.043 rounded to five significant digits.

Combination of individual estimated uncertainties in relative atomic masses from table 1.2 .1 are calculated by quadrature (square root of the sum of the squares) as recommended by Armstrong and Jobe $[12 a, b]$. The uncertainty of the relative molecular mass of methane will then be the square root of the sum of the uncertainty in the value of atomic mass for carbon (0.001) squared plus four times the uncertainty in the values of atomic mass for hydrogen $(0.00007)$ squared. Results of this calculation for important components of natural gas rounded to five significant figures) are given in table 1.2.2.

Table 1.2 .2

Relative Molecular Mass of Selected Substances [12a,b]

\begin{tabular}{|c|c|c|c|c|}
\hline Hydrocarbon & Formula & $\begin{array}{l}\text { Relative } \\
\text { Molecular } \\
\text { Mass }\end{array}$ & $\begin{array}{c}\text { Uncertainty } \\
\times 10^{4}\end{array}$ & $\begin{array}{c}\text { Relative } \\
\text { Uncertainty } \\
\times 10^{4} \\
\end{array}$ \\
\hline $\begin{array}{l}\text { methane } \\
\text { ethane } \\
\text { propane } \\
\text { n-butane } \\
\text { iso-butane } \\
\text { n-pentane } \\
\text { iso-pentane } \\
\text { neo-pentane } \\
\text { n-hexane } \\
\text { 2-methylpentane } \\
\text { 3-methylpentane } \\
\text { 2,2-dimethylbutane } \\
\text { 2,3-dimethylbutane }\end{array}$ & $\begin{array}{l}\mathrm{CH}_{4} \\
\mathrm{C}_{2} \mathrm{H}_{6} \\
\mathrm{C}_{3} \mathrm{H}_{8} \\
\mathrm{C}_{4} \mathrm{H}_{10} \\
\mathrm{C}_{4} \mathrm{H}_{10} \\
\mathrm{C}_{5} \mathrm{H}_{12} \\
\mathrm{C}_{5} \mathrm{H}_{12} \\
\mathrm{C}_{5} \mathrm{H}_{12} \\
\mathrm{C}_{6} \mathrm{H}_{12} \\
\mathrm{C}_{6} \mathrm{H}_{14} \\
\mathrm{C}_{6} \mathrm{H}_{14} \\
\mathrm{C}_{6} \mathrm{H}_{14} \\
\mathrm{C}_{6} \mathrm{H}_{14}\end{array}$ & $\begin{array}{l}16.043 \\
30.070 \\
44.097 \\
58.123 \\
58.123 \\
72.150 \\
72.150 \\
72.150 \\
86.177 \\
86.177 \\
86.177 \\
86.177 \\
86.177\end{array}$ & $\begin{array}{l}10 \\
20 \\
31 \\
41 \\
41 \\
51 \\
51 \\
51 \\
61 \\
61 \\
61 \\
61 \\
61\end{array}$ & $\begin{array}{l}0.62 \\
0.67 \\
0.70 \\
0.71 \\
0.71 \\
0.71 \\
0.71 \\
0.71 \\
0.71 \\
0.71 \\
0.71 \\
0.71 \\
0.71\end{array}$ \\
\hline \multicolumn{5}{|l|}{ Non-hydrocarbon } \\
\hline $\begin{array}{l}\text { carbon dioxide } \\
\text { nitrogen } \\
\text { oxygen } \\
\text { water }\end{array}$ & $\begin{array}{l}\mathrm{CO}_{2} \\
\mathrm{~N}_{2} \\
\mathrm{O}_{2} \\
\mathrm{H}_{2} \mathrm{O}\end{array}$ & $\begin{array}{l}44.0098 \\
28.0134 \\
31.9988 \\
18.0153\end{array}$ & $\begin{array}{l}12 . \\
1.4 \\
4.2 \\
3.3\end{array}$ & $\begin{array}{l}0.15 \\
0.05 \\
0.13 \\
0.18\end{array}$ \\
\hline
\end{tabular}

The values in table 1.2 .2 based on those of table 1.2 .1 represent a recent assessment by the Commission on Atomic Weights of the values from the relevant literature. Recent historical changes and sources of this assessment are given in Armstrong and Jobe $[12 a, b]$.

\subsubsection{Data Reference States}

Definitions of reference conditions for measurement of fluids are currently in a state of flux. A selection has been made based on Armstrong and Jobe [12a,b] and other industrial requirements. The reference conditions of pressure and temperature selected for this manual are shown in table 1.2 .3 . The recent recommendation of the International Union for Pure and Applied Chemistry (IUPAC) that $100.000 \mathrm{kPa}$ be adopted as a new standard pressure for thermodynamic standard state [39] is not followed here as its use will be quite limited until some time in the future. The previously adopted IUPAC reference condition [42] is used in this manual and is listed and referenced in the table.

Table 1.2.3 Reference Conditions of Measurement

\begin{tabular}{|l|c|c|c|}
\cline { 2 - 4 } \multicolumn{1}{c|}{} & $\begin{array}{c}\text { Temperature } \\
\mathrm{K}\end{array}$ & $\begin{array}{c}\text { Pressure } \\
\mathrm{kPa}\end{array}$ & $\begin{array}{c}\text { Reference } \\
\text { Source }\end{array}$ \\
\hline IUPAC & 298.15 & 101.325 & {$[42]$} \\
ASTM-ANSI-API & 288.71 & 101.560 & {$[3,7,45]$} \\
(U.S. Customary) & & 101.325 & {$[45]$} \\
ASTM-ANSI-ISO/metric & 288.15 & 101.325 & {$[47]$} \\
JIS/also STP & 273.15 & \multicolumn{2}{c}{} \\
\hline
\end{tabular}


The reference state of the Japanese Industrial Standard (JIS) [47] is identical to the reference state referred to traditionally as Standard Temperature and Pressure (STP).

In the following sections, pure fluid data are given for the components of LNG with relative molecular masses through the hexanes. Nitrogen is included as it is the most important impurity found in LNG. Sources of data and state equations are included. In general, reference data at standard conditions are found from the virial equation form to be consistent with later developed combustion enthalpy data while broad ranges of pressure and temperature are provided when available by references to the more complex mathematical forms.

The reference data values for each fluid can be generated at temperatures and pressures other than those shown is table 1.2.3. This can be done by reference to section 1.3 .4 on Real Gas Enthalpy of Combustion. Equations (6), (7), (9), (10) of section 1.3 and the equation constants of table 1.3 .4 along with the descriptive text should allow calculation of the second virial coefficient and the real gas molar volume within the stated temperature range and at moderate pressures near one atmosphere.

As an example, the density of methane at the reference condition of $273.15 \mathrm{~K}$ and $101.325 \mathrm{kPa}$ (JIS/STP) can be found by combining equation (10) of section 1.3 , data from table 1.3 .3 and the value of the gas constant $\mathrm{R}$ as defined from Armstrong and Jobe $[12 \mathrm{a}, \mathrm{b}]$,

$$
\text { Gas constant } R=8.31441(26) \mathrm{J} / \mathrm{mol} \mathrm{K}
$$

where the number in parentheses indicates the uncertainty in the last two figures. The actual density may be calculated from:

$$
\text { Reference density }=(1000)\left(M_{W}\right) /\left[( V _ { m } ^ { i d } ) \left(0.5+0.5\left(1+4 B(T) /\left(V_{m}^{i d}\right)^{1 / 2}\right] \mathrm{kg} / \mathrm{m}^{3}\right.\right.
$$

where $V_{m}^{i d}=R T / P, M_{W}$ is the relative molecular mass and $B(T)$ is the second virial coefficient.

Temperature (T) and pressure (P) are defined and can be considered exact. For methane; molecular mass $M_{W}=16.043$ from table 1.2.2, the gas constant $R$ is defined from equation ( 1 ) and the second $v i r i a l$ coefficient $B(T)$ is found from table 1.3 .4 .

$$
\begin{aligned}
\text { Reference density } & =\left[16.043 /\left[(22414)\left(0.5+0.5(1+4(-54.4) / 22414)^{1 / 2}\right] 1000\right.\right. \\
& =0.71751 \mathrm{~kg} / \mathrm{m}^{3}
\end{aligned}
$$

The uncertainty in the value of density calculated from equation (2) can be found from the stated uncertainties of the molecular mass (table 1.2.2), gas constant $R$ (equation 1 ) and the second virial coefficient (table 1.3.4).

Continuing the above example; the uncertainty in molecular mass of methane is $0.001 \mathrm{~g} / \mathrm{mol}$ and from equation (2) amounts to an error in density of $0.000045 \mathrm{~kg} / \mathrm{m}^{3}$. The uncertainty in gas constant $\mathrm{R}$ is $0.00026 \mathrm{~J} / \mathrm{mol} \mathrm{K}$ which amounts to an error in density of $0.000023 \mathrm{~kg} / \mathrm{m}^{3}$. Finally, the uncertainty in the value of the second virial coefficient $\mathrm{B}$ at $273.15 \mathrm{~K}$ is $1 \mathrm{~cm} 3 / \mathrm{mol}$ and amounts to an error in density of $0.000032 \mathrm{~kg} / \mathrm{m}^{3}$. Since these errors are independent values, they may be summed in quadrature (square root of the sum of the squares of the individual values). The density value for methane at $273.15 \mathrm{~K}$ and $101.325 \mathrm{kPa}$ calculated from equation (3) is $0.71751 \mathrm{~kg} / \mathrm{m} 3 \mathrm{and} \mathrm{has}$ an uncertainty of $0.00006 \mathrm{~kg} / \mathrm{m}^{3}$ or 0.01 percent. These equations and procedures are used to generate all of the reference state densities and estimated errors for the following pure substances.

\subsubsection{Pure component Properties}

The term "liquefied natural gas" or LNG refers by common practice to a liquid phase non-ideal m $1 x t u r e$ of low molecular mass hydrocarbons with nitrogen as an inert impurity. The major component of L:IG $1 \mathrm{~s}$ methane, which sets this hydrocarbon mixture apart from others such as "liquefled petroleum gas" or LPG which is predominantly propane or butane.

LNG is manufactured in the sense that the liquid mixture does not occur naturally as does LPG. Although the methane content of LNG is generally greater than eighty percent, the mixture composit $10 n$ is variable and contains minor components of ethane, propane, iso-butane and normal butane w! th nitrogen at less that two percent. Also present in some LNG mixtures at very low rractlong aro heavier hydrocarbons such as the pentanes and hexanes. These constituents have only a small 1 nfluence on the density of the LNG, but, because of high relative heating value, must be cons!dered In establishing the total value of the mixture. 
The single most attractive characteristic of LNG is the increased density over that of the natural gas at atmospheric pressure and temperature. To achieve and maintain this high density of about 600 times normal density, the liquid is maintained at low pressures and temperatures in insulated containers both for storage and shipment. Boiling points for the LNG mixtures do not normally exceed $150 \mathrm{~K}$ and primary containment vessels are especially designed to limit heat input (see section 2.5 ).

Commerce in LNG is conducted by establishing the value of the LNG at the point of sale. The value, of course, depends on the end point use of the fluid, either as a primary heat source or as a feed stock in a chemical process. If the LNG is purchased as a heat source, the total heating value must be determined. If the LNG is purchased as a feed stock, then the total mass and constituent fractions must be established. No single direct measurement device is currently available to accomplish these objectives. In either case, the value of the LNG is established through a series of measurements which are then combined to give the desired result. Each individual measurement ultimately requires a precise knowledge of fluid properties.

The prediction of density or other derived properties of the non-ideal mixtures of the light hydrocarbons with nitrogen has been a long term research effort conducted by research groups in the U.S. and abroad: One approach has been to determine the thermophysical properties of the pure components of the mixture and then to establish the value of the interaction quantities which influence the mixture properties. A mathematical model of the mixture can then be generated, tested and used to provide the necessary derived thermophysical properties. Several mathematical models or equations of state have been used to derive properties of the pure fluid state and of the mixtures. Simple models such as the virial form [21] with one or two coefficients are used to develop a theoretical basis for the gas phase properties of pure fluids and mixtures. This form gives best results at moderate pressures and temperatures and can be used to predict properties where only a minimum amount of experimental data are available. With larger quantities of experimental data, more powerful multi-constant mathematical models may be used if large main frame computers are available.

It is not the purpose of this manual to included a detailed discussion of the above fluid properties modeling process. A description and examples of the use of several mixture models will be found under section 2.4 of this manual. The cited references should be consulted for more detailed information on the development of the thermophysical properties of the LNG pure fluids and mixtures.

In order to assure greatest accuracy and precision in assessing the value of LNG, it is necessary to consider the real gas properties as opposed to ideal gas properties which are an approximation and may introduce significant errors. Mathematical models which represent existing experimental fluid property data become increasingly complex as the range of pressure and temperature is extended to include liquefaction, mixture and storage processes. The major pure fluid components of LNG such as methane, ethane and propane have been examined by experimentalists in great detail in respect to thermophysical properties (pressure, temperature, density), thermodynamic properties (enthalpy, entropy, specific heats, etc.) and transport properties (thermal conductivity, viscosity, etc.) properties. State equations and tabular data are available as will be shown in the following sections. The more minor LNG components such as the butanes, pentanes and hexanes have received less attention by the experimentalists, and broadly based experimental property data are less available. This is also shown in the following sections. However, every effort has been made to include the most recent reliable values and references for all the components of LNG.

\subsubsection{Methane}

The primary reference for the thermophysical properties of methane is the work of Goodwin [24]. This work was the first in a series designed to provide broad range, internally consistent thermophysical properties data for pure hydrocarbon fluids and LNG mixtures. The derived properties tables have been designated an ASTM Standard [8]. Thermophysical properties of methane are tabulated at uniform temperatures from $90.68 \mathrm{~K}$ to $500 \mathrm{~K}$ along isobars to $70 \mathrm{MPa}(700 \mathrm{bar})$. A novel equation of state is employed for the first time, having origin on the vapor-liquid coexistence boundary. Tabulated data for each isobar include temperature, specific volume, density, internal energy, enthalpy, entropy, specific heats, velocity of sound and the pressure derivatives in respect to temperature and density. Computer program listings for the tabulations are provided. other treatments of these data are in the literature $[51,52]$. Other properties data for methane appear in the 1 iterature $[2,10,30,35,52,56,58,64,65,67,77]$. 
The estimated absolute error of the pressure- temperature- density data of Goodwin [22] is 0.1 percent except in the critical region. The absolute error of the derived thermodynamic functions is greater than that of the pressure- temperature- density data because of the first and second order derivatives and derivative differences used in calculating the derived functions. Enthalpy differences are estimated to be accurate within 2 percent except in the region of the critical point. Experimental, constant-volume specific heats have an estimated inaccuracy of 0.5 percent.

The primary reference for the gas density at reference conditions is Armstrong and Jobe [12a,b]. The reference density equation (2) is used to calculate the density at all reference conditions. The gas constant $\mathrm{R}$ is defined by equation (1). The molecular mass and uncertainty are found from table 1.2 .2 . and the second virial coefficient at reference temperatures with uncertainties are found from table 1.3.4. The variation of density uncertainty at different reference temperatures is not significant. The error in density at the reference conditions is estimated to be 0.01 percent.

Table 1.2.4 Methane

\begin{tabular}{|l|c|c|c|}
\hline Base Quantity & $\begin{array}{c}\text { Temperature } \\
\mathrm{K}\end{array}$ & $\begin{array}{c}\text { Pressure } \\
\mathrm{kPa}\end{array}$ & $\begin{array}{c}\text { Density } \\
\mathrm{kg} / \mathrm{m}\end{array}$ \\
\hline \hline Fixed Points [22] & 190.555 & 4598.83 & 163. \\
Critical Point & 111.63 \\
Normal Boiling Point & 90.680 & 101.325 \\
Triple Point & & 11.744 & 422.63 \\
Reference Conditions [12a,b] & & & 451.56 \\
IUPAC & 298.15 & 101.325 & 0.65689 \\
ASTM-ANSI-API & 288.71 & 101.560 & 0.68012 \\
(U.S. Customary) & 288.15 & 101.325 & 0.67986 \\
ISO-ASTM-ANSI/Metric & 273.15 & 101.325 & 0.71751 \\
JIS/also STP & & & \\
\hline
\end{tabular}

\section{2 .3 .2 Ethane}

Ethane, a valuable commodity as a by-product of petroleum production, is also a important constituent of most LNG. The primary thermophysical property source data is Goodwin, Roder and Straty [29]. In this work the thermophysical properties of ethane are tabulated at integral temperatures over the entire range of fluid states at temperatures from 90 to $600 \mathrm{~K}$ along isobars to $70 \mathrm{MPa}(700$ bar).

Isobaric tabulation includes temperature, density, internal energy, enthalpy, entropy, the specific heats and sound velocity. This source document incorporates many new physical properties measurements to obtain more accurate tables of thermodynamic functions and provides the first of such tables available for liquid ethane below its normal boiling temperature (184.55 K). 0ther properties data for ethane appear in the literature $[29,35,60,68,69,71,75]$.

Estimates of the uncertainties of the densities calculated from the state equation are generally less than 0.2 percent except near the critical point where the uncertainty may approach 2 percent. The uncertainty in derived properties of enthalpies is 4 percent in compressed liquid domalns at low temperature, increasing up to 10 percent very close to the critical point, and diminishing toward 2 percent at low densities and for all densities at temperatures well above critical. The specific heats are uncertain by 4 percent. The speeds of sound are accurate within 1 percent except for the saturated liquid, where deviations approach 10 percent near the critical point.

The primary reference for the gas density at reference conditions is Armstrong and Jobe [12a,b]. The reference density equation (2) is used to calculate the density at all reference conditions. The 8 as constant $R$ is defined by equation (1). The molecular mass and uncertainty are found from table 1.2 .2$. and the second virial coefficients at reference temperatures with uncertainties are found rrom table 1.3.4. The variation of density uncertainty at different reference temperatures is not significant. The error in density at the reference conditions is estimated to be 0.01 percent. 
Table 1.2 .5 Ethane

\begin{tabular}{|l|c|c|c|}
\hline Base Quantity & $\begin{array}{c}\text { Temperature } \\
\mathrm{K}\end{array}$ & $\begin{array}{c}\text { Pressure } \\
\mathrm{kPa}\end{array}$ & $\begin{array}{c}\text { Density } \\
\mathrm{kg} / \mathrm{m} 3\end{array}$ \\
\hline Fixed Points [29] & & & \\
Critical Point & 305.33 & 4871.4 & 204.5 \\
Normal Boiling Point & $\begin{array}{c}184.55 \\
\text { Triple Point }\end{array}$ & $\begin{array}{c}101.325 \\
0.001\end{array}$ & $\begin{array}{l}544.09 \\
\text { Reference Conditions [12a,b] }\end{array}$ \\
IUPAC & & & \\
ASTM-ANSI-API & 298.15 & 101.325 & \\
(U.S. Customary) & 288.71 & 101.560 & 1.2387 \\
ISO-ASTM-ANSI/Metric & 288.15 & 101.325 & 1.2833 \\
JIS/also STP & 273.15 & 101.325 & 1.2829 \\
\hline
\end{tabular}

\subsubsection{Propane}

The primary reference of Goodwin and Haynes [31] tabulates the thermophysical properties of propane at integral temperatures over the entire range of fluid states from 85 to $700 \mathrm{~K}$ along isobars to $70 \mathrm{MPa}$ by using a modified form of a nonanalytic equation of state. These tables, along with a table for the saturated liquid, include values for density, compressibility factor, internal energy, enthalpy, entropy, heat capacities, fugacity, sound velocity, dielectric constant, and isochore and isotherm derivatives. In addition to the equation of state, equations are presented for vapor pressures, orthobaric vapor and liquid densities, ideal gas properties virial coefficients, dielectric constants, heats of vaporization, melting pressures, and orthobaric liquid specific heats, enthalpies, and entropies. Coefficients were determined by a least squares fit of selected experimental data, including several new sets of data not included in previous propane correlations. Comparisons between experimental and calculated values are given, including those for sound velocities, heat capacities, $\mathrm{P}-\mathrm{V}-\mathrm{T}$ data, etc. Additional data on pure propane are available in the literature $[25,26,27,28,78]$.

Densities from the equation of state in most regions are within 0.2 percent of the experimental data except near the critical point where the uncertainty may approach 2 percent. Uncertainties in enthalpy differences along listed isobars agree in general with experimental specific heats to within 3 percent.

The primary reference for the gas density at reference conditions is Armstrong and Jobe [12a,b]. The reference density equation (2) is used to calculate the density at all reference conditions. The gas constant $\mathrm{R}$ is defined by equation (1). The molecular mass and uncertainty are found from table 1.2.2., and the second virial coefficient at reference temperatures with uncertainties are found from table 1.3.4. The error in density at the reference conditions is estimated to be 0.1 percent.

Table 1.2 .6 Propane

\begin{tabular}{|l|c|c|c|}
\hline Base Quantity & $\begin{array}{c}\text { Temperature } \\
\mathrm{K}\end{array}$ & $\begin{array}{c}\text { Pressure } \\
\mathrm{kPa}\end{array}$ & $\begin{array}{c}\text { Density } \\
\mathrm{kg} / \mathrm{m} 3\end{array}$ \\
\hline \hline Fixed Points [31] & & & \\
Critical Point & 369.8 & 4242.04 & 218.7 \\
Normal Boiling Point & 231.071 & 101.325 & 580.67 \\
Triple Point & 85.47 & $3 . \times 10^{-11}$ & 732.89 \\
\hline Reference Conditions [12a,b] & & & 1.8322 \\
IUPAC & 298.15 & 101.325 & 1.9006 \\
ASTM-ANSI-API & 288.71 & 101.560 & 1.9001 \\
(U.S. Customary) & 288.15 & 101.325 & 2.0131 \\
ISO-ASTM-ANSI/Metric & 273.15 & 101.325 & \\
JIS/also STP & & & \\
\hline
\end{tabular}




\section{2 .3 .4 n-Butane}

The primary source of data, Haynes and Goodwin [33], provides a modified version of the nonanalytic equation of state to generate thermophysical properties of n-butane from physical properties data and are tabulated at intergral temperatures from 135 to $700 \mathrm{~K}$ along isobars at pressures to 70 MPa. These isobar tables, along with a table for the saturated liquid, give values for densities, compressiblility factors, internal energies, enthalpies, entropies, heat capacities, fugacities, sound velocities, dielectric constants, and isochore and isotherm derivatives. Equations, whose coefficients are determined from a least squares fit of selected experimental data, are also presented for vapor pressures, orthobaric liquid and vapor densities, ideal gas properties, second virial coefficients, dielectric constants, heats of vaporization, melting pressure, and orthobaric liquid specific heats, enthalpies, and entropies. Comparisions between experimental and calculated values for all properties considered here are reported in detail. Additional data on n-butane are included in the references $[34,35]$.

The primary reference for the gas density at reference conditions is Armstrong and Jobe [12a,b]. The reference density equation (2) is used to calculate the density at all reference conditions. The gas constant $\mathrm{R}$ is defined by equation (1). The molecular mass and uncertainty are found from table 1.2 .2 , and the second virial coefficients at reference temperatures with uncertainties are found from table 1.3.4. The variation of density uncertainty at different reference temperatures is not significant. The error in density at the reference conditions is estimated to be 0.1 percent.

Table 1.2 .7 n-Butane

\begin{tabular}{|l|c|c|c|}
\hline Base Quantity & $\begin{array}{c}\text { Temperature } \\
\mathrm{K}\end{array}$ & $\begin{array}{c}\text { Pressure } \\
\mathrm{kPa}\end{array}$ & $\begin{array}{c}\text { Density } \\
\mathrm{kg} / \mathrm{m} 3\end{array}$ \\
\hline \hline Fixed Points [33] & & & 227.85 \\
Critical Point & 425.16 & 3796. & 601.09 \\
Normal Boiling Point & 272.638 & 101.325 & 735.27 \\
Triple Point & 134.86 & $6.74 \times 10^{-4}$ & \\
\hline Reference Conditions [12a,b] & & & 2.4504 \\
IUPAC & 298.15 & 101.325 & 2.5486 \\
ASTM-ANSI-API & 288.71 & 101.560 & 2.5481 \\
(U.S. Customary) & 288.15 & 101.325 & 2.7154 \\
ISO-ASTM-ANSI/Metric & 273.15 & 101.325 & \\
JIS/also STP & & & \\
\hline
\end{tabular}

\section{$1 \cdot 2 \cdot 3 \cdot 5$ iso-Butane}

Using a modified version of the nonanalytic equation of state of Goodwin and Haynes [32], thermophysical properties of iso-butane are derived from physical properties data and are tabulated at integral temperatures over the entire range of fluid states from 114 to $700 \mathrm{~K}$ along isobars at pressures to $70 \mathrm{MPa}$. Results included dielectric constants, densities, enthalpies, entroples. equation of state, internal energies, isobars, isochores, isotherms, Joule-Thomson inverslon, heats of vaporization, melting line, orthobaric densities, specific heats, sound velocities, vapor pressures, and virial coefficients. In addition to the equation of state, equations are given for vapor pressures, orthobaric vapor and liquid densities, ideal gas properties, second v1rlal coerflclents, dielectric constants, heats of vaporization, melting pressures, and orthobaric liquld speclflc heats. enthalpies, and entropies. Several new sets of data have been used in this correlat $10 n$ and comparisons between experimental and calculated values are given. Additional data on 1so-butane are included in the references [35].

The primary reference for the gas density at reference conditions is Armstrong and Jobe [12a,b]. The reference density equation (2) is used to calculate the density at all reference conditions. The 833 constant $\mathrm{R}$ is defined by equation (1). The molecular mass and uncertainty are found from table i. 2 .? and the second virial coefficients at reference temperatures with uncertainties are found rrom table 1.3.4. The variation of density uncertainty at different reference temperatures is not slentricant. The error in density at the reference conditions is estimated to be 0.2 percent. 
Table 1.2 .8 iso-Butane

\begin{tabular}{|l|c|c|c|}
\hline Base Quantity & $\begin{array}{c}\text { Temperature } \\
\mathrm{K}\end{array}$ & $\begin{array}{c}\text { Pressure } \\
\mathrm{kPa}\end{array}$ & $\begin{array}{c}\text { Density } \\
\mathrm{kg} / \mathrm{m} 3\end{array}$ \\
\hline \hline Fixed Points [32] & & & \\
Critical Point & 407.85 & 3640. & 224.36 \\
Normal Boiling Point & 261.517 & 101.325 & 593.71 \\
Triple Point & 113.55 & $1.95 \times 10^{-5}$ & 741.38 \\
\hline Reference Conditions [12a,b] & & & \\
IUPAC & 298.15 & 101.325 & 2.4421 \\
ASTM-ANSI-API & 288.71 & 101.560 & 2.5381 \\
(U.S. Customary) & 288.15 & 101.325 & 2.5376 \\
ISO-ASTM-ANSI/Metric & 273.15 & 101.325 & 2.6996 \\
JIS/also STP & & & \\
\hline
\end{tabular}

\section{2 .3 .6 n-Pentane}

Primary source data is Das, Reed and Eubank [16]. Selected values of vapor pressure, specific volume, enthalpy, and entropy are presented for the saturated liquid and vapor states of n-pentane from normal boiling point $(309.19 \mathrm{~K})$ to the critical point $(469.65 \mathrm{~K})$. Experimental values of the density, together with the saturated vapor densities, were fitted to a differential regional BenedictWebb-Rubin(DR-BWR) equation of state; compressibility factors were generated from 309 to $600 \mathrm{~K}$ with pressure to $70.9 \mathrm{MPa}$. Thermodynamic properties of the real gas, enthalpy, specific heats, Helmholtz function, entropy and Gibbs function were also calculated from the classical relationships.

The vapor pressure equation correlates the published data with a standard deviation of 3.7 kPA. Published liquid volumes were fitted to a correlating equation and were represented with a standard deviation of $0.24 \mathrm{~kg} / \mathrm{m}^{3}$. Liquid volumes are considered to have maximum inaccuracies of 0.1 percent from the NBP to $320 \mathrm{~K}, 0.3$ percent from 320 to $410 \mathrm{~K}$, and 0.2 percent from 410 to $450 \mathrm{~K}$.

The densities of the real gas are not given as n-pentane is liquid phase at the reference conditions.

Table 1.2 .9 n-Pentane

\begin{tabular}{|l|c|c|c|}
\hline Base Quantity & $\begin{array}{c}\text { Temperature } \\
\mathrm{K}\end{array}$ & $\begin{array}{c}\text { Pressure } \\
\mathrm{kPa}\end{array}$ & $\begin{array}{c}\text { Density } \\
\mathrm{kg} / \mathrm{m} 3\end{array}$ \\
\hline \hline Fixed Points [16] & & & \\
Critical Point & 469.65 & 3369. & 237. \\
Normal Boiling Point & 309.19 & 101.325 & 610.4 \\
Triple Point & 143.429 & & 761. \\
\hline
\end{tabular}

\section{$1.2 \cdot 3 \cdot 7$ iso-Pentane}

Primary source data is Das, Reed and Eubank [17]. Selected values of vapor pressure, specific volume, enthalpy, and entropy are presented for the saturated liquid and vapor states of iso-pentane from normal boiling point $(301.025 \mathrm{~K})$ to the critical point $(460.39 \mathrm{~K})$. Experimental values of the density, together with the saturated vapor densities, were fitted to a differential regional Benedict-Webb-Rubin(DR-BWR) equation of state; compressibility factors were generated from 301 to 600 $\mathrm{K}$ with pressure to $30.4 \mathrm{MPa}$. Thermodynamic properties of the real gas, enthalpy, specific heats, Helmholtz function, entropy and Gibbs function were also calculated from the classical relationships.

The vapor pressure equation correlates the published data with a standard deviation of 21 kPa. Published liquid volumes were fitted to a correlating equation and were represented with a standard deviation of $0.46 \mathrm{~kg} / \mathrm{m}^{3}$. Liquid volumes are considered to have maximum inaccuracies of 0.3 percent from the NBP to $360 \mathrm{~K}, 0.4$ percent from 360 to $440 \mathrm{~K}$, and 0.2 percent from 440 to $460 \mathrm{~K}$.

Densities for the real gas are not given as iso-pentane is liquid phase at the reference conditions. 
Table 1.2 .10 iso-Pentane

\begin{tabular}{|l|c|c|c|}
\hline Base Quantity & $\begin{array}{c}\text { Temperature } \\
\mathrm{K}\end{array}$ & $\begin{array}{c}\text { Pressure } \\
\mathrm{kPa}\end{array}$ & $\begin{array}{c}\text { Density } \\
\mathrm{kg} / \mathrm{m}^{3}\end{array}$ \\
\hline \hline Fixed Points [17] & & & \\
Critical Point & 460.39 & 3381. & 236. \\
Normal Boiling Point & 301.025 & 101.325 & 612.5 \\
Triple Point & 113.25 & & 787. \\
\hline
\end{tabular}

\subsubsection{8 neo-Pentane}

Primary source data is Das, Reed and Eubank [18]. Selected values of vapor pressure, specific volume, enthalpy, and entropy are presented for the saturated liquid and vapor states of neo-pentane from normal boiling point $(282.628 \mathrm{~K})$ to the critical point $(433.75 \mathrm{~K})$. Experimental values of the density, together with the saturated vapor densities, were fitted to a differential regional BenedictWebb-Rubin(DR-BWR) equation of state; compressibility factors were generated from 282.628 to $600 \mathrm{~K}$ with pressure to $40.5 \mathrm{MPa}$. Thermodynamic properties of the real gas, enthalpy, specific heats, Helmholtz function, entropy and Gibbs function were also calculated from the classical relationships.

The vapor pressure equation is believed to be accurate to 0.49 percent from $300 \mathrm{~K}$ to the critical point, with a maximum uncertainty of $8.1 \mathrm{kPa}$ near the critical point. Published liquid volumes were fitted to a correlating equation and were represented with a standard deviation of $0.27 \mathrm{~kg} / \mathrm{m} 3$ from 256 to $433 \mathrm{~K}$. Liquid Volumes are considered to have maximum inaccuracies of 0.2 percent from the NBP to $370 \mathrm{~K}, 0.1$ percent from 370 to $420 \mathrm{~K}$, and 0.2 percent from 420 to $433 \mathrm{~K}$.

The primary reference for the gas density at reference conditions is Armstrong and Jobe [12a,b]. The reference density equation (2) is used to calculate the density at all reference conditions. The gas constant $\mathrm{R}$ is defined by equation (1). The molecular mass and uncertainty are found from table 1.2 .2$. and the second virial coefficients at reference temperatures with uncertainties are found from table 1.3.4. The variation of density uncertainty at different reference temperatures is not significant. The error in density at the reference conditions is estimated to be 0.2 percent. Neo-pentane is liquid phase at the reference condition of $273.15 \mathrm{~K}$ and $101.325 \mathrm{kPa}$.

Table 1.2.11 neo-Pentane

\begin{tabular}{|l|c|c|c|}
\hline Base Quantity & $\begin{array}{c}\text { Temperature } \\
\mathrm{K}\end{array}$ & $\begin{array}{c}\text { Pressure } \\
\mathrm{kPa}\end{array}$ & $\begin{array}{c}\text { Density } \\
\mathrm{kg} / \mathrm{m}^{3}\end{array}$ \\
\hline \hline Fixed Points [18] & & & 232. \\
Critical Point & $\begin{array}{l}433.75 \\
282.628 \\
\text { Normal Boiling Point }\end{array}$ & $\begin{array}{c}3196 . \\
101.325\end{array}$ & 603.3 \\
Triple Point & 256.6 & & 3.0693 \\
\hline Reference Conditions [12a,b] & & 101.325 & 3.1974 \\
IUPAC & 298.15 & 101.560 & 3.1971 \\
ASTM-ANSI-API & 288.71 & 101.325 & \\
(U.S. Customary) & 288.15 & & \\
ISO-ASTM-ANSI/Metric & & & \\
\hline
\end{tabular}

\subsubsection{9 n-Hexane and Hexane Isomers}

The primary source of data for n-hexane is Weber [74]. Thermodynamic propert1es of n-hexane have bern calculated over a a temperature range of $273.15 \mathrm{~K}$ to $556 \mathrm{~K}$ and up to a pressure of $4.1 \mathrm{MPa}$. These properties were determined from vapor pressure, volumetric heat capacity, and latent heat of vaporization data through the application of rigorous thermodynamic relationships according to hieber and the calculated data have been found to be internally consistent. A Benedict-Webb-Rubln (Bir) state equation was used and resulted in an estimated average error of 0.74 percent.

Densities of the real gas are not given as the hexanes are liquid phase at the reference condltons. 
Table 1.2.12a n-Hexane

\begin{tabular}{|l|c|c|c|}
\hline Base Quantity & $\begin{array}{c}\text { Temperature } \\
\mathrm{K}\end{array}$ & $\begin{array}{c}\text { Pressure } \\
\mathrm{kPa}\end{array}$ & $\begin{array}{c}\text { Density } \\
\mathrm{kg} / \mathrm{m}^{3}\end{array}$ \\
\hline \hline Fixed Points [59] & & & \\
& & 3031. & 233.8 \\
Critical Point & 507.8 & 101.325 & 629.0 \\
Normal Boiling Point & 341.9 & & \\
Triple Point & 177.8 & & \\
\hline
\end{tabular}

Table 1.2.12b 2-methylpentane

\begin{tabular}{|l|c|c|c|}
\hline Base Quantity & $\begin{array}{c}\text { Temperature } \\
\mathrm{K}\end{array}$ & $\begin{array}{c}\text { Pressure } \\
\mathrm{kPa}\end{array}$ & $\begin{array}{c}\text { Density } \\
\mathrm{kg} / \mathrm{m}^{3}\end{array}$ \\
\hline \hline Fixed Points [59] & & & \\
& & 3009. & 234.8 \\
Critical Point & 397.5 & 101.325 & \\
Normal Boiling Point & 333.4 & & \\
Triple Point & 119.5 & & \\
\hline
\end{tabular}

Table 1.2.12c 3-methylpentane

\begin{tabular}{|l|c|c|c|}
\hline Base Quantity & $\begin{array}{c}\text { Temperature } \\
\mathrm{K}\end{array}$ & $\begin{array}{c}\text { Pressure } \\
\mathrm{kPa}\end{array}$ & $\begin{array}{c}\text { Density } \\
\mathrm{kg} / \mathrm{m}^{3}\end{array}$ \\
\hline \hline Fixed Points [59] & & & \\
Critical Point & 504.4 & 3121. & 234.8 \\
Normal Boiling Point & 336.4 & 101.325 & \\
Triple Point & 155. & & \\
\hline
\end{tabular}

Table 1.2.12d 2,2-dimethylbutane

\begin{tabular}{|l|c|c|c|}
\hline Base Quantity & $\begin{array}{c}\text { Temperature } \\
\mathrm{K}\end{array}$ & $\begin{array}{c}\text { Pressure } \\
\mathrm{kPa}\end{array}$ & $\begin{array}{c}\text { Density } \\
\mathrm{kg} / \mathrm{m}^{3}\end{array}$ \\
\hline \hline Eixed Points [59] & & & \\
& & 3080. & 240.1 \\
Critical Point & 488.7 & 101.325 & \\
Normal Boiling Point & 322.9 & & \\
Triple Point & 173.3 & & \\
\hline
\end{tabular}

Table 1.2.12e 2,3-dimethylbutane

\begin{tabular}{|l|c|c|c|}
\hline Base Quantity & $\begin{array}{c}\text { Temperature } \\
\mathrm{K}\end{array}$ & $\begin{array}{c}\text { Pressure } \\
\mathrm{kPa}\end{array}$ & $\begin{array}{c}\text { Density } \\
\mathrm{kg} / \mathrm{m}^{3}\end{array}$ \\
\hline \hline Fixed Points [59] & & & \\
& & 3131. & 240.7 \\
Critical Point & 499.9 & 101.325 & \\
Normal Boiling Point & 331.2 & 144.6 & \\
Triple Point & 14.2 & \\
\hline
\end{tabular}




\subsubsection{Nitrogen}

Nitrogen is an undesirable impurity present in most freshly liquefied natural gas. The source of the nitrogen is generally the natural gas mixture from the well head, and because the normal boiling point of nitrogen is near that of methane, a fraction of the nitrogen is carried through the liquefaction process, where it is miscible with LNG in the proportions present. Nitrogen is an undesirable impurity in that it is inert, has no fuel value, causes depression of the boiling point, and causes anomalous density variations in LNG mixtures. Weathering of the LNG dissipates the nitrogen by selective vaporization as it has a lower boiling point temperature than other constituents in the LNG mixture.

The primary reference of Jacobsen, et al. [46] provides tables of thermophysical properties of nitrogen for temperatures from the fusion line to $1944 \mathrm{~K}$ for pressures to $20 \mathrm{MPa}$, and from the fusion line to $833 \mathrm{~K}$ for pressures above $20 \mathrm{MPa}$ to $1034 \mathrm{MPa}$. The tables include values of entropy, enthalpy, internal energy, density, specific volume, velocity of sound, specific heats, thermal conductivity, viscosity, thermal diffusivity, Prandtl number, and dielectric constant for selected isobars.

The equation of state is estimated to be accurate within 0.5 percent in the 1 iquid region for pressures to $50 \mathrm{MPa}$, except in the vicinity of the critical point. For pressures and temperatures above the critical point to pressures of $135 \mathrm{MPa}$ the estimated inaccuracy of the state equation is 0.1 percent. A simple form of the state equation is given by strobridge [70] which covers a more restricted range of pressure and temperatures but with about the same density uncertainty as Jacobsen, et al.

Table 1.2.13. Nitrogen

\begin{tabular}{|l|c|c|c|}
\hline Base Quantity & $\begin{array}{c}\text { Temperature } \\
\mathrm{K}\end{array}$ & $\begin{array}{c}\text { Pressure } \\
\mathrm{kPa}\end{array}$ & $\begin{array}{c}\text { Density } \\
\mathrm{kg} / \mathrm{m} 3\end{array}$ \\
\hline \hline Fixed Points [46] & & & \\
Critical Point & 126.2 & 3399.96 & 314.03 \\
Normal Boiling Point & 77.347 & 101.325 & 808.75 \\
Triple Point & 63.148 & 12.53 & 867.85 \\
\hline Reference Conditions [46] & & & 1.1454 \\
IUPAC & 298.15 & 101.325 & 1.1856 \\
ASTM-ANSI-API & 288.71 & 101.560 & 1.1852 \\
(U.S. Customary & 288.15 & 101.325 & 1.2504 \\
ISO-ASTM-ANSI/Metric & 273.15 & 101.325 & \\
JIS/also STP & & & \\
\hline
\end{tabular}





\section{-}





\subsection{Heating Value of Natural Gas Components}

Introduction. Heat energy in the form of enthalpy change is made available upon combustion of natural gas with air. Measurement or calculation of this thermal energy or heating value is a basic and necessary quantity in assessing the value of LNG as a potential thermal energy commodity in commerce.

Direct measurement of the heat of combustion is accomplished by mixing a vaporized LNG gas sample with air and burning the mixture completely while measuring the temperature rise of the combustion products. This temperature rise for the gas mixture is compared to the temperature rise found from a calibration gas of known heating value under the same mixing and combustion conditions. The accuracy of the measurement process depends almost entirely on the knowledge of uncertainties in the heating value of the calibration gas.

Calculation of the heat of combustion depends on the analysis of a vaporized LNG sample with respect to the individual pure components, assignment of combustion heats based on component fraction and the combination of the pure component combustion heats to reflect properties of the real gas mixture. Here again the accuracy of the process depends almost entirely on the knowledge of the uncertainties in the heating value of a reference gas or in this case a number of component gases.

A description and discussion of these two processes can be found in Part 2 of this manual. Variations of the processes are possible. In the case of the direct measurement process, the calibration gas is generally methane of known and certified purity [10]. By using a pure gas, it is possible to reduce error caused by analysis procedures. Methane has by tradition been used as a calibration gas in the U.S. because the heating value of the pure gas is accurately defined and its heating value is within 85 to 90 percent of that of pipeline quality natural gas in interstate pipelines. This is not the case for LNG, as some of the vaporized liquid samples may have heating values which may be nearly twice the pure methane value. The range of the instrumentation must then be increased to accommodate these new values, and this is accomplished generally with a loss in measurement precision and accuracy. An alternate method would use a gas mixture which more closely approximates the unknown vaporized LNG fluid. This, of course, introduces additional error associated with gas analysis and could result in a net increase in measurement error of the heating value.

In either case, accurate and precise values for natural gas pure components and mixtures are necessary to establish the heat energy potential of the LNG at the point of sale.

\subsubsection{Enthalpy of Combustion}

The enthalpy of combustion is the difference between the appropriate sum of the enthalpies of formation of the products and that of the reactants. It is a negative number. The constant temperature, constant pressure enthalpy of combustion $\Delta_{\mathrm{C}} H(T, P)$, is tabulated in this document. In all cases the reactants, hydrocarbon and oxygen, are gaseous, the product carbon dioxide is gaseous and the product water is a liquid.

The standard enthalpy of combustion is the value calculated (from experiment or from tables) for the isothermal process with each reactant and product in the standard state. The standard state for a gas is the ideal gas at the designated standard state pressure. The standard state for a liquid $1 \mathrm{~s}$ the liquid under a total pressure of the standard state pressure. Two different standard state pressures are used in this document.

Several other terms, all positive, are used to describe the energy released dur1ng combustion. Usually they depend upon the definition of particular test methods. The total (or gross) calorlflc value and the higher (or superior) heating value approximate $-\Delta_{\mathrm{C}} \mathrm{H}(\mathrm{T}, \mathrm{P}$ ) when the product water 19 a liquid, and if the data are reduced to constant pressure conditions. (Caloriflc values for liquid and solid fuels, however, usually are determined under constant volume conditions and then correspond to a change in internal energy, $-\Delta_{C} U(T, P)$. The net calorific value and the lower (or inferlor) heating value approximate $-\Delta_{\mathrm{c}} \mathrm{H}(\mathrm{T}, \mathrm{P})$ when the product water is gaseous, and the data have been reduced to constant pressure conditions. The "heat of combustion" usually means $\Delta_{C} H$ or $-\Delta_{C} H$ dependlng upon whether the value is negative or positive.

An historical review of the NBS work on hydrocarbons is given by Armstrong [11] where the areas of interest are discussed in terms of the three major classes of natural hydrocarbonaceous fuels: natural gas, petroleum and coal. Portions of this review by Armstrong are included in sectlon 2.3 or inla manual. The work done by NBS on the measurement of properties of the pure components has lncluded measurement of the values of the properties themselves and development of pract $1 \mathrm{cal}$ and $3 c \mathrm{c}$ (r) $\mathrm{e}$ measurement procedures and instruments. 
Additional values of combustion energies, densities, viscosities, vapor pressures, refractive indices, elemental compositions and other parameters have been determined for complex fuel mixtures and correlated to find methods of estimating properties. Extensive standard reference data tables have been compiled, and a number of standard reference materials have been developed. For the light hydrocarbons of interest to the natural gas industry, the detailed work on methane properties seemed to be adequate in establishing the values for this important fuel.

Early in the development of the LNG industry, ethane, propane, butane, pentane and hexane in addition to methane became important when defining the value of this fuel in the liquid state. Some time was to pass before it was found that additional critically evaluated data were needed to accomplish this measurement process. The quantity and quality of LNG were found to depend strongly on more accurate values of enthalpy of combustion for hydrocarbons in addition to methane, and since the measurement process for LNG was composed of several submeasurement processes, a measure of accuracy and precision for each substance was found necessary in calculating total uncertainty or error of measurement. As part of the task to develop this LNG Measurement manual, Groupe International des Importateurs de Gaz Naturel Liquefie (G .I.I.G.N.L) contracted with NBS to develop the required information.

Dr. G. T. Armstrong of NBS, an internationally recognized authority on the subject, directed the work which was first published in 1982 [12a]. This document provides the basic data, the recommended procedures and illustrative calculations for computing heating values of natural gas pure components and mixtures. The composition of the mixtures and the properties of the components are given at commonly used reference conditions for gas measurement within the range $273.15 \mathrm{~K}$ to $298.15 \mathrm{~K}$. Symbols, terms and units of measurement are defined, and conversion factors and physical constants are given.

Selected values for standard enthalpies of combustion and heat capacities of the pure hydrocarbon gases $C_{1}$ through $C_{6}$ are tabulated at the reference temperatures $273.15 \mathrm{~K}\left(0^{\circ} \mathrm{C}\right), 288.15 \mathrm{~K}\left(15^{\circ} \mathrm{C}\right)$, $288.71 \mathrm{~K}\left(60^{\circ} \mathrm{F}\right)$ and $298.15 \mathrm{~K}\left(25^{\circ} \mathrm{C}\right)$ on a molar and a volumetric basis. Both the dry gases and the ideal water-saturated gas are treated. The calculation of enthalpies of combustion of gas mixtures on a molar, mass, or volumetric basis is described.

Second virial coefficients are presented as functions of temperature for the pure substances and for binary interactions with methane. Tables are given for molar volumes, enthalpic effects and the heating values of the dry real-gas hydrocarbons on a molar, mass and volumetric basis at two reference conditions, $288.15 \mathrm{~K}\left(15^{\circ} \mathrm{C}\right), 101.325 \mathrm{kPa}$; and $288.71 \mathrm{~K}\left(60^{\circ} \mathrm{F}\right), 101.560 \mathrm{kPa}(14.73 \mathrm{psia})$. An analysis is presented of the effects of errors in the data on calculated heating values.

The recommended values in this publication by Armstrong and Jobe [12a] were revised, in 1984, in order to give more emphasis to recent measurements and to develop a common set of basic data to be used by G.I.I.G.N.L. and the Gas Producers Association (GPA)(U.S.) [12b,37]. This re-evaluation slightly changes the values for the $C_{1}$ to $C_{6}$ alkanes, except for $n$-pentane, previously recommended to both groups. These revised values are quoted here and are implied whenever Armstrong and Jobe are cited. The revision was carried out by a joint working party from the NBS Chemical Thermodynamics Data Center and the Texas A\&M University Thermodynamics Research Center. The revision will be documented separately. For now it should be kept in mind that this revision affects all tables of enthalpies and virial coefficients in reference [12a].

The content of this publication by Armstrong and Jobe is much more extensive than required for this measurement manual. It will serve as a basic reference document for this section on heating values. Selected calculation procedures and tabular data have been taken directly from Armstrong and Jobe [12a] and are reproduced here. The following discussion will emphasize only the origin and uncertainty of the standard enthalpy of combustion for the hydrocarbons C1 through C6 and the calculation methods required to present the values in terms of the required reference states. only pure component data will be considered in this section. Gas mixture properties and enthalpies of combustion will be considered in Section 3 .

\subsubsection{Standard Enthalpy of Combustion}

The standard enthalpy of combustion is the enthalpy of combustion calculated for the isothermal process with all reactants and products in their thermodynamic standard state. The standard thermodynamic properties for a gaseous substance, whether pure or in a gaseous mixture, apply to the pure substance at the standard state pressure and in a hypothetical state in which the gas exhibits ideal gas behavior. A gas which conforms to the ideal gas behavior follows the Ideal Gas Law of $\mathrm{PV}=\mathrm{nRT}$. 
Table 1.3.1 lists the standard enthalpy of combustion for the pure components considered in this LNG study. The values and uncertainties are those recommended in Armstrong and Jobe [12b] and the cited 1 terature is included in the table. These are the base data for LNG calculations. They are either selections of outstanding measurements or combinations of measurements from several laboratories. All values are for the gaseous state.

Table $1 \cdot 3 \cdot 1$

Standard Enthalpy of Combustion

of the Hydrocarbons at Ideal Gas Conditions and $298.15 \mathrm{~K}$ With Estimated Uncertainties [12b]

\begin{tabular}{|c|l|l|}
\hline Hydrocarbon & $\begin{array}{c}-\Delta \mathrm{c}^{\mathrm{HO}} \\
\mathrm{kJ} / \mathrm{mol}\end{array}$ & Reference \\
\hline \hline methane & $890.65 \pm 0.37$ & {$[79,80]$} \\
ethane & $1560.69 \pm 0.25$ & {$[79]$} \\
propane & $2219.17 \pm 0.45$ & {$[79]$} \\
n-butane & $2877.40 \pm 1.00$ & {$[79,81,82]$} \\
2-methylpropane (iso-butane) & $2868.20 \pm 1.00$ & {$[79,81,83,84]$} \\
n-pentane & $3535.77 \pm 0.5$ & {$[23]$} \\
2-methylbutane (iso-pentane) & $3528.83 \pm 0.58$ & {$[23]$} \\
2,2-dimethylpropane (neo-pentane) & $3514.61 \pm 0.50$ & {$[23]$} \\
n-hexane & $4194.95 \pm 0.67$ & {$[85]$} \\
2-methylpentane & $4187.32 \pm 1.00$ & {$[86]$} \\
3-methylpentane & $4189.90 \pm 1.00$ & {$[86]$} \\
2,2-dimethylbutane & $4177.52 \pm 1.00$ & {$[83,86,87,88]$} \\
2,3-dimethylbutane & $4185.28 \pm 1.00$ & {$[83,86,87,88]$} \\
\hline
\end{tabular}

\subsubsection{Standard Enthalpy of Combustion at Reference Conditions}

The standard enthalpy of combustion for the specified temperature of $298.15 \mathrm{~K} 1$ isted in table 1.3 .1 is the most basic heating value reference used in this manual. Armstrong and Jobe have defined source data accuracy by analysis of individual experiments and comparison of the results from several laboratories, where possible. Therefore, the following discussion will deal only with applications of the data of table 1.3 .1 to the standard states at temperatures other than $298.15 \mathrm{~K}$ and the calculation of the enthalpy of combustion on a mass and volumetric basis for the real gas.

The following procedures suggested by Armstrong can be used to define the enthalpy of combustion at any selected temperature and pressure. The four reference conditions described in table 1.2 .2 will be used to develop basic property data and as examples if other reference conditions are required. It should be noted that the specified temperature of table 1.3 .1 (298.15 K) is one of the reference condition temperature values.

The combustion of a gaseous hydrocarbon can be represented by the following equation:

$$
\mathrm{C}_{\mathrm{a}} \mathrm{H}_{\mathrm{b}}(\text { gas })+(\mathrm{a}+\mathrm{b} / 4) \mathrm{O}_{2}(\text { gas })=(\mathrm{a}) \mathrm{CO}_{2}(\text { gas })+(\mathrm{b} / 2) \mathrm{H}_{2} \mathrm{O}(\text { liquid })
$$

where the stoichiometric coefficients a and b depend on the composition of the hydrocarbon. The standard enthalpy of combustion at temperature $\mathrm{T}=298.15 \mathrm{~K}$ shown in table 1.3 .1 represents the enthalpy of combustion with each reactant and each product of combustion in its standard state at the stated temperature. Further, the enthalpy of reaction (including formation) at a temperature other than the specified temperature is the sum of the changes in enthalpy of reactants and products plus the enthalpy of reaction at the initial temperature. The following example using methane will illustrate the process of calculating the change in standard enthalpy of combustion for temperatures other than that originally specified.

Equation (1) when applied to methane becomes:

$$
\mathrm{CH}_{4}(\text { gas })+(2) \mathrm{O}_{2} \text { (gas) }=\mathrm{CO}_{2}(\text { gas })+(2) \mathrm{H}_{2} \mathrm{O}(\text { liquid }) \text {. }
$$

In this case $a=1 ; b=4 ; b / 2=2$ and $a+b / 4=2 ;$ giving the stoichiometric coefflcients for carbon dioxide, water and oxygen for the combustion of one mol of methane. The standard enthalpy of combustion at other temperatures for example $\mathrm{T}=288.15 \mathrm{~K}$ can be calculated from the rollowl nf relationships: 


$$
\begin{aligned}
& \Delta \mathrm{c}^{\mathrm{O}}(298.15 \mathrm{~K})-\Delta_{\mathrm{c}^{\mathrm{O}}}(288.15 \mathrm{~K})= \\
& \mathrm{a}\left[\mathrm{H}^{\circ}\left(\mathrm{CO}_{2} \text {, gas, } 298.15 \mathrm{~K}\right)-\mathrm{H}^{\circ}\left(\mathrm{CO}_{2} \text {, gas, } 288.15 \mathrm{~K}\right)\right] \\
& +\mathrm{b} / 2\left[\mathrm{H}^{\circ}\left(\mathrm{H}_{2} \mathrm{O}, \text { liquid, } 298.15 \mathrm{~K}\right)-\mathrm{H}^{\circ}\left(\mathrm{H}_{2} \mathrm{O}, \text { liquid, } 288.15 \mathrm{~K}\right)\right] \\
& -(\mathrm{a}+\mathrm{b} / 4)\left[\mathrm{H}^{\circ}\left(\mathrm{O}_{2} \text {, gas, } 298.15 \mathrm{~K}\right)-\mathrm{H}^{\circ}\left(\mathrm{O}_{2} \text {, gas, } 288.15 \mathrm{~K}\right)\right] \\
& -\left[\mathrm{H}^{\circ}\left(\mathrm{C}_{\mathrm{a}} \mathrm{H}_{\mathrm{b}} \text {, gas, } 298.15 \mathrm{~K}\right)-\mathrm{H}^{\circ}\left(\mathrm{C}_{\mathrm{a}} \mathrm{H}_{\mathrm{b}} \text {, gas, 288.15) }\right]\right.
\end{aligned}
$$

Standard heat capacities at constant pressure can now be used to calculate the ideal enthalpy difference indicated in equation (3). These values and references to their sources were provided by Armstrong and Jobe $[12 a, b]$ for the hydrocarbons and a number of auxiliary substances. Enthalpy differences between $298.15 \mathrm{~K}$ and $288.71,288.15$ and $273.15 \mathrm{~K}$ are also provided in [12a].

The heat capacity data is presented in the form of polynomials of degree two and are valid in the temperature range $268 \mathrm{~K}$ to $308 \mathrm{~K}$. The polynomials have the dimensionless form:

$$
C_{p}^{O} / R=a+b(T-273.15 K)+c(T-273.15 K)^{2}
$$

for which the values of $a, b$ and $c$ are determined using the method of least squares to fit the selected data.

The standard state enthalpy difference at temperatures other than $298.15 \mathrm{~K}$ is calculated as the integral of the standard state heat capacity of equation (4) over the change in temperature. The integrated equation is:

$$
H^{\circ}\left(T_{2}\right)-H^{\circ}\left(T_{1}\right)=R\left[a\left(t_{2}-t_{1}\right)+(b / 2)\left(t_{2}^{2}-t_{1}^{2}\right)+(c / 3)\left(t_{2}^{3}-t_{1}^{3}\right)\right]
$$

where $t=T-273.15 \mathrm{~K}$ is the Celsius temperature.

The values for the polynomial constants for the substances of interest 4 are 4 given in table 1.3 .2 . For water (*), add the term $d(T-273.15 K)^{3}$ to equation $(4)$ and $(d / 4)\left(t_{2}^{4} t_{1}^{4}\right)$ to equation $(5)$ with $d=$ $-1.378 \times 10^{-6}$.

Table 1.3 .2

Polynomial Constants for Hydrocarbons and Auxiliary Substances for Calculation of Heat Capacities for the Temperature Range of $268 \mathrm{~K}$ to $308 \mathrm{~K}$. [12a,b]

\begin{tabular}{|l|c|c|c|}
\hline \multirow{2}{*}{ Substance } & \multicolumn{3}{|c|}{ Constants for Equations (4) } \\
\cline { 2 - 4 } & $\mathrm{a}$ & $\mathrm{b} \times 10^{2}$ & $\mathrm{c} \times 10^{5}$ \\
\cline { 2 - 4 } methane (gas) & 4.1947 & 0.3639 & 1.49 \\
ethane (gas) & 5.9569 & 1.377 & 1.69 \\
propane (gas) & 8.2671 & 2.286 & 1.90 \\
n-butane (gas) & 11.109 & 2.875 & 1.82 \\
iso-butane (gas) & 10.824 & 3.153 & 0.82 \\
n-pentane (gas) & 13.587 & 3.288 & 2.98 \\
iso-pentane (gas) & 13.412 & 3.540 & 1.40 \\
neo-pentane (gas) & 13.584 & 3.846 & 0.45 \\
n-hexane (gas) & 16.134 & 3.986 & 3.60 \\
2-methylpentane (gas) & 15.768 & 4.351 & 1.11 \\
2,2-dimethylbutane (gas) & 15.877 & 4.579 & 0.52 \\
2,3-dimethylbutane (gas) & 15.739 & 4.139 & 1.77 \\
oxygen (gas) & 3.520 & 0.044 & 0.28 \\
carbon dioxide (gas) & 4.324 & 0.580 & -0.65 \\
water (liquid) * & 9.1398 & -0.78588 & 25.3814 \\
\hline
\end{tabular}


The Individual terms of equation (3) can now be evaluated using equation (5) and the data of table 1.3 .2 .

$$
\begin{aligned}
& \mathrm{a}\left[\mathrm{H}^{\circ}\left(\mathrm{CO}_{2} \text {, gas, } 298.15 \mathrm{~K}\right)-\mathrm{H}^{\circ}\left(\mathrm{CO}_{2} \text {, gas, } 288.15 \mathrm{~K}\right)\right]= \\
& 8.31441\left[4.324(25-15)+(0.00580 / 2)\left(25^{2}-15^{2}\right)+\right. \\
& \left.(-0.0000065 / 3)\left(25^{3}-15^{3}\right)\right]=368.94 \mathrm{~J} / \mathrm{mol} \\
& \mathrm{b} / 2\left[\mathrm{H}^{\circ}\left(\mathrm{H}_{2} \mathrm{O} \text {, liquid, } 298.15 \mathrm{~K}\right)-\mathrm{H}^{\circ}\left(\mathrm{H}_{2} \mathrm{O} \text {, liquid, } 288.15 \mathrm{~K}\right)\right] \\
& 2(8.31441)\left[9.1398(25-15)-(0.0078588 / 2)\left(25^{2}-15^{2}\right)+\right. \\
& \left(0.0002538 / 3\left(25^{3}-15^{3}\right)-(0.000001378 / 4)\left(25^{4}-15^{4}\right)\right] \\
& =1509.1 \mathrm{~J} / \mathrm{mol}
\end{aligned}
$$

$(\mathrm{a}+\mathrm{b} / 4)\left[\mathrm{H}^{\circ}\left(\mathrm{O}_{2}\right.\right.$, gas, $\left.298.15 \mathrm{~K}\right)-\mathrm{H}^{\circ}\left(\mathrm{O}_{2}\right.$, gas, $\left.\left.288.15 \mathrm{~K}\right)\right]=$

$$
\begin{gathered}
(2)(8.31441)\left[3.52(25-15)+(0.00044 / 2)\left(25^{2}-15^{2}\right)+\right. \\
\left.(0.0000028 / 3)\left(25^{3}-15^{3}\right)\right]=586.98 \mathrm{~J} / \mathrm{mol}
\end{gathered}
$$

$$
\begin{gathered}
{\left[\mathrm{H}^{\circ}\left(\mathrm{CH}_{4}, \text { gas, } 298.15 \mathrm{~K}\right)-\mathrm{H}^{\circ}\left(\mathrm{CH}_{4}, \text { gas, } 288.15\right)\right]} \\
8.31441\left[4.1947(25-15)+(0.003639 / 2)\left(25^{2}-15^{2}\right)+\right. \\
\left.(0.00000149 / 3)\left(25^{3}-15^{3}\right)\right]=355.32 \mathrm{~J} / \mathrm{mol}
\end{gathered}
$$

Combining these individual terms of equation (3) gives;

$$
\begin{aligned}
& \Delta \mathrm{cH}^{\circ}(298.15 \mathrm{~K})-\Delta \mathrm{c}^{\mathrm{H}}(288.15 \mathrm{~K})= \\
& (368.94)+(1509.1)-(586.99)-(355.32)=935.7 \mathrm{~J} / \mathrm{mol}
\end{aligned}
$$

For this calculation, a value of $935.7 \mathrm{~J} / \mathrm{mol}$ is the difference between the standard enthalpy of combustion of methane gas at $288.15 \mathrm{~K}$ and the standard enthalpy of combustion at $298.15 \mathrm{~K}$. The standard enthalpy of combustion of methane gas at this new temperature can be found by combining the calculated difference of $935.7 \mathrm{~J} / \mathrm{mol}$ and the standard enthalpy of combustion for methane from table 1.3 .1 of $890.65 \mathrm{~kJ} / \mathrm{mol}$ to give a value of $891.58 \mathrm{~kJ} / \mathrm{mol}$ at the new temperature of $288.15 \mathrm{~K}$. The above calculation method and data have been used to generate table 1.3 .3 which provides standard enthalpy of combustion values at the four specified reference temperatures.

Table $1 \cdot 3 \cdot 3$

Standard Enthalpy of Combustion

of the Hydrocarbons at Ideal Gas Conditions and at Specified Reference Temperatures [12a,b].

\begin{tabular}{|l|r|r|r|r|}
\hline \multirow{2}{*}{ Hydrocarbon } & \multicolumn{4}{|c|}{$-\Delta_{c^{H}} \mathrm{H}^{\mathrm{kJ}} / \mathrm{mol}$} \\
\cline { 2 - 5 } & $298.15 \mathrm{~K}$ & $288.71 \mathrm{~K}$ & $288.15 \mathrm{~K}$ & $273.15 \mathrm{~K}$ \\
\hline \hline methane & 890.65 & 891.53 & 891.58 & 892.99 \\
ethane & 1560.69 & 1562.06 & 1562.14 & 1564.34 \\
propane & 2219.17 & 2220.99 & 2221.10 & 2224.02 \\
n-butane & 2877.40 & 2879.63 & 2879.76 & 2883.82 \\
iso-butane & 2868.20 & 2870.45 & 2870.59 & 2874.20 \\
n-pentane & 3535.77 & 3538.45 & 3538.60 & 3542.89 \\
iso-pentane & 3528.83 & 3531.52 & 3531.68 & 3535.98 \\
neo-pentane & 3514.61 & 3517.28 & 3517.44 & 3521.72 \\
n-hexane & 4194.95 & 4198.06 & 4198.24 & 4203.23 \\
2-methylpentane & 4187.32 & 4190.43 & 4190.62 & 4195.61 \\
3-methylpentane & 4189.90 & 4193.03 & 4193.22 & 4198.25 \\
2,2-dimethylbutane & 4177.52 & 4180.64 & 4180.83 & 4185.84 \\
2,3-dimethylbutane & 4185.28 & 4188.42 & 4188.60 & 4193.63 \\
\hline
\end{tabular}

\subsubsection{Real Gas Enthalpy of Combustion}

Custody transfer measurements of LNG require accurate data for properties of the real fluld. To establish and maintain equity in the market place, these property data must be valid over a broad range of fluid pressures, temperatures and composition states. To develop the baslc physlcal and thermodynamic property data, the procedure most generally followed consists of the definition of fundamental quantities such as molecular and atomic masses, description of the properties ln ldeal states in terms of these and other fundamental standard state thermal quantitles and the development of ideal-real property relationships for the pure substance in the most simple real property state. This procedure is generally followed by property data definition over a broad range of pressure, 
temperature and state and then the definition of properties of mixtures with other substances in binary and higher order combinations.

Molecular and atomic masses and the development of standard state enthalpies of combustion on a molar basis for several reference temperature states have been considered in previous sections. This section will summarize the methods used by Armstrong and Jobe [12a,b] to define the effects of non-ideality on the calorific properties of the real gases which are of interest to the LNG and natural gas industry.

Armstrong and Jobe [12a] use the virial form of an equation of state to define the differences in ideal and real gas properties and to calculate the molar volume of the real gas. The equation is:

$$
P V_{m} / R T=1+B(T) / V_{m}+C(T) / V_{m}^{2}
$$

At the low pressures represented by the selected reference conditions (101.325 or $101.65 \mathrm{kPa}$ ) the second virial coefficient is adequate to represent the interactions that occur, and so for this document the virial equation is truncated at the $B(T)$ term such that equation (6) becomes:

$$
\mathrm{PV}_{\mathrm{m}} / \mathrm{RT}=1+\mathrm{B}(\mathrm{T}) / \mathrm{V}_{\mathrm{m}}
$$

Equation (7) is used by Armstrong and Jobe [12a] to calculate the difference in enthalpy of the real gas and the ideal gas by:

$$
\left.\left(H_{m}-H_{m}^{0}\right) / R T=\{B(T)-T(d B / d T)\} / V_{m}\right)
$$

Rearrangement of equation (7) will also allow calculation of real gas molar volume.

$$
\mathrm{V}_{\mathrm{m}}=(\mathrm{RT} / \mathrm{P})\left\{1+\mathrm{B}(\mathrm{T}) / \mathrm{V}_{\mathrm{m}}\right\}
$$

This is done by solving the quadratic equation in terms of $V_{m}$ the real gas molar volume, $v_{m}$ the ideal gas molar volume and of course the second virial coefficient $B(T)$. The solution to equation ( 9 ) is:

$$
V_{m}=V_{m}^{i d}\left[0.5+0.5\left\{1+4 B(T) / V_{m}^{i d}\right\}^{1 / 2}\right]
$$

The negative solution of equation (9) is ignored as being physically meaningless. $\mathrm{V}_{\mathrm{m}}^{\text {id }}$ is a constant for a given value of $P$ and $T$, and so the equation can be solved exactly if $B(T)$ is $k n o w n$.

Two additional data terms are needed to allow calculation of real gas molar volumes and combustion enthalpies using the equations (8), (9) and (10). These are the second virial coefficient B(T)and its temperature derivative, $\mathrm{dB} / \mathrm{dT}$. For this report, both are obtained from a correlation of $\mathrm{B}(\mathrm{T})$ and the critical temperature and volume. This correlation is used for calculation of real gas properties for both pure substances and for mixtures in section 3 . It is based on fitting values for $B(T)$ of pure substances and binary mixtures similar to those selected by Armstrong and Jobe [12a,b]. The values of $\mathrm{B}(\mathrm{T})$ and $\mathrm{dB} / \mathrm{dT}$ obtained from the correlation are intended only for use in calculating the volume and enthalpy of natural gas. They are not a substitute for measurements when making calculation on pure substances or mixtures in which methane is not the dominant component.

Table 1.3.4 lists the correlated values of $B(T)$ for the hydrocarbons of interest in this report and the uncertainties for $B(T)$ assigned in [12a] on the basis of an evaluation of published measurement.

The correlating equation and its temperature derivative are:

$$
\begin{aligned}
\mathrm{B}(\mathrm{T})= & \mathrm{V}_{0}\left(\mathrm{a}_{1} / \mathrm{T}_{r}+\mathrm{a}_{2} / \mathrm{T}_{r}{ }^{2}+\mathrm{a}_{3} / \mathrm{T}_{r}{ }^{3}+\mathrm{a}_{4} / \mathrm{T}_{r}{ }^{4}\right)+\mathrm{a}_{5} e^{\left(\mathrm{a}_{6} / \mathrm{T}_{r}\right)} \\
\mathrm{dB} / \mathrm{dT}= & \left(\mathrm{dB} / \mathrm{dT}_{r}\right)\left(\mathrm{dT}_{r} / \mathrm{dT}\right)=\left(1 / \mathrm{T}_{0}\right)\left(\mathrm { V } _ { 0 } \left[-\mathrm{a}_{1} / \mathrm{T}_{r}{ }^{2}-2 \mathrm{a}_{2} / \mathrm{T}_{r}{ }^{3}\right.\right. \\
& \left.\left.\left.-3 \mathrm{a}_{3} / \mathrm{T}_{r} 4-4 \mathrm{a}_{4} / \mathrm{T}_{r}{ }^{5}\right]-\mathrm{a}_{5} \mathrm{a}_{6} / \mathrm{T}_{r}{ }^{2}\left(\mathrm{a}_{6} / \mathrm{T}_{r}\right)\right)\right)
\end{aligned}
$$

in which $V_{0}=\left(V_{C}(i)^{1 / 3}+V_{C}(i)^{1 / 3}\right)^{3 / 8}$

$\mathrm{T}_{\mathrm{r}}=\mathrm{T} / \mathrm{T}_{\mathrm{O}}$

$\mathrm{T}_{0}=\left(\left(\mathrm{T}_{\mathrm{C}}(\mathrm{i}) \mathrm{T}_{\mathrm{C}}(\mathrm{j})\right)^{1 / 2}\right) \mathrm{F}_{\mathrm{a}}$

and $i$ and $j$ are two components of a mixture (or are the same for a pure substance), $T_{C}$ and $V_{c}$ are the critical point temperature and pressure, and $\mathrm{F}_{\mathrm{a}}$ is a correlation factor for a mixture containing water vapor and is specific for each substance paired with water. Table 1.3 .51 ists the parameters $a_{i}$, $V_{C}$, $\mathrm{T}_{\mathrm{c}}$ and $\mathrm{F}_{\mathrm{a}}$ for equation (11). 
Table $1.3 \cdot 4$

Correlated Values of Second Virial Coefficients for Pure Substances at Specified Reference Temperatures for Use in Calculations on Natural Gas Samples at $273.15 \mathrm{~K}$ to $298.15 \mathrm{~K}$

\begin{tabular}{|c|c|c|c|c|c|}
\hline \multirow[b]{2}{*}{ Substance/K } & \multicolumn{4}{|c|}{$-\mathrm{B}(\mathrm{T}) \mathrm{cm} 3 / \mathrm{mol}$} & \multirow{2}{*}{$\begin{array}{c}\text { Error } \\
\mathrm{cm} 3 / \mathrm{mol}\end{array}$} \\
\hline & 298.15 & 288.71 & 288.15 & 273.15 & \\
\hline methane & 42.5 & 46.7 & 47.0 & 54.4 & 1 . \\
\hline ethane & 188.3 & 202.6 & 203.5 & 230.4 & 2. \\
\hline propane & $391 \cdot 3$ & 426.0 & 428.3 & 497.2 & 20. \\
\hline n-butane & 722.7 & 800.0 & 805.1 & 963.2 & 20 \\
\hline iso-butane & 647.1 & 712.3 & 716.5 & 848.8 & 40. \\
\hline n-pentane & 1180 & 1325. & 1334 & 1634. & 60. \\
\hline iso-pentane & 1107. & 1240 & 1248. & 1522. & 40. \\
\hline neo-pentane & 920.5 & 1022 . & 1028. & 1236 . & 35. \\
\hline n-hexane & 1878. & 2131. & 2147 . & 2675 . & 100 \\
\hline 2-methylpentane & 1742 & 1971. & 1986. & 2464 . & 100 \\
\hline 3-methylpentane & 1826. & 2071 . & 2087 . & 2596 . & 100 \\
\hline 2,2-dimethylbutane & 1588 . & 1792. & 1806 . & 2231. & 100 \\
\hline 2,3-dimethylbutane & 1724 & 1953. & 1968. & 2444 & 100 \\
\hline oxygen & 16.7 & 18.9 & 19.1 & 23.1 & 0.1 \\
\hline nitrogen & 5.2 & 7.0 & 7.1 & 10.4 & 0.1 \\
\hline carbon dioxide & $127 \cdot 9$ & $137 \cdot 7$ & 138.4 & 156.8 & 2 \\
\hline water vapor [38] & 1166.3 & 1368.6 & 1381.9 & 1817.0 & 60. \\
\hline
\end{tabular}

Table $1.3 \cdot 5$

Parameters for the Correlation Equation (11) for second virial Coefficients. Valid From $273.15 \mathrm{~K}$ to $298.15 \mathrm{~K}$

$$
\begin{array}{lll}
a_{1}=2.058978, & a_{2}=-7.020596, & a_{3}=5.953652, \\
a_{4}=-2.057299, & a_{5}=-1.76 \mathrm{~cm} / \mathrm{mol} & a_{6}=2.52
\end{array}
$$

\begin{tabular}{|l|c|c|c|}
\hline \multicolumn{1}{|c|}{ Substance } & $\begin{array}{c}\mathrm{V}_{\mathrm{C}} \\
\mathrm{cm} / \mathrm{mol}\end{array}$ & $\mathrm{K}$ & $\mathrm{T}_{\mathrm{C}}$ \\
\hline \hline methane & 99. & 190.55 & 0.835 \\
ethane & 147. & 305.33 & 0.790 \\
propane & 200. & 369.85 & 0.759 \\
n-butane & 255. & 425.16 & 0.737 \\
iso-butane & 259. & 407.85 & 0.728 \\
n-pentane & 304. & 469.7 & 0.715 \\
iso-pentane & 306. & 460.43 & 0.706 \\
neo-pentane & 310. & 433.75 & 0.706 \\
n-hexane & 369. & 507.4 & 0.688 \\
2-methylpentane & 369. & 497.5 & 0.688 \\
3-methylpentane & 367. & 504.4 & 0.688 \\
2,2-dimethylbutane & 358.9 & 488.7 & 0.688 \\
2,3-dimethylbutane & 358. & 499.9 & 0.688 \\
oxygen & 73.3 & 154.58 & 0.995 \\
nitrogen & 89.1 & 304.21 & 0.837 \\
carbon dioxide & 94.2 & 647.14 & 1.0 \\
water vapor & 55.9 & & 1.0 \\
\hline
\end{tabular}

It should be noted in table 1.3 .5 that $B(T)$ is calculated in the same units as $V_{C}$ and as as cm 3 . To obtain $B(T)$ in terms of $\mathrm{m} 3 / \mathrm{mol}$, divide $V_{C}$ and $a_{5}$ by the quantity $1 \times 10^{6}$.

In section 1.3 .3 sample calculations were shown which demonstrate the procedure and should allow calculation of the required standard enthalpy of combustion at any temperature in the range or 268 i $308 \mathrm{~K}$. Methane was used in the sample calculation. This procedure w111 be continued here in demonstrating the calculation methods leading to real gas values at the specifled reference condlinns or at other reference conditions.

The objective, of course, is to determine the difference in enthalpy of the real and 1 doal solatilu in equation (8). This difference calculated for each fluid of interest and for each sponlfio: reference condition will be combined with the ideal gas Standard Enthalpy of Combustion or tablo 1 . 3. ? 
to give the real gas enthalpy at the specified reference conditions. Additional calculations will provide data in terms of mass and volume as well as on the molar basis.

The ideal molar volume for methane at $298.15 \mathrm{~K}$ and $0.101325 \mathrm{MPa}$ is:

$$
\begin{aligned}
V_{m}^{i d} & =R T / P=(8.3144 i)(298.15) /(0.101325) \\
& =24465 \mathrm{~cm} 3 / \mathrm{mol}
\end{aligned}
$$

where the gas constant $\mathrm{R}=8.31441 \mathrm{~cm}^{3}-\mathrm{MPa} / \mathrm{mol} \mathrm{K}$.

It is now possible to calculate the real gas molar volume $V_{m}$ from equation (10). At $298.15 \mathrm{~K}$ the value of $B(T)$ for methane is found from table 1.3 .4 to be $-42.5 \mathrm{~cm} 3 / \mathrm{mol}$.

$$
\begin{aligned}
V_{m} & =(24465)\left[0.5+0.5\{1+4(-42.5) / 24465\}^{1 / 2}\right] \\
& =24423 \mathrm{~cm} 3 / \mathrm{mol}=2.4423 \times 10^{-2} \mathrm{~m} 3 / \mathrm{mol}
\end{aligned}
$$

The value of $\mathrm{dB} / \mathrm{dT}$ for the fitting function of table 1.3 .5 is found by differentiating the function of equation (11) using:

$$
\begin{aligned}
i & =j=C_{4}, V_{0}=V_{C}=99 \mathrm{~cm}^{3} / \mathrm{mol}, T_{0}=T_{C} \times F_{a}=190.55 \mathrm{~K}, \\
T_{r} & =298.15 / 190.55=1.56468,
\end{aligned}
$$

resulting a value for $\mathrm{dB} / \mathrm{dT}=0.42305 \mathrm{~cm} 3 / \mathrm{mol}$.

The enthalpy difference between the ideal and the real gas can now be calculated using equation ( 8 ) with the values of $V_{m}$ and $d B / d T$ from above and $B(T)$ from table 1.3 .4 .

$$
\begin{aligned}
\mathrm{H}-\mathrm{H}^{\mathrm{O}} & =(8.31441)(298.15)\{-42.5-298.15(0.42305)\} / 24423 \\
& =-17.12 \mathrm{~J} / \mathrm{mol}
\end{aligned}
$$

The real gas enthalpy of combustion can now be found from these calculated differences and the standard enthalpy of combustion of table 1.3.1. The method is similar to that previously used to find the standard enthalpies of combustion at temperatures other than the standard state.

The combustion of a gaseous hydrocarbon can be represented by the expression:

$$
\mathrm{C}_{\mathrm{a}} \mathrm{H}_{\mathrm{b}}(\text { gas })+(\mathrm{a}+\mathrm{b} / 4) \mathrm{O}_{2}(\text { gas })=(\mathrm{a})\left(\mathrm{CO}_{2}\right)(\text { gas })+(\mathrm{b} / 2) \mathrm{H}_{2} \mathrm{O}(1 \text { iquid })
$$

where the stoichiometric coefficients a and b depend on the composition of the hydrocarbon. This equation when applied to methane becomes:

$$
\mathrm{CH}_{4} \text { (gas) }+(2) \mathrm{O}_{2} \text { (gas) }=\mathrm{CO}_{2}(\text { gas })+(2) \mathrm{H}_{2} \mathrm{O}(\text { liquid) }
$$

The real gas enthalpy of combustion for the example of methane at $298.15 \mathrm{~K}$ is then found from:

$$
\begin{aligned}
\left.\Delta_{\mathrm{C}} \mathrm{H}(\text { real gas })\right) & =-\left[\mathrm{H}-\mathrm{H}_{\mathrm{O}}\right](\mathrm{CH} 4, \mathrm{~g}, 298.15 \mathrm{~K}) \\
& -(2)\left[\mathrm{H}-\mathrm{H}_{\mathrm{O}}\right](\mathrm{O} 2, \mathrm{~g}, 298.15 \mathrm{~K})+\left[\mathrm{H}-\mathrm{H}_{\mathrm{O}}\right](\mathrm{CO} 2, \mathrm{~g}, 298.15 \mathrm{~K}) \\
& +(2)\left[\mathrm{H}-\mathrm{h}_{\mathrm{O}}\right](\mathrm{H} 2 \mathrm{O}, 1 \mathrm{iq}, 298.15 \mathrm{~K}) \\
& +\Delta_{\mathrm{c}} \mathrm{H}\left(\mathrm{CH}_{4},\right. \text { ideal gas, 298.15 K) }
\end{aligned}
$$

Substituting values calculated from equation ( 8 ) for all reactants and combustion products (Armstrong and Jobe consider $\mathrm{H}-\mathrm{H}_{\mathrm{O}}$ for $\mathrm{H}_{2} \mathrm{O}$ liquid to be zero) gives:

$$
\begin{aligned}
\Delta_{\mathrm{C}} \mathrm{H}(\text { real gas })= & -(-0.0171)-(2)(-0.00859)+(-0.0427)+(-890.65) \\
& =-890.658=-890.66 \mathrm{~kJ} / \mathrm{mol}
\end{aligned}
$$

The difference between the real and ideal gas enthalpy of combustion of methane is quite small. For the other hydrocarbons the difference is less than the uncertainty in the enthalpy of combustion.

To calculate enthalpy of combustion in terms of mass, the molar values are divided by the molecular masses from table 1.2.2. The resulting value for methane is $\Delta_{\mathrm{C}} \mathrm{Hm}=-890.658 / 16.043=-55.5169 \mathrm{~kJ} / \mathrm{g}=$ $-55.5169 \mathrm{MJ} / \mathrm{kg}$. 
To calculate enthalpy of combustion in terms of volume, the molar values are divided by the real gas molar volume found from equation (10). For the example of methane then $\Delta_{\mathrm{c}^{H}} \mathrm{v}=-890.658 / 24423=$ $-0.036468 \mathrm{~kJ} / \mathrm{cm}^{3}=-36.4680 \mathrm{MJ} / \mathrm{m}^{3}$.

The results of a these calculations are presented in the following four tables of values of real gas enthalpy of combustion at the selected reference conditions. Some of the hydrocarbons are $l i q u i d s$ at these reference temperatures and pressures. Real gas properties are not given for them.

Table 1.3 .6

Enthalpy of Combustion of the Real Gas Hydrocarbons [12b]

Molar, Mass and Volume SI Units

IUPAC Reference Conditions of: $101.325 \mathrm{kPa}, 298.15 \mathrm{~K}$

\begin{tabular}{|l|c|c|c|}
\hline Hydrocarbon & $-\Delta_{c} \mathrm{H} \frac{\mathrm{kJ}}{\mathrm{mol}}$ & $-\Delta_{c} \mathrm{H} \frac{\mathrm{MJ}}{\mathrm{kg}}$ & $-\Delta_{c^{\mathrm{H}}} \frac{\mathrm{MJ}}{\mathrm{m}^{3}}$ \\
\hline \hline methane & 890.66 & 55.5169 & 36.4680 \\
ethane & 1560.7 & 51.902 & 64.290 \\
propane & 2219.1 & 50.323 & 92.204 \\
n-butane & 2877.2 & 49.502 & 121.30 \\
iso-butane & 2868.1 & 49.345 & 120.51 \\
n-pentane & $*$ & $*$ & $*$ \\
iso-pentane & $*$ & 48.7 & 149.50 \\
neo-pentane & 3514.4 & $*$ & $*$ \\
n-hexane & $*$ & $*$ & $*$ \\
2-methylpentane & $*$ & $*$ & $*$ \\
3-methylpentane & $*$ & $*$ & $*$ \\
2,2-dimethylbutane & $*$ & $*$ & \\
2, 3-dimethylbutane & $*$ & & $*$ \\
\hline
\end{tabular}

* Liquid at this temperature and pressure.

Table $1 \cdot 3 \cdot 7$

Enthalpy of Combustion of The Real Gas Hydrocarbons [12b]

Molar, Mass and Volume U.S. Customary Units

ANSI/ASTM/API Reference Conditions of : $101.560 \mathrm{kPa}, 288.71 \mathrm{~K}$

\begin{tabular}{|c|c|c|c|c|}
\hline Hydrocarbon & $-\Delta_{c^{H}} \frac{\mathrm{Btu}}{\mathrm{mol}}$ & $-\Delta_{c} H \frac{B t u}{1 b}$ & $-\Delta_{c} H \frac{M J}{m^{3}}$ & $-\Delta_{c}{ }^{H} \frac{B t u}{f^{3}}$ \\
\hline methane & 845.02 & 23891.7 & 37.7953 & 1014.4 \\
\hline ethane & 1480.5 & 22333 . & 66.666 & 1789.2 \\
\hline propane & 2105.0 & 21653 . & 95.723 & 2569.1 \\
\hline n-butane & 2729.1 & 21298 & 126.25 & 3388.5 \\
\hline iso-butane & 2720.5 & 21231 & $125 \cdot 34$ & 3363.9 \\
\hline n-pentane & * & $*$ & * & $*$ \\
\hline iso-pentane & * & * & * & * \\
\hline neo-pentane & 3333.4 & 20970 . & 155.86 & 4183.0 \\
\hline n-hexane & * & * & * & * \\
\hline 2-methylpentane & * & * & * & * \\
\hline 3-methylpentane & * & * & * & * \\
\hline 2,2 -dimethylbutane & * & * & * & * \\
\hline 2,3 -dimethylbutane & * & * & * & * \\
\hline
\end{tabular}

* Liquid at this temperature and pressure 
Table 1.3 .8

Enthalpy of Combustion of the Real Gas Hydrocarbons [12b]

Molar, Mass and Volume SI Units

ASTM/ANSI/ISO Metric Reference Conditions: $101.325 \mathrm{kPa}, 288.15 \mathrm{~K}$

\begin{tabular}{|l|c|c|c|}
\hline & $-\Delta_{\mathrm{c}} \mathrm{H} \frac{\mathrm{kJ}}{\mathrm{mol}}$ & $-\Delta_{\mathrm{c}^{\mathrm{H}}} \frac{\mathrm{MJ}}{\mathrm{kg}}$ & $-\Delta_{\mathrm{c}^{\mathrm{H}}} \frac{\mathrm{MJ}}{\mathrm{m}^{3}}$ \\
\hline \hline Hydrocarbon & 891.59 & 55.5752 & 37.7831 \\
ethane & 1562.1 & 51.950 & 66.646 \\
propane & 2221.0 & 50.367 & 95.699 \\
n-butane & 2879.5 & 49.542 & 126.24 \\
iso-butane & 2870.4 & 49.385 & 125.32 \\
n-pentane & $*$ & $*$ & $*$ \\
iso-pentane & $*$ & $*$ & $*$ \\
neo-pentane & 3517.1 & $*$ & $* 777$ \\
n-hexane & $*$ & $*$ & $*$ \\
2-methylpentane & $*$ & $*$ & $*$ \\
3-methylpentane & $*$ & $*$ & $*$ \\
2,2-dimethylbutane & $*$ & $*$ & \\
2,3-dimethylbutane & $*$ & & $*$ \\
\hline
\end{tabular}

* Liquid at this temperature and pressure.

Table $1.3 \cdot 9$

Enthalpy of Combustion of the Real Gas Hydrocarbons [12b]

Molar, Mass and Volume SI Units

JIS/STP Reference Conditions: $101.325 \mathrm{kPa}, 273.15 \mathrm{~K}$.

\begin{tabular}{|l|c|c|c|}
\hline & $-\Delta_{c^{H}} \frac{\mathrm{kJ}}{\mathrm{mol}}$ & $-\Delta_{\mathrm{c}^{\mathrm{H}}} \frac{\mathrm{MJ}}{\mathrm{kg}}$ & $-\Delta_{\mathrm{c}} \mathrm{H} \frac{\mathrm{MJ}}{\mathrm{m}^{3}}$ \\
\hline \hline Hydrocarbon & 893.00 & 55.6632 & 39.9388 \\
ethane & 1564.3 & 52.023 & 70.526 \\
propane & 2223.9 & 50.432 & 101.53 \\
n-butane & 2883.0 & 49.602 & 134.69 \\
iso-butane & 2874.0 & 49.446 & 133.49 \\
n-pentane & $*$ & $*$ & $*$ \\
iso-pentane & $*$ & $*$ & $*$ \\
neo-pentane & $*$ & $*$ & $*$ \\
n-hexane & $*$ & $*$ & $*$ \\
2-methylpentane & $*$ & $*$ & $*$ \\
3-methylpentane & $*$ & $*$ & $*$ \\
2,2-dimethylbutane & $*$ & $*$ & $*$ \\
2,3-dimethylbutane & $*$ & & \\
\hline
\end{tabular}

* Liquid at this temperature and pressure.

\subsubsection{Total Uncertainty Estimates for Combustion Enthalpy}

The enthalpy of combustion for the pure gaseous hydrocarbon can be used as the starting point for calculation of the combustion enthalpy of natural gas mixtures. Armstrong [10] provided a documented reference for the combustion enthalpy of methane as a calibration gas for flow combustion calorimetry. That work and an estimate of uncertainty in the combustion enthalpy for methane provided estimates of error for this relative method of measuring calorific value of hydrocarbon mixtures such as natural gas. It does not provide independent uncertainties for the enthalpies of combustion of the other hydrocarbons.

Armstrong and Jobe $[12 a, b]$ do provide this information on individual uncertainties of the pure gases. The information and data given in table 1.3 .1 are the standard enthalpy of combustion on a molar basis at a common temperature $(298.15 \mathrm{~K})$ and at ideal gas conditions. These recommended data are based on measurements of different laboratories for different gases, occasionally are weighted averages of studies by several investigators and use consistent corrections different from those of the original authors. The uncertainties take into account the spread of the data both within each study and among the studies, the purities of the samples and the reliabilities of the methods. Some of the uncertainties reported here are larger than reported by the original authors because of the additional 


\begin{abstract}
factors considered. For data at temperatures other than $298.15 \mathrm{~K}$ (see table 1.3.1) and for the real gases there are additional uncertainties in the heat capacities and the virial coefficients. These are, however, smaller than those in the enthalpies of combustion. For details, the reader is referred to Armstrong and Jobe $[12 \mathrm{a}, \mathrm{b}]$.

The basic thermal data of table 1.3.6 through table 1.3.9, when combined with the data on molecular mass of table 1.2 .1 and the pure component real gas densities at various reference states of tables 1.2. 4 through 1.2.12e, are the required base physical and thermal properties data and uncertainties necessary to support the measurements considered in the following parts of this manual.
\end{abstract}





\subsection{References}

[1] Ackerson, B. J. and G. C. Straty, Rayleigh scattering from methane, J. Chem. Phys. 69, No. 3, 1207-12 (1978).

[2] Ackerson, B. J. and H. J. M. Hanley, The thermal diffusivity of methane in the critical region, Chem. Phys. Letters 53, 596 (1978).

[3] American National Standards Institute, Base conditions of pressure and temperature for the volumetric measurement of natural gas, ANSI Standard Z 132.1 1969, API Standard 2562, Am. Pet. Inst., New York, NY(1969).

[4] American Petroleum Institute, Measuring, sampling, testing and base conditions for natural gas fluids, Am. Pet. Inst., Publ. 2529, 3rd Ed., p 1 (1979).

[5] American Petroleum Institute, Research Project 44, Selected values of properties of hydrocarbons and related compounds, data sheets, Texas A\&M University, College Station, TX(1973).

[6] American Petroleum Institute, Technical Data Book- Petroleum Refining, American Petroleum Institute, Washington, D. C. (1970).

[7] American Society for Testing and Materials Committee D-3 on Gaseous Fuels, Standard methods for volumetric measurement of gaseous fuel samples, ANSI/ASTM D 1071-78a, Part 26, Am. Soc. Testing and Materials, Philadelphia, Pa (1980).

[8] American Society for Testing and Materials Committee D-3 on Gaseous fuels, Standard methane thermophysical property tables, ASTM D 3956-82, Am. Soc. Testing and Materials, Philadelphia, PA (1982).

[9] American Society for Testing and Materials, Standard methods for volumetric measurement of gaseous fuel samples, ANSI/ASTM D 1071-78a, Am. Soc. Testing and Materials, Philadelphia, PA (1980).

[10] Armstrong, G. T., Calculation of the heating value of a sample of high purity methane for use as a reference material, Nat. Bur. Stand. (U.S.), Tech. Note 299, 19 pp (December, 1966 ).

[11] Armstrong, G. T., Hydrocarbons for fuel- 75 years of research at NBS, Nat. Bur. Stand. (U.S), Special Publ. 434, 20 pp (May, 1976).

[12] a) Armstrong, G. T. and T. L. Jobe, Jr., Heating values of natural gas and its components, Nat. Bur. Stand. (U.S.), NBSIR 82-2401, 168 pp (August, 1982).

b) Garvin, D., E. S. Domalski, G. R. Somayajulu, R. C. Wilhoit and K. N. Marsh, Physical Properties of Pure Components of Natural Gas, (to be presented at the International Congress on Gas Quality, Groningen, Netherlands, April 22-25, 1986).

[13] Armstrong, G. T.,E. S. Domalski and J. I. Minor,Jr., Standard combustion data for the fuel gas industry, Am. Gas Assoc. Operating Section Proc., pp D-74 to D-88 (1972).

[14] Cameron, A. E. and Wichers, E. J., Report of the international commission on atomic weights 1961, J. Am. Chem. Soc., 84, pp 4175 (1962).

[15] Canjar, L. N. and F. S. Manning, Thermodynamic properties and reduced correlations for gases, Gulf Publishing Co., Houston, TX(1967).

[16] Das, T. R., C. O. Reed and P. T. Eubank, PVT surface and thermodynamic properties of n-pentane, J. Chem. Eng. Data, 22, No. 1, 3-9(1977).

[17] Das, T. R., C. O. Reed and P. T. Eubank, PVT surface and thermodynamic properties of 1sopentane, J. Chem. Eng. Data, 22, No. 1, 9-15 (1977).

[18] Das, T. R., C. O. Reed and P. T. Eubank, PVT surface and thermodynamic properties of neopentane, Chem. Eng. Data, 22, No. 1, 16-21 (1977). 
[19] Diller, D. E., Editor, LNG Materials and fluids users manual, Supplement 1 (see [50,55]), Nat. Bur. Stand.(U.S.), Boulder, Co 80303 (1979).

[20] Domalski, E. S., J. Phys. Chem. Reference Data, 1, pp 221-77 (1972).

[21] Dymond, J.H. and E. B. Smith, The virial coefficients of pure gases and mixtures, Clarendon Press, Oxford UK, 536 pp (1980).

[22] Goldman, D. T. and R. J. Bell, Editors, The international system of units (SI), Nat. Bur. Stand. (U.S.), Special Pub. 330, 48 pp (December, 1981).

[23] Good, W. D., J. Chem. Thermodynamics, 2, pp 237-244 (1970).

[24] Goodwin, R. D., The thermophysical properties of methane from 90 to $500 \mathrm{~K}$ at pressures to 700 bar, Nat. Bur. Stand.(U.S.), Tech. Note 653, $280 \mathrm{pp}$ (April 1974).

[25] Goodwin, R. D., Provisional thermodynamic properties of propane, from 85 to $700 \mathrm{~K}$ at pressures to 700 bar, Nat. Bur. Stand.(U.S.), NBSIR 77-860, 239 pp, (July 1977).

[26] Goodwin, R. D., On the non-analytic equation of state for propane, Adv. Cryo. Eng. 23, 611, Plenum Press, NY(1978).

[27] Goodwin, R. D., Specific heats of saturated and compressed liquid propane, J. Res. Nat. Bur. Stand. (U.S.) 83, No. 5, 449-58(Sept.1978).

[28] Goodwin, R. D., The nonanalytic equation of state for pure fluids applied to propane, Adv. in Chem., Ser. No. 182, Am. Chem. Soc., Washington, D. C., 345-64 (1979).

[29] Goodwin, R. D., H. M. Roder and G. C. Straty, Thermophysical properties of ethane from 90 to 600 $\mathrm{K}$ at pressures to 700 bar, Nat. Bur. Stand. (U.S.), Tech. Note 684, 320 pp(August 1976 ).

[30] Goodwin, R. D. and R. Prydz, Densities of compressed liquid methane and the equation of state, J. Res. Nat. Bur. Stand. (U.S.), 76A, 81 (1972).

[31] Goodwin, R. D. and W. M. Haynes, Thermophysical properties of propane from 85 to $700 \mathrm{~K}$ at pressures to $70 \mathrm{MPa}$, Nat. Bur. Stand. (U.S.), Monograph 170, $249 \mathrm{pp}$ (April, 1982).

[32] Goodwin, R. D. and W. M. Haynes, Thermophysical properties of isobutane from 114 to $700 \mathrm{~K}$ at pressures to $70 \mathrm{MPa}$, Nat. Bur. Stand.(U.S.), Tech. Note 1051, $196 \mathrm{pp}$, (January,1982).

[33] Haynes, W. M. and R. D. Goodwin, Thermophysical properties of normal butane from 135 to $700 \mathrm{~K}$ at pressures to $70 \mathrm{MPa}$, Nat. Bur. Stand. (U.S.), Monograph 169, $197 \mathrm{pp}$ (April, 1982).

[34] Haynes, W. M., Orthobaric liquid densities of normal butane from 135 to $300 \mathrm{~K}$, paper M-6, Adv. Cryo. Eng. 21, 516-21, Plenum Press, NY(1976).

[35] Haynes, W. M. and M. J. Hiza, Measurements of the orthobaric liquid densities of methane, ethane, propane, isobutane \& normal butane, J. Chem. Thermodynamics 9 , 179 (1977).

[36] Hiza, M. J., An empirical excess volume model for estimating LNG densities, Fluid Phase Equilibria, 2, pp 27-38, Elsevier Scientific Publishing Company, Amsterdam(1978).

[37] Gas Producers Association Publication 2172, Calculation of gross heating value, relative density and compressibility factor for natural gas mixtures from composition analysis, Appendix (1984).

[38] Haar,L., J. S. Gallagher and F. S. Kell, NBS/NCA steam tables, Hemisphere Pub. Corp. Washington, D.C. $(1984)$.

[39] IUPAC Commision on Thermodynamics, Manual of symbols and terminology for physicochemical quantities and units, Pure and Applied Chem., 51, pp 393-400 (1979).

[40] IUPAC Commission on Atomic Weights and Isotopic Abundances, Atomic weights of the elements 1981, Pure and Applied Chem., 55, 1119 (1983).

[41] IUPAC Commission on Atomic Weights, Atomic weights of the elements, Pure and Applied Chem., 30 , pp 637-49 (1972). 
[42] IUPAC Commission on Thermodynamics and Thermochemistry, A guide to procedures for the publication of thermodynamic data, J. Pure and Applied Chem., 29, 399-408(1972).

[43] IUPAC Commission on Thermodynamics, Manual of symbols and terminology for physiochemical quantles and units, Appendix IV, 54, 1239-1250 (1982).

[44] International Bureau of Weights and Measures (BIPM), Le systeme international d'unites, 1970, OFFILIB, 84 Rue Gay-Lussac, F. 75005 Paris (1977).

[45] International Organization for Standardization, Petroleum liquids and gases - measurement standard reference conditions, ISO 5024-1976 (E), Int. Org. Std. (1976).

[46] Jacobsen, R. T., R. B. Steward, R. D. McCarty and H. J. M. Hanley, Thermophysical properties of nitrogen from the fusion line to $3500 \mathrm{R}$ for pressures to 150,000 psia, Nat. Bur. Stand.(U.S.), Tech. Note $648,162 \mathrm{pp}$ (December 1973).

[47] Japanese Industrial Standards Committee, Methods for chemical analysis and testing of fuel gases and natural gas, Japanese, Ind. Stand., JIS K 2301-1980, Japanese Stand. Assoc., Tokyo, Japan (1980).

[48] Kelso, E. A. and W. A. Felsing, The pressure-volume-temperature relations of n-hexane and of 2-methylpentane, J. Am. Chem. Soc., 62(11), 3132 (1940).

[49] Kudchadker, A. P., G. H. Alani and B. J. Zwolinski, The critical constants of organic substances, Chem Rev. 68, No. 6, 659-673 (1968).

[50] Mann, D. B., Editor, LNG Materials and fluids user's manual, first edition, Nat. Bur. Stand. (U.S.), Boulder, Co 80303(1977).

[51] McCarty, R. D., Interactive FORTRAN IV computer programs for the thermodynamic and transport properties of cryogens, Nat. Bur. Stand. (U.S.), Tech. Note 1025, 112 pp(October, 1980).

[52] McCarty, R. D., A modified Benedict-Webb-Rubin equation of state for methane using recent experimental data, Cryogenics 14, No. 5, 276-80 (May, 1974).

[53] McClune, C. R., Measurement of the densities of liquefied hydrocarbons from -100 to -180 C ( 173 - 93 K), Cryogenics 16, 289-95(1976).

[54] Mopsik, F. I., Dielectric constant of n-hexane as a function of temperature, pressure and density, J. Research Nat. Bur. Stand., 71A(4)(1967).

[55] Olien, N. A., Editor, LNG Materials and fluids user's manual, Supplement 2, Nat. Bur. Stand. (U.S.), Boulder, CO 80303(1980).

[56] Olson, J. D., The refractive index and Lorenz-Lorentz function of fluid methane, J. Chem. Phys. 63, $474(1975)$.

[57] Orrit, J. and J. M. Laupretre, Density of liquefied natural gas constituents, Adv. Cryo. Eng., 23, 573-9, Plenum Press, NY(1978).

[58] Prydz, R., and R. D. Goodwin, Experimental melting and vapor pressures of methane, J. Chem. Thermodynamics $\underline{4}, 127$ (1972).

[59] Reid, R. C., J. H. Prausnitz and T. K. Sherwood, The properties of gases and 11qu1ds, 3rd ed., McGraw-Hill Book Co., New York, NY, 688 pp (1977).

[60] Roder, H. M., Heats of transition of solid ethane, J. Chem. Phys. 65, 1371 (1976).

[61] Roder, H. M., Measurements of the specific heats, $C$ and $C_{V}$ of dense gaseous and 11 quld methane, J. Res. Nat. Bur. Stand (U.S.), 80A, p 739 (1976).

[62] Shim, J. and J. P. Kohn, J. Chem. Eng. Data, 7(1), pp 3-8(1962).

[63] Stewart, D. E., Volumetric behavior of n-hexane in the liqu1d phase, Ind. Eng. Chem.. $46(1 \geq 1$. 2529 (1954). 
[64] Straty, G. C., Hypersonic velocities in saturated and compressed fluid methane, Cryogenics 15, 729 (1975).

[65] Straty, G, C., Velocity of sound in dense fluid methane, Cryogenics, 14, 367 (1974).

[66] Straty, G, C. and D. E. Diller, PVT properties of saturated and compressed fluid nitrogen, J. Chem. Thermodynamics, 12, 927(1980).

[67] Straty, G. C. and R. D. Goodwin, Dielectric constant and polarizability of saturated and compressed fluid methane, Cryogenics 13, 712 (1973).

[68] Straty, G. C. and R. Tsumura, Phase transition and melting pressures of solid ethane, J. Chem. Phys. 64, $859(1976)$.

[69] Straty, G. C. and R. Tsumura, PVT and vapor pressure measurements on ethane, J. Res. Nat. Bur. Stand.(U.S.), 80a, 35 (1976).

[70] Strobridge, T. R., The thermodynamic properties of nitrogen from 64 to $300 \mathrm{~K}$ between 0.1 and 200 atmospheres, Nat. Bur. Stand.(U.S.), Tech. Note 129, $85 \mathrm{pp}$ (January, 1962).

[71] Tsumura, R. and G. C. Straty, speed of sound in saturated and compressed fluid ethane, Cryogenics 17,193 (1977).

[72] U.S. Department of Commerce, National Bureau of Standards, Guidlines for use of the modernized metric system, Nat. Bur. Stand. (U.S.), Dimensions, LC 1120, pp 12-19 (December, 1979).

[73] Vargaftik, N. B., Tables on the thermophysical properties of liquids and gases, John Wiley \& Sons, NY, Second Ed. 262-65(1975).

[74] Weber, J.H., Thermodynamic properties of n-hexane, AICHE J. 2 (4), 514 (1954).

[75] Weber, L. A., Dielectric constant data and derived Clausius-Mossotti function for compressed gaseous \& liquid ethane, J. Chem. Phys. 65, 446 (1976).

[76] Weber, L. A., Some vapor pressure and PVT data on nitrogen in the range 65 to $140 \mathrm{~K}$, J. Chem. Thermodynamics $\underline{2}, 839$ (1970).

[77] Younglove, B. A., The specific heats, $C$ and $C_{V}$ of compressed and liquefied methane, J. Res. Nat. Bur. Stand.(U.S.), 78A, 401 (1974).

[78] Younglove, B, A., Velocity of sound in liquid propane, J. Res. Nat. Bur. Stand. (U.S.) 86, No. 2, 165-70(March, 1981).

[79] Pittam, D.A. and G. Pilcher, J. Chem. Soc., Faraday Trans. I, 68, 2225-2229 (1972).

[80] Rossini, F. D., J. Res. Nat. Bur. Stand. (U.S.), 6, 37-49, , 329-330 (1931).

[81] Prosen, E. J., F. W. Maron and F. D. Rossini, J. Res. Nat. Bur. Stand. (U.S.), 46, 106-112 (1951).

[82] Rossini, F. D., J. Res. Nat. Bur. Stand. (U.S.), 12, 735-750 (1934).

[83] Scott, D. W., J. Chem. Phys., 60, 3144-3165 (1974).

[84] Pines, H., B. Kvetinskas, L. S. Kassel, and V. N. Ipatieff, J. Am. Chem. Soc., 67, 631-637 $(1945)$.

[85] Good, W. D. and N. K. Smith, J. Chem. Eng. Data, 14, 102-106 (1969).

[86] Prosen, E. J. and F. D. Rossini, J. Res. Nat. Bur. Stand. (U.S.), 27, 289-310 (1941).

[87] Somayajulu, G. R. and B. J. Zwolinski, J. Chem. Soc. Faraday Trans. (2), 72, 2213 (1976).

[88] Evering, B. L. and E. L. d'Ouville, J. Am. Chem. Soc., 71, 440-445 (1949). 


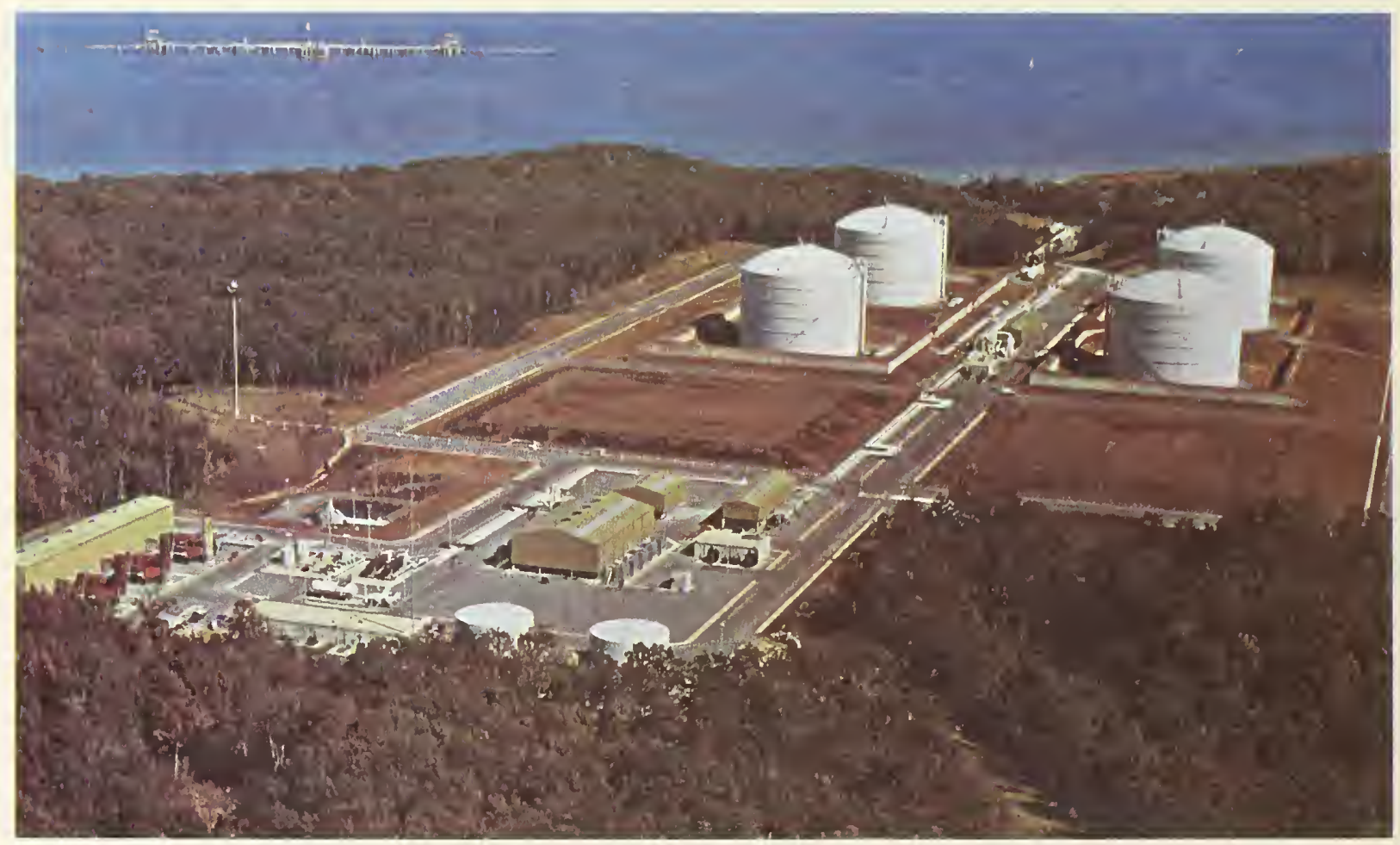

\section{COVE POINT TERMINAL}

The Cove Point Terminal is located in Cove Point, Maryland, which is about 60 $\mathrm{km}$ south of Washington, D.C., and faces the Chesapeake Bay, where berthing facilities for two 125,000 cubic meter LNG ships are 10cated. The terminal is jointly owned by Columbia LNG, a wholly owned subsidiary of Columbia Gas System, and Consolidated System LNG, also a wholly owned subsidiary of the Consolidated Natural Gas. LNG was imported under a sales agreement with El Paso and Algeria's Sonatrach at a daily amount equivalent to 18 milion cubic meters of gas.

Construction was started in 1973, and the first shipment was received in March 1978. Because of the location, great care was taken to blend the facility into the existing environment. Large sections of forest surround the actual terminal as can be seen in the photograph. In order to protect the shoreline, the berthing facilities are located well into the Bay and are connected to the shore storage with a $2 \mathrm{~km}$ submarine tunnel containing the off-loading lines. The photograph also shows the four tanks of $60,000 \mathrm{~kL}$ capacity and the ten submerged combustion type vaporizers having a total capacity $0 f 900$ t/h (center of photograph). The facility is equipped to provide all internal electrical power requirements through three gas-turbine type generators located at the left center of the photograph. Currently, no LNG is being imported through this terminal. (Photograph by courtesy of The Japan Gas Association, Tokyo, Japan, on behalf of the 1981 Japan LNG Congress) 


\section{CONTENTS}

\subsection{MEASUREMENT ELEMENTS}

2. 1

2.2

$2.2 \cdot 1$

2.2 .2

2.2 .3

2. 2.4

2.2 .5

$2 \cdot 2 \cdot 6$

2.3

$2 \cdot 3 \cdot 1$

2.3 .2

2.3 .3

$2 \cdot 4$

2. $4 \cdot 1$

2.4 .2

2 . 4.3

2. 4.4

2.4 .5

2. 5

2. 5 . 1

2. 5 . 2

2. 5.3

2.5 .4

2.5 .5

2.5 .6
The Measurement Process Applied to LNG .

Sampling and Analysis

Sampling Measurement Program Summary

Sampling Measurement Program Scope

Previous Work

Gas Analysis

LNG Sampling

References

LNG Calorific Value

Hydrocarbon Gaseous Fuel Research

Calorific Value Instrumentation and Measurements

References

LNG Density

LNG Density Reference System

Measured LNG Density

Calculated LNG Density

Mathematical Models for the Prediction of LNG Densities

Interlaboratory Comparison of LNG Density Measurements
Page

2. $1-1$

2. $2-1$

$2 \cdot 2-2$

$2 \cdot 2-3$

$2.2-4$

2. 2-7

$2 \cdot 2-24$

2. $2-71$

2. $3-1$

2. 3-1

2. $3-3$

2. 3-7

2. 4. 1-1

2. 4. 1-1

2. 4. 2-1

2. 4. 3-1

2. 4. 4-1

2. 4. 5-1

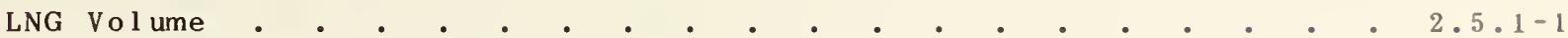

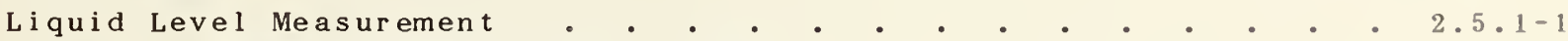

Volume of Membrane-Type LNG Ship Tanks . . . . . . . . . . 2.5.2-1

Volume of Freestanding Prismatic LNG Ship Tanks . . . . . . . 2.5.3-1

Volume of Spherical LNG Ship Tanks . • . . . . . . . . . . $2.5 .4-1$

Volume of Cylindrical LNG Shore Tanks . . . . . . . . . . . $2.5 .5-1$

Vol ume From Totalized Rate Metering . . . . . . . . . . . 2.5.6-1 



\section{CONTENTS}

\section{0 MEASUREMENT ELEMENTS}

\begin{tabular}{|c|c|c|c|c|c|c|c|c|c|c|c|c|c|c|c|c|}
\hline Measu & ent Process & & & & & & & & & & & & & & & Page \\
\hline 2.1 & The Measurement & rocess & Appli ed & to & LNG & - & & & - & - & - & - & - & - & & $2.1-1$ \\
\hline $2 \cdot 1 \cdot 1$ & Introduct ion & - & - & - & - & - & & & - & - & - & - & & & & $2 \cdot 1-1$ \\
\hline $2 \cdot 1 \cdot 2$ & Components of the & LNG M & as uremer & t s & Pro & es s & & & - & - & - & - & & & & $2 \cdot 1-4$ \\
\hline $2 \cdot 1 \cdot 3$ & Statistical Terms & - & - & - & - & - & 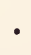 & 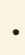 & - & - & - & - & & 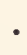 & 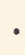 & $2 \cdot 1-4$ \\
\hline $2 \cdot 1 \cdot 4$ & References & - & - & - & - & • & ${ }^{\circ}$ & $\bullet$ & . & - & - & - & • & • & • & $2 \cdot 1-6$ \\
\hline
\end{tabular}






\subsection{The Measurement Process Applied to LNG}

Measurement is a process operating on a system [1]. The application of the measurement process to 11 quefled natural gas (LNG) is limited in this manual to establishing the quantity and composition of LNG relative to an end-point use such as a fuel or as a feed stock. However, the system is the LNG commodity in liquid form, even though the quantitative or qualitative value may be derived at some later date as a result of some undefined process or chemical reaction. The key to assessment of these values is the accurate and precise definition of LNG thermophysical properties, mixture laws and storage and transport characteristics.

\subsubsection{Introduction}

LNG is a complex mixture of low molecular weight hydrocarbons with nitrogen as the principal inert impurity. Liquefied natural gas is characterized by methane as the major component, but the mixture fractions vary with the source of the raw gas, the liquefaction pretreatment, and the liquefaction process and storage. Table 2.1.1 shows analyses of LNG samples from a number of different geographic sources.

Table 2.1.1 LNG Mixture Variations in Mole Percent As a Function of Source

\begin{tabular}{|l|c|r|r|r|r|r|r|r|}
\hline Hydrocarbon & Alaska & Brunei & \multicolumn{1}{c|}{$\begin{array}{c}\text { Das } \\
\text { Island }\end{array}$} & Badak & Arum & $\begin{array}{c}\text { Algeria } \\
\text { Arzew }\end{array}$ & $\begin{array}{l}\text { Libyan } \\
\text { light }\end{array}$ & $\begin{array}{l}\text { Libyan } \\
\text { medium }\end{array}$ \\
\hline \hline methane & 99.81 & 89.83 & 82.07 & 89.91 & 86.96 & 87.00 & 81.0 & 71.9 \\
ethane & 0.07 & 5.89 & 15.86 & 5.44 & 8.40 & 8.14 & 10.0 & 18.0 \\
propane & & 2.92 & 1.89 & 3.16 & 3.66 & 2.30 & 5.0 & 6.0 \\
n-butane & & 0.74 & 0.07 & 0.75 & 0.39 & 0.68 & 1.2 & 1.4 \\
i-butane & & 0.56 & 0.06 & 0.67 & 0.53 & 0.46 & 0.8 & 0.8 \\
n-pentane & & 0.04 & & 0.03 & & 0.02 & & 0.3 \\
i-pentane & & & & & & & & 0.5 \\
hexanes+ & & & & & & & & 0.1 \\
nitrogen & 0.12 & 0.02 & 0.05 & 0.04 & 0.06 & 1.40 & 2.0 & 1.0 \\
\hline
\end{tabular}

Differences in the mixture are important in determining the exact quantity and composition of the commodity being measured. The differences are a matter of degree and, in general, do not materially change the overall measurement process, but can add to the process detail and complexity. LNG is maintained as a commodity at a very low temperature, generally less than $150 \mathrm{~K}$., and at low stat $1 \mathrm{c}$ pressure in order to achieve the highest practical fluid density.

In addition, LNG is stored in large volume containers to benefit from economies of size and to achieve maximum storage efficiency. The containers have double walls with insulation between the walls in order to limit heat transport to the cold liquid. Simply weighing the container is not practical, and other techniques of determining quantity must be used.

Assessment of quality is also a problem with LNG. There is no simple, inexpensive, currently available device, technique or procedure which will directly measure any property of the LNG fluid which in turn could provide an accurate and precise measure of the the heating value of the LNG from this measurement in the liquid mixture state.

These restrictive and possibly unique characteristics of the LNG commodity have resulted 1 n a separation of the measurement process into simpler and more basic measurement forms. The measurement process becomes more complicated because of the number of additional measurements, but each of the individual measurements is more basic, such as length, mass concentration, time, volume, sampling and gas analysis. With these additional measurements, redundant processes can be used to verlfy each of the individual measurements. A general overview of each of the measurement elements and the individual instrumentation is given in this section. Specific references to more detalled descriptions are included in the following sections. 


\subsubsection{Components of the LNG Measurement Process}

The quantity of LNG can be measured in terms of mass or of volume. If the fluid density is known, then total mass may be calculated from volume measurements. This method is generally the most commonly used with LNG for two reasons; (1) LNG is quite difficult to measure directly because of the very low fluid temperature, the combustible nature of the the vapor and the fact that the fluid is at or near its normal boiling point, (2) the LNG storage containers are generally so large in volume that it is either impractical or impossible to accurately weigh the fluid. As a result, container volume and LNG density are basic, necessary elements of the LNG measurement process. If the LNG is to be used as a fuel, then the calorific value either on a mass basis or a volume basis will also be a required measurement element. Thus:

\section{Total calorific value $=$ (calorific value)(density) (volume)}

Sampling and Analysis Sampling for analysis of the LNG pipeline flow or the contents of the large containers may be used to meet the requirements of the measurement process both in quantity and quality. The sample must, of course, be representative of the entire fluid volume. As the size of the container is increased, it becomes more difficult to demonstrate that the sample is truly representative. A detailed discussion of this very important phase of the LNG measurement problem will be found in section 2.2 .

Calorific Values Qualitative assessment of LNG requires some type of measurement of each individual component fraction or the calorific or heating value of the actual fluid mixture. The measurement of volume and fluid density allows calculation of a mass of fluid but does not address either the amount of the individual components which may have value as feed stock or the fuel heating value of the fluid mixture. LNG sampling and analysis will provide quantitative measurements which, when combined with the total mass measurement, will give the amount of an individual component available from the total mass.

A second measure of quality, the calorific or heating value, may be found from the assessment of the combustion enthalpy of the mixture or by a sum of the individual component combustion enthalpies. As will be shown in the following sections on the elements of volume and density, there are also two methods of finding specific combustion enthalpies of the LNG mixture. The two methods, if used on the same mixture, should give the same result within the experimental error.

The first method is direct and simple in concept. A representative LNG sample is taken and vaporized at near ambient temperature and pressure. A volume of the mixture is measured and mixed with a known volume of air and burned in a specially designed combustion cell. The temperature rise caused by the combustion process is measured and compared with the temperature rise found with a calibration gas of known calorific or heating value. The device which accomplishes this process is called a combustion calorimeter and may also contain a "specific gravity" meter which relates the density of the LNG mixture to that of the calibration gas or to the density of the ambient air. This additional measurement of LNG mixture density will allow calculation of the heating value of the mixture in terms of mass.

The calibration gas used in the combustion calorimeter may be a pure fluid such as methane or a precisely characterized mixture which closely resembles the actual LNG mixture. The latter calibration gas should be used with LNG containing relatively large amounts of the heavier hydrocarbons, because the accuracy of the combustion calorimeter measurement can be improved if the known heating value of the calibration gas is near that of the actual LNG mixture. Values of the enthalpy of combustion and other related property data are given in sections 1.2 and 1.3 of this manual. A description of current devices and methods of operation are given in section 2.3 .

The second method of determining the calorific or heating value of the LNG fluid mixtures requires LNG sampling and mixture component analysis. Combustion enthalpies for each of the components can be found from section 1.3 and combined to give a predicted heating value of the real gas mixture. A discussion of the methods are given in sections $2.2,2.3$ and 3 .

Density Measurement Direct measurement of LNG density can be accomplished by placing the density measuring device (densimeter) directly in the LNG. At the present time, all available commercial field-type LNG densimeters are of the relative type and must be calibrated at the conditions of intended use.

Several different types of densimeters have been successfully tested and used in LNG service. The least complicated device from the standpoint of a simple primary measurement element is the capacitance type. Electrically isolated plates are installed in the liquid container, and 
measurements of the dielectric constant of the liquid are made and compared to known correlations of LNG density and dielectric properties. Precision of the device can be quite good, but the accuracy has been found to depend on the mixture and is sensitive to the quantity of nitrogen. A continuous mixture analysis is required to achieve highest accuracy.

A second type of LNG densimeter is based on a primary element which is caused to vibrate at a measured frequency. The actual frequency can be shown to be a function of the density of the fluid in which the primary element is immersed. Two different physical configurations of vibrating element densimeters have been used with LNG. These are a flat plate and a cylinder. Both configurations have performed well under test.

A third type of LNG densimeter is based on the Archimedes principle which relates the buoyancy of a fluid to its density. The actual instrument consists of a float which is weighed by an electric balance. The density of the LNG is found by comparing the apparent weight of the float in LNG to that of the calibration fluid. This device has the potential to perform as an absolute densimeter by careful calibrations in vacuum and has been configured in this way in the laboratory environment. $A$ more detalled description of this and the other densimeters is given in section 2.4 .

LNG density may be found by calculation using mathematical models which relate various properties of the fluid mixture. Pressure and temperature of the liquid LNG are certainly two of the most of ten used independent variables. Identification of the components of the mixture and an accurate and precise quantitative analysis has been found to be necessary to predict LNG mass concentration or density. However, even with these measured independent variables of pressure (P), temperature ( $T$ ) and mixture fraction $(X)$, mixing rules must be considered. LNG is a non-ideal mixture, and, therefore, the ideal mixing rules do not apply. Liquid density errors of up to 3 percent have been found in comparing experimental mixture density with that predicted from ideal solution calculations.

Major research efforts have been made to establish the real mixing relationships between density and pressure, temperature and mixture fraction. Techniques have varied from simple empirical curve fitting of experimental data to theoretical model optimization to pure fluid property data in addition to binary, ternary and higher-order component property data. A summary of this work is presented in section 2.4 .

Volume Measurement Two methods of determining the volume of LNG are possible with existing technology. The first method is the traditional "strapping" or in-place physical length measurement, starting generally at the lowest point in the container and resulting in a series of plane areas which, when combined with an incremental height, provide volume sums as a function of container helght or depth. Tabular summaries of these measurements and calculations may be referred to as tank tables, although modifications must be made to the tables before actual use.

These in-place measurements are generally made during the construction of the container and may be internal, external or a combination of the two. The containers may be designed for shipment as well as storage or may be simply for storage alone. If the container is for shipment, such as part of a LNG tanker, then additional measurements and calculations must be made to take into account the relative orientation of the container and the liquid. It should be noted that in all likelihood no repeat measurements will ever be made to verify either the original dimensions or to ascertain changes caused by damage.

Liquid level devices are used to provide an external indication of the internal liquld level. Generally, two different level devices are required with the second as a redundant backup for the primary device. The liquid level must be referenced to a bench-mark or reference system within the tank so that the indicated level accurately corresponds to the tank tables derived by "strapping".

Finally, the tank tables and liquid level indications must be adjusted to account for thermal contraction of the container materials and tank distortions caused by hydrostatic loads. These adjustments directly modify the volume-height relationships of the tank tables. Temperature effects on the liquid level measurement device may involve both changes in device performance and change $t \mathrm{n}$ physical dimensions. The calculations are complicated if the container and level device are not at uniform temperature.

Each of these physical and electrical measurements and adjustments have assoclated measiurement err r . and these errors must be summed to define the total uncertainty of the volume measurement. Sect 1 in 2.5 provides some examples of actual field measurements and estimated values of error.

The second method of determining volume of LNG is to totalize the flow into or out of the lug tha This method utilizes a dynamic rather than a static technique. In act. ion, the process is con icr.". 
an indirect primary measurement which relies on calculations and inferred physical relationships such as flowmeter calibrations.

The measurement process involves a flowmeter installed in the LNG container supply piping which will indicate total flow per unit time. The actual flowmeter type is not important other than it must have a known precision and a stable performance. The meter may be an orifice meter, turbine, vortex device, positive displacement meter or any other process device which develops an output indication which has a known relationship to a quantity of LNG passing through the piping during some period of time. Mass flow meters have been adapted for cryogenic service [2] but are not available in the larger line sizes of interest in LNG service.

At this time, all of the flowmetering devices are relative in that all must be calibrated using some type of flow reference system. Water calibration is the most commonly used method but does not provide performance adjustments caused by low temperature operation. It is possible to define flowmeter performance at low temperatures for small line sizes and to relate this performance to water flow calibrations. Scaling of the meters to larger pipe diameters has been done by careful step by step performance evaluations. A description of the research in this subject area is given in section 2.5 .

The following sections of this portion of the LNG Measurement Manual are designed to provide engineering detail of instrumentation and technology developed to address the measurement process for LNG. Estimates of error have been given, and extensive detailed explanations have been provided in order to give the reader a general knowledge of the process and also to provide a gauge of the important and critical elements of the measurement.

With this background it will then be possible to discuss actual field-type measurements and to provide detailed explanations of the actual methods of combining the individual measurement elements. This will be done in section 3 .

\subsubsection{Statistical Terms}

In all of the measurement elements presented above, it is assumed that adequate experimental evidence and verification are available in order to completely define the accuracy and precision of each of the measurement processes. Extensive use of statistical techniques is required to evaluate the experimental data. This is particularly true in the following sections on LNG sampling and analysis as well as the sections dealing with density and volume measurements. Because of this extensive general use of statistical techniques in this section and throughout the balance of the manual, the following is a summary list of the definitions of statistical terms which may be useful in understanding the techniques and procedures used.

Accuracy denotes the closeness of computations or estimates to the exact or true value.

Average value $\overline{\mathbf{x}}$ is the arithmetic average of replicate measurements. The average value represents the most likely value of the mean.

Bias (B) is a systematic offset between the mean and true value.

Confidence interval is the interval, based on replicate measurements, for a parameter which will include the true value of the parameter at a specified percentage of the time.

Mean $(X)$ is a value, usually unknown, about which the measured values of replicate measurements randomly scatter.

Measurement process is a series of steps used to assign a numerical value to a physical property.

Precision is the closeness with which the results of independent replicate measurements agree - usually quantified by the estimated standard deviation.

Quadrature is an average which involves the squares of the values being averaged. The square root of the average reduces it to the same dimensions as the values being averaged. The quadratic mean which is also used in propagation of error calculations. 
Random error is an error related only to the random behavior within a measurement process; i.e., not a systematic offset.

Replicate measurements are two or more measurements made under conditions where all controllable variables are identical for all measurements.

Standard deviation $\sigma$ is the most widely used measure of dispersion or scatter of a frequency distribution. If $x_{i}$ is a series of $n$ measurements, $\bar{x}$ is the average value of these measurements then;

$$
\sigma=s=\left[\sum_{i=1}^{n}\left(x_{i}-\bar{x}\right) /(n-1)\right]^{1 / 2}
$$

The symbol o generally refers to the total population while the symbol s refers to a particular sample estimate.

Systematic error is an estimate of the upper limit of the unknown but fixed error of a measurement process.

Total uncertainty, for the purposes of this manual, is the estimated maximum error in a measurement process and is computed by summing the known sources of systematic error and the random error including the confidence interval.

The following general comments apply directly to the the following section on sampling and analysis but also apply to other sections which included discussions of data and error estimates. For additional information on this subject, the reader is referred to Natrella [3], and the work of Mandel [1] and other standard statistical texts on treatment of experimental data.

Replicate measurements yield estimates of both bias and precision. The difference between the average value, $\bar{x}$, of the measured values, $x_{i}$, and the true value is an estimate of the bias in the measurement process. Scatter in the data values about the average value is an estimate of the precision; normally the scatter is given in terms of the estimated standard deviation, a It is assumed that the random errors follow the statistics of a normal distribution.

The values of $\bar{x}$ and $\sigma$ will vary among different sets of the same measurement. However, they wlll vary about the true mean, $X$, and standard deviation $\sigma$. The values of $x$ and o are considered known only after making a very large number of measurements. Where only a limited number of measurements are available, special statistical estimating techniques must be used. The values of $x$ and $\sigma$ may be estimated by using $\bar{x}$ and confidence intervals; for example, a 99 percent confidence interval for the mean value signifies that 99 percent of the time the interval will include the true value. In this case the interval is given by

$$
\bar{x}-t \cdot s / \sqrt{n}<x<\bar{x}+t \cdot s / \sqrt{n}
$$

where $t$ is the Student's statistic for a 99 percent confidence of n repeated measurements.

The concept of total uncertainty was used in assuming a limit on the error in a measurement process. Total uncertainty reflects two errors -- systematic and random. The systematic error represents an estimate of the upper limit of the unknown, nonrandom errors of the measurement process. Its est1mate is based on understanding the nonrandom errors of the components within the measurement process. The random error may be defined to be three times the estimated standard deviation - this roughly corresponds to a 99.7 percent confidence interval.

One of the major goals of the sampling and analysis study was to determine which operating variables affected the precision of composition measurement. To determine if a given varlable changed tho precision, measurements would be made at two (or more) values of the varlable. Confldencr ln detecting the change in precision depended upon the number of samples taken under each 3 e: of conditions and upon the observed change in the egtimated standard devlatlon. 
As shown in table 2.1.3, there is a 65 percent chance of detecting a difference in the precision when there is a factor of four difference between the observed standard deviation and when the measurement is repeated four times with each value of the operating variable; for a 62 percent chance of detecting a difference when there is a factor of two difference in the observed standard deviation it would take 10 measurements with each value of the operating variable.

Table 2.2 .3

Probability of detecting a difference in the standard deviation between two sets of conditions ( $A$ and $B$ ) when the measurements have a factor difference in standard deviations and they are repeated $N$ times under each set of conditions [3]

\begin{tabular}{|c|c|c|c|c|}
\cline { 2 - 5 } \multicolumn{1}{c|}{} & \multicolumn{4}{|c|}{$\begin{array}{c}\text { Probability, percent } \\
\lambda=\sigma_{\mathrm{B}} / \sigma_{\mathrm{A}}\end{array}$} \\
\hline $\mathrm{N}$ & 1 & 2 & 3 & 4 \\
\hline 4 & 5 & 25 & 49 & 65 \\
6 & 5 & 41 & 71 & 87 \\
8 & 5 & 52 & 87 & 95 \\
10 & 5 & 62 & 93 & 99 \\
\hline
\end{tabular}

other experimental test plans are presented in the individual measurement sections.

\subsubsection{References}

[1] Mandel, J., The statistical analysis of experimental data, 410 pp, John Wiley (1967).

[2] Brennan, J. A.,R. W. Stokes, C. H. Kneebone and D. B. Mann, An evaluation of selected angular momentum, vortex shedding and orifice cryogenic flowmeters, NBS Tech. Note 650, Nat. Bur. Stand. (U.S.), $69 \mathrm{pp}$ (March, 1974).

[3] Natrella, M. G., Experimental Statistics, Handbook 91, National Bureau of Standards (1963). 



\section{LNG MEASUREMENT}

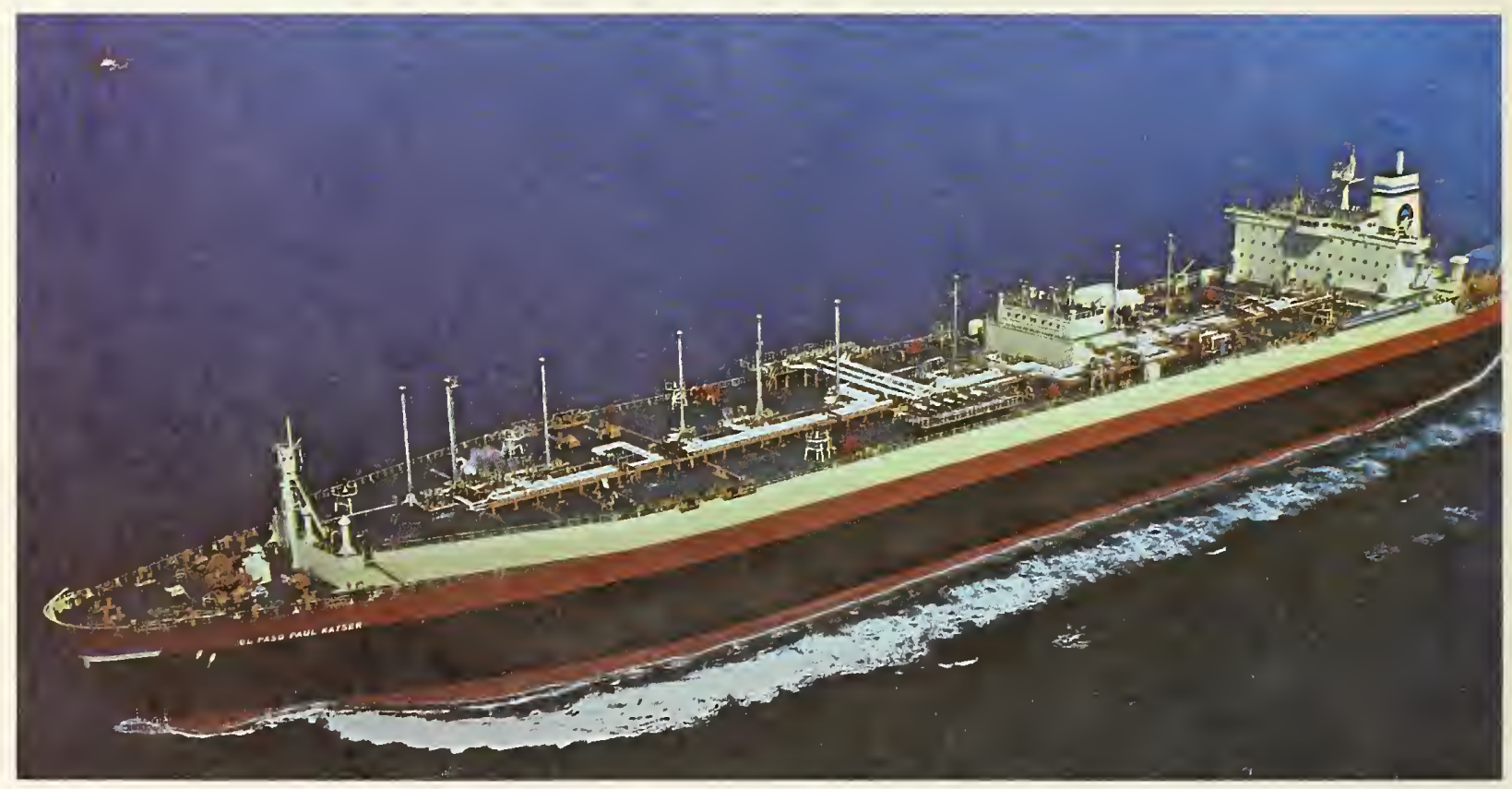

\section{LMG EL PASO PAUL KAISER}

The LNG tanker El Paso Paul Kaiser was one of three Gaz Transport membrane type 125,000 cubic meter ships built by Ateliers et Chantiers de Dunkerque (France). The ships were intended to deliver LNG from Arzew and Skikda, Algeria, to Everret, Massachusetts (U.S.), Elba Island, Georgia (U.S) and Cove Point, Maryland (U.S).

The field tests of the LNG sampling and analysis equipment were conducted on a sister ship, the El Paso Consolidated, and are described in the Measurement Manual. In addition, the methods of volume measurement of the individual membrane tanks are described in the Manual, and a value for estimated error is presented. (Photograph by courtesy of The Japan Gas Association, Tokyo, Japan, on behalf of the 1981 Japan. LNG Congress) 


\section{2 . 0 MEASUREMENT ELEMENTS}

Sampling and Analys is

2.2 LNG Sampling and Analysis

2.2.1 Sampling Measurement Program Summary

$2 \cdot 2-2$

2.2.2 Sampling Measurement Program Scope

$2 \cdot 2-3$

$2 \cdot 2 \cdot 3$

Previous Work

$2 \cdot 2-4$

$2 \cdot 2 \cdot 4$

2. 2.4 .1

2. 2.4 .2

2. 2.4 .3

2.2 .4 .4

2. 2.4 .5

2. 2.4 .6

2.2 .4 .7

2.2 .4 .8

2. 2.4 .9

2.2 .4 .10

2 . 2.4 . 11

2. 2.5

2.2.5. 1

2.2 .5 .2

2.2 .5 .3

2.2 .5 .4

2.2 .5 .5

2.2 .5 .6

2.2 .5 .7

2. 2.5 .8

2.2 .5 .9

2.2 .5 .10

2.2 .6
Gas Analysi

Comparison Between Gas Analysis Methods

Gas Chromatograph (GC) System

Mass spectrometer (MS) System

Results of Comparison Tests

Detailed Evaluation of GC Analysis System.

GC Column Configurations and Digital Integrators

GC Column Confiqurations and Column Packings

Digital Integrator Evaluation.

GC Analysis Accuracy and Precision

Sample Handling Procedures

Conclusions and Recommendations

LNG Sampling

Laboratory Test

Ins t r umen $\mathbf{t}$ a $\mathbf{t}$ i on

Safety Features

Experimental Procedure

Experimental Results.

Probe Evaluation

Vaporizer Evaluation.

Field Tests at NBS Flow Facility.

Shipboard Test s

Conclusions and Recommendations

References
$2 \cdot 2-7$

$2 \cdot 2-7$

2. $2-7$

$2.2-11$

2. 2-11

2. 2- 13

2. 2-15

2. 2-16

2. $2-21$

2. 2-2 1

2. 2-2 2

2. 2-23

2. $2-24$

2. 2-25

2. 2-26

2. 2-27

2. 2- 27

2. 2-29

2. 2-29

2. 2-35

2. $2-47$

2. 2-5 1

2. $2-68$

2. 2-71 



\subsection{LNG Sampling and Analysis}

The Importance of accurate sampling and analysis of LNG cannot be overemphasized. The results of these measurements are used in several of the other elements which provide a measure of LNG quantity and qual1ty. Sampling means the acquiring of a relatively small quantity of LNG which is identical in all respects to the bulk liquid mixture. Direct analysis of the liquid phase is, at the present time, not within the contemporary state of the art, and, therefore, this representative LNG sample must be vaporized in such a way that the mixture composition is not changed in any manner. The gas phase must be 1 dentlcal to the liquid phase in respect to composition. State of the art gas analysis is then able to identify the molecular species and to establish the fraction of each component in the mixture sample.

Mixture composition is used directly to calculate the salorific or heating value of the LNG mixture. The calculation method is described in section 2.3 of this manual. In like manner the mixture fraction may be used with measured LNG pressures and temperatures to predict the liquid density over a broad range of conditions both in the liquid and gas phases. A number of calculation methods are described in section 2.4 .3 of this manual, and specific examples are given in section 3 .

This section of the manual contains a detailed description of the development and evaluation of an LNG sampling measurement system. The text is an edited version of the work of Parrish, Arvidson and LaBrecque [1]. The choice of this particular work was based on the completeness of the treatment of the sampling and analysis measurement process as applied to LNG. The project, conducted by the Boulder Laboratories of NBS, was sponsored by the LNG Sampling Measurement Committee, a natural gas industry consortium. It should be noted that some of the tables and figures contain dimensions which are not in SI terms. Duplicate units were used in the original publication to accommodate the intended audience and are included here to provide continuity with the original work.

The concept of LNG sampling, vaporization and analysis is basically quite simple and straightforward. The complete process, including all three sub-components, had not been treated extensively, if at all, in the technical literature. Since the objective of the study was the development of a recommended fleld-type measurement system, extreme care was exercised by Parrish, et al. in examining the separate components of sampling, vaporizing and gas analysis and just how the individual components would operate when assembled into a single self-contained measurement system. Because of the importance of the sampling and analysis process, it was deemed necessary to include most of the original study which contained the selection decisions and supporting data.

The main body of the study begins with the selection of the gas analysis method in sections 2.2 .4 .1 through 2.2.4.4. Of the various methods available, gas chromatography and nass spectrometry were chosen as having the necessary accuracy, precision and industry acceptance as reliable measurement devices.

Gas chromatography is based on the separation of mixtures into their constituents by preferential adsorption on a solid or liquid, and mass spectrometry is based on the separation of mixtures based on relative mass of the ionized constituent or molecular fragment of the constituent. Each method has attractive features, and available equipment was assessed by Parrish, et al. in order to select the one method best suited for use as a field-operated LNG analysis system. Comparison testing was described and criteria for selection of the gas chromatagraph was given.

The analysis method was selected, and it was then necessary to establish the accuracy and precision of the instrument over a broad range of anticipated operating conditions and with different kinds of auxillary equipment. This included operation with various adsorption column conflgurations, column packings and digital integrators used to integrate the response curve of the detector. The extensive testing and selection process is described in sections 2.2.4.5 through 2.2.4.9.

It was then possible to begin the assessment of various types of sampling and vaporizlng methods and equipment. These devices are generally custom fabricated for field installation. The study limitnd the sampling to that of flowing LNG in a pipeline, but it was believed that the equipment and techniques which were developed could be used in other applications. The proven gas analysis equipment was used to experimentally verify the sampling process. 
The actual description of the sampling investigation is covered in section 2.2.5. Different sampling tap configurations and several types of vaporizer designs were tested and evaluated. Testing was conducted under controlled laboratory conditions, then in a controlled field-type facility and finally with an actual LNG shipboard installation which consisted of the most favorable configurations of sampling tap, vaporizer and gas analysis equipment.

The conclusions and recommendations are extensive and detailed as are most of the evaluation testing. However, the document is the most comprehensive work on the subject, and to accept only the shipboard design as a recommended system could lead to problems in application where design constraints are significantly different, such as with a land-based LNG peakshaving storage tank. A broad base of data and technique result in an understanding of the entire process and should allow useful applications.

\subsubsection{Sampling Measurement Program Summary [1]}

Laboratory and field test results showed that the total uncertainty in the computed heating value of samples taken with the recommended sampling system could be made routinely to within \pm 0.3 percent: this included the \pm 0.1 percent uncertainty in analysis by gas chromatography. Three sample probes and two vaporizer designs were considered. Of the ten operating variables considered, six were found to be important in sampling. Test results were used to establish recommended design and operating criteria.

Accurate LNG composition determination requires:
a) a sample probe for drawing a sample representative of the fluid passing the sampling point,
b) a sample conditioner for completely vaporizing the sample,
c) a gas analyzer for accurately and precisely analyzing the sample composition, and
d) a correct set of operating parameters and procedures.

A systematic study evaluating all of the above components was confined to the problem of sampling from a flowing LNG stream. Instead of directly measuring the heating value by combustion calorimetry, the gas composition was analyzed so that both heating value and liquid density could be computed.

Gas chromatography was selected for analyzing vaporized LNG samples over mass spectrometry, because it was found to be more precise and accurate. By using a gas chromatograph-programmable integrator system, the total uncertainty in the heating value (on a unit volume basis), as computed from the analyzed composition, routinely can be less than \pm 0.1 percent. This figure includes a \pm 0.03 percent uncertainty in the calibration gas composition; this uncertainty, which neglects the uncertainty of the individual components heating value (see section 1.3 of this manual), is based only on the uncertainty in the weighing process used to prepare the mixtures. The \pm 0.1 percent uncertainty also includes a random error of \pm 0.06 percent. The random error is three times the estimated standard deviation of \pm 0.02 percent. This value represents the maximum value obtained from over 100 measurements of three or more repetitive analyses employing a properly operating gas chromatograph-programmable integrator system. Procedures and equipment are recommended for analyzing vaporized LNG samples.

The results of sampling tests at a NBS field site (see 2.2.5.8) show that the total uncertainty of a single measurement in sampling and analyzing LNG mixtures can be less than \pm 0.3 percent in the computed heating value. This uncertainty includes the \pm 0.03 percent uncertainty in the calibration gas composition plus an uncertainty of \pm 0.27 percent for random error. The \pm 0.27 percent figure represents three times the upper limit of the standard deviation determined for the combined sampling plus analysis measurement process. The recommended sampling system consists of a side tap probe, a steam or electrically heated tube vaporizer, a gas mixing chamber and a sampling manifold. Sampling system design criteria are recommended, including ranges of the operating variables found to affect sampling precision.

Data obtained during a shipboard test has limits of precision of \pm 0.30 percent. very favorably to the \pm 0.27 percent obtained on the flow facility. 


\subsubsection{Sampling Measurement Program Scope}

Composition plays an important role in determining the total dollar value of LNG shipments. Sampling LNG presents special problems because of possible fractionation during the sampling process. Accurate and precise composition determination of LNG mixtures (and other cryogenic liquid mixtures) requires a Sampling-Measurement System (SMS) which contains:

a) a sampling probe which draws a sample without altering the composition,

b) a sample conditioner which completely vaporizes the sample, and

c) a gas analyzer which accurately and precisely analyzes the sample.

Also, the SMS may include facilities for collecting representative samples of vaporized LNG for transport from the sampling point to the analytical laboratory. The proper operating parameters and procedures must be specified to minimize the total uncertainty in the measurement process.

The project consisted of two primary tasks:

a) the evaluation of vaporized LNG analysis methods, and

b) the evaluation of LNG sampling techniques.

Possible alternatives were carefully evaluated for each of the components of the SMS. Because of the time limitations, only the most promising alternatives were considered in depth. The study covered only sampling from flowing LNG streams. Emphasis was on compositions typical of Algerian LNG as shown in table 2.2.1. The values in parentheses are the range of compositions included in this study.

Table 2.2.1

The Anticipated Composition Range of Algerian LNG

\begin{tabular}{|l|l|}
\hline Component & Composition Range, mol percent \\
\hline \hline nitrogen & 0.60 to $1.40(0$ to 11$)$ \\
methane & 84 to $92(65$ to 90$)$ \\
ethane & 6 to $9.5(5$ to 18$)$ \\
propane & 2.2 to $3.5(1$ to 5.4$)$ \\
isobutane & 0.3 to $0.5(0$ to 0.8$)$ \\
normal butane & 0.3 to $0.7(0.2$ to 4.8$)$ \\
pentanes and heavier & 0 to $0.02(0$ to 0.3$)$ \\
\hline
\end{tabular}

The initial phase of work evaluated the relative merits of analyzing vaporized LNG samples by gas chromatography (GC) and mass spectrometry (MS); accuracy, precision and simplicity were the primary factors considered. The second phase of work was a study of the effect of various parameters on the accuracy and precision of analysis by GC; the work included an evaluation of GC columns and GC data processors (i.e.,integrators). Gas mixtures prepared by weight were used in all of these tests.

The sampling portion of the study contained laboratory tests and field confirmation tests; these tests were performed at the NBS LNG Flow Facility and aboard the LNG tanker El Paso Consolidated.

The laboratory tests evaluated three probes - a pitot tube, a side tap and a reference probe - and two continuous vaporizers - vaporizer A and a reference vaporizer. To separate effects caused by the vaporizer from those of the probes, the probes were used with a reference vaporizer and vaporizer A with the reference probe. The laboratory evaluation provided a rapid way to determine the effect of a given operating variable while holding other variables constant. The variable's effect upon sampling accuracy was determined by using mixtures of known composition. In addition to the three probes and two vaporizers, the following operating variables were considered:

a) flow rate of the liquid stream,

b) flow rate through the sampling device,

c) amount of subcooling (pressure differential between the sample point and the saturated liquid),

d) temperature of the LNG,

e) pressure drop upstream of the sample vaporizer,

f) time-averaging the sample,

g) vaporizer outlet temperature, and

h) composition, particularly the effect of pentane and higher hydrocarbons. 
The field confirmation tests provided a scale-up test for the conclusions obtained from the laboratory tests. Two series of tests were made in the NBS flow facility. Here, operating conditions were closer to the conditions of actual LNG facilities in respect to size, pressures and flow rates. However, there was no way to directly determine the correct composition of the mixture in the flow facility. Also, operating conditions were not as constant and well-defined as in the laboratory apparatus.

The shipboard tests provided an evaluation of a LNG sampling system under conditions comparable to those in LNG custody transfer operations. The primary variable evaluated in these tests was sampling rate. The actual LNG composition was unknown. However, comparisons were made between liquid samples taken from the inlet header to the ship's vaporizer and gas samples taken from the vaporizer outlet header. This provided a check on the liquid sampler performance.

In LNG custody transfer, the composition is important because it is needed to compute the heating value and sometimes liquid density. Experimental results are compared using compositions, computed heating values and computed liquid densities. Several sets $[2,3]$ of ideal gas heating values are available.

The values proposed by the American Gas Association [2] were chosen because, with their method of correcting for gas nonidealities, computed heating values could be directly compared with the results of combustion calorimetry. For convenience, the non-ideality correction was omitted in most of the work. However, in the LNG flow facility tests, the non-ideality correction was necessary to make direct comparison with calorimeter measurements. The work of Armstrong and Jobe, which is the basis of section 1.2 and 1.3 of this manual, present ideal and real gas combustion enthalpy values based on the current assessment of basic properties and other data and supersedes the data in table 2.2 .2 . However, to maintain the consistency of the original Parrish, et al.study, the A.G.A. values are presented and used here (editor's note).

Table 2.2.2 Pure Component Ideal Gas Heating Values [2]

\begin{tabular}{|l|c|c|}
\hline \multirow{2}{*}{ Component } & \multicolumn{2}{|c|}{ Heating Value } \\
& Btu/scf & MJ/m3 \\
\cline { 2 - 3 } & - & $-56 \mathrm{kPa}, 288.71 \mathrm{~K}$ \\
\hline nitrogen & 1012.1 & 37.701 \\
methane & 1773.0 & 66.045 \\
ethane & 2523.3 & 93.994 \\
propane & 3260.7 & 121.46 \\
iso butane & 3269.8 & 121.80 \\
normal butane & 4008.7 & 149.33 \\
iso pentane & 4018.9 & 149.71 \\
normal pentane & 4764.4 & 177.48 \\
hexane, average & 5509.7 & 205.34 \\
heptane, average & & \\
\hline
\end{tabular}

\subsubsection{Previous Work}

A search of the literature has not produced a systematic evaluation of a complete LNG Sampling Measurement System (SMS). However, there are publications on individual parts of the (SMS) such as gas analysis and on LNG sampling techniques.

Gas Analysis Publications comparing gas chromatographic (GC) and mass spectrometric (MS) analyses of natural gas have not been found in the open literature. However, ASTM standards exist for natural gas analyses by gas chromatography [7] and mass spectrometry [8]. Each standard gives the estimated reproducibility of analyses; values are given for the case of one laboratory and apparatus, and for the case of different laboratories. The ASTM standards indicate that the analyses should be considered suspect if differences between replicate analyses are greater than those given in table 2.2.3; also, the table gives the estimated error in heating values for these differences assuming the composition range given in table 2.2 .1 . 
Table 2.2 .3

Est Imates of Reproducibility between Replecate Natural Gas Analysis Using ASTM $[7,8]$ Procedures for Same Operator and Apparatus

(a) Same Operator and Apparatus

\begin{tabular}{|l|l|l|l|l|}
\hline \multirow{2}{*}{ Component } & \multicolumn{2}{|c|}{ Gas Chromatagraph } & \multicolumn{2}{c|}{ Mass Spectormeter } \\
\cline { 2 - 5 } & mol \% & $\begin{array}{l}\text { \% error in } \\
\text { heating value }\end{array}$ & mol \% & $\begin{array}{l}\text { \% error in } \\
\text { heating value }\end{array}$ \\
\hline \hline nitrogen & 0.03 & 0.07 & 0.2 & 0.4 \\
methane & 0.3 & 0.4 & 0.2 & 0.3 \\
ethane & 0.15 & 0.07 & 0.1 & 0.05 \\
propane & 0.05 & 0.01 & 0.02 & 0.01 \\
i-butane & 0.03 & 0.03 & - & - \\
n-butane & 0.03 & 0.03 & - & - \\
pentanes & 0.03 & $0.04^{*}$ & - \\
\hline
\end{tabular}

(b) Different Operators and Apparatus

\begin{tabular}{|l|l|l|l|l|}
\hline nitrogen & 0.06 & 0.1 & 0.3 & 0.7 \\
methane & 0.6 & 0.8 & 0.5 & 0.6 \\
ethane & 0.20 & 0.1 & 0.3 & 0.1 \\
propane & 0.1 & 0.02 & 0.06 & 0.02 \\
i-butane & 0.06 & 0.06 & - & - \\
n-butane & 0.06 & 0.06 & - & - \\
pentanest & 0.06 & $0.04 *$ & - & - \\
\hline
\end{tabular}

* Error in pentanes+ limited to 0.02 mol percent which is the maximim anticipated composition.

There are several papers discussing different GC column configurations; these are discussed in section $2 \cdot 2 \cdot 4 \cdot 6$ and $2 \cdot 2 \cdot 4 \cdot 7$

LNG Sampling Dalmaze [9] and Trigo [10] discussed two similar continuous SMS which are used in Europe. Figure 2.2.1 is a schematic of Dalmaze's system. Both use an upstream facing pitot tube located at the LNG pipe centerline as the probe. To prevent premature vaporization the probe line is cooled by an outer jacket through which LNG is circulated and returned to the main line. The sample then flows through a check valve to prevent backflashing and past an electrically heated vaporizer. Trigo's system replaces the check valve with a filter. The vaporized sample goes through a vessel to dampen pressure pulsations (if operating at less than 30 psig $(0.3 \mathrm{MPa})$ ), past a pressure controller, and to a chromatograph. Neither author describes the details of the gas analyses. Dalmaze's data showed a precision of \pm 0.6 percent in the computed heating value of liquid samples taken upstream of a LNG vaporizer; by sampling the totally vaporized LNG stream the precision was 0.4 percent. (The precision is defined here as three times the estimated standard deviation.) The difference in the average computed heating values between the two sampling methods is about 0.01 percent when 30 sets of samples were averaged over seven hours. Dalmaze reports that this difference is well within the error due to gas analyses alone.

There are at least two batch sampling devices which have been tried. One is the cryogenic sampler [11] shown in figure 2.2.2. LNG passes through a cooling passage which surrounds a cup contained $1 n$ an evacuated outer container. Once the cup is at LNG temperature the sample valve is opened and the cup is filled with liquid. After the cup is full, the sample valve is closed, the sample lines are disconnected, and the sampler is allowed to warm to ambient temperature. The ratio of the cup volume to the total chamber volume is such that the final pressure will be approximately 500 psig ( 3.6 Mpa) for LNG. A valve at the bottom of the sampler is used to draw a gas sample for analyses.

The other batch sampling device which collects the liquid is described by Cook [12] and is shown In figure 2.2.3. To take a sample, both valves are opened to allow LNG to flow through the sampler whlch is well insulated. Once the sampler is at LNG temperature, as evidenced by a continuous l1quld 10 w from the device, both valves are closed. Then the device is removed from the sampling line and allowed to warm up. After the initial pressure rise, a pressure relief valve which is set above the cricondenbar (the maximum pressure at which a mixture may exist in two phases) of the mixture (>2000 psi (13.8 MPa)) keeps the sample at a constant pressure and in one phase. Once the sampler $1 \mathrm{~s}$ warm $1 \mathrm{t}$ can be connected directly to an analytical instrument. 


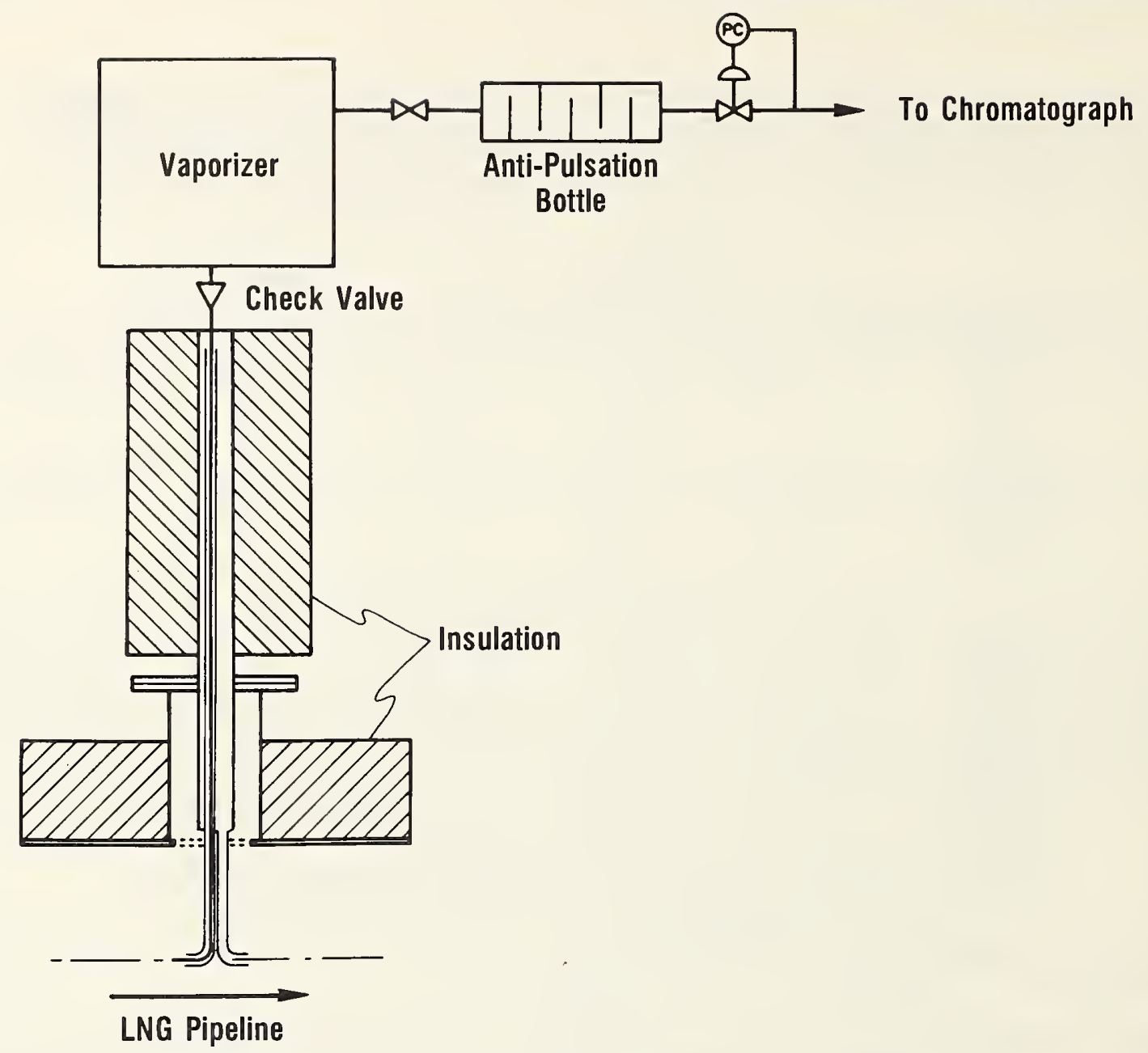

Figure 2.2.1 Schematic of continuous sampling system of Dalmaze [9].

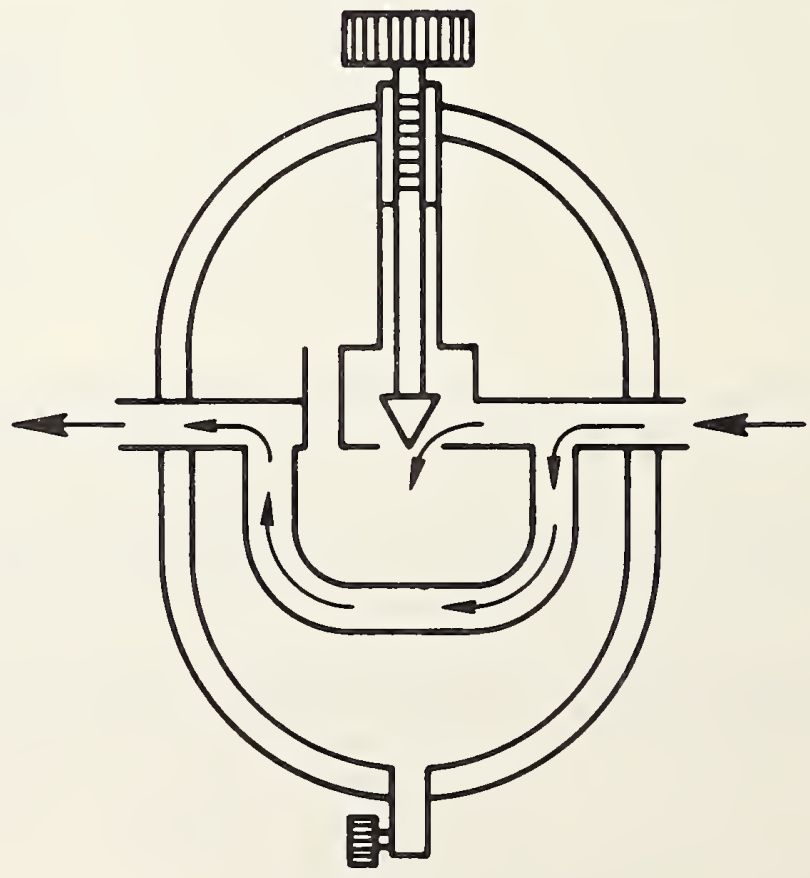

Figure 2.2.2 Schematic of cryogenic sampler [11]. 


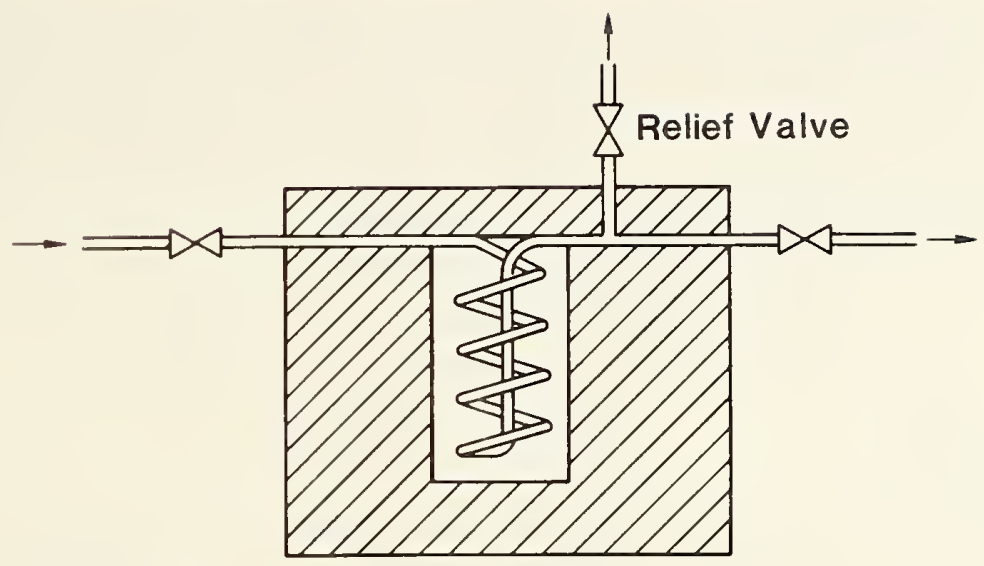

Figure 2.2.3 Schematic of LNG sampler described by Cook [12].

Methods for collecting a gas sample from a pipeline have been discussed by Miller [13]; also, an ASTM standard exists for natural gas sampling from pipelines [14]. Miller concludes that all of the following filling methods give good results;
a) Iiquid displacement,
b) purge by fill and empty,
c) purge at a constant flow rate, and
d) fill of evacuated cylinder with no purge.

Also, he briefly describes the two batch sampling devices used for collecting LNG as a liquid which were described above.

Finally, Chapman and Payne [15] give some general guidelines for sampling flowing streams. Although they do not describe cryogenic sampling systems, many of their guidelines still apply.

\subsubsection{Gas Analysis}

The uncertainty involved in gas analyses was determined prior to taking any sampling data. This evaluation and a comparison between gas chromatography (GC) and mass spectrometry (MS) were made early in the project. Later, more careful evaluation was made of the effect of various operating variables, column configurations, and digital integrators on analyses precision and accuracy. The appendix of the original study [1] lists all of the data obtained from the gas analyses tests.

\subsubsection{Comparison Between Gas Analysis Methods}

A comparison of precision and accuracy of GC and MS analyses of LNG type mixtures was made by hav 1 ng a local MS laboratory analyze some prepared mixtures; these mixtures also were analyzed using a GC. The MS laboratory was capable of general quantitative analyses, including hydrocarbon analyses, on a routine basis.

\subsubsection{Gas Chromatograph System (GC) System}

In this phase of the work a gas chromatograph was used which contained two columns and a f1lament-type thermal conductivity detector, in conjunction with a programmable, digital integrator (integrator A). Traditionally, whenever feasible, peak heights were preferred over peak areas for comput 1 ng compositions from GC analyses. The reasons for this were that peak heights were easler to measure and were often more precise than the mechanical devices available for measuring peak areas. However. programmable integrators eliminate the drawbacks of using peak areas. Several sets of cal1bratlons and analyses were made using both peak heights and peak areas. (Integrator A reports peak areas and/or peak heights.) These tests showed that the compositions based on peak helghts had an estlmatrd standard deviation which was four to five times greater than the estimated standard dev1ations cor the compositions computed from peak areas. Based on these results and the fact that the 11 near responso between peak height and composition has a limited range, peak areas were used throughout th1s work. 
Table 2.2.4 Iists the GC operating conditions. Figure 2.2.4 is a schematic of the GC and ancillary equipment while figure 2.2 .5 shows a chromatogram (on a logarithmic scale) for a gas mixture typical of Algerian LNG. The GC contained a sample valve which was used to inject the gas sample from either of two sample loops into the carrier gas stream. Helium carrier gas was maintained at a constant flow by using two stages of pressure regulation. The column bypass valve was used to bypass Column 2 . In the bypass mode the carrier gas passed through a needle valve set to have the same pressure drop as Column 2; this minimized the baseline upset during valve switching.

Table 2.2 .4

Gas Chromatograph Operating Conditions

\begin{tabular}{|lc|}
\hline Carrier gas flow rate & $27 \mathrm{cc} / \mathrm{min}(14.7 \mathrm{psia}(0.1 \mathrm{MPa}))$ \\
Sample loop volumes & approx. $0.1 \mathrm{~cm}$ each \\
Sample loop temperature & $90^{\circ} \mathrm{C}$ \\
Column 1 temperature & $25^{\circ} \mathrm{C}$ \\
$\left(10\right.$ feet $(3.0 \mathrm{~m})$ of $1 / 8$ inch $\left(3.2^{\circ} \mathrm{mm}\right)$ of Durapak*, 60/80 mesh) \\
Column 2 temperature & $25^{\circ} \mathrm{C}$ \\
$\left(6\right.$ feet $(1.8 \mathrm{~m})$ of $1 / 8$ inch $(3.2 \mathrm{~mm})$ of Porapak $\left.Q^{*}, 60 / 80 \mathrm{mesh}\right)$ \\
Detector temperature & $120^{\circ} \mathrm{C}$ \\
Filament current & $220^{\circ} \mathrm{mA}$ \\
\hline
\end{tabular}

* Certain commercial equipment, instruments, or materials are identified in this study in order to adequately specify the experimental procedure. In no case does such indentification imply recommendation or endorsement by the National Bureau of Standards, nor does it imply that the material or equipment identified is necessarily the best available for the purpose.

The integrator had a variety of operating parameters and timed events which could be used to maximize the precision of the data reduction of a chromatogram. The use of these parameters is illustrated in the next section.

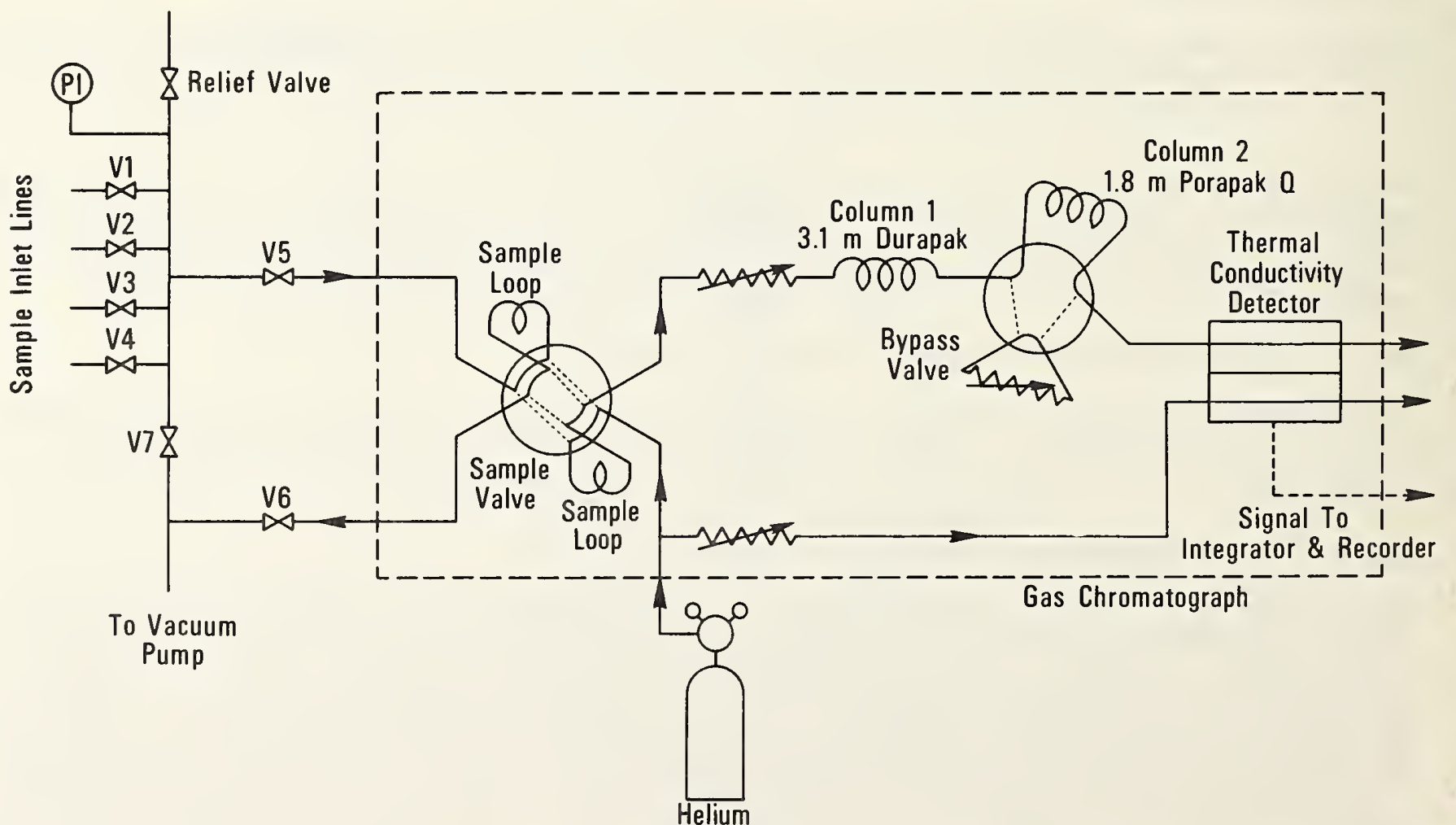

Carrier Gas

Figure 2.2.4. Flow schematic of two column gas chromatography system. 
Calloration Procedure All GC's require calibration with one or more mixtures of known composition; these mixtures should have a composition similar to that of the unknown mixtures. In this work all of the calibration mixtures were prepared by weight using high purity components; table 2.2 .6 ists the component concentrations and their estimated uncertainties for all the prepared mixtures used here.

To make a calibration run, the sample loop first was purged by alternately evacuating and filling with the calibration gas. It was found that one to three purges were adequate, provided that the manifolding was arranged so that all of the gas passed through the sample loop during purging (see figure 2.2.4). However, it was imperative that all connections were leak-tight.

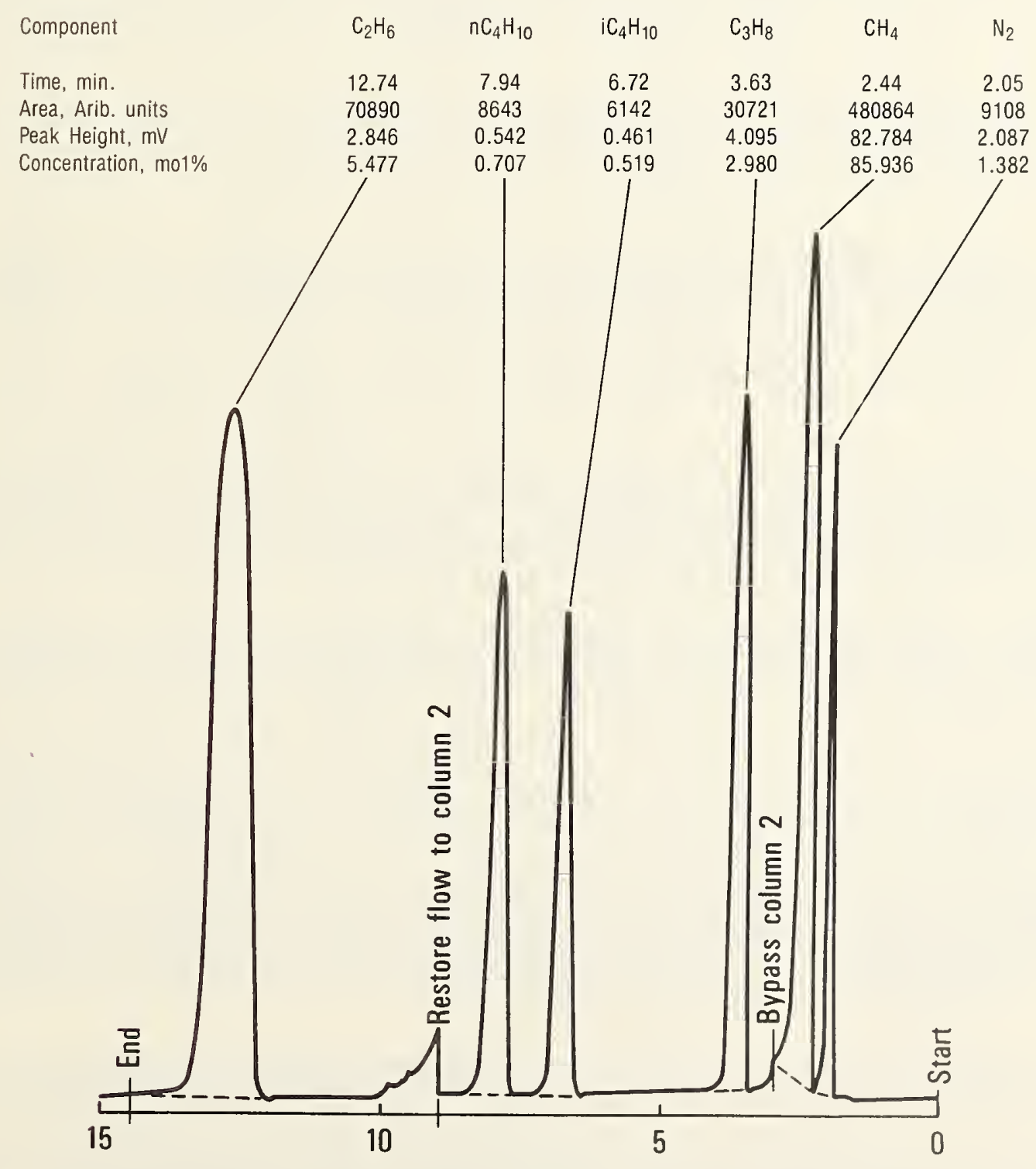

TIME, mIn

Figure 2.2.5. A chromatogram of a natural gas mixture. The peak heights are shown on a logarithmic scale. 
Table 2.2.6. Compositions, Ideal Gas Heating Values with Estimated Uncertainty for All Mixtures Prepared by Weight Used in This Study

\begin{tabular}{|c|c|c|c|c|c|c|c|c|c|c|}
\hline \multirow[t]{2}{*}{ Mixture } & \multicolumn{8}{|c|}{ Composition in mol percent } & \multicolumn{2}{|c|}{ Heating Value 2} \\
\hline & $\mathrm{N}_{2}$ & $\mathrm{CH}_{4}$ & $\mathrm{C}_{2} \mathrm{H}_{6}$ & $\mathrm{C}_{3} \mathrm{H}_{8}$ & $i_{4} \mathrm{H}_{10}$ & $\mathrm{nC}_{4} \mathrm{H}_{10}$ & $i_{C_{5}} \mathrm{H}_{12}$ & $\mathrm{nC}_{5} \mathrm{H}_{12}$ & $\mathrm{Btu} / \mathrm{scf}$ & $\mathrm{MJ} / \mathrm{m}^{3}$ \\
\hline A & $\begin{array}{r}4.316 \\
\pm 0.005\end{array}$ & $\begin{array}{l}81.249 \\
\pm 0.008\end{array}$ & $\begin{array}{r}4.746 \\
\pm 0.005\end{array}$ & $\begin{array}{r}4.866 \\
\pm 0.001\end{array}$ & $\begin{array}{r}2.409 \\
\pm 0.002\end{array}$ & $\begin{array}{r}2.413 \\
\pm 0.002\end{array}$ & nnon & $\cdots$ & $\begin{array}{r}1186.7 \\
\pm 0.3\end{array}$ & $\begin{array}{l}44.205 \\
\pm 0.011\end{array}$ \\
\hline B & $\begin{array}{r}1.349 \\
\pm 0.002\end{array}$ & $\begin{array}{l}88.118 \\
\pm 0.003\end{array}$ & $\begin{array}{r}8.392 \\
\pm 0.002\end{array}$ & $\begin{array}{r}0.978 \\
\pm 0.001\end{array}$ & $\begin{array}{c}0.481 \\
\pm 0.0004\end{array}$ & $\begin{array}{c}0.682 \\
\pm 0.0004\end{array}$ & - & nno & $\begin{array}{r}1103.3 \\
\pm 0.1\end{array}$ & $\begin{array}{l}41.098 \\
\pm 0.004\end{array}$ \\
\hline c & $\begin{array}{r}0.601 \\
\pm 0.002\end{array}$ & $\begin{array}{l}90.622 \\
\pm 0.002\end{array}$ & $\begin{array}{r}6.027 \\
\pm 0.002\end{array}$ & $\begin{array}{r}2.154 \\
\pm 0.001\end{array}$ & $\begin{array}{c}0.300 \\
\pm 0.0004\end{array}$ & $\begin{array}{c}0.306 \\
\pm 0.0004\end{array}$ & nnon & -non & $\begin{array}{r}1098.2 \\
\pm 0.1\end{array}$ & $\begin{array}{l}40.909 \\
\pm 0.004\end{array}$ \\
\hline D & $\begin{array}{l}0.0 \\
0.0\end{array}$ & $\begin{array}{l}85.317 \\
\pm 0.008\end{array}$ & $\begin{array}{r}5.077 \\
\pm 0.004\end{array}$ & $\begin{array}{r}4.855 \\
\pm 0.001\end{array}$ & $\begin{array}{l}0.0 \\
0.0\end{array}$ & $\begin{array}{r}4.751 \\
\pm 0.002\end{array}$ & $\cdots$ & $\cdots$ & $\begin{array}{r}1231.4 \\
\pm 0.2\end{array}$ & $\begin{array}{l}45.870 \\
\pm 0.007\end{array}$ \\
\hline E & $\begin{array}{r}1.382 \\
\pm 0.002\end{array}$ & $\begin{array}{l}85.936 \\
\pm 0.003\end{array}$ & $\begin{array}{r}8.477 \\
\pm 0.002\end{array}$ & $\begin{array}{r}2.980 \\
\pm 0.001\end{array}$ & $\begin{array}{c}0.519 \\
\pm 0.0004\end{array}$ & $\begin{array}{c}0.707 \\
\pm 0.0004\end{array}$ & -no- & & $\begin{array}{r}1135.3 \\
\pm 0.1\end{array}$ & $\begin{array}{l}42.290 \\
\pm 0.004\end{array}$ \\
\hline F & $\begin{array}{r}0.973 \\
\pm 0.002\end{array}$ & $\begin{array}{l}88.225 \\
\pm 0.003\end{array}$ & $\begin{array}{r}7.259 \\
\pm 0.002\end{array}$ & $\begin{array}{r}2.561 \\
\pm 0.001\end{array}$ & $\begin{array}{c}0.490 \\
\pm 0.0004\end{array}$ & $\begin{array}{c}0.492 \\
\pm 0.0004\end{array}$ & -non & -...... & $\begin{array}{r}1118.3 \\
\pm 0.1\end{array}$ & $\begin{array}{l}41.657 \\
\pm 0.004\end{array}$ \\
\hline G & $\begin{array}{r}11.090 \\
\pm 0.002\end{array}$ & $\begin{array}{l}65.732 \\
\pm 0.003\end{array}$ & $\begin{array}{l}16.283 \\
\pm 0.002\end{array}$ & $\begin{array}{r}5.443 \\
\pm 0.001\end{array}$ & $\begin{array}{c}0.490 \\
\pm 0.0004\end{array}$ & $\begin{array}{c}0.963 \\
\pm 0.0004\end{array}$ & - & - & $\begin{array}{r}1138.8 \\
\pm 0.1\end{array}$ & $\begin{array}{l}42.421 \\
\pm 0.004\end{array}$ \\
\hline $\mathrm{H}$ & $\begin{array}{c}0.599 \\
\pm 0.0004\end{array}$ & $\begin{array}{l}90.070 \\
\pm 0.0007\end{array}$ & $\begin{array}{c}6.537 \\
\pm 0.0004\end{array}$ & $\begin{array}{c}2.200 \\
\pm 0.0001\end{array}$ & $\begin{array}{r}0.2912 \\
\pm 0.0002\end{array}$ & $\begin{array}{r}0.2837 \\
\pm 0.0002\end{array}$ & $\begin{array}{r}0.0101 \\
\pm 0.0002\end{array}$ & $\begin{array}{r}0.0113 \\
\pm 0.0002\end{array}$ & $\begin{array}{c}1102.7 \\
\pm 0.03\end{array}$ & $\begin{array}{l}41.075 \\
\pm 0.001\end{array}$ \\
\hline
\end{tabular}

1 The uncertainty is based only on the error in the weight measurement; details of this error analysis are given in [32].

${ }^{2}$ The ideal gas heating value is computed as the sum of the mol fraction of each component times its ideal gas heating value at standard conditions [2]. The uncertainty in the heating value is computed as the sum of uncertainties in the mol fraction of each component times its ideal gas heating value.

After purging, the sample loop was filled to the desired pressure - in this case 2 psig (0.11 MPa) and the sample valve was switched, injecting the mixture into the carrier gas stream. During the first three minutes of the run Column 2 remained in series; this allowed the nitrogen and methane to travel through both columns and the detector. (These columns did not resolve oxygen and nitrogen; therefore, any oxygen present was included in the nitrogen peak.) Once the methane was out of Column 2 , the column was bypassed, trapping the ethane. The integrator made a "forced baseline" just prior to the valve switch and ignored the tailing baseline after the switch. (The dotted lines under each peak in figure 2.2.5 represent the baseline as determined by the integrator.) The integrator then picked the bottom of the baseline drift as the baseline for the propane peak. Column 2 remained bypassed until the butanes passed through Column 1. At 9 minutes Column 2 was switched in again, allowing ethane to elute from the column. As before, the integrator ignored the baseline upset due to valve switching. The tailing after switching valves was caused by a temporary decrease in carrier gas flow as pressure gradients in the line were being re-established. The two peaks on the second tail were caused by minute air leakage during the valve switch. This leakage is caused by diffusion and can occur in pressurized helium and hydrogen systems.

The total analyses time was 14.5 minutes. If pentanes were present the same procedure was used, but the analyses time increased to 24 minutes. The prepared mixtures in this study did not contain hexanes.

Using the areas under each peak and the known composition of the calibration gas, relative response factors were computed for each component. Normally, two or three calibration runs were made and the response factors averaged for each component. The GC was calibrated at the beginning of each operating day.

Analysis procedure The procedure for an analyses of an unknown mixture was the same as a calibration run. However, in this case the relative response factors were used with the peak areas to determine the composition. In this work the results were normalized; this eliminated the need to know the exact 
amount of sample injected. However, effort was made to keep the sample amount, i.e., sample pressure, constant to with \pm 5 percent because variations in sample size may cause subtle effects which could d'minish the analytical accuracy.

\subsubsection{Mass Spectrometer (MS) System}

The mass spectrometer used for this work was a 180 degree sector, high precision gas mass spectrometer [16]. It electronically scanned a mass range of two to 200 with the output recorded on a strip chart. By adjusting the sample pressure, the pressure at the controlled leak could be set between 1 to 100 microns $(0.1$ to $13 \mathrm{~Pa})$. The controlled leak permitted a small amount of sample to continuously enter the lon chamber; the leak rate was proportional to the pressure upstream of the controlled leak.

Calibration procedure prior to running a calibration (or analysis), a background scan was made by closing the valve upstream of the controlled leak. Any peaks from the residual gas were noted. Next, high purity hydrocarbons (> 99.99 percent) were injected one at a time at pressures comparable to the expected partial pressures in the unknown mixture. The MS response was slightly nonlinear; however, this effect was negligible as long as the calibration pressure was within 3 to $4 \mathrm{microns}(0.4$ to 0.5 $\mathrm{Pa}$ ) of the components partial pressure in the unknown mixture.

Hydrocarbons fracture inside the ion chamber. Each hydrocarbon has a characteristic cracking pattern and at least one mass peak which is unique to that hydrocarbon. The response factor was computed for each component by dividing the characteristic peak height (corrected for background) by the known calibration gas pressure. (Resolving the iso and normal butane required solving two simultaneous equations.)

Analysis Procedure After evacuating the line connecting the sample cylinder to the MS, the sample pressure was reduced to the desired level by a series of expansions into evacuated volumes. Then, the sample was opened to the controlled leak and a mass scan was made. Using response factors, peak heights (corrected for background) and sample pressure, the composition of each component was calculated.

\subsubsection{Results of Comparison Tests}

Two sets of six samples were analyzed. The first set contained duplicate samples of mixtures A, $B$ and $D$, while the second set contained duplicate samples of mixtures $B, C$ and $D$; the samples were labeled with the approximate composition of the sample. Two analyses were made on each sample. Table 2.2 .7 gives the results of the MS and GC analysis. The composition analyses and the raw data are 1 isted in the original work [1]. Since this was not a statistically balanced test, only a comparison of the bias (i.e., accuracy) and precision could be made; there was no way to determine what factors affected the accuracy and precision. However, it was found that the second set of MS analyses showed a significant improvement in the standard deviation, whereas there was little change in the bias. In all cases both biases and standard deviations for the GC analysis were much smaller than those of the MS analysis.

The MS is an important qualitative and quantitative tool [16]. However, for routine gas analysis, where all of the components are known, a properly designed and operated GC-digital integrator system will be more precise than the MS.

The precision of the MS analysis results obtained in the study is poorer than would be expected based on the ASTM standard [8] (see table 2.2.3). One possible reason is that the reference mixture used In the ASTM standard to determine analytical precision did not include butanes; butanes and heavier hydrocarbons made the data reduction of the mass spectrum much more difficult. Also, the concentration levels of components other than methane were lower in the ASTM mixture than in this study.

In addition to this comparison a tabulation was available of roughly 30 sets of analyses of natural gas mixtures containing 95 mol percent or more methane. Samples of each gas mixture were analyzed by MS in two different industrial laboratories and by GC. Also, the heating value of the m1xture was measured by using combustion calorimetry. Table 2.2 .8 lists the average error and its estimated standard deviation between the computed heating values, based on gas analysis, and the measured values. Clearly, the GC analysis is much better than one MS laboratory and has a better estimated standard deviation than the second MS laboratory. 
Table 2.2 .7

Summary of Biases (B) 1 and Standard Deviations (s) in the Heating value from Gas Chromatographic (GC) and Mass Spectrometer (MS) Analysis

\begin{tabular}{|c|c|c|c|c|}
\hline Mixture & $\begin{array}{c}\text { Analysis } \\
\text { Method }\end{array}$ & $\begin{array}{l}\text { Number } \\
\text { of } \\
\text { Analyses }\end{array}$ & \multicolumn{2}{|c|}{$\begin{array}{l}\text { Heating Value } \\
\text { Btu/scf } \\
\left(\mathrm{MJ} / \mathrm{m}^{3}\right)\end{array}$} \\
\hline \multirow{3}{*}{ A } & & & (B) & (s) \\
\hline & MS & 4 & $-37.3 \pm 9.2$ & 5.8 \\
\hline & GC & 6 & $\begin{array}{c}1.38 \pm 1.13 \\
(0.051 \pm 0.042)\end{array}$ & $\begin{array}{c}0.91 \\
(0.034)\end{array}$ \\
\hline \multirow[t]{2}{*}{ B } & MS & 8 & - & $5.0^{2}$ \\
\hline & GC & 14 & - & $\begin{array}{c}0.50 \\
(0.019)\end{array}$ \\
\hline \multirow[t]{2}{*}{ c } & MS & 4 & $\begin{array}{l}-31.3 \pm 18.0 \\
(-1.16 \pm 0.67)\end{array}$ & $\begin{array}{l}11.3 \\
(0.42)\end{array}$ \\
\hline & GC & 13 & -- & $\begin{array}{c}0.58 \\
(0.022)\end{array}$ \\
\hline \multirow[t]{2}{*}{ D } & MS & 8 & - & $8 \cdot 3^{2}$ \\
\hline & GC & 8 & - & $\begin{array}{l}1.04 \\
(0.039)\end{array}$ \\
\hline$E^{3}$ & GC & 3 & - & $\begin{array}{l}0.95 \\
(0.035)\end{array}$ \\
\hline
\end{tabular}

1 The bias is defined here as the difference between the mean of the measured values and the correct value. The \pm interval is the 99 percent confidence interval for the value.

${ }^{2}$ These values represent the best of two sets of analyses.

3 This mixture was used as the GC calibration gas.

${ }^{4}$ The dashed line (-) means that the confidence interval was larger than the bias.

Table 2.2 .8

Comparison of Average Error and Standard Deviation Between Heating Values Computed from GC, MS and the Heating value Measured by Combustion Calorimetry for Gas Mixtures Containing 95 or Greater Mol Percent Methane

\begin{tabular}{|c|c|c|}
\hline $\begin{array}{c}\text { Analytical } \\
\text { Method }\end{array}$ & $\begin{array}{c}\text { Average Error } \\
\mathrm{Btu} / \mathrm{scf} \\
\left(\mathrm{MJ} / \mathrm{m}^{3}\right) \\
\end{array}$ & $\begin{array}{c}\text { Standard Deviation } \\
\mathrm{BTU} / \mathrm{scf} \\
\left(\mathrm{MJ} / \mathrm{m}^{3}\right)\end{array}$ \\
\hline GC & $\begin{array}{c}1.27 \\
(0.0473)\end{array}$ & $\begin{array}{c}0.55 \\
(0.0205)\end{array}$ \\
\hline MS I & $\begin{array}{c}5.05 \\
(0.1881)\end{array}$ & $\begin{array}{c}3.63 \\
(0.1352)\end{array}$ \\
\hline MS II & $\begin{array}{c}1.17 \\
(0.0436)\end{array}$ & $\begin{array}{c}1.46 \\
(0.0544)\end{array}$ \\
\hline
\end{tabular}

The average error is defined here as the average of the absolute value of the difference between the computed and measured heating value. 


\subsubsection{Detalled Evaluation of GC Analysis System}

Based on the above results the GC was selected to be used in this project. Therefore, a statistically defined test was required to determine the analytical precision and accuracy of the GC; also, the effect of various operating parameters needed to be defined. This test was directed toward finding operator, composition, and day-to-day effects. Two operators were used, four mixtures (mixtures C, E, $F$ and $G$, table 2.2.6) and tests were run for four days. Figure 2.2.6 shows the computed ideal gas heating values for mixture $E$ (the calibration gas) as a function of chronological order and operator; the o error bar represents one estimated standard deviation ( 0.20 percent) in the heating value. Although there was no operator dependence, there was a day-to-day shift in the average value; this probably was due to calibration shifts. Also, there were apparent changes in the scatter from day to day which could not be explained; however, excluding the outlier on the last day, the total scatter was 0.09 percent.

The outlying point on the fourth day illustrates an instrument malfunction discovered in this test. Roughly 20 percent of the analyses contained one component, usually nitrogen, methane or ethane, whose composition was more than four estimated standard deviations from the average value. During this period, the integrator started malfunctioning and had to be returned to the factory. After receiving the repaired instrument another test was performed, this time using only one operator and one gas mixture.
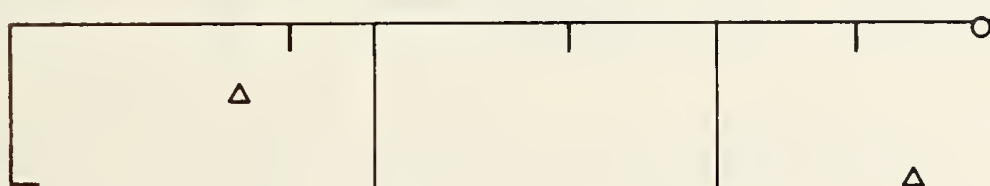

1135.9

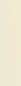

$1135.8-$

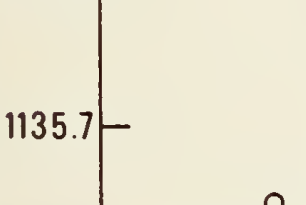

$\Delta$

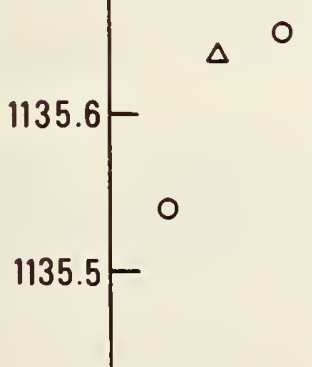

1135.4

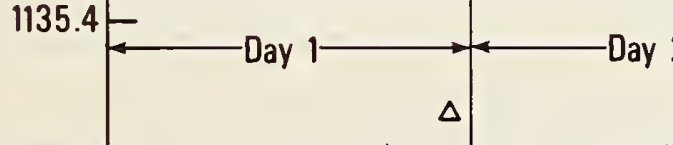

$\Delta$

$\Delta$

$1135.3 \frac{5}{0}$
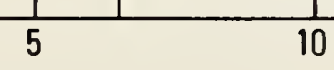
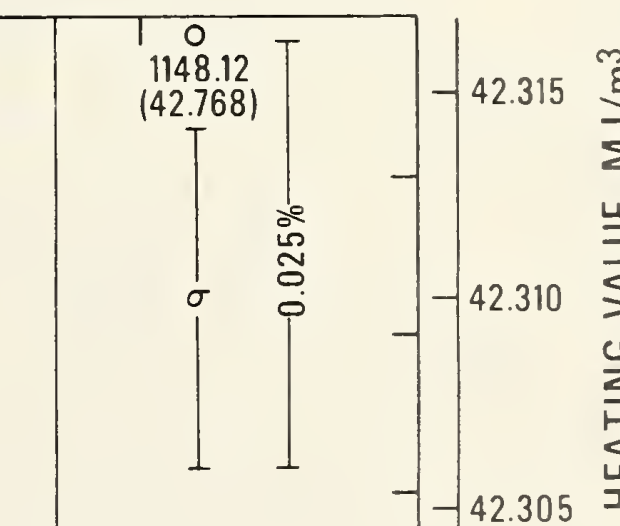

\section{ANALYSIS NUMBER}

Figure 2.2.6. Computed ideal gas heating value for mixture E. The symbols o and $\Delta$ denote operators 1 and 2 , respectively. The true value for this mixture 13 1 35.29 Btu/scf or $42.2906 \mathrm{MJ} / \mathrm{m}^{3}$. 


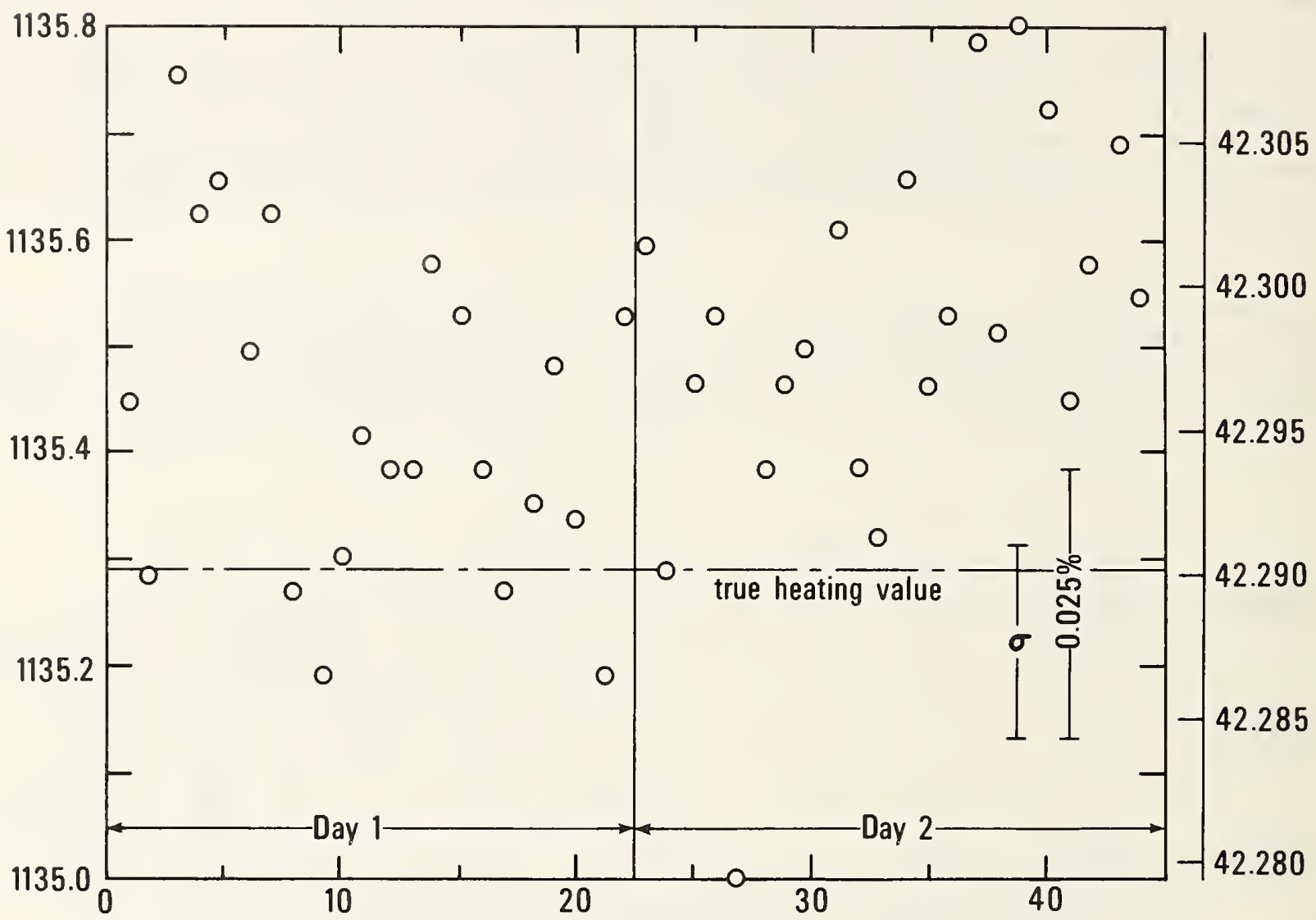

ANALYSIS NUMBER

Figure 2.2.7. Computed ideal gas heating values for mixture E after integrator repair.

Figure 2.2.7 shows the computed heating values for the 44 analyses which were taken over a two-day period. This time the total spread of computed heating values as 0.07 percent and the largest deviation in heating values was 2.8 estimated standard deviations; 75 percent of the analyses were within one estimated standard deviation of the average. This indicated that the malfunctioning integrator had caused the outliers.

Table 2.2.9 lists the biases and estimated standard deviations obtained from the results of the above two tests; values differing from the average measured value by more than four estimated standard deviations were omitted in the computations. Composition error is given in the original publication $[1]$.

Comparing the biases of mixture $G$ with those of the other mixtures points out the necessity of using a calibration gas which is similar to that of the unknown mixture. Also, the estimated standard deviations were generally larger for mixture G; this indicates that the operating variables would require some modification when analyzing mixtures containing large amounts of nitrogen and/or ethane.

Figures 2.2.6 and 2.2.7 illustrate how an insufficient number of calibration runs can introduce biases into the computed heating values. Since the calibration gas was the analyzed gas in these figures, the biases had to be caused by the calibration. In the first test all results were based on a single calibration run at the beginning of the day, while two calibrations were made at the beginning of each day in the second test. 
Table 2.2 .9

Summary of Biases (B) 1 and Standard Deviations ( $s$ ) in the Ideal Gas Heating Values for Four Mixtures Analyzed By Gas Chromatography

\begin{tabular}{|c|c|c|c|}
\hline Mixture & $\begin{array}{l}\text { Number } \\
\text { of } \\
\text { Analyses }\end{array}$ & $\begin{array}{l}\text { Heating Vo } \\
\text { Btu/scf } \\
\left(\mathrm{MJ} / \mathrm{m}^{3}\right)\end{array}$ & \\
\hline C & $24^{3}$ & $\begin{array}{c}(B) \\
0.1 \pm 0.1 \\
(0.004 \pm 0.004)\end{array}$ & $\begin{array}{c}(s) \\
0.16 \\
(0.006)\end{array}$ \\
\hline$E^{2}$ & $24^{3}$ & $\begin{array}{l}0.31 \pm 0.14 \\
(0.012 \pm 0.005)\end{array}$ & $\begin{array}{l}0.22 \\
(0.008)\end{array}$ \\
\hline$F$ & $24^{3}$ & $\begin{array}{c}0.46 \pm 0.13 \\
(0.017 \pm 0.005\end{array}$ & $\begin{array}{c}0.22 \\
(0.008)\end{array}$ \\
\hline G & $24^{3}$ & $\begin{array}{c}1.27 \pm 0.28 \\
(0.047 \pm 0.005)\end{array}$ & $\begin{array}{c}0.39 \\
(0.015)\end{array}$ \\
\hline$E^{2}$ & $44^{4}$ & $\begin{array}{l}0.10 \pm 0.07 \\
(0.007 \pm 0.003)\end{array}$ & $\begin{array}{c}0.17 \\
(0.006)\end{array}$ \\
\hline
\end{tabular}

\footnotetext{
${ }^{1}$ Biases are given with 99 percent confidence interval.

2This mixture was used as the calibration gas.

3 Analyses taken prior to integrator repair and on four different days; outlying points were omitted from computation of bias and estimated standard deviation.

${ }^{4}$ Analyses taken after integrator repair and on two different days.
}

Biases were computed for the second test by using the first three runs of each day for calibration; the bias dropped from 0.009 to 0.005 percent. Thus it appears that at least three good ( $i . e$. , not a set that includes an outlier) calibrations runs should be used to minimize biases introduced by calibration. This assumes that the other parameters in the GC system remain constant. Since this may not always be the case, calibrations should be made several times during the day as an additional check.

\subsubsection{GC Column Configurations and Digital Integrators}

The previous section determined the precision and accuracy of one gas chromatograph (GC)-1ntegrator system. Additional tests employing different column configurations and integrators were needed to determine the factors limiting the precision of GC analysis. This section describes tests using two different three column configurations and two different GC's, and tests directly comparing two different integrators. Table 2.2.10 summarizes the chromatograph-integrator combinations used in all of the analyses tests.

Table 2.2 .10

Summary of Gas Chromatogram-Integrator Systems Used in Analysis Tests

\begin{tabular}{|l|c|c|c|}
\cline { 3 - 4 } \multicolumn{1}{c|}{} & Two Column & \multicolumn{2}{c|}{ Three Column Configuration } \\
\cline { 3 - 4 } \multicolumn{1}{c|}{ Configuration } & Configuration A & Configuration B \\
\hline Chromatograph & I & II & I \\
Detector & Hot-wire & Thermistor & Hot-wire \\
Auto-Valve Switch & no & yes & no \\
Integrator & A & A \& B & A \\
\hline
\end{tabular}




\subsubsection{GC Column Configurations and Column Packings}

Obtaining the most accurate and precise analysis of vaporized LNG samples requires:
a) resolving oxygen, nitrogen and methane through pentane and column back flushing (i.e., carrier gas flow reversal) to obtain a composite hexane+ peak,
b) analyzing for all components in a single sample injection,
c) completing the analysis within approximately 30 minutes,
d) operating the column ovens in an isothermal mode,
e) minimizing the effect of baseline upset by valve switches, and
f) computing the composition by using peak areas.

The primary purpose in resolving the nitrogen and oxygen peaks is to detect contaminated samples, since the most likely contaminant is air. Also, it is a good way to detect leaks in the GC plumbing. The need for good resolution between the hydrocarbons is obvious. The anticipated concentrations of hexane and heavier components is small (> 0.01 mol percent); therefore, combining the hexane+ peak fraction into one peak by backflushing causes an insignificant error in the computed heating value.

The GPA [18] and ASTM [7] analysis procedures suggest using two sample injections -- one injection into a column to resolve air and methane through pentane followed by a backflush for the hexane+ peak: a second injection into a different column to resolve nitrogen, oxygen and methane (the column then is backflushed to remove the remaining hydrocarbons). However, the two analyses must be combined. This is done through sample size or methane peak area. Both methods diminish analytical precision.

The primary reason for minimizing the analysis time is that the peak height to peak width ratio decreases with increasing residence time. Maximum accuracy and precision in peak area measurement decreases as the ratio decreases. It was found that, for the column configurations and operating parameters considered here, the analysis times fell in two ranges -- one was 40 minutes or greater and the other was 24 to 30 minutes. Configurations having long analysis times elute the hexane+ peak last; the other configurations elute methane last.

Temperature programming is an attractive way of reducing analysis time and sharpening peaks of the heavier components. However, whenever feasible, isothermal column oven operation is normally preferred over temperature programming when using thermal conductivity detectors. This is because flow variations caused by changing column temperature produce baseline drift when using these detectors. Temperature programming becomes attractive if the baseline drift can be compensated for reliably by the integrator or if flame ionization detectors are used. These detectors are insensitive to flow variations but also do not detect nitrogen, oxygen and carbon dioxide.

Multicolumn configurations require valve switching with its associated carrier gas rlow upsets; these, in turn, produce baseline upsets. The time duration of baseline upsets are minimized by;

a) adjusting flow restrictors in column bypass lines so that the steady-state carrier gas flow rate remains constant after switching the column in or out, and

b) using the carrier gas inlet pressure instead of mass flow to control the flow rate [18].

To minimize peak integration problems, each valve switch should occur far enough away from the nearest peaks to allow baseline establishment between the peaks and the valve switch.

Traditionally, peak height measurement was preferred over peak area measurement for computing composition from chromatograms. Peak height was used because it was easy, quick and usually more precise than peak area. However, the peak height response is linear over a narrow concentration range, using a $0.5 \mathrm{~cm}^{3}$ sample loop volume, peak height response is linear at concentrations below roughly $10 \mathrm{~mol}$ percent (peak area response is close to linear over the entire composition range). Also, peak heights cannot be used with composite backflush peaks. The currently available programmable integrators eliminate the drawbacks to using peak areas. Also, since both methane and $\mathrm{C}_{6}{ }^{+}$peaks must be measured, peak area measurement was selected. 
Compromises are necessary to meet the requirements listed above. It is possible to make the complete analysis on one column [19], but it requires temperature programming between $-70^{\circ} \mathrm{C}$ and $145^{\circ} \mathrm{C}$ and the hexane+ backflush peak retention time is about 22 minutes; the hexane+ peak width is roughly 3 minutes whlch, in combination with the baseline drift caused by temperature programming, can cause errors in the peak area measurement. If the separation of oxygen and nitrogen is not required, the analysis can be made by temperature programming between $35^{\circ} \mathrm{C}$ and $145^{\circ} \mathrm{C}$. In this case the analysis time is roughly 15 minutes. Based on the data for five replicate natural gas analyses [20], the estimated standard deviation in the computed heating value for this method is 0.04 percent.

The most commonly used column packing for isothermally resolving nitrogen and oxygen is molecular sleve 5A [19]. It makes a good separation between nitrogen and methane, but cannot be used with the higher hydrocarbons. This means that at least two columns in series are needed -- one to separate oxygen, nitrogen and methane (molecular sieve) and one to resolve the higher hydrocarbons (DC $200 / 500$ on Chromasorb PAW). Using this configuration without temperature programming, the retention time for the pentanes is roughly 20 minutes. After eluting the pentanes, the DC $200 / 500$ column is backflushed with the hexane+ peak eluting in another 20 minutes; this peak becomes so broad that at the 0.01 mol percent level it would not be detected using a TC detector and a $0.5 \mathrm{~cm}^{3}$ sample loop.

When operating the columns isothermally, the preferred way to shorten the pentane and hexane+ retention times is to use two columns to resolve the $\mathrm{C}_{2}+$ hydrocarbons. By selecting the proper column packings, lengths, and temperatures, and by using column bypassing, the components can be eluted in almost any sequence desired. In this work two three-column configurations were considered -- one which eluted the pentanes and hexanet peaks midway through the analysis (configuration A) and one which eluted them at the beginning of the analysis (configuration $B$ ).

Column configuration A Figure 2.2 .8 shows a schematic of this three-column arrangement and figure 2.2.9 is a chromatogram of a sample taken during a flow facility sampling test; table 2.2 .11 lists the operating parameters for this configuration. The vaporized LNG samples were analyzed using the following automatic valve switching sequence:

Step 1. Switch valve V1 for sample injection -- all columns are in series.

Step 2. Switch valve V2 to bypass columns 2 and 3 after oxygen, nitrogen and methane have entered these columns; ethane through pentanes are eluted in this step. (Restrictor $A$ is adjusted so that its pressure drop is comparable to the pressure drop of columns 2 and 3; this minimizes baseline upset after valve switchings.)

Step 3. Switch valve V1 to original position to stert backflush.

Step 4. Switch valve v2 to put columns 2 and 3 back in series with column 1.

Step 5. Switch valve V3 to bypass column 3 which now contains oxygen, nitrogen and methane; ethane (and carbon dioxide, if present) are eluted in this step. (Restrictor B is set to provide the same pressure drop as column 3.)

Step 6. Switch valve 22 to bypass column 2; the $\mathrm{C}_{6}+$ peak elutes in this step.

step 7. Switch valves V2 and V3 to put column 2 and 3 in series; oxygen, nitrogen and methane elute in this step.

Table 2.2.11

Operating Conditions for Column Configuration A

\begin{tabular}{|c|c|}
\hline $\begin{array}{l}\text { Carrier gas flow } \\
\text { Sample loop volume }\end{array}$ & $\begin{array}{l}27.5 \mathrm{scm}^{3} / \mathrm{min}^{-} \\
0.5 \mathrm{~cm}^{3}\end{array}$ \\
\hline $\begin{array}{l}\text { Column } 1 \text { temperature } \\
(10 \mathrm{ft} .(3 \mathrm{~m}) \times 1 / 8 \text { inch }(3.2 \mathrm{~mm}) \text { of } 15 \text { percent squalane } \\
\text { on Chromosorb PAW, } 60 / 80 \text { mesh) }\end{array}$ & $66^{\circ} \mathrm{C}$ \\
\hline $\begin{array}{l}\text { Column } 2 \text { temperature } \\
\qquad(5 \mathrm{ft} .(1.5 \mathrm{~m}) \times 1 / 8 \text { inch }(3.2 \mathrm{~mm}) \text { of Porapak } \mathrm{N}, 60 / 80 \text { mesh })\end{array}$ & $66^{\circ} \mathrm{C}$ \\
\hline $\begin{array}{l}\text { Column } 3 \text { temperature } \\
\text { ( } 5 \mathrm{ft} .(1.5 \mathrm{~m}) \times 1 / 8 \mathrm{inch}(3.2 \mathrm{~mm}) \text { of Molecular } \\
\text { sieve } 5 \mathrm{~A}, 60 / 80 \mathrm{mesh})\end{array}$ & $66^{\circ} \mathrm{C}$ \\
\hline $\begin{array}{l}\text { Detector temperature } \\
\text { Detector current }\end{array}$ & $\begin{array}{l}66^{\circ} \mathrm{C} \\
\text { nistor detec }\end{array}$ \\
\hline
\end{tabular}




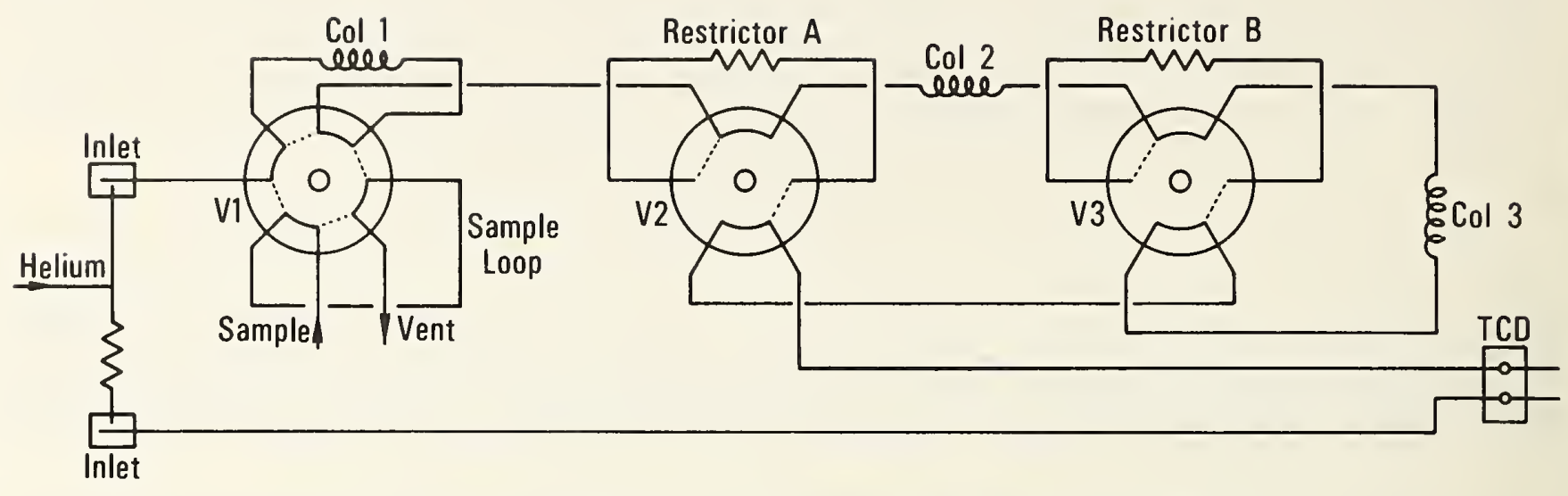

Figure 2.2.8. Schematic of three column configuration A.

A series of analyses were made using this configuration and prepared mixture H (see table 2.2.6). A comparison of these results with results given in table 2.2 .8 shows that the precision of the two different column configurations was comparable. No biases were found in this test since the same mixture was used for both calibration and analysis; three calibrations were made prior to running the replicate samples. It is encouraging that configuration A, with eight valve switches, had about the same precision as the much simpler two column system which had only three valve switches.

Unfortunately, the backflush peak in configuration A was so broad that one of the two integrators (integrator $A$ ) had difficulties in accurately measuring the $\mathrm{C}_{6}+$ peak area at the 0.10 mol percent level. Although integrator $B$ reliably integrated the $\mathrm{C}_{6}{ }^{+}$peak, the broad backflush peak affected the reliability of integrating the following two peaks. It is extremely unlikely that the integrators would reliably detect a $\mathrm{C}_{6}+$ peak at the 0.01 mol percent level. (Missing a $\mathrm{C}_{6}{ }^{+}$concentration of 0.01 mol percent produces a 0.04 error in the computed heating value.)

The only way to obtain a relatively precise $\mathrm{C}_{6}+$ analysis is to reduce the retention time; this can be done by choosing a different three-column configuration.

Column configuration $B$ If precise determination of pentanes and $\mathrm{C}_{6}+$ concentrations in the 0.01 mol percent range is required, these components must be eluted at the beginning of the analysis. This is done by using the columns listed in table 2.2 .13 and the flow scheme shown in figure 2.2 .8 . Configuration $\mathrm{B}$ uses the following valve sequence:

Step 1. Switch valve V1 for sample injection - all columns are in series.

Step 2. Switch valve V2 to bypass columns 2 and 3 after all components except $C_{5}+$ have entered these columns; the iso and normal pentane elute in this step.

Step 3. Switch valve $\mathrm{V} 1$ back to original position to backflush the $\mathrm{C}_{6}{ }^{+}$component.

Step 4. Switch valve V2 to put columns 2 and 3 into series again.

Step 5. Switch valve V3 to isolate oxygen, nitrogen and methane in column 3 . Ethane through the butanes are eluted in this step.

Step 6. Switch valve V3 to elute oxygen, nitrogen and methane.

Table 2.2 .13

operating Conditions for Column Configuration B

\begin{tabular}{|l|l|}
\hline Carrier gas flow & $28 . \mathrm{scm} / \mathrm{min}$ \\
Sample loop volume & $0.5 \mathrm{~cm} 3$ \\
Column 1 temperature $(6 \mathrm{ft}(1.8 \mathrm{~m}) \times 1 / 8$ inch $(3.2 \mathrm{~mm})$ & 62 to $68^{\circ} \mathrm{C}$ \\
of DC $200 / 500$ on Chromasorb PAW, $60 / 80 \mathrm{mesh})$ & $60^{\circ} \mathrm{C}$ \\
Column 2 temperature $(25 \mathrm{ft}(7.6 \mathrm{~m}) \times 1 / 8$ inch $(3.2 \mathrm{~mm})$ & \\
of DC $200 / 500$ on Chromasorb PAW, $60 / 80 \mathrm{mesh})$ & $65^{\circ} \mathrm{C}$ \\
Column 3 temperature $(6 \mathrm{ft}(1.8 \mathrm{~m}) \times 1 / 8$ inch $(3.2 \mathrm{~mm})$ & \\
$\quad$ of Molecular sieve, $5 \mathrm{~A}, 60 / 80 \mathrm{mesh})$ & $120^{\circ} \mathrm{C}$ \\
Detector temperature & $220 \mathrm{~mA}$ \\
\hline
\end{tabular}


Either of the latter two problems could produce the observed scatter in the computed heating value. Additional tests should be made to determine the precision of this configuration under better operating conditions.

Comparison of pentane peak heights showed that the peak heights using configuration $B$ were twice that of configuration A. A sample containing hexane + was analyzed using configuration B; the hexane + peak eluted as a single sharp peak instead of the broad double peak produced by configuration A. The full results of testing were hampered by experimental problems and additional work in this comparison was suggested by the authors [1].

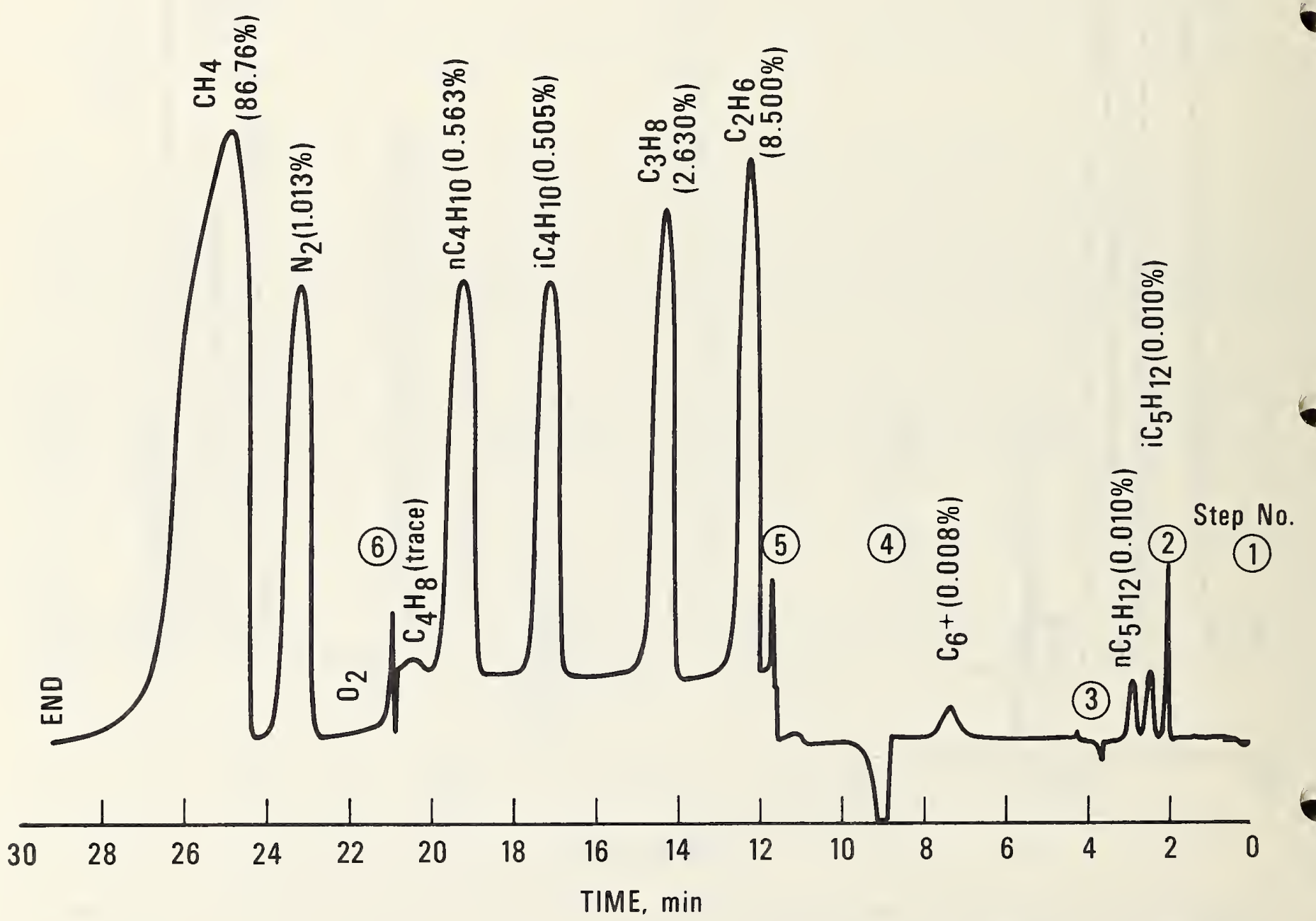

Figure 2.2.10 A chromatogram of a natural gas mixture using column configuration B. (The peak heightg are shown on a logarithmic scale). 


\subsubsection{Digital Integrator Evaluation}

The purpose of this work was to directly compare the accuracy and precision of two different programmable digital integrators. All digital GC integrators use voltage to frequency converters and counters to convert the detector output voltage into peak areas. The primary difference in integrators is the way they treat the converted signal. Both integrators used in this evaluation employed sophisticated software programs; these programs identified peaks, baseline segments and small peaks riding on the tail of larger peaks. Also, they compensated for transient baselines when computing peak areas. Both integrators were programmable so that automatic adjustments, which were unique to the particular analysis, could be made during the analysis. The primary differences between the two integrators were that the software programs used different peak detection algorithms and that integrator $B$ had considerable more programing capabilities; however, this versatility was unnecessary for analyzing vaporized LNG samples.

During the last 2 days of a 4 day test period both integrators produced one or more extraneous results. The outliers appeared to occur randomly among the integrators which excluded the GC as the cause. It is suspected that the outliers were caused by power fluctuations since the integrators were on different circuits; the GC detector power supply was connected to a constant voltage transformer. Shortly after these tests there were several power fluctuations severe enough to shut down the integrators.

\subsubsection{GC Analysis Accuracy and Precision}

Variation in any of the operating parameters 1 isted in table 2.2 .11 could affect analysis precision and accuracy. However, variables such as column temperature are electronically controlled. Therefore, they will be constant unless the set point is changed. The variables most subject to change are sample amount (i.e., sample pressure since a fixed volume sample loop is used) and carrier gas flow rate because it must be set manually.

A test was conducted using column configuration $A$ and both integrators. In one test injected samples were used at pressures of $13.7,21.1$ and 8.6 psia $(94,145$ and $59 \mathrm{kPa})$; the calibration sample pressure was $13.7 \mathrm{psia}(95 \mathrm{kPa})$. The results of the test showed that the precision was insensitive to sample pressure. However, there was an apparent linear relationship between bias and sample pressure. This test indicates that maintaining the sample pressure within \pm 10 percent of that used for calibration will keep the bias to less than 0.025 percent in the computed heating value.

The observed bias was caused by the method the integrators used to determine the peak area. Unless the peak was very large and sharp, part of the peak area was lost during the baseline determination. The absolute amount of area lost remained nearly constant as the peak area changed, provided that the peak width at the half-height did not change. Therefore, the relative amount of area lost decreased with increasing peak size. Since the methane peak was so large there was a negligible amount of area lost during baseline determination. However, this was not the case for all other components except the pentanes. (The pentane peak areas were so small that the effect was within the data scatter.) This explains why the apparent methane content decreased and the ethane through butane contents increased with increasing sample injection pressure.

In the second test the carrier gas flow was varied by \pm 7 percent. Integrator A results were insensitive to the flow variation, whereas, integrator B showed a bias in the computed heating value. This bias was caused by the method used to establish a baseline between the nitrogen and methane peaks. During these tests integrator B was programmed to force a baseline at a specifled time. The time was set at the bottom of the valley between the two peaks (see figure 2.2.9) when the carrier gas flow rate was $26.5 \mathrm{scm} 3 / \mathrm{min}$. Increasing the carrier gas flow rate made the valley bottom occur before the forced baseline. Therefore, the forced baseline caused the integrator to 1 gnore part of the methane peak; this lowered the apparent methane content and raised the apparent composition or tho other components. This problem can be eliminated by having the integrator automatically select tho valley bottom as the baseline when two peaks are not completely resolved.

Based on these tests it appears that the precision and accuracy of the computed heating values aro unaffected by changes of \pm 7 percent in the carrier gas flow rate. This conclusion strictly applios only to configuration A. However, it should apply to other column configurations whlch have comparable time spacings between valve switches and peak elutions. 


\subsubsection{Sample Handing Procedures}

Typically, the LNG sampling procedure will include the filling of gas sample containers. Therefore, it was necessary to determine which filling procedures are the most reliable and if there are any effects of storage which could change the sample composition over a period of time. Considering the filling procedures described by Miller [13] (see discussion in Section 2.2.3) and the experience developed at NBS, it was felt that the purge and fill technique along with purging at a constant flow rate are the simplest and most reliable; however, when using the latter technique, some method of flow measurement is desirable.

In using the purge and fill technique it is important to adequately purge the cylinder to remove the original gas. Miller [13] states that 3 to 8 purge and fill operations are sufficient; the ASTM standard [14] recommends 10 complete operations at cie point and 3 to 8 in another. The number depends upon the line pressure, because the important factor is the number of sample container volumes of gas at ambient pressure (assuming ideal gas behavior), which pass through the sample container. Assuming complete mixing, 10 volumes of gas should be adequate. However, it was felt that testing was necessary to determine:
a) the number of purges required, both in filling the sample cylinder and in purging the lines to the GC, and
b) the effect of sample "aging" which could change the apparent position.

Test Apparatus and Procedure Figure 2.2.11 shows a schematic of the apparatus for evaluating sample handling techniques. In the first test, the number of purges necessary to reduce the air concentration to below the detectable limit on the GC was determined. Several $150 \mathrm{~cm}^{3} \mathrm{stainless}$ steel sample cylinders with valves at each end were used; the valves contained teflon packing and Kel-F stem tips. The valves were fitted with a gland and nut for making an 0-ring coupling to the sample line. The sample cylinders were not pretreated in any way. However, they were pressure checked to 1500 psi (10 $\mathrm{MPa}$ ) and vacuum checked with a helium leak detector. In this test, a sample cylinder was attached to valve V5. Initially, several analyses were made directly from the prepared mixture cylinder. In addition to noting the nitrogen content of the mixture, the ratio of the methane to nitrogen peak areas was also recorded. (This is a sensitive method for detecting air leaks.) To make sure that air was in the sample cylinder initially, the manifolding was evacuated between valves V2 and V3, with V5 and V6 open, but V7 closed; after closing V5, V7 was opened to let air in. Next, the manifolding was pressurized to $30 \mathrm{psig}(0.3 \mathrm{MPa})$ with the mixture by opening valves V1 and V2. To purge and fill the sample cylinder a procedure was developed to alternately open valve V5 with V7 closed, then close V5 and open V7; V7 was left open just long enough to bleed the pressure to near ambient. After the desired number of purges, valve V2 was shut and the manifolding evacuated through V8. During this evacuation, valve V5 was open but V6 closed. V6 was then cracked open to fill the manifold to 2 psig. $(0.11 \mathrm{MPa})$. After filling the manifold, valve $\mathrm{V} 5$ was closed and $\mathrm{V} 6$ opened. Opening valves $\mathrm{V} 3$ and $\mathrm{V} 9$ evacuated the manifold and GC sample loop. After V9 was closed, V5 was cracked open to fill the line with sample gas and the analysis started. Originally the GC sample loop was purged several times, but tests showed that one 20 to 30 second evacuation and fill was sufficient. However, if there was sufficient pressure in the sample cylinder, the evacuation and fill was repeated at least once.

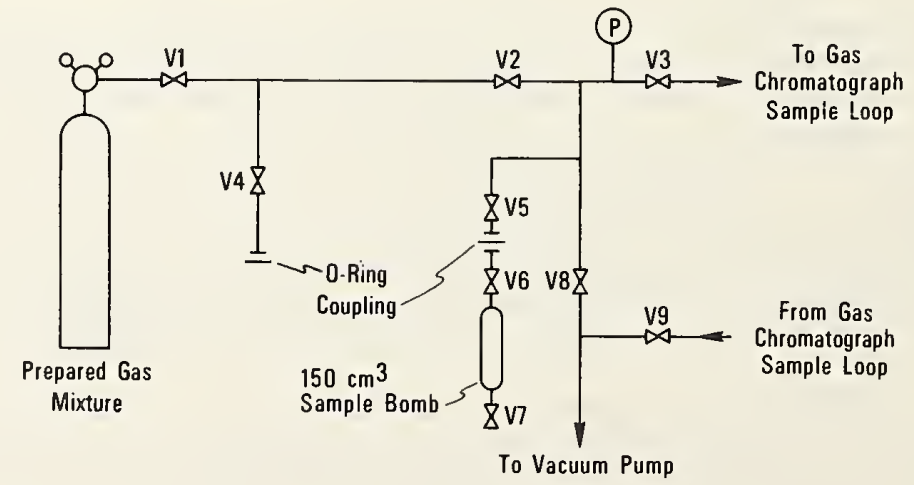

Figure 2.2.11 Flow Schematic for Sample Handing Tests. 
Evaluation of Results Analyses were taken on samples from cylinders purged and filled 4 , 6 , 10 and 14 tlmes. Only the last one indicated that all of the air was removed. This is equivalent to 42 sample comtalner volumes of gas at ambient conditions, four times more than would be expected if there was coliplete mixing.

In the second test sample aging was tested to see if processes such as adsorption on sample cylinder walls would alter sample composition. Again sample cylinders initially containing air were used after 14 purge and fills with a sample pressure of $30 \mathrm{psig}(0.3 \mathrm{MPa})$. However, this time the sample contalners were filled at the O-ring coupling adjacent to valve V4; for analysis, the sample cylinders were attached to valve V5 and followed the line and GC sample loop purging procedure outlined above. To ensure that the change in composition, if any, was not due to biases in the GC analysis, the prepared mixture from the cylinder was analyzed before and after each sample cylinder analysis. Analyses were made at $1 / 2,4,24,48$ hours and 50 days after filling; in all cases the samples were within one estimated standard deviation of the original mixture for all components.

Additional tests (described in following sections) showed that continuous purging also was a satisfactory sample collecting technique. However, the sample container was purged the equivalent of 14 or more purge and fills. The gas throughput was estimated by monitoring the pressure, flow rate (5 to 11 standard liters per minute) and time.

\subsubsection{Conclusions and Recommendations}

Accuracy and Precision of Analysis by Gas Chromatography Based on the results of comparative tests it was found that a gas chromatograph-digital integrator system was more accurate and precise than mass spectrometry. Additional tests showed that the gas chromatograph produced a bias of 0.02 percent, or less, in the computed heat value provided that:
a) the response factors for computing the composition are the average of three or more calibrations,
b) the gas chromatograph and digital integrator operating parameters remain constant during calibration and analysis runs, and
c) the calibration and unknown mixture compositions are comparable.

Test results showed that gas mixtures within the anticipated LNG composition range given in table 2.2.1 can be analyzed by gas chromatography using only one calibration gas (which is within the same range) without producing biased results.

The total uncertainty in computing the heating value routinely can be less than \pm 0.1 percent. This assumes:
a) taking three or more replicate analyses and averaging the computed heating value,
b) making three or more calibration runs prior to the analysis and averaging the resulting response factor for each component,
c) using a calibration gas which has a total uncertainty in the composition which, neglecting the specific heating value of each component, corresponds to an accuracy of \pm 0.03 percent in the computed heating value (if the calibration gas is prepared by d) using a gas chromatograph-integrator system with a known precision of 0.06 percent, or better (based on three standard deviations), in the computed heating value, and
e) following the sample injection procedures described previously (sections 2.2 .4 ) for gas analysis.

If precise analysis of $\mathrm{C}_{5}+$ is unnecessary, column configuration A should be used for analyzlng vaporized LNG samples. If the pentanes and $\mathrm{C}_{6}+$ peak areas need to be precisely known, column configuration $\mathrm{B}$ is recommended.

To maintain the maximum accuracy and precision, the sample amount and carrier gas flow rate should be kept constant to within \pm 7 percent. It is recommended that a high quality rotometer be used to monitor the flow rate; the rotometer can be calibrated with a simple soap bubble flowmeter. 
Direct comparison of two different integrators showed that the two instruments had comparable precision. Based on all of the tests (see section 2.2.4), it was concluded that, at present, the digital integrator is less precise than the gas chromatograph employing a thermal conductivity detector. Therefore, the most precise analysis will be one in which the integrator "sees" the sharpest peaks and the least baseline fluctuations before and after peaks. To maximize the precision, a programmable integrator should be used.

Sample Handling Tests showed that both the purge and fill technique and the continuous purge technique were accurate methods for collecting vaporized LNG samples. However, both techniques require some care to insure that the cylinder has been purged properly. Commercially available stainless steel sample cylinders are recommended for collecting gas samples. No special preparation of the cylinders is required prior to their use. Tests showed that stainless steel cylinders do not alter the hydrocarbon content over a six week period (However, stainless steel is not inert to sulphur compounds.)

Selection of a Gas Chromatograph-Integrator System It is recommended that the following features be included in any gas chromatograph-integrator system dedicated to routine analysis of vaporized LNG samples:

a) Automatic valve switching - this is required to insure that the right valve is switched at the right time.

b) Thermal conductivity detector - this is the only detector that is sensitive to all the components in natural gas and is linear in response over the anticipated concentration ranges (i.e., the output signal is directly proportional to the concentration). Hot wire filaments are preferred over thermistors because they provide superior stability, longer operating life and fewer anomalies of operation [20]. The instrument should contain a thermal protecting device to minimize the $r i s k$ of oxidizing the filaments. To maximize sensitivity the detector cell volume should be as small as possible.

c) Isolated power supply - this is necessary to prevent electrical noise generated by oven temperature controllers from entering the detector circuitry. The detector power input should be connected to a constant voltage transformer to minimize effect of line power fluctuations.

d) Carrier gas flow control by inlet pressure - this is preferable to mass flow control when the analysis involves valve switching [18]. A two-stage pressure regulator should be used to keep the inlet pressure constant.

e) Readily accessible valve and column connections - this is needed to make routine maintenance and leak checking easy.

f) Programmable digital integrator - this is necessary to maximize the analytical precision. A memory protect feature should be used to prevent losing programs during momentary power failures. A constant voltage transformer should be connected to the integrator to minimize noise due to line power fluctuations.

\section{2 .5 LNG Sampling}

The sampling tests included both laboratory and field tests. The laboratory tests provided close control of all operating variables whereas the field tests permitted evaluation of a full scale sampling system.

The chronological order of the tests and the variables considered for their effect on sampling accuracy and precision were:

a) laboratory tests (Probe Evaluation) - sampling rate, LNG flow rate, temperature, pressure, degree of subcooling and three probes;

b) laboratory tests (Vaporizer Evaluation) - sampling rate, temperature, degree of subcooling, LNG flow rate and two vaporizer designs,

c) field tests (LNG Flow Facility Test 1) - three probes and three vaporizers,

d) laboratory tests (General Tests) - accumulator residence time, vaporizer outlet temperature, pressure drop upstream of vaporizer, heat leak to sample probe and presence of heavy components $\left(\mathrm{C}_{5}+\right)$ in the LNG,

e) field tests (Shipboard Tests) - sampling rate and comparison between liquid and vapor sampling,

f) field tests (LNG Flow Facility Test 2) - sampling rate, heat leak, start-up transients and three variations of a vaporizer design. 


\section{2 .5 .1 Laboratory Tests}

Laboratory Scale Apparatus Figure 2.2.12 shows a schematic of the laboratory scale apparatus used in this work. The cryogenic portion of the apparatus was contained in an evacuated double walled dewar which was 33 inches ( 838 millimeters) deep and had a 10-3/4 inch (273 millimeters) inside diameter. The LNG vessel, which was 8-1/2 inch (215 millimeters) long with an outer diameter of 8 inches ( 203 millimeters), had an inner volume of roughly 6 liters; the wall was copper and the two end plates were brass. A centrifugal pump circulated LNG through a turbine flowmeter, with a range of 0.25 to 2.5 galions per minute ( 0.016 to $0.16 \mathrm{liters} / \mathrm{second})$, and through the test section between valves T1 and T2. All of the lines in this loop were $1 / 2$ inch $(12.7$ millimeters) $0 . D$. copper tubing except for a 3 Inch (76 millimeters) long section containing 1 inch ( 25 millimeters) 0.D. copper tubing just downstream of valve $\mathrm{T} 1$. This short section acted as a mixing chamber to reduce flow turbulence caused by the valve. The test section was a 6-3/4 inch (1;1 millimeters) long straight section. It contained two probes -- an upstream facing pitot tube and a side tap. Both probes were mounted vertically and were made of $1 / 16$ inch ( 1.59 millimeters) O.D. by 0.023 inch (0.58 millimeter) I.D. stainless steel tubing. There was roughly 6 inches (150 millimeters) of line between the probes and valves $\mathrm{T} 4$ and $\mathrm{T} 5$.

Besides the pitot tube and side tap, a "reference" probe was located in the straight-run leg of a tee positioned just upstream of valve T1. Since the flow into the tee was downward, the reference probe was in essence located on the bottom of the line. There were 8 inches (200 millimeters) of 0.023 inch (0.58 millimeter) I.D. stainless steel tubing between this probe and valve T3.

Valves T1 through T6 were bellows valves which were located inside the insulating vacuum space at LNG temperature. They were actuated by stem extensions protruding through the top plate of the apparatus. Valves T3 through T6 were mounted on a brass plate which was soldered to the top of the mixing chamber.

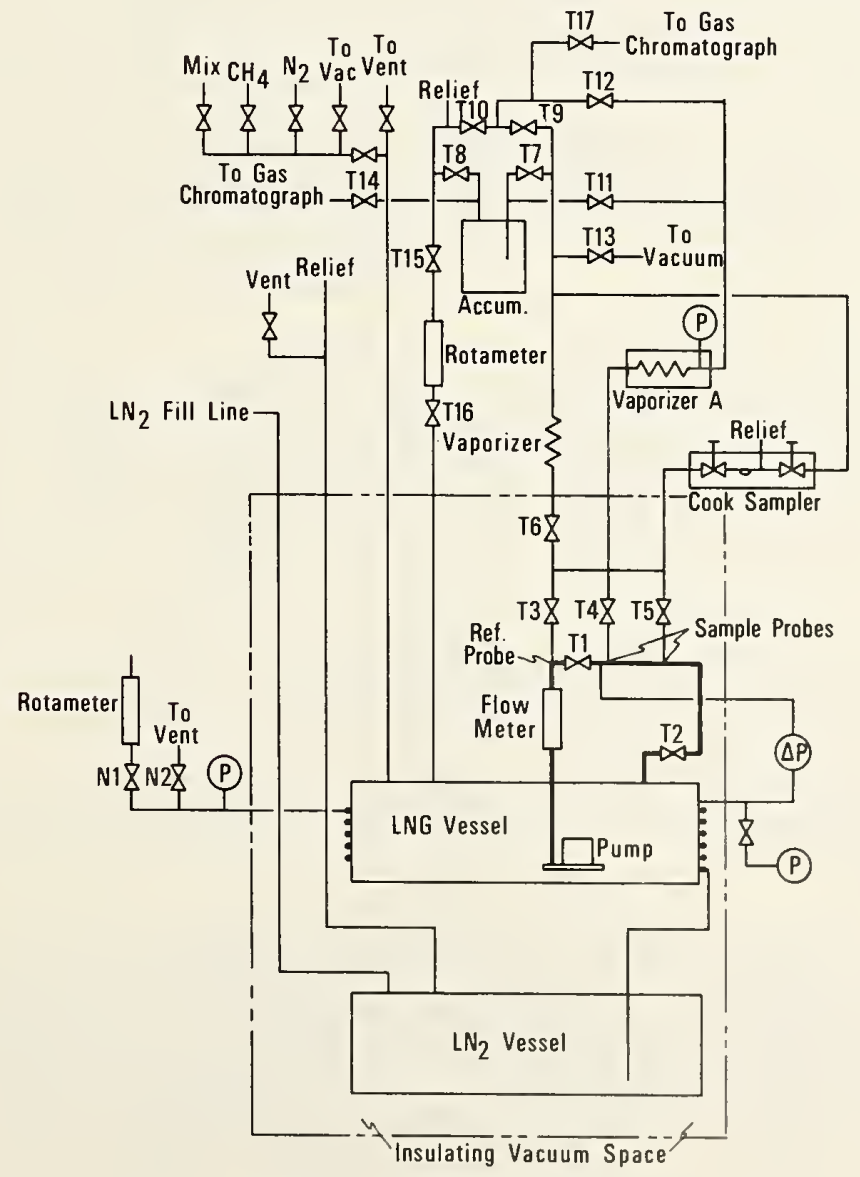

Figure 2.2.12. Schematic of laboratory scale apparatus. 
After a probe was selected by opening either valve T3, T4, or T5, the sample passed through $1 / 16$ inch (1.59 millimeters) O.D. by 0.023 inch ( 0.58 millimeter) I.D. stainless steel tubing to one of three vaporizers. If the sample went to the reference vaporizer, it first passed through valve T6 and 8 inches (203 millimeters) of tubing; valve T6 was a needle valve used to provide additional pressure drop to prevent sample backflash, i.e., flow reversal due to vaporization. The reference vaporizer consisted of a 5 inch (127 millimeters) long section of $1 / 16$ inch ( 1.58 millimeters) $0 . D$. by 0.051 inch ( $1.28 \mathrm{millimeters)} \mathrm{I.D.} \mathrm{stainless} \mathrm{steel} \mathrm{tubing} \mathrm{and} \mathrm{a} 12$ inch (304 millimeters) long section of $1 / 8$ inch ( 3.18 millimeters) O.D. by 0.101 inch $(2.56$ millimeters) I.D. stainless steel tubing. The sample was vaporized by passing a low voltage, high amperage de current through the two sections of tubing. To prevent shorts, the two ends of the vaporizer were epoxied into micarta couplings. The reference vaporizer was located less than $1 / 2$ inch (13 millimeters) from where the sample line left the vacuum insulated space.

After the sample left the vaporizer it went to a manifold containing a 2 liter accumulator, through a 0 to 11.4 liter per minute rotometer and back to the LNG vessel. The lines between the vaporizer and rotometer were $1 / 4$ inch ( 6.35 millimeters) 0.D. by 0.190 inch ( 4.83 millimeters) I.D. copper tubing; the copper tubing downstream of the rotometer was $3 / 8$ inch $(9.53$ millimeters) 0.D. by 0.343 inch ( 8.71 millimeters) I.D. The accumulator could be isolated by closing valves T7 and T8; to maintain the sample flow, valves $\mathrm{T} 9$ and $\mathrm{T} 10$ (a regulating valve) were opened when the accumulator was isolated. Samples could be taken from the accumulator through valve T14 and from the bypass line through valve T17.

The second vaporizer, vaporizer A, contained an electrically heated vaporizing element, an adjustable back pressure regulator and a 0 to $30 \mathrm{psig}(0.1$ to $0.3 \mathrm{MPa}$ ) pressure gauge in a single unit. The heating element was a 1-1/4 inch ( 31.8 millimeters) wide by 28 inch ( 71.1 centimeters) long section of 100 mesh, 316 stainless steel screen wrapped around a $1 / 2$ inch ( 12.7 millimeters) 0. D. stainless steel tube. The diameter of the tube plus screen was 0.71 inches ( 18 millimeters). The vaporizer was designed to have the sample flow through the screen perpendicular to the axis of the tube. Also, the vaporizer contained a bypass valve and a pressure relief valve to protect the pressure gauge; both of these valves were capped in this study. Approximately 15 inches (381 millimeters) of stainless steel tubing connected the probes to vaporizer A. To prevent premature vaporization the line was vacuum jacketed to within 1 inch ( 25 millimeters) of the vaporizer inlet. When using this vaporizer, valve $\mathrm{T} 11$ and $\mathrm{T} 12$ replaced the function of valves $\mathrm{T} 7$ and $\mathrm{T} 9$. The third vaporizer was a Cook sampler, while the reference vaporizer and vaporizer A were for continuous sampling, the Cook sampler was designed for the batch sampling. Since the LNG sample was used to cool the sampler, the lines were piped to return the vapor to the LNG vessel; otherwise, the mixture would be depleted too quickly. The line from the probes to the sampler was roughly 15 inches ( 381 millimeters) long and vacuum jacketed.

Liquid nitrogen ( $\mathrm{LN}_{2}$ ) was used as the refrigerant in this study. The LN 2 vessel was $9-7 / 8$ inches (251 millimeters) in diameter and 10-3/4 inches (273 millimeters) long and could contain about 10 liters of liquid. The LNG vessel was cooled by liquid flowing from the $\mathrm{LN}_{2}$ vessel and through a coil wrapped and soldered on the LNG vessel. Cold nitrogen vapor leaving the coil went to another coil soldered on a $1 / 8$ inch ( 3.2 millimeters) thick copper plate located roughly 2 inches (50 millimeters) below the top plate. All lines into the vacuum space were in thermal contact with this plate to reduce heat leakage. After leaving the copper plate the nitrogen went to valve N1 and to a 0-44 liter per minute full scale rotometer and/or to bypass valve $\mathrm{N}_{2}$. When the apparatus was temporarily shut down, e.g., overnight, nitrogen gas was vented through another line which contained a relief valve set at 8 psig $(0.16 \mathrm{MPa})$. During these periods the $\mathrm{LN}_{2}$ vessel needed to be filled every 24 to 28 hours. The LNG vessel cooled at a rate of 1 to $2 \mathrm{~K}$ per day during idle periods.

To minimize radiation heat leak in the vacuum space, the apparatus was surrounded by a copper radiation shield. This shield was fastened to the $\mathrm{LN}_{2}$ vessel and extended up to the copper plate.

\section{2 .5 .2 Instrumentation}

The temperature was measured using a platinum resistance thermometer (PRT) calibrated on the IPTS-68 temperature scale. The PRT was encased in a copper sheath pressurized with helium gas and positioned near the bottom of the LNG vessel. To measure the resistance, a one milliampere constant current source was used with a potentiometer and a nanovolt null detector. The thermometer current was monitored by measuring the voltage drop across a 100 ohm standard resistor. Initially, the output from the null detector was directed to a power regulator; this regulator powered either a 120 or a 230 ohm heater wrapped on the LNG vessel. Although the temperature control was good ( $\pm 0.01 \mathrm{~K}$ ), crosstalk developed between the power regulator and the null detector. This control method was dropped and thereafter the temperature was controlled by manually adjusting the $\mathrm{LN}_{2}$ flow rate; using this method, it was possible to control the temperature to $\pm 0.03 \mathrm{~K}$ or better. 
The saturation pressure of the LNG was measured with a 0 to 100 psia (0 to 6.9 MPa) quartz bourdon tube pressure gauge; this gauge was calibrated against an air dead weight gauge. The difference between the LNG pressure in the test section and in the LNG vessel was measured with a 0-300 inches of water ( 0 to $0.075 \mathrm{MPa}$ ) differential pressure gauge; this gauge was not calibrated during this study.

\section{2 .5 .3 Safety Features}

Since the study involved flammable fluids, all vents on the LNG system were tied to the room vent system. In addition, all vacuum pump outlets were connected to the same vent system. A relief valve, set at $120 \mathrm{psig}(0.93 \mathrm{MPa})$, was located on the sample return line; this relief valve led to the vent system. Finally, the insulating vacuum system had a relief valve connected to the vent and a pressure switch-solenoid valve combination. If the vacuum pressure increased to $100 \mathrm{microns}$ (13 Pa), or, if there was a power failure, the solenoid closed, isolating the vacuum space from the vacuum pump.

Recirculation Pump Motor In the early stages of the study some problems developed with the motors selected to drive the recirculation pump. The same pump had been operated in Iiquid helium using a small three phase induction motor [21]. However, this motor had too much slippage when used with LNG (the density of LNG is four times greater than that of liquid helium). Two small motors in tandem still did not provide sufficient torque. A $1 / 7 \mathrm{HP}$ (106 watt) dc brush motor was then tried, and the motor had sufficient torque but operated only 100 hours before the brushes failed. The brushes were replaced and the same motor operated for another 90 hours. Finally, a larger capacity three phase induction motor ( $1 / 2 \mathrm{HP}$ ( 373 watt) at $10,000 \mathrm{rpm}$ ) was purchased and installed; it worked satisfactorily for the remainder of the study.

\subsubsection{Experimental Procedure}

After checking for leaks the insulating vacuum space was evacuated for 15 or more hours. By this time the pressure was down to $10^{-4}$ millimeters $\mathrm{Hg}(0.1 \mathrm{~Pa})$ or less. The complete LNG system was evacuated and back filled several times with high purity nitrogen which passed through a molecular sieve purifier. The cooldown was started by filling the $\mathrm{LN}_{2}$ vessel with liquid nitrogen. With bypass valve N2 (see figure 2.2.12) wide open it took about 2 hours to bring the apparatus to $110 \mathrm{~K}$.

Once at LNG temperature, all instruments and the pump were tested, and the system was checked for cold leaks. If all was well, the prepared mixture was condensed into the LNG vessel. Using a pressure of 40 to 50 psi $(0.27$ to $0.34 \mathrm{MPa}$ ) above the mixture's saturation pressure it took about. one hour to fll the LNG vessel; when the LNG vessel was full there was a sudden rise in the pressure of the LNG system. At this point the startup was complete.

Preparation of Known Mixture Because the accuracy of Sampling Measurement Systems (SMS) needed to be known, prepared mixtures of known composition were required in the laboratory scale apparatus. Of the several preparation methods considered, it was decided that the fastest and most accurate method was to fill a large ( $1.75 \mathrm{ft}^{3}$ ( 49.6 liters)) gas cylinder with pure fluids and analyze the resulting mixture. (Size and weight limitations of the mass balance made preparation by weight unfeasible.) Partial pressures were used as a guide for estimating how much of each component should be added. Prior to filling, the cylinder was evacuated while being warmed with a heat lamp for at least 48 hours.

The cylinder was filled to $1100 \mathrm{psi}(7.59 \mathrm{MPa})$, which was well below the dew point pressure of the mixtures used here, and warmed with heat lamps for at least 6 hours. To enhance mixing, the cyl 1 nder was alternately heated in vertical and horizontal positions; when in the horizontal position a heat lamp was placed at each end of the cylinder. Next, a sample of the mixture was analyzed on the cc: the cylinder then was heated for several more hours before making another analysis. If the analyses did not agree, the procedure was repeated until they did. (It never took more than 48 hours to obtaln complete mixing.) Next, a series of at least 6 analyses were made on the mixture and the results averaged to determine the composition as accurately as possible.

After filling the LNG vessel the cylinder pressure was 100 to 150 psig (0.79 to $1.14 \mathrm{MPa}$. The mixture remaining in the cylinders was analyzed again to see if the composition had changed. Alao. this gas mixture was analyzed several times during each operating day so that day-to-day sh1rts $1 \mathrm{n}$ tho analysis could be detected. Table 2.2 .15 gives the a summary of the average values of 1 des 1 a heating value and liquid density from the final sets of analyses for the prepared mixturn. The analyses are included in the original publication [1]. 
Table 2.2.15

Summary of Average Value and Estimated Standard Deviation in

Ideal Gas Heating Value and Liquid Density of the Prepared Mixtures Charged into the Laboratory Apparatus*

\begin{tabular}{|c|c|c|c|c|}
\hline $\begin{array}{l}\text { Mixture } \\
\text { Number }\end{array}$ & $\begin{array}{l}\text { When } \\
\text { Analyzed }\end{array}$ & $\begin{array}{l}\text { Number of } \\
\text { Analyses }\end{array}$ & $\begin{array}{l}\text { Ideal Gas } \\
\text { Heating Value } \\
\text { Btu/scf } \\
\left(\mathrm{MJ} / \mathrm{m}^{3}\right)\end{array}$ & $\begin{array}{l}\text { Liquid } \\
\text { Density } \\
\text { g/ } \mathrm{cm}^{3}\end{array}$ \\
\hline \multirow[t]{2}{*}{1} & $\begin{array}{l}\text { Before } \\
\text { filling }\end{array}$ & 6 & $\begin{array}{c}1151.0 \\
0.06 \\
(42.876) \\
(0.002)\end{array}$ & $\begin{array}{l}0.4692(113 \mathrm{~K}) \\
0.00002 \\
0.4593(120 \mathrm{~K}) \\
0.00002\end{array}$ \\
\hline & $\begin{array}{l}\text { After } \\
\text { filling }\end{array}$ & 15 & $\begin{array}{c}1150.77 \\
0.68 \\
(42.867) \\
(0.024)\end{array}$ & $\begin{array}{l}0.4691(113 \mathrm{~K}) \\
0.0002 \\
0.4592(120 \mathrm{~K}) \\
0.0002\end{array}$ \\
\hline \multirow[t]{2}{*}{5} & $\begin{array}{l}\text { Before } \\
\text { filling }\end{array}$ & 6 & $\begin{array}{c}1151.6 \\
1.1 \\
(42.897) \\
(0.040)\end{array}$ & $\begin{array}{l}0.4788(111 K) \\
0.0003 \\
0.4662(120 K) \\
0.0003\end{array}$ \\
\hline & $\begin{array}{l}\text { After } \\
\text { filling }\end{array}$ & 25 & $\begin{array}{c}1151.4 \\
0.3 \\
(42.891) \\
(0.011)\end{array}$ & $\begin{array}{l}0.4788(111 \mathrm{~K}) \\
0.0001 \\
0.4682(120 \mathrm{~K}) \\
0.0001)\end{array}$ \\
\hline \multirow[t]{2}{*}{6} & $\begin{array}{l}\text { Before } \\
\text { filling }\end{array}$ & 9 & $\begin{array}{c}1141.2 \\
0.6 \\
(42.510) \\
(0.022)\end{array}$ & $\begin{array}{l}0.4537(123 \mathrm{~K}) \\
0.0002\end{array}$ \\
\hline & $\begin{array}{l}\text { After } \\
\text { filling }\end{array}$ & 39 & $\begin{array}{c}1141.6 \\
0.4 \\
(42.525) \\
(0.015)\end{array}$ & $\begin{array}{l}0.4538(123 K) \\
0.0001\end{array}$ \\
\hline
\end{tabular}

* The day-to-day scatter caused by calibration error has been subtracted from the estimated standard deviations presented in this and all of the following tables.

Operating Procedure The daily startup consisted of bringing the system to the desired operating condition and calibrating the gas chromatograph. The reference vaporizer and vaporizer A required 15 to 30 minutes, depending upon the sample flow rate, to establish steady-state conditions. The flow through the vaporizer had to be stable to obtain temperature control because the sample stream constituted 25 to 50 percent of the heat load on the system. (The only other major heat input was from the pump motor.)

After establishing the desired operating conditions the accumulator was isolated by closing valve T8 and valve T7 or T11 (see figure 2.2.12), depending upon the vaporizer used. (Operating the Cook sampler is discussed below.) To ensure a representative sample of the mixture leaving the vaporizer, the sample passed through the accumulator for at least 10 minutes under steady-state conditions. Immediately after isolating the accumulator either valve T9 or T12 was opened (valve T10 remained open) to avoid upsetting the system. At this time the operating variables could be changed so that the system would be stable when the next sample was ready to be taken. Under normal operation the power input to the reference vaporizer was set so that the tubing just downstream was warm to the touch; vaporizer A contained a thermostated heater set at $125 \mathrm{~F}$ (40 $\mathrm{C}$ ).

To analyze the trapped sample in the accumulator the line between valve T14 and the GC was evacuated by opening valves V1, V5 and V6 (see figure 2.2.4). After closing valve V6, the line then was pressurized to about $2 \mathrm{psig}(0.11 \mathrm{MPa})$ by opening valve T14. This purge and evacuation procedure was repeated three times; next, valve T14 was left open and V1 closed. Then the analysis procedure described in section 2.2 .4 was followed. 
To draw a sample from the accumulator bypass line, valves V2 and V7 were opened to evacuate the 1 ine connecting V2 and T16. After closing V7, valve T17 was opened to pressurize the line to about 2 psig $(0.11 \mathrm{MPa})$. After repeating the purge procedure three times, valve V2 was closed and $\mathrm{T} 17 \mathrm{left}$ open. Next, the analysis procedure was followed. The sample flow in the bypass line was uninterrupted during the purging procedure.

Inlt lally, at least two analyses were made on each sample. Since each analysis took 14.5 minutes, this limited the analysis to a maximum of two samples per hour. Once the integrator was repaired, only one analysis was made on each sample unless the results were more than three estimated standard deviations, for gas analysis alone, from the expected values. In this case, another analysis was made; In all cases, duplicate analyses were within one estimated standard deviation of the first analysis for all components. The prepared mixture from the cylinder was analyzed several times a day. Most often these were made during a temperature change and/or a $\mathrm{LN}_{2}$ vessel filling -- each of which took about 30 minutes. The prepared mixture also was analyzed at the end of the day after shutting down the apparatus.

Sample flow to the Cook sampler was obtained by closing valves $\mathrm{T} 6$, $\mathrm{T} 11$, and $\mathrm{T} 12$ and by opening valves $\mathrm{T} 7$ and $\mathrm{T} 8$ and the valves on the Cook sampler (see figure 2.2.12). Once the sampler contained only liquid the valves on the sampler were closed, and it was allowed to warm up. Next the accumulator was evacuated by closing valve T8 and opening valve T13 for several minutes. After closing valve T13, the downstream valve on the Cook sampler was opened. Then the accumulator was isolated by closing valve T7 and the mixture analyzed following the procedures described above.

Control of Operating Variables The operating variables initially considered in this study were temperature, Reynold's number in the test section (Re), difference between the pressure at the sampling point and saturation pressure ( $p p)$ and sampling rate (Q). The temperature was maintained by balancing the refrigerant flow against the heat input. Usually temperature control was maintained by having only valve $N 1$ open (see figure 2.2.12); however, when operating at high sample flow rates, bypass valve $\mathrm{N} 2$ had to be opened as much as $1 / 4$ turn. Both Re, i.e., flow rate in the test section, and $\mathrm{pp}$ were set by adjusting pump speed and valve T2. (In all tests reported here valve T1 remained wide open.) Valve T15 controlled the sampling rate.

\subsubsection{Experimental Results}

The laboratory tests were divided into three phases:

a) Probe Evaluation

b) Vaporizer Evaluation

c) General Tests.

The first two phases were conducted prior to the first flow facility tests. The last series of tests were made to explain the unexpectedly poor results obtained at the flow facility. The second flow facility tests and the shipboard tests followed the general laboratory tests.

\subsubsection{Probe Evaluation}

The purpose of this phase of work was to:

a) determine which, if any, operating variables affected the reliability of the three probes,

b) determine which of the test probes was the most reliable,

c) show that the reference probe produced samples of the same composition as the test probes, and

d) establish the precision and accuracy of the reference probe.

The reason for using a reference probe was to determine the mixture composition. This probe design is unfeasible for most industrial applications. However, proving the probe's reliability required tests using mixtures of known composition. In the probe evaluation three different compositions were used. and two of these were mixtures of known composition.

In addition to the composition, the following operating variables were considered:

a) three sample flow rates (Q),

b) four Reynold's numbers (Re) in the test section,

c) two operating pressures, i.e., temperatures, and

d) five differential pressures ( $p p)$ i.e., the difference between the pressure at the sampling point and the saturation pressure of the mixture. 
The original document of Parrish, Arvidson and Labrecque [1] contains the raw data for evaluation tests of the reference, side tap and pitot tube probes in conjuction with the reference vaporizer. Table 2.2.16 gives a summary of the estimated standard deviations in ideal gas heating value and liquid density for each probe. The variables for the tests 1 through 4 were Reynolds Number, heat input, differential pressure and temperature for test 1; Heat input for test 2 and Reynolds Number, differential pressure and temperature for tests 3 and 4 . Except where noted in the text, average values of composition, ideal gas heating value, and liquid density for these tests are indistinguishable statistically from the corresponding values given in table 2.2.15. ( The appendix of the original publication [1] lists the raw data for all sampling tests.) Except for Test 2, the estimated standard deviation in heating value and density for the sampling data is presented as a percentage of the average values obtained from analysis of the prepared mixture in the gas cylinder. In test 2 the percentage is based on the average of all of the probe analyses.

Table 2.2 .16

Summary of Estimated Standard Deviation in Heating Value and Liquid Density for Probe Tests

\begin{tabular}{|c|c|c|c|c|}
\hline \multirow[b]{2}{*}{$\begin{array}{l}\text { Test } \\
\text { Number }\end{array}$} & \multirow[b]{2}{*}{ Probe $^{1}$} & \multirow[b]{2}{*}{$\begin{array}{c}\text { Number } \\
\text { of } \\
\text { Points }\end{array}$} & \multicolumn{2}{|c|}{ Standard Deviation (\%) } \\
\hline & & & $\begin{array}{l}\text { Heating } \\
\text { value }\end{array}$ & $\begin{array}{l}\text { Liquid } \\
\text { Density }\end{array}$ \\
\hline 1 & $\begin{array}{l}\mathrm{R} \\
\mathrm{S} \\
\mathrm{P}\end{array}$ & $\begin{array}{r}6 \\
5 \\
14\end{array}$ & $\begin{array}{l}0.04 \\
0.06 \\
0.09\end{array}$ & $\begin{array}{l}0.02 \\
0.03 \\
0.06\end{array}$ \\
\hline 2 & $\begin{array}{l}R \\
S \\
P\end{array}$ & $\begin{array}{l}9 \\
8 \\
8\end{array}$ & $\begin{array}{l}0.04 \\
0.09 \\
0.11\end{array}$ & $\begin{array}{l}0.02 \\
0.06 \\
0.08\end{array}$ \\
\hline 3 & $\begin{array}{l}R \\
S \\
P\end{array}$ & $\begin{array}{l}13 \\
13 \\
13\end{array}$ & $\begin{array}{l}0.04 \\
0.03 \\
0.08\end{array}$ & $\begin{array}{l}0.03 \\
0.03 \\
0.07\end{array}$ \\
\hline 4 & $\begin{array}{l}R \\
S \\
P\end{array}$ & $\begin{array}{l}12 \\
12 \\
12\end{array}$ & $\begin{array}{l}0.03 \\
0.02 \\
0.07\end{array}$ & $\begin{array}{l}0.01 \\
0.02 \\
0.05\end{array}$ \\
\hline
\end{tabular}

1 Reference probe ( $R$ ), side tap probe (S) and pitot tube probe (P).

2 These standard deviations are given as a percentage of the mean value determined by analyses of the prepared mixture in the gas cylinder. However, the values for test 2 are a percentage of the mean value of all probe analyses in the test.

Test 1 The purpose of this test was to establish the feasible operating range of the variables and to identify operating variables which affect the reliablility of the probes. Prepared mixture 1 was used in this test. Figure 2.2.13 shows the deviation in the computed ideal gas heating values as a function of probe and run number. The deviation is the difference between the heating value obtained via the probe sample and the heating value given in table 2.2.15 for mixture 1 , all divided by the tabular value. Replicate points for a given run number represent the results of replicate gas analyses. The occasional large scatter between replicate analyses was caused by a GC integrator malfunction (see section 2.2.4). Although this scatter confuses the results somewhat, figure 2.2 .13 shows that the pitot tube tends to be more erratic. The results showed that it is possible to obtain representative samples at a pp as low as $1.8 \mathrm{psi}(0.012 \mathrm{MPa})$; the pressure drop in the sampling system prevented us from trying a lower pp.

A statistical analysis of the results from the reference probe and the prepared mixture indicates that the reference probe collected a representative sample. The figure indicates that most of the computed heating values were slightly higher than the prepared mixture; this bias apparently was caused by not making three independent GC calibrations each day. Figure 2.2.13 shows that all three probes sampled the same liquid.

Test 2 The first test was not a statistically designed experiment; therefore, the effect of each operating variable could not be quantitatively determined. Nitrogen was added to the mixture to bring the nitrogen content to roughly $1.34 \mathrm{~mol}$ percent for Test 2. (Two intermediate concentrations of 0.55 


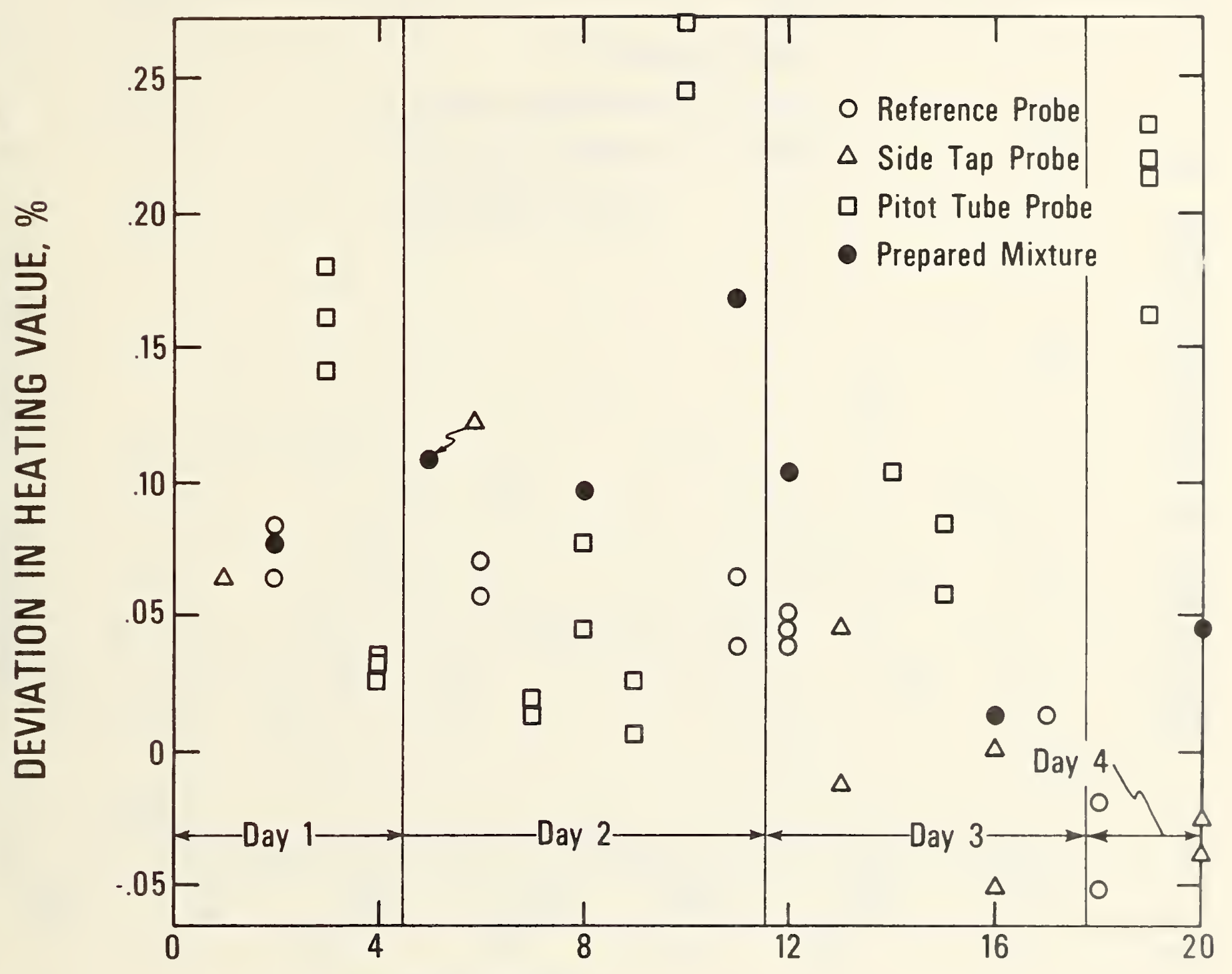

RUN NUMBER

Figure 2.2.13. Deviations in the computed ideal gas heating values for probe test 1 .

and $4.34 \mathrm{~mol}$ percent nitrogen content were made before reaching the 1.34 mol percent level: the data taken with these mixtures are given in the original publication [1].) In this test the sampling rate was varied between 2.4 and $7.2 \mathrm{slpm}$ (standard liters per minute, at $70^{\circ} \mathrm{F}\left(21{ }^{\circ} \mathrm{C}\right.$ ) and $14.7 \mathrm{ps} 1 \mathrm{a}$ $(0.101 \mathrm{MPa}))$. Figures 2.2.14, 2.2.15 and table 2.2.17 show the scatter, on a percentage bas $1 \mathrm{~s}$ ab 12 the mean, in the computed heating value and density as a function of run number and probe. There seems to be a day-to-day effect in the scatter with the first and last days being the worst and bes.. respectively. The only plausible explanation for this behavior is that the contents may not hive the 7 well mixed on the first day (this same trend seems apparent in figure 2.2.13).

In these tests the prepared mixture was condensed into the LNG vessel from the trp. unfortunately minimized the mixing during the filling process. The same procedure was followed when

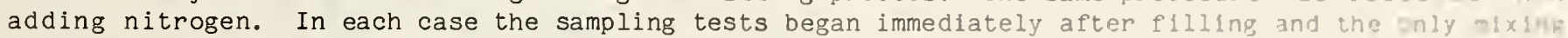
occurred during the recirculation of the LNG. 


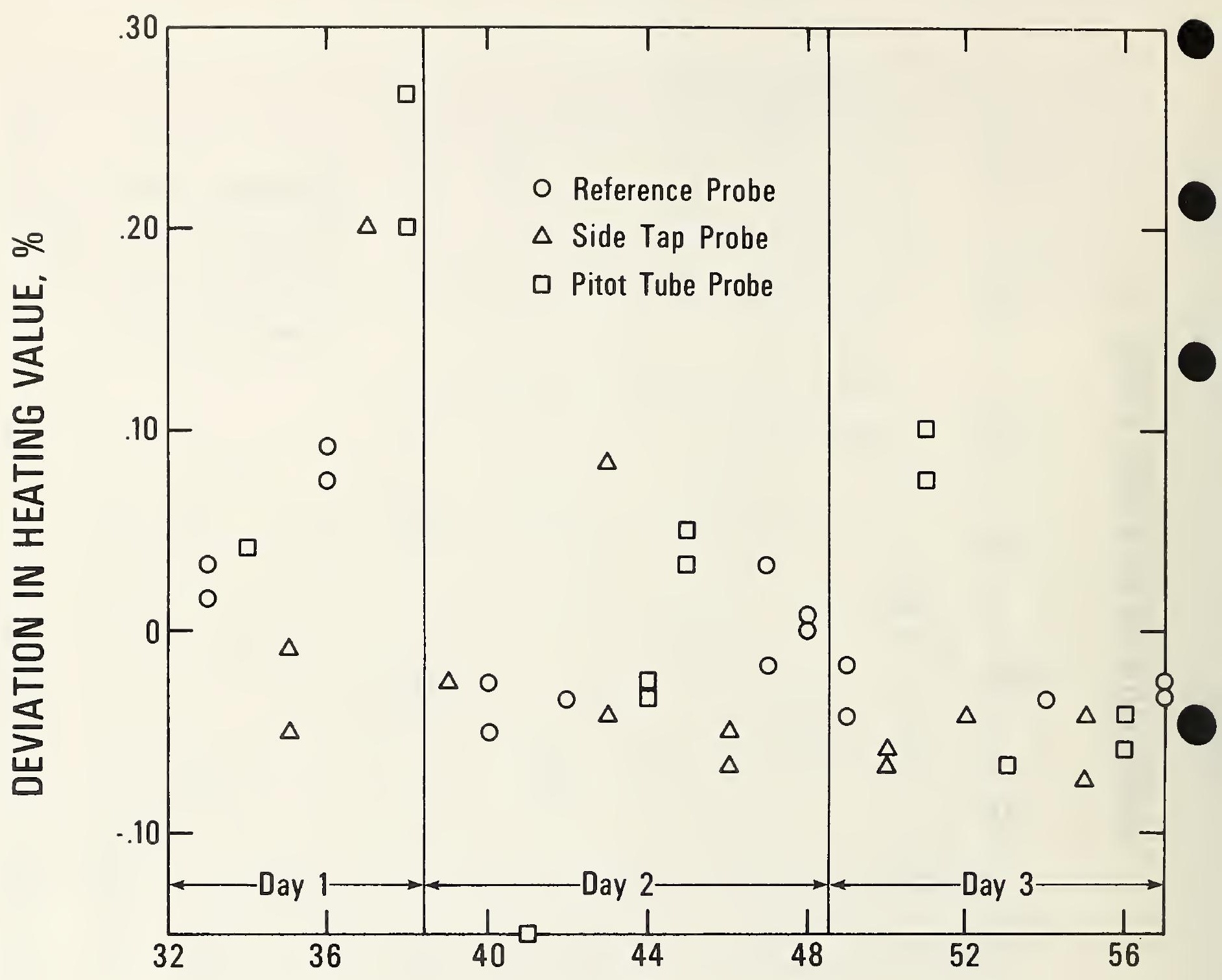

\section{RUN NUMBER}

Figure 2.2.14. Deviations in the computed ideal gas heating values for probe test 2 .

Table 2.2.17

Summary of Estimated Standard Deviation in Heating value and Liquid Density for Probe Type and Flow Rate

\begin{tabular}{|c|c|c|c|c|}
\hline \multirow{2}{*}{$\begin{array}{c}\text { Sampling } \\
\text { Rate } \\
\text { slpm }\end{array}$} & Probe & $\begin{array}{c}\text { Number } \\
\text { of } \\
\text { Points }\end{array}$ & $\begin{array}{c}\text { Standard Deviation (\%) } \\
\text { Value }\end{array}$ & $\begin{array}{c}\text { Liquid } \\
\text { Density }\end{array}$ \\
\hline \hline 2.4 & $\mathrm{R}$ & 5 & 0.04 & 0.03 \\
& $\mathrm{~S}$ & 4 & 0.12 & 0.08 \\
7.2 & $\mathrm{P}$ & 4 & 0.16 & 0.11 \\
& $\mathrm{R}$ & 4 & 0.007 & 0.00 \\
& $\mathrm{~S}$ & 4 & 0.016 & 0.02 \\
& $\mathrm{P}$ & 4 & 0.015 & 0.01 \\
\hline
\end{tabular}




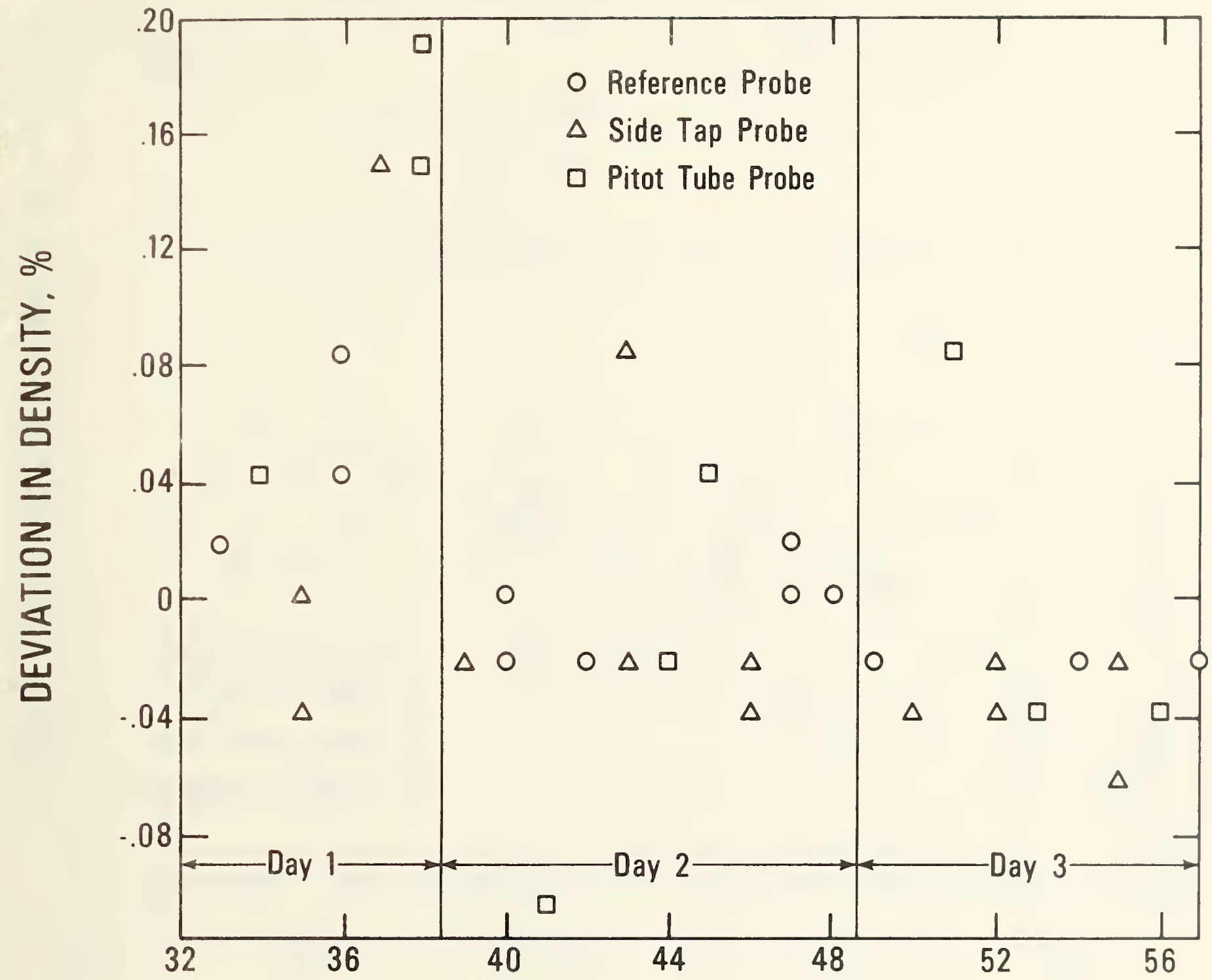

\section{RUN NUMBER}

Figure 2.2.15. Deviations in the computed liquid phase densities for probe test 2 .

Test 3 In this test the temperature, flow rate and pressure difference were varied but the sampling rate was held constant at 4.2 or $5.6 \mathrm{slpm}$. Prepared mixture 4 was used in this test. Recirculat $10 n$ pump characteristics permitted operation at both low and high flow rates (Reynolds numbers of 17.000 to 31,500$)$ at a low pp $(3.6 \mathrm{psi}(25 \mathrm{kPa}))$, but only the low flow rate at the high pp (10.1 psi ( 70 $\mathrm{kPa}))$. Shortly after starting this test the turbine meter stopped working. However, the pump spees required to give the desired flow rates at each pp when operating at $111 \mathrm{~K}$ were unknown and ihls information was used to set the flow rate. It was assumed that the same pp and pump speed worn required to obtain the desired flow rates at $120 \mathrm{~K}$. This was verified later when the turblne meier began working again.

Figure 2.2.16 shows the deviation in the ideal gas heating value for this test. Heither tho rlgre nor a statistical analysis of the data (see table 2.2.17) show an effect due to Re, $p p$ or $T$. A $A 1 \cap$, the pitot tube had the most scatter. The nitrogen content in the liquid was 0.024 mol percent 1 ingr than in the prepared mixture 2; calculation showed that this could have been caused by nitropon go 1 no into the vapor phase during condensation into the apparatus. 


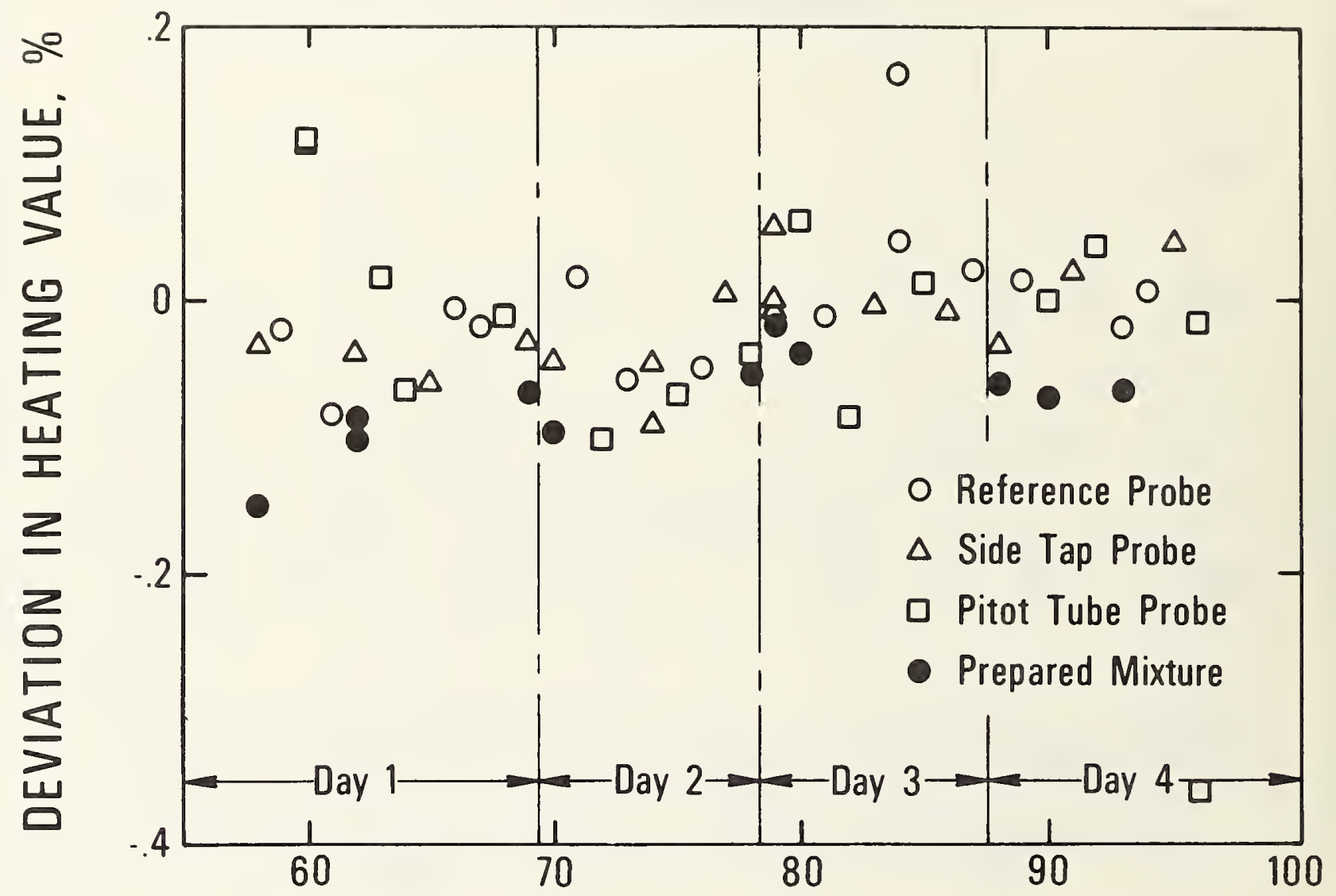

RUN NUMBER

Figure 2.2.16. Deviations in the computed ideal gas heating values for probe test 3 .

Test 4 The purpose of this short test was to ascertain the effect of laminar flow on sampling precision. The difference in operating variables between this test and test 3 was that the Reynold's number was 1500 or 17,000 instead of 17,000 or 31,500 . In this test the low flow rate was maintained the first day while pp and temperature were varied; the second day the flow rate and pp were varied while the temperature was held constant at $111 \mathrm{~K}$. Figure 2.2.17 shows the deviation in ideal gas heating value. During the first part of the second day the power supply for the pump frequently tripped the circuit breaker. Each shutdown caused the vaporizer to warm up. The increased scatter during the first part of day 2 was caused by the reference vaporizer not being at steady state conditions when the gas sample was trapped in the accumulator. Once the power supply problem was fixed (prior to run number 177) the scatter became comparable to that of the previous day. 


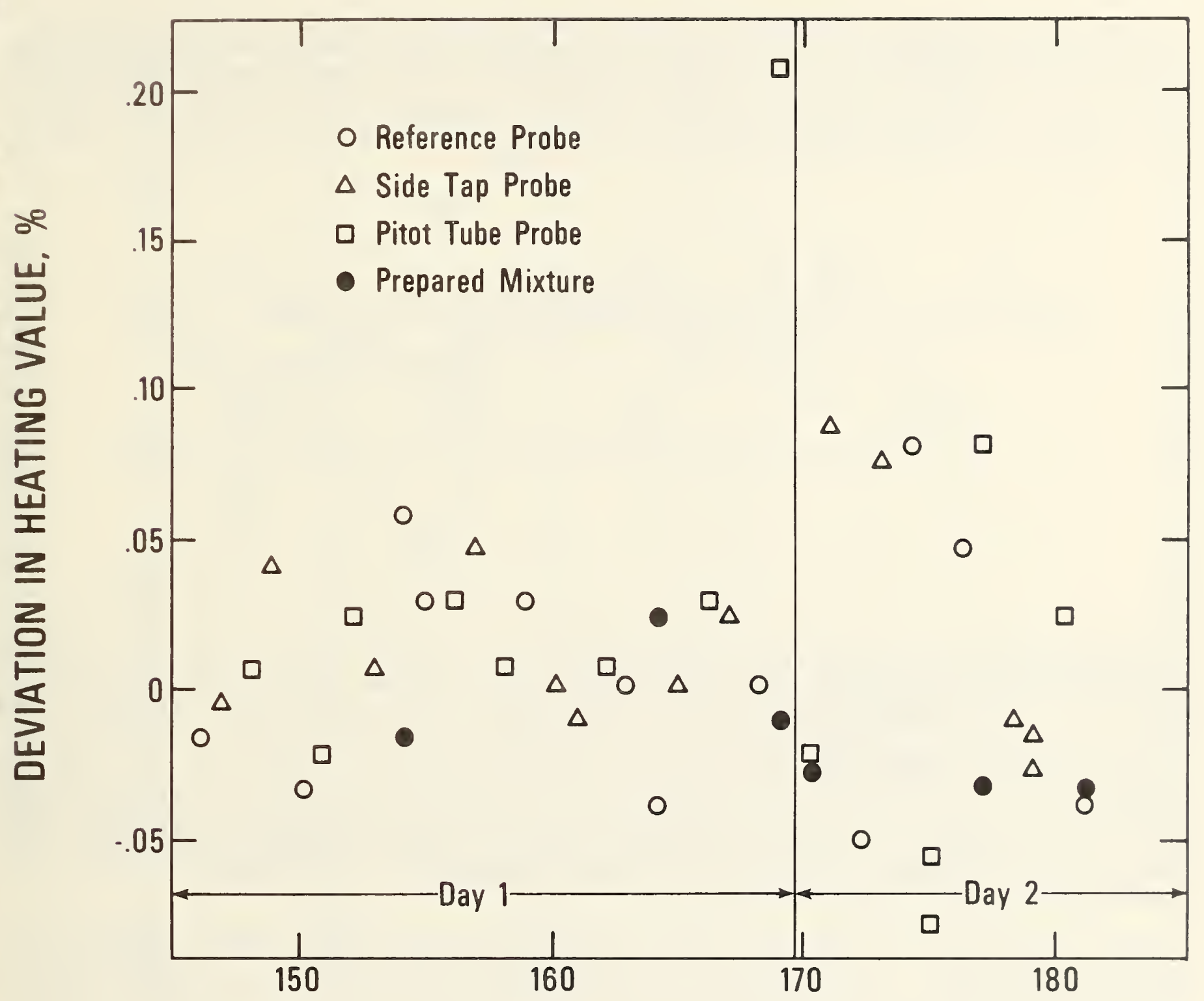

RUN NUMBER

Figure 2.2.17. Deviations in the computed ideal gas heating values for probe test 4 .

The results indicate that the probes work equally well in laminar and turbulent flow. The statlstlcal analysis shows that the nitrogen content decreased by 0.006 mol percent when going from 111 to $120 \mathrm{~K}$. This effect had not been detected in any other test, nor for any other component. However, by the time of this test, roughly 100 samples had been withdrawn from the apparatus, thus increas 1 ng the vapor space in the LNG vessel. Calculations showed that this apparent temperature effect could have been caused by the increased amount of nitrogen in the vapor phase at $120 \mathrm{~K}$.

\subsubsection{Vaporizer Evaluation}

The probe tests showed the reference probe to be the most reliable of the three probes teatod. Therefore, the reference probe was used in the evaluation of vaporizer A and the Cook sampler. Stnce the Cook sampler is designed for batch sampling, it is not a continuous vaporizer $11 k e$ the refornnce vaporizer and vaporizer A. Preliminary testing of the Cook sampler showed that $1 \mathrm{t}$ would not work whl on the laboratory apparatus. Cook [23] indicated that it is designed for use wlth hlgh sagplan. rates, so that the total sampling time would be less than 30 seconds. 
In these tests the sampling rate was so low that it took at least 30 minutes to cool the sampler down. By this time the sampler valves were very cold and leaked when closed. Because of this problem and because the primary interest was in continuous sampling systems, the Cook sampler was not tested further on the laboratory apparatus. However, vaporizer A was found to work at low sampling rates; it was evaluated in the same manner as the probes. Prepared mixture 4 was used in both tests. Table 2.2.18 lists the estimated standard deviation in ideal gas heating value and liquid density. The raw test data is include in the original work [1].

Table 2.2.18

Summary of Estimated Standard Deviation in Heating Value and Liquid Density for Vaporizer ( $R$ ) and (A)

\begin{tabular}{|c|c|c|c|c|}
\hline \multirow{2}{*}{$\begin{array}{c}\text { Test } \\
\text { Number }\end{array}$} & \multirow{2}{*}{ Vaporizer } & \multirow{2}{*}{$\begin{array}{c}\text { Number } \\
\text { of } \\
\text { Points }\end{array}$} & $\begin{array}{c}\text { Heating } \\
\text { Value }\end{array}$ & $\begin{array}{c}\text { Siquid } \\
\text { Density }\end{array}$ \\
\hline \hline 1 & A & 29 & 0.76 & 0.8 \\
& R & 10 & 0.11 & 0.06 \\
2 & A & 8 & 0.25 & 0.19 \\
\hline
\end{tabular}

Test 1 The purpose of this test was to evaluate vaporizer A at two sampling rates (2.0 and 5.6 slpm), temperatures ( 111 and $120 \mathrm{~K}$ ), pressure differences (3.6 to $10.1 \mathrm{psi}$ (25 to $70 \mathrm{kPa})$ ) and flow rates (Re of 18,000 to 35,000$)$; these conditions are comparable to those used for the probe evaluations. Figure 2.2.18 shows the deviation in heating values for this test. The estimated standard deviation in the heating value for this vaporizer was about 20 times greater than in any other test. However, the precision of the reference system was poorer in this test than in any other test. This increased scatter was due to either incomplete accumulator purging and/or to taking a sample too quickly after starting the reference vaporizer. Three points on the third day showed that the reference system precision was a factor of three worse than usual. During these runs the accumulator residence time was 40 to 120 seconds; the samples were analyzed after 10 minutes of purging the accumulator. The fourth reference sample was taken after the accumulator was purged for 20 minutes. This sample was representative of the prepared mixture.

Once it was obvious that vaporizer A was not performing well, several minor changes were made to try to reduce the scatter. Originally the sampling rate was set by using valve T15 (see figure 2.2 .12 ); starting with run 137, the sample rate was set by adjusting the pressure regulator on the vaporizer. This did not improve the results. During most of this test the throttling valve (valve T6, figure 2.2.12) for the reference vaporizer was roughly $1 / 4$ open. Two reference vaporizer runs (runs 108 and 109) were made with the valve wide open, assuming that the valve might be preventing backflashing (this was the purpose of the valve). With the valve wide open, the pressure drop in the lines between the probe and the two vaporizers was comparable. Having the valve wide open did not diminish the performance of the reference probe. Near the end of the test the sampling rate was increased to 16 slpm (runs 137, 140 and 141) or $10 \mathrm{slpm} \mathrm{(the} \mathrm{last} \mathrm{five} \mathrm{runs).} \mathrm{Since} \mathrm{the} \mathrm{higher} \mathrm{sampling} \mathrm{rate} \mathrm{seemed}$ to improve the vaporizer performance, another test was planned to determine the effect of high sampling rate.

Test 2 This test was conducted at a temperature of $111 \mathrm{~K}$ and a pp of roughly $3.8 \mathrm{psi}$ (27 kPa), and the Reynold's number was varied between 16,000 and 33,000 and sampling rate between 6.7 and 19 slpm. Figure 2.2.19 shows the deviation in the heating values for this test. The estimated standard deviation in heating value for vaporizer $A$ in this test was 0.25 percent as compared to 0.76 percent for the first test but still high compared to the typical 0.05 percent obtained for the reference vaporizer. Runs 188 and 189 are different from the rest of the data for unknown reasons. 


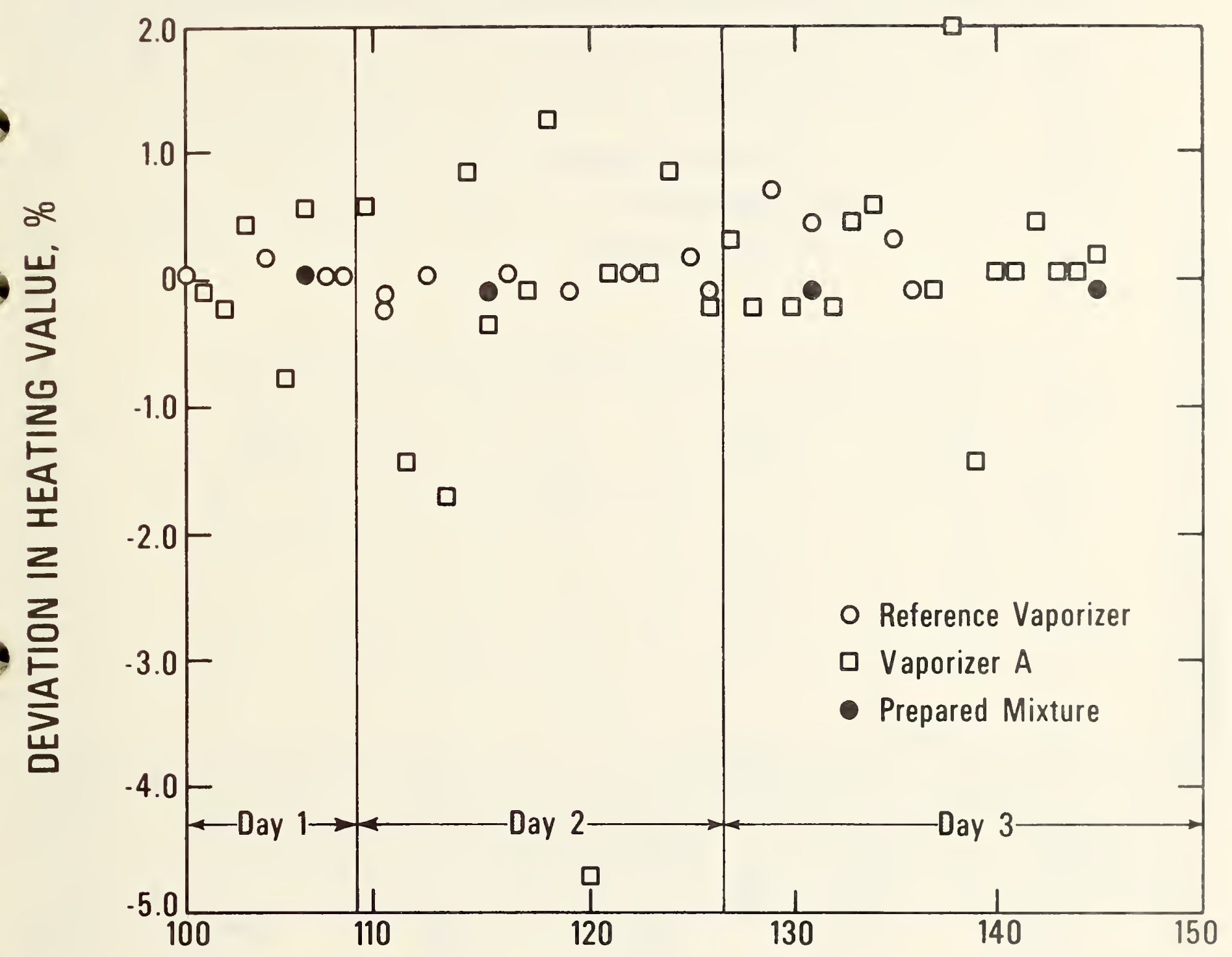

\section{RUN NUMBER}

Figure 2.2.18. Deviations in the computed ideal gas heating values for vaporizer test 1 .

General Tests To explain the poor results obtained in the first flow facility test (see sectlon 2.2.5) it was necessary to conduct additional laboratory measurements. These tests cons1dered whether sampling precision could be affected by:
a) eliminating the sample accumulator,
b) increasing or decreasing the vaporizer outlet temperature,
c) heat leak to the sample probe,
d) minimizing the pressure drop upstream of the vaporizer,
e) decreasing the sample residence time in the accumulator, and
f) having relatively high concentrations of heavy hydrocarbons ( $\mathrm{C}_{5}{ }^{\prime} \mathrm{s}$ through $\mathrm{Cg}^{\prime} \mathrm{s}$ ) in the LNG. 


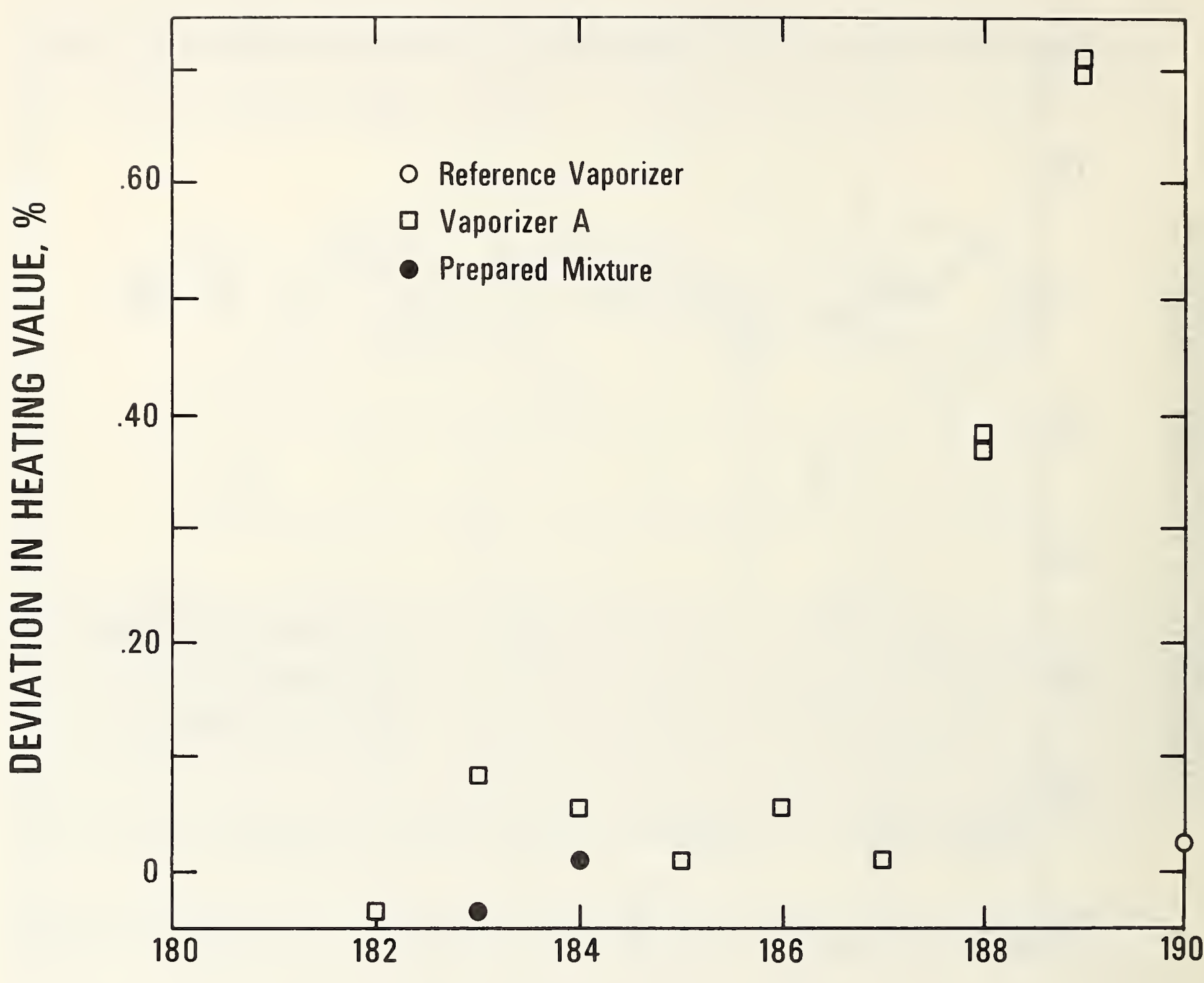

\section{RUN NUMBER}

Figure 2.2.19. Deviations in the computed ideal gas heating values for vaporizer test 2 .

The following operating variables were held constant during each test;

a) temperature and pressure,

b) sampling rate, $Q$ (except when varying accumulator residence time),

c) Reynold's number (Re) in the test section, and

d) differential pressure.

These variable values were in the range known to give good results in the previous laboratory tests. Prepared mixture 6 (see table 2.2.15) was used in tests 1 through 5; LNG, which was prepared by condensing natural gas containing $\mathrm{C}_{6}{ }^{+}$was used in the last test. The reference vaporizer was used in all of the tests. 
Test 1 The purpose of this test was to determine the sampling precision when drawing samples from the accumulator bypass line while having the accumulator bypassed. In all previous laboratory tests (but not the first flow facility test) samples had been taken only from the accumulator. Table 2.2.19 and figure 2.2.20 show that the scatter increased markedly when the accumulator was not used. (The symbols $0, \Delta$, and $\square$ denote samples taken using the reference, side tap and pitot tube probes. The symbols , and denote samples taken using the reference, side tap and pitot tube probes with the accumulator bypassed. The symbol + denotes analysis of the prepared mixture.)

This indicates that the vaporization process generates inhomogeneities in the sample which can be eliminated by time averaging the sample in an accumulator. The accumulator was used in all remaining tests.

Table 2.2 .9

Summary of Estimated Standard Deviation in Heating Value and Liquid Density for Different Probe and Sample Points

\begin{tabular}{|c|c|c|c|c|}
\hline \multirow{2}{*}{$\begin{array}{c}\text { Sampling } \\
\text { Point }\end{array}$} & Probe & $\begin{array}{c}\text { Number } \\
\text { of } \\
\text { Points }\end{array}$ & $\begin{array}{c}\text { Seating } \\
\text { Value }\end{array}$ & $\begin{array}{c}\text { Liquid } \\
\text { Density }\end{array}$ \\
\cline { 4 - 5 } & & 8 & 0.03 & 0.02 \\
Accumulator & $\mathrm{R}$ & 8 & 0.02 & 0.009 \\
& $\mathrm{~S}$ & 6 & 0.05 & 0.04 \\
& $\mathrm{P}$ & 10 & 0.18 & 0.15 \\
& $\mathrm{R}$ & 8 & 2.01 & 1.61 \\
& $\mathrm{~S}$ & 8 & 0.26 & 0.20 \\
\hline
\end{tabular}

In addition to taking discrete samples, a small gas sample stream (approximately 10 cc/min) was continuously fed to an ultrasonic detector. It was hoped that by continuously monitoring overall composition fluctuations (there was no easy way to selectively monitor one component) a periodic behavior could be found. Samples from the bypass line, accumulator, prepared mixture cylinder and from a pure methane cylinder were analyzed continuously. Only the pure methane sample showed no composition fluctuations. The other samples showed random fluctuations with the bypass sample having the largest scatter. The fluctuations in the prepared mixture and accumulator samples were comparable in magnitude. This indicates that even gas mixtures withdrawn from cylinders will contaln some composition variations. Unfortunately, it was not possible to quantify the fluctuations in terms of mol percent.

Test 2 The dew point temperature of the LNG mixtures in the first flow facility test was well below ambient temperature; however, it was thought that a possible problem could have been the use of a long vaporizer warmed only by cold water. To simulate this condition in the laboratory, the power to the vaporizer was varied; this, in effect, changed the vaporizer length. Three different power levels, 0 , 62 and 98 watts, were used. With no input power, the vaporizer plus approximately 5 feet ( $1.5 \mathrm{~m}$ ) of the 1.4 inch 0.D. (6.4 millimeters) copper tubing downstream of the vaporizer became coated w1th frost. At 62 watts the vaporizer outlet temperature was warm to the touch; this was the temperature that was used in all other laboratory tests. At 100 watts, the tubing just downstream of the vaporizer was too hot to touch for more than a second. At all three power levels the accumulator remained at ambient temperature. Only the side tap probe was used in this test and the values ror the 62 watt case were taken from the previous test.

Table 2.2.20 shows that only the 0 power case affected the sampling precision. These results iend $t$ indicate that it is better to vaporize the sample quickly. It was thought that the hlgher heat 1 nplit might offset the effect of bypassing the accumulator. However, table 2.2 .20 and f1gure 2.2 .21 shaw that the increased heat input did not improve the sampling precision when the accumulator was bypassed. (The symbols $\Delta$ and $\Delta$ denote 100 watt power to the vaporizer with and without ising tha accumulator, respectively. The symbols $\nabla, \nabla$ and denote power input, wlth and without tho accumulator and prepared mixture analysis, respectively.) 


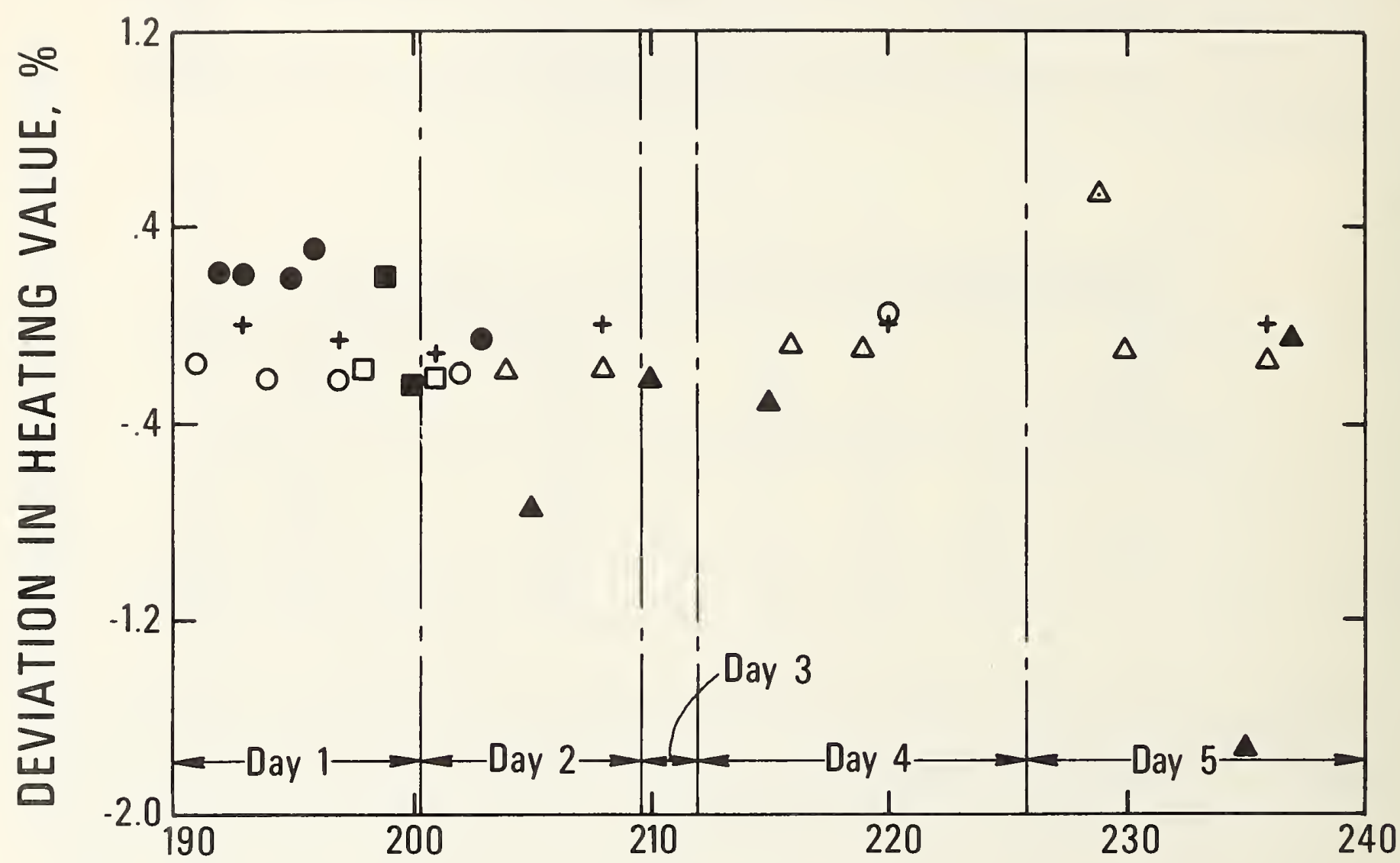

RUN NUMBER

Figure 2.2.20. Deviations in the computed ideal gas heating values for general test 1 .

Test 3 In the first flow facility test the lines from the probes to the vaporizers were uninsulated. Therefore, it was thought that heat leak could cause the observed sampling problems. To test the effect of heat leak 42 ohm heaters were attached to the pitot and side tap probe. Since the probe line was 1.16 inch ( 1.6 millimeters) O.D. tubing it was impractical to wrap heater wire directly on the tubing. The heater wire was wrapped on a copper cylinder which was soft soldered to the probe lines approximately 1.5 inches ( 38 millimeters) from where they entered the test section. The heaters covered approximately 0.5 inches ( 13 millimeters) of the $1 / 16$ inch ( $1 / 6$ millimeter) stainless steel tube giving a heat transfer area of 0.10 in $^{2}$ (63 millimeters $\left.{ }^{2}\right)$. 
Table 2.2.20

Summary of Estimated Standard Deviation in Heating Value and Liquid Density for Different Heat Input to Reference Vaporizer

\begin{tabular}{|c|c|c|c|}
\hline \multirow{2}{*}{$\begin{array}{c}\text { Heater Input } \\
\text { Power, watts }\end{array}$} & $\begin{array}{c}\text { Number } \\
\text { of } \\
\text { Points }\end{array}$ & $\begin{array}{c}\text { Heating } \\
\text { Value }\end{array}$ & $\begin{array}{c}\text { Siquid } \\
\text { Density }\end{array}$ \\
\cline { 2 - 4 } & 6 & 0.12 & 0.10 \\
0 & 12 & 0.02 & 0.02 \\
62 & 6 & 0.02 & 0.02 \\
98 (with accumulator) & 5 & 0.18 & 0.14 \\
98 (accumulator bypassed) & 5 & & \\
\hline
\end{tabular}

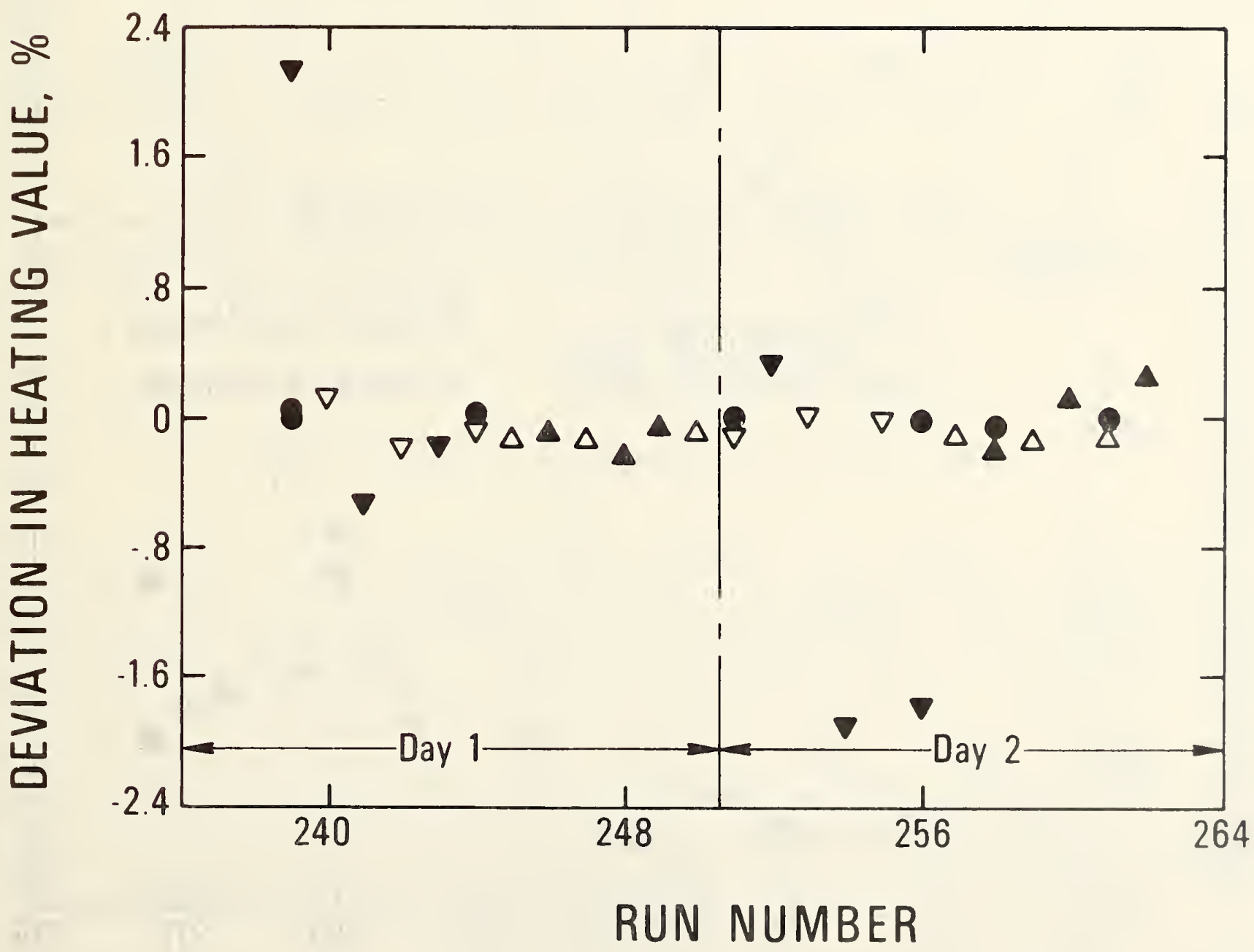

Figure 2.2.21. Deviations in the computed ideal gas heating values ror general test 2 . 
In the first part of the test, the side tap was heated and samples were taken at input powers of 0.10 , $0.42,0,1.7$ and 6.7 watts - this corresponds to $0.2,0.7,0,3$ and 11 percent of the power ( 62 watts) to the vaporizer. As figure 2.2.22 shows, there was no apparent effect on sampling. At 6.7 watts the heater shorted out. The test was repeated for the pitot tube. Although, statistically, there was no apparent change in the precision, increasing the heat seemed to decrease the methane and nitrogen content (runs 269 through 275 in figure 2.2.23). Therefore, it was decided to repeat the test with the heat input randomized instead of systematically increasing the input power. Table 2.2.21 lists the estimated standard deviations. This test showed that the apparent trend noted before for nitrogen was coincidence and that there was no detectable effect on the sampling precision.

Table 2.2.21

Summary of Estimated Standard Deviation in Heating Value and Liquid Density for Pitot Tube Probe with Probe Line Heater

\begin{tabular}{|c|c|c|c|}
\hline \multirow{2}{*}{$\begin{array}{c}\text { Heater Input } \\
\text { Power, watts }\end{array}$} & \multirow{2}{*}{$\begin{array}{c}\text { Number } \\
\text { of } \\
\text { Points }\end{array}$} & $\begin{array}{c}\text { Standard Deviation (\%) } \\
\text { Value }\end{array}$ & $\begin{array}{c}\text { Liquid } \\
\text { Density }\end{array}$ \\
\cline { 3 - 4 } & 4 & 0.06 & 0.04 \\
1.7 & 4 & 0.03 & 0.03 \\
6.7 & 4 & 0.04 & 0.03 \\
\hline
\end{tabular}

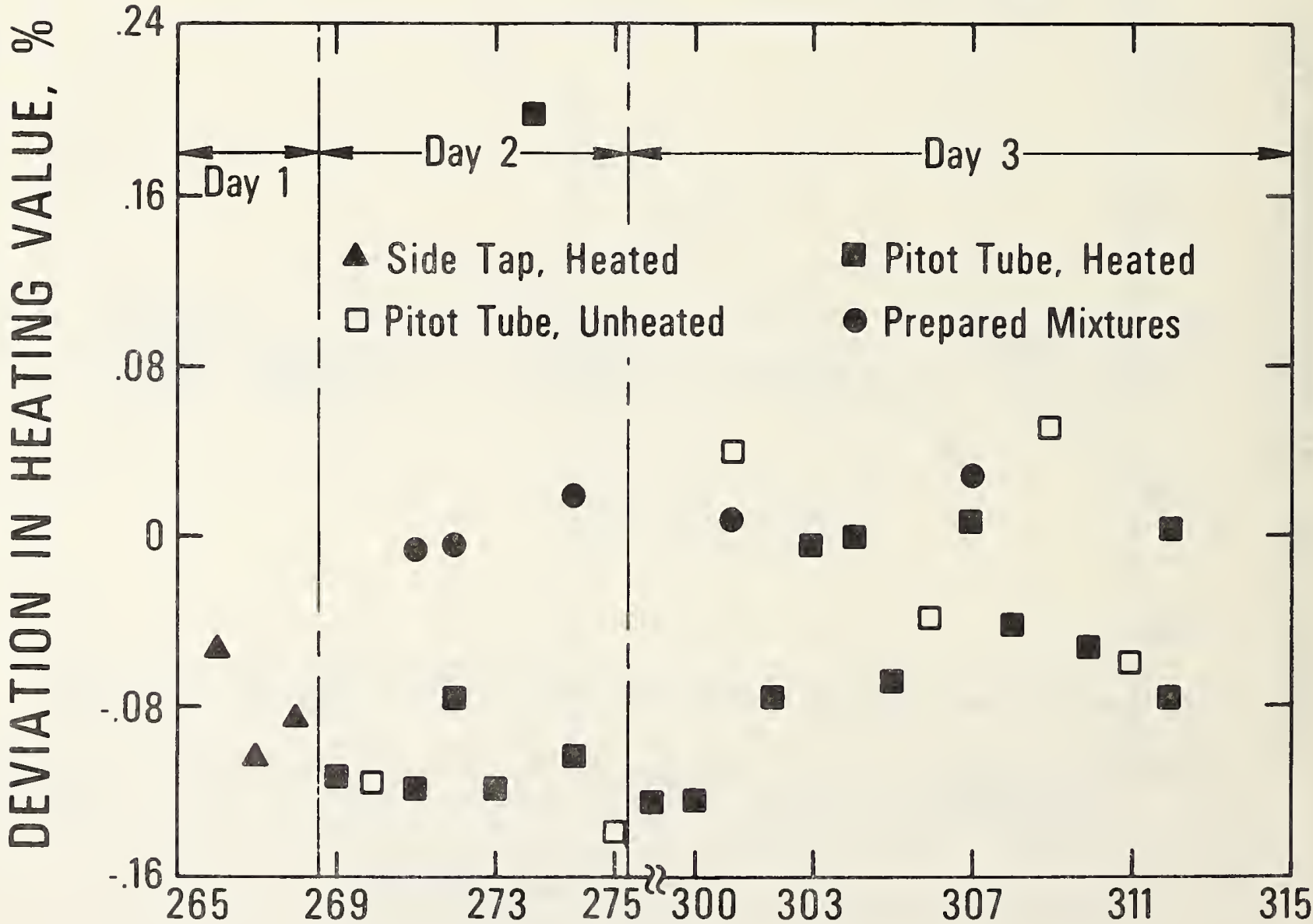

\section{RUN NUMBER}

Figure 2.2.22, Deviations in the computed ideal gas heating values for general test 3 . 


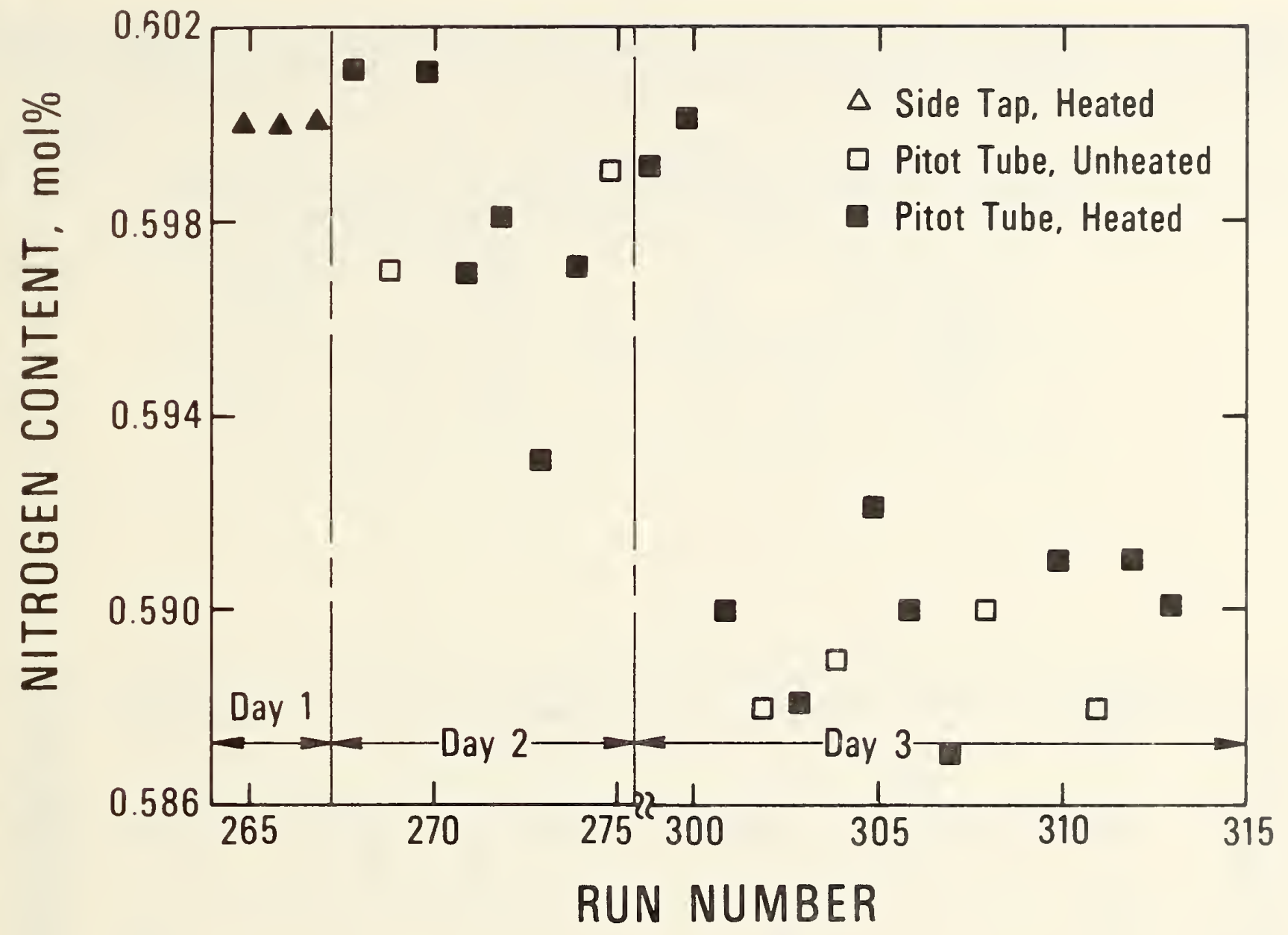

Figure 2.2.23. Nitrogen content for general run 3.

During the last run of this test, this heater shorted out too. When the apparatus was disassembled, both heaters were found to be unsoldered from the tubing. Soft solder ( 50 percent Sn, 50 percent Pb) melts at $490 \mathrm{~K}$ [24] and the thermal conductivity for 304 stainless steel is roughly 0.12 watts/cm-K [25]. Using these values, the heater length and tube dimension, and 6.7 watts input power, thermal conductivity calculations show that the temperature of the inside tube wall could have been as high as $480 \mathrm{~K}$. Therefore, there had to be film boiling inside the probe line. The effect of heat leak was studied in the second flow facility test.

Test 4 During previous tests the needle valve upstream of the reference vaporizer was used to provide some pressure drop in the liquid phase which would reduce the possibility of backflashing. In the vaporizer evaluation (see section 2.2.5) the needle valve was opened and no apparent change in the sampling precision was found. If the needle valve could be omitted, it would eliminate an operatinf. variable in the LNG sampling system. Therefore, it was important to confirm the previous results $1 \mathrm{n}$ this test using the side tap instead of the reference probe.

When wide open there was an estimated $0.03 \mathrm{psi}(0.2 \mathrm{kPa}$ ) across the needle valve (Valve T6, $\mathrm{rlfur}$ 2.2.12); in this case regulating valve T15 was used to control the sample flow rate. The max $1 \mathrm{~m} 1 \mathrm{~m}$ pressure drop (approximately 1.5 psi $(10.3 \mathrm{kPa})$ ) across the needle valve was obtalned by uslinf. $1 \mathrm{t}$ ( control the sampling rate; in this case all valves downstream of the needle valve were wlde open. (The pressure drop in the vaporizer and the line to the vaporizer was estimated to be 1.0 p 1 ( $h$. ? $\mathrm{kPa})$ and $1.8(12.4 \mathrm{kPa})$, respectively.) When controlling the sampling rate with the needle valvo, thr sampling rate had fluctuations of approximately \pm 50 percent of the average reading. 
Table 2.2.22 and figure 2.2.24 show that the amount of pressure drop across the needle had no apparent effect on the sampling precision. However, there may have been sufficient resistance in the 1 ine to the vaporizer to prevent backflashing. This test was repeated in the second flow facility test.

Table 2.2 .22

Summary of Estimated Standard Deviation in Heating Value and Liquid Density for Different Needle Valve Pressure Drop

\begin{tabular}{|c|c|c|c|}
\hline \multirow{2}{*}{$\begin{array}{c}\text { Pressure Drop } \\
\text { Across Needle } \\
\text { Valve }\end{array}$} & $\begin{array}{c}\text { Number } \\
\text { of } \\
\text { Points }\end{array}$ & $\begin{array}{c}\text { Heating } \\
\text { Value }\end{array}$ & $\begin{array}{c}\text { Siquid } \\
\text { Density }\end{array}$ \\
\cline { 3 - 4 } & 14 & 0.03 & 0.03 \\
high & 14 & 0.05 & 0.04 \\
\hline
\end{tabular}

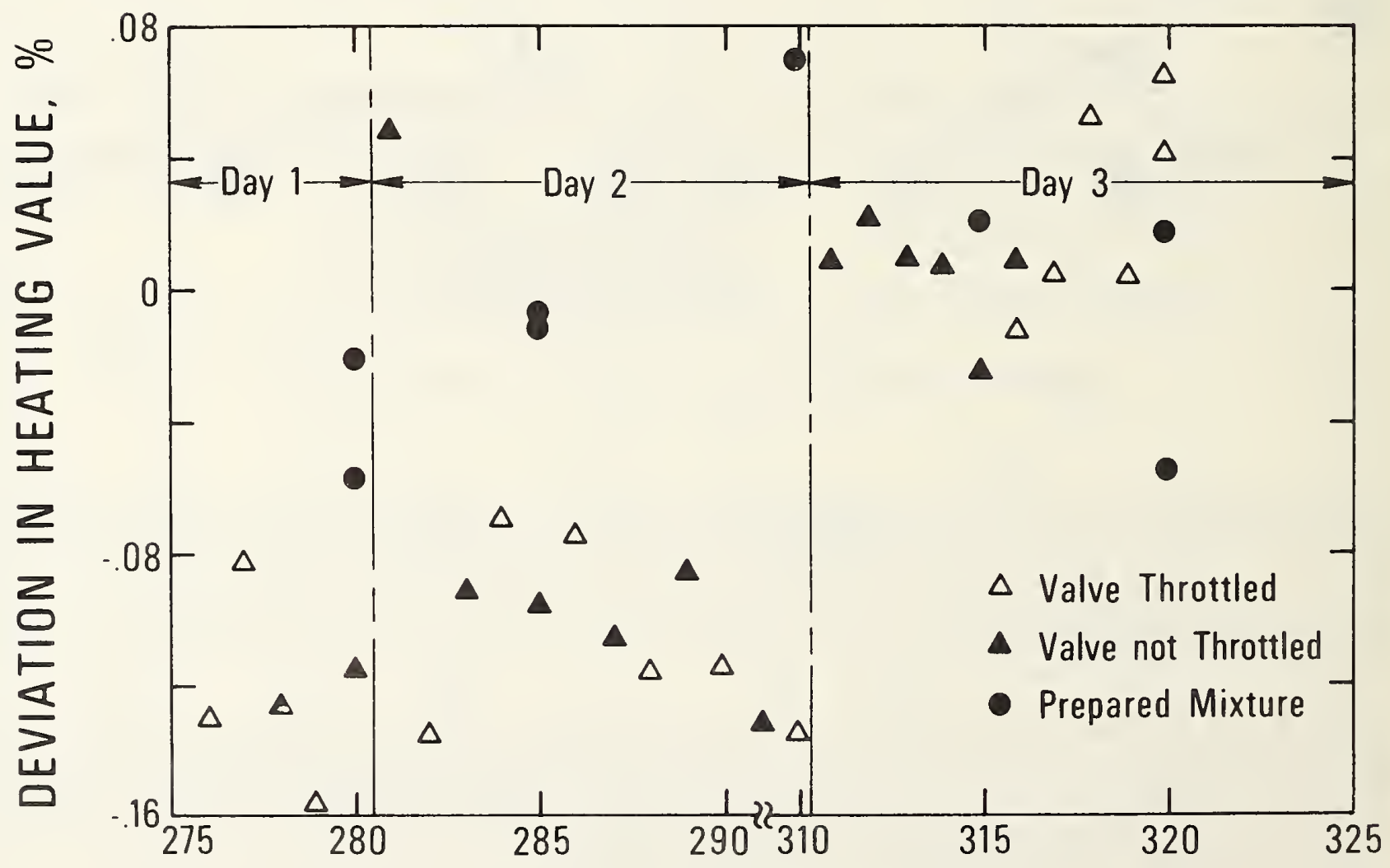

RUN NUMBER

Figure 2.2.24. Deviations in the computed ideal gas heating values for general test 4 . 
Test 5 The first test in this phase of work clearly indicated that an accumulator was needed. However, the minimum acceptable residence time in the accumulator was not determined. In this short test, the resldence time was varied between 10 and 22 seconds. Residence times for past test were in the range of 15 to 30 seconds. The 10 -second residence time corresponded to a full scale reading on the rotometer used to measure the sampling rate. Figure 2.2 .25 shows the heating values for this short test. Although the first point (293) appears too high, it still is well within the error limits of gas analysis alone. There were too few data points to do a statistical analysis on each residence time. However, an analysis of the whole group indicated that the estimated standard deviation in computed heating values was comparable to the best values obtained in other laboratory tests (approximately 0.02 percent).

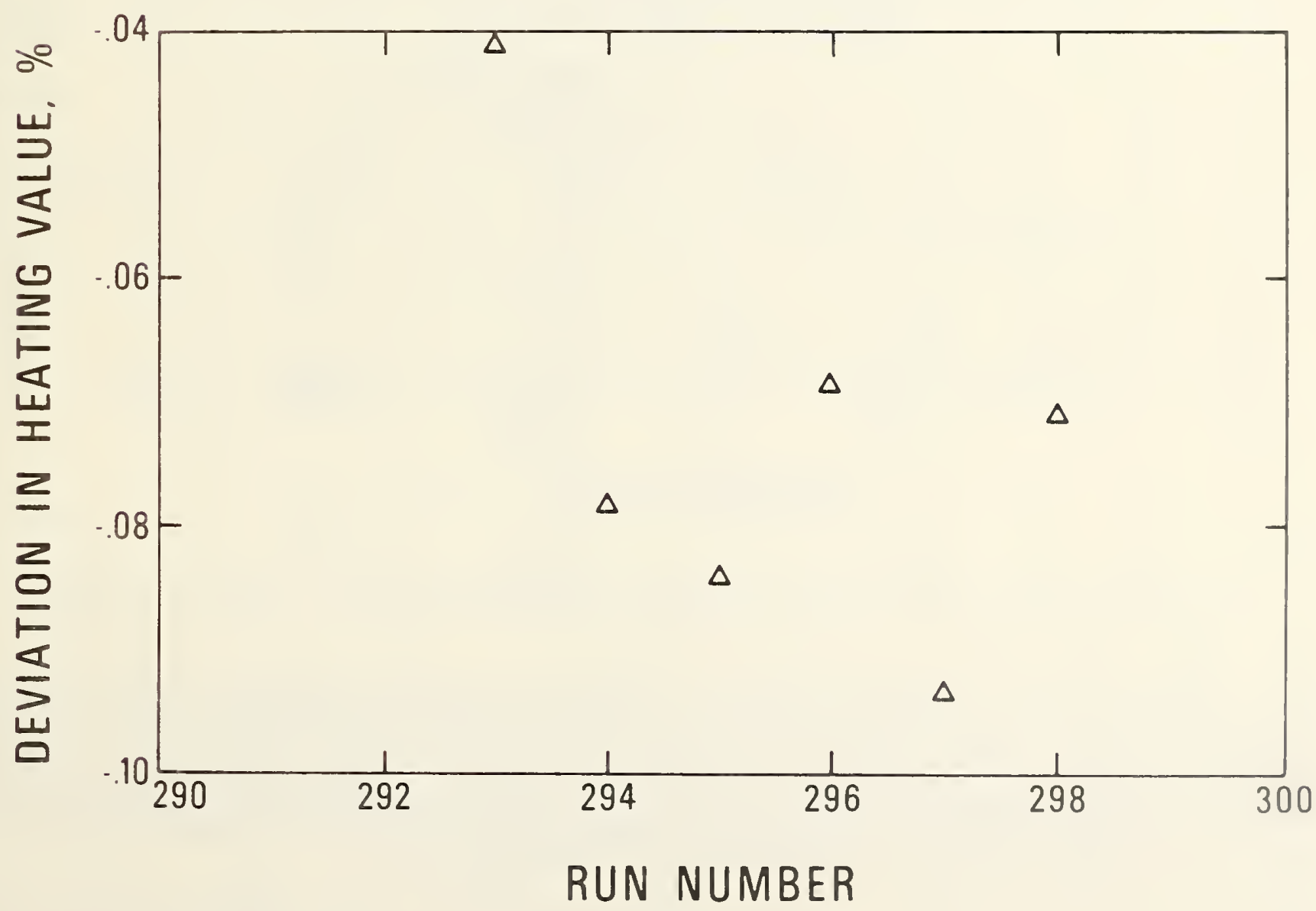

Figure 2.2.25. Deviations in the computed ideal gas heating value ror general test 5 . 
Test 6 In all of the previous laboratory tests, prepared mixtures containing nitrogen and methane through butane were used. The LNG for the first flow facility test was prepared by condensing natural gas obtained from the local gas utility. The liquid contained roughly 0.2 mol percent hexanes and higher hydrocarbons. It was thought that the presence of heavy components may have caused some of the sampling problems experienced in the first flow facility test. Therefore, additional natural gas from the local utility was condensed and transferred into the laboratory apparatus. While holding all operating variables constant, a series of runs was made to determine the effect of heavy components on the sampling precision.

Table 2.2.23 lists the average values and estimated standard deviations for this test; figure 2.2 .26 shows the deviation in the heating value. (The deviation is defined here as the difference between the measurement and average value divided by the average value for the given mixture.) Runs were made with different nitrogen levels. All of these analyses wer made using column configuration A (see section 2.2.4). The estimated standard deviations for the first two days were comparable to previous laboratory tests. (The $0.005 \mathrm{~mol}$ percent value for the estimated standard deviation of nitrogen was typical for the gas chromatograph used on this test.) However, the imprecision was much greater in the heating value on the last day. This was due to an increase in the scatter of the normal pentane and the $\mathrm{C}_{6}+$ component peaks. An estimated standard deviation of 0.04 mol percent in the $\mathrm{C}_{6}{ }^{+}$peak corresponds to nearly $2 \mathrm{Btu} / \mathrm{scf}\left(0.08 \mathrm{MJ} / \mathrm{m}^{3}\right)$ or 0.17 percent in the ideal gas heating value. The problem was not in sampling but in the inability of the digital integrator to correctly determine the area of the peak. (This problem is discussed in section 2.2.4).

Table 2.2 .23

Summary of Average Values and Estimated Standard Deviations in Ideal Gas Heating Value and Liquid Density for LNG Mixtures Containing Heavy Components

\begin{tabular}{|c|c|c|c|}
\hline \multirow[b]{2}{*}{ Date } & \multirow[b]{2}{*}{$\begin{array}{c}\text { Number } \\
\text { of } \\
\text { Points }\end{array}$} & \multicolumn{2}{|c|}{$\begin{array}{l}\text { Average Value and } \\
\text { Standard Deviation }\end{array}$} \\
\hline & & $\begin{array}{c}\text { Heating Value } \\
\text { Btu/scf } \\
(\mathrm{MJ} / \mathrm{m} 3)\end{array}$ & $\begin{array}{l}\text { Liquid } \\
\text { Density } \\
(\mathrm{g} / \mathrm{cm} 3)\end{array}$ \\
\hline $5 / 11 / 77$ & 6 & $\begin{array}{c}1175.6 \\
0.4 \\
(43.791) \\
(0.015)\end{array}$ & $\begin{array}{l}0.4607 \\
0.0001\end{array}$ \\
\hline $5 / 12 / 77$ & 8 & $\begin{array}{c}1167.9 \\
0.2 \\
(43.505) \\
(0.007)\end{array}$ & $\begin{array}{l}0.4646 \\
0.00004\end{array}$ \\
\hline $5 / 16 / 77$ & 8 & $\begin{array}{c}1150.8 \\
1.4 \\
(42.868) \\
(0.052)\end{array}$ & $\begin{array}{l}0.4707 \\
0.0001\end{array}$ \\
\hline
\end{tabular}

At the time of these tests the calibration gas did not contain pentanes; therefore, response factors for the pentanes and $\mathrm{C}_{6}{ }^{+}$(assuming the peak contained equal parts of $n$-hexane and $n$-heptane) were estimated by taking the ratio of the response factor for the given component to that of n-butane [26] and multiplying that by the response factor for n-butane as determined from calibration. The same approach was used for the oxygen and carbon dioxide peaks except that nitrogen, instead of n-butane, was used in the ratio. Based on the results of this test, the presence of heavy components does not affect the sampling precision, but they can affect the analytical precision. This test pointed out the analytical problem of correctly integrating the $\mathrm{C}_{6}+$ backflush peak. 


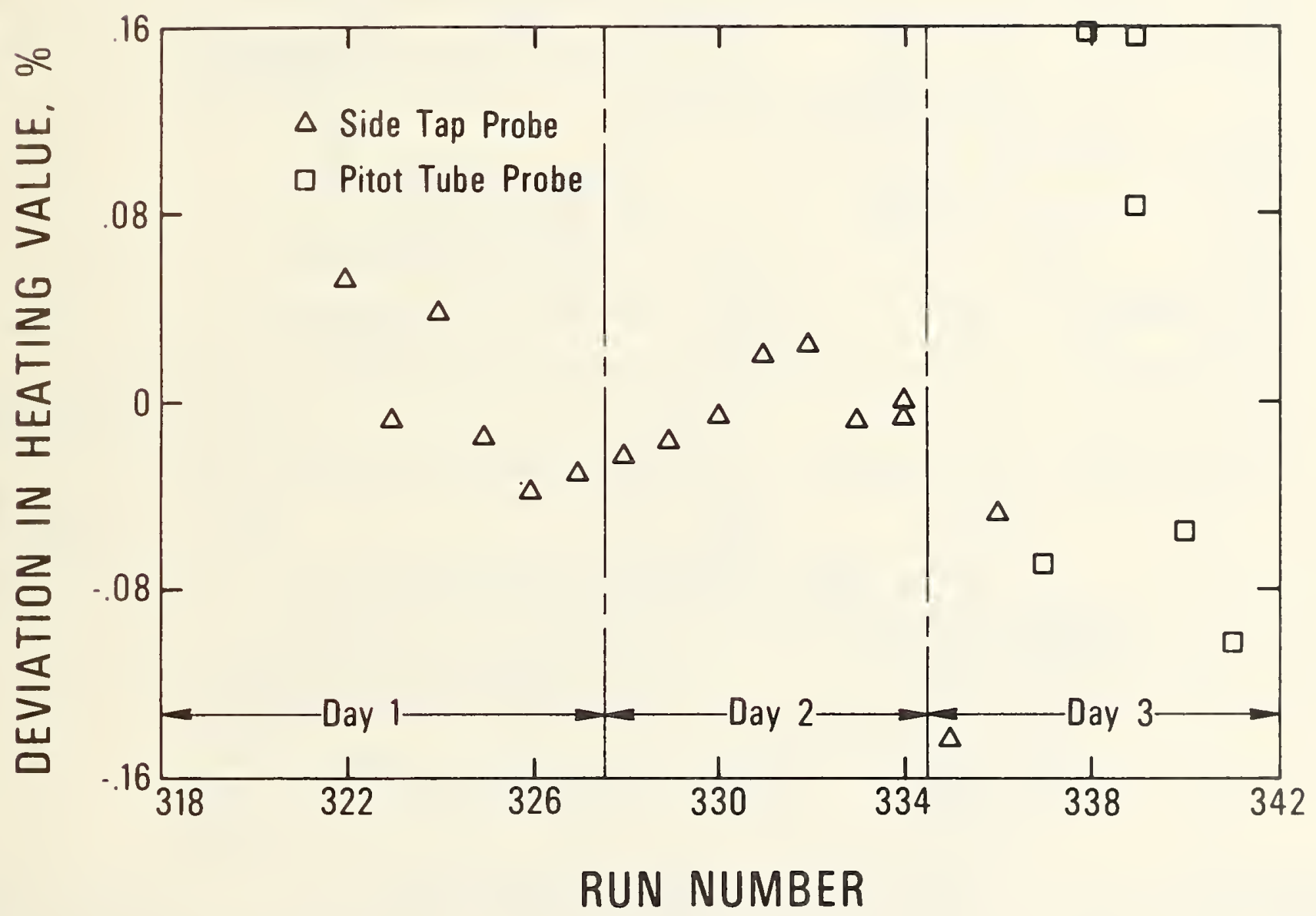

Figure 2.2.26. Deviations in the computed ideal gas heating values for general test 6 .

\subsubsection{Field Tests at NBS Flow Facility}

It was important that the conclusions obtained from the small scale laboratory tests be confirmed in a facility comparable to the proposed industrial installation. Three confirmation tests were made -two at the NBS LNG Flow Facility and one aboard the LNG tanker El Paso Consolidated. The shipboard test was made after completing the laboratory tests and between the two facility tests.

NBS LNG Flow Facility- Test 1 The LNG flow facility [27] is an outdoor pilot plant which has been used primarily for LNG flowmeter measurements. Figure 2.2.27 is a schematic of the facllity and table 2.2.24 lists the pertinent operating data. For the sampling tests a 3 foot (0.9 meter) lonf, test section was inserted roughly 10 foot (3 meters) downstream of the recirculat $10 n$ pump. All 11 nes 1 n the flow loop were 2.5 in (72 millimeters) diameter stainless steel plpe and all 1 ines except the test section were vacuum jacketed. 


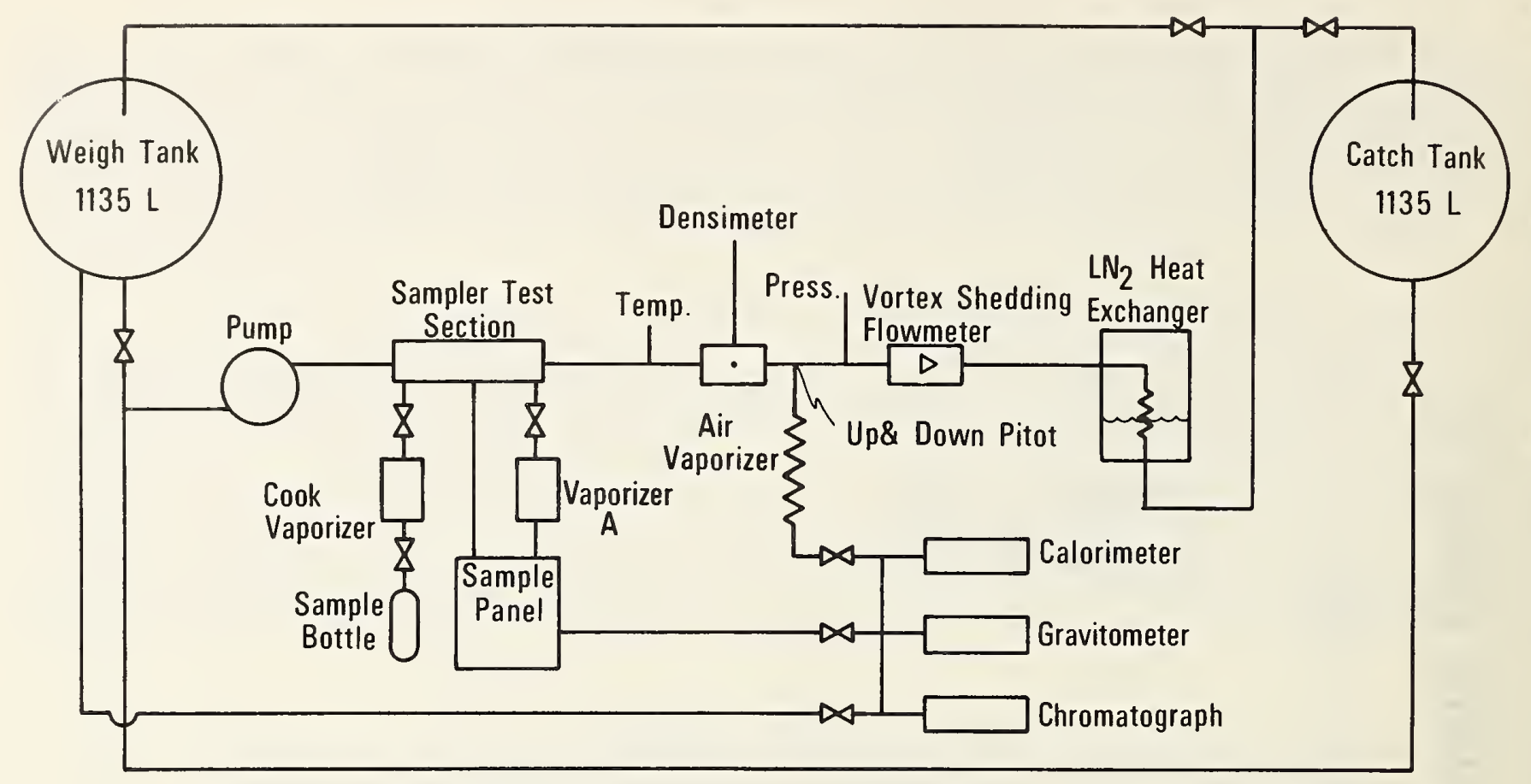

Figure 2.2.27. Flow schematic for the first LNG flow facility test.

$\mathrm{Tab} 1 \mathrm{e} 2.2 .24$

LNG Flow Facility Operating Characteristics

\begin{tabular}{|l|l|}
\hline Temperature range & $95-140 \mathrm{~K}$ \\
Temperature stability & $\pm 1 \mathrm{~K}$ or better \\
Pressure range & $19-110 \mathrm{psia}(0.13-0.76 \mathrm{MPa})$ \\
Pump discharge Pressure & $2.7-30 \mathrm{psid}(19-207 \mathrm{kPa})$ \\
$\quad$ (above weight tank pressure) & $20-200 \mathrm{gpm}(76-760 \mathrm{Lpm})$ \\
LNG flow rate & $10,000-100,000$ \\
Reynold's Number & \\
\hline
\end{tabular}

Vaporized samples from the test section were collected in $150 \mathrm{~cm}^{3}$ sample cylinders for analyses in the laboratory GC. Column configuration A was used in these tests. The vaporized samples also could be sent to a gas gravitometer and combustion calorimeter. These units were located approximately 50 ft (15 meters) away in a constant temperature room. The residence time of gas in the line between the sample panel and calorimeter was 1.6 minutes. Additional gas samples could be taken just upstream of the calorimeter and gravitometer.

It was possible to send gas samples directly to a process gas chromatograph. But since it measured only the concentration of nitrogen, methane, ethane, carbon dioxide and propane it was not used in these tests. Also, there was a pitot tube (up and downstream facing pitot)-air vaporizer sampling system [28] downstream of the sampling test section. It was used only in the first test.

During the initial startup the flow loop was purged with dry nitrogen and then cooled down with liquid nitrogen which was recirculated through the flow loop. After cooldown, LNG was transferred into the weigh tank. For the sampling tests the LNG was pumped from the bottom of the weigh tank and returned to the top of the weigh tank. The temperature was controlled by adjusting the liquid level in the liquid nitrogen heat exchanger. 


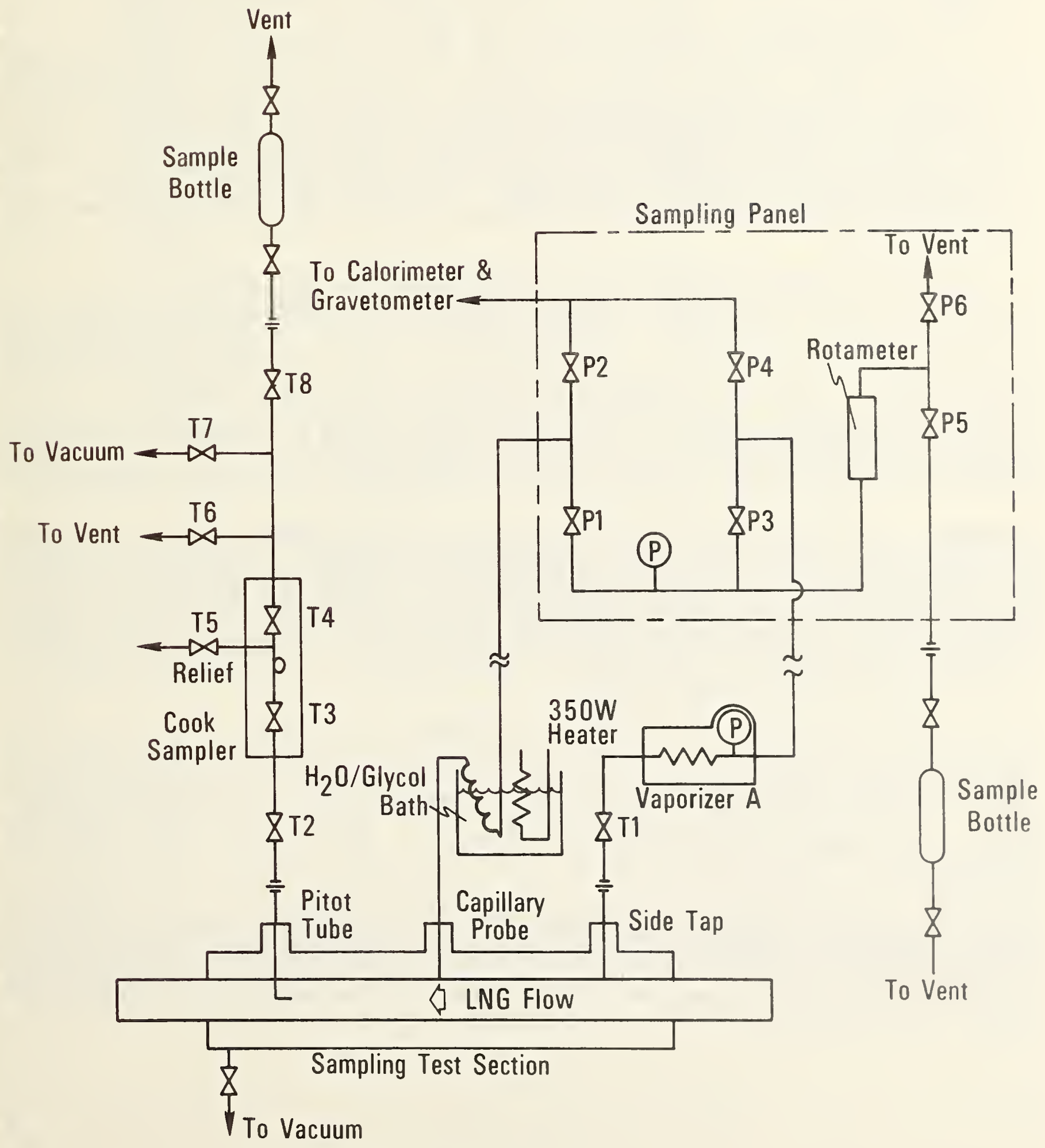

Figure 2.2.28. Schematic of sampling system used in LNG flow facll lty test 1 . 
The "true" composition of the LNG in the flow facility could not be measured. This limited the tests to comparing only the precision of the various sampling systems. However, the composition could be held constant throughout an operating day. System venting during shutdown caused day to day shifts in the composition. LNG for this test was made by condensing the natural gas supplied by the local gas utility. The gas was dried with a desiccant and the carbon dioxide was removed by filtering the liquid [28].

In Test 1, three probes were compared. These probes were a side tap, a side tap with a capillary opening and an upstream facing pitot tube; two vaporizers which were a water heated coil and vaporizer $A$; and the Cook sampler. Figure 2.2.28 shows a schematic of the sampling system (a sample accumulator was not used). All three probes were mounted horizontally in the test section and were 6 inch (152 millimeters) apart. The test section was enclosed in a 5 inch (127 millimeters) section of copper tubing. The annular space was evacuated to provide insulation. (However, during the tests it became obvious that the vacuum was very poor because a heavy layer of frost formed on the outer tube.) All three probes were made of 1.8 inch ( 3.2 millimeters) $0 . D$. by 0.101 inch ( $2.56 \mathrm{millimeters)} \mathrm{I.D.}$ stainless steel tubing. The probe lines were vacuum jacketed $6-1 / 2$ inch ( 165 millimeters) beyond the 5 inch (127 millimeters) tube. The pitot tube had a 90 degree bend with the intake on the flow section center line.

It was thought that if part of the pressure drop between the probe and vaporizer could be taken in the liquid phase there would be no danger of backflash; hence, it would not matter how the sample was vaporized downstream. Therefore, a capillary probe was built which contained a $1 / 4$ inch ( 6.4 millimeters) long section of 0.007 inch $(0.18 \mathrm{millimeter)} \mathrm{I.D.} \mathrm{tubing.} \mathrm{This} \mathrm{was} \mathrm{soldered} \mathrm{into} \mathrm{the} 1 / 8$ inch ( 3.2 millimeters) O.D. tubing. The capillary section produced roughly 5-1/2 psi (38 kPa) pressure drop for a liquid flow corresponding to 8 standard liters per minute of gas flow.

The water heated vaporizer consisted of 10 feet $(3 \mathrm{~m})$ of $1 / 4$ inch $(6.35$ millimeters $) 0 . D$. $x$ 0.190 inch (4.83) I.D. copper tubing immersed in a water-glycol bath which was at approximately $30 \%\left(-10^{\circ}\right)$. During operation the coil would have an ice layer which extended about 3 feet ( $0.9 \mathrm{~m})$ along the tubing in the bath.

Test Results The results of this test were extremely poor; the best agreement between two samples was 0.04 percent in the heating value. However, this was fortuitous because the typical scatter was on the order of one percent. The scatter was unaffected by the probe and vaporizer used. It was found that the heating values of the samples taken five minutes apart from the same sampling system varied by 0.7 percent; samples taken simultaneously from the same stream at the panel and at the gravitometer inlet differed by 1.5 percent. However, during all of these tests the calorimeter and gravitometer remained steady. (The gas specific gravity is much less sensitive to composition than the heating value, e.g., in the composition range studied a 1.5 percent change in the heating value corresponds to only 0.4 percent change in the specific gravity.)

Initially, it was thought that the scatter could be caused by the different methods of filling the sample cylinder. Samples taken at the panel were purged and filled 14 times; the sample cylinders at the gravitometer/calorimeter inlet were filled by continuous purge. To test the effect of sample cylinder filling procedure on precision, one of the calibration gases was connected to the sample panel. Using comparable sample flows and pressures to those used in the other tests, samples were taken at both the panel and the gravitometer/calorimeter inlet, The resulting sample compositions were within the error of the gas analysis alone; therefore, the sample cylinder filling procedure was not a problem.

The possibility that the scatter was caused by either liquid composition fluctuations or by the presence of vapor in the test section was considered. The stability of the densimeter in the LNG flow facility indicated that there were no composition fluctuations. The possibility of two phase flow was eliminated because the vortex shedding flowmeter would have behaved erratically in two phase flow. At this point it was decided to make additional laboratory tests (see section 2.2.5) to determine the cause of the poor sampling precision. 
Even with the erratic sampling precision, the following qualitative results were obtained:

1) It was not possible to obtain reliable samples from the Cook sampler as tested; this sampler should have been insensitive to the problems experienced with the continuous samplers. However, it is possible that valve T2 (figure 2.2.28) was leaking during the warm up period or that the reduction in sample pressure when filling the sample cylinder caused partial condensation. (The ambient air during these tests was 30 to 40 F $\left(-1.1\right.$ to $\left.4.4^{\circ} \mathrm{C}\right)$ ).

2) Based on the band width of the gravitometer trace, the up and down pitot-air vaporizer and the capillary probe-water vaporizer systems produced more precise samples than did the side tap-vaporizer A system. The gravitometer band width for the capillary probe-water vaporizer samples and gravitometer calibration gas (air) were comparable. Also, it was found that the heating values for the side tap-vaporizer A samples were 0.5 to 1 percent higher than those from the other two systems; the heating value difference between these two systems was less than 0.2 percent.

3) The capillary probe is not a feasible probe in industrial applications where entrained solids could cause plugging. In the flow facility there were small amounts of solid carbon dioxide which plugged the capillary line.

\subsubsection{Shipboard Tests}

Using the information obtained in the laboratory and first flow facility tests, a full scale sampling system was designed for tests aboard the LNG tanker El Paso Consolidated. Sampling tests were made at Canvey Island, England during the ship's cargo system trials. Two sampling systems were installed -a liquid sampling system was installed on the 3 inch (76 millimeters) diameter header to the ship's LNG vaporizer; a gas sampling system was placed on the 16 inch (406 millimeters) diameter vaporizer outlet header. Sampling the gas phase provided a check on the liquid sampling system.

Figure 2.2.29 shows a schematic of the liquid sampling system and table 2.2 .25 lists the component dimensions. The system had a 1.4 inch, schedule 80 pipe ( 13.7 millimeters 0.0 . x $7.67 \mathrm{millimeters}$ I.D.) side tap probe mounted horizontally on the header. All valves, fittings and tubing upstream of the sample panel were stainless steel; tubing in the panel was copper and the other components were brass. Except for the filter connections and union just upstream of the vaporizer, all connections upstream of the vaporizer were socket welds. The line between the header and vaporizer was wrapped with a one inch (25 millimeters) layer of fiberglass pipe insulation and sealed with duct tape.

Valves $\mathrm{T} 1$ and $\mathrm{T} 2$ were bellows valves with the valve components welded together. Valve $\mathrm{T} 2$ was $\mathrm{a}$ metering valve; it and the back pressure regulator were used to control the sampling rate.

The nitrogen purge connection (which was insulated up to valve T3) was used for displacing air from the sampling system; it was located downstream of the 60 micron filter so that nitrogen could be used to back purge the filter, if necessary. However, the filter never clogged during these tests.

Because of film boiling, a precise ( \pm 20 percent) design of the sample vaporizer would have been extremely tedious. Therefore, the design was based on the warming of methane gas from 115 to $310 \mathrm{~K}$. A flow rate of 65 standard liters per minute was used. It was assumed that the steam side temperature was constant at $340 \mathrm{~F}\left(171^{\circ} \mathrm{C}\right)$; this corresponds to the temperature of $205 \mathrm{psia}$ ( $1.41 \mathrm{MPa}$ ) steam isenthalpically expanded to $60 \mathrm{psia}(0.4 \mathrm{MPa})$. The heat transfer resistances on the steam slde and $1 \mathrm{n}$ the tube wall were negligible compared to the gas phase resistance; properties of methane [29,30] at $150 \mathrm{~K}$ were used in the calculations. The final vaporizer tube length was twice the length computed using the above assumptions. 


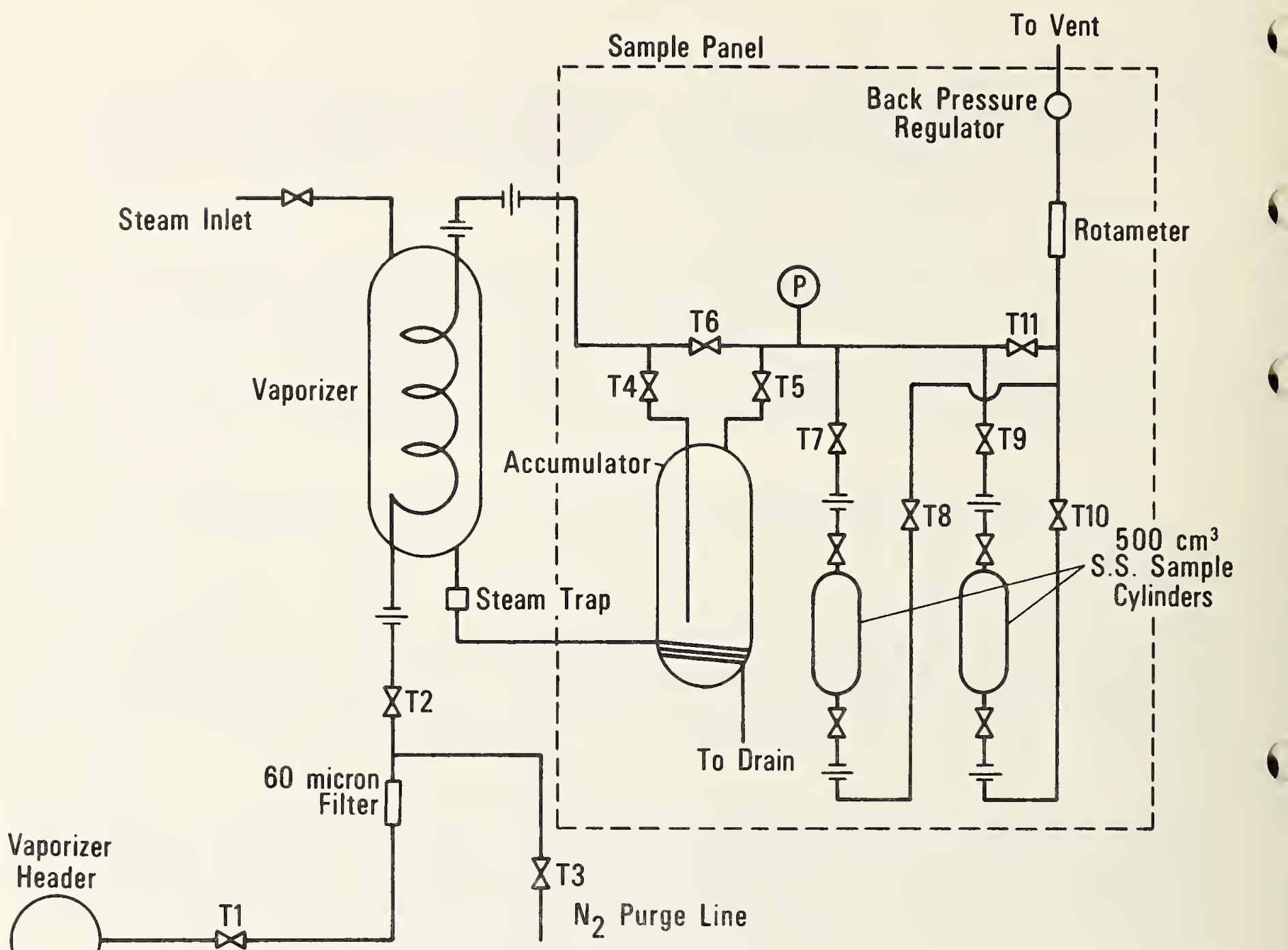

Figure 2.2.29. Schematic of LNG liquid sampling system used in shipboard tests.

The steam inlet line, vaporizer and condensate line were insulated; this was done primarily for personnel safety. Condensate from the vaporizer was used to warm the bottom of the accumulator to provide additional mixing.

The accumulator was sized to provide a 30 second residence time for a sample rate of 65 standard liters per minute and sample pressure of $50 \mathrm{psig}(0.45 \mathrm{MPa})$. The length to diameter ratio was 2.5 and the volume was 11 liters.

The sample panel layout was designed so that the sample flow could be maintained with or without sample cylinders in the system. The back pressure regulator was extremely useful for making minor changes in the sampling rate and setting the sample cylinder pressure.

The gas sampling system consisted of a 1.4 inch, schedule 80 pipe ( 13.7 millimeters 0.0$) x 7.67$ millimeters I.D.) side tap mounted vertically on the top of the outlet header, a block valve and 1.4 Inch (6.35 millimeters) O.D. by 0.190 inch ( 4.83 millimeters) I.D. copper tubing going to the sample panel. This panel had the same layout as the liquid sample panel but included a needle valve upstream of the accumulator for flow control. This system was designed for a vaporizer outlet pressure comparable to the inlet pressure (65 psig ( $0.055 \mathrm{MPa})$ ). However, the outlet pressure was only 1.5 to $2 \mathrm{psig}(0.112$ to $0.115 \mathrm{Mpa})$; this reduced the sampling rate to approximately 1 standard 1 iters per minute from the anticipated 10 or more standard liters per minute. 
Table 2.2 .25

Dimensions of LNG Sampling System Used on Shipboard Tests

\begin{tabular}{|c|c|c|c|c|}
\hline $\begin{array}{l}\text { Diameter } \\
\text { Component }\end{array}$ & $\begin{array}{l}\text { Length } \\
\text { inches ( } \mathrm{mm})\end{array}$ & $\begin{array}{l}\text { Outer Diameter } \\
\text { inches }(\mathrm{mm})\end{array}$ & inches & $\begin{array}{l}\text { Inner } \\
(\mathrm{mm})\end{array}$ \\
\hline Vaporizer Header & & $\left(3^{3 \cdot 5}\right.$ inch pipe) & & \\
\hline $\begin{array}{l}\text { Side tap and line } \\
(7.67) \\
\text { between header and } \\
\text { Valve T1 }\end{array}$ & $4.5(114)$ & $\begin{array}{l}0.540(13.7) \\
(1 / 4 \text { inch Sch. } \\
80 \text { pipe })\end{array}$ & & 0.302 \\
\hline $\begin{array}{l}\text { Line between Valve } \\
(4.57) \\
\text { T1 and filter (con- } \\
\text { tained a long radius } \\
90 \text { degree bend) }\end{array}$ & $11(279)$ & $0.25 \quad(6.4)$ & & 0.180 \\
\hline $\begin{array}{l}\text { Line between filter } \\
(4.57) \\
\text { and Valve T2 }\end{array}$ & (76) & $0.25 \quad(6.4)$ & & 0.180 \\
\hline $\begin{array}{l}\text { Line between valve } \\
(2.56) \\
\text { T2 and union at } \\
\text { vaporizer }\end{array}$ & $18(460)$ & $0.125(3.18)$ & & 0.101 \\
\hline $\begin{array}{l}\text { Vaporizer tubing } \\
\text { ( } 4.15) \\
\text { formed into a } 4 \\
\text { inch ( } 102 \mathrm{~mm}) \text { dia- } \\
\text { meter helix) }\end{array}$ & $45 \quad(1140)$ & $0.188(4.76)$ & & 0.164 \\
\hline $\begin{array}{l}\text { Vaporizer casing } \\
\text { (with welded cap } \\
\text { ends) }\end{array}$ & $20 \quad(508)$ & $6.00 \quad(150)$ & 5.94 & (151) \\
\hline $\begin{array}{l}\text { Line from vaporizer } \\
(4.57) \\
\text { to union (contained } \\
\text { a } 90 \text { degree long } \\
\text { radius bend) }\end{array}$ & 11 (279) & $0.25 \quad(6.4)$ & & 0.180 \\
\hline$\underset{(4.57)}{\operatorname{Line} \text { to valve } \mathrm{T} 3}$ & $14(356)$ & $0.25 \quad(6.4)$ & & 0.180 \\
\hline $\begin{array}{l}\text { Accumulator } \\
\text { (with welded end } \\
\text { cap) }\end{array}$ & $24 \quad(610)$ & $6.00 \quad(152)$ & 5.94 & (151) \\
\hline
\end{tabular}

Gas analyses were made using a gas chromatograph containing a 30 foot ( $9.1 \mathrm{~m}$ ) long column of DC $200 / 500$ on Chromasorb $P$ and a 5 foot $(1.5 \mathrm{~m}$ ) molecular sieve 5A (MS) column. The column valve configuration permitted backflushing the DC 200/500 column and bypassing the MS column. Nitrogen and methane were resolved on the MS column. Ethane through the butanes were separated on the DC $200 / 500$ volume; the column was backflushed to obtain a $\mathrm{C}_{5}{ }^{+}$peak. Digital integrator $\mathrm{A}$ was used to measure peak areas. The precision of the analytical system was found to be 0.06 percent in the computed heating value.

Test Results The primary purpose of this test was to examine the precision of the LNG sampling system. The LNG was totally vaporized downstream of the sampling probe and the analysig of this gas was to serve as the basis on which to evaluate the liquid sample analysis. However, the analysis of the vaporized gas produced erratic results that are felt to be more a function of the low presgure of the gas than composition change in the pipeline. of the six sets of sampling data taken, only the second set contained sufficient samples taken under constant vaporizer conditions to directly compare the gas and liquid sample compositions (see figure 2.2.30). Since composition varlations were probably less of a factor than the gas analysis, separate means and standard deviatlons were computed for the liquid samples and for the gas samples for each of the six data sets (see table 2.2 .26 ). The heating values are computed using the A.G.A. method [2] and assumes equal parts of 190 and normal pentane; the liquid phase densities were computed from the extended corresponding $9 t a t e s$ model [4] (the original publication [1] lists the compositions and operating parameters for all of the tests). 


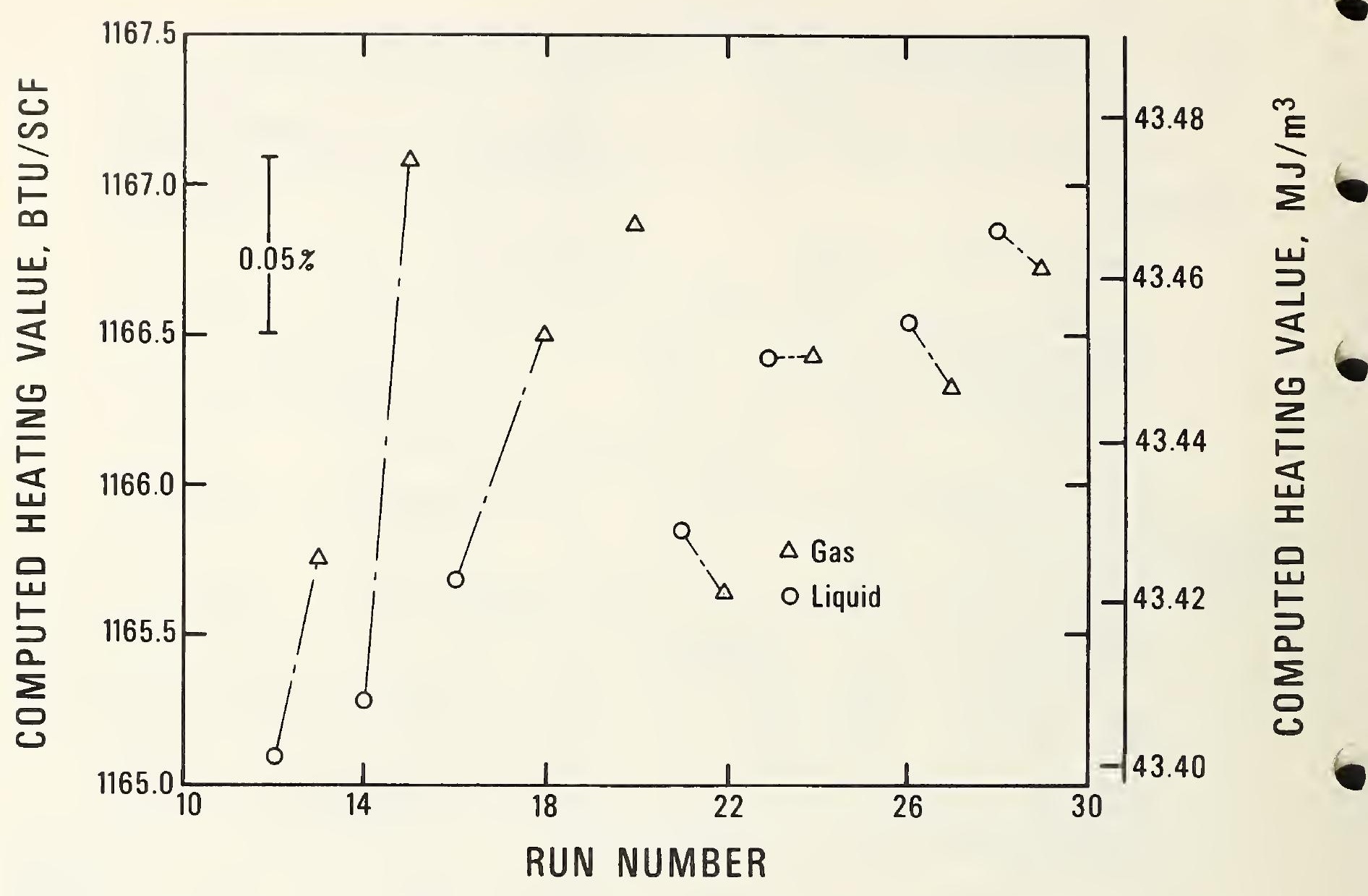

Figure 2.2.30. Computed real gas heating values of shipboard data set 2 samples.

During the first data set the vaporizer inlet pressure varied erratically (30-50 psig). Therefore, these samples were taken only to gain experience in operation the sampling system. For data set 6 , the chromatograph-integrator system was behaving erratically with respect to the butane peaks. For these reasons, the results for these two data sets, as given in table 2.2.26, do not represent a properly operating system under stable operating conditions and were not included in subsequent data analyses.

Although the conditions under which liquid samples were taken for data sets 2 , 3 , 4 and 5 are considered acceptable for sampling purposes, some points were deleted from the data analyses. It was found that the LNG sampling system worked well at sampling rates of 12.5 standard liters per minute or greater; however, two samples, run No. 19 at 3.4 standard liters per minute and run No. 57 at 6.2 standard liters per minute, yielded poor results and were omitted from the analyses. Also during these tests the accumulator residence time of the "good" liquid samples ranged between 28 and 76 seconds, and on this basis run No. 25 was also omitted. Run No. 31 was omitted from the data analyses because it did not go through the accumulator and run No. 35 was omitted as the evidence indicated that it was not representative. For data set 4 , run No. 51 was analyzed three times; the first analysis, being compltely wrong, was omitted. 
Table 2.2.26

Summary of Average Values and Estimated Standard Deviations

in Real Gas Heating Value and Liquid Density for

Shipboard Tests

\begin{tabular}{|c|c|c|c|c|}
\hline \multirow[b]{2}{*}{ Data Set } & \multirow[b]{2}{*}{ Sample } & \multirow[b]{2}{*}{$\begin{array}{l}\text { Number } \\
\text { of } \\
\text { Points } \\
\end{array}$} & \multirow{2}{*}{\multicolumn{2}{|c|}{ 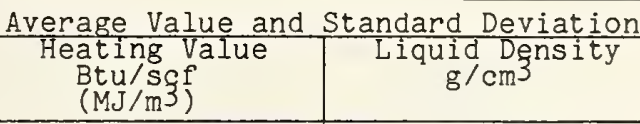 }} \\
\hline & & & & \\
\hline \multirow[t]{2}{*}{$\mathrm{T}=114^{*} .6 \mathrm{~K}$} & liq & 7 & $\begin{array}{r}1165.8 \\
35.9 \\
(43.428) \\
(1.338)\end{array}$ & $\begin{array}{l}0.4682 \\
0.0107\end{array}$ \\
\hline & gas & 1 & $\begin{array}{l}1176.7 \\
(43.833)\end{array}$ & 0.4752 \\
\hline \multirow[t]{2}{*}{$T=114.6$} & liq & 7 & $\begin{array}{r}1165.8 \\
0.5 \\
(43.428) \\
(0.020)\end{array}$ & $\begin{array}{l}0.4712 \\
0.0001\end{array}$ \\
\hline & gas & 8 & $\begin{array}{r}1166.3 \\
0.5 \\
(43.446) \\
(0.020)\end{array}$ & $\begin{array}{l}0.4713 \\
0.0001\end{array}$ \\
\hline \multirow[t]{2}{*}{$T=114.6$} & liq & 4 & $\begin{array}{r}1195.8 \\
0.41 \\
(44.547) \\
(0.015)\end{array}$ & $\begin{array}{l}0.4799 \\
0.0001\end{array}$ \\
\hline & gas & 4 & $\begin{array}{r}1198.3 \\
5.6 \\
(44.638) \\
(0.211)\end{array}$ & $\begin{array}{l}0.4806 \\
0.0016\end{array}$ \\
\hline \multirow[t]{2}{*}{$T=115.0$} & liq & 8 & $\begin{array}{r}1236.2 \\
0.9 \\
(46.050) \\
(0.035)\end{array}$ & $\begin{array}{l}0.4914 \\
0.0009\end{array}$ \\
\hline & gas & 4 & $\begin{array}{r}1237 \cdot 9 \\
2.8 \\
(46.112) \\
(0.102)\end{array}$ & $\begin{array}{l}0.4916 \\
0.0007\end{array}$ \\
\hline \multirow[t]{2}{*}{$T=19.0$} & liq & 2 & $\begin{array}{r}1242.6 \\
0.7 \\
(46.289) \\
(0.027)\end{array}$ & $\begin{array}{l}0.4917 \\
0.0002\end{array}$ \\
\hline & gas & 2 & $\begin{array}{r}1243.0 \\
0.9 \\
(46.304) \\
(0.035)\end{array}$ & $\begin{array}{l}0.4919 \\
0.0003\end{array}$ \\
\hline \multirow[t]{2}{*}{$\mathrm{T}=116.5$} & liq & 7 & $\begin{array}{r}1247.5 \\
0.9 \\
(46.471) \\
(0.035)\end{array}$ & $\begin{array}{l}0.4926 \\
0.0006\end{array}$ \\
\hline & gas & 4 & $\begin{array}{r}1242.6 \\
5.6 \\
(46.287) \\
(0.209)\end{array}$ & $\begin{array}{l}0.4912 \\
0.0015\end{array}$ \\
\hline
\end{tabular}

* These tests occured during unstable conditions (see text) and the values for the means and standard deviations are not meaningful. 
An estimate of the standard deviation was calculated based on data sets 2 , 3 , 4 , and 5. For the liquid samples the value obtained was 0.72 Btu/scf with 17 degrees of freedom. An upper 99 percent confidence bound for the standard deviation based on this estimate is $1.18 \mathrm{Btu} / \mathrm{scf}$, or 0.1 percent based on a heating value of 1200 Btu/scf.

A commercial "primary standard" calibration gas was used for gas chromatograph calibrations. During the calibrations it became obvious that the reported and actual calibration gas compositions were different. This discrepancy was found by comparing the nitrogen to methane response factor ratio with the ratio obtained from Dietz's data [26]. Table 2.2.27 gives the manufacturer's stated composition and error limits along with the composition determined in the NBS laboratory. The statistical confidence interval given in table 2.2.27 is at 99 percent and is based on four gas calibrations and five gas analyses of the mixture. The confidence interval assumes no composition error in the calibration gas used to calibrate the GC and that the standard deviations of both calibrations and analyses are equal. Column configuration $A$ was used with mixture H as the calibration gas. The difference between the stated and actual composition represents a 0.5 percent error in the heating value. This means that if the stated compositions had been used there would have been a bias of 0.5 percent in the computed heating value. This experience points out the importance of accurately knowing the composition of calibration gases used in custody transfer. If possible the stated composition should be verified by an independent gas analysis laboratory.

Table 2.2 .27

Reported and Measured Compositions of Commercially Prepared Standard Calibration Gas

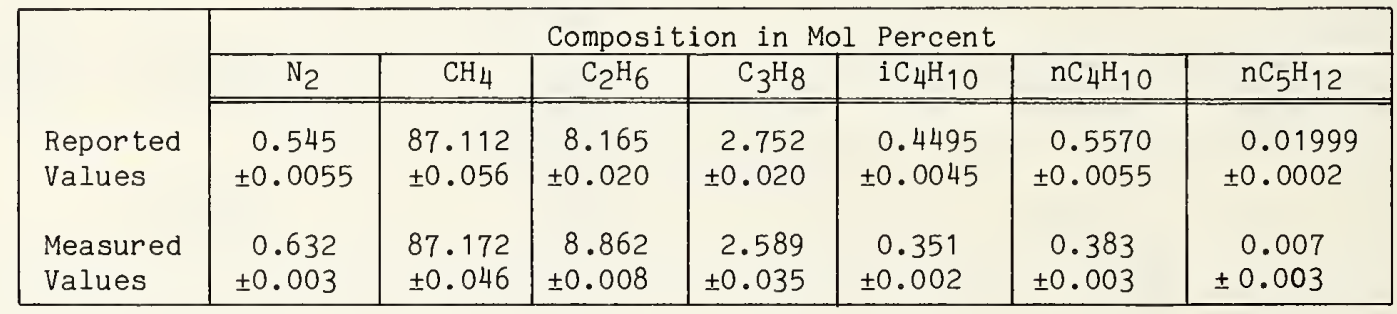

LNG Flow Facility- Test 2 At this point, a full scale LNG sampling system was developed which had worked in the field. However, another field test was necessary to determine:

a) the criteria for establishing sampling rate,

b) the effect of using steam versus electricity for sample vaporization,

c) the effect of not using a needle valve to control sampling rate, and

d) the effect of heat leak.

Figure 2.2.31 shows the flow schematic for this test. Three vaporizers were constructed -- two steam heated and one electrically heated. One of the steam vaporizers had the same dimensions as the electric vaporizer; the second steam vaporizer had a 50 percent larger tube inner diameter and the tube length was 40 percent longer than the other two vaporizers. Table 2.2 .28 gives the important dimensions of the vaporizers and sampling systems; figure 2.2.32 is a schematic of one of the sampling systems. The old test section was replaced with a test section containing two side taps mounted horizontally. The side taps were $1 / 4$ inch schedule 80 (13.7 millimeters 0.D. x 7.67 millimeters I.D.) stainless steel pipe. Except for the vaporizers, all dimensions on both sampling systems were identical. Therefore, by operating two vaporizers simultaneously direct comparisons could be made between vaporizer designs. 


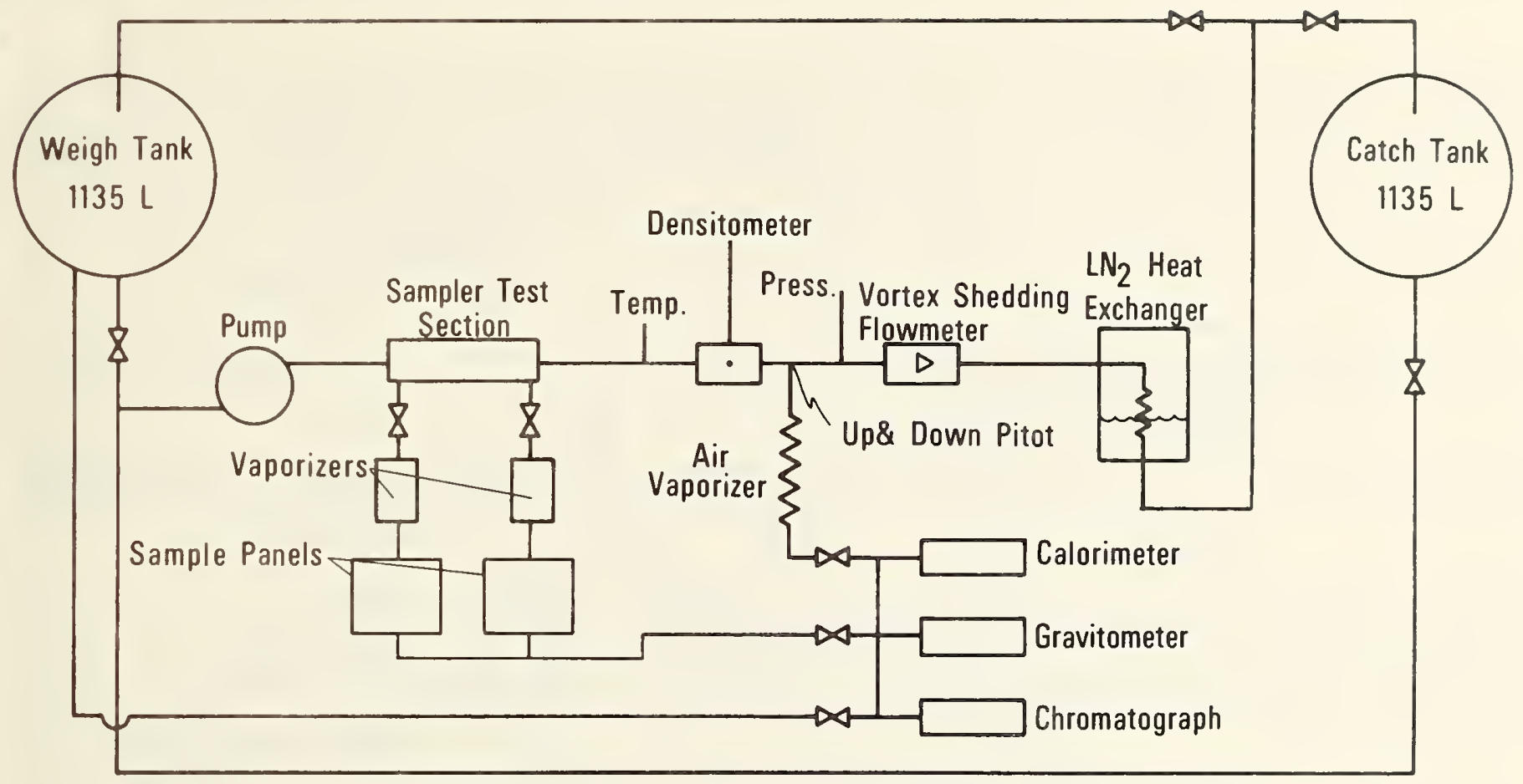

Figure 2.2.31. Flow schematic for the second LNG flow facility test.

Table 2.2.28

Dimensions of LNG Sampling System Used in second LNG Flow Facility Tests

\begin{tabular}{|c|c|c|c|c|c|c|}
\hline \multirow{2}{*}{$\frac{\text { Component }}{\text { Test section }}$} & \multicolumn{2}{|c|}{$\begin{array}{c}\text { Length } \\
\text { Inches }(\mathrm{mm})\end{array}$} & \multicolumn{2}{|c|}{$\begin{array}{l}\text { Outer Diameter } \\
\text { Inches }(\mathrm{mm})\end{array}$} & \multicolumn{2}{|c|}{$\begin{array}{c}\text { Inner Diameter } \\
\text { Inches (mm) }\end{array}$} \\
\hline & 36 & $(914)$ & $3-1 / 8$ & $(79.4)$ & 2.812 & $(71.42)$ \\
\hline $\begin{array}{l}\text { Side tap and line } \\
\text { between test section } \\
\text { and valve S1 }\end{array}$ & $4-1$ & $(114)$ & $\begin{array}{l}0.540 \\
(i / 4 \\
80 \mathrm{pip}\end{array}$ & (13.7) & 0.302 & $(7.67)$ \\
\hline $\begin{array}{l}\text { Line between valve S1 } \\
\text { and filter (contained } \\
\text { a short radius } 90 \\
\text { degree bend) }\end{array}$ & 11 & (279) & 0.25 & $(6.4)$ & 0.180 & $(4.57)$ \\
\hline $\begin{array}{l}\text { Line between filter } \\
\text { and valve } \mathrm{S} 21\end{array}$ & 2 & $(51)$ & 0.25 & $(6.4)$ & 0.180 & $(4.57)$ \\
\hline $\begin{array}{l}\text { Line between valve } \mathrm{S} 2 \\
\text { and union at vaporizer } 1\end{array}$ & $13-$ & $2(343)$ & 0.25 & $(6.4)$ & 0.180 & $(4.57)$ \\
\hline Vaporizer 1 tubing & 36 & $(914)$ & $1 / 8$ & $(3.18)$ & 0.101 & $(2.56)$ \\
\hline Vaporizer 2 tubing & 50 & $(1270)$ & $3 / 16$ & $(4.76)$ & 0.156 & $(3.96)$ \\
\hline Vaporizer 3 tubing & 36 & $(914)$ & $1 / 8$ & $(3.18)$ & 0.101 & $(2.56)$ \\
\hline $\begin{array}{l}\text { For vaporizers } 1 \text { and } 2 \\
\text { steam casing (with } \\
\text { flat welded ends) }\end{array}$ & 10 & $(254)$ & 6 & $(152)$ & 5.5 & $(140)$ \\
\hline $\begin{array}{l}\text { Inpingement chamber } \\
\text { (with flat welded ends) }\end{array}$ & $1-1$ & $(38)$ & $1-1 / 2$ & $(38)$ & 1.376 & $(34.95)$ \\
\hline $\begin{array}{l}\text { Line between impinge- } \\
\text { ment chamber and down- } \\
\text { stream union }\end{array}$ & 8 & $(200)$ & 0.25 & $(6.4)$ & 0.180 & $(4.57)$ \\
\hline Accumlator 1 & 16 & $(406)$ & 5.125 & $(130.2)$ & 4.805 & (122) \\
\hline
\end{tabular}

1 These dimensions apply to both sampling systems. 


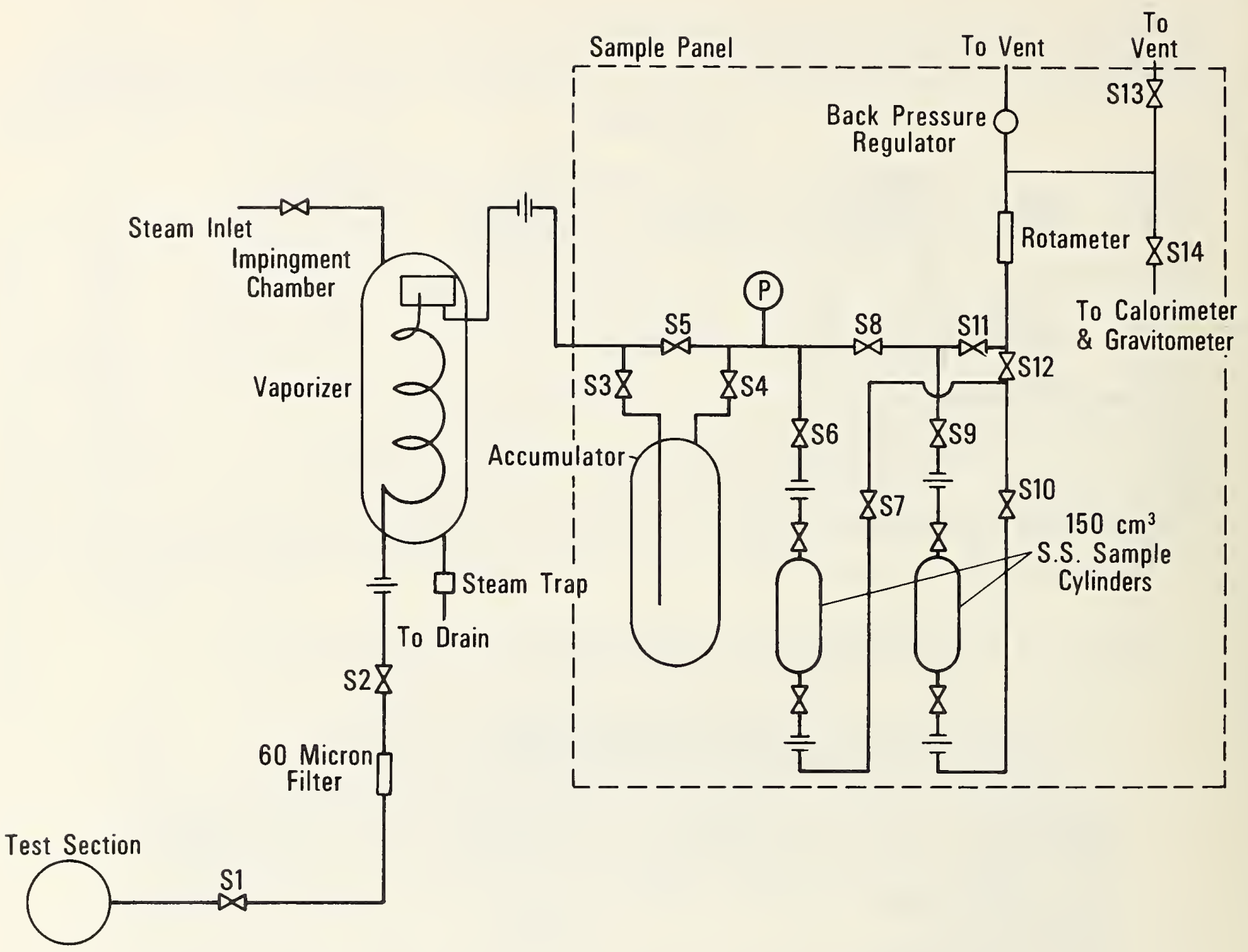

Figure 2.2.32, Schematic of liquid sampling system used in second LNG flow facility test.

The LNG sample lines to the vaporizers were wrapped with a $1 / 2$ inch ( 13 millimeters) thick layer of fiberglass pipe insulation and sealed with duct tape. Also, the steam vaporizers, steam inlet lines and condensate lines were insulated for personnel safety.

Valve S1 was a block valve (see figure 2.2.32) and valve S2 a metering valve; both valves were all welded stainless steel bellows valves. Neither valve was degreased before use, and stem extensions were not used.

The two steam vaporizer tube lengths were twice the tube length computed by considering only the heat transfer resistance of a pure methane gas phase (see section 2.2 .5 for more details). For this test, a steam generator was used which produced roughly 50 psia ( $0.34 \mathrm{MPa})$ saturated steam.

To prevent the entrainment of fine droplets in the steam vaporizer outlet stream, an impingement chamber was included in each steam vaporizer. The stainless steel chamber consisted of a 1-1/2 inch (38 millimeters) diameter cylinder 1-1/2 inches (38 millimeters) long. It was constructed so that the vapor plus mist leaving the vaporizer tube contacted the upper surface of the chamber. It was hoped that the combination of impingement, sudden flow decrease and flow reversal would eliminate any remaining liquid droplets. 
The electrlc vaporizer dimensions were chosen to correspond to one of the steam vaporizers so that a direct comparison between vaporizers could be made. However, the impingement chamber was not used with the electric vaporizer. This vaporizer consisted of thin wall ( 0.012 inch ( 0.30 millimeter)) stalnless steel tubing with electrical leads attached at each end. Since the tubing was used as the resist1ve heater, a high amperage ( 18 amps) low voltage ( 4.3 volts) direct current was used. To prevent possible shorts, the vaporizer tube was epoxied into micarta couplings at each end.

Inltilly, the accumulator volume was two liters, but the first day's test results showed that the accumulator was too small. Thereafter, a four liter accumulator was used.

Analyses of the LNG used in the first flow facility test showed relatively high concentration of $\mathrm{C}_{6}{ }^{+}$. To obtain a more representative LNG mixture, the LNG for this test was purchased from San Diego Gas and Electric Company. However, even this LNG contained approximately 0.08 mol percent $\mathrm{C}_{6}{ }^{+}$.

For sample analysis, column configuration A was used. Prepared mixture H (table 2.2.6) was used as the callbration gas. Both integrators A and B were connected to gas chromatograph II so that their results could be compared.

Test Results A total of seven tests were made in the flow facility over a four day period. Table 2.2.29 lists the pertinent operating parameters and lists a comparison of measured and computed heating values and specific gravities. The computed quantities are real gas values computed from the compositions using the A.G.A methods [2]. Table 2.2.29 gives the average values and estimated standard deviation of the compositions and the liquid densities computed using the extended corresponding states method [4].

Test A Because the sampling rates would be comparable to those used in laboratory tests, two liter accumulators were used in test A. However, the test results suggested that the accumulator was too small. Figure 2.2.33 shows the deviation from the average heating value for this test. At the sample rates used in this test the accumulator residence time ranged between 10 and 18 seconds; this same range gave good results in laboratory tests. All remaining tests were made using four liter accumulators.

Test B As shown in figure 2.2.34, results of test B clearly confirmed the need for the larger accumulator. Possible reasons for this include:
a) the use of a constant wall temperature vaporizer (as opposed to the constant heat flux vaporizer used in the laboratory apparatus, or
b) the presence of a finite heat leak into the sample line.

Unfortunately, there was not sufficient time to identify the reason for needing the longer res 1 dence time.

Two points were eliminated from figure 2.2 .34 and from the statistical analysis of test B results. For no obvious reason, one sample had a methane content five standard deviations higher than the average value for the test (both integrators gave the same result). However, the second outlier was the first sample taken after changing the sampling rate; it was taken less than 20 m 1 nutes after the change. This suggested the possibility of flow transients affecting the sampling precislon.

Test $C$ Test $C$ was a short test to determine if sampling system startup transients affected sampling precision. Figure 2.2.35 shows the deviation in heating value for this test. The dev1atlons are relative to the average values obtained under steady conditions in test $\mathrm{D}$, which was run on the same day. In this test, samples were taken every 15 minutes after putting the sampling system 1 n operation. Although the scatter was greater, especially in the nitrogen, methane and ethane contents, there was no obvious trend with time. 
Table 2.2.29

Comparison of Measured and Computed Heating Values and Specific Gravities of Second LNG Flow Facility Test

\begin{tabular}{|c|c|c|c|c|c|c|c|c|}
\hline \multirow[b]{2}{*}{ Test } & \multirow{2}{*}{$\begin{array}{l}\text { Vapor- } \\
\text { izer }\end{array}$} & \multirow{2}{*}{$\begin{array}{c}\text { Sampling } \\
\text { Rate } \\
\text { slpm }\end{array}$} & \multirow{2}{*}{$\begin{array}{c}\text { Number } \\
\text { of } \\
\text { points }\end{array}$} & \multicolumn{2}{|c|}{ Heating Value Btu/scf $\left(\mathrm{MJ} / \mathrm{m}^{3}\right)$} & \multicolumn{2}{|c|}{ Specific Gravity } & \multirow[t]{2}{*}{ Remarks } \\
\hline & & & & Measured & Computed $^{\top}$ & Measured & Computed & \\
\hline \multirow[t]{2}{*}{ A } & 1 & $\begin{array}{l}14 \\
19\end{array}$ & $\begin{array}{l}3 \\
6\end{array}$ & $\begin{array}{l}1159 \cdot 5^{6}(43 \cdot 192) \\
1159 \cdot 5^{5}(43 \cdot 192)\end{array}$ & $\begin{array}{rr}1158.6 & (43.159) \\
3.9 & (0.145) \\
1162.2 & (43.293) \\
4.7 & (0.175)\end{array}$ & $\begin{array}{l}0.650 \\
0.650\end{array}$ & $\begin{array}{l}0.647 \\
0.002 \\
0.650 \\
0.003\end{array}$ & $\begin{array}{l}\text { Two liter sample } \\
\text { accumulator used } \\
\text { in this test }\end{array}$ \\
\hline & 2 & $\begin{array}{l}13 \\
16\end{array}$ & $\begin{array}{l}4 \\
3\end{array}$ & $\begin{array}{l}1159.5^{6}(43.192) \\
1159.5^{5}(43.192)\end{array}$ & $\begin{array}{rr}1163.7 & (43.349) \\
7.9 & (0.294) \\
1161.0 & (43.248) \\
1.4 & (0.052)\end{array}$ & $\begin{array}{l}0.650 \\
0.650\end{array}$ & $\begin{array}{l}0.651 \\
0.005 \\
0.649 \\
0.001\end{array}$ & \\
\hline$B^{2}$ & 2 & $\begin{array}{l}13 \\
22\end{array}$ & $\begin{array}{r}7 \\
13\end{array}$ & $\begin{array}{l}1171.5^{6}(43.639) \\
1169.0^{6}(43.546)\end{array}$ & $\begin{array}{rr}1170.6 & (43.606) \\
0.8 & (0.030) \\
1169.7 & (43.572) \\
0.6 & (0.022)\end{array}$ & $\begin{array}{l}0.658 \\
0.658\end{array}$ & $\begin{array}{l}0.655 \\
0.0007 \\
0.654 \\
0.0005\end{array}$ & $\begin{array}{l}\text { Increased acc- } \\
\text { mulator volume } \\
\text { to } 4 \text { liters }\end{array}$ \\
\hline$c^{3}$ & 1 & 16 & 5 & $1174.0^{4}(43.733)^{6}$ & $\begin{array}{rr}1173.9 & (43.729) \\
1.4 & (0.052)\end{array}$ & 0.680 & $\begin{array}{l}0.674 \\
0.0007\end{array}$ & $\begin{array}{l}\text { Samples taken at } \\
15 \text { minute inter- } \\
\text { vals after start- } \\
\text { up of sampling } \\
\text { system }\end{array}$ \\
\hline D3 & 2 & $\begin{array}{l}6.0 \\
9.8 \\
4.9 \\
9.5\end{array}$ & $\begin{array}{l}3 \\
4 \\
2 \\
4\end{array}$ & $\begin{array}{l}1184.5-1186.0^{6} \\
(44.124-44.180) \\
1176.5-1178.0^{6} \\
(43.826-43.882) \\
1184.5-1186.0^{6} \\
(44.124-44.180) \\
1176.5-1178.06 \\
(43.826-43.882)\end{array}$ & $\begin{array}{rr}1178.6 & (43.804) \\
6.9 & (0.257) \\
1174.6 & (43.755) \\
0.7 & (0.026) \\
1172.4 & (43.673) \\
5.8 & (0.216) \\
1175.8 & (43.788) \\
1.3 & (0.048)\end{array}$ & $\begin{array}{l}0.680- \\
0.690 \\
0.680 \\
0.680- \\
0.690 \\
0.680\end{array}$ & $\begin{array}{l}0.675 \\
0.001 \\
0.674 \\
0.0 \\
0.674 \\
0.004 \\
0.674 \\
0.0008\end{array}$ & \\
\hline$E^{2}$ & 1 & 19 & 4 & $1182.5^{5}(44.049)$ & $\begin{array}{rr}1183.8 & (44.098) \\
0.1 & (0.004)\end{array}$ & 0.717 & $\begin{array}{l}0.707 \\
0.1\end{array}$ & $\begin{array}{l}\text { Needle Value } 52 \text {, } \\
\text { fig. } 2.2 .32 \text { wide } \\
\text { open }\end{array}$ \\
\hline$F^{2}$ & $\begin{array}{l}1 \\
3\end{array}$ & $\begin{array}{l}16.8 \\
15 \cdot 3\end{array}$ & $\begin{array}{l}3 \\
8\end{array}$ & $\begin{array}{l}1182.0-1183.07 \\
(44.031-44.068) \\
1182.0-1183.07 \\
(44.031-44.068)\end{array}$ & $\begin{array}{rr}1184.2 & (44.098) \\
0.8 & (0.030) \\
1184.6 & (44.127) \\
0.6 & (0.022)\end{array}$ & $\begin{array}{l}0.717 \\
0.717\end{array}$ & $\begin{array}{l}0.707 \\
0.0006 \\
0.707 \\
0.0005\end{array}$ & $\begin{array}{l}\text { Put electric } \\
\text { vaporizer in } \\
\text { system }\end{array}$ \\
\hline$G^{2}$ & 1 & 16.9 & 4 & $1186.57(44.198)$ & $\begin{array}{rr}1197.3 & (44.600) \\
4.2 & (0.156)\end{array}$ & 0.715 & $\begin{array}{l}0.706 \\
0.005\end{array}$ & $\begin{array}{l}\text { Removed insula- } \\
\text { tion from sample } \\
\text { line to vapor- } \\
\text { izer } 1\end{array}$ \\
\hline
\end{tabular}

\footnotetext{
1 Values given are the average values and the estimated standard deviations computed from the composition using the A.G.A. method [2].

2Integrator A results used in this test.

Integrator $B$ results used in this test.

${ }^{4}$ This is the steady state value; the value at the beginning of the test was $1188 \mathrm{Btu} / \mathrm{scf}$ ( $44.254 \mathrm{MJ} / \mathrm{m}^{3}$ ) and decreasing.

5Flow from vaporizer 1 to calorimeter and gravitometer.

6 Flow from vaporizer 2 to calorimeter and gravitometer.

7 Flow from vaporizer 3 to calorimeter and gravitometer.
} 
Table 2.2 .30

Average Values and Estimated Standard Deviations in

Compositions and Liquid Densities for the Second

LNG Flow Facility Test

\begin{tabular}{|c|c|c|c|c|c|c|c|c|c|c|c|c|}
\hline \multirow[b]{2}{*}{ Test } & \multirow{2}{*}{$\begin{array}{l}\text { Vapor- } \\
\text { izer }\end{array}$} & \multirow{2}{*}{$\begin{array}{l}\text { Sampling } \\
\text { Rate } \\
\text { slpm }\end{array}$} & \multicolumn{9}{|c|}{$\begin{array}{c}\text { Average Value and Estimated Standard Deviation in } \\
\text { Mol Percent Composition }\end{array}$} & \multirow{2}{*}{$\begin{array}{l}\text { Density } \\
\mathrm{g} / \mathrm{cm}^{3}\end{array}$} \\
\hline & & & $\mathrm{N}_{2}$ & $\mathrm{CH}_{4}$ & $\mathrm{C}_{2} \mathrm{H}_{6}$ & $\mathrm{C}_{3} \mathrm{H}_{8}$ & $\mathrm{iC}_{4} \mathrm{H}_{10}$ & $\mathrm{nC}_{4} \mathrm{H}_{10}$ & $i C_{5 H} 12$ & $\mathrm{nC}_{5} \mathrm{H}_{12}$ & $\mathrm{C}_{6^{+}}$ & \\
\hline \multirow[t]{4}{*}{$A^{1}$} & 1 & 14 & 0.0 & $\begin{array}{r}84.997 \\
0.413\end{array}$ & $\begin{array}{r}12.005 \\
0.334\end{array}$ & $\begin{array}{l}2.414 \\
0.067\end{array}$ & $\begin{array}{l}0.190 \\
0.005\end{array}$ & $\begin{array}{l}0.226 \\
0.006\end{array}$ & $\begin{array}{l}0.048 \\
0.0004\end{array}$ & $\begin{array}{l}0.037 \\
0.001\end{array}$ & $\begin{array}{l}0.083 \\
0.0008\end{array}$ & $\begin{array}{l}0.4593 \\
0.0015\end{array}$ \\
\hline & & 19 & $\begin{array}{l}0.0009 \\
0.002\end{array}$ & $\begin{array}{r}84.609 \\
0.486\end{array}$ & $\begin{array}{r}12.324 \\
0.390\end{array}$ & $\begin{array}{l}2.469 \\
0.077\end{array}$ & $\begin{array}{l}0.193 \\
0.006\end{array}$ & $\begin{array}{l}0.232 \\
0.007\end{array}$ & $\begin{array}{l}0.049 \\
0.002\end{array}$ & $\begin{array}{l}0.038 \\
0.002\end{array}$ & $\begin{array}{l}0.084 \\
0.003\end{array}$ & $\begin{array}{l}0.4607 \\
0.0016\end{array}$ \\
\hline & 2 & 13 & 0.0 & $\begin{array}{r}84.454 \\
0.831\end{array}$ & $\begin{array}{r}12.439 \\
0.673\end{array}$ & $\begin{array}{l}2.506 \\
0.132\end{array}$ & $\begin{array}{l}0.196 \\
0.010\end{array}$ & $\begin{array}{l}0.233 \\
0.013\end{array}$ & $\begin{array}{l}0.049 \\
0.002\end{array}$ & $\begin{array}{l}0.039 \\
0.002\end{array}$ & 0.084 & $\begin{array}{l}0.4610 \\
0.0022\end{array}$ \\
\hline & & 16 & 0.0 & $\begin{array}{r}84.669 \\
0.080\end{array}$ & $\begin{array}{r}12.302 \\
0.019\end{array}$ & $\begin{array}{l}2.466 \\
0.080\end{array}$ & $\begin{array}{l}0.187 \\
0.004\end{array}$ & $\begin{array}{l}0.220 \\
0.005\end{array}$ & $\begin{array}{l}0.044 \\
0.0008\end{array}$ & $\begin{array}{l}0.035 \\
0.001\end{array}$ & $\begin{array}{l}0.077 \\
0.001\end{array}$ & $\begin{array}{l}0.4603 \\
0.0006\end{array}$ \\
\hline \multirow[t]{2}{*}{$B^{1}$} & 2 & 13 & $\begin{array}{l}0.007 \\
0.005\end{array}$ & $\begin{array}{r}83.772 \\
0.075\end{array}$ & $\begin{array}{r}12.968 \\
0.064\end{array}$ & $\begin{array}{l}2.604 \\
0.013\end{array}$ & $\begin{array}{l}0.205 \\
0.001\end{array}$ & $\begin{array}{l}0.244 \\
0.001\end{array}$ & $\begin{array}{l}0.052 \\
0.0008\end{array}$ & $\begin{array}{l}0.058 \\
0.001\end{array}$ & $\begin{array}{l}0.090 \\
0.001\end{array}$ & $\begin{array}{l}0.4537 \\
0.0005\end{array}$ \\
\hline & & 22 & $\begin{array}{l}0.011 \\
0.003\end{array}$ & $\begin{array}{r}83.866 \\
0.050\end{array}$ & $\begin{array}{r}12.904 \\
0.037\end{array}$ & $\begin{array}{l}2.585 \\
0.016\end{array}$ & $\begin{array}{l}0.203 \\
0.002\end{array}$ & $\begin{array}{l}0.242 \\
0.002\end{array}$ & $\begin{array}{l}0.052 \\
0.0008\end{array}$ & $\begin{array}{l}0.057 \\
0.001\end{array}$ & $\begin{array}{l}0.090 \\
0.001\end{array}$ & $\begin{array}{l}0.4534 \\
0.0005\end{array}$ \\
\hline$c^{2}$ & 1 & 16 & $\begin{array}{l}1.611 \\
0.024\end{array}$ & $\begin{array}{r}80.026 \\
0.118\end{array}$ & $\begin{array}{r}14.712 \\
0.119\end{array}$ & $\begin{array}{l}2.959 \\
0.018\end{array}$ & $\begin{array}{l}0.293 \\
0.006\end{array}$ & $\begin{array}{l}0.345 \\
0.007\end{array}$ & $\begin{array}{l}0.067 \\
0.002\end{array}$ & $\begin{array}{l}0.053 \\
0.001\end{array}$ & $\begin{array}{l}0.114 \\
0.006\end{array}$ & $\begin{array}{l}0.4802 \\
0.0038\end{array}$ \\
\hline \multirow[t]{4}{*}{$D^{2}$} & 1 & 6.0 & $\begin{array}{l}1.444 \\
0.424\end{array}$ & $\begin{array}{r}79.903 \\
0.302\end{array}$ & $\begin{array}{r}14.932 \\
0.241\end{array}$ & $\begin{array}{l}3.012 \\
0.043\end{array}$ & $\begin{array}{l}0.238 \\
0.003\end{array}$ & $\begin{array}{l}0.280 \\
0.005\end{array}$ & $\begin{array}{l}0.052 \\
0.006\end{array}$ & $\begin{array}{l}0.042 \\
0.002\end{array}$ & $\begin{array}{l}0.095 \\
0.008\end{array}$ & $\begin{array}{l}0.4797 \\
0.0012\end{array}$ \\
\hline & & 9.8 & $\begin{array}{l}1.611 \\
0.024\end{array}$ & $\begin{array}{r}80.026 \\
0.118\end{array}$ & $\begin{array}{r}14.712 \\
0.119\end{array}$ & $\begin{array}{l}2.959 \\
0.018\end{array}$ & $\begin{array}{l}0.233 \\
0.002\end{array}$ & $\begin{array}{l}0.274 \\
0.003\end{array}$ & $\begin{array}{l}0.051 \\
0.001\end{array}$ & $\begin{array}{l}0.042 \\
0.002\end{array}$ & $\begin{array}{l}0.090 \\
0.005\end{array}$ & $\begin{array}{l}0.4768 \\
0.0010\end{array}$ \\
\hline & 2 & 4.9 & $\begin{array}{l}1.665 \\
0.718\end{array}$ & $\begin{array}{r}79.978 \\
0.819\end{array}$ & $\begin{array}{r}14.771 \\
0.086\end{array}$ & $\begin{array}{l}2.889 \\
0.036\end{array}$ & $\begin{array}{l}0.228 \\
0.013\end{array}$ & $\begin{array}{l}0.269 \\
0.002\end{array}$ & $\begin{array}{l}0.050 \\
0.003\end{array}$ & $\begin{array}{l}0.040 \\
0.004\end{array}$ & $\begin{array}{l}0.102 \\
0.009\end{array}$ & $\begin{array}{l}0.4795 \\
0.0007\end{array}$ \\
\hline & & 9.5 & $\begin{array}{l}1.491 \\
0.024\end{array}$ & $\begin{array}{r}80.103 \\
0.132\end{array}$ & $\begin{array}{r}14.757 \\
0.082\end{array}$ & $\begin{array}{l}2.956 \\
0.025\end{array}$ & $\begin{array}{l}0.234 \\
0.014\end{array}$ & $\begin{array}{l}0.275 \\
0.003\end{array}$ & $\begin{array}{l}0.052 \\
0.003\end{array}$ & $\begin{array}{l}0.042 \\
0.002\end{array}$ & $\begin{array}{l}0.089 \\
0.005\end{array}$ & $\begin{array}{l}0.4770 \\
0.0008\end{array}$ \\
\hline$E^{1}$ & 1 & 19 & $\begin{array}{l}4.122 \\
0.008\end{array}$ & $\begin{array}{r}74.153 \\
0.013\end{array}$ & $\begin{array}{r}17.161 \\
0.013\end{array}$ & $\begin{array}{l}3.662 \\
0.006\end{array}$ & $\begin{array}{l}0.291 \\
0.0003\end{array}$ & $\begin{array}{l}0.347 \\
0.003\end{array}$ & $\begin{array}{l}0.077 \\
0.0007\end{array}$ & $\begin{array}{l}0.054 \\
0.0006\end{array}$ & $\begin{array}{l}0.133 \\
0.001\end{array}$ & $\begin{array}{l}0.5015 \\
0.0010\end{array}$ \\
\hline \multirow[t]{2}{*}{$F^{1}$} & 1 & 16.8 & $\begin{array}{l}4.138 \\
0.018\end{array}$ & $\begin{array}{r}74.083 \\
0.062\end{array}$ & $\begin{array}{r}17.202 \\
0.059\end{array}$ & $\begin{array}{l}3.669 \\
0.015\end{array}$ & $\begin{array}{l}0.292 \\
0.001\end{array}$ & $\begin{array}{l}0.349 \\
0.001\end{array}$ & $\begin{array}{l}0.077 \\
0.0002\end{array}$ & $\begin{array}{l}0.055 \\
0.0004\end{array}$ & $\begin{array}{l}0.134 \\
0.0003\end{array}$ & $\begin{array}{l}0.5020 \\
0.0010\end{array}$ \\
\hline & 3 & 15.3 & $\begin{array}{l}4.043 \\
0.026\end{array}$ & $\begin{array}{r}74.223 \\
0.049\end{array}$ & $\begin{array}{r}17.174 \\
0.038\end{array}$ & $\begin{array}{l}3.654 \\
0.020\end{array}$ & $\begin{array}{l}0.292 \\
0.0006\end{array}$ & $\begin{array}{l}0.348 \\
0.001\end{array}$ & $\begin{array}{l}0.077 \\
0.0003\end{array}$ & $\begin{array}{l}0.054 \\
0.0005\end{array}$ & $\begin{array}{l}0.134 \\
0.0005\end{array}$ & $\begin{array}{l}0.5020 \\
0.0012\end{array}$ \\
\hline$G^{1}$ & 1 & 16.9 & $\begin{array}{l}3.231 \\
0.279\end{array}$ & $\begin{array}{r}74.597 \\
0.129\end{array}$ & $\begin{array}{r}17.517 \\
0.139\end{array}$ & $\begin{array}{l}3.729 \\
0.019\end{array}$ & $\begin{array}{l}0.298 \\
0.003\end{array}$ & $\begin{array}{l}0.356 \\
0.002\end{array}$ & $\begin{array}{l}0.078 \\
0.001\end{array}$ & $\begin{array}{l}0.056 \\
0.0006\end{array}$ & $\begin{array}{l}0.136 \\
0.002\end{array}$ & $\begin{array}{l}0.5002 \\
0.0005\end{array}$ \\
\hline
\end{tabular}

1 Integrator A results used in this test.

2Integrator $B$ results used in this test. 


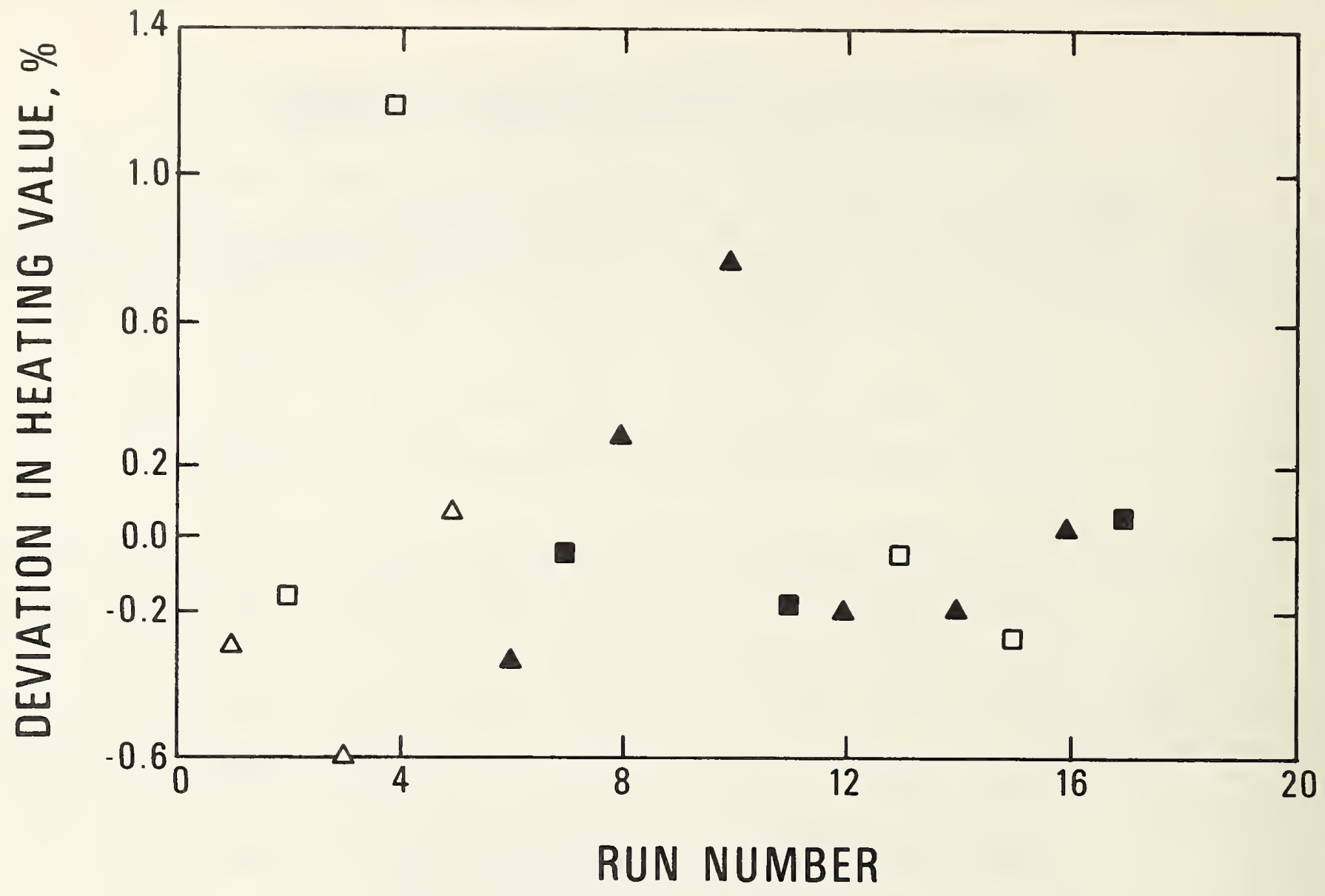

Figure 2.2.33. Deviations in the computed real gas heating value for flow facility test A. The symbols $\Delta$ and $\Delta$ denote vaporizer 1 samples taken at 14 and 19 standard liters per minute while taken at 13 and 16 standard liters per minute, respectively.

Another sample was rejected in Test $D$ which was one percent higher than the average heating value in that test; it too was drawn less than 30 minutes after changing sample rates. These results suggest that the sampling rate should be established at least 30 minutes before taking a sample.

Test $D$ Test $D$ was made to determine the sampling precision of the two steam vaporizers at 10 w sampling rates. The initial sampling rates were 9.8 and 9.5 slpm in vaporizers 1 and 2 , respectively. At these rates neither the gravitometer nor the calorimeter indicated the precision being diminished for either vaporizer. These sampling rates corresponded to a linear liquid flow rate of 4.0 centimeters per second and 1.6 centimeters per second in vaporizer 1 and 2 , respectively. Next, the sampling rates were lowered so that the linear flow rates were 2.4 centimeters per second and 0.8 centimeters per second in vaporizers 1 and 2, respectively. For both liquid vaporizers at these rates the gravitometer indicated a poorer sampling precision but the calorimeter did not. Figure 2.2 .36 shows the change in precision of the computed heating value when going to the lower sampling rate. If the linear flow rate in the sample vaporizer was the important factor in determining sampling precision at low sample rates, the vaporizer 2 results at 9.5 slpm would have been much poorer than actually observed. This suggests that the poor precision at low sample rates originates at some point in the sampling system, other than in the vaporizer. It seemed plausible that the low sample flows could have been too low to provide adequate mixing in the accumulator. However, this probably was not the case because the accumulator residence time was 68 seconds in these tests; good results were obtained during shipboard tests with residence times of 120 seconds. However, the shipboard accumulator was heated to induce mixing. 


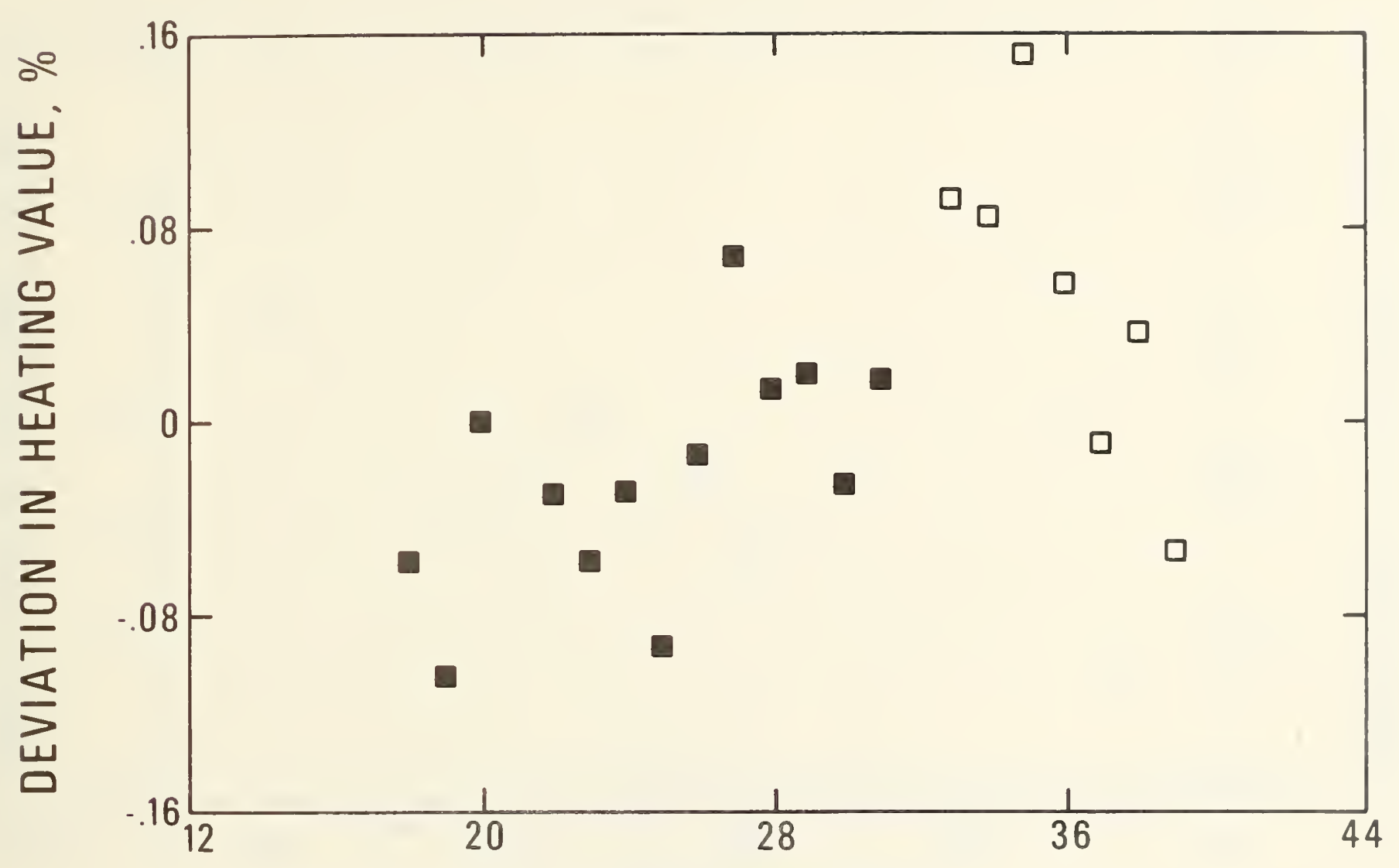

\section{RUN NUMBER}

Figure 2.2.34. Deviations in the computed real gas heating values for flow facility test B. The symbols and adenote vaporizer 2 samples taken at 13 and 22 standard liters per minute, respectively.

Test E Figure 2.2.37 shows the deviation in computed heating values for test E; this was a short test to see if the needle valve is necessary in a sampling system to prevent backflow. In this test the needle valve (valve 52 , figure 2.2.32) was fully open. These results confirmed the laboratory results in that a needle valve is not necessary, at least when using a tube vaporizer where there is a finite pressure drop in the vaporizer. (However, the needle valve is useful for controlling sampling rates.) The pressure difference between the test section and sample panel varied between $0.5 \mathrm{psi}(3 \mathrm{kPa}$ ) and $1.5 \mathrm{psi}(10 \mathrm{kPa})$ during this test; when using the needle valve to control the sample rate the pressure difference was typically 20 psi (0.14 MPa) to $35 \mathrm{psi}(0.24 \mathrm{MPa})$. (No significance should be put on the unusually high precision of this test; these estimated standard deviations are smaller than that of analysis alone.)

Test $\mathbf{F}$ Test $\mathbf{F}$ was a comparison of electric (vaporizer 3 ) and steam (vaporizer 1) vaporizers. Figure 2.2.38 shows the deviation in computed heating value for this test.

In this test the electric vaporizer had a heat input of 77 watts; at this power level, roughly half of the vaporizer was frostfree and the outlet temperature was roughly $100^{\circ} \mathrm{F}\left(38^{\circ} \mathrm{C}\right.$ ). As expected, the precision of the steam and electric vaporizers was comparable. 


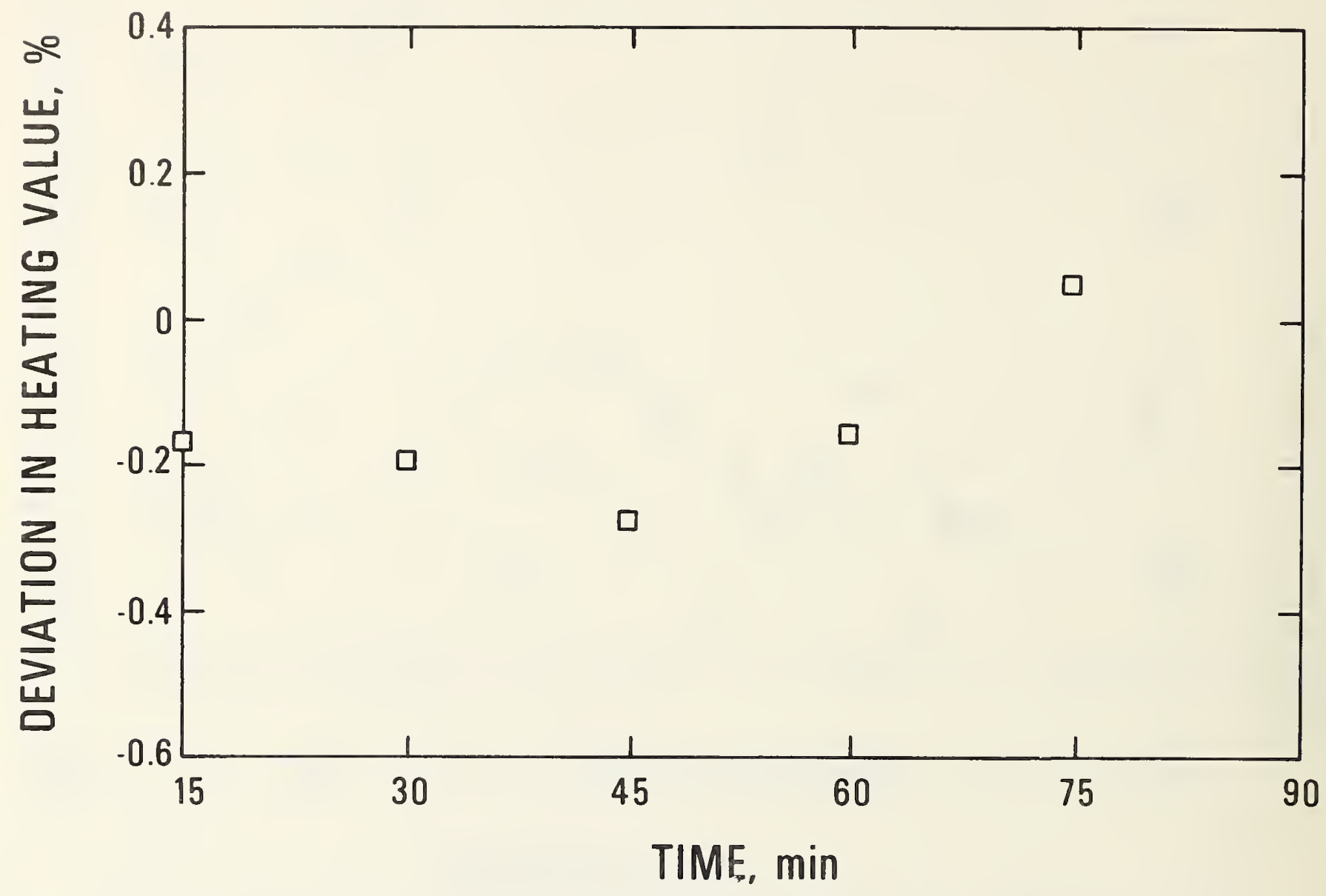

Figure 2.2.35. Deviations in the computed real gas heating values for flow facility test $C$.

Test $G$ The last test considered the effect of heat leak on precision. Up to this point the sample lines had been covered with fiberglass pipe insulation. During operation the lines felt slightly cool to the touch in most places; at the 90 degree bend between the block valve (Valve S1) and needle valve (Valve S2) there was a frost spot on the line to vaporizer 2 (or 3). No frost spots appeared on the sample line to vaporizer 1. Samples from this vaporizer were going to the calorimeter and gravitometer during the insulation removal. Before all of the insulation had been stripped away the calorimeter went off scale, indicating a heating value of greater than $1195 \mathrm{Btu} / \mathrm{scf}$ (44.515 MJ/m3). As figure 2.2.39 shows, the computed heating values were significantly higher for the uninsulated sampling system. (The deviations are referenced to the average values obtained in test $\mathrm{F}$.) The sample analysis indicated a $0.8 \mathrm{~mol}$ percent reduction in nitrogen which was statistically significant. Also, there was a 0.3 mol percent increase in the ethane content; but based on the limited number of samples, this increase could not be considered statistically significant. This indicates that backflashing occurred in the sample line and that it enriched the heavy components in the observed samples. 


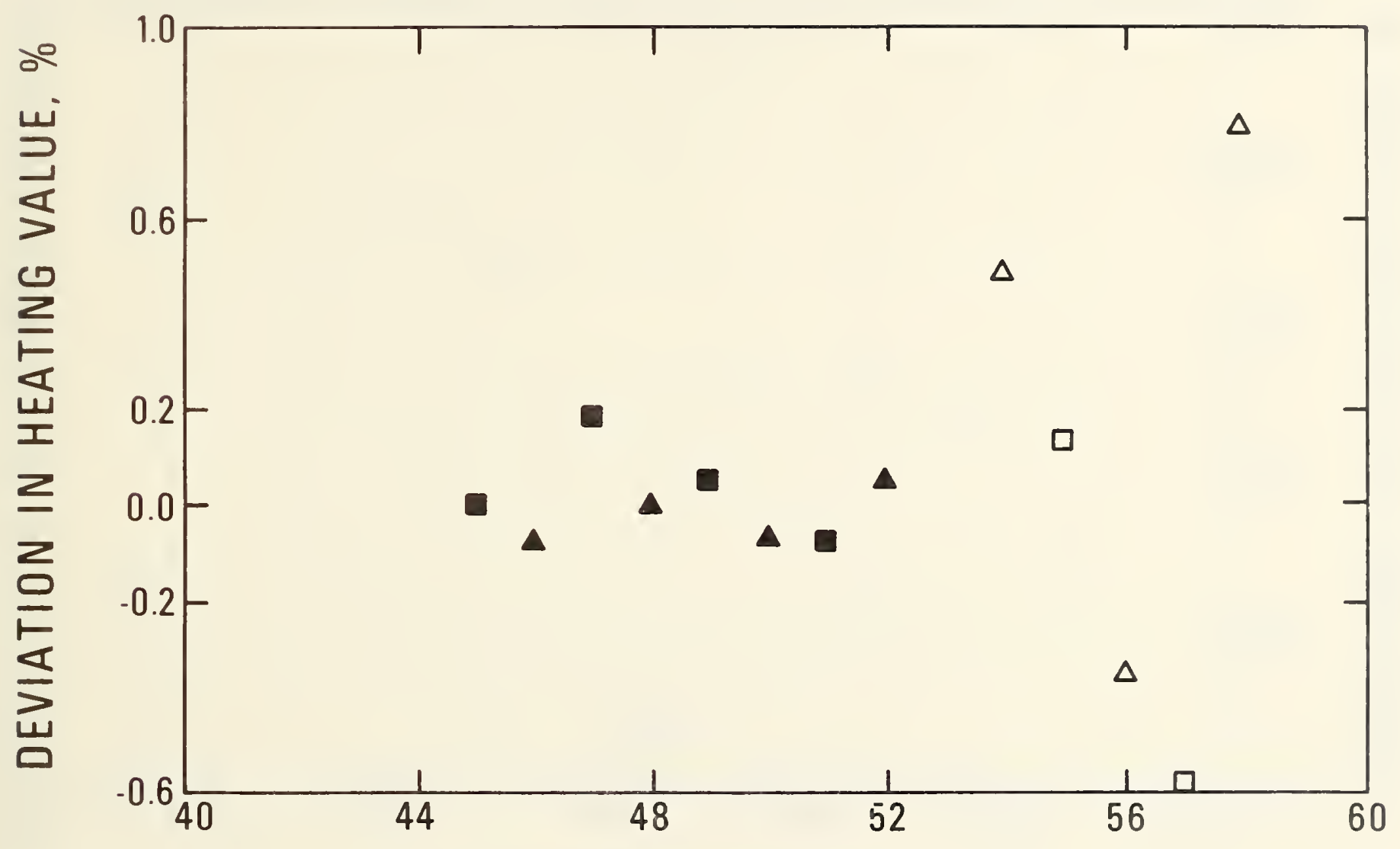

RUN NUMBER

Figure 2.2.36. Deviations in the computed real gas heating values for flow facility test D. The symbols $\Delta$ and $\Delta$ denote vaporizer 1 samples taken at 6.0 and 9.8 standard liters per minute while a and adenote vaporizer 2 samples taken at 4.9 and 9.5 standard 1 iters per minute, respectively.

General Comments Based on the shipboard tests, frost was expected to accumulate at the 90 degree bend on the steam vaporizer sample outlet lines at sample rates above 10 slpm. The impingement chamber apparently worked satisfactorily, because there never was any indication of frost at the bend. The sample lines were felt at and near the bends several times throughout the tests; there was never an indication that the line was cooler at the bend.

Throughout these tests, the comparison between the measured and computed heating values was ralrly good considering that the $\mathrm{C}_{6}{ }^{+}$fraction ranged between 0.08 and 0.13 mol percent. An analys 13 of the heavy fraction indicated that it had roughly equal parts of $\mathrm{C}_{6}{ }^{\prime} \mathrm{s}$ and $\mathrm{C}_{7}{ }^{\prime} \mathrm{s}$. Therefore, the response factor for $\mathrm{C}_{6+}$ peaks, $\mathrm{K}_{\mathrm{C} 6}+$ was computed by

$$
\mathrm{K}_{\mathrm{C}^{+}}=\mathrm{K}_{\mathrm{nC}_{4}}\left(\mathrm{D}_{\mathrm{nC}_{6}}+\mathrm{D}_{\mathrm{nC}}\right) / 2 \mathrm{D}_{\mathrm{nC}_{4}}
$$

where KnC4 is the measured response factor for normal butane and the D's are the response factor reported by Dietz [25].

Except for runs $E, F$ and $G$ (the final day's tests), the agreement between measured and computad specific gravities was excellent. There is no explaination as to why the final day's results wora consistently low by 1.3 to 1.4 percent, especially since the heating values agreed to 0.14 percent or better. 


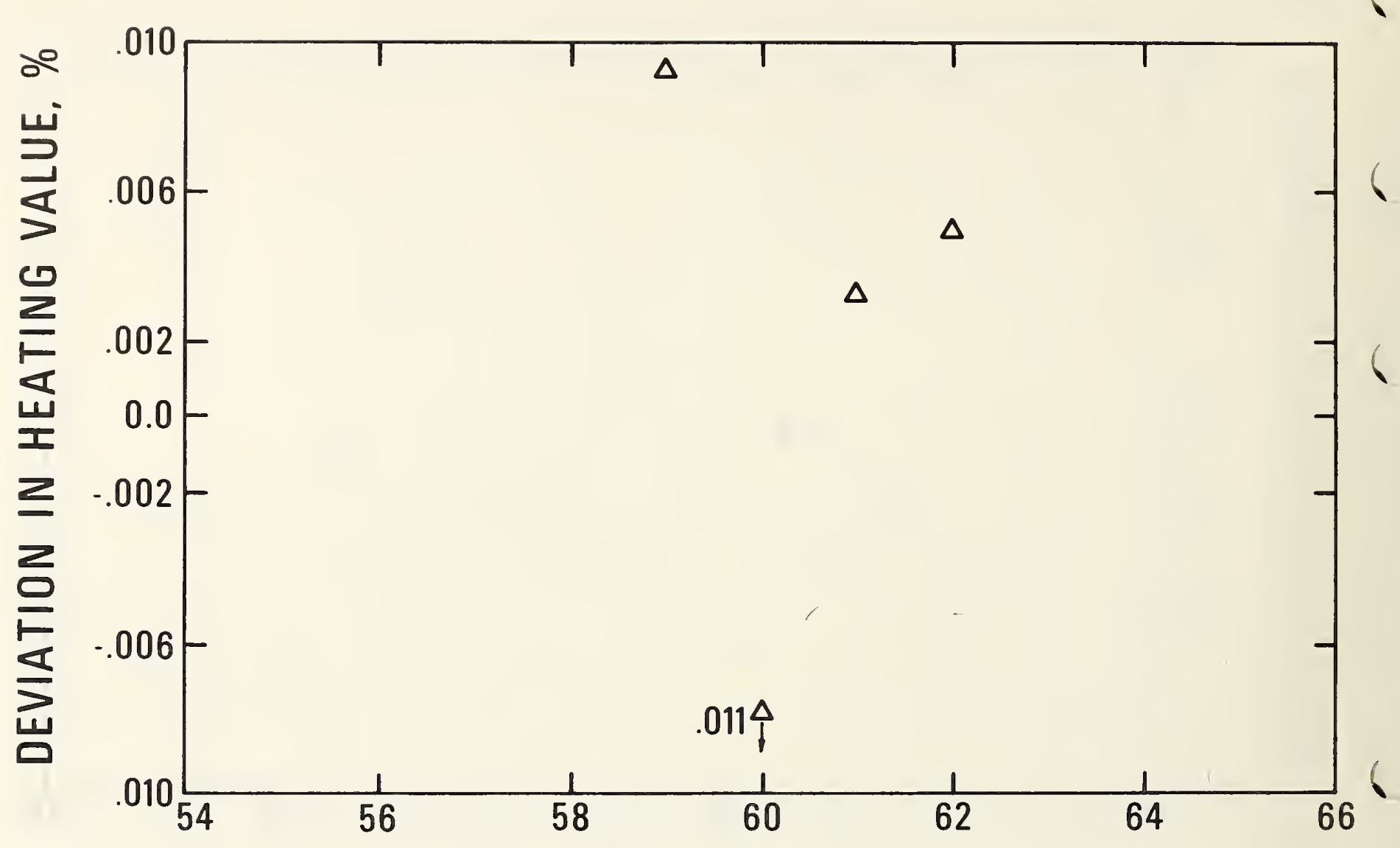

RUN NUMBER

Figure 2.2.37. Deviations in the computed real gas heating values for flow facility test E.

The estimated standard deviation in the computed liquid densities listed in table 2.2 .30 is much larger than observed in previous tests. However, the densities in the flow facility tests reflected both temperature and composition fluctuations. The temperature fluctuations, which were as large as $1.5 \mathrm{~K}$, make comparisons based on density meaningless.

Throughout these tests, integrator A had difficulties in reliably measuring the C6+ peak area because the peak had a small peak height and wide peak width compared to the other peaks (see section 2.2 .4 for more details). Except for the first day integrator B reported consistent $6_{6}{ }^{+}$peak areas. Therefore, the $\mathrm{C}_{6}+$ peak area for integrator $\mathrm{A}$ was computed by assuming that the ratio of the $\mathrm{C}_{6}{ }^{+}$peak area to that of the normal butane peak area was equal for both integrators; this ratio for integrator $B$ was constant to within \pm 2 percent throughout the tests. (a check showed that the ratios of iso to normal butane peak areas for the two integrators were equal to within one percent or better.)

During the first two days the two integrators reported comparable compositions for all components except iso and normal pentane; this difference was traced to calibration problems with integrator B. The typical spread between the calculated heating values for the two integrators was 0.05 to 0.14 percent; the difference in pentane contents reported by the two integrators accounted for this heating value difference. 


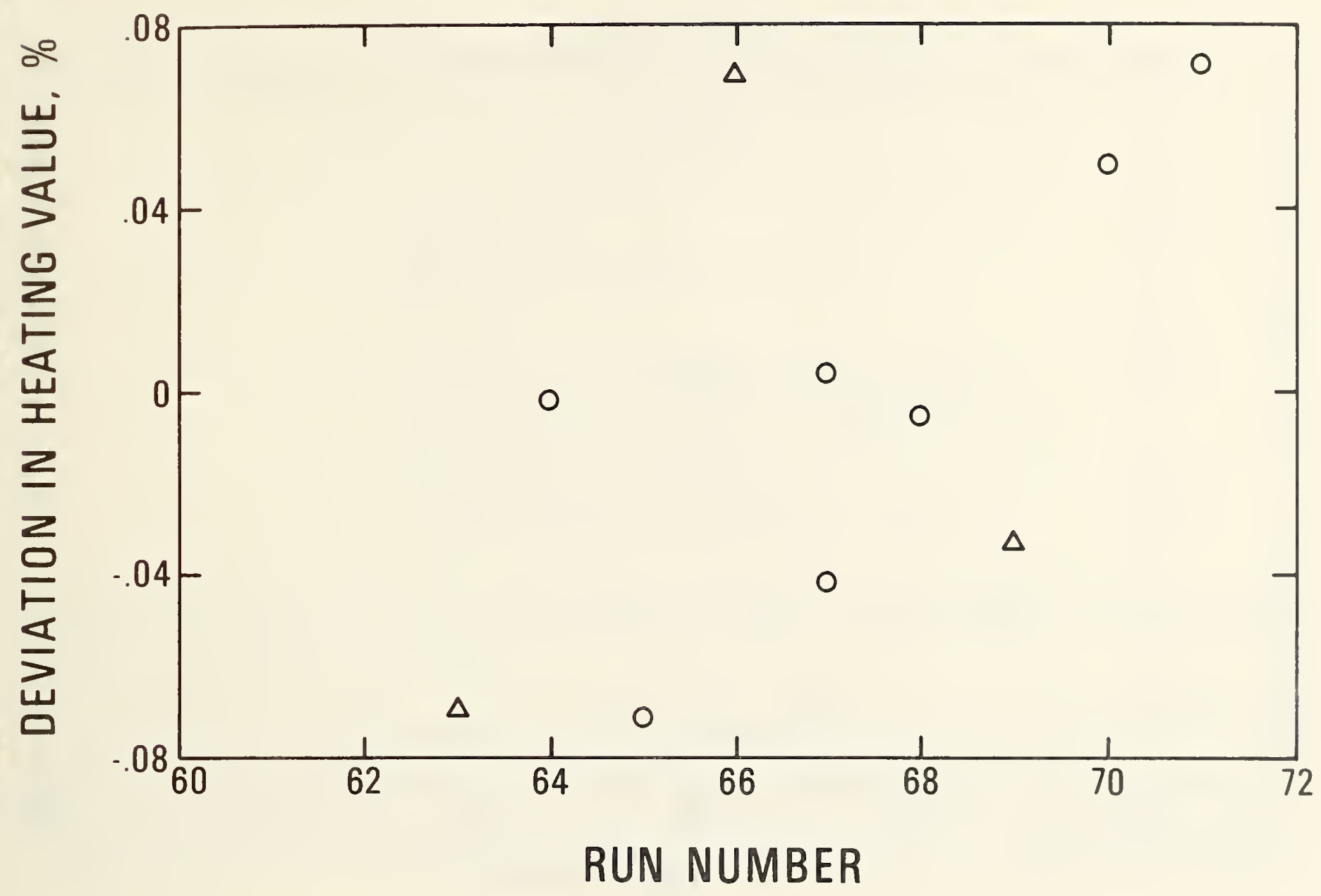

Figure 2.2.38. Deviations in the computed real gas heating values for LNG flow facility test F. The symbols $\Delta$ and 0 denote samples for vaporizers 1 and 3 , respectively.

On the third day (Test D) the program in integrator A was altered to eliminate the scatter in detecting the $\mathrm{C}_{6}{ }^{+}$peak. This change not only did not improve the $\mathrm{C}_{6}{ }^{+}$peak detection but it caused increased scatter in integrating the nitrogen and methane peaks. (In this chromatographlc column arrangement, nitrogen and methane eluted after $\mathrm{C}_{6}{ }^{+}$.) The differences in computed heating values differed by as much as one percent with integrator A showing the most scatter.

On the last day the integrator A program was identical to the program used the second day. On this day integrator B gave erratic nitrogen contents even though no program changes were made. However. 1 t was noted that the peak width parameter (which was automatically updated after each peak) varled between 20 and 66 seconds. This variation affected the next (nitrogen) peak area measurement but not the methane peak area measurement. This is because the typical peak widths for nitrogen and methann were 20 and 60 seconds, respectively. Agreement between the two integrators occurred only whon the updated peak width parameter was between 20 and 30 seconds. 


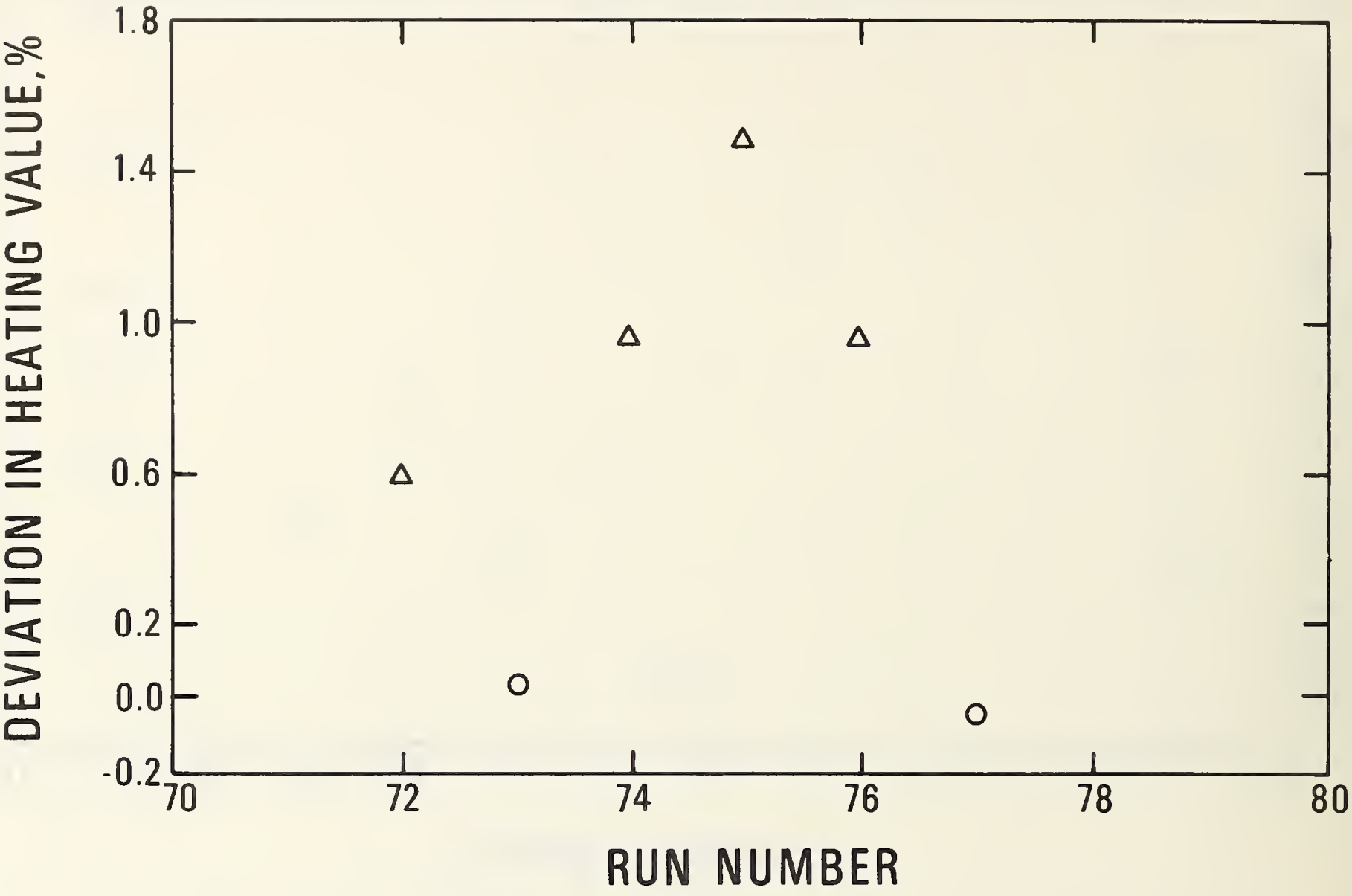

Figure 2.2.39. Deviations in the computed real gas heating values for flow facility test $G$. The symbols $\Delta$ and 0 denote samples from vaporizers 1 and 3 respectively.

\subsubsection{Conclusions and Recommendations}

This section of the manual has systematically considered many factors which could affect the precision and accuracy of the composition of samples withdrawn from a flowing LNG stream. Laboratory and field tests showed that it is feasible to obtain and analyze representative samples with a precision (based on three standard deviations) of better than \pm 0.30 percent in the computed heating value; this includes the gas analysis precision of \pm 0.06 percent. The accuracy of the measurement depends only on the gas analysis accuracy; both laboratory and shipboard tests showed that a properly designed and operated sampling system produces no statistically significant bias. A comparison of measured and computed heating values during the second flow facility test verified that the difference between the two values was within the combined uncertainty of the calorimetric measurement ( \pm 0.3 percent [31]) and the sampling measurement process.

Table 2.2.31 summarizes the variables evaluated in the sampling tests and groups them according to their effect on sampling precision and accuracy. 


\author{
Table 2.2.31 \\ Variables Evaluated for Their Effect on \\ LNG Sampling Precision and Accuracy \\ * Variables affecting both sampling precision and accuracy: \\ Heat leak to liquid sample line. \\ - Variables affecting sampling precision but not accuracy: \\ Sampling rate, \\ Vaporizer design, \\ Time-averaging the vaporized sample, \\ Sample rate transients, \\ Probe design. \\ - Variables not affecting sampling precision and accuracy: \\ Temperature and pressure at the sampling point, \\ Pressure differential between the sampling point \\ pressure and the equilibrium LNG pressure, \\ Flow rate past the sampling point, \\ Pressure drop in the liquid sample line between \\ the sampling point and the vaporizer, \\ composition of the liquid being sampled.
}

The test results indicated that heat leak causes backflashing of nitrogen (and probably methane) which enriches the samples in the heavy $\left(\mathrm{C}_{2}+\right)$ components. However, field tests showed that insulating the sample line with one inch (25 millimeters) thick layer of fiberglass pipe insulation sealed with duct tape eliminates the heat leak problem.

Five variables were found to adversely affect sampling precision. Both laboratory and field tests showed that below a certain sampling rate, the sampling precision diminishes; this minimum sampling rate varied among various sampling system designs. A mechanism for sampling rate's importance is unknown. Attempts to correlate the sampling rate with heat leak, accumulator residence time, linear flow rate in the liquid sample line and linear flow rate in the vaporizer failed, However, criteria for setting the minimum allowable sample rate must be found before general guidelines for LNG sampling system design can be established. The next section gives sampling rates for the recommended sampling system design.

Laboratory results clearly indicated that a small diameter tube vaporizer was better than a vaporizer with a large cross sectional area. Tests also showed that rapid sample vaporization gave the best results. However, there is no advantage in having the vaporizer outlet temperature above $100^{\circ} \mathrm{F}$ ( 38 ${ }^{\circ}$ ). Both steam and electrically heated vaporizers produce comparable results.

The need to time-average the sample stream leaving the vaporizer in an accumulator arises from fractionation during vaporization. The minimum residence time necessary to obtain precise results depended upon the sampling system; residence times of 20 to 120 seconds gave good results.

Although the field tests were inconclusive, they indicated that taking samples within $30 \mathrm{~m} 1$ nutes of a major change in sampling rate diminishes sampling precision. However, minor fluctuations and slow drifts in sampling rates did not affect the results.

The side tap probe was found to be more reliable than an upstream facing pitot tube. For unknown reasons the pitot tube occasionally gave erratic results.

The conclusion that certain variables did not affect sampling error must be considered valid only ovr the ranges tested; however, this range usually included the anticipated operating ranges of on and off-loading of LNG tankers.

There is no reason to expect the temperature and pressure to affect sampling error provided tho conditions are far from the critical point of the LNG.

At some low value the difference between the pressure of the sampled 11 quid and the satiration pressure of the LNG (i.e., subcooling) in combination with heat leak should create prematirn vaporization which could adversely affect sampling precision; however, this effect was not detected at pressure differentials as low as 3.9 psi ( $25 \mathrm{kPa}$ ) (this corresponds to approxlmatrly 0.5 k sub-cooling). 
LNG flow rates corresponding to Reynold's numbers ranging between 1500 and 50,000 did not affect sampling precision; it is unlikely that there would be any adverse effect on precision at the higher Reynold's numbers anticipated in on and off loading operations.

The fact that a pressure drop in the liquid sample line which is comparable to the pressure drop in the vaporizer is unnecessary eliminates the need for liquid flow control valve. However, this conclusion is known to be valid only when using a tube vaporizer.

Finally, there is no problem in sampling LNG mixtures which contain up to 0.15 percent $\mathrm{C}_{5}+$. The heavies would be a problem if they raised the sample dew point temperature to near ambient temperature.

Recommended LNG Sampling System Design and Operating Criteria Based on the laboratory and field test results, the following sampling system design criteria are recommended:

Probe - side tap mounted horizontally with the probe flush with the wall of the LNG pipe; probe inner diameter should be as small as feasible ( $<0.25$ inch ( 6.4 millimeters)).

Liquid phase needle valve - optional, but useful for flow control.

Liquid sample line - use as small a bore tubing as feasible ( $\leqq 0.20$ inch (5.1 millimeters)) and make the line as short as possible. (A filter is recommended to prevent line plugging.)

Sample vaporizer - use a coiled tube vaporizer with the tube inner diameter comparable to that of the sample line tubing. If steam is the vaporizing medium, use the design procedure outlined in section 2.2.5 with an impingement chamber. Electrically heated vaporizers can be designed using the same procedure as steam vaporizers; however, thin walled tubing is necessary to minimize the necessary amperage. Also, a thermostatic control is necessary to prevent the vaporizer from overheating if the sample flow rate decreases.

Accumulator - size for a residence time of at least 45 seconds; the length to diameter ratio of the accumulator should be greater than 1.5. The sample inlet line should extend to near the bottom of the vessel but the outlet line should not penetrate the vessel volume. Heating the bottom of the accumulator may improve mixing.

Insulation - the probe and liquid sample line should be insulated sufficiently to prevent moisture from condensing on the lines.

The following sample system operating conditions are within the range of values found to give good results in the field tests and therefore are recommended;

Sampling rate - greater than $20 \mathrm{slpm}$; this criteria applies only to the recommended sampling system.

Sample pressure - preferrably greater than 20 psig (0.24 MPa) to provide sufficient gas sample for purging and replicate analyses from a $500 \mathrm{~cm}^{3}$ sample cylinder.

Vaporizer outlet temperature -80 to $100^{\circ} \mathrm{F}\left(27\right.$ to $\left.38^{\circ} \mathrm{C}\right)$.

The shipboard tests and flow facility test 2 sampling systems both worked satisfactorily and may be considered proven sampling systems. 


\subsubsection{References}

[i] Parrish, W. R., J. M. Arvidson and J. F. LaBrecque, Development and evaluation of an LNG Sampling Measurement System, Nat. Bur. Stand. (U.S.), NBSIR 78-887, 199 pp(July, 1978 ).

[2] Caldwell, B. J., Fuel gas energy metering, Transmission Measurement Committee Report Number 5 (revised), page 55 (1976), American Gas Association, Inc., Arlington, VA.

[3] Armstrong, G. T., E. S. Domalski and J. I. Minor, standard combustion data for the fuel gas Industry, American Gas Association 1972 Operating Section Proceedings, page D-72 (1972).

[4] McCarty, R. D., A comparison of mathematical models for the prediction of LNG densities, Nat. Bur. Stand. (U.S.) Internal Report, NBSIR 77-867 (Oct. 1977).

[5] Hiza, M. J., An empirical excess volume model for estimating liquefied natural gas densities, Fluid Phase Equilibria, 2(2), pp 27-38 (August 1978).

[6] Natrella, M. G., Experimental Statistics, Handbook 91, National Bureau of Standards (1963).

[7] "Standard Method for Analysis of Natural Gas by Gas Chromatography," ASTM Designation: D1945-64 (Reapproved 1973).

[8] "Standard Method for Analysis of Natural Gases and Related Types of Gaseous Mixtures by the Mass Spectrometer," ASTM Designation: D1137-53.

[9] Dalmaze, M. R., LNG sampling technique, Gas Aujourdhui 96, 448 (Nov. 1972 ).

[10] Trigo, J., System samples LNG accurately, Hydrocarbon Proc. p. 83 (April 1976).

[11] The Cosmodyne Corporation, Cryogenic Sampler Bulletin TDS-8064, Torrance, Calif.

[12] Cook, H. L., Method and apparatus for sampling refrigerated volatile liquids, U.S. Patent No. 3, 487,692 (Jan. 1970).

[13] Miller, A. J., Techniques for sampling natural gas, SNG, and LNG, Pipe Line Industry 39 ( 3 ), 70 (Sept. 1973).

[14] "Standard Method of Sampling Natural Gas," ASTM Designation: D1145-53.

[15] Chapman, J. A. and G. D. Payne, Sampling system guidelines, paper presented at the 19 th Annual ISA Analysis Instrumentation Symp., April 24-26, 1973, St. Louis, MO.

[16] Turbett, J.R., Rockwell International, Atomics International Division, Golden, Co (private communication, 1977).

[17] Conn, A. P., Transco, Houston, TX (private communication, 1976).

[18] GPA Publication 2261-72, Method of analysis for natural gas and similar gaseous mixtures by gas chromatography,, Gas Processors Association, Tulsa, OK.

[19] Thompson, B., Fundamentals of Gas Analysis by Gas Chromatography, Varian Associates, Inc., Palo Alto, CA (1977).

[20] Purcell, J. E. and C. P. Gilson, Improved analysis of natural gas, Chromatography Newsletter 1 (2), 45 (Nov. 1972), published by Perkin-Elmer Corp., Norwalk, CT.

[21] Lawson, A. E., A versatile, low cost gas chromatograph, American Laboratory (May 1969).

[22] Ludtke, P. R., Performance characteristics of a liquid helium pump, Nat. Bur. Stand. (U.S.) Internal Report, NBSIR 75-816 (July 1975).

[23] Cook, H. L., Transco, Houston, TX (private communication, 1977).

[24] Handbook of Chemistry and Physics, 45th Ed., p. D86, The Chemical Rubber Co., Cleveland, OH. 
[25] Childs, G. E., L. J. Ericks, and R. L. Powell, Thermal conductivity of solids at room temperature and below, Nat. Bur. Stand. (U.S.) Monograph 131, p. 257 (Sept. 1973).

[26] Dietz, W. A., Response factors for gas chromatographic analyses, J. of Gas Chromatography, p. 68 (Feb. 1967).

[27] Mann, D. B., Cryogenic flowmetering research at NBS, Cryogenics 11, 179 (1971).

[28] Brennan, J. A., Cryogenics Division, National Bureau of Standards, Boulder, Co (private communication, 1976).

[29] Goodwin, R. D., The thermophysical properties of methane from 90 to $500 \mathrm{~K}$ at pressure to 700 bar, Nat. Bur. Stand. (U.S.) Technical Note 653 (April 1974).

[30] Hanley, H. J. M., W. M. Haynes, and R. D. McCarty, The viscosity and thermal conductivity coefficients for dense gaseous and liquid methane, J. of Phys. and Chem. Ref. Data 6 (2), 597 (1977).

[31] "Standard Method of Test for Calorific Value of Gases in Natural Gas Range by Continuous Recording Calorimeter," ASTM Designation: D1145-53.

[32] Hiza, M. J., W. M. Haynes and W. R. Parrish, Orthobaric liquid densities and excess volumes for binary mixtures of low molar-mass alkanes and nitrogen between 105 and $140 \mathrm{~K}$, $\mathrm{J}$. Chem. Thermodynamics $\underline{9}, 873(1977)$. 





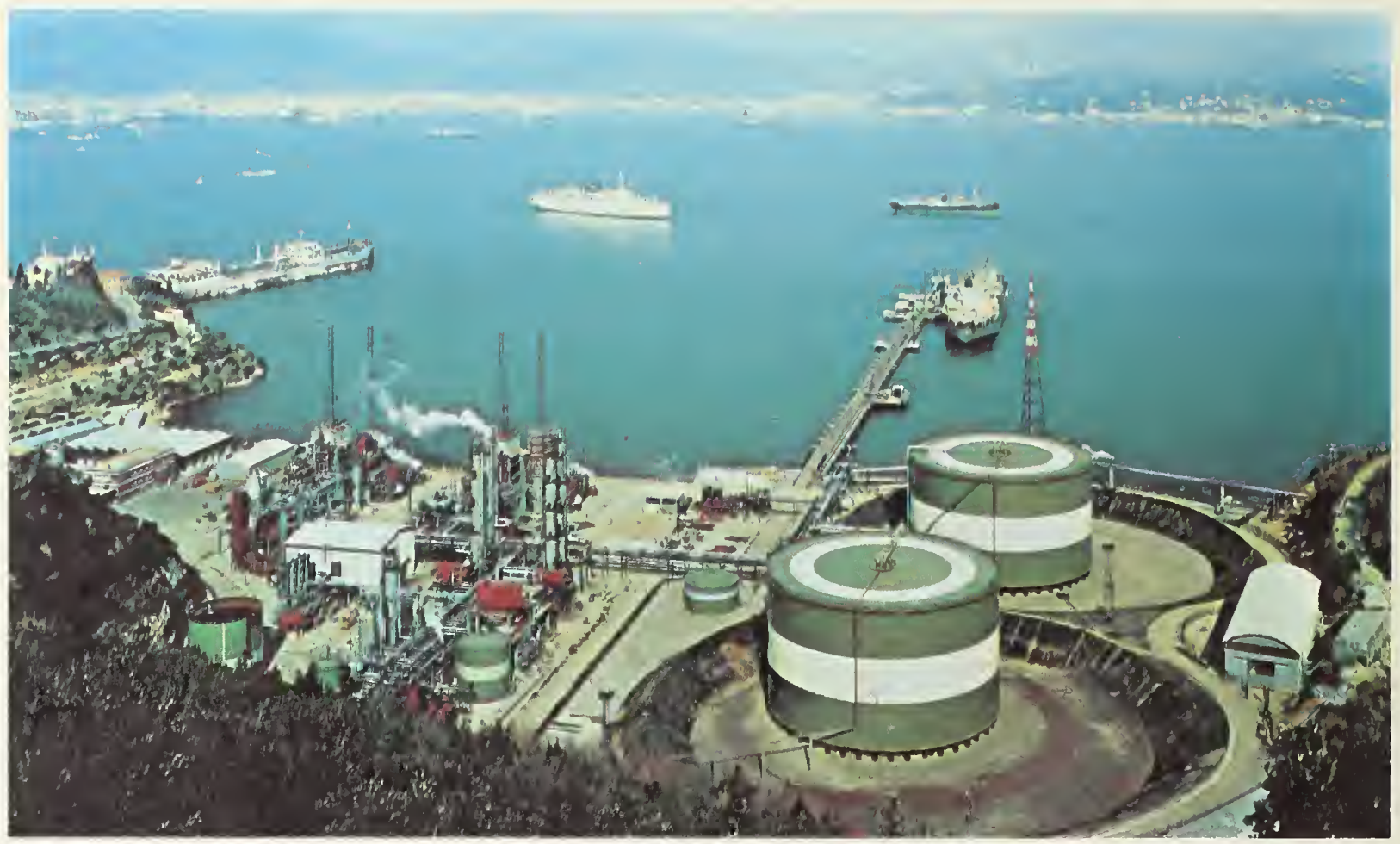

\section{PAN I GAGLIA TERMINAL}

The Panigaglia Terminal, constructed and operated by SNAM, a company belonging to the state-run ENI group, is located in La Spezia, Italy. The terminal was completed in 1969 with regular deliveries from Algeria started in 1971. 1981 , LNG from the Marsa el Brega Liquefaction Plant in Libya was being received at a rate of 2 millions tons annually. Receiving facilities include two 50,000 kL LNG tanks, carrier berth, pumps and vaporization equipment. The length of the unloading lines are typical of import terminals.

Because of the high calorific value of the Libyan LNG (27 percent of hydrocarbons with combustion enthalpies greater than methane), part of the LNG is reformed by steam to make it interchangeable with natural gas from the Po Valley. Waste heat from steam reforming is used for LNG vaporization. (Photograph by courtesy of The Japan Gas Association, Tokyo, Japan, on behalf of the 1981 Japan LNG Congress) 


\section{CONTENTS}

\section{0 MEASUREMENT ELEMENTS}

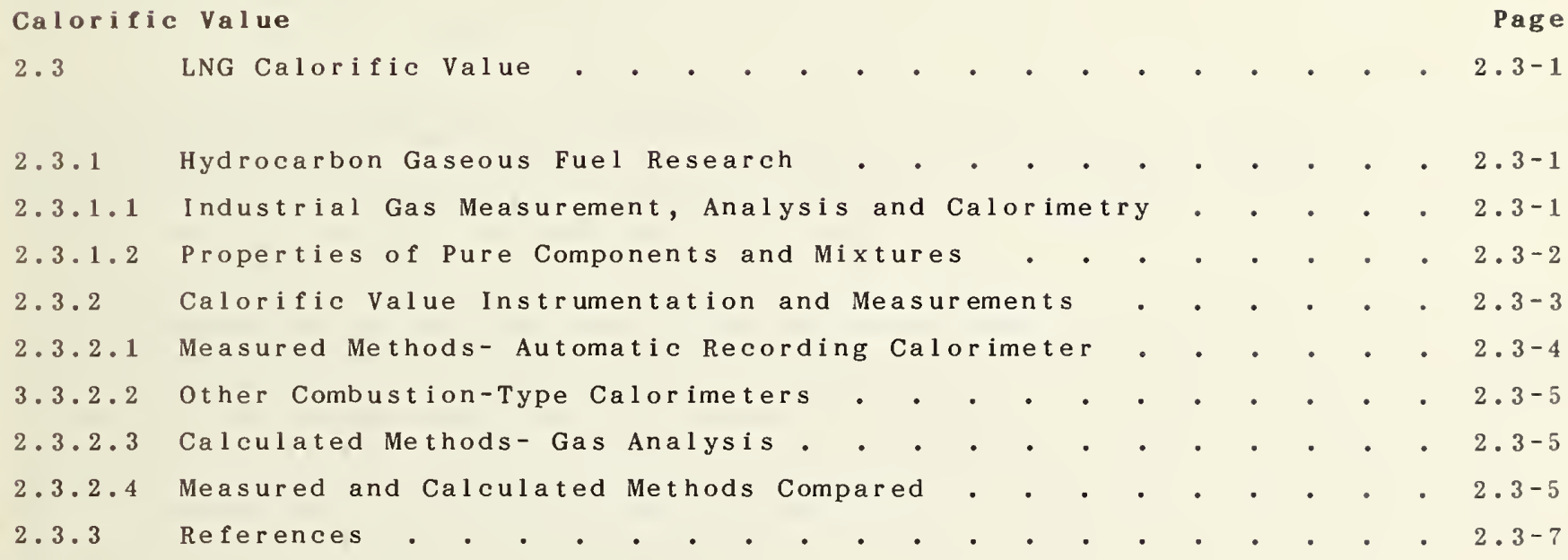




\subsection{LNG Calorific Values}

It is the purpose of this section of the manual to provide information on the methods of measurement of the calorific or heating value of natural gas and the accuracy and precision of these methods. Instrumentation and properties data necessary for determination of the actual values are also discussed in section 2.2 and 1.3. However, these sections deal with specific methods developed for LNG or LNG mixtures and describe relatively recent technological advances.

The general problem of assessing heating values of gaseous fuels dates back to the beginning of the current century, and the methods and techniques developed over the years since then are still very much in use. Advances certainly have been made in accuracy and precision, but in general very little has changed in the techniques used. The historical perspective and a discussion of the accuracy and precision of the methods, both direct and calculated, are presented.

\subsubsection{Hydrocarbon Gaseous Fuel Research}

A principal value of natural gas is the quantity of thermal energy derived when the natural gas is burned in air. The following is an edited portion of an historical review by Armstrong [32] published on the occasion of the $75 \mathrm{th}$ anniversary of the National Bureau of Standards. Only that portion of the publication dealing with natural gas is included here. The complete publication deals with hydrocarbon fuels in general, and the reader is referred to the original document for details.

In the early days of NBS, which was founded in 1901, natural gas was just coming into service as a domestic heating and cooking fuel, its prior use having been mainly for illumination. Early NBS work was directed to the goals of safety and quality of this new fuel and to measurement of its properties. Studies included methods of measurement of gas quantities (volumetrically) and of density, measurement of heating value and candlepower, and determination of impurities. Circulars were prepared describing how these could be determined accurately. For the United States the NBS also tested calorimeters for measuring the heating value of gaseous fuels. In later years NBS evaluated automatic calorimeters for continuous recording of gas heating values. One important result of NBS work has been the adoption of heating value as the measure of a gaseous fuel in trade; this is the current practice. In order to provide measurement validity, a reference material (a good quality methane) is certified for real-gas heating value provided at intervals by NBS for the Institute of Gas Technology, which is the present source of reference fuels for the fuel gas industry.

\subsubsection{Industrial Gas Measurement, Analysis, and Calorimetry}

Study of natural gas began at NBS as part of an extensive program guided by E. B. Rosa (1901-1921) in the early years of NBS to establish standards of safety, quality, measurement, and performance in the public utilities industries. Natural gas was in transition from its principal use for illumination to its later use for heating [1]. At the turn of the century gas was measured to individual customers by volume and charged for on that basis. Important properties were "candlepower" and "heating value". E. B. Rosa almost immediately began advocating the use of heating value as a measure of quality and value of natural gas [2]. Heating value was ultimately adopted as the measure of the commodity as a result of this NBS activity [1]. The procedure is still in practice.

Early NBS work included studies of the measurement of quantity of gas [3, 4], measurement of heating value [5], determination of candlepower [6], determination of impurities, in particular ammonia [7] and hydrogen sulfide [8], and determination of gas density [9]. Other aspects of commerclal gas technology were also involved, including safety and fittings for gas service.

Calorimetry of gaseous methane on a routine basis required the conduct of the combustion process in a reproducible way, the use of commercial gas calorimeters (such as the Junkers or the Boys calorimeters) ${ }^{1}$ together with commercial gas metering devices (usually the wet test meter), and associated temperature and mass measurements. The process was fairly complex for a routine quality-test procedure. It consisted of measuring volumetrically the amount of gas flowing into the system, burning the gas with a controlled amount of combustion air in the calorimeter, in which the heat was absorbed in flowing water, and observing the heat effect of the combustion when a steady state was achieved.

${ }^{1}$ Certain trade names and company products are identified in order to adequately specify the experimental procedures. In no case does such indentification $1 \mathrm{mply}$ recommendation or endorsement ty the National Bureau of Standards, nor does it $1 \mathrm{mply}$ that the products are necessarily the best available for the purpose. 
The NBS studies established the conditions for carrying out the measurements accurately, with inaccuracies lying between 0.2 and 0.5 percent. Factors requiring measurement or control include temperature, pressure, and humidity of the gas, amount of gas burned, amount of combustion air used, degree of completeness of combustion, rate of water flow in the calorimeter, temperature rise of the flowing water in the calorimeter, heat losses due to extraneous processes, and mass of water condensed from the combustion gases.

With heating value proposed as a criterion of value for trade in natural gas, methods for determining it and adequate auxiliary data for interpreting the measurements were needed. These were prepared as NBS Circulars: Standard Methods of Gas Testing (Circular No. 48 [4]) and Gas Calorimeter Tables (Circular No. 65, revised and reissued at intervals as Circulars C417 and C464) [10].

The NBS also undertook to test calorimeters for the states that adopted heating value as a measure for pricing fuel. This was to provide regulatory commissions with the necessary equipment and measurement verification to enable them to test fuels and approve of charges by public utilities corporations. Although the gas-flow calorimeter is an absolute instrument for which calibration is not appropriate, demonstration of the accuracy of the complete process in a particular calorimeter required analysis of combustion gas and products and a careful mathematical treatment of the results. This was a relatively time consuming process. The testing of manually operated calorimeters for the states continued until about 1955, when the number tested annually had become very small. The gas industry by that time had almost universally adopted the automatic recording calorimeter, and this accounted for the absence of manually operated instruments.

The automatic recording gas calorimeter of the type principally in use in the United Stated was the subject of two extensive investigations at NBS. R. S. Jessup [11] compared the Thomas Recording Calorimeter (later called Cutler-Hammer Automatic Recording Calorimeter) with the Junkers calorimeter, and established the reliability of the former as being well within 1.0 percent.

The calorimeter suffered to a certain extent from a lag in responding to sudden changes of heating value of the gas. However, the convenience of use and the continuous record obtained provided sufficient incentive to cause this calorimeter and its later versions to replace the manually operated calorimeters.

J. H. Eiseman and E. A. Potter [12] later established that with gas of high heating value (methane-like) the calorimeter could provide measurements with uncertainties of 0.25 percent or less. Their study was necessitated by the higher heating value of gases that had come into the market since the earlier study.

In the period of World War I (see [18]) the NBS became involved intensively in measures for conservation and rationing of natural gas. Toward the end of the war period the necessity of obtaining helium from natural gas created a need for measurement of physical and thermodynamic properties of methane as a cryogenic fluid in order to establish conditions for separation of methane from the helium. This led to an initiation of studies on the physical properties of methane [16] such as the vapor pressure measurements by Cragoe $[19,20 \mathrm{~b}]$.

\subsubsection{Properties of Pure Components and Mixtures}

With the discovery of deuterium in 1932 by Urey, Brickwedde, and Murphy [17], the theoretical interest in isotope effects led Brickwedde and Scott to undertake a study of the thermodynamic properties of deuterated methanes. This work, including the original vapor pressure work on $\mathrm{CH}_{4}$ by $\mathrm{Crag} \mathrm{Ce}$ unpublished for many years, was finally summarized by Armstrong and published [20]. The vaporpressure study of the deuteromethanes is unique in the number of closely related deuterium-substituted molecules for which precise data were reported, and showed a strong and very regular isotope substituent effect.

Three of the most important thermochemical studies made by F. D. Rossini, and among his earliest studies at NBS, were the definitive determinations of the enthalpies of combustion of hydrogen [13], methane [14], and carbon monoxide [14]. Not only are these substances important ingredients of commercial fuels, but the products of combustion, water and carbon dioxide, are universal products of combustion of hydrocarbon fuels. The enthalpies of formation of the products are essential in fuel energy calculations. 
Rossini's studies reduced the uncertainties in the combustion energies by a factor of three or more. These studies were followed by other studies covering the principal hydrocarbon constituents of gaseous fuels, the low molecular weight aliphatic hydrocarbons ethane, propane, and the butanes. The measured values are generally accepted as standard reference data for the ideal gas hydrocarbons.

In further support of the combustion calorimetry of natural gas as a pricing measurement, a standard reference material consisting of a natural gas of good quality was certified as to its heating value and 1ssued by NBS for some years. This reference material was used in the industry to verify the accuracy of calorimeters in the natural gas range of heating values. The responsibility for distribution of this reference material was transferred to the Institute of Gas Technology in 1961 , with the provision that NBS would supply a certification of a reference fuel as needed to provide traceability of the energy measurement to the national standards.

The last certification of such a reference material was prepared in 1965 [15]. The material certified was analyzed by mass spectrometric and gas chromatographic techniques by E. E. Hughes and J. K. Taylor. It was about 99.95 percent methane, with 0.04 percent ethane and much smaller amounts of non-combustible constituents. The heating value was calculated for unit volume of the real gas both dry and water-saturated under the standard pressure and temperature conditions in use by the gas industry. The calculation, which was done by G. T. Armstrong, was based upon Rossini's determination of the standard enthalpies of combustion of methane and ethane (adopted as standard reference data), the compressibility data of Douslin (Bureau of Mines) and the vapor pressure of water measured by $\mathrm{H}$. F. Stimson (NBS).

Calculation of the heating value of gas mixtures from composition has long been considered attractive as an alternative to calorimetry for many purposes requiring less than the highest accuracy. To meet the needs of the gas industry in particular, and to prepare users for coming metric practice, a recommended set of values of combustion energies of the ideal gases was prepared in 1972 [16]. This compilation gave heating values on a dry and on a water-saturated basis for ideal gases: $\mathrm{H}_{2}$, $\mathrm{H}_{2} \mathrm{~S}$, $\mathrm{CO}_{\text {, }}$ $\mathrm{CH}_{4}$, and 49 other selected gaseous hydrocarbons ( $C_{1}$ to $C_{6}$ ) in kilojoules per mole, megajoules per cubic meter, and British thermal units per cubic foot. Values were tabulated under two standard conditions: $288.71 \mathrm{~K}, 101591.3 \mathrm{~N} \cdot \mathrm{m}^{-3}\left(60^{\circ} \mathrm{F}, 30\right.$ inches $\left.\mathrm{Hg}.\right)$ used for many years by ASTM; and $288.71 \mathrm{~K}$, $101559.8 \mathrm{~N} \cdot \mathrm{m}^{-3}\left(60^{\circ} \mathrm{F}, 14.73 \mathrm{psia}\right)$ recently adopted by ANSI. An outline was given of a procedure to derive data for the real gases, and to adjust temperature and pressure bases.

In deriving these values, advantage was taken of a recent critical review and compilation by $E$. S. Domalski [21] of the enthalpies of combustion of organic substances including many hydrocarbons. This review was a partial updating of the well known compilation made at NBS by Kharasch [22], which for many years stood as the only extensive authoritative collection of enthalpies of combustion of organlc compounds.

Finally, as a direct result of the project to generate this measurement manual, a review and reassessment of the basic physical properties of natural gas was generated by Armstrong and Jobe [23]. The pure components and mixtures were primarily of interest to the LNG industry but have wlde applicability to the natural gas industry as a whole. This publication, although similar to the Armstrong [16] work includes discussions of atomic and molecular constants in addition to combustion enthalpies of pure components for hydrocarbons $C_{1}$ through $C_{6}$. A summary of the Armstrong and Jobe work [23] is included in this manual in section 1.2 and 1.3 .

\subsubsection{Calorific Value Instrumentation and Measurements}

The automatic recording calorimeter has, until recently, dominated the process of measurement of the natural gas calorific value. With the develpment of more accurate properties data and the microprocessor, a number of new instrumentation techniques have been developed to compete with the more traditional combustion calorimeter.

These devices can be separated into two general catagories. The first is the type referenced in the preceding section, the combustion calorimeter. Natural gas is mixed with air and burned, and the resulting temperature rise is measured and compared to that found using a calibration gas. The result of this process can be considered a measured calorific value in the sense that the magnitude of the value is related directly to the combustion process. 
The second category is based on more indirect methods such as gas analysis and calculation of the calorific value from pure component combustion enthalpies. Combustion of the fuel and air are not necessary. Other methods in this category would include the measurement of one or more state properties of the natural gas mixture (dielectric, acoustic, etc.) and correlating these properties with combustion enthalpies of the gas. These methods may have difficulty in assessing the contribution of the inert nitrogen impurity and may require an additional measurement of the amount of nitrogen present in the LNG mixture.

\subsubsection{Measured Methods-Automatic Recording Calorimeter}

A description of the operation of this device is taken from Armstrong [32]. "The Cutler-Hammer calorimeter operates on the following principle: Gas is burned at a constant rate, and the heat developed is absorbed by a stream of air. The rates of flow of the gas, the air for combustion, and the air for absorption of heat are regulated by metering devices similar to the ordinary wet-gas meter. These metering devices are geared together and driven by an electric motor so that the ratios of the three rates of flow are constant. The products of combustion are kept separate from the heat-absorbing air and are cooled very nearly to the initial temperature of the air. The water formed in the combustion is condensed to the liquid state. The rise in temperature of the heat-absorbing air is proportional to the total or gross heating value of the gas. The rise in temperature is measured by means of resistance thermometers and is recorded graphically. Gas calorimeters of the above type, with public reliance justified by NBS studies, give the basis for calculating heating values of fuels delivered to the homeowner and exchanged in trade between producers, transmission companies, and industrial or public utilities consumers."

The calorimeter requires a stable environment and, in many cases, a dedicated room or building is necessary. Stable temperatures are critical and are specified to be controlled in the range $22-24^{\circ} \mathrm{C}$ $\pm 0.56^{\circ} \mathrm{C}$.

Several detailed studies of the accuracy and precision have been made, particularly the work of Eiseman and Potter [12] in which the calorific value of a sample of propane was very carefully calculated and compared to the value found by the calorimeter. This publication provides detailed descriptions of the preparation of a standard gas of high calorific value, a comparison of hydrogen and methane as calibrating gases, and the effect upon the instrument of changes in room and tank water temperature. In addition, the accuracy of the measurement instrument when burning propane is given as to within 0.25 percent (which is approximately $0.24 \mathrm{MJ} / \mathrm{m}^{3}$ ), and the effects of bleeder burner height differences and instrument performance differences caused by changes in inlet gas pressures are presented. However, this information was developed nearly 30 years ago, and changes in the equipment and process may not reflect current findings.

Accuracy and precision of this device operating with LNG-type mixtures are difficult to assess. The manufacturer states an accuracy of \pm 0.5 percent of full scale $\left(44.71 \mathrm{MJ} / \mathrm{m}^{3}\right)$ or $\pm 0.22 \mathrm{MJ} / \mathrm{m}^{3}$. Warner [30] provides support for this value at the 99.7 percent confidence level. Gilbert, Ludwig and Warner [31] provide additional installation and maintenance information.

The recording combustion calorimeter is a volumetric device and gives calorific values in terms of cubic meters. A sample gas density is required to give this information on a mass basis for use in the total LNG measurement process. In many recording calorimeter installations, a density value is found by comparing the gas sample density to that of air at standard conditions. Blanchard [28] describes a simple beam balance method, and Lewis [29] describes a dynamic system which the manufacturer claims to have an error of \pm 0.5 percent. It is assumed that this is a maximum error and includes the uncertainty in applying the density of standard air.

An estimate of total error of the calorific value of the recording combustion calorimeter and the gas sample density measurements can be found by combining the individual estimates of \pm 0.5 percent (calorimeter) and \pm 0.5 percent (density) in quadrature. The total estimate is then \pm 0.71 percent.

The unexpectedly large uncertainty in measured calorific value is probably a result of limited published research quality data. In Europe, where combustion calorimetry is, in many cases, the official accounting method, supporting data is believed to be adequate to show a lower error value. Actual performance data beyond that cited in the open literature is not available at this time. 


\section{3 .2 .2 other Combustion-Type Calorimeters}

Although the recording combustion calorimeter is unquestionably the most widely recognized calorific value instrumentation, the device has at least two serious limitations.

1) The device requires a closely controlled environment in which to operate. The controlled ambient temperature requirement of $\pm 0.55^{\circ} \mathrm{C}$ in the range of $22-24^{\circ} \mathrm{C}$ means as a practical matter that a separate building with $28 \mathrm{~m}^{3}$ or more of space complete with heating and air conditioning must be considered part of the installation [26]. Further, separate additional instrumentation is required to determine the gas sample density, a necessity for determining calorific value for LNG.

2) The response time of the calorimeter is quite slow. This time varies but has been reported as 20 to 45 minutes to show a $7.5 \mathrm{MJ} / \mathrm{m}^{3}$ change in gas sample calorific value [25].

For these and other reasons, such as high maintenance cost [26], alternate methods are under study. In addition to the obvious miniaturization possibilities, stoichiometric combustion calorimetry has been suggested as a possible alternative $[25,28]$.

The operation of the stoichiometric combustion calorimeter, sometimes referred to as a thermal titrator, takes advantage of the fact that the ratio of air to fuel that results in the maximum flame temperature is directly related to the calorific value of the fuel. Combustion air flow through the instrument is generally fixed while the fuel flow is controlled to give the maximum measured flame temperature.

Shimps [27] reports results of some applications and Light [25] presents results of a performance comparison of the titrator with that of the recording combustion colorimeter. The reported response time has been shortened to 1 minute to show a $7.5 \mathrm{MJ} / \mathrm{m}^{3}$ change in gas sample calorific value. The device required no special ambient temperature control, small floor space (0.6 by 1.2 meters) and was portable. A separate gas density measurement was required.

The manufacturer claims an accuracy after calibration in which the standard deviation between measured and stated values of a standard calibration gas is \pm 0.5 percent. If the calibration gas is methane with a calorific value of $37.7804 \mathrm{MJ} / \mathrm{m}^{3}(101.560 \mathrm{kPa}$ and $288.71 \mathrm{~K})$, the error estimate is $\pm 0.57 \mathrm{MJ} / \mathrm{m}^{3}$, assuming three standard deviations.

\subsubsection{Calculated Methods-Gas Analysis}

Gas chromatography has developed as a field type measurement process for the determination of calorific value on both a volume and mass basis. Mass spectrometry is a traditional analytical instrument which provides a similar result but requires a more rigidly controlled environment and higher skill of the operator. Both methods are discussed in detail in section 2.2 .4 of th1s manual, with the gas chromatograph showing greater accuracy and precision for the particular application to LNG.

Although it was shown in section 2.2 that it was possible to routinely compute the caloriflc value from chromatographically analyzed composition to less than \pm 0.1 percent, this was performed 1 n a controlled laboratory environment. It is further shown as a result of field site sampling tests that the total uncertainty of a single measurement in sampling and analyzing LNG mixtures can be less than \pm 0.3 percent in the computed calorific value. Section 2.2 should be consulted for details. This estimate of error does not include the uncertainty in the values of combustion enthalpy of the individual components which are contained in 1.2 and 1.3 . The combination of these and other error summaries are presented in the examples of 3.0 .

\subsubsection{Measured and Calculated Methods Compared}

A study of the potential uncertainty of the calculated and measured calorific values is provided by Melrose [24]. Although this publication is somewhat dated, it does provide a falrly contemporary assessment of the uncertainty of the measurement process. 
In the Melrose publication, analytical data for 879 samples of natural gas were studied to correlate the difference in calorific value found with a recording combustion calorimeter and that calculated from a composition analysis using a gas chromatograph. In addition to the data for calorific value, Melrose presented data on specific gravity, range of calorific value, concentrations of various inert gases and classification of gaseous mixtures.

The differences in measured and calculated specific gravities were found to be similar in magnitude and algebraic sign to the corresponding differences in calorific values. The similarities may be a result of the resolution characteristics of both the higher hydrocarbons and the water that are present in the chromatographic back-flush which was part of the gas analysis instrumentation.

The differences of measured and calculated calorific values were found in the Melrose study to be independent of nitrogen concentrations from 0 percent to 7 percent but may be dependent upon concentrations of carbon dioxide that are greater than the 1.5 percent limit of most of the samples in the study.

The greatest differences in calorimeter and calculated calorific values were for samples of production gases and for samples with either a calorific value greater than $39 \mathrm{MJ} / \mathrm{m}^{3}$ or specific gravities greater than 0.63 , or both.

In the Melrose study, the average absolute difference in calorimeter calorific value and calculated calorific value was $74.5 \mathrm{~kJ} / \mathrm{m}^{3}$, and the average algebraic difference was $111.7 \mathrm{~kJ} / \mathrm{m}^{3}$. The standard deviation of all calorific data over the range investigated was \pm 0.28 percent. A conservative statistical confidence level of three standard deviations would give a maximum estimated error of $\pm 0.32 \mathrm{MJ} / \mathrm{m}^{3}$ ( 99.7 percent confidence level), which is somewhat greater than that found in the Eiseman and Potter [12] study or that claimed by the calorimeter manufacturer. However, this value applies to the difference in the two measurement techniques, and a portion of the error must be allocated to the gas analysis instrumentation.

Broadwater [26] provides recent data comparing the performance of the recording combustion calorimeter and the gas chromatograph in measurement of natural gas heating values. The data was the result of studies made by a major natural gas transmission company to select instrumentation and techniques for the measurement of natural gas on an energy basis. The instrumentation considered under the study consisted of the recording combustion calorimeter, two gas chromatography analyzers and a "gravitometer" (gas density relative to standard air density). The two chromatographs differed as to the number of natural gas constituents which could be analyzed. The simpler device analyzed for methane, ethane, propane, iso and normal butane, iso, normal and neo pentane, hexanes + , nitrogen and carbon dioxide. The second chromatograh also resolved a number of higher molecular mass constituents. The data from the simpler chromatograph is adequate for the purposes of this manual.

A much smaller number of samples were reported in the Broadwater work as compared to the Melrose study. However, enough data were presented to provide a fairly good statistical basis for analysis.

On the basis of 22 samples with calorific values ranging from $36 \mathrm{MJ} / \mathrm{m}^{3} \mathrm{to} 48 \mathrm{MJ} / \mathrm{m}^{3}$, the average difference in calorific value between the recording combustion calorimeter and the simpler of the two gas chromatographs was found to be -0.002 percent with a standard deviation of \pm 0.18 percent. On a basis of three standard deviations, this amounts to $\pm 0.21 \mathrm{MJ} / \mathrm{m}^{3}$.

On the basis of 22 samples with specific gravities ranging from 0.5750 to 0.6930 , the average difference in specific gravity between a gravitometer and the simpler of the two gas chromatographs was 0.12 percent with a standard deviation of 0.14 percent [26]. Although the two measurement differences are not independent observations, it is possible to estimate the combined error in calorific value on a mass basis by quadrature. This procedure gives a value of \pm 0.68 percent on the basis of three standard deviations. The value is somewhat less than the value found in section 2.3.2.1 above but is comparable in the absence of additional critical evaluations. 


\subsubsection{References}

[1] Weaver, E.R., History of the gas chemistry section of the National Bureau of Standards, 1910-1957, manuscript, NBS Archives, Oct. 1964.

[2] Rosa, E.B., Use of gas for heat, and power testing of gas, J. Franklin Institute 6 , $157-86$ (1912).

[3] Stillman, M.H., A portable cubic foot standard for gas, Nat. Bur. Stand. (U.S.), Tech. Paper 114 (1919).

[4] Anon. (McBride, R.S., Edwards, J.D., Weaver, E.R., et al., see [11]), Standard methods of gas testing, Nat. Bur. Stand. (U.S.), Circ. 48 (1916).

[5] Waidner, C.W. and Mueller, E.F., Industrial gas calorimetry, Nat. Bur. Stand. (U.S.), Tech. Paper 36 (Mar. 1914).

[6] Crittenden, E.C., An experimental study of flame standards, Trans. Illumin. Eng. Soc. 6, 413-37 (1911).

[7] Edwards, J.D., Determination of ammonia in illuminating gas, Nat. Bur. Stand (U.S.), Tech. Paper 34 (1919).

[8] McBride, R.S. and Weaver, E.R., Determination of sulphur in illuminating gas, Nat. Bur. Stand. (U.S.), Tech. Paper $20(1913)$.

[9] Edwards, J.D., The determination of gas density, J. Ind. Eng. Chem. 9, 790-2 (1914).

[10] Anon. Gas calorimeter tables, Nat. Bur. Stand. (U.S.)., Circ. 65 (July 1917); Anon., Nat. Bur. Stand. (U.S.), Circ. C1 47 (Mar. 1938); Jessup, R.S. and Weaver, E.R., Nat. Bur. Stand. (U.S.), Circ. 464 (March 1948).

[11] Jessup, R.S., The Thomas recording calorimeter, J. Res. Nat. Bur. Stand. (U.S.) 10, 99-122 (1933) RP 519 .

[12] Eiseman, J.H. and Potter, E.A., Accuracy of the Cutler-Hammer recording gas calorimeter when used with gases of high heating value, J. Res. Nat. Bur. Stand. (U.S.) 58, 213-226 (1957) RP 1605 .

[13] Rossini, F.D., The heat of formation of water, J. Res. Nat. Bur. Stand. (U.S.) 6, 1-36 (1931) RP 259 .

[14] Rossini, F.D., The heats of combustion of methane and carbon monoxide, J. Res. Nat. Bur. Stand (U.S.) $6,37-50$ (1931) RP 260 .

[15] Armstrong, G.T., Calculation of the heating value of a sample of high purity methane for use as a reference material, Nat. Bur. Stand. (U.S.), Tech. Note 299, 19 pp. (Dec. 1966).

[16] Armstrong, G.T., Domalski, E.S., and Minor, J.I., Jr., Standard combustion data for the fuel gas industry, American Gas Association, Operating Section Proceedings, Atlanta, GA, 1972, pp. D-74-87. (American Gas Association, Arlington, VA (1972)).

[17] Urey, H. C., F. G. Brickwedde and G. M. Murphy, A hydrogen isotope of mass 2, Phys. Rev. 39 $164-65(1932) ; 40$ 1-15 (1932).

[18] Anon., War Work of the Bureau of Standards, Nat. Bur. Stand (U.S.), Misc. Pub1. 46, (1921). (a) pp. 23-24, aviation gasoline; (b) pp. 179-183, natural gas; (c) pp. 291-293, toluene recovery.

[19] Cragoe, C.S., unpublished work, See, for instances: International Crit1cal Tables (E.h. Washburn, ed.), Vol. V (1929). Vapor pressure tables.

[20] Armstrong, G.T., Brickwedde, F.G., and Scott, R.B.: (a) The vapor pressures of the deuteromethanes, J. Chem. Phys. 21, 1297-8 (1953); (b) Vapor pressures of the methanes, 3. Rej. Nat. Bur. Stand. (U.S.), 55, 39-5 (1955) RP 2603. 
[21] Domalski, E.S., Selected values of heats of combustion and heats of formation of organic compounds containing the elements C, H, N, O, P, and S., J. Phys. Chem. Ref. Data 1, 221-277 (1972).

[22] Kharasch, M.S., Heats of combustion of organic compounds, J. Res. Nat. Bur. Stand. (U.S.), 2, 359 (1929) RP 41.

[23] Armstrong, G. T. and T. L. Jobe, Jr., Heating values of natural gas and its components, Nat. Bur. Stand. (U.S.), NBSIR 82-2401, 168 pp (May, 1982).

[24] Melrose, D. C., Comparison of calculated and measured heating value of natural gas, Paper 72-D-2, Am. Gas Assoc. 1972 Operating Section Proceedings, American Gas Association, 1515 Wilson Blvd., Arlington, VA 22209 (1972).

[25] Light J., How does the therm-titrator compare?, Paper 80-t-47, Am. Gas Assoc. 1980 Operating Section Proceedings, American Gas Association, 1515 Wilson Blvd., Arlington, VA 22209, pT191-93 (1982).

[26] Broadwater, S. R., One company's approach to energy measurement, Paper 80-T-78, Am. Gas Assoc. 1980 Operating Section Proceedings, American Gas Association, 1515 Wilson Blvd., Arlington, VA 22209 pT369-88 (1982).

[27] Shimps, A. J., Applications of the stoichiometric combustion calorimeter, Paper 84-DT-26, Am. Gas Assoc. 1984 Operating Section Proceedings, American Gas Association, 1515 Wilson Blvd., Arlington, VA 22209, pD16-25 (1984).

[28] Blanchard, E. F., Specific gravity instruments- installation \& operation, Proc. 55th International School of Hydrocarbon Meas., Univ. Oklahoma Center for Cont. Ed., Norman, OK, 198-204 (April, 1980).

[29] Lewis, H. E., Kinetic type indicating \& recording instruments for determing specific gravity, Proc. 55 th International School of Hydrocarbon Meas., Univ. Oklahoma Center for Cont. Ed., Norman, OK, 205-210 (April, 1980).

[30] Warner, C. W., Cutler-Hammer calorimeter accuracy and maintenance requirements, American Gas Association Distribution Conference, Atlanta, GA, May $8-10,1972$.

[31] Gilbert, T. M., J. B. Ludwig and C. W. Warner, Installation and maintenance of Cutler-Hammer, Inc. recording calorimetrer for accuracy and reliability, Proceed. Thirteenth An. Appalachian Gas Measurement Short Course, West Virginia University, Morgantown, WV.

[32] Armstrong, G. T., Hydrocarbons for fuel- 75 year's of research at NBS, Nat. Bur. Stand. (U.S.), Special Publ. 434, 20 pp (May 1976) 


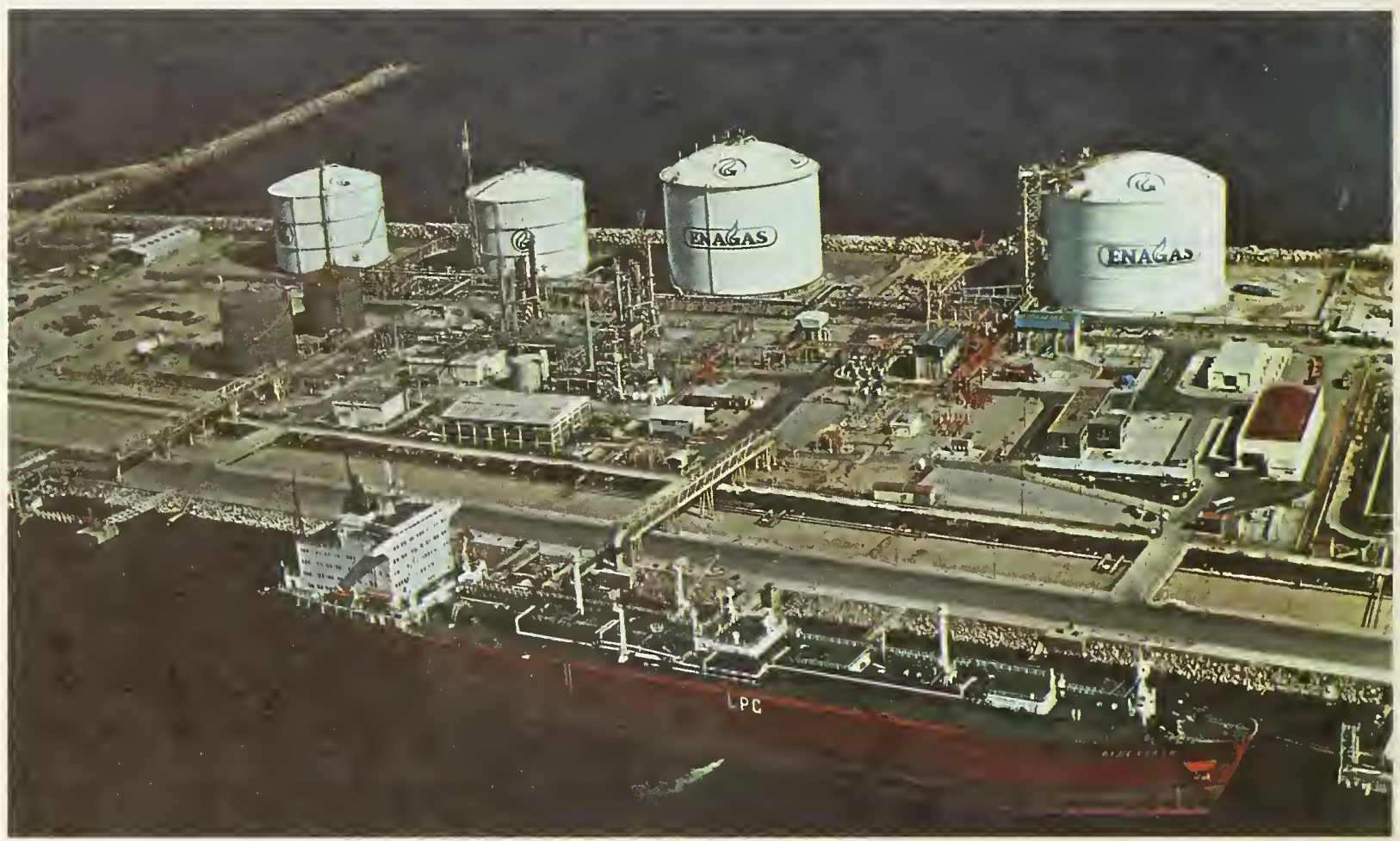

\section{BARCELONA TERMINAL}

The Barcelona Terminal, located in southern Spain on the Mediterranean and run by ENAGAS, was constructed to cope with industrial and household gas demands of the surrounding area. Facilities shown in the photograph include two $40,000 \mathrm{~kL}$ pre-stressed concrete storage tanks and two $80,000 \mathrm{~kL}$ tanks. $0 \mathrm{pen}$ rack type vaporizers are shown in the photograph located between the two larger storage tanks and the carrier berth. LNG was first received from Libya $(830,000$ tons annually) in 1971 , and in 1974 the terminal began receiving LNG from Algeria at an annual rate of 380,000 tons.

Through fractionation of the LNG, the terminal supplies methane and ethane as town gas, and also sells propane and butane separately. Fractions heavier than butane are used inside the terminal as fuel for boilers. A portion of the LNG is also used by satellite terminals and is carried there by tanker truck. (Photograph by courtesy of The Japan Gas Association, Tokyo, Japan, on behalf of the 1981 Japan LNG Congress) 


\section{CONTENTS}

\section{0 MEASUREMENT ELEMENTS}

Dens I t y

2.4

LNG Density

2.4 .1

$2.4 \cdot 1 \cdot 1$

$2.4 \cdot 1 \cdot 2$

$2.4 \cdot 1.3$

$2.4 \cdot 1.4$

2.4 .1 .5

2.4 .1 .6

2.4 .1 .7

$2 \cdot 4 \cdot 1.8$

2.4 .2

2.4 .2 .1

2.4 .2 .2

2.4 .2 .3

$2.4 \cdot 2.4$

$2.4 \cdot 2.5$

2.4 .2 .6

2.4 .2 .7

2.4 .2 .8

2.4 .2 .9

2.4 .2 .10

2.4 .3

$2 \cdot 4 \cdot 3 \cdot 1$

2.4 .3 .2

2.4 .3 .3

$2.4 \cdot 3 \cdot 4$

2.4 .3 .5

2.4 .3 .6

2.4 .3 .7

2.4 .3 .8

2.4 .3 .9

2.4 .3 .10

2.4 .4

2.4 .4 .1

2.4 .4 .2

2.4 .4 .3

2.4 .4 .4

2.4 .4 .5

2.4 .4 .6

2.4 .4 .7

$2 \cdot 4 \cdot 4 \cdot 8$

2.4 .5

2.4 .5 .1

2.4 .5 .2

2.4 .5 .3

2.4 .5 .4

2.4 .5 .5

$2 \cdot 4 \cdot 5 \cdot 6$

Summa r y

References

Conclusions

References

References

References

Int roduct ion

Conclusions

References
LNG Density Reference System

Introduction.

Cryogenic Fluids Density Reference System

Description of Improved Density Reference System Densimeter and Instrumentation

Measurement Process Parameters

Measurement Process Control

Measured LNG Density

In troduction

LNG Densimetry

Vibrating Element Densimeter

Dielectric Cell Densimeter

Displacement or Archimedes Densimeter

Testing Commercial Densimeters

Test Results

$\mathrm{Ca} l$ ibrat ion

Calculated LNG Density

General Comments

In troduction

Major Accomplishments- Experimental Apparatus

Experimental Measurements-Pure Fluid Data

Binary Mixture Data

Multicomponent Mixture Data

Mathematical Models

LNG Density Research at Other Laboratories

Acknowl edgmen $\mathrm{s}$

Mathematical Models for the Prediction of LNG Densities In troduct ion

Extended Corresponding $S$ tates Method

Hard Sphere Method

A Revised Klosek and McKinley Method

The Cell Model.

Use of the Methods

Conclusions

Inter laboratory Comparison of LNG Density Measurements

The Density Calibration Systems

Intercomparison of Calibration Systems

Experimental Results.
Page

$2.4 \cdot 1^{-1}$

$2 \cdot 4 \cdot 1-1$

$2.4 .1-1$

2. 4. 1- 1

$2.4 .1-2$

$2 \cdot 4 \cdot 1-6$

2. $4.1-8$

$2 \cdot 4 \cdot 1-14$

2. 4. 1-14

$2.4 .1-15$

2. 4. 2-1

$2 \cdot 4 \cdot 2-1$

$2 \cdot 4 \cdot 2-1$

2. $4.2-1$

2. $4.2-2$

2. $4.2-3$

$2.4 .2-4$

2. $4.2-4$

$2.4 .2-8$

2. 4. 2-11

2. 4. 2-12

2. 4. 3-1

2. 4 . 3-1

2. 4. 3-1

2. 4 . $3-2$

2. $4.3-3$

2 . $4.3-3$

2. 4. 3-4

2. 4. 3-4

2. 4. 3-5

2. 4. 3-6

2. 4. 3-7

2. 4. 4-1

2. 4. 4-1

2. 4. 4-1

$2.4 .4-4$

2. $4.4-5$

2.4. 4-7

$2 \cdot 4 \cdot 4-8$

$2 \cdot 4 \cdot 4-8$

2.4.4-10

2. 4. 5-1

2. 4. 5-1

2.4. 5-2

2. 4. 5-5

$2.4 .5-5$

2. $4.5-8$

$2.4 .5-9$ 


\section{4 LNG Density}

\subsubsection{LNG Density Reference System}

\subsubsection{Introduction}

Full shiploads or entire shore storage tanks cannot be weighed directly to the accuracy and precision consistent with the requirements of the measurement process. The tare weight portion or the tank welght itself may be many times greater than the active weight of the LNG. The uncertainty in the tare welght will dominate the uncertainty in the weighing process. In addition, routine weighing of these containers would certainly be difficult to justify on an economic basis and in some clrcumstances might be impossible and therefore could jeopardize the entire measurement process. Alternatives to gross weighing involve representative measurements on a much smaller scale and extrapolation of these representative measurements to the total contents of the LNG container.

A measure of LNG density or the mass concentration per unit volume, if established as representative of the entire contents, can be combined with a volume measurement to give a total weight or mass of the contents of the LNG container. This measure of LNG density is commonly performed either directly with a LNG densimeter, or by calculation, by sampling, analysis and the use of mathematical correlations. The latter methods are discussed in sections $2.2,2.4 .3$ and 2.4 .4 . The methods involving direct density measurement using densimeters will be presented in the following sections of this part of the measurement manual.

LNG density may vary throughout the contents of a large LNG container, and it may be necessary to place more than one density measurement element within the container volume to assure that the measurement is representative of the total contents. Several commercial densimeters are available for use with LNG and are discussed in section 2.4.2. No matter which device is selected, it is necessary at the present time to calibrate these commercial devices in a reference system specifically designed for use at cryogenic temperatures.

As a part of the overall LNG program at NBS, a LNG Density Reference System (DRS) was constructed and evaluated [1]. Improvements were made in the DRS as it was used to calibrate individual commercial densimeters. A description of the improved DRS, the major design characteristics and the estimated accuracy and precision are given by Siegwarth and LaBrecque [7]. The general subject is so important to the understanding of the total LNG measurement process that an edited version of the reference [7] is presented here in the following sections.

\subsubsection{Cryogenic Fluids Density Reference System [7]}

In an earlier report [1], a density reference system (DRS) was described which had been used to test some commercially available densimeters in liquefied natural gas (LNG). These tests involved comparing the density measurement by the densimeter under test to the density measured by the DRS densimeter in a well-characterized liquid sample. This earlier report gives an estimate of the uncertainty associated with a density measurement by the DRS densimeter used as standard for comparison. The uncertainty of a single determination of the density at the instrument under test was taken to be \pm 0.076 percent. This includes a systematic error of 0.028 percent and a random error of \pm 0.048 percent, which is three times the estimated standard deviation of \pm 0.016 percent. Four different commercial densimeters were tested and the results reported [2]. The major problem with the commercial densimeters found from these tests and some later measurements was in the callbration of the commercial instrument by the manufacturer. None of the factory calibrations were entirely suitable at LNG temperatures at the time of these tests.

Because of this calibration problem, NBS offered a calibration service through "transfer standards." NBS would calibrate a manufacturer's densimeter, which was then installed in a calibration system at the manufacturer's plant and used to calibrate production densimeters. The densimeter manufacturer would then periodically return the transfer standards to NBS for recalibration. The process would eventually yield data on the stability as a function of time of the instruments used as transfer standards. The manufacturer's calibration system and methods could be evaluated by testing in the DRS some of the instruments calibrated by the manufacturer.

The DRS sample container used in the tests of these first four commercial denslmeters was designed around the densimeters tested. The densimeters in some cases were specially bullt. The first verslon of the DRS was too inflexible to deal with any design modifications of the commerclal denstmetors. 
A more flexible sample container dewar system was constructed for use in densimeter evaluation and calibration work. This DRS design with a companion liquid sample storage dewar should be suitable for a commercial calibration facility. This new sample container was found to be more temperature stable than that of the original DRS. The DRS densimeter has been modified several times before, during and since the rebuild. Details of these modifications and a shift in the measured density after the rebuild are discussed in 2.4 .1 .4 , along with the other measuring devices.

In 2.4.1.5, the measurement process is discussed. The associated systematic errors are identified, their magnitudes estimated and the overall error estimate of a density measurement is given. This estimated deviation of the density for one measurement from the true value is \pm 0.055 percent for pure methane at the normal boiling point. The maintenance of this accuracy is discussed in 2.4 .1 .6 .

Since the improved version of the DRS was completed, a number of additional commercial densimeters have been tested. Each test consisted of a series of measurements in pure liquid methane then approxamately about 5 percent propane and 1 percent to 2 percent nitrogen are added to simulate an LNG mixture and another series of measurements was made. This mixture was used, because both propane and nitrogen can be added without any danger of solids forming in the fill line, since both have freezing points below $110 \mathrm{~K}$. Measurements in an LNG-like fluid permit LNG densimeters to be tested in a fluid around the temperature and density at which they will be used. The tests in liquid methane provide a fluid with a known temperature density relationship, provided 99.97 percent pure methane is used, to which the DRS density value can be compared. Also, the methane density data along with the LNG-like liquid density data provide a much wider range of densities for determining the calibration equation for the instruments under test.

\subsubsection{Description of the Improved Density Reference System}

The improved DRS operates on the same principle as the original. Densimeters under test are compared to the density reference system densimeter in a very nearly isothermal liquid sample. The DRS densimeter is an Archimedes-type densimeter consisting of a single crystal silicon float weighed while immersed in the sample liquid by an electronic balance. Density measurements are made over an approximately $15^{\circ} \mathrm{C}$ temperature range along the saturation curve of the sample $\mathrm{liquid}$. The measurements are made with the sample at equilibrium after a change of sample temperature has been affected either by a liquid nitrogen cooling coil or an electrical resistance heater. The sample equilibrium is obtained by stirring the liquid with a turbine pump. Equilibrium conditions remain for the period of time required for measurement without stirring, because the heat leak to the sample has been reduced to a small value by means of radiation shields and other heat flux interrupting devices.

Sample Container A schematic of this improved DRS is shown in Figure 2.4.1.1. The sample container consists of a 25.4 centimeters OD, 1.6 millimeters wall stainless steel tube extending 760 millimeters down from an ambient temperature stainless steel flange to a 3 millimeters thick dished bottom head. A similar stainless steel vessel or vacuum jacket 35.6 centimeters OD forms an outer wall enclosing the sample container.

The space between these two vessels contains radiation shields and is maintained at a pressure below $10^{-4} \mathrm{~Pa}$ by an oil diffusion type vacuum pump. All the stainless steel to stainless steel joints are heliarc welded. All flanges and other gasketed joints in both the insulating vacuum jacket and sample container employ "O" ring seals. Unlike the sample container of the original system, which required a low temperature indium wire vacuum seal to close it, all demountable seals on the new DRS are located at ambient temperature. A 73 millimeters high aluminum ring, clamped between the sample vessel and vacuum jacket flanges, provides six 38 millimeters ID flange-covered access ports into the vacuum space. Through these flanges passes the liquid nitrogen line to the various cooling coils, the gas vent lines from these coils and the electrical leads for heaters and thermocouples. The connection to the vacuum pump is also through one of these ports. All lines and leads can be readily disconnected to permit the counterbalanced dewar assembly to be lowered enough so that the top flange is below the bottom of the sample space. The time required to disconnect the few lines, remove the nuts from the twelve 13 millimeters studs clamping the sample container to the top flange and lower the dewar is only a few minutes. A valve between the vacuum jacket and the vacuum line disconnect permits the dewar to be lowered without breaking the insulating vacuum.

Another 73 millimeters high aluminum ring is located between the top of the sample container flange and the top cover of the sample container. Again, six flange-covered 3.8 millimeters diameter access ports provide locations to introduce electrical leads, the vapor pressure bulb tubes shown in figure 2.4.1.1 and for pressure gauge connections. The sample vessel seals are "0" rings. The sample pressure is maintained above atmospheric pressure in case small leaks are present. 


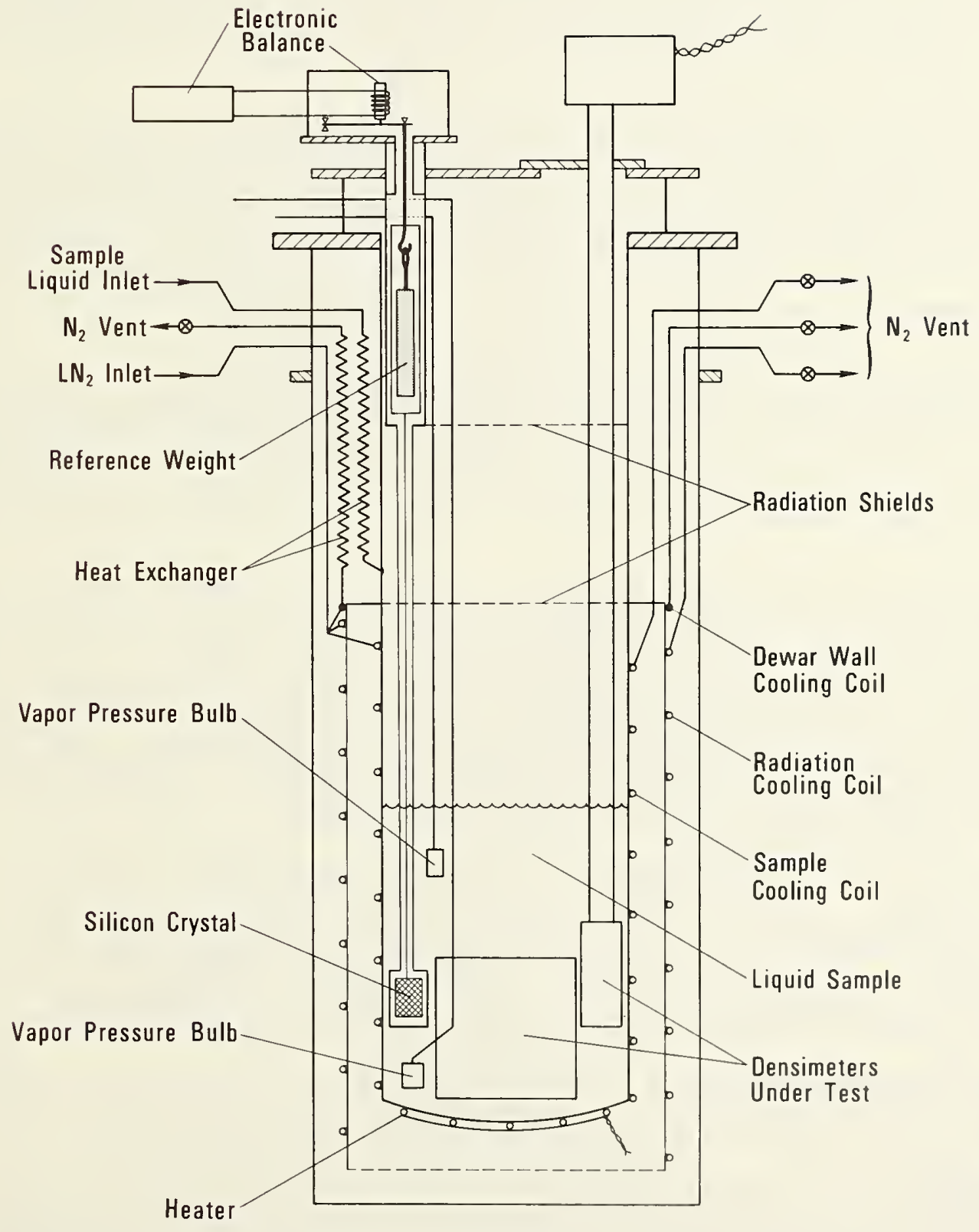

Figure 2.4.1.1. Schematic diagram of the improved Density Reference System. 
The top plate of the sample container has a port for the DRS densimeter, a 153 millimeters access port shown in figure 2.4.1.1 and a port for the stirrer drive shaft (not shown). The access port flange cover can be modified as required to provide mountings for various insertion-type densimeters. Figure 2.4.1.1 illustrates the mount for a vibrating cylinder densimeter. An "O" ring sealed gland on the access flange provides a gas tight stem seal. To avoid a seal on a rotating shaft, the stirrer drive shaft is coupled magnetically from the exterior to the interior of the sample chamber.

The evacuated annular space between the sample container and the vacuum jacket contains all the refrigeration systems used to condense and maintain the sample at cryogenic temperature. All the refrigeration is supplied from an adjacent liquid nitrogen storage dewar through a vacuum-insulated transfer line into the liquid nitrogen inlet shown in figure 2.4.1.1. This inlet is connected to all the various cooling coils, and the amount of cooling by each coil is regulated by throttling the independent nitrogen gas vent lines from the various coils.

The coil on the radiation shield, figure 2.4.1.1, is vented continuously, and the temperature of this polished copper shield is maintained below the sample temperature. The dewar wall cooling coil attached to the shield mounting ring a few centimeters above the sample level is maintained at a temperature a few degrees above the sample temperature to minimize the heat conducted down the sample vessel wall. Within the sample vessel, a copper radiation shield, with an aluminum foil shield on top, interrupts the heat radiated down from the top of the sample holder. A second radiation shield is placed a few centimeters above the lower shield. The operating temperature of the lower shield is normally a few degrees above the liquid temperature. The cooling coil and heater on the sample vessel, figure 2.4.1.1, are used only to change the sample temperature.

The sample is condensed into the DRS from gas bottles using liquid nitrogen via the counterflow heat exchanger. This parallel tube heat exchanger, composed of two 8 millimeters I.D. copper tubes about 7 meters long soft soldered together along their length, will condense the 16 iters required in the sample holder in about an hour. The actual time depends on the liquid nitrogen supply rate. Most joints in these coils and lines are soft soldered, and all coils and heaters are soft soldered to the surfaces to which they transfer heat.

Each time the density of an LNG or methane sample is measured, the temperatures of the sample near the top and bottom are also determined by measuring the methane vapor pressure in the vapor pressure bulbs shown in figure 2.4.1.1. The vapor lines are connected through a selector valve to a quartz spiral Bourdon tube pressure gauge. The temperature difference is nearly always less than $20 \mathrm{mK}$ and usually less than $10 \mathrm{mK}$. This low temperature gradient is obtained without the low speed stirring used in the original system [1]. The temperature drift during the measuring period is comparable in magnitude to the temperature difference cited above. Temperatures of the radiation shields and the annular shield support ring are monitored by thermocouples to provide information for minimizing the heat leak to the sample. The temperature and pressure of the gas surrounding the reference weight are recorded and used to provide the gas buoyancy correction to this weight reading.

A larger system of similar design should be suitable for a manufacturer's calibration facility. To speed up the operation, a manufacturer could employ additional cryogenic vessels into which he could transfer the calibration liquids. Heated nitrogen gas or additional electrical heaters could be used to rapidly warm the sample holder and to change densimeters; and a liquid nitrogen spray nozzle could be used to rapidly cool the calibration dewar. Or the instruments calibrated could be introduced and removed from the top, without warming the whole dewar. Either way, some modest care is required to ascertain that the dewar is properly purged and inerted before introducing a cryogenic sample.

Safety Considerations in the DRS Design Manufacturers of densimeters suitable for cryogenic use have demonstrated some reluctance to employ calibration methods using cryogenic liquid combustible gases. In this section, safety considerations incorporated by NBS in the DRS are presented.

An experimental system using combustible gases can be made safe by eliminating any opportunity for a combustible mixture of air and the gas to form, or by eliminating all ignition sources in any area where combustible mixtures might form. The elimination of combustible mixtures is the preferred way to make a system safe. Thus, the DRS sample handling system is gas tight and evacuated before introducing the combustible gas. However, should a leak develop in the system or an accident occur, a combustible mixture could result; so ignition sources have been minimized in the laboratory. Since it is not possible to be assured that all existing or potential ignition sources are completely 
eliminated, the possibility of a combustible mixture forming is minimized by a laboratory exhaust fan. This fan has the capacity to change the room air about every 12 minutes.

The schematic of the combustible gas handling system for the DRS shown in figure 2.4 .1 .2 illustrates the essential safety features. All lines that may or do involve gas exhausting from the sample container are connected to a stack that vents above the roof of the laboratory building. The sample container, approved for operating pressures to 10 bar, has a 10 millimeters ID relief line connected to the vent with a relief valve set just above the highest operating pressure. In the very unlikely event that the sample vessel ruptures and dumps the contents of the sample into the vacuum jacket, a 25 milimeters diameter relief line ducts the resulting vapor through a low pressure relief valve to the vent. The vacuum pumps maintaining the insulating vacuum also exhaust to the vent line. The vessel vacuum pump exhaust is connected to the vent, because the system fill procedure requires the vessel to be evacuated, filled with the sample gas, then evacuated and filled twice more before condensing the sample. When the tests are completed, the liquid is blown to the vent through valve 5 via the evaporating coils. The gas remaining is pumped out, and the sample container is back filled with nitrogen gas during warm-up. This pump also serves to purge the gas sample supply lines when changing or starting gas supplies. Valve 6 provides a route to release sample gas into the vent. Check valves could be placed in both the valve 5 and 6 lines to preclude air entering the sample container if these valves should be accidentally opened with lower than atmospheric pressure in the sample container. The solenoid valve 12 prevents complete loss of vacuum in the event of a power fallure.

The liquid sample is often left in the sample container overnight unattended. Liquid nitrogen is passed through the sample and radiation cooling coils at a rate sufficient to remove the heat leak to the sample. The vapor pressure is held at about 1.5 bar absolute by a feedback system that senses this pressure and supplies sufficient additional heat to the sample heater, figure 2.4.1.1, to maintain the set pressure.

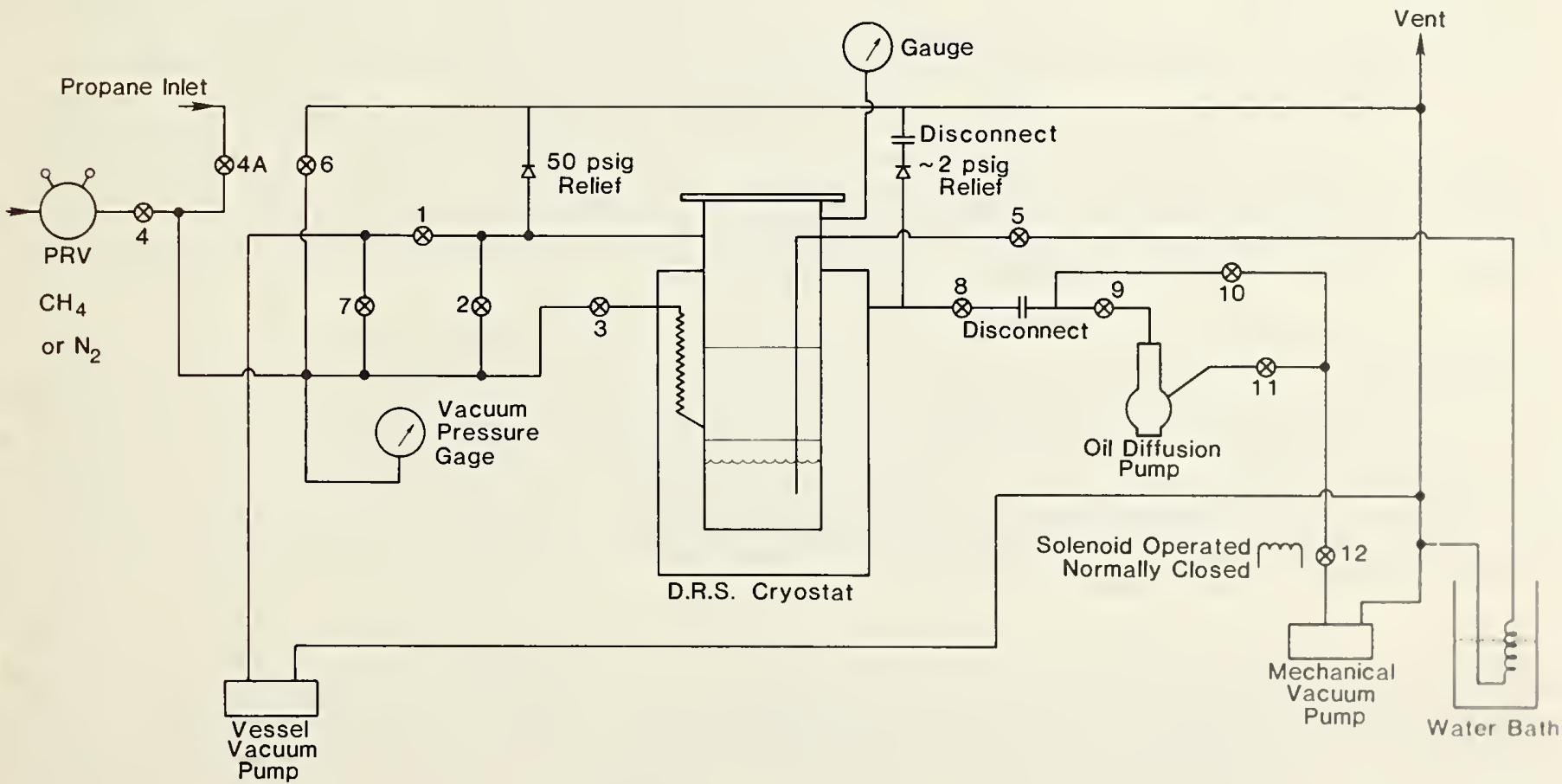

Figure 2.4.1.2. DRS combustible gas handing system. 
Small quantities of gas escaping from the DRS would be swept out of the room by the laboratory celling vent fan. To reduce the chances of igniting any larger spill, mainly "explosion proof" electrical equipment operates in the room during tests. The stirrer motor case is purged with compressed air. All the electrical cabinets are enclosed and purged with compressed air. Access to the laboratory is limited, and since the floor is conducting, conducting shoes or ground straps are used by anyone regularly in the laboratory. A combustion gas detector connected to a warning horn is located near the DRS.

The vapor pressure bulbs contain less than a liter of methane at ambient temperature and pressure, but this can result in a very high vapor bulb pressure if not properly vented to the storage cylinder during warmup. Two bypass relief valves between the vapor bulbs and the storage cylinder eliminate this potential hazard.

\subsubsection{Densimeter and Instrumentation}

The DRS densimeter described in the previous publication [1] has undergone several modifications since that report was prepared. A major modification occurred when the electronic balance used to weigh the single crystal was replaced.

Since manually disconnecting and connecting the silicon single crystal and reference weight caused occasional discontinuities in the electrical balance reading, pneumatic drive cylinders were connected to both actuators. These cylinders were mounted on the scale case and were sufficiently slow-moving to gently place the weights on the balance while not shaking the balance mounting.

This modification decreased the scatter of the methane density values determined by the DRS relative to the Haynes-Hiza [3] density values calculated from the average sample temperature.

When the sample containment portion of the DRS was reconstructed, as described in 2.4 .1 .3 , the silicon crystal disconnect mechanism was rebuilt. The suspension wire in the vapor region was replaced by a 1.6 millimeters diameter thin wall stainless tube, and the assembly connecting the suspension to the bottom of the balance was modified. The balance was readjusted for corner load effects. These adjustments, when properly made, make the balance weight reading independent of the position on the pan a weight is placed. The density of methane in the new sample holder as determined by the DRS densimeter was offset by approximately 0.07 percent relative to the Haynes-Hiza [3] and Goodwin [4] values for the density calculated from average temperature for six subsequent methane fillings.

The vapor bulb positions were altered and the methane in the bulbs changed without affecting this offset. To ascertain that the pressure gauge had not shifted calibration, it was compared to the barometric pressure and found to be in agreement. The temperature difference represented by the difference between the barometric pressure and the vapor pressure gauge reading amounted to less than $0.01 \mathrm{~K}$. The methane density difference corresponding to $0.01 \mathrm{~K}$ is too small for the DRS densimeter to measure. This offset will be discussed in more detail below.

Addition of a New Balance Recently an electronic balance with sufficient range to directly weigh the 128 gram crystal to 1 milligram has become commercially available. This balance has other advantages; it is more compact and much less sensitive to vibration than the original balance. It is lightweight and can be transported in any orientation without securing the moving parts. The DRS densimeter has been rebuilt to incorporate one of these electronic balances. Conceptually, and in actual design, this densimeter differs little from the original densimeter. However, because it has sufficient capacity to weigh the silicon directly, a reference weight [1] with the attendant buoyancy correction is no longer required. The reference weight has been retained as a means of calibrating the balance and monitoring the calibration during tests.

A suspension system consisting of two cages, one for a reference weight and one for the silicon crystal, is attached to the underside of the balance. The reference weight cage is about 20 centimeters below the balance and in the sample gas space, while the silicon crystal cage is 76 centimeters below the balance and completely immersed in the sample liquid. The section of the suspension system passing through the liquid surface is a wire. The suspension is always attached to the balance. Lifting mechanisms permit the weights to be attached and detached from the suspension independently. Surface tension effects on the suspension should cancel, since weight off (zero) readings are included in the measurements and subsequent density calculations. 
The liquid density $\rho$ is now given by the relation (equation 4 , Reference 1 ) with $M_{a}$ replaced by $\left(\mathrm{M}_{\mathrm{a}}-\mathrm{M}_{\mathrm{aO}}\right)$ :

$$
\rho=\rho_{s}\left[1-\left(M_{a}-M_{a o}\right) / M_{S}\right]
$$

where $\rho_{s}$ is the silicon density, $M_{S}$ is the silicon crystal mass in vacuum, $M_{a}$ is the apparent mass when welghed in the $l i q u i d$ and $M_{a o}$ is the balance reading with the crystal and reference weight disconnected. This latter value nearly always is zero to \pm 1 milligram as the balance is tared (zeroed) with the silicon crystal removed before the density readings are taken.

The systematic offset in the observed density of liquid methane compared to Haynes-Hiza discussed in 2.4.1.4 remained and even increased slightly with the introduction of the new balance. The previously reported data [1] are probably in error because of an undetected corner loading error in the balance. This error cannot be directly confirmed as it is not possible to recover the old balance adjustment. Because of the way the silicon crystal and the reference weight were placed on the balance in the original system, any corner loading error must have had some effect on density measurement. The reference weight was placed on the pan, and the crystal was suspended from a part of the balance assembly well below the pan. This vertical of set in attachment points probably did not contribute a systematic error to the density determination, but the relative displacement in the horizontal plane of the attachment points that existed could affect the results. 100 gram weights were placed on the pan which were weighed relative to a counterweight so that the balance reading was on scale on the 20 gram range of the balance. However, the pan was only corner load adjusted so 20 grams could be read to within a milligram, thus a mass value error approaching 5 milligrams at the edge of the pan was possible for a 100 gram weight even for a properly adjusted balance. The results of a series of tests of the balance indicated some corner loading error was still present even after the balance was readjusted.

Comparison tests between the DRS densimeter and a silicon crystal densimeter built for a commercial calibration facility showed agreement to about 0.01 percent. These results are shown in the appendix of the original publication [7] and lend added support to the contention that the systematic shift in the measured methane densities relative to the measurements reported in reference [1] resulted from an error in the adjustment of the original electronic balance.

In some later tests, a third silicon densimeter, the Portable Reference Densimeter (PRD) was compared to the DRS densimeter in the DRS [5]. The PRD and DRS densimeters are similar in design, but the silicon single crystal was obtained from a different source. The density readings were indistinguishable.

Calibration of the Balance Even though the present DRS densimeter no longer requires a reference weight to determine LNG density, the weight has been retained for calibrating the balance and monitoring the calibration. The balance is calibrated by weighing the reference weight $1 \mathrm{n}$ alr and adjusting the balance to read the apparent true mass in air. The reference weight stlli welghs approximately the same as the silicon crystal when the latter is submerged in liquid methane.

In the course of taking data at each sample temperature setting, this reference weight is measured and recorded. The apparent mass is corrected for gas buoyancy and compared to the true mass value. This correction is readily made for methane or methane-nitrogen samples but not for methane-propane-nitrogen samples, because propane suppresses the methane vapor pressure by an uncertain amount, and because the gas mixture is slow to reach equilibrium. The method of calculating the buoyancy correction is detailed in the appendix of the original publication [7]. The reference weight is located in a region in which the gas temperature is -10 to $-20^{\circ}$, whlch contributes to the buoyancy correction uncertainty in the ternary mixture, even though the gas temperature is measured and applied to the buoyancy calculation.

The reference weight is weighed periodically in air to monitor the balance cal1bratlon. The calibration can be adjusted if needed, or a calibration factor can be introduced. In the slx months of operation, the calibration has not been adjusted. A calibration correction amounting to 1.7 milligrams was included in the calculations for two fillings. For later fillings, a correction was not needed.

The results of monitoring the reference weight during tests are presented In 2.4.1.5. Corner $10 a d 1$ f errors are unlikely in the new silicon crystal welghing system since both the reference welght and ing silicon weight are suspended below and placed on the suspension axial to the suspenslon wlre, therrby eliminating essentially all possibility of corner loading errors. 
Sample Temperature Temperature is measured using vapor pressure thermometers. One thermometer bulb was located at the bottom of the sample volume and the other near the top. Each thermometer contains a few cubic centimeters of liquid methane. Pressure communicates from each thermometer bulb via a 1.6 millimeters 0.D. stainless capillary tubing through a selector valve to a quartz spiral Bourdon tube pressure gauge. The temperature range used is $110 \mathrm{~K}$ to $126 \mathrm{~K}$.

The accuracy of the quartz spiral gauge is 0.01 percent of full-scale pressure as determined by a master piston gauge calibrated by NBS. This pressure uncertainty is equivalent to $0.01 \mathrm{~K}$ at $110 \mathrm{~K}$ and $0.003 \mathrm{~K}$ at $125 \mathrm{~K}$.

Sample Vapor Pressure The liquid sample vapor pressure was measured using a Bourdon tube pressure gauge with a 0 to 4 bar range and an 0.2 percent of full scale accuracy according to the manufacturer. The vapor pressure is used only for a small buoyancy correction to the reference weight and need not be accurately known.

\subsubsection{Measurement Process Parameters}

Bounds for Systematic Errors The primary sources of uncertainty in the density measurements can be obtained from examination of the measurement equation. The knowledge of the basic uncertainties of the separately measured quantities, such as the masses and densities, can be combined to give an estimate of the systematic errors in the density as measured by the density reference system.

Equation (1), the measurement equation, gives the density as a function of several independently measured variables.

$$
\rho=\rho\left(M_{S}, M_{a}, M_{a 0}, \rho_{S}, T\right)
$$

The temperature is included here, because the density at the test densimeter is the quantity required and a temperature gradient may exist.

The uncertainty in liquid density arising from uncertainty in some variable $x$, for example, can be evaluated from

$$
\delta \rho=\frac{\partial \rho}{\partial x} \delta x
$$

The total uncertainty in density is taken to be the square root of the sum of the squares of the various contributions,

$$
\frac{\delta \rho}{\rho}=\frac{1}{\rho}\left[\Sigma\left(\frac{\partial \rho_{i}}{\partial x_{i}} \delta x_{i}\right)^{2}\right]^{1 / 2}
$$

This is the propagation of error relationship for systematic error when the variables are independent, and the magnitudes of the relative errors are small, so that second-order terms are negligible [6].

Table 2.4.1.1 is a summary of the most significant contributions to the total systematic uncertainty in density as estimated from the various contributing sources. The first column, labelled $x$, identifies the variable for which the uncertainty is calculated. The second column is the partial derivative of the liquid density with respect to the variable of the first column, and the third column gives its maximum magnitude. The column labelled $\delta \mathrm{x}$ is the estimated largest error of that variable.

The systematic error, $\delta \mathrm{x}$, of $\mathrm{M}_{\mathrm{S}}$, is estimated from the standard deviation of the weighings and the uncertainties of the 5 weights used (Appendix [7]). The estimate of the systematic error for $M_{a}$ and $\mathrm{M}$ ao is the resolution of the electronic balance or 1 milligram. The calibration of the electronic balance is monitored by weighing the reference weight, as discussed earlier, which permits holding this uncertainty within the balance resolution. The error estimate of the silicon crystal density covers uncertainties because of the temperature and pressure effects as well as uncertainties in published densities (Appendix [7]). 
Table 2.4.1.1

Summary of Systematic Errors

\begin{tabular}{|c|c|c|c|c|}
\hline Variable, $x$ & $\partial \rho / \partial \mathrm{x}$ & $\begin{array}{l}\text { Maximum } \\
\text { Magnitude }\end{array}$ & $\delta \mathrm{x}$ & $\delta / \rho(\%)^{*}$ \\
\hline \hline $\mathrm{M}_{\mathrm{S}}$ & $\left(\rho-\rho_{\mathrm{S}}\right) / \mathrm{M}_{\mathrm{S}}$ & $-0.015 \mathrm{~cm}^{-3}$ & 0.0005 & 0.0019 \\
$\mathrm{M}_{\mathrm{a}}$ & $-\rho_{\mathrm{S}} / \mathrm{M}_{\mathrm{S}}$ & $-0.0184 \mathrm{~cm}^{-3}$ & $0.001 \mathrm{~g}$ & \pm 0.0046 \\
$\mathrm{M}_{\mathrm{aO}}$ & $\rho_{\mathrm{S}} / \mathrm{M}_{\mathrm{S}}$ & $0.0184 \mathrm{~cm}^{-3}$ & $0.001 \mathrm{~g}$ & \pm 0.0046 \\
$\rho_{\mathrm{S}}$ & $\rho / \rho_{\mathrm{S}}$ & 0.17 & $6 \times 10^{-5} \mathrm{~g} / \mathrm{cm}^{3}$ & \pm 0.0026 \\
$\mathrm{~T}$ & $\partial \rho / \partial \mathrm{T}$ & $0.0016 \mathrm{gm} / \mathrm{cm}^{3}$ & 0.05 & \pm 0.02 \\
Total, from from equation (4) & - & & $\pm 0.022 \%$ \\
\hline
\end{tabular}

* At the smallest measured density, $\sim 0.4 \mathrm{gm} / \mathrm{cm}^{3}$

The combined temperature gradients and drift during data acquisition measured by the vapor pressure thermometers rarely exceed the value of $0.05 \mathrm{~K}$. It is assumed for the purposes of error estimation that a $\Delta \mathrm{T}$ no larger than $0.05 \mathrm{~K}$.

This temperature difference is dependent on the heat input of the instruments under test, and $\Delta \mathrm{T}$ is usually less than half this value. A density difference resulting from this temperature difference can be calculated from the methane properties $[3,4]$ and is shown in table 2.4.1.2.

Table 2.4 .1 .2

Density Differences for Small Temperature Changes

\begin{tabular}{|c|c|}
\hline $\begin{array}{l}\text { Average System } \\
\text { Temperature }\end{array}$ & $\Delta \rho / \rho$ for $\Delta \mathrm{T}=0.05 \mathrm{~K}$ \\
\hline \hline $110 \mathrm{~K}$ & $\begin{array}{l}0.017 \text { percent } \\
0.02 \text { percent }\end{array}$ \\
\hline
\end{tabular}

These uncertainties are the same size as claimed for the original DRS and are most certainly overestimated. To confidently reduce these values, however, it would be better to have a more detailed knowledge of the temperature distribution in the sample during a density measurement. The present accuracy needs do not warrant increasing the complexity of the system by the addition of more thermometers. An additional systematic error results from the uncertainty in the absolute value of $\mathrm{T}$. This is not included in the table, since it makes no contribution to the systematic error when densimeters are being compared. When liquid methane density measurements are being compared with those of other workers, however, the 0.01 percent contribution to the systematic error at $110 \mathrm{k}$ decreases to less than 0.001 percent at $127 \mathrm{~K}$.

Calculation of the Randon Uncertainty The accuracy and precision of the DRS densimeter is essentially independent of liquid composition. Its accuracy and precision depend primarily upon the accuracy and precision of its balance and how well the weight and density of the silicon crystal are known. The ability to make an accurate reading will, of course, depend upon conditions within the sample holder. The liquid needs to be relatively quiet, well mixed and of a fairly uniform temperature in the area in which the densimeters are being tested. It should be sufficient to test the DRS densimeter in a liquid whose density is known. Saturated liquid methane was chosen, as its density, as a function of temperature, has been determined by Goodwin [4] and Haynes and Hiza (3]; and it is the major constituent of LNG.

The present evaluation of the improved DRS is based on data taken for fifteen fillings of the 11 quld sample holder. Ten of these fillings were made after the new balance was installed.

The major interest in commercial densimeters is their performance in an LNG-like mixture. The DRS sample holder was first filled with liquid methane, then a series of measurements were taken over the range of about 110 to $125 \mathrm{~K}$. Then nitrogen (about 1 percent to 2 percent) and propane (about 4 percent to 6 percent) were added to simulate LNG and another series of measurements were taken $1 \mathrm{n}$ the same temperature range. This data of the DRS was then compared to the Haynes-Hiza methane dens\&tins, and an evaluation of the densimeter under test was made in an LNG-like mixture. 
The values of density as determined by the Haynes-Hiza method for saturated liquid methane were compared with the corresponding values determined by the DRS densimeter. The vapor pressure bulbs, from whose average value the Haynes-Hiza density is calculated, bracket the volume containing the silicon crystal. The Haynes-Hiza value, then, is more of an average reading, while the DRS densimeter registers a point value.

A continuing calibration test of the new DRS balance is made as discussed in 2.4.1.4. The silicon crystal apparent mass in the sample liquid is 100 grams to 107 grams for the mixtures being considered, and the mass value of the reference weight is approximately 103 grams. This weight is used before filling the system, during the density measurements, and after the system is emptied to determine the calibration of the DRS balance.

Figure 2.4.1.3 is a plot in chronological order of the differences between the Haynes-Hiza values for saturated liquid methane and the corresponding values determined by the DRS densimeter. All the methane data for the time period April 1979 through February 1980 are shown. The symbols alternate from filling to filling to distinguish the separate fillings. Solid diamonds show the average difference for each filling. The measurements in the first five fillings, counting the groups from the left, were by the old DRS balance after readjusting it for corner loading. The jump between the fourth and fifth filling is attributed to a change in the calibration of the balance since the densimeter had been dismantled and reassembled between these fillings. The apparent shift occurring after the installation of the new DRS balance probably results from some remaining corner loading effects for the old balance as discussed in 2.4.1.4.

The average difference between the Haynes-Hiza and the DRS densimeter values using the new balance is approximately 0.09 percent larger than the 0.01 percent difference reported in reference [1]. The difference for the old balance readjusting was approximately 0.07 percent larger than previously reported [1].

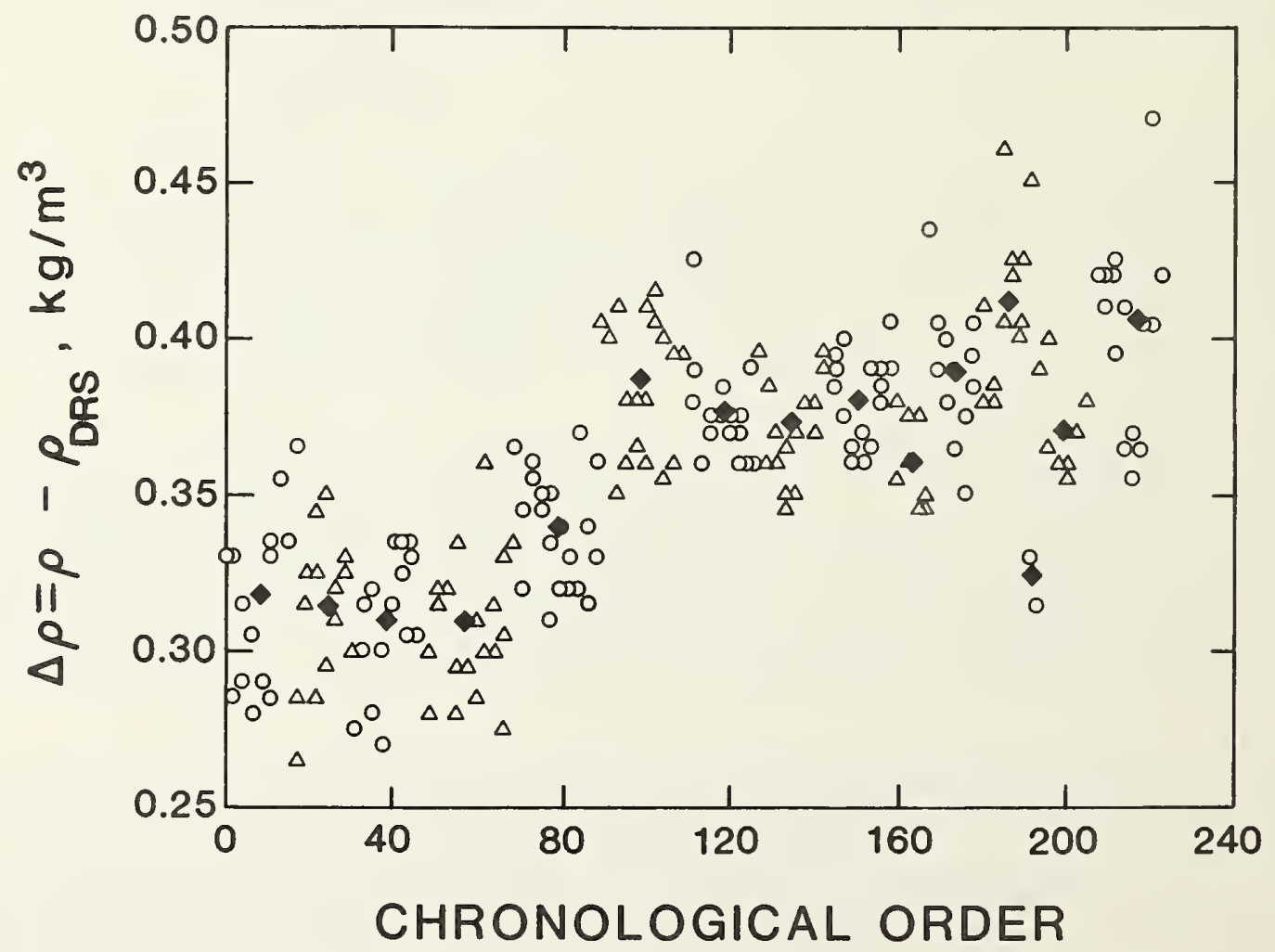

Figure 2.4.1.3. Liquid methane density difference between DRS and Haynes-Hiza. 
The average difference between the methane densities determined by the Haynes-Hiza method and those determined by the DRS densimeter shows the latter to be approximately 0.1 percent lower. That the DRS denslmeter is now measuring density correctly is supported by the data for fillings 11 through 14. The meter under test for these fillings was also an Archimedes densimeter using a silicon crystal. See Append1x 9.1 [7] for details of this comparison.

The average difference between the corresponding density values given by this meter and the DRS densimeter is 0.004 percent \pm 0.017 percent (95 percent confidence interval), i.e., the density values were not statistically distinguishable. The comparison between the DRS and Portable Reference Densimeter, to be reported later, also shows the present DRS densimeter to be correctly measuring the density.

Each time a commercial densimeter was tested in pure methane, data were taken to permit comparing the DRS measured density to the Haynes-Hiza density calculated from the average temperature. A series of density measurements with each liquid sample were taken over a range of temperatures by either heating or cooling the sample to a target temperature, waiting for equilibrium while stirring the sample, and taking the desired schedule of readings with the stirrer of . The target temperature was only nominally achieved at equilibrium. The sample was stirred again and the readings duplicated. The duplicate readings provided assurance that the DRS and test densimeters had reached equilibrium. A lack of equilibrium was never noted. The second reading also reduced the chance of losing a data point through a reading error or a malfunction of a test densimeter, which are often sensitive to bubbles. The temperature drift between the duplicate sets of readings for the new DRS had a standard deviation of approximately $0.1 \mathrm{~K}$ with the second reading averaging $0.05 \mathrm{~K}$ higher.

The random errors of the duplicate density values obtained from a densimeter as described above would be expected to correlate more closely with each other than with values at different temperatures. In fact, the correlation between duplicates for the difference between the Haynes-Hiza and DRS densit1es is about 0.5 . A correlation of 1.0 would mean the duplicates provide the same information, while a correlation of 0 would mean the duplicates provide independent information on the process. Since there is much more data than needed for the error estimate, and the correlation between the duplicate data at each temperature complicates the analysis, only the first of each duplicate reading has been used to analyze the DRS.

The model used to analyze the methane data is:

$$
H(T)-Y(T)_{i j}=\mu(T)+\alpha_{i}+e_{i j}
$$

where $Y(T)_{i j}$ is the $j$ th density determination by the DRS on the ith methane filling for temperature $T$; $\mathrm{H}(\mathrm{T})$ is the corresponding density value derived from the Haynes-Hiza relationship, $\mu$ ( $T$ ) represents any systematic difference between the two methods at temperature $T, \alpha_{i}$ represents a shift in the mean for measurements on the ith filling, and $e_{i j}$, the random contribution to the $j$ th measurement made on the ith filling.

The solid diamonds in figure 2.4.1.3 show the average difference for each filling and show the sh1fts $\alpha_{i}$ in the mean of the $Y_{i j}$ from one filling to the next. Considering the data before the new balance, the only significant shift is between the 4 th and 5 th fillings, and this probably resulted from dismantling the densimeter between the two fillings. The data taken after the installation of the new balance show the shifts from filling to filling to be rather small for the first flve fllilngs, but somewhat larger for the last five.

The $\alpha_{i}$ appear to be random in nature and have an estimated standard deviation of $0.013 \mathrm{~kg} / \mathrm{m} 3(0.0031$ percent). A 99 percent upper confidence bound for this value is $0.036 \mathrm{~kg} / \mathrm{m}^{3}$ ( 0.0082 percent). What accounts for the shifts in the $\alpha_{i}$ is at present unknown. The deviations of the measured values of the calibration weight from the true mass value of 102.9022 grams, $\Delta W$, are shown versus chronological order in figure 2.4.1.4. A buoyancy correction must be made to the balance reading to obtaln the true mass value (see Appendix 9.3 [7]). The changes in the mean of the observed true mass values of tho calibration weight from filling to filling are statistically signlflcant, but they are not well correlated with the $\alpha_{i}$, and if they were, their estimated standard deviation could account for only a 0.0020 percent component in the variation of the $\alpha i$, leaving additional effects to account for 0.0024 percent. A difference between the temperature of the liquid at the crystal and the average temperature given by the two vapor pressure bulbs might also contribute to this uncertalnty 1 f the difference were to persist over the time of the run (filling). Based on the differences shown ln figure 2.4.1.3, the within filling standard deviation for flllings through the 15 th $1 \mathrm{~g}$ estimated to bn $0.0216 \mathrm{~kg} / \mathrm{m} 3(.0051$ percent) with a 99 percent upper bound of $0.0277 \mathrm{~kg} / \mathrm{m} 3(.0065$ percent). Th13 13 a conservative estimate. 


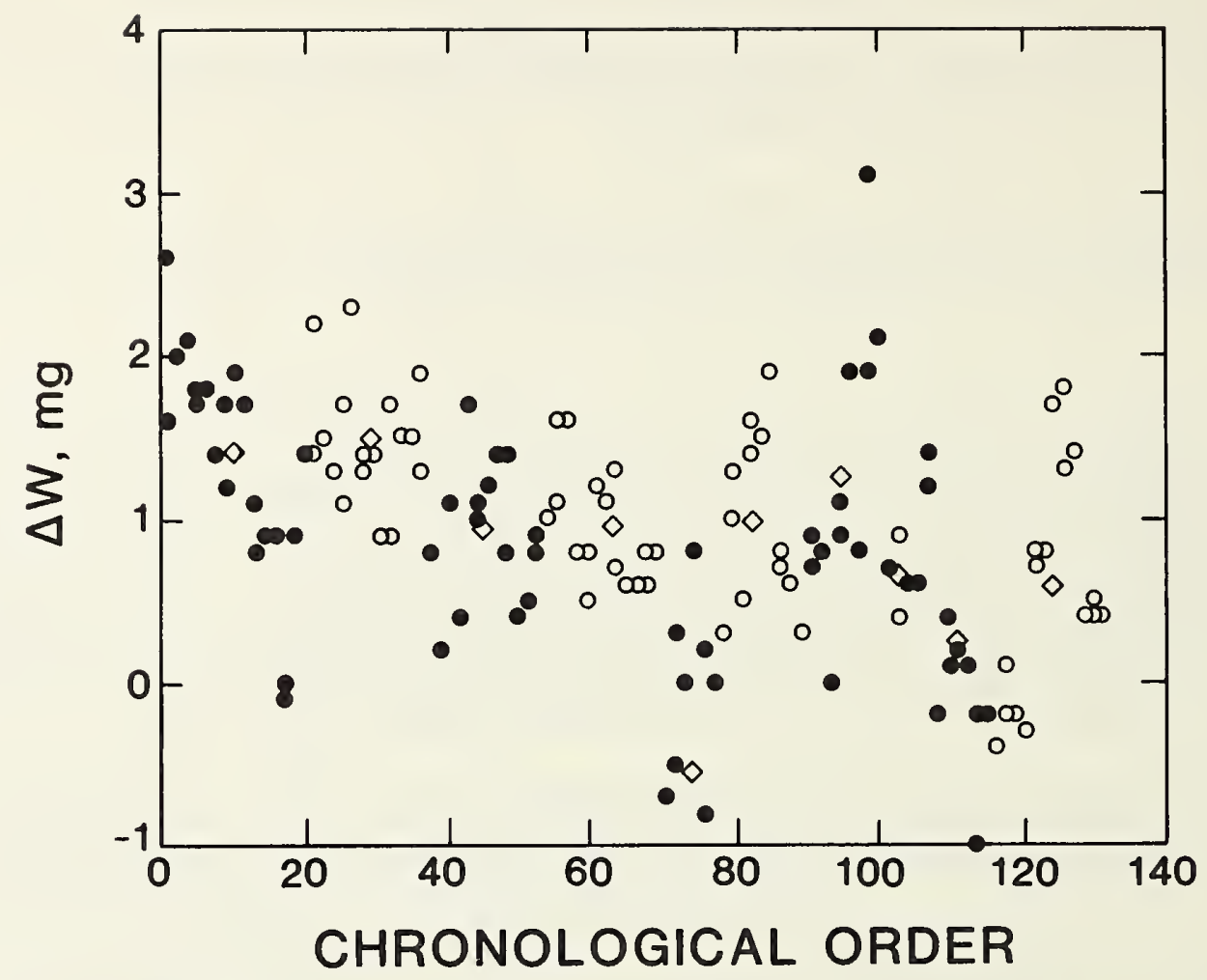

Figure 2.4.1.4. Reference weight during test compared to vacuum value.

A least squares fit of the DRS density data for fillings 1 through 5 to the temperature of the saturated methane after removing the filling-to-filiing shifts results in the equation,

$$
D(T)=526.935-0.410996 T-0.0047017 T^{2}
$$

where $D(T)$ is the predicted density in $\mathrm{kg} / \mathrm{m}^{3}$ for $\mathrm{T}$ in kelvin. The fit was made assuming that only the DRS density data were subject to error. However, T being the average of the upper and lower temperature of the liquid sample, it will differ from the temperature of the liquid at the densimeter. This assumption is likely to bias the estimated coefficients in the equation and also the estimated standard deviation for the DRS values.

The residual standard deviation is $0.027 \mathrm{~kg} / \mathrm{m}^{3}$ (37 degrees of freedom) and is comparable to the within standard deviation discussed above. The range for $\mathrm{T}$ was $109 \mathrm{~K}$ to $128 \mathrm{~K}$. A similar fit for the DRS density data for fillings 6 through 15 results in the equation:

$$
D(T)=525.092-0.382535 \mathrm{~T}-0.0048151 \mathrm{~T}^{2}
$$

The residual standard deviation for this fit is $0.019 \mathrm{~kg} / \mathrm{m}^{3}$ ( 53 degrees of freedom). The range for $\mathrm{T}$ was $109 \mathrm{~K}$ to $130 \mathrm{~K}$. The differences between the predicted values of the two equations over the range $110 \mathrm{~K}$ to $130 \mathrm{~K}$ is given for selected temperatures in the table 2.4.1.3. The last column of the table gives the differences less the $0.07 \mathrm{~kg} / \mathrm{m}^{3}$ estimated of fset between the old balance values and the new balance values. 
Table 2.4.1.3

Predicted Temperature Differences

\begin{tabular}{|c|c|c|c|c|}
\hline Temperature & Equation (6) & Equation (7) & Eq (6)- Eq (7) & Less 0.07 \\
\hline \hline 110 & 424.83 & 424.75 & 0.08 & 0.01 \\
115 & 417.49 & 417.42 & 0.07 & 0.00 \\
118 & 412.97 & 412.91 & 0.06 & -0.01 \\
120 & 409.91 & 409.85 & 0.06 & -0.01 \\
125 & 402.10 & 402.04 & 0.06 & -0.01 \\
130 & 394.05 & 393.99 & 0.06 & -0.01 \\
\hline
\end{tabular}

The percentage deviation, $100(\mathrm{H}(\mathrm{T})-\mathrm{D}(\mathrm{T})) / \mathrm{D}(\mathrm{T})$, between the Haynes-Hiza relationship [3] and equation (7) is shown in figure 2.4.1.5. The same comparison is also shown for equation (7) and the Goodwin results [4].

Uncertainty statement Three times the estimated standard deviation is commonly used in reporting the limits of random error. This derives partly from simplicity and partly from the fact that if the true standard deviation were being used,

$$
m(T) \pm 3 S D
$$

would contain 99 percent (almost all) of the measured $Y(T)_{i j}$, where $m(T)$ is the systematic error.
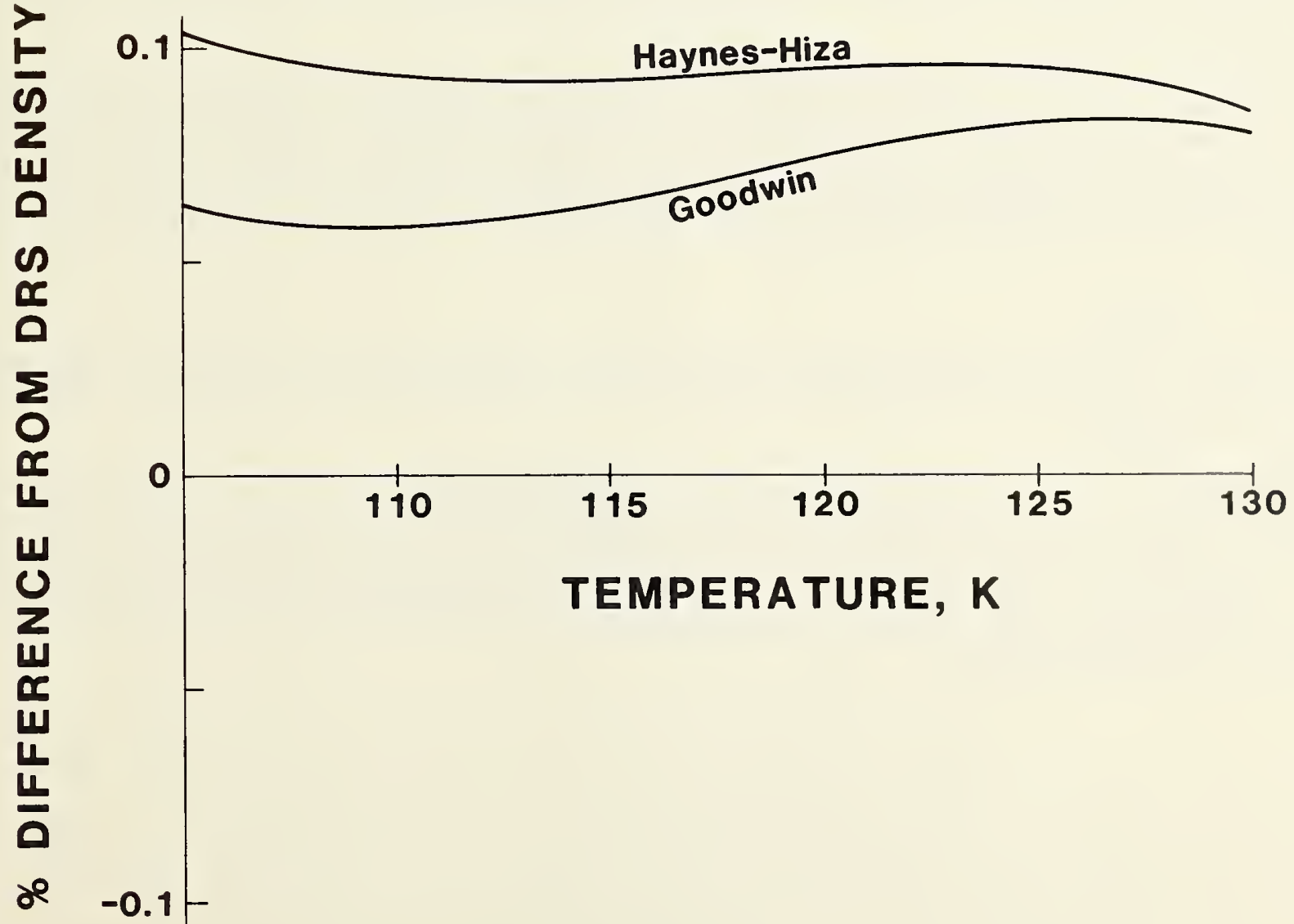

Figure 2.4.1.5. Methane densities of Haynes-Hiza, Goodwin and DRS compared. 
Table 2.4.1.3 gives bounds to this value of \pm 0.022 percent. From figure 2.4 .1 .4 , the filling-to-filling standard deviations 99 percent upper bound is $0.036 \mathrm{~kg} / \mathrm{m}^{3}$ and that for within a filling has a 99 percent upper bound of $0.028 \mathrm{~kg} / \mathrm{m}^{3}$. Combining these two to obtain an upper bound on the standard deviation of a single determination of Haynes-Hiza minus DRs, gives ( (.036) ${ }^{2}+$ $\left.(.028)^{2}\right)^{1 / 2}=0.046 \mathrm{~kg} / \mathrm{m}^{3}$. Although this value contains the uncertainty for both density determinations (Haynes-Hiza and DRS), all of the uncertainty is assigned to the DRS. The 30 value for this uncertainty is $0.138 \mathrm{~kg} / \mathrm{m}^{3}$.

Transforming the estimated standard deviaton of $\pm 0.046 \mathrm{~kg} / \mathrm{m}^{3}$ to percent at $422.63 \mathrm{~kg} / \mathrm{m}^{3}$, the normal boiling point density of methane, gives a value of \pm 0.011 percent. The sum of $3 \times 0.011$ percent plus 0.022 percent gives the present estimated limit of total uncertainty for a single density measurement made on pure methane with the DRS as \pm 0.055 percent. This uncertainty decreases for LNG mixtures because of the increased density.

\subsubsection{Measurement Process Control}

As future measurements are made, the random errors will be continually monitored, and the systematic error estimates will be confirmed experimentally as described below.

Each time a set of density reference measurements is made using the system, measurements on at least two separate fillings of pure liquid methane will be included in the tests. Density measurements will be made at temperatures around $110 \mathrm{~K}, 115 \mathrm{~K}, 120 \mathrm{~K}$ and $125 \mathrm{~K}$ with some randomness in the order. The remeasured methane densities will be compared to the Haynes-Hiza results and to the earlier reference system measurements to determine whether the system has shifted. This reliability of the system will be checked only in conjunction with density comparison work rather than periodically because of the expense of making density measurements. The reference weight, of course, now permits a continuing monitoring of the balance performance and can provide information to correct any drift within a run. The methane tests will primarily monitor the silicon crystal itself and the vapor pressure bulb thermometer performances.

For future occasions, the within standard deviations will be compared statistically to those of previous occasions to test for any change in this measure of precision. Tests will be made to detect the presence of occasion-to- occasion shifts or signs of non-random behavior (e.g., drifting). If there are no apparent changes, the data from previous occasions will be combined with the new data to obtain updated estimates. Any statistically verifiable changes in the DRS process will call for corrective action with respect to the process. Revised uncertainty statements will be presented as needed.

\section{$2.4 \cdot 1 \cdot 7$ summary}

The density reference system of the National Bureau of Standards is described. The procedure for determining the density from weighing, zeroing and calibrating the balance and the calculational method is discussed.

The uncertainty of the improved density reference system for pure methane at the normal boiling point is \pm 0.055 percent. The contribution from the estimated systematic error in density was \pm 0.022 percent. The estimated uncertainty caused by random error is three times the standard deviation of 0.011 percent and is based on sixty-three measurements of the densities of saturated liquid methane. This total density uncertainty is taken to be the sum of the systematic and random errors and should vary approximately as $\rho_{\mathrm{m}} / \rho$ where $\rho_{\mathrm{m}}$ is the density of normal boiling point liquid methane and $\rho$ is the density of the sample liquid. 
2.4.1.8 References

[1] Siegwarth, J. D., B. A. Younglove and J. F. LaBrecque, Cryogenic fluids density reference system: Provisional Accuracy Statement, Nat. Bur. Stand. (U.S.), Tech. Note 698,(November 1977).

[2] Siegwarth, J. D., B. A. Younglove, and J.F. LaBrecque, An evaluation of commercial densimeters for use in LNG, Nat. Bur. Stand. (U.S.), Tech. Note 697, (October, 1977).

[3] Haynes, W. M., Hiza, M. J., Measurements of or thobaric liquid densities of methane, ethane, propane, isobutane and normal butane, J. Chem. Thermodynamics 9, 179 (1977).

[4] Goodwin, R. D., The thermophysical properties of methane, from 90 to $500 \mathrm{~K}$ at pressures to 700 bar, Nat. Bur. Stand. (U.S.), Tech. Note 653, (April, 1974).

[5] Siegwarth, J. D. and J. F. LaBrecque, A portable calibration densimeter for use in cryogenic liquids, Nat. Bur. Stand. (U.S.), Tech. Note 1035, (1981).

[6] Cook, N. H. and E. Rabinowitz, Physical Measurement and Analysis, Addison Wesley Publishing Co., Inc., 1963.

[7] Siegwarth, J. D. and J. F. LaBrecque, Cryogenic fluids density reference system: provisional accuracy statement (1980), Nat. Bur. Stand. (U.S.), Tech. Note 1041, 60pp (April, 1091). 



\subsubsection{Introduction}

The measurement of the density of a complex mixture of liquid phase light hydrocarbons can be accomplished directly by placing the active element of a densimeter in contact with the fluid. At the present time, all densimeters used in LNG service must be calibrated in order to provide the accuracy and precision required in measurement of LNG density. The design, construction, performance and operation of a Density Reference System (DRS) used to calibrate LNG densimeters is described in part 2.4.1 of this manual. The contributions of Dewardt [14] and Siegwarth et al. [1,7-12,15] in this specialized area have resulted in excellent progress in establishing the degree of uncertainty in this very important element of the measurement of LNG.

This part of the manual describes the performance of the field-type densimeter while under test in the Density Reference system (DRS). In order to treat this subject in detail, the edited work of Siegwarth and LaBrecque [1], which deals primarily with calibration equipment and techniques, is combined with descriptions of the generic types of commercial densimeters evaluated [9,12]. A description of a portable calibration densimeter is also given [15], which will allow field calibrations using base physical properties of the liquid hydrocarbon.

\subsubsection{LNG Densimetry [1]}

Custody transfer of hydrocarbon liquids based on mass rather than volume is growing in importance. Since accurate large mass-flowmeters are not available presently, and large quantities of liquids or liquids flowing in pipelines cannot be weighed, the mass is determined from volume and density measurements. The volume is determined either from calibrated tanks or by flowmeters. Density can be calculated from pressure and temperature measurements for pure fluids when data are available, and from pressure, temperature and composition measurement for mixtures for which data exist. For liquids of unknown or changing composition or for which thermophysical property data are unavailable, direct measurement of the density can be provided by a densimeter. Even for cases where the composition is known or can be measured, the densimeter measurement can be more accurate than calculated density and can provide a continuous value for the density.

A number of instruments are commercially available for density measurement: instruments using the Archimedes principle, instruments using variation of vibration frequency with density, and instruments measuring change of dielectric constant. None of the instruments tested are based entirely on fundamental measurements, and thus require calibration. The following is a brief description of the generic types of densimeters currently used for LNG. More detailed information can be found by reference to Siegwarth and LaBrecque $[9,12]$.

\subsubsection{Vibrating Element Densimeter}

The basic principle relating density to frequency is directly equivalent to the classical mass and spring oscillator. The vibrating member of the densimeter, a rectangular plate in one instance (figure 2.4.2.1) and a cylinder in the other instance (figure 2.4.2.2), has a natural frequency determined by the stiffness constant and apparent mass of the vibrating element. The apparent mass of

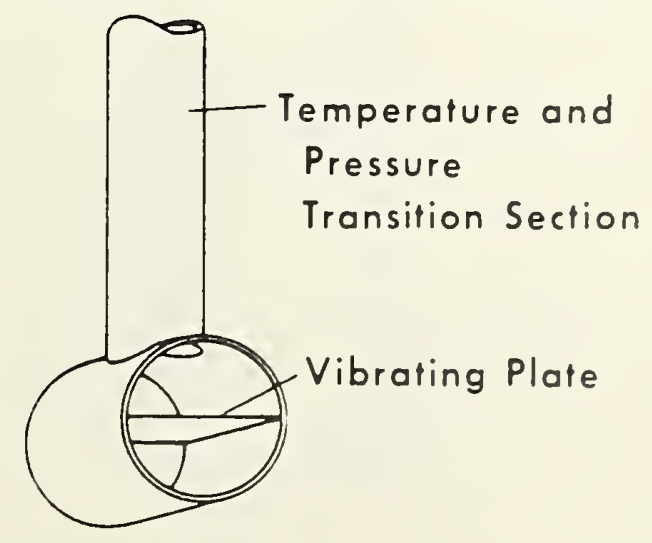

Figure 2.4.2.1. Primary elements of vibrating plate denslmeter. 
the element depends on the density of the fluid surrounding it, since in vibration a portion of this liquid is also forced to move. The density, $\rho$, is related to the period of oscillation, $\tau$, by

$$
\rho=A+B \tau^{2}
$$

$\mathrm{A}$ and $\mathrm{B}$ are constants determined empirically at cryogenic temperatures.

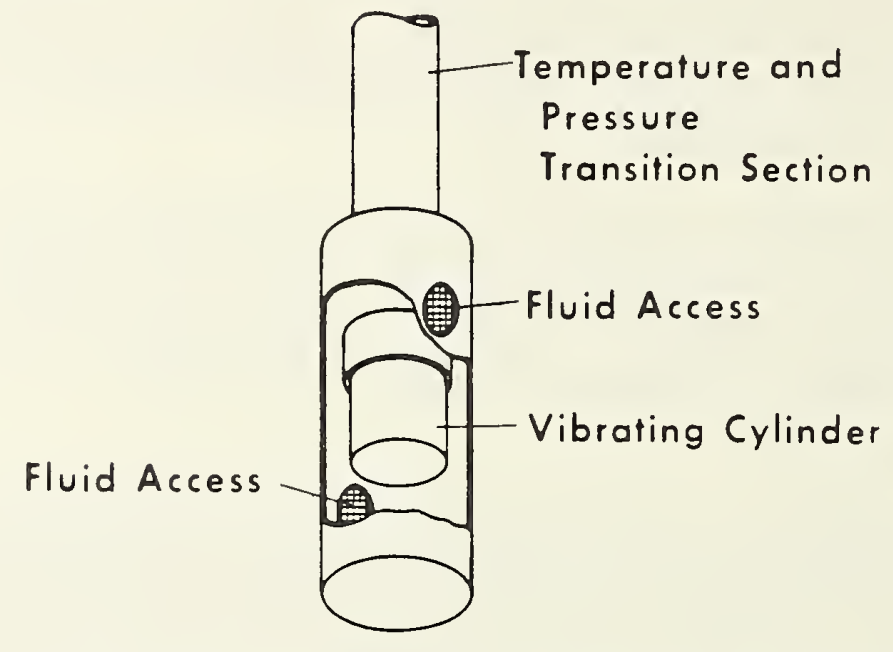

Figure 2.4.2.2. Primary elements of vibrating cylinder densimeter.

\subsubsection{Dielectric Cell Densimeter}

The Clausius-Mossitti relation [17] is

$$
\rho=\frac{(\varepsilon-1)}{(\varepsilon+2) \alpha}
$$

where $\varepsilon$ is dielectric constant and $\alpha$ is the polarizability, generally taken to be a constant, independent of density.

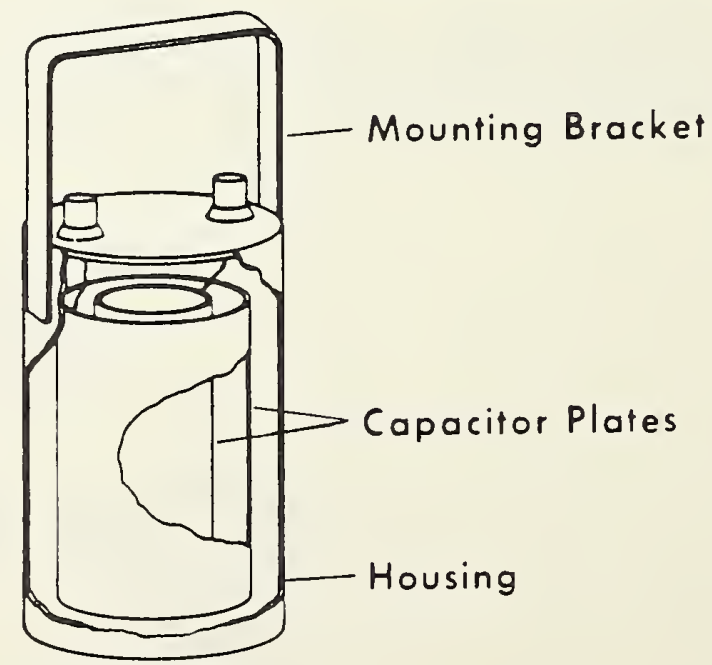

Figure 2.4.2.3. Primary elements of dielectric cell densimeter. 
Over a limited range of composition, however, the density can be represented by a linear function of the temperature and dielectric constant rather than using equation (2). This linear relation can be used along the saturation line of the liquid. The densimeter studied in this work (figure 2.4.2.3) uses a bilinear relation to relate dielectric constant and temperature to density.

\subsubsection{Displacement or Archimedes Densimeter}

The operation of this densimeter is based on the Archimedes principle (figure 2.4.2.4); that is, an object immersed in a still fluid experiences a lifting force equal to the weight of the fluid it displaces.

The measurement equation for this device is

$$
\rho=\rho_{0}\left(M-M_{a}\right) / M
$$

where $\rho_{0}$ is the density of the submerged object and $M$ its mass. $M_{a}$ is its apparent mass. The two unknowns $\rho_{O}$ and $M$ are determined for this instrument by calibration at two or more well defined densities.

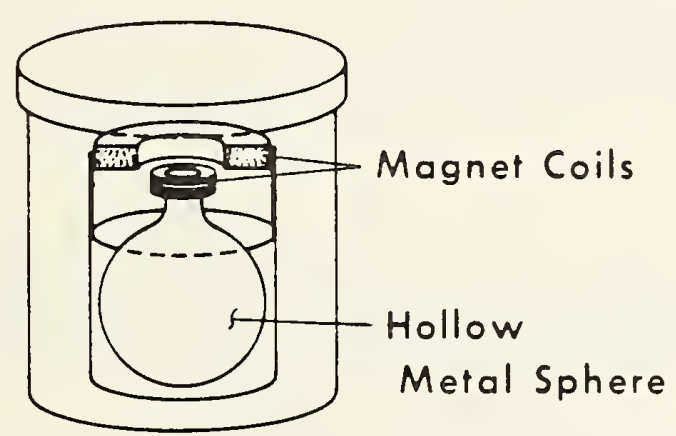

Figure 2.4.2.4. Primary elements of displacement densimeter.

Calibration of any of these densimeters can be accomplished by placing the instrument in liquids of known density and generating a calibration curve giving density as a function of the densimeter output parameter. Ideally, the output parameter of a densimeter would be independent of any quantity except density. However, this is not the case. Because the densimeters may be affected by temperature, pressure, and composition, they are best calibrated in liquids similar, if not identical, to those 1 , which they are to be used, and by a calibration procedure which provides the desired accuracy under normal operating conditions. Also, a calibration procedure that can be used at the densimeter installation is necessary to maintain the densimeter accuracy as it continues in service.

The American Petroleum Institute has specified a calibration method in the Manual of Petroleum Measurement Standards [2] using a pycnometer. The pycnometer is a vessel of known volume that is filled with the liquid and weighed. The density is calculated from the liquid weight and vessel volume. The temperature at which the measurement is to be done is ambient. The requirement that the pycnometer be filled completely with the liquid renders the method unsuitable for refrigerated liquids, since the filled and sealed vessel is likely to explode as it warms.

The LNG industry recognized the need for a different method of testing and calibrating LNG densimeters, and, through first the American Gas Association and then the Gas Research Institute, has provided funding for the LNG Density Reference System Project. The purpose of this project was to test the performance of a number of commercial densimeters in LNG. A test apparatus, the denslly reference system (DRS), was developed to do this work. When it became evident that the densimetrrs tested all lacked adequate calibrations, a calibration apparatus and methods were developed. This paper provides a summary of that work.

Though this work was directed towards density measurement of LNG, the apparatus and test aquipant developed could be used with little or no modification for densimeter testing and callbratlon avn a wide range of liquids. 


\subsubsection{Testing Commercial Densimeters}

Liquefied natural gas is predominately methane and has a density and boiling point near those of methane. However, pure methane, whose density can be computed from temperature and pressure, is generally not an adequate calibration fluid. This is true, because one calibration fluid does not provide sufficient information to determine the sensitivity of a densimeter to temperature, pressure and composition. Additional pure fluids with properties near that of methane could provide such information, but none exist. The use of calculated values of pure methane density for calibration also has the disadvantage that methane is easily contaminated by air, which alters the density.

The need for standard calibration fluids can be eliminated with a densimeter using the Archimedes principle to measure density. If a solid body of known volume $V_{O}$ and true mass $M_{0}$ has an apparent mass $M_{a}$ while immersed in a liquid, the liquid density

$$
\rho=\left(M_{0}-M_{a}\right) / V_{O}
$$

The quantity $\left(M_{O}-M_{a}\right)$ is the mass of liquid displaced by the solid body of volume $V_{O}$. The dominant uncertainty in $\rho$ is often the uncertainty of measuring $v_{O}$, but the need to measure the volume of the body is eliminated by using a body with an accurately known density.

The standard densimeter in the density reference system uses a silicon single crystal immersed in the test liquid that is weighed by an electronic balance located at ambient temperature to measure density. The $\rho_{\mathrm{s}}$ of single crystal silicon is known to about 3 parts per million [3,4], so $V_{0}$ can be calculated from $\rho_{\mathrm{S}}$ and $\mathrm{M}_{\mathrm{O}}$. Silicon has a number of other advantages: the density is approximately 2.3 $\mathrm{g} / \mathrm{cm}$, not far above liquid densities, and the thermal expansion coefficient [5] and compressibility [6] are known and are small.

The present DRS [7] is shown schematically in section 2.4.1 figure 2.4.1.1. It has a sample container of 16 liters in volume, which contains both the DRS densimeter and one or more densimeters under test. The sample liquid must be brought to a homogeneous, isothermal condition for several minutes in order to take readings on all the instruments. This is accomplished by containing the sample in the specially constructed 25 centimeter ID metal dewar.

Radiation shields, in some cases cooled by cold nitrogen gas, reduce the heat leak into the sample. A cooling coil around the vessel wall a short distance above the sample surface intercepts the heat conducted down the inner vessel wall. A counterflow heat exchanger, using liquid nitrogen as a coolant, introduces the sample liquid. An electric heater and a cooling coil provide sources of heat and cooling to change the temperature of the sample along the saturation line so that a range of densities can be obtained with one sample. The sample container is gas tight and able to withstand an internal pressure of 10 bar, though no measurements over about 3 bar have been made. A turbine pump, not shown in figure 2.4.1.1, vigorously mixes the liquid. The two vapor bulbs measure the temperature and the degree to which the sample is isothermal. Usually the two thermometers differ by less than m10 K. The stirrer is stopped during the time measurements are made.

Densimeter manufacturers calibrate densimeters in ambient temperature liquids and liquid nitrogen but are reluctant to use liquefied combustible gases for safety reasons. This seriously degrades the accuracy of the densimeters used in LNG as will be shown below. The DRS shown in $\mathrm{figure} 2.4 .1 .1$ was also designed to demonstrate a calibration system in which manufacturers and users can safely use LNG. The application of a system of this design to a commercial calibration facility has been discussed in reference [7]. This reference also details the safety features incorporated in the design.

\subsubsection{Test results}

To date, 20 densimeters have each been installed one or more times in the DRS for testing or calibration. A total of more than 1350 data sets were recorded. For a single measurement, the density measured by the DRS densimeter is estimated to give the density seen by the test densimeter to within \pm 0.055 percent more than 99 percent of the time. This uncertainty was estimated to be \pm 0.076 percent for the first densimeter tests [8].

Results are presented in this section to emphasize the need for better calibration procedures than are now used for commercial densimeters. Some of the densimeters tested were not provided with a cryogenic calibration at the factory. Of those provided with a cryogenic calibration, the calibration constants for LNG was usually extrapolated from liquid nitrogen and ambient temperature hydrocarbon measurements. 
Flgure 2.4.2.5 shows the results of tests of a calibrated dielectric type densimeter [9,10]. The capacitance between two coaxial cylinders varies with the dielectric constant of the liquid in the annular gap. The dielectric constant of LNG is assumed to have a dependence on composition similar to that of density on composition. The density is related to dielectric constant and temperature by a calibration equation. In figure 2.4.2.5, the difference in the density measured by the capacitance densimeter and the DRS densimeter is shown as a function of density.

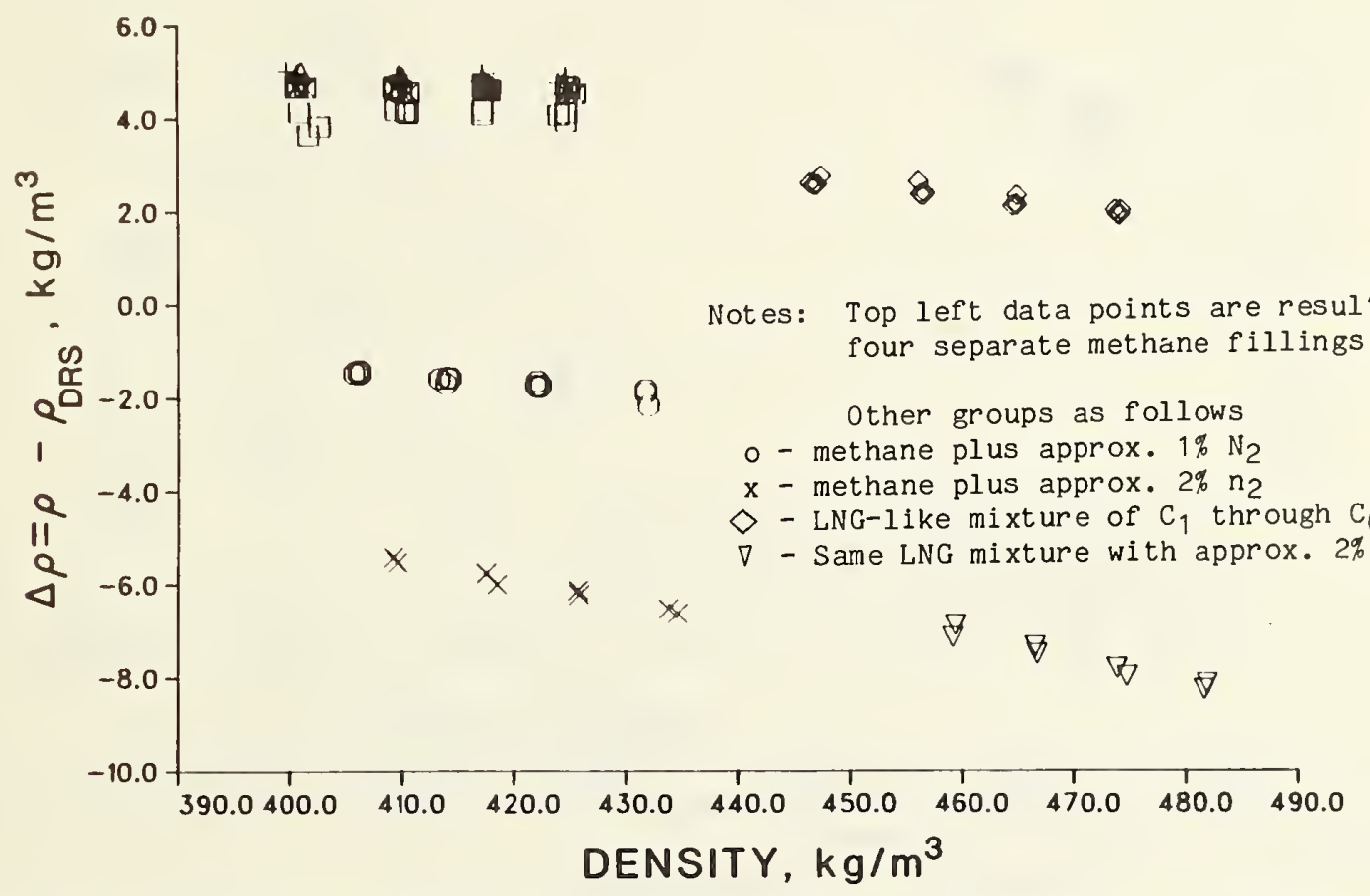

Figure 2.4.2.5. Comparison of factory calibrated capacitance densimeter to the NBS DRS densimeter.

The methane densities are about 1 percent high and the LNG-like mixture of $C_{1}, C_{2}, C_{3}$ and $C_{4} 1 s$ about 0.6 percent high. When about 1 percent nitrogen is added to liquid methane the density is now about 0.5 percent low. Adding about 2 percent nitrogen causes the dielectric densimeter to read about 1.5 percent low. The addition of about 2 percent nitrogen to the LNG-like mixture causes the dielectric densimeter to read about 1.6 percent low. The small slope in the differences as a function of density could be removed by adjusting for temperature. A filling-to-filling variation of the calibration of about \pm 0.08 percent was observed for the methane tests.

The dielectric densimeter will obviously not give accurate values for density without introducing composition into the calibration equation. Composition is introduced into the calibration of newer versions of the capacitance densimeter, but none of these have been tested in the DRS.

Figure 2.4.2.6 shows the results of the test of a vibrating cylinder densimeter [11, 12]. The vibrating cylinder forms the hole portion of a sealed doughnut shaped evacuated case so the 11 quid only contacts the inside of the vibrating tube. Basically, the vibrating frequency $f$ is related to the liquid density $\rho$ by equation (1) above. However, corrections were included by the manufacturer in the calibration equation for pressure, temperature, and velocity of sound. The cal1bration equat1on was derived from measurements in liquids other than LNG or methane. The difference between the density measured by this and the DRS densimeter for pure methane as a function of density is shown at the left of figure 2.4.2.6. The calibration is in error by 1.5 to 2 percent, and a large temperature and/or pressure effect is evident. The larger scatter in the first two f1llings resulted because frequency instead of period was measured, and the scatter results from the $0.1 \mathrm{~Hz}$ resolution. The density reading repeats well from filling to filling. The LNG like mixture results ror the same densimeter are shown on the right side figure 2.4.2.6. The offset is slightly smaller. a larger temperature or pressure dependence is evident; and each filling lies on a different curve presumably because the composition of the methane, 5 percent propane, 1 percent nitrogen, comprising the mixture, varied from filling to filling. The offset between straight line fits to the methane and to the Lic data suggests that composition should be an input parameter. 
No retesting has been done on later models of the above two densimeter types, but more extensive measurements have been done on two other densimeter types: the displacement densimeter and a different type vibrating cylinder instrument.

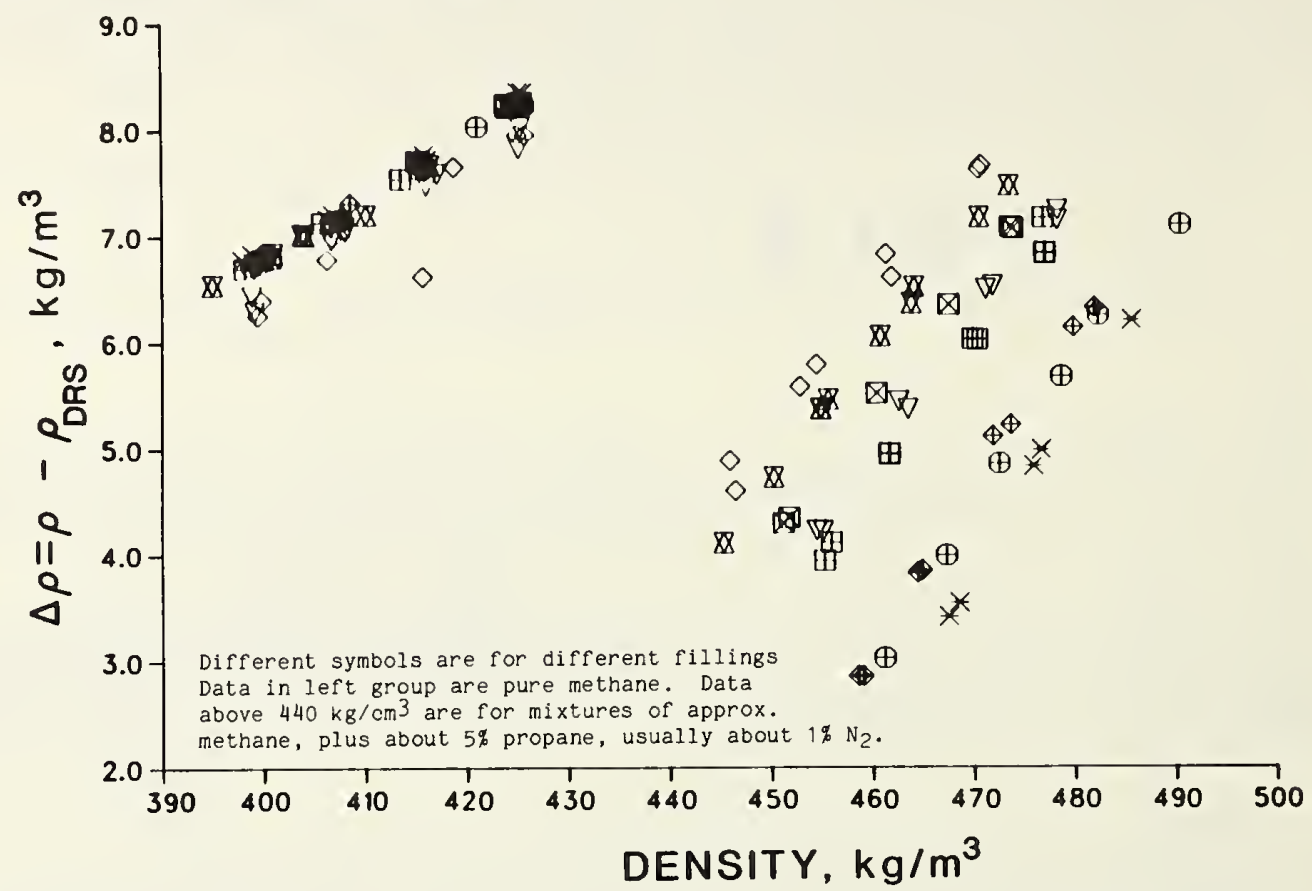

\section{Figure 2.4.2.6. Comparison of a factory calibrated vibrating cylinder densimeter to NBS DRS densimeter.}

The displacement densimeter consists of a hollow spherical float with an approximate density of 0.5 $\mathrm{g} / \mathrm{cm}^{3}$. An electromagnetic force coil counterbalances the buoyant force. The current in the force coil and the liquid temperature are combined to give the density. The first densimeters tested showed 0.1 percent shifts in calibration from test to test. The factory calibration also had errors, often above 0.5 percent.

Three later versions were calibrated in the DRS by calculating the constants in the calibration equation relating the liquid temperature and current in the force coil to the density provided by the DRS. The densimeters were removed and transported by automobile from 300 to 800 kilometer, then retested. The results for one of the three tested is shown in figure 2.4.2.7.

The difference between the displacement and DRS densimeters DRS is shown as a function of density. The average difference between the DRS and displacement densimeter density for the first test is zero, since this data provided the calibration. The densities measured by the displacement densimeter in the second test were obtained from the calibration equation. Shifts in calibration from 0.01 to 0.03 percent were noted for the three densimeters upon retesting.

Three vibrating cylinder densimeters to be used as transfer standards were calibrated in the DRS. Two of the three were later retested. These densimeters are a vibrating cylinder with liquid in contact with both surfaces of the cylinder. The readings were found to be quite noisy until the filter screens in the circulation path of the liquid through the instrument were removed. These apparently trapped bubbles in the vicinity of the cylinder. This problem may not occur when the liquid is subcooled.

The calibration equation is nominally equation (1). These densimeters, however, show a definite temperature dependence in the calibration amounting to 0.1 percent over a $15 \mathrm{~K}$ range. This can be removed by adding a term linear in $\mathrm{T}$ to equation (1). 


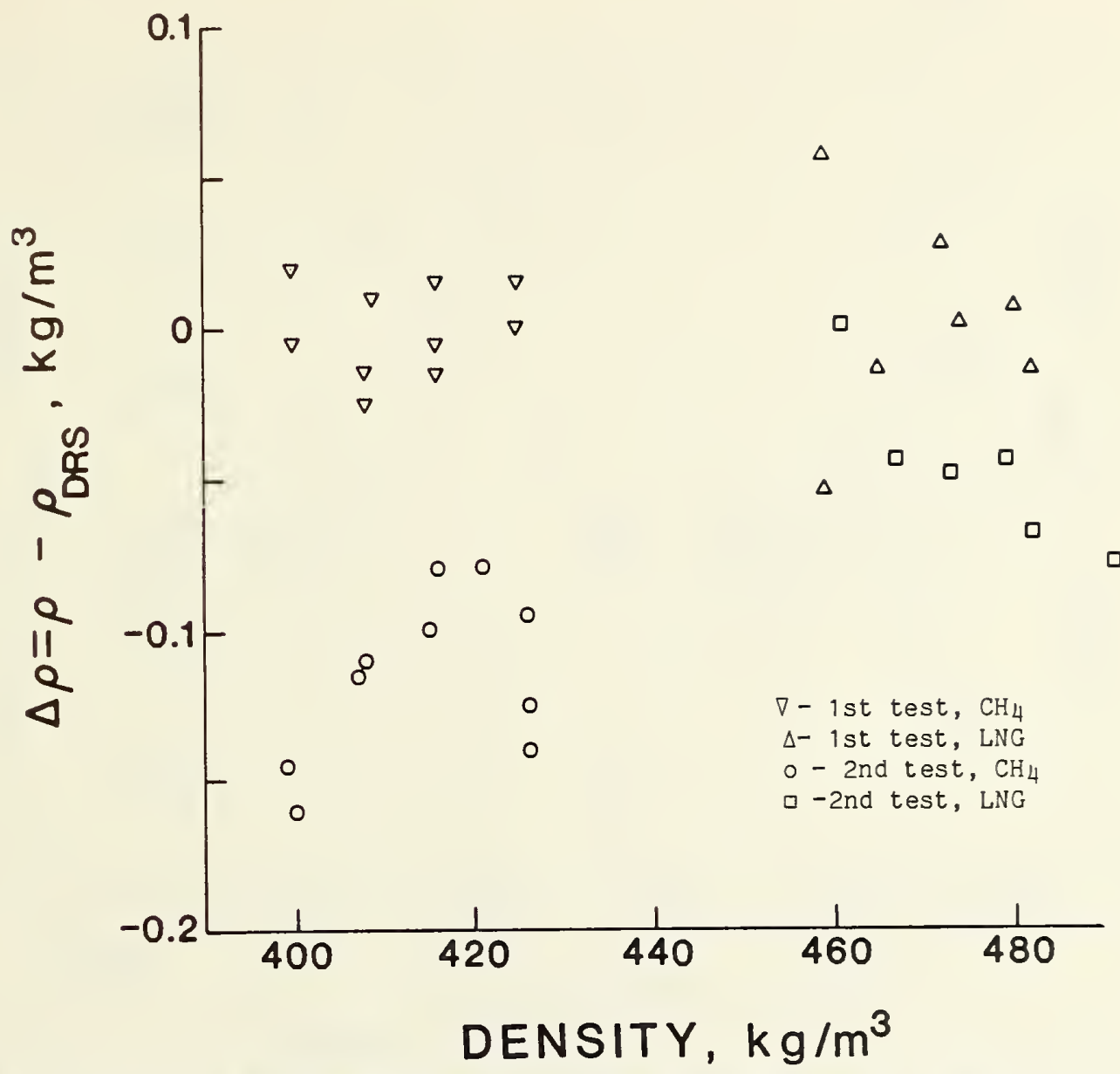

Figure 2.4.2.7. Comparison of uncalibrated displacement densimeter with NBS DRS densimeter.

Both of the instruments retested were cooled to $77 \mathrm{~K}$ a number of times in the one and a half years between tests. Only 非 2 was operated when cold and also used in other liquids. The original test data of $\equiv^{2}$ along with the retest data are shown in figure 2.4.2.8. The calibration changed. The results for densimeter $\#_{1}$ are shown in figure 2.4.2.9. This densimeter has held its calibration to within the estimated uncertainty over the elapsed year and a half. Densimeter 非 showed better stabll1ty and precision in the first tests. Whether the poorer stability of 非 or its greater use over the year and a half is responsible for its calibration shift is not known.

Both this vibrating cylinder densimeter and the displacement densimeter, for selected instruments at least, can be provided with calibrations precise to better than 0.1 percent and stable to better than 0.1 percent over the limited sequence of tests described here. Further testing is needed. Thls testing should be done on instruments installed in the field.

The results of the tests of the commercial densimeters in LNG demonstrate that the densimeters should be calibrated in the liquids in which they are to be used. Assuming a calibration equation with $n$ coefficients, then using only $\mathrm{n}$ calibration points is also hazardous though often done. Th1s method is not satisfactory unless the densimeter has been thoroughly tested over the range of liquids and operating conditions to which it will be subjected and has adequately demonstrated its insens1tiv1ty to parameters absent from the calibration equation. Based on these LNG tests, extrapolation of densimeter calibrations via the calibration equations to untested liquids is inadvisable.

The stability of densimeters over time and use has been touched upon in this work. but surricient information is not presently available to establish the long-term stability of any of the Instruments tested. 


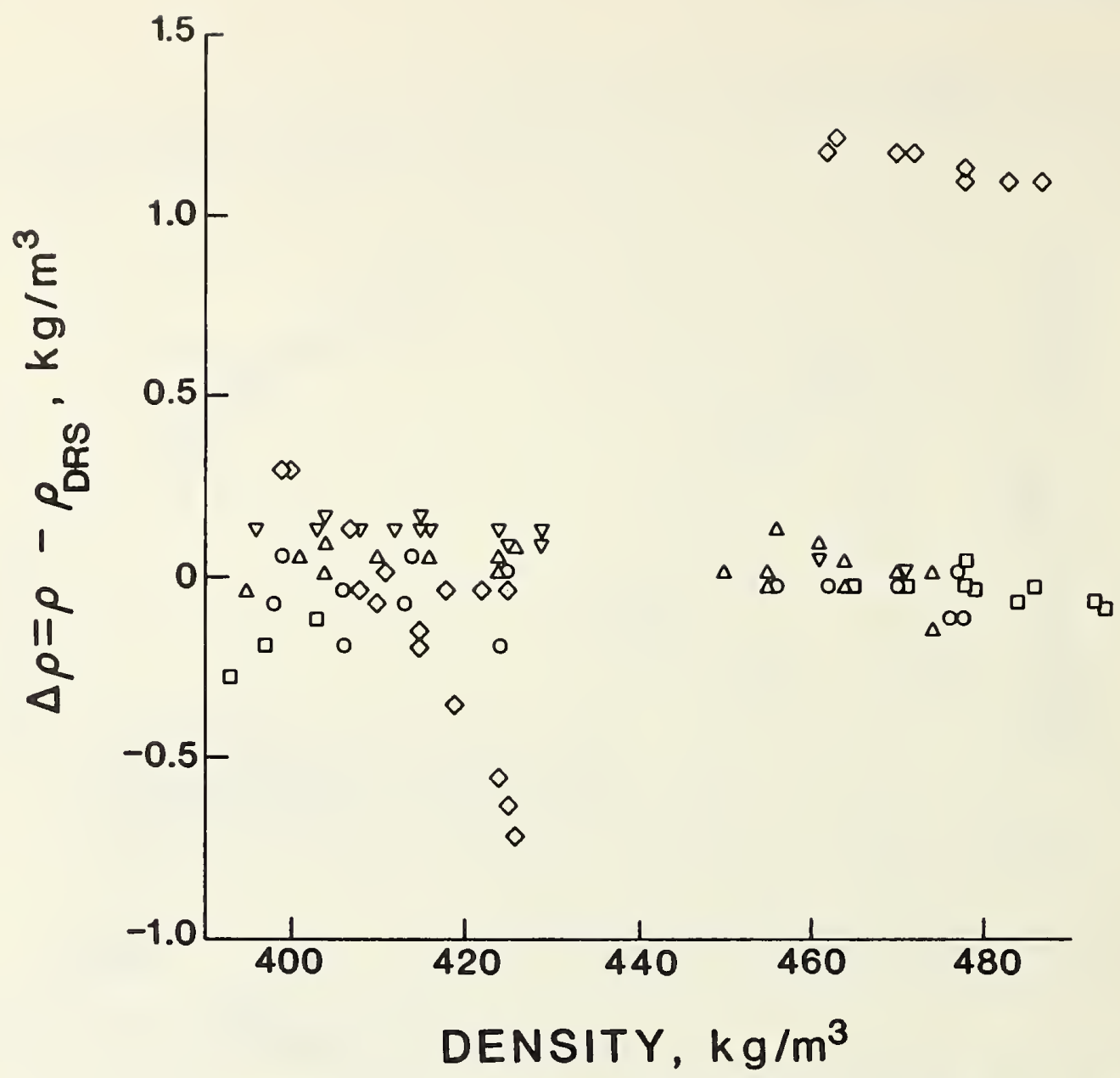

Figure 2.4.2.8. Comparison of uncalibrated vibrating cylinder densimeter 2 to NBS DRS densimeter. The calibration was provided from the first four tests, shown by symbols $\nabla, \Delta, 0$, and $\square$. The retest is shown by $\diamond$.

\subsubsection{Calibration}

The tests of the various commercial densimeters emphasized the need for an accurate calibration method. The density reference system provides a means of accurately calibrating densimeters, especially those used in refrigerated gas service. The DRS cannot provide and maintain calibrations of all cryogenic densimeters but can maintain a few instruments as transfer standards which can be used to calibrate and maintain field instruments. Densimeters of the DRS densimeter design could be used as portable standards, and two additional silicon single crystal densimeters have been built for this purpose.

The first versions of the DRS densimeter used a balance that did not have sufficient range to weigh the silicon to a milligram, so a reference weight had to be used. A more sensitive balance became commercially available, and one was installed in the DRS densimeter. The density measured for a pure methane sample can also be compared to the density calculated from the average sample temperature using the Haynes-Hiza relationship of density as a function of temperature [13].

Figure 2.4.2.10 shows the difference between the measured density and that calculated from temperature before and after the new balance was installed. The difference between the mean levels for the two different balances used in the DRS densimeter is within its \pm 0.028 percent systematic error uncertainty estimated for the first balance. The DRS now measures a density for liquid methane about 0.1 percent less than the values obtained by Haynes and Hiza [13]. The estimated uncertainty for one measurement is \pm 0.055 percent with the new balance [7]. 


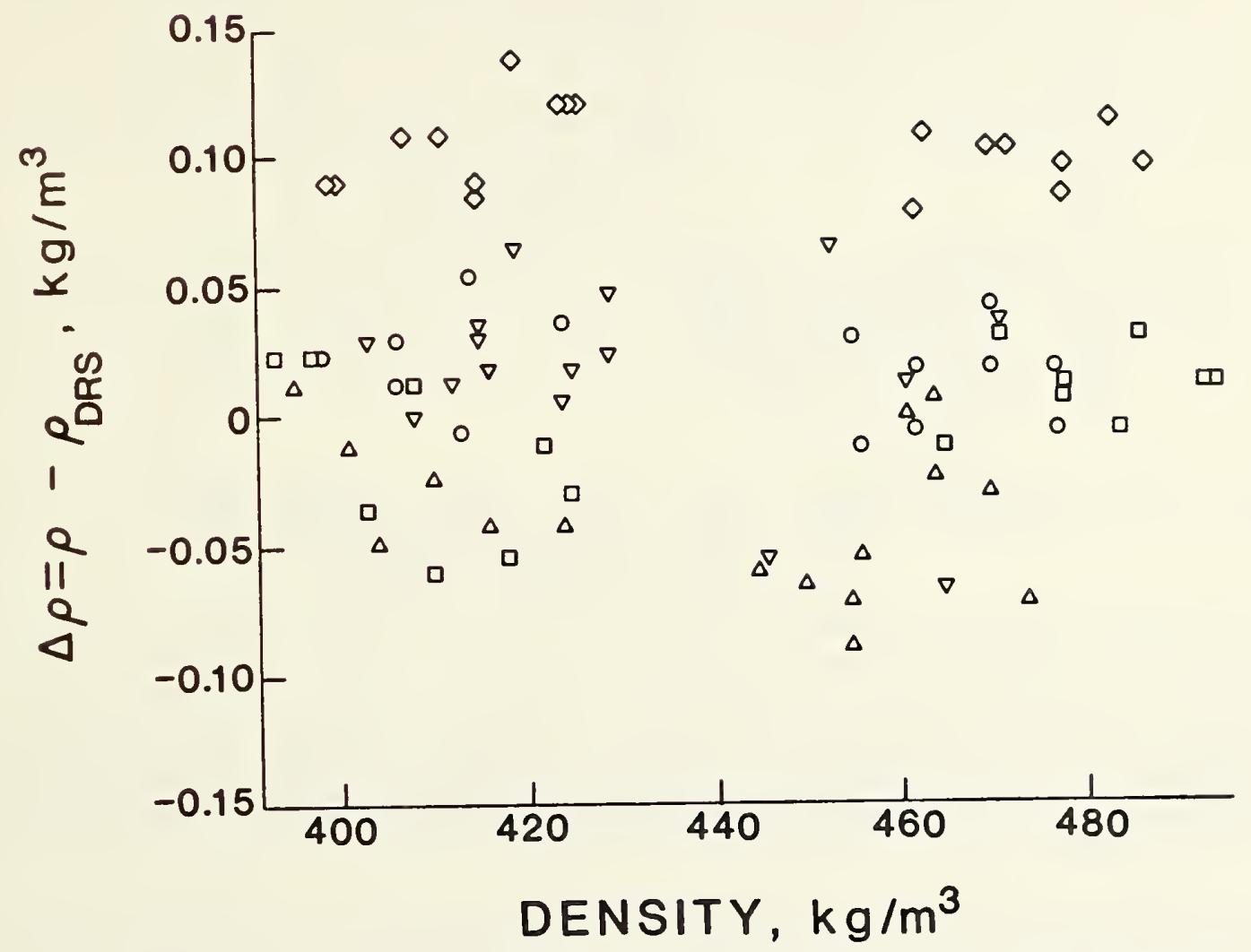

\begin{abstract}
Figure 2.4.2.9. Comparison of uncalibrated vibrating cylinder densimeter 1 to NBS DRS densimeter. This was tested at the same time as 2 and the symbols are the same as for figure 2.4.2.8.
\end{abstract}

The reference weight was retained from the earlier version of the densimeter but now serves as a means of calibrating and monitoring the calibration during measurements. Liquid methane density measurements by temperature and a silicon densimeter in an entirely different apparatus confirmed the approximately 0.1 percent of fset as will be discussed below.

Based on the accuracy to which silicon density is known and the accuracy of the balance, the silicon densimeter can now be expected to have combined systematic errors within \pm 0.01 percent. Two additional silicon densimeters have been built and both tested in the DRS. The first had a different balance and used the reference weight method. This instrument was built by an LNG transporter to calibrate densimeters for shipboard use. The results of the tests in the DRS were reported in [7]. The difference of the measured densities was less than 0.01 percent with no adjustable parameters. Within the limits of statistical error, the densities measured by the two densimeters were the same.

Calibration installations need to be compared to obtain a worldwide conformity in dens!ty measurement. Also, when a calibration facility requires calibration, the best calibration is obtalned by installing a standard in it, thereby calibrating the combination of facility and calibration densimeter rather than just the calibration densimeter.

The new balance installed on the DRS densimeter was found to be both compact and rugged, two necessary requirements for a portable unit. Such a unit has been designed and built [15]. It differs from the DRS densimeter mainly in that the calibration weight and silicon crystal are both held capt 1 ve when lifted from the balance suspension. The results of testing this instrument in the DRS are shown in figure 2.4.2.11. 


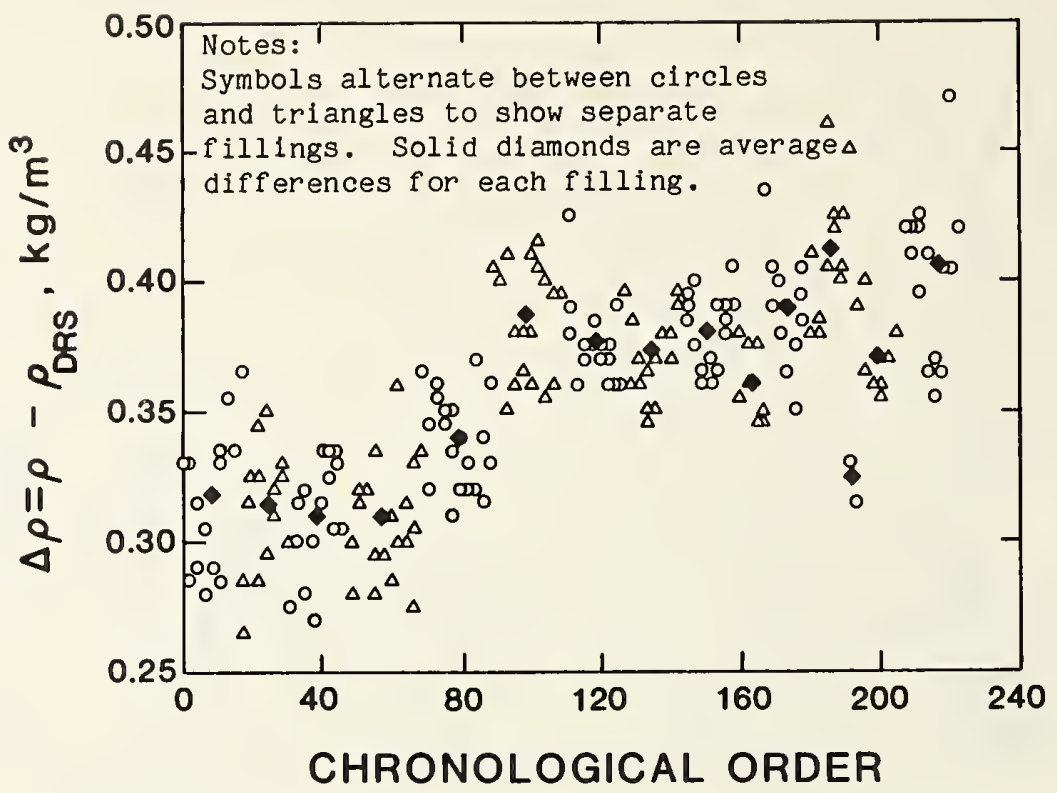

Figure 2.4.2.10. Differences between measured and calculated density.

The densities measured by the DRS densimeter and the portable reference densimeter (PRD) are indistinguishable. The nearly identical results with three Archimedes densimeters using silicon single crystals suggest that the systematic difference between these densities and densities from the Haynes-Hiza results is not an error in weighing the crystal.

The PRD has been used once to compare the DRS and the LNG densimeter test system [14] of Gaz de France (GDF) located near Paris at the Centre d'Etudes et de Recherches sur les Installations Gazie. The PRD was air freighted from Denver, Colorado to Paris. The balance required an adjustment for the change in the acceleration of gravity between Paris and Boulder. Otherwise the densimeter was unaffected.

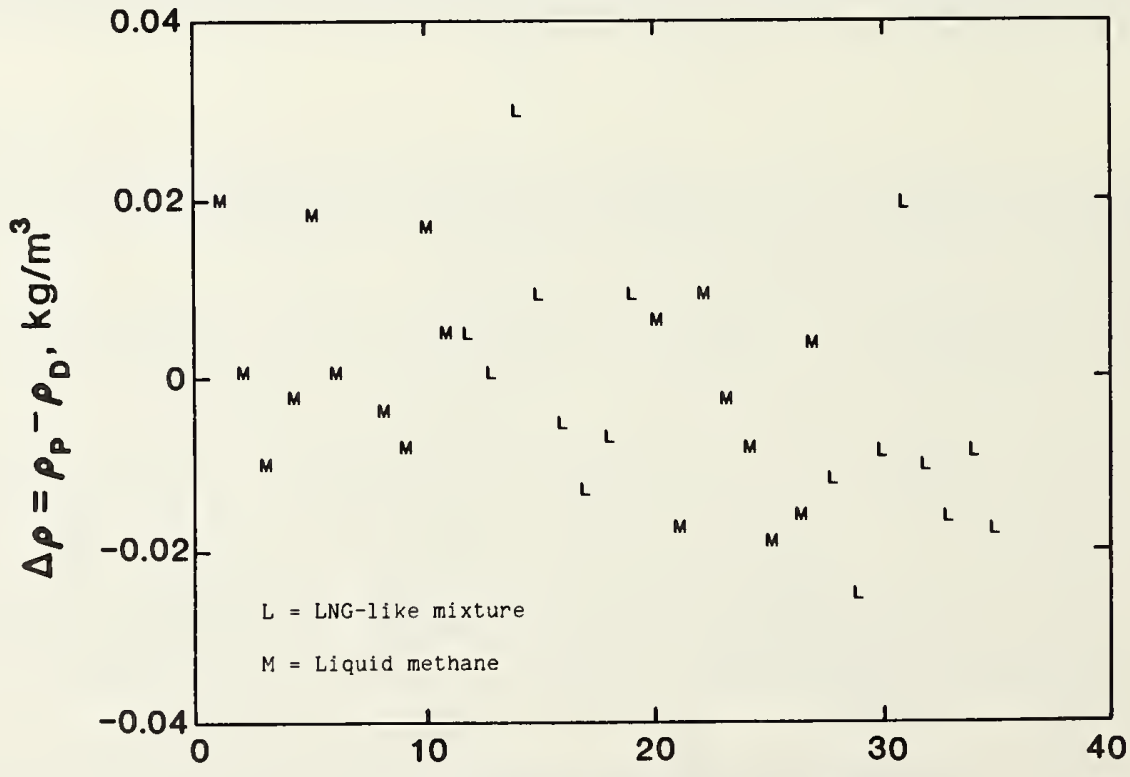

\section{CHRONOLOGICAL ORDER}


The PRD was adjusted and installed by NBS personnel then operated over a period of five months by Gaz de France personnel with no difficulty during tests in pure liquid methane and LNG-like mixtures. The GDF test facility uses density calculated from temperature for their true methane density and the density calculated from temperature and composition, using the extended corresponding states method for mixtures. The density from temperature in pure methane was about 0.12 percent higher than that given by the PRD. This tends to confirm the difference of 0.1 percent found with the DRS, see figure 2.4.2.10. The reason for this systematic difference has not been traced. The present agreement of the sllicon densimeters seems to point toward a systematic error in the $\rho(T)$ equation of Haynes-Hiza $[13]$.

The random uncertainty in the comparisons in liquid methane was within \pm 0.05 percent, while the random uncertainty for the mixture data was within \pm 0.25 percent ( 95 percent of the values 1 ie within these limits). The larger part of this uncertainty is assumed to arise from the sampling and analysis of the mixture to determine composition. This uncertainty may be even larger outside of the laboratory, as Parish [16] found in his heating value studies.

\subsubsection{Conclusions}

The testing and calibration of densimeters for use in liquefied natural gas has been the object of the densimeter studies at NBS. To do this study, a calibration system and accurate self calibrating densimeters suitable for a density standard were developed.

Though the commercial densimeters tested all functioned in LNG, that is, all showed unambiguous variations in the parameter affected by density variations, they all required calibrations in liquids of known density and were found to have inadequate calibrations. Their calibration error was about ten times larger than their precision. The calibration error arose because the densimeter was sensitive to parameters not included in the calibration equation and also because the densimeters were never calibrated in LNG or methane for reasons of safety. Tests of calibration stability with time and use, performed on some of the commercial densimeters, indicated they had short term stability. The measurements of longer term stability were not done for some and were inconclusive for those for which some tests were done.

Though the measurement of LNG density must be done under the severest environmental conditions, it is probable that similar problems are incurred even at ambient temperatures. For instance, commercial densimeters for ambient temperature measurement are of en not calibrated at actual operating conditions and at only sufficient points to determine unambiguously the constants of the assumed calibration equation.

The density standard developed has definite advantages over the pycnometer standard [2] for cryogenic liquids for safety reasons. The measurement vessel has little potential of exploding. It could offer advantages over the pycnometer for ambient temperature density measurement because, as an example, any contamination of the density sensor that would cause an erroneous measurement is visible. Whether the electronic balance used to weigh the silicon would maintain its accuracy at the high pressures encountered in the measurement of natural gas liquids has yet to be established. The electronic balance used in the present instruments is expected to be insensitive to the pressures often encountered when measuring densities of such liquids. 


\subsubsection{References}

[1] Siegwarth, J. D. and J. F. LaBrecque, Calibration of densimeters for liquefied light hydrocarbons, $0 i 1$ \& Gas Journal, pp 64-9 (December 20, 1982).

[2] API Manual of Petroleum Measurement Standards, Chapter 14, Section 6, Installing and proving density meters used to measure hydrocarbon liquid with densities between 0.3 to $0.7 \mathrm{gm} / \mathrm{cc}$ at $15.56^{\circ} \mathrm{C}\left(60^{\circ} \mathrm{F}\right)$ and saturation vapor pressure, (1979).

[3] Bowman, H. A., R. M. Schoonover and M. V. Jones, Procedure for high precision density determinations by hydrostatic weighting, J. Research, NBS, 71C, 178 (1967).

[4] Henins, I. and J. A. Bearden, Silicon crystal determination of the absolute scale of x-ray wavelengths, Phys. Rev. 135, A890 (1964).

[5] Corruccini, R. J. and J. J. Gniewek, Thermal expansion of technical solids at low temperatures, Nat. Bur. Stand. (U.S.), Monograph 29, (May, 1961).

[6] McSkimin, H. J., Measurement of elastic constants at low temperatures by means of ultrasonic waves--data for silicon and germanium single crystals and fused silica, J. Appl. Phys. 24,988 (1953).

[7] Siegwarth, J. D., B. A. Younglove and J. F. LaBrecque, Cryogenic fluids density reference system: provisional accuracy statement (1980), Nat. Bur. Stand. (U.S.), Technical Note 1041 $(1981)$.

[8] Siegwarth, J. D., B. A. Younglove and J. F. LaBrecque, Cryogenic fluids density reference system: provisional accuracy statement, Nat. Bur. Stand. (U.S.), Technical Note 698 (November, 1977).

[9] Siegwarth, J. D. and J. F. LaBrecque, An evaluation of commercial densimeters for use in LNG, Nat. Bur. Stand. (U.S.), Technical Note 697 (October, 1977).

[10] Siegwarth, J. D., J. F. LaBrecque and B. A. Younglove, Test of densimeters for use in custody transfer of LNG, Proceedings, 53rd International School of Hydrocarbon Measurement, Norman, Oklahoma, p 385(1978).

[11] Siegwarth, J. D. and J. A. Brennan, LNG instrumentation for custody transfer, AGA Operating Section Proceedings, Proceedings of the Transmission Conference, Atlanta, Georgia, T-119 (1981).

[12] Siegwarth, J. D. and J. F. LaBrecque, Tests of commercial densimeters, Nat. Bur. Stand. (U.S.), Technical Note 1055 (June, 1982).

[13] Haynes, W. M. and M. J. Hiza, Measurements of orthobaric liquid densities of methane, ethane, propane, isobutane and normal butane, J. Chem. Thermodynamics 9, 179, (1977).

[14] Dewardt, F. and P. Mousset, Contribution a l'Amelioration de Comptage du GNL - Evaluation de densimetres et echantillonneur pour GNL, Sixth International Conference on Liquefied Natural Gas, Appendex Paper 8, Kyoto, Japan (1980).

[15] Siegwarth, J. D. and J. F. LaBrecque, A portable calibration densimeter for use in cryogenic liquids, Nat. Bur. Stand. (U.S.), Technical Note 1035 (1981).

[16] Parrish, W. R., J. M. Arvidson and J. F. LaBrecque, Development and evaluation of an LNG sampling measurement system, Nat. Bur. Stand., NBSIR 78-887, (July, 1978).

[17] Handbook of Physics, Condon, E. U. and N. V. Frederick, Editors, 4-112, Mcgraw-Hill Book, Inc. (1967). 


\subsubsection{Calculated LNG Density}

\subsubsection{General Comments}

The measurement process by which the density of the LNG mixture is calculated from measurements of other properties, such as pressure, temperature and constituent fraction is considered in this section of the manual. The process requires some type of mathematical structure which relates the measured properties to that of density. In the case of LNG, the structure can vary from a purely empirical relationship to very complex models having extensive theoretical basis.

A recently completed program, which focused exclusively on LNG mixture density, has provided extensive, accurate and precise experimental data upon which both empirical and theoretical models were optimized and tested. Haynes, McCarty and Hiza [51] have summarized the work of this program, and this edited summary is reproduced in the following sections. The program was truly an international effort by natural gas companies and agencies who supported and guided the research.

The report summarizes the results of a project concerning the densities of liquefied natural gas (LNG) and its components. This project was initiated in the Properties of Fluids Section of the Cryogenics Division of the National Bureau of Standards in July, 1972 and was carried out under the sponsorship of a consortium of eighteen energy companies, through a grant administered by the American Gas Association, Inc.

The experimental part of this project has included the following accomplishments: (a) development of a magnetic suspension densimeter for absolute density measurements on liquids, including liquid mixtures in equilibrium with their vapor, at temperatures from 90 to $300 \mathrm{~K}$; (b) orthobaric 1 iquid density measurements on the major components of LNG, which include nitrogen ( $95-120 \mathrm{~K}$ ), methane $(105-160 \mathrm{~K})$, ethane $(100-270 \mathrm{~K})$, propane $(100-288.7 \mathrm{~K})$, isobutane $(115-300 \mathrm{~K})$, and normal butane (135-300 K); (c) orthobaric liquid density measurements on approximately thirty-five binary mixtures of the above components for all combinations except nitrogen + butane systems, primarily in the temperature range of 105 to $130 \mathrm{~K}$; and (d) orthobaric liquid density measurements on twenty-seven multicomponent mixtures $(105-120 \mathrm{~K})$, including several LNG-like mixtures with up to eight components. The total uncertainty of a single density measurement is approximately 0.1 percent at low temperatures and decreases to approximately 0.06 percent at room temperature. The estimated standard deviation of a single density measurement is less than 0.02 percent.

The density data have been used to optimize, test, and compare several mathematical models as to their suitability for the calculation of LNG densities for custody transfer. Models selected for optimization and testing included an extended corresponding states method, a hard sphere model, a cell model, and an empirical model by Klosek and McKinley. The ultimate goal of this project was to produce one or more mathematical models that could be used to predict the density of any LNG mixture to within an uncertainty of 0.1 percent from an input of pressure, temperature, and composition. Each of the models investigated here satisfies this goal within their inherent limitations. The limitations and ranges of validity of the various models are discussed. Also presented are techniques for predicting LNG densities from dielectric constant measurements and from excess volume calculations.

The last section of the original publication [51] provides a complete and detailed account of the results of the project. This section is not included as a part of this measurement manual, 3 and the reader is referred to the program summary papers which are references 1-4.

\section{4 .3 .2 Introduction}

During the past decade liquefied natural gas has become an increasingly important commodity on the world energy market. This trend is expected to continue into the foreseeable future. In the buy 1 . and selling of LNG, the basis of custody transfer is its heating value. The determinat $10 n$ or the heating value of LNG requires a knowledge of its density, which in turn $1 \mathrm{~s}$ dependent upon 1 ts composition, temperature, and pressure. Since, for example, an error of one percent in dens 1 ty can result in an inequity of between $\$ 100,000$ and $\$ 200,000$ (at 1983 prices) per $125,000 \mathrm{~m}^{3} \mathrm{sh} 1 \mathrm{pload}$ of LNG, the accuracy to which the density of the liquid can be determined is extremely $1 \mathrm{mportant} 1 \mathrm{n}$ transactions involving LNG. A one percent error in density was not uncommon at the time th1s project was initiated.

There are, at least, two means for determining the density of a large volume of LNG. One method $1 \mathrm{~s}$ by direct field measurements using commercially available densimeters. A second method cons 1 stg of the use of a mathematical model or correlation to calculate a density based on direct measurements or the liquid composition and temperature. 
This report [51] has been concerned with the determination of the density by the second method. In actual transfer situations it is likely that both methods will be used to compliment each other. By either method, the accuracy to which the density can be determined is limited by both practical and state-of-the-art considerations. This has resulted in setting a goal of 0.1 percent for the total uncertainty in the determination of the density of LNG.

In July 1972, a project was initiated with an ultimate goal of providing one or more mathematical models that could be used to calculate the density of any LNG mixture with a total uncertainty of less than 0.1 percent based on a knowledge of the pressure, temperature, and composition of the liquid mixture. The mathematical models would be developed and/or optimized using density data for the major components of LNG and for binary mixtures of these components. Density data for multicomponent mixtures of LNG components would be used to evaluate and compare the performance of the mathematical models. Some of the test mixtures would be selected to simulate commercial LNG compositions. LNG consists primarily of methane (concentration level typically greater than 75 percent) with lesser amounts of ethane (up to 15 percent), propane (up to 5 percent), butanes (up to 2 percent), nitrogen (up to 2 percent), and pentanes plus heavier hydrocarbons (up to 0.5 percent).

The accuracy of a calculational technique based on experimental data can be no better than the input data from which the technique is developed. At the time this project was initiated, comparisons of saturated (orthobaric) liquid density data for the pure components of LNG exhibited differences as large as 0.5 percent, while each investigator was generally claiming inaccuracies of 0.1 percent or less. Furthermore, there were only a few sets of published data for liquid mixtures containing LNG components, especially at low temperatures. These data were generally limited in scope or had claimed inaccuracies larger than the desired 0.1 percent. (The pure fluid and mixture data for LNG components from other investigations are summarized in section 2.4.3.8 of this report.) Thus, a major task of the LNG density project was to provide an accurate and internally consistent set of density data for the major components of LNG and for mixtures of these components. (These data could also serve as a basis for calibration or development of gauging and metering methods of LNG.)

This project was sponsored by a consortium of eighteen international energy companies, five of which were from countries outside the United States. The sponsors represented both buyers and sellers of LNG. The American Gas Association, Incorporated has provided administration of project funds. In carrying out this research project, the National Bureau of Standards was serving in its traditional role as an independent third party. All results of this project would be published in the open literature and hopefully gain wide acceptance throughout the LNG industry, since qualified sponsor representatives from all parts of the international LNG market would have closely followed the progress of this project throughout its entirety.

\subsubsection{Major Accomplishments-Experimental Apparatus}

The experimental technique selected for performing the density measurements for this project had to satisfy relatively stringent criteria. It must be capable of absolute density measurements of high accuracy and precision on a liquid, including mixtures, in equilibrium with vapor at cryogenic temperatures and at pressures to approximately $4 \mathrm{MPa}$ using an optical cell. A magnetic suspension densimeter based on an application of Archimedes' principle was developed for this purpose.

Two apparatuses $[5,6]$, each incorporating a magnetic suspension technique in the density determination, were constructed for the measurements on liquids, including liquid mixtures. For both apparatuses, the total uncertainty of a single density measurement, which is taken as three times the standard deviation plus the systematic error, is approximately 0.1 percent at low temperatures and decreases to approximately 0.06 percent at room temperature. The estimated standard deviation of a single density measurement is less than 0.02 percent.

A brief description of the method for determining densities with either apparatus is as follows. A small magnetic buoy is freely suspended in the fluid whose density is to be determined. The density of the buoy is significantly greater (by approximately an order of magnitude) than the density of the fluids investigated here. The force necessary to lift the buoy is supplied by one or more air-core solenoids. The buoy is maintained at a stable (vertical) position through the automatic regulation of an electronic servocircuit containing the lift coil and either a differential transformer [5] or a differential capacitor [7] that detects the motion of the buoy. The horizontal position of the buoy is maintained by the axially symmetrical, diverging field of the lift coil.

When the buoy is supported at the same position in vacuum and in the fluid of interest, the upward magnetic force on the buoy supplied by the air-core solenoids gives a means for measuring the density of the fluid, since this is the force that must be added to the buoyant force to balance the downward 
gravitational force. Thus, the density is deduced from measurements of the currents in the support colls necessary to support the buoy in vacuum and in the fluid of interest at the same temperature and at the same vertical position (determined with a $125 \mathrm{X}$ microscope).

The mass and volume of the buoy must be determined from independent measurements. The mass was determined using an analytical microbalance. The volume of the buoy at room temperature was determined by using distilled water as a reference fluid of known density. Thermal expansion data [8] for barlum ferrite, the material from which the buoy was fabricated, were obtained so that the volume of the buoy at low temperatures could be calculated.

The first apparatus [5] could be used at temperatures between 90 and $300 \mathrm{~K}$ and at pressures to 5 MPa. After initial tests with a three-coil arrangement to support the buoy, the densimeter was simplified considerably by reducing to a system employing only one coil [9]. The second apparatus [6], which employed exactly the same technique for determining density, was significantly different from, and more versatile than, the first apparatus. The second apparatus contained a concentric cylinder capacitor that was used to make dielectric constant measurements on the same liquid samples for which density data were obtained. The major reason for the construction of the second apparatus was a need in other research projects (e.g., PVT and dielectric constant measurements on liquid propane [10], isobutane [11], and normal butane [12]) for a higher pressure capability (35 MPa) for the magnetic suspension densimeter. The extension to higher pressures was not needed in the LNG density project.

It should be noted that the second apparatus gave no improvement over the first in the accuracy to which the density could be determined. The consistency of the density data from both apparatuses should be equivalent to that for either apparatus alone. To insure that this was the case, measurements on liquid methane were used as a control throughout the project with both apparatuses.

\subsubsection{Experimental Measurements- Pure Fluid Data}

The first experimental measurements for the LNG density project consisted of the acquisition of orthobaric liquid density data for the major components of LNG. Comprehensive results were obtained for nitrogen $(95-120 \mathrm{~K})$ [5], methane $(105-160 \mathrm{~K})[5,13]$, ethane $(100-270 \mathrm{~K})$ [13], propane (100-288.7 K) [13], isobutane $(115-300 \mathrm{~K})$ [13], and normal butane $(135-300 \mathrm{~K})$ [13, 14]. Detailed comparisons between the data from this project and independent results demonstrated the need for an accurate and internally consistent set of data for the major components of LNG. Differences as large as 0.5 percent between the density data of independent investigators were common. At the time this project was initiated, there were no published data for normal butane at low temperatures. (In the course of evaluating the performance of the magnetic suspension densimeter, density data were also obtained for saturated liquid argon (100-120 K) and ethylene (105-200 K) [15].)

\subsubsection{Binary Mixture Data}

Orthobaric liquid density measurements were then carried out on thirty-five binary mixtures [16-18] containing the major components of LNG. Most of the binary mixture data were taken in the temperature range of 105 to $140 \mathrm{~K}$. All binary combinations of the six major components of LNG were invest1gated in this project, with the exception of the nitrogen + isobutane and nitrogen + normal butane systems.

Prior to the LNG density project, no low temperature, liquid density data could be found in the literature for the following systems: nitrogen + ethane, nitrogen + propane, ethane + 1sobutane, propane + isobutane, propane + normal butane, and isobutane + normal butane, all of which have been investigated under this program. All of the binary mixture measurements were carried out on liquld samples condensed into the cell from gravimetrically prepared gas mixtures. This was considered to be the most accurate method to fix the compositions of the liquid mixtures. For all mixtures containing nitrogen and/or methane, total vapor pressures have also been measured. For the methane-rich binary mixtures containing either isobutane or normal butane, dielectric constant data [17] were obta1ned simultaneously with the density results.

Extensive comparisons of the binary mixture data from this study have been made with avallablo literature data. In general, differences were less than 0.1 percent, except that the dens1ty data ror the ethane + propane system exhibited approximately an 0.8 percent discrepancy when compared w1th tho data of Shana'a and Canfield [19]. This was inconsistent with comparisons with the same authors ror the methane + ethane and methane + propane systems as well as for pure fluid results, where tho agreement was typically better than 0.1 percent. 


\subsubsection{Multicomponent Mixture Data}

In order to evaluate and test the mathematical models that have been developed and optimized using the binary mixture and pure component data, orthobaric liquid density measurements were performed on twenty-seven multicomponent mixtures $[20,21]$ of LNG components, primarily in the temperature range of 110 to $120 \mathrm{~K}$. The multicomponent mixtures investigated in this project ranged from ternary mixtures containing methane and binary combinations of nitrogen, ethane, propane, and normal butane to four to eight component methane-rich ( $75-90$ mole percent) mixtures containing up to 5 mole percent nitrogen, 15 mole percent ethane, 7 mole percent propane, 5 mole percent butanes, and 0.44 mole percent pentanes.

The compositions of some of the six to eight component mixtures were selected to simulate commercial LNG mixtures. The compositions of other multicomponent mixtures were chosen to provide severe tests of the mathematical models and to complement the use of binary mixture data in the optimization of the models. Except for three of the multicomponent mixtures, the compositions were determined from the preparation of the gas mixtures by gravimetric means. For the other three mixtures, the compositions were determined by gas chromatographic analysis using a thermal conductivity detector. The chromatograph was calibrated with a gravimetrically prepared mixture. Vapor pressure data have also been obtained for the multicomponent mixtures. For those multicomponent mixtures investigated with the second apparatus, dielectric constant measurements [22] were performed simultaneously with the density measurements.

Although the pure fluid and binary mixture measurements from this project have not included data for pentanes, five multicomponent mixtures containing pentanes [21] have been investigated. It was thought that densities of these systems could be predicted with the mathematical models using existing pure fluid data for the pentanes from other sources [23,24] and predicting the interaction parameters for binary mixtures containing pentanes from the behavior of the binary mixtures studied in the LNG density project. (Expressions for representing the pentane data of orrit and Laupretre [24] are reported by Hiza [25].) High accuracy in these predictions is not required, since LNG mixtures contain relatively small fractions of pentanes.

\subsubsection{Mathematical Models}

As mentioned earlier, one method of determining the density of LNG is to predict that density with a mathematical model of the equation of state, given the pressure, temperature, and composition of the LNG. The ultimate goal of this study was to produce such a model (or models) that would be accurate to within 0.1 percent of the true density. The scope of this study was limited to saturated liquids over a temperature range of 105 to $140 \mathrm{~K}$ with pressures to $2 \mathrm{MPa}$. The components of LNG were assumed to be nitrogen, methane, ethane, propane, isobutane, normal butane, isopentane and normal pentane.

Four different models were chosen to fit to the experimental data. Each of the selected models represent a different approach to modeling the equation of state of a fluid. These are an extended corresponding states model [26-27], a hard sphere model [28], a cell model [29-31], and a graphical model by Klosek and McKinley [32]. During the course of optimizing the four models using the experimental data, it became necessary to revise the functional form of the model proposed by Klosek and McKinley. No revisions of the functional form of the other three models were necessary, and only the adjustable parameters were changed to achieve the desired fit of the experimental data. The final result was that all four of the models originally chosen can be used to predict the density of LNG to within 0.1 percent of the true density, given the temperature, pressure, and composition of the LNG. Details of the fitting procedures, comparisons to experimental data, and computer program listings are given in references [33-35].

Also during the course of this study, an excess volume model was developed by Hiza [25]. This model also achieves the 0.1 percent criteria outlined above under the same restrictions as the other models. Lastly, a model has been developed by Haynes and McCarty [22] that does not require either the temperature or pressure as input information (all of the other models require a minimum of temperature as input and most require both temperature and pressure). This model requires an input of dielectric constant and composition of the LNG and predicts the density to within 0.15 percent of the experimental value.

All of the models have restrictions on pressure, temperature, and composition ranges, which must be defined for each model. The models are of widely ranging computational complexity. The selection of a particular model is dependent on the needs and objectives of the individual user. However, on the basis of the accuracy of the calculated density of a commercial LNG mixture, each of the models appears to be equally acceptable. 
One final comment about accuracy should be emphasized. No mathematical model of the equation of state can be more accurate than the experimental data to which it has been fit. Therefore, all of the accuracy claims are dependent upon the accuracy of the experimental data from this study, which were used almost exclusively to optimize and test the models.

\subsubsection{LNG Density Research at other Laboratories}

Because of the widespread interest in reliable data and the prediction of properties of multicomponent liquefied natural gas mixtures, in particular saturated liquid (bubble point) densities, a number of independent research programs were conducted from which a significant amount of density results have been published in the open literature. These include the studies at Gaz de France (Morlett [36]), Air Products and Chemicals, Inc. (Klosek and McKinley [32]), Institute of Gas Technology (Gonzalez, et al. [37,38], Huebler, et al. [39]), University of Kansas (Huang, et al. [40], Jensen and Kurata [41]), University of Oklahoma (Shana'a and Canfield [19]), Shell Research, Ltd. (Boyle and Reece [42], McClune [23]), University of Wyoming (Miller, et al. [28,43-46]), and Societe Nationale Elf Aquitane (Roche, et al. [47,48], Orrit and Laupretre [24,49], and Orrit [50]).1 There is considerable variation in the precision and accuracy of measurement and in the completeness of the data published from these studies. Therefore, the contributions of these programs to the precise knowledge of the density behavior of LNG mixtures are also quite variable.

From comparisons with these independent results, it appears that the data from the University of Wyoming are the most precise and are uniformly consistent with the results of the NBS program. Direct comparisons of measurement precision were also made between the NBS densimeter and the Wyoming apparatus. Measurements were made at the University of Wyoming on one binary mixture and four multicomponent mixtures, prepared at NBS, with the apparatus calibrated with pure methane densities determined with the NBS densimeter [46]. Densities for the binary mixture and three of the multicomponent mixtures were also measured with the NBS densimeter. Comparisons were made through the extended corresponding states model when measured temperatures were not exactly the same. The maximum difference found was 0.10 percent for one multicomponent mixture point; the remaining differences were less than 0.03 percent and random. Though the Wyoming program was carefully planned, measurements for some of the important binary mixtures (e.g., nitrogen + ethane, nitrogen + propane, ethane + propane, etc.) were not included, and the accuracy of the molar volume results (though not that of derived excess volumes) depends directly on the accuracy of the density data of the low temperature liquid used to calibrate the apparatus.

The most extensive experimental measurements are those from the Elf Aquitane program. The precision of measurement does not appear to be quite as good as the measurements of the University of Wyoming, and there is a systematic bias of about -0.1 percent in a sample of multicomponent mixtures data [49] compared to the extended corresponding states model optimized to the NBS results. Though not all of the important binary mixtures were included in their study, it is notable that measurements were made on a nearly equimolar mixture of ethane + propane [50]. Excess volumes derived from these data and their pure fluid data between 105 and $140 \mathrm{~K}$ are in excellent agreement with those of the NBS program. Most of their $V E$ values are between -0.01 and $-0.04 \mathrm{~cm} 3 / \mathrm{mol}$ compared to -0.03 to $-0.05 \mathrm{~cm} 3 / \mathrm{mol}$ for a comparable mixture from the present study and about $-0.49 \mathrm{~cm} 3 / \mathrm{mol}$ from the University of Oklahoma data at $108.15 \mathrm{~K}$ for a mixture containing 58.52 mole percent ethane.

With the exception of this large discrepancy in the University of Oklahoma data for the ethane propane mixture, the remainder of the data from the University of oklahoma, as well as those from Shell Research, Ltd., are of comparable precision as those from the University of Wyoming and from Elf Aquitane. From the Shell Research, Ltd. program, only the pure component density data has been published. None of the remaining programs have provided data approaching the precision and accuracy goals desired for custody transfer.

The programs of Elf Aquitane and Shell Research, Ltd. provide the only low temperature experimental densities for pure isopentane and normal pentane, and these data sets are in good agreement. $1 . e$., within about 0.1 percent. With the small concentrations of pentanes normally encountered in Lif mixtures, and the fact that the densities from these investigations are generally within about o.

${ }^{1}$ Certain companies are identified in this paper for the purpose of clarlty only. Such identification does not imply any type of endorsement by the Nat1onal Bureau of Standards. 
percent of the NBS value for the lower molar mass alkanes, it was felt that additional measurements on the pure pentanes were unnecessary. In the NBS program, the pentanes were included only in multicomponent mixtures, with compositions much like LNG mixtures that would be encountered in practice. These measurements were made to provide proof that the mathematical models developed could properly account for the presence of pentanes.

Compared to the LNG research programs noted above, the NBS program is unique in that a single set of data are provided of uniformly high precision and accuracy for all of the pure components (i.e., nitrogen and the lower mass alkanes through the butanes), all the possible binary combinations where liquid phase separation does not occur, the important ternary combinations, and multicomponent mixtures that contain the highest probable amounts of nitrogen, butanes, and pentanes. The measurement method employed is a sophisticated state- of-the-art method for which a detailed analysis of the measurement uncertainties has been made and published, and statistical control of the experiments was assured throughout the program by repeating measurements of the density of liquid methane with each experimental run. The mathematical models optimized to the data are representative of existing theoretical and empirical models in wide use from the most simple to the most complex. The concurrent experimental and mathematical modeling efforts also served to guarantee that sufficient data were obtained to optimize and to identify the inherent limitations of each.

\subsubsection{Acknowledgments [51]}

The contributions of the members of the LNG Density Project steering Committee are gratefully acknowledged. The Steering Committee, comprised of representatives from each of the sponsoring companies, met quarterly from May 11, 1972 to February 15, 1979. The critique of research progress and numerous suggestions offered by the members were extremely valuable. Special thanks are due Martin R. Cines of the Phillips Petroleum Company who served as Chairman of the LNG Density Project Steering Committee during that time, and who, in fact, was chiefly responsible for obtaining financial support for this work. Thanks are also due Louis A. Sarkes of the American Gas Association for his contributions to this project and for his general support of LNG properties research. L. James Kemp of Southern California Gas Company assumed Chairmanship of the Steering Committee following the retirement of Martin R. Cines from Phillips Petroleum Company. 


\subsubsection{References}

[1] Haynes, W. M., M. J. Hiza, and R. D. McCarty, Densities of LNG for custody transfer. Proceedings of Fifth International Conference on LNG, 1977 August 29-September 1, Dusseldorf, Germany. Institute of Gas Technology, Chicago, Ill. Paper 11, Section III. 36 p.

[2] Haynes, W. M. and R. D. McCarty, The density of liquefied natural gas, Proceeding of the Fifty-E1ghth Annual (GPA) Convention of the Gas Processors Association, 1979 March 19-21, Denver, Colo. Gas Processors Association, Tulsa, Okla.

[3] Diller, D. E., LNG density determination, Hydrocarbon Processing 56(4), 142-144, 1977 April.

[4] McCarty, R. D. LNG densities for custody transfer. Proceedings of the Fifty-Sixth International School of Hydrocarbon Measurements, Norman, OK, pp 515-17 (April 14-16, 1981 ).

[5] Haynes, W. M.,M. J. Hiza and N. V. Frederick, Magnetic suspension densimeter for measurements on fluids of cryogenic interest, Rev. Sci. Instrum. 47(10), pp 1237-50 (October, 1976).

[6] Haynes, W. M., Apparatus for density and dielectric constant measurements to 35 MPa on fluids of cryogenic interest, J. Res. Nat. Bur. Stand. (U.S.), 84(4): 241-252; (July-August, 1983).

[7] Frederick, N. V. and W. M. Haynes, Differential capacitance sensor as position detector for a magnetic suspension densimeter, Rev. Sci. Instrum. 50(9),pp 1154-55 (September, 1979 ).

[8] Clark, A. F., W. M. Haynes, V. A. Deason and R. J. Trapani, Low temperature thermal expansion of barium ferrite, Cryogenics 16(3), pp 267-70, (May, 1976).

[9] Haynes, W. M., Simplified magnetic suspension densimeter for absolute density measurements, Rev. Sci. Instrum. 48(1), pp 39-41, (January,1977).

[10] Haynes, W. M., Measurements of densities and dielectric constants of liquid propane from 90 to $300 \mathrm{~K}$ at pressures to $35 \mathrm{MPa}$, J. Chem. Thermodynamics, 15(5): 419-424 (May, 1983).

[11] Haynes, W. M., Measurements of densities and dielectric constants of 1 iquid isobutane from 120 to $300 \mathrm{~K}$ at pressures to $35 \mathrm{MPa}$, J. Chem. Eng. Data. 28(4), pp 367-69 (0ctober, 1983 ).

[12] Haynes, W. M., Measurements of densities and dielectric constants of Iiquid normal butane from 140 to $300 \mathrm{~K}$ at pressures to $35 \mathrm{MPa}$, J. Chem. Thermodynamics, 15(9): 801-805 (September, 1983 ).

[13] Haynes, W. M. and M. J. Hiza, Measurements of the orthobaric liquid densities of methane, ethane, propane, isobutane, and normal butane, J. Chem. Thermodynamics 9(2), pp 179-87 (February, 1977)

[14] Haynes, W. M. and M. J. Hiza, Orthobaric Iiquid densities of normal butane from 135 to $300 \mathrm{~K}$ as determined with a magnetic suspension densimeter, Advances in Cryogenic Engineering, Vol $21, \mathrm{~K}$. D. Timmerhaus and D. H. Weitzel, ed., pp 516-21, Plemum Press, New York, NY (1976).

[15] Haynes, W. M., Measurements of the orthobaric liquid densities of argon (100-120 K) and ethylene (105-200 K), Cryogenics 18(10), pp 621-23 (October, 1978).

[16] Hiza, M. J., W. M. Haynes and W. R. Parrish, Orthobaric Iiquid densities and excess volumes ror binary mixtures of low molar-mass alkanes and nitrogen between 105 and $140 \mathrm{~K}$, J. Chem. Thermodynamics 9(9), pp 873-96, (September, 1977).

[17] Haynes, W. M., Orthobaric liquid densities and dielectric constants of (methane + 1sobutane) and (methane + normal butane) at low temperatures, J. Chem. Thermodynamics, 15(10): 903-911 (October, 1983).

[18] Hiza, M. J. and W. M. Haynes, Liquid mixture excess volumes and total vapor pressures using a magnetic suspension densimeter with compositions determined by chromatograph1c analys1s: methane plus ethane, Advances in Cryogenic Engineering, Vol 23, K. D. Timmerhaus, ed. pp 594-601, Plnn im Press, New York, NY (1976).

[19] Shana'a, M. Y. and F. B. Canfield, Liquid density and excess volume of light hydrocarbon mixtures at $-165 \mathrm{C}$, Trans. Faraday Soc. 64(549),pp 2281-2286 (September, 1968 ). 
[20] Hiza, M. J. and W. M. Haynes, Orthobaric liquid densities and excess volumes for multicomponent mixtures of low molar-mass alkanes and nitrogen between 105 and $125 \mathrm{~K}$, J. Chem. Thermodynamics 12(1), pp 1-10 (January, 1980).

[21] Haynes, W. M. Measurements of orthobaric-liquid densities of multicomponent mixtures of LNG components (N2, $\mathrm{CH} 4, \mathrm{C} 2 \mathrm{H} 6, \mathrm{C} 3 \mathrm{H} 8, \mathrm{CH} 3 \mathrm{CH}(\mathrm{CH} 3) \mathrm{CH} 3, \mathrm{C} 4 \mathrm{H} 10, \mathrm{CH} 3 \mathrm{CH}(\mathrm{CH} 3) \mathrm{C} 2 \mathrm{H} 5$, and $\mathrm{C} 5 \mathrm{H} 12$ ) between 110 and $130 \mathrm{~K}$, J. Chem. Thermodynamics 14(7), pp 603-12 (July, 1982).

[22] Haynes, W. M. and R. D. McCarty, Prediction of liquefied-natural-gas (LNG) densities from dielectric constant measurements. Cryogenics, 23(8): 421-426 (August, 1983).

[23] McClune, C. R., Measurement of the densities of liquefied hydrocarbons from -100 to $-180 \mathrm{C}$ (173 to $93 \mathrm{~K}$ ), Cryogenics 16(5), pp 289-95 (May, 1976).

[24] Orrit, J. and J. M. Laupretre, Density of Iiquefied natural gas constituents, Advances in Cryogenic Engineering, Vol. 23, K. D. Timmerhaus, ed., pp 573-79, Plenum Press, New York, NY (1978).

[25] Hiza, M. J., An empirical excess volume model for estimating liquefied natural gas densities, Fluid Phase Equilibria 2(2), pp 27-38 (August, 1978).

[26] Rowlinson, J. S. and I. D. Watson, The prediction of the thermodynamic properties of fluids and fluid mixtures - I. The principle of corresponding states and its extensions, Chem. Eng. Sci. 24(10), pp 1565-74 (October, 1969).

[27] Mollerup, J. and J. S. Rowlinson, The prediction of the densities of liquefied natural gas and of lower molecular weight hydrocarbons, Chem. Eng. Sci. 29(6), pp 1373-82 (June, 1974 ).

[28] Rodosevich, J. B. and R. C. Miller, Calculation of LNG excess volumes by a modified hard-sphere model, Advances in Cryogenic Engineering, Vol. 19, K. D. Timmerhaus, ed., pp 339-45, Plenum Press, New York, NY (1974).

[29] Renon, H., C. A. Eckert and J. M. Prausnitz, Molecular thermodynamics of simple liquids, Ind. Eng. Chem. Fundam., 6(1), pp 52-8 (Eebruary, 1967).

[30] Eckert, C. A., H. Renon and J. M. Prausnitz, Molecular thermodynamics of simple liquids, Ind. Eng. Chem. Fundam, 6(1), pp 58-67 (February, 1967).

[31] Albright, M. A., A model for the precise calculation of liquefied natural gas densities. NGPA Tech. Pub. TP-3, (1973).

[32] Klosek, J. and C. McKinley, Densities of liquefied natural gas and of low molecular weight hydrocarbons. White, J. W., Neumann, A. E. S., ed. Proceeding of the Eirst International Conference on LNG, Session 5 Paper 21, 15 pp, Chicago, IL (April, 1968).

[33] McCarty, R. D., A comparison of mathematical models for the prediction of LNG densities, Nat. Bur. Stand. (U.S.), NBSIR 77-867, 60 pp (October,1977).

[34] McCarty, R. D., Four mathematical models for the prediction of LNG densities, Nat. Bur. Stand. (U.S.) Tech. Note 1030, 76 pp (December, 1980).

[35] McCarty, R. D., Mathematical models for the prediction of liquefied- natural-gas densities, J. Chem. Thermodynamics, 14(9), 837-54 (September, 1982).

[36] Morlet, J., The density and miscibility of liquefied hydrocarbon gases at low temperatures, Rev. Inst. Francais Petrole, 18, pp 127-43 (January,1963).

[37] Gonzalez, M. H., T. K. Subramanian, R. L. Kao and A. L. Lee, Physical properties of natural gases at cryogenic conditions, White, J.W., Neumann, A. E. S., ed., Proceedings of the First International Conference on LNG, Session 5 Paper 21, 38 pp, Chícago, IL (April, 1968).

[38] Gonzalez, M. H., A. L. Lee, Dew and bubble points of simulated natural gases, J. Chem. Eng. Data ,13(2), pp 172-6 (April, 1968). 
[39] Huebler, J., B. E. Eakin, A. L. Lee, Physical properties of natural gas at cryogenic conditions. White, J. W., ed., Proceedings of the Second International Conference on LNG, Vol. I, Session 2 Paper 9, 10 pp, Paris, France (October, 1970).

[40] Huang, E. T. S., G. W. Swift, and F. Kurata, Viscosities and densities of methane-propane mixtures at low temperatures and high pressures, AIChE J. 13(5), pp 846-50 (September, 1967).

[41] Jensen, R. H. and F. Kurata, Density of liquefied natural gas, J. Petrol. Tech. 21, pp 683-91 (June, 1969).

[42] Boyle, G. J. and D. Reece, Bulk measurement of LNG. White, J. W., ed. Proceedings of the Second International Conference on LNG, Vol. I, Session 2 Paper 5, 14 pp, Paris, France (October, 1970).

[43] Rodosevich, J. B. and R. C. Miller, Experimental liquid mixture densities for testing and improving correlations for liquefied natural gas, AIChE J. 19(4), pp 729-35 (1973, July).

[44] Miller, R. C., Estimating the densities of natural gas mixtures, Chem. Eng. 81(23), pp 134-35 (October, 1974).

[45] Pan, W. P., M. H. Mady, R. C. Miller, Dielectric constants and Clausius-Mossotti functions for simple liquid mixtures: systems containing nitrogen, argon and light hydrocarbons, AIChE J. $21(2)$, pp 283-89 (March, 1975).

[46] Milier, R. C. and M. J. Hiza, Experimental molar volumes for some LNG-related saturated liquid mixtures, Fluid Phase Equilibria 2(1), pp 49-57 (August, 1978).

[47] Roche, Y., J. Perret, and L. Bochirol, Density of LNG and of its constituents, White, J. W., ed., Proceedings of the Second International Conference on LNG, Vol I, Session 2 Paper 8,8 pp, Paris, France, (October, 1970).

[48] Roche, Y., J. Perret, L. Bochirol and P. Brauns, Density of LNG and of its constituents, White, J. W., ed., Proceedings of the Third International Conference on LNG, Session II Paper 13 , 11 pp, Washington, D.C. (September, 1972).

[49] Orrit, J., and J. M. Laupretre, A calculational method for obtaining the density of a liquefled natural gas, Advances in Cryogenic Engineering, Vol. 23, K. D. Timmerhaus, ed., pp 566-79, Plenum Press, New York, NY (1978).

[50] Orrit, J. E., Orthobaric Iiwuid densities of natural gas component mixtures, Fluid Phase Equilibria, 12(3), pp253-81 (September, 1983).

[51] Haynes, W. M., R. D. McCarty and M. J. Hiza, Liquefied natural gas densities: summary of research program at the National Bureau of Standards, Nat. Bur. Stand. (U.S), Monograph 172, 241 pp (October, 1983). 



\subsubsection{Mathematical Models for the Prediction of LNG Densities}

A general description of the extensive study program on the development of data for thermophysical properties of LNG is summarized in 2.4.3. Accurate and precise experimental data on this complex fluld mixture was necessary in order to provide a basis for data correlation programs which would give useful accurate density data from measurements of other properties. of the numerous possible mathematical relationships considered, just four were chosen for the NBS program. These four models represented a spectrum of methods from the purely empirical to the more theoretical. The brief summary of 2.4.3.7 does not provide the scope or depth to be useful for measurements and calculations of LNG density. The work of McCarty [39] provides a detailed description of the development, and resulting accuracy and precision of each of these models. Because of the importance of these methods and techniques, re-publication of McCarty has been included in this section of the manual. It is reproduced in detail (except for the original experimental data) with only minor editing.

\subsubsection{Introduction}

The purpose of the study was to present in final form the four mathematical models which were optimized to the experimentally determined orthobaric liquid PVTx data of Miller and Hiza [25], Haynes and Hiza [12], Haynes, et al. [11], Hiza, et al. [14], Hiza and Haynes [15] and Haynes [9]. Interim results of the project have been reported by Haynes, et al. [13] and by McCarty [23]. The models reported in these two interim publications differ only slightly from those presented here. A companion archival document, McCarty [24], with the same results but in much less detail, has been submitted to the Journal of Chemical Thermodynamics. The intent of the documentation here is more in the veln of a user's handbook. The above experimental data are for the liquid phase of nitrogen, methane, ethane, propane, normal and isobutane and various mixtures thereof.

The goal of the project (the project included the above referenced experimental work) was to produce one or more computer models which would predict the density of LNG to within 0.1 percent of the true value from a knowledge of the temperature, pressure and composition of the LNG. At the beginning of this study LNG was defined as mixtures of the above components (nitrogen, methane, ethane, propane, normal and isobutane), and only the saturated liquid between $95-150 \mathrm{~K}$ was to be considered. Near the end of the project, normal and isopentane were added to the list of allowable components, but no experimental PVTx of pure normal and isopentane or binary systems containing normal and isopentane were measured as part of the project. The inclusion of these two components is based on data from orrit, et al. [29] and Orrit, et al. [30].

Four models were considered: the extended corresponding states model, a hard sphere model, a cell model and a Revised Klosek and McKinley model. With the exception of the Revised Klosek and McKinley model, only pure fluid and binary system data were used to optimize the models. In the case of the Revised Klosek and McKinley model, multicomponent PVTx data were used in the optimization process.

Over a normal range of LNG composition and temperature, all four of the models predict densities which agree to within 0.1 percent of experiment. This is true of all of the experimental PVTx measurements on LNG like mixtures made as part of this project.

No equation or mathematical model based on experimental data can be more accurate than the original data, and, therefore, the extent to which the original goal of 0.1 percent accuracy has been met depends entirely upon the accuracy of the experimental data referenced above.

There is no reason to doubt the experimental data, and, therefore, there is every reason to bel1eve that the goal of the project has been achieved.

\subsubsection{Extended Corresponding States Method}

The thermodynamic equations for the extended corresponding states method are developed in a paper by Rowlinson and Watson [35], and only a very brief description will be given here. Leach [20] developed transformation functions for hydrocarbons which are called shape factor functions. Mollerup [27] and Mollerup and Rowlinson [26] combined the earlier work with the equation of state for methane by Goodwin [8] to produce a computer program to calculate the density of LNG mixtures, which was further modified by Mollerup [28].

The computer programs for the calculation of LNG densities based on the extended corresponding stateg method are an extensive revision of the Mollerup program. The program listings are 1 ncluded $1 \mathrm{n}$ McCarty [39]. Earlier versions were reported by McCarty [23] and Haynes, Hiza and McCarty [13]. 
The extended corresponding states method is defined by the following equations:

$$
\begin{aligned}
& Z_{i}[P, T]=Z_{o}\left[P h_{i i, o} / f_{i i, o}, T / f_{i i, o}\right] \\
& G_{i}[P, T]=f_{i i, o} G_{o}\left[P h_{i i, o} / f_{i i, o}, T / f_{i i, o}\right]-R T \ell n\left(h_{i i, o}\right)
\end{aligned}
$$

where $\mathrm{Z}$ is the compressibility factor, $\mathrm{G}$ is the Gibbs free energy, $\mathrm{P}$ is pressure and $\mathrm{T}$ is temperature.

The subscript "o" denotes the reference fluid, and the subscript "i" denotes the fluid for which properties are to be obtained via the equation of state for the reference fluid and the transformation functions $\mathrm{f}_{i \mathrm{i}, 0}$ and $\mathrm{h}_{\mathrm{i} i, 0}$. The double subscript "ii" is introduced now to allow extension of the method to mixtures. The two defining equations (1) and (2) are necessary since there are two transformation functions. In this case the equation of state for methane by McCarty [22] was chosen for the reference fluid. During the course of the study it was necessary to modify the equation of state by MCCarty [22] to give a realistic vapor liquid phase boundary down to a temperature of $43 \mathrm{~K}$. This modification was necessary to accommodate the very low reduced temperatures of the heavier hydrocarbons and was accomplished without changing the performance of the equation of state above the triple point of methane. The equation of state is given in McCarty [39].

The $f_{i i, o}$ and $h_{i}, o$ are defined as

$$
\mathrm{f}_{i \mathrm{i}, 0}=\left(\mathrm{T}_{\mathrm{i} i}^{c} / \mathrm{T}_{0}^{c}\right) \theta_{i \mathrm{i}, 0}\left(\mathrm{~T}_{\mathrm{r}}, \mathrm{V}_{\mathrm{r}}\right)
$$

and

$$
\mathrm{h}_{\mathrm{i} i, \mathrm{o}}=\left(\mathrm{V}_{\mathrm{i} i, \mathrm{o}}^{\mathrm{C}} / \mathrm{V}_{\mathrm{o}}^{\mathrm{c}}\right) \phi_{\mathrm{i} i, \mathrm{o}}\left(\mathrm{T}_{\mathrm{r}}, \mathrm{V}_{\mathrm{r}}\right)
$$

where

$$
\begin{aligned}
& \theta_{i, 0}=1+\left(w_{i}-w_{0}\right)\left[n_{1}-n_{2} \ell n T_{r_{i}}+\left(n_{3}-n_{4} / T_{r_{i}}\right)\left(V_{r_{i}}-n_{5}\right)\right] \\
& \phi_{i i, 0}=\frac{z_{o}^{c}}{z_{i}^{c}}\left[1+\left(w_{i}-w_{0}\right)\left[n_{6}\left(v_{r_{i}}-n_{7}\right)-n_{8}\left(v_{r_{i}}-n_{9}\right) \ell n T_{r_{i}}\right]\right]
\end{aligned}
$$

The $\mathrm{V}_{\mathrm{r}_{\mathrm{i}}}$ and $\mathrm{T}_{\mathrm{r}_{\mathrm{i}}}$ are reduced temperature and volume such that

$$
\begin{aligned}
& \mathrm{T}_{\mathrm{r}_{\mathrm{i}}}=\mathrm{T} / \mathrm{T}_{\mathrm{i} i}^{\mathrm{C}} \\
& \mathrm{V}_{\mathrm{r}_{\mathrm{i}}}=\mathrm{V} / \mathrm{V}_{\mathrm{i} i}^{\mathrm{C}}
\end{aligned}
$$

and each fluid requires a unique $w_{i}$ which was estimated using pure fluid experimental data. A single set of the $n_{i}$ 's are used for all fluids. The $n_{i}$ 's were estimated using all of the pure fluid experimental data from this study.

The factor

$$
\frac{z_{o}^{c}}{z_{i}^{C}}
$$

is the ratio of the compressibility factors $\left(\mathrm{Z}^{\mathrm{C}}=\mathrm{P}_{\mathrm{C}} \mathrm{V}_{\mathrm{C}} / \mathrm{RT}_{\mathrm{C}}\right.$ ) at the critical point. The parameters $\mathrm{n}_{j}$, $n_{j}, w_{i}$ and $Z_{i i}$ are given in McCarty [39]. All of these parameters were estimated using the experimental PVT data set from this laboratory and least squares estimation techniques.

The extension of the above to mixtures is now accomplished by the application of the following combining rules:

$$
\begin{aligned}
& h_{x, 0}=\sum_{i} \sum_{j} x_{i} x_{j} h_{i j, o} \\
& f_{x, 0} h_{x, 0}=\sum_{i} \sum_{j} x_{i} x_{j} f_{i j, o} h_{i j, o} \\
& f_{i j, o}=\xi_{i j}\left(f_{i i, o} f_{j j, o}\right)^{1 / 2}
\end{aligned}
$$




$$
n_{1 j, 0}=n_{1 j}\left(\frac{1}{2} n_{i i, 0}^{1 / 3}+\frac{1}{2} n_{j j, 0}^{1 / 3}\right)^{3}
$$

The $\xi_{1 j}$ and the $n_{1 j}$ are binary interaction parameters determined by least squares from the PVTx data for binary mixtures. These parameters are given in McCarty [39].

Th1s method works quite well as may be seen in the comparisons with the original experimental data [39]. It has indeed reproduced all of the present experimental data set to within \pm 0.1 percent except for 14 out of a total of 285 experimental data points. Of these 14 points, 11 are judged to have an uncertalnty greater than 0.1 percent. Figure 2.4.4.1 presents the deviations between the calculated and experimental densities for these 14 points. The original publication [39] contains comparisons of calculated and experimental densitles for the entire data set. This is the best performance of the four models presented here. No pressure, temperature or composition restrictions have been placed on this model.

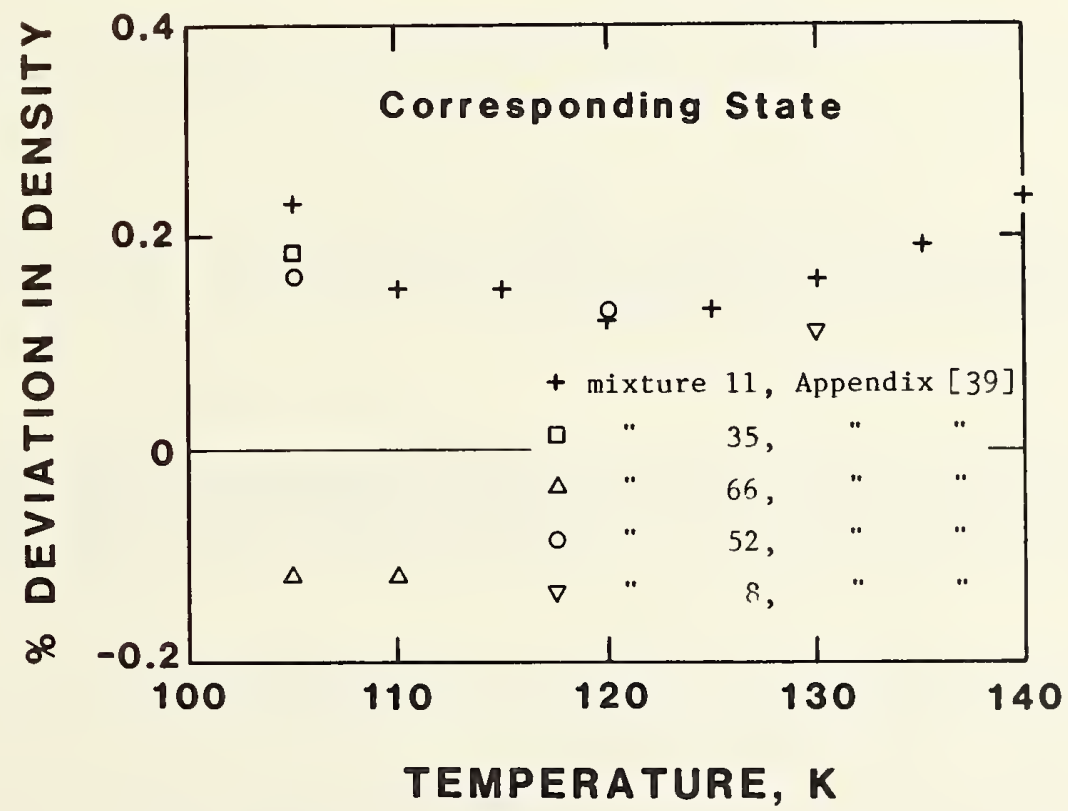

\footnotetext{
Figure 2.4.4.1. All deviations greater than 0.1 percent between experimental and calculated densities using the Extended corresponding States method.
}

In the interim publications by McCarty [23] and Haynes, Hiza and McCarty [13] some doubt about the accuracy of the calculated densities was expressed because of the disagreement with a few b1nary and multicomponent systems containing methane and butane.

This disagreement has since been resolved by additional measurements (Haynes [9], Haynes [10] and Miller and Hiza [25] on some of the systems which agree with the predictions of the model but disagree with the previous measurements. The net result of the new measurements $1 \mathrm{~s}$ a very slight change $1 \mathrm{n}$ binary interaction coeficients of the methane-butane and nitrogen-butane system. These changes have no practical effect on LNG-like mixtures where the concentrations of nitrogen, isobutane and normal butane are individually less than 5 percent. In other words, elther the models presented here on those in the interim publications may be used to predict the density of a LNG-I1ke m1xture to w1th1n 0.1 percent of the true density. 


\subsubsection{Hard Sphere Method}

The model of Rodosevich and Miller [33] is one of many modifications of the Longuet-Higgins and Widom [21] model, and was chosen to be included in this study as a representative example of the application of the hard sphere equation of state concept to the correlation of PVTx data. The equation of state by Rodosevich and Miller [33] is

$$
\frac{\mathrm{PV}}{\mathrm{RT}}=\mathrm{c} \frac{1+\mathrm{y}+\mathrm{y}^{2}}{(1-\mathrm{y})^{3}}-\frac{\mathrm{a}}{\mathrm{RTV}}
$$

where the $\mathrm{y}=\mathrm{b} / 4 \mathrm{~V}$ and $\mathrm{a}, \mathrm{b}$, and $\mathrm{c}$ are adjustable parameters, $\mathrm{P}$ is pressure, $\mathrm{V}$ is specific volume, $\mathrm{T}$ is temperature and $R$ is the gas constant. The equation is applied to mixtures by assuming the one-fluid theory and applying the following combining rules.

$$
\begin{aligned}
& a_{m}=\sum_{i} \sum_{j} a_{i j} x_{i} x_{j} \\
& b_{m}=\sum_{i} \sum_{j} b_{i j} x_{i} x_{j} \\
& c_{m}=\sum_{i} \sum_{j} c_{i j} x_{i} x_{j}
\end{aligned}
$$

The mixing rules are:

$$
\begin{aligned}
& b_{i j}=\left[\frac { b _ { i j } ^ { 1 / 3 } + b _ { j j } ^ { 1 / 3 } ( 1 - j _ { i j } ) ] ^ { 3 } } { 2 } \left(a_{\left.i i a_{j j}\right)^{1 / 2}}\left[\frac{b_{j j}^{2}}{b_{i i} b_{j j}}\right]^{1 / 2}\left(1-k_{i j}\right)\right.\right. \\
& a_{i j}=\left(c_{j i}+c_{j j}\right. \\
& c_{i j}
\end{aligned}
$$

The parameters $j_{i j}$ and $k_{i j}$ are in this case the binary interaction parameters. The a's, b's, c's, $j_{i j}$ 's and $k_{i j}$ 's are given in McCarty [39]. The excess volume is now calculated using the equation of state and

$$
V_{E}=\tilde{V}_{m}-\tilde{V}_{i} x_{i}
$$

where $\tilde{V}_{m}$ and the $\tilde{V}_{i}$ are calculated via the equations 11 through 17 and then

$$
v_{m}=v_{i} x_{i}+v_{E}
$$

where the $V_{E}$ is from equation (18) and the $V_{i}$ are from experimental data. The values of $V_{i}$ in this case were calculated from the equations for the liquid density of the pure fluids given in McCarty [39].

The above equations are those of Rodosevich and Miller [33] and Rodosevich [34], and only the $j_{i j}{ }^{\prime} s$ and $k_{i j}$ 's have been revised on the basis of the present new data set; and only binary systems data were used to estimate via least squares the $j_{i j}$ 's and $k_{i j}$ 's.

As the method is used, here it is an excess volume method, and consequently when the temperature of the mixture approaches the critical temperature of one of the component fluids, the method fails. Since the critical temperature of nitrogen is about $126 \mathrm{~K}$, this method should not be used for mixtures containing nitrogen at temperatures above $120 \mathrm{~K}$. Eliminating the data points for mixtures which contain nitrogen at temperatures above $120 \mathrm{~K}$ reduces the set from 285 to 251 PVTx points. Figure 2.4.4.2 is a percentage deviation plot containing all of the data points from the set of 251 for which densities calculated by the hard sphere method differ from the experimental density by more than 0.1 percent. Two things are readily seen in comparing figures 2.4.4.1 and 2.4.4.2; first, even though the total number of points has been reduced in the comparison set, the number of points for which deviations exceed 0.1 percent in the hard sphere comparison, figure 2.4.4.2, is far more than for the extended corresponding states comparison, figure 2.4.4.1. Second, the hard sphere method becomes more uncertain for all mixtures, regardless of components as the temperature exceeds $115 \mathrm{~K}$. 


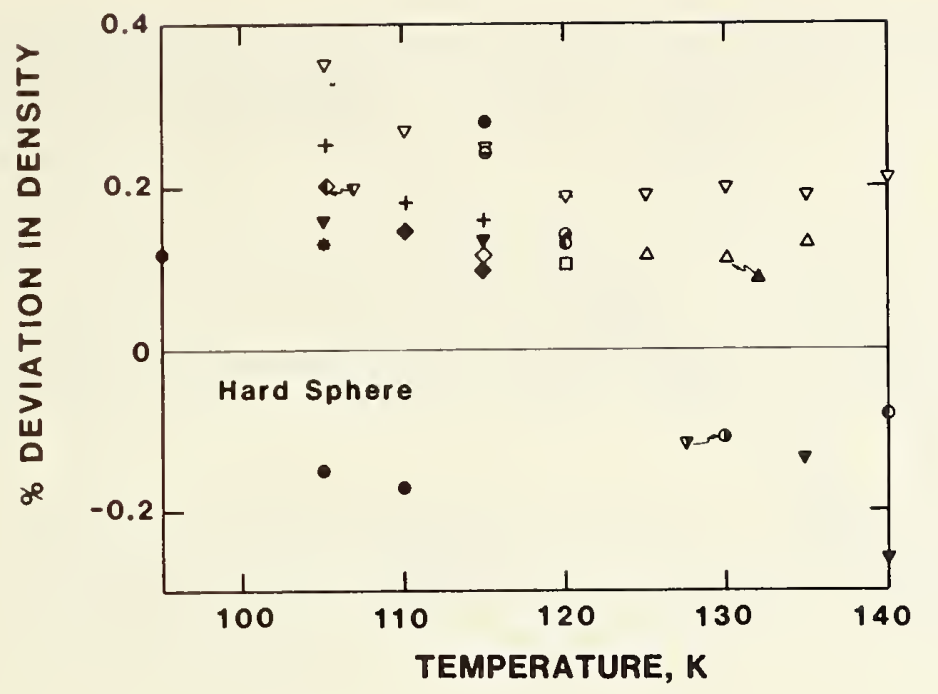

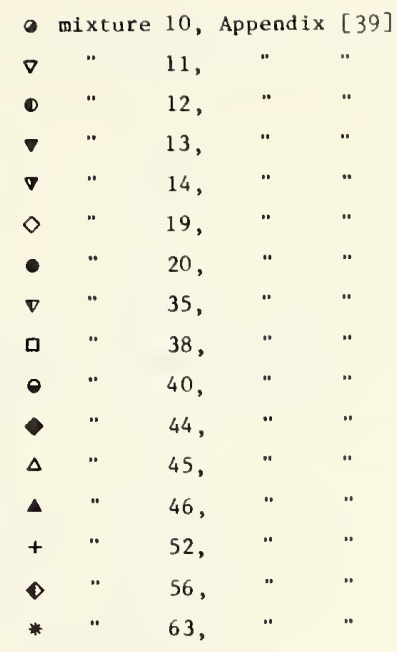

Figure 2.4.4.2. Deviations greater than 0.1 percent between experimental and calculated densities using the Hard Sphere model.

\subsubsection{A Revised Klosek and Mckinley Method}

The Klosek and McKinley method [18] is a totally empirical recipe for calculating the density of a LNG-like mixture given the temperature and composition. Pressure is not taken into account. However, this does not seem to be a serious omission. The procedure proposed by Klosek and McKinley [18] is as follows:

$$
v_{\operatorname{mix}}=\sum x_{i} v_{i}-k x_{C H}
$$

where $V_{\operatorname{mix}}$ is the volume of the mixture, $X_{i}$ and $V_{i}$ are the mole fraction and volume of the 1 th component, $\mathrm{X}_{\mathrm{CH}_{4}}$ is the mole fraction of methane and $\mathrm{k}$ is a correction factor obtained from a table or graph. The $V_{i}$ and $k$ are obviously temperature dependent, and, in addition, $k$ is dependent upon the molecular weight of the mixture.

Using the present data set, $\mathrm{k}$ was calculated for all of the experimental data points where methane was present in the mixture and excluding all data points where nitrogen was present in greater than 5 percent concentration. Figure 2.4.4.3 shows a typical isotherm for $k$, with nitrogen present ( 1 abeled $\mathrm{k}_{2}$ ) and without nitrogen present (labeled $\mathrm{k}_{1}$ ). All of the isotherms available show similar behavior, i.e., all of the $\mathrm{k}^{\prime} \mathrm{s}$ for mixtures containing nitrogen (of about 5 percent) fall on one 1 ine, and all of those for mixtures without nitrogen fall on another. Since all of the mixtures with nitrogen have about the same amount of nitrogen present (about 4.5 percent), the method was modified by adding a term to take into account the nitrogen when it is present. The equation becomes

$$
\mathrm{V}_{\operatorname{mix}}=\sum \mathrm{X}_{\mathrm{i}} \mathrm{V}_{\mathrm{i}}-\left[\mathrm{k}_{1}+\left(\mathrm{k}_{2}-\mathrm{k}_{1}\right) \mathrm{x}_{\mathrm{N}_{2}} 10.0425\right] \mathrm{x}_{\mathrm{CH}_{4}}
$$

where everything is the same as in equation (20) except that $k_{1}$ is read from one curve and $k_{2} 1 \mathrm{~s}$ read from the other. Mccarty [39] gives tables of values for the $V_{i}, k_{1}$ and $k_{2}$ which are spaced so that linear interpolation is adequate in both variables (i.e., temperature or molecular welght). These tables have been reproduced in section 3.2 as tables $3.2 .6,3.2 .7$ and 3.2 .8 . The $\mathrm{k}$ factors $1 \mathrm{n}$ McCarty [39] have been obtained graphically from the multicomponent PVTx data of Hiza and Haynes [15] and Miller and $\mathrm{Hiza}$ [25] as well as densities calculated from the extended corresponding states method of section 2.4 .4 .2 .

The limits of compositions of the revised Klosek and McKinley method are the most severe of any of the methods given here. This method should not be used for mixtures other than LNG-11ke mixtures and ror LNG-like mixtures only when they contain at least 60 percent methane, less than 4 percent nltrogen, less than 4 percent each of isobutane and normal butane and less than 2 percent total or 1 sopentane and normal pentane. 


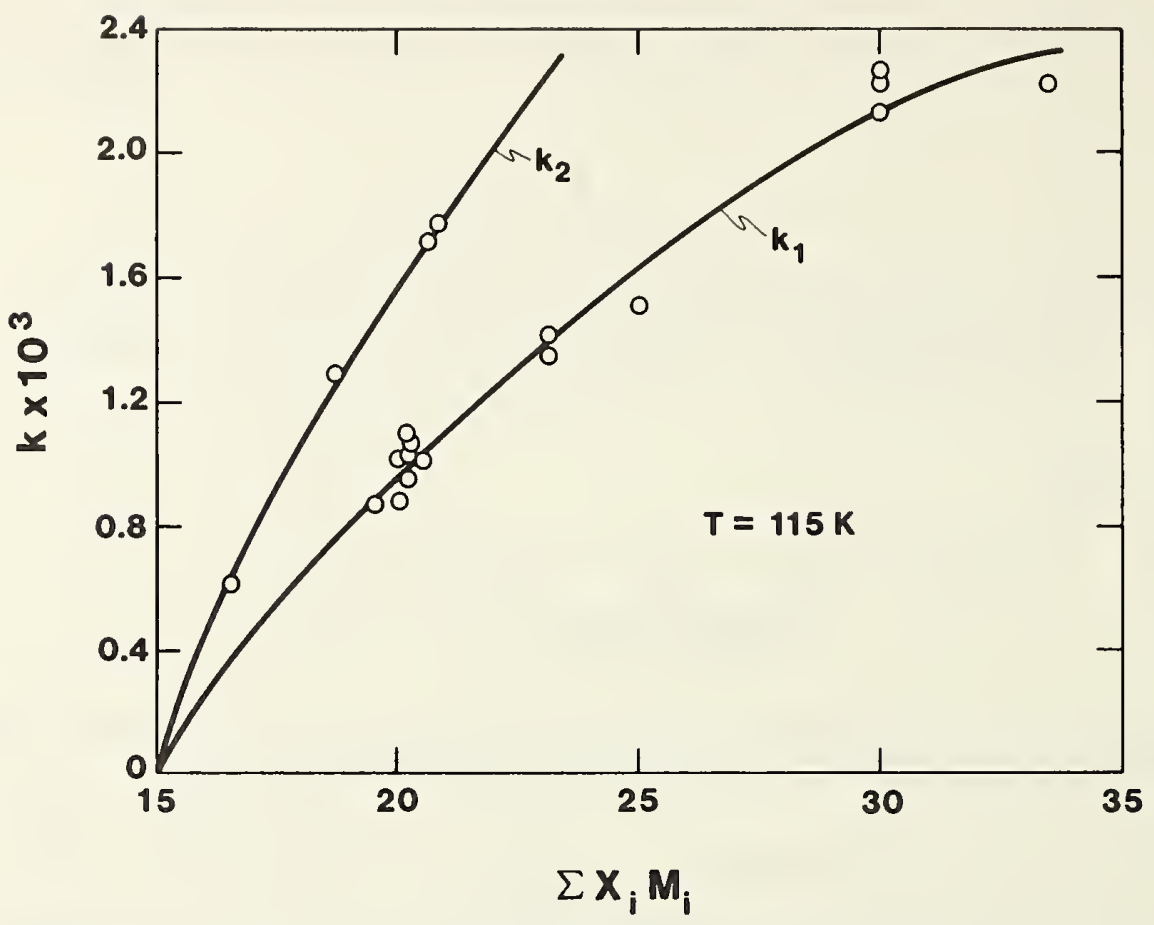

Figure 2.4.4.3. Correction factors ( $k_{1}$ and $\left.k_{2}\right)$ for the 115 kelvin is otherms.

There are 40 experimental PVTx points from the original set of 285 which may be considered LNG-1ike and fall within the composition limits outlined above. Figure 2.4.4.4 shows all of the deviations between calculated and experimental densities in this 40 point comparison set which exceeds the 0.1 percent criterion.

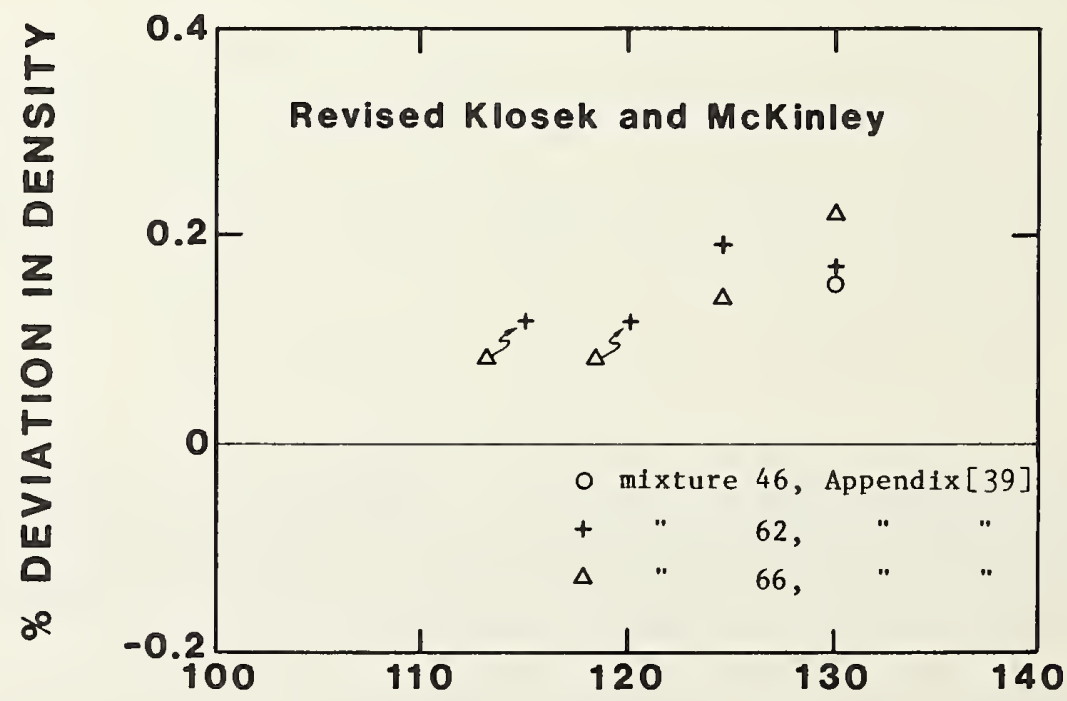

TEMPERATURE, $K$

Figure 2.4.4.4. Deviations greater than 0.1 percent between experimental and calculated densities using the Revised Klosek and McKenley model. 
The deviation trends for the revised Klosek and McKinley method (figure 2.4.4.4) are very similar to those of the hard sphere method (figure 2.4.4.2) and in fact all of the deviations in $f i g u r e 2.4 .4 .4$ occur at temperatures at or above $115 \mathrm{~K}$, therefore the method can only be considered as accurate as the others for LNG like mixtures at temperatures below $115 \mathrm{~K}$.

\subsubsection{The Cell Model}

The cell model considered here was originally proposed by Renon, et al. [32]. In a paper by the same three authors which appeared simultaneously (Eckert, et al. [7]), the cell model was applied to mixtures via scott's [36] two-fluid theory and a three parameter corresponding states theory. Albright [2] further modified the method by modifying the mixing rules on the basis of a proposal by Yuan [38] and by inserting a pressure dependence based on the experimental liquid ethane data by Pope [31].

The optimization of this method was carried out by M. Albright [1] at Phillips Petroleum Company in Bartlesville, Oklahoma, and the details of this work will be published elsewhere. The model is included here because it was optimized to the same data set as the others, and, therefore, the comparisons between experimental and calculated densities given here in figure 2.4 .4 .5 together with figures $2.4 .4 .1,2.4 .4 .2$ and 2.4 .4 .4 provide a common basis of comparison with the other three methods.

The same data set that was used in the hard sphere method for comparison has been used here, i.e., all of the data points for mixtures containing nitrogen at temperatures $120 \mathrm{~K}$ and above have been taken out of the original 285 points leaving a total of 251 data points. As in the case of the other methods figure 2.4 .4 .5 shows all of the points for which the calculated and experimental densities differ by more than 0.1 percent.

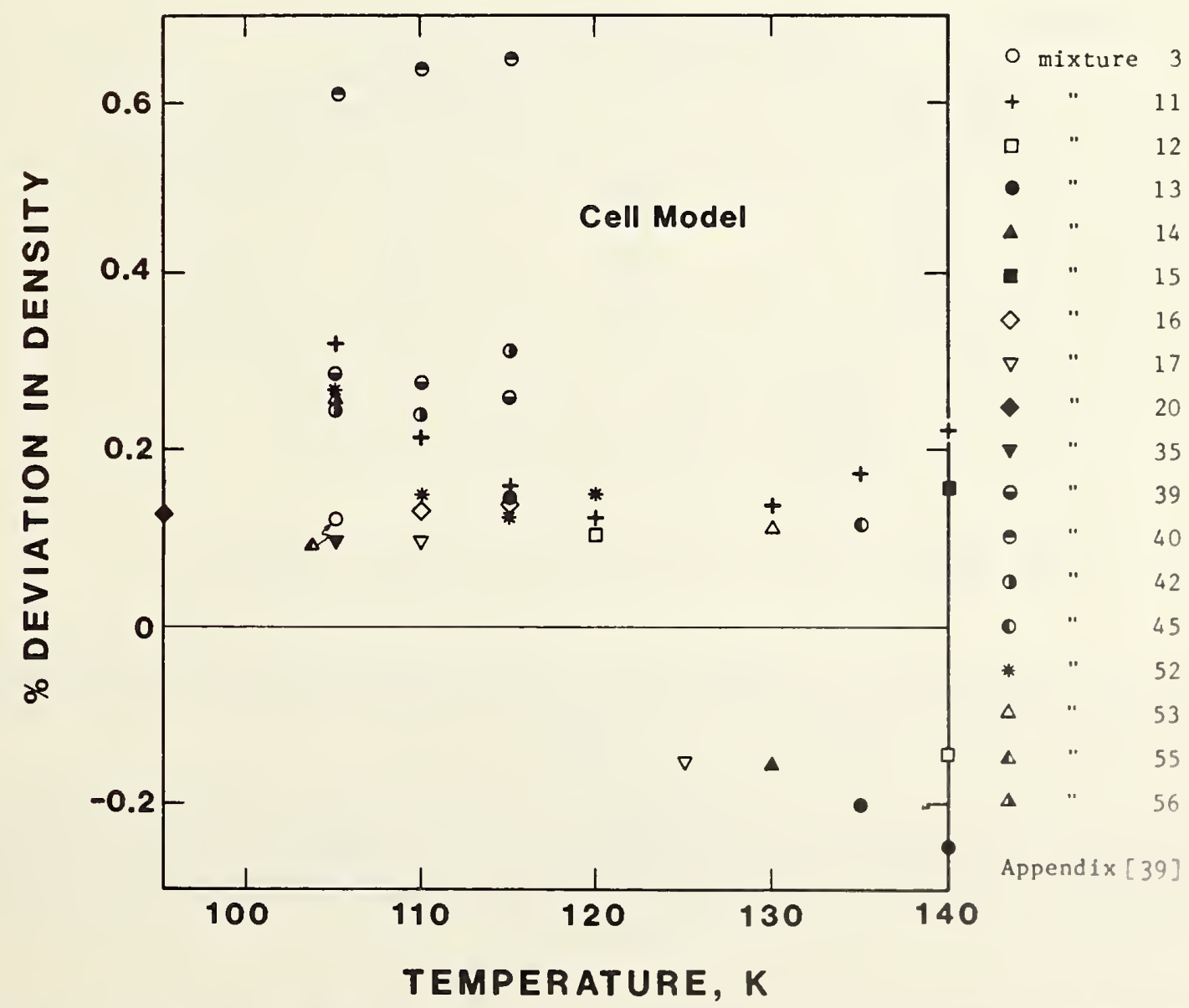

Figure 2.4.4.5. Deviations greater than 0.1 percent between experimental and calculated densities using the Cell model. 


\subsubsection{Use of the Methods}

When the project started in 1972, the atomic weights of nitrogen, carbon and hydrogen were taken from the 1961 carbon 12 scale, IUPAC [16]. During the course of the investigation a revision, Atomic Weights of the Elements [3], to this scale appeared. The revision changed slightly the atomic weights of carbon and hydrogen, but since the changes were small (the maximum difference in any of the densities used here is 0.003 percent), and because changing the atomic weights would not change the relative results, the changes were not made. Therefore, when using the tables and programs included herein, the molecular weights given in the tables and programs should be used to maintain consistency.

The critical parameters used here are from: methane, McCarty [22]; ethane, Sliwinski [37]; propane, Das, et al. [4]; isobutane, Das, et al. [5]; normal butane, Das, et al. [6]; isopentane, Kudchadker, et al. [19]; and nitrogen, Jacobsen, et al. [17].

Errors in the input variables will, of course, cause errors in the density predicted by the models. In general, the error in density caused by an error in the input variables is a function of those input variables, and must be treated on an individual basis. However, for LNG-like mixtures certain general trends are found. An error in the pressure must be at least 50 percent before it will have any effect at all on the resulting density. An error in composition, unless it is of the order of several percent, will cause the same relative error in density as it will cause in the molecular weight of the mixture, i.e., if an error in composition causes a 0.1 percent error in the resulting molecular weight, it will also cause a 0.1 percent error in the predicted density.

The error in the calculated density due to an error in the input temperature is a function of the composition and the temperature. Table 2.4.4.1 gives resulting errors in density for a 1 percent error in temperature, for three hypothetical LNG like mixtures.

Table 2.4.4.1 Errors in Density Caused by an Error in the Input Temperature of 1 percent.

\begin{tabular}{|c|c|c|c|}
\hline \multirow{2}{*}{$\begin{array}{c}\text { Temperature } \\
K\end{array}$} & \multicolumn{3}{|c|}{ Error in Density } \\
\cline { 2 - 4 } & Mix A & Mix B & Mix C $^{*}$ \\
\hline \hline & 0.28 & 0.25 & 0.20 \\
100 & 0.30 & 0.27 & 0.22 \\
105 & 0.32 & 0.29 & 0.24 \\
110 & 0.35 & 0.32 & 0.29 \\
115 & 0.39 & 0.34 & 0.31 \\
\hline
\end{tabular}

$\begin{aligned} \operatorname{Mix} A^{*}= & 0.95 \mathrm{CH}_{4}, 0.05 \mathrm{~N}_{2} \\ \operatorname{Mix} B^{*}= & 0.9 \mathrm{CH}_{4}, 0.02 \mathrm{C}_{2} \mathrm{H}_{6}, 0.02 \mathrm{C}_{3} \mathrm{H}_{8}, 0.02 \mathrm{iC}_{4} \mathrm{H}_{10}, \\ \operatorname{Mix~} C^{*}= & 0.02 \mathrm{nC}_{4} \mathrm{H}_{10}, 0.02 \mathrm{~N}_{2} \\ & 0.6 \mathrm{CH}_{4}, 0.3 \mathrm{C}_{2} \mathrm{H}_{6}, 0.02 \mathrm{C}_{3} \mathrm{H}_{8}, 0.02 \mathrm{iC}_{4} \mathrm{H}_{10}, \\ & 0.02 \mathrm{nC}_{4} \mathrm{H}_{10}, 0.02 \mathrm{~N}_{2}, 0.02 \mathrm{iC}_{5} \mathrm{H}_{12} .\end{aligned}$

* Arbitrary LNG-like compositions assumed for the purpose of illustrating the effect of an error in the input temperature.

In general the errors in density caused by an error in temperature are the largest for mixtures containing a high concentration of the most volatile fluids, methane and nitrogen, and correspondingly the errors decrease as the concentration of the heavier hydrocarbons increases in the mixture. These errors are not a function of which model is being used.

When using the extended corresponding states method, one should keep in mind that twelve significant figures are required by the methane equation of state. The hard sphere model also uses the methane equation from McCarty [22] and the nitrogen equation of Jacobsen, et al. [17] to calculate compressibilities and therefore requires twelve significant figures to insure the accuracy of the calculated density.

\subsubsection{Conclusions}

On the basis of the performance of the four models given here and subject to the composition and temperature restrictions already noted, it is estimated that given the pressure, temperature and composition of LNG, any one of the four models may be used to predict the density to within 0.1 percent of the true value. As has already been mentioned (see section 2.4.4.1) the above accuracy 


\begin{abstract}
statement is dependent entirely upon the accuracy of the experimental data in Haynes, et al. [11], Haynes, et al. [13], Hiza, et al. [14], Haynes [9], Hiza and Haynes [15], Miller and Hiza [25] and Haynes [10]. These data have been estimated by the authors to be accurate to within 0.1 percent of the true value with a precision of a few hundredths of a percent. The work on the models given here have provided no basis for questioning the claims of the experimenters, in fact the ability of the models to predict the densities of the multicomponent mixtures to within 0.1 percent of the measured values tends to support the accuracy claims of the experimenters.
\end{abstract}

Interim results of this study were reported by Haynes, et al. [13] and McCarty [23], both of which contain earlier versions of the mathematical models given here. These earlier versions are only slightly different from the final ones, and, for the purposes of calculating LNG densities, either of the versions may be used. The reader is, however, cautioned to read the limitations of each model as defined in the earlier sections.

Computer programs for the four models are available at the Thermophysical Properties Division of the National Bureau of Standards in Boulder, Colorado. 


\subsubsection{References}

[1] Albright, M. A., Private communication (1976).

[2] Albright, M. A., A Model for the precise calculation of liquefied natural gas densities, NGPA Technical Publication TP-3 (1973).

[3] Atomic weights of the elements 1973, Pure and Applied Chemistry, Vol 37, 589-603 (1974).

[4] Das, T. R. and P. T. Eubank, Advances in Cryogenic Engineering Vol 18, Plenum Press, New York (1973), p. 208 .

[5] Das, T. R. and P. T. Eubank, Advances in Cryogenic Engineering Vol 18, Plenum Press, New York (1973), p. 253 .

[6] Das, T. R. and P. T. Eubank, Advances in Cryogenic Engineering Vol 18, Plenum Press, New York (1973), p. 244 .

[7] Eckert, C. A., H. Renon and J. M. Prausnitz, Molecular Thermodynamics of Simple Liquids, IEC Fund 6 , 58 (1967).

[8] Goodwin, R. D., The thermophysical properties of methane, from 90 to $500 \mathrm{~K}$ at pressures up to 700 bars, Nat. Bur. Stand. (U.S.), Tech. Note 653 (1974).

[9] Haynes, W. M., Orthobaric liquid densities and dielectric constants of (methane + 2-methylpropane) and (methane + n-butane) at low temperatures, J. Chem. Thermodynamics 15(10), pp 903-11 (Oct 1983).

[10] Haynes, W. M., Measurements of orthobaric liquid densities of multicomponent mixtures of LNG components $\left(\mathrm{N}_{2}, \mathrm{CH}_{4}, \mathrm{C}_{2} \mathrm{H}_{6}, \mathrm{C}_{3} \mathrm{H}_{8}, \mathrm{iC}_{4} \mathrm{H}_{10}, \mathrm{nC}_{4} \mathrm{H}_{10}, \mathrm{iC}_{5} \mathrm{H}_{12}, \mathrm{nC}_{5} \mathrm{H}_{12}\right)$ between 110 and $130 \mathrm{~K}$, J. Chem. Thermodynamics 14(7), pp 603-12 (July 1982).

[11] Haynes, W. M., M. J. Hiza and N. V. Frederick, A magnetic suspension densimeter for measurements on fluids of cryogenic interest, Review of Scientific Instruments 47, No. 10, 1237-50 (0ct 1976).

[12] Haynes, W. M. and M. J. Hiza, Measurements of the orthobaric liquid densities of methane, ethane, propane, isobutane, and normal butane, J. Chem. Thermodyn., 9, 179 (1977).

[13] Haynes, W. M., M. J. Hiza and R. D. McCarty, Densities of LNG for custody transfer, (Proc. Fifth International Conference of LNG, Dusseldorf, Germany), J. W. White, W. McGrew, S. Farmer, D. Hansen, H. Jacobshagen, editors; Institute of Gas Technology, Chicago, Ill., Vol. 2, Paper II, Section III (1977).

[14] Hiza, M. J., W. M. Haynes and W. R. Parrish, Orthobaric liquid densities and excess volumes for binary mixtures of low molecular weight alkanes and nitrogen between 105 and $140 \mathrm{~K}$, J. Chem. Thermodyn. 9 , 873 (1977).

[15] Hiza, M. J. and W. M. Haynes, Orthobaric liquid densities and excess volumes for multicomponent mixtures of low molar mass alkanes and nitrogen between 105 and $125 \mathrm{~K}$, J. Chem. Thermodyn. 12 , 1 $(1980)$.

[16] International Union of Pure and Applied Chemistry, 1961 Carbon 12 Scale.

[17] Jacobsen, R. T., R. B. Stewart, R. D. McCarty and H. J. M. Hanley, Thermophysical properties of nitrogen from the fusion line to $3500 \mathrm{R}(1944 \mathrm{~K})$ for pressures to $150,000 \mathrm{psia}\left(10342 \times 10^{5}\right.$ $\mathrm{N} / \mathrm{m}^{2}$ ), Nat. Bur. Stand. (U.S.), Technical Note No. 648 (Dec 1973).

[18] Klosek, J. and C. McKinley, Densities of liquefied natural gas and of low molecular weight hydrocarbons, Proc. First Int. Conf. on LNG, IGT, Chicago (1968).

[19] Kudchadker, A. P., G. H. Alani and B. J. Zwolinski, The Critical Constants of Organic Substances, Chem. Rev. 68, 659-735 (1968).

[20] Leach, J. W., Molecular structure correction for applying the theory of corresponding states to non-spherical pure fluids and mixtures, Ph.D. Thesis, Rice University (1967). 
[21] Longuet-Higgins, H. C. and B. Widom, A rigid sphere model for melting of Argon, Mol. Phys. 8 , $549(1964)$.

[22] McCarty, R. D., A modified Benedict-Webb-Rubin equation of state for methane using recent experimental data, Cryogenics 14, No. 5, 276-80 (May, 1974).

[23] McCarty, R. D., A comparison of mathematical models for the prediction of LNG densities, Nat. Bur. Stand. (U.S.), Interagency Report NBSIR 77-867 (Oct 1977).

[24] McCarty, R. D., Mathematical models for the prediction of liquefied natural gas densities, J. Chem. Thermodynnamics 14(9), pp 837-54 (Sept 1982).

[25] Miller, R. C. and M. J. Hiza, Experimental molar volumes for some LNG related saturated liquid mixtures, Fluid Phase Equilibria 2, 49-57 (1978).

[26] Mollerup, J. and J. S. Rowlinscn, The prediction of densities of liquefied natural gas and of lower molecular weight hydrocarbons, Chem. Engng. Sci. 29 (1973).

[27] Mollerup, J., Correlated and predicted thermodynamic properties of LNG and related mixtures in the normal and critical region, Adv. Cryog. Eng. 20, 172 (K. D. Timmerhaus, editor, Plenum Press, New York, 1975).

[28] Mollerup, J,. Thermodynamic properties of natural gas, petroleum gas, and related mixtures enthalpy predictions, Adv. Cryog. Eng. 23550 (K. D. Timmerhaus, editor, Plenum Press, New York, 1978).

[29] Orrit, J. and J. F. Olives, Density of liquefied natural gas and its components, Distributed at the 4 th International Conference on Liquefied Natural Gas, Algeria (1974).

[30] Orrit, J. E. and T. M. Laupretre, Density of liquefied natural gas components, Adv. Cryog. Eng. 23573 (K. D. Timmerhaus, editor, Plenum Press, New York, 1978).

[31] Pope, G. A., Calculation of argon, methane and ethane virial coefficients at low reduced temperature, Ph.D. Thesis, Rice University (1971).

[32] Renon, H., C. A. Eckert and J. M. Prausnitz, Molecular Thermodynamics of Simple Liquids, IEC Fund. 6 , $52(1967)$.

[33] Rodosevich, J. B. and R. C. Miller, Calculation of LNG excess volumes by a modified hard-sphere model, Adv. Cryog. Eng. 19, 339 (1974).

[34] Rodosevich, J. B., Measurements and prediction of liquefied natural gas densities, M.Sc. Thesis, Univ. Wyoming (1973).

[35] Rowlinson, J. S. and I. D. Watson, The prediction of the thermodynamic properties of fluids and fluid Mixtures - I. The Principle of Corresponding States and Its Extensions, Chem. Eng. Sc1. 24, 1565 (1969).

[36] Scott, R. L., J. Chem. Phys. 25, 193 (1956).

[37] Sliwinski, P. Z., Die Lorentz-Lorenz funktion von dampfformigem und fluessiyem athan, propan and butan, Phys. Chem. (Frankfurt) 63263 (1969).

[38] Yuan, I. C., Correlation and prediction of excess properties for selected binaries in the $C_{6}$ C $_{7}$ hydrocarbon boiling range of conformal solution theory, D.Sc. Dissertation, Washington University (1971).

[39] McCarty, R. D., Four mathematical models for the prediction of LNG densities, Mat., Bur., Stand. (U.S.), Technical Note 1030, 84 pp (December, 1980). 



\subsubsection{Interlaboratory Comparison of LNG Density Measurements}

It has been shown in the previous sections of this part of the manual that LNG density may be determined both by direct measurement and by calculation. However, most commercial direct density measurement devices are relative in the sense that a calibration is required to establish and maintain the densimeter's accuracy and precision. In many cases the calibrations rely on calculation techniques and mathematical models, and, as a result, the two density measurement techniques are highly interdependent. In order to establish and maintain a dependable measurement system, it is necessary to develop tests to insure against error.

One such test is the interlaboratory comparison, in which one or more portable density measurement devices are calibrated at different density reference systems, and the results compared. Both in situ meter performance and density calculation can be performed. The following sections describe an international interlaboratory LNG densimetry comparison which was conducted at NBS in Boulder, Colorado, and Gaz de France, Saint-Denis, France. The text is an edited version of a the work of Roncier, Philippe, Saint-Just and Dewerdt of Gaz de France and Siegwarth and LaBrecque of NBS [17].

The comparison between the two test facilities, using a portable version of an absolute densimeter, provides an experimental estimate of the uncertainty of the indirect method of density measurement for the first time, on a large (32 liter) sample. The two test facilities agree for pure methane to within about 0.02 percent. For the LNG-like mixtures consisting of methane, ethane, propane, and nitrogen with the methane concentrations always in excess of 86 percent, the calculated density is within 0.25 percent of the directly measured density for a confidence level of 95 percent.

\subsubsection{Introduction}

The density of 1 iquefied natural gas (LNG) is one of the factors needed to establish the value of a quantity of LNG for custody transfer. Two methods are available to determine density: by calculation from correlations of density as a function of composition, pressure and temperature, and by direct measurement.

The calculational method is one of several commonly used. The density is inferred from measurements of pressure, temperature and composition using correlations such as one of those developed during the LNG density project at the National Bureau of Standards (NBS) [1]. These calculational methods are based on a series of density measurements of LNG pure components [2] and mixtures [1] done at NBS. The composition is determined by gas chromatographic analysis of vaporized LNG samples [3,4,5].

Direct density measurement can be accomplished by determining the effect of a change in the liquid density on some physical property which is sensed by the density measuring instrument or densimeter immersed in the liquid. This property might be a buoyancy, frequency, or capacitance. Ideally, no other property of the liquid, such as temperature or composition, will affect the density measurement.

Though direct density measurement is not now used for custody transfer measurement, the measurement simplicity and the concurrent promise of a greater accuracy have generated an interest in applying densimeters to LNG density measurement.

A number of commercially built densimeters are presently available. Some of these instruments are capable of making cryogenic density measurements. In fact, some were designed specifically for LNG service. However, those built for LNG service are not necessarily tested or calibrated in LNG. LNG calibrations, when provided for the densimeters, are generally extrapolated from measurements in liquid nitrogen and ambient temperature hydrocarbons.

Gaz de France (GDF) has built a facility to test and calibrate commercial densimeters for LNG service [6]. The National Bureau of Standards, at the request of and with the support of the American gas industry [7], also built a test facility, the density reference system (DRS) [5,8-12].

Both test facilities are similar in that the densimeters are tested in nominally isothermal samples or pure methane and LNG-like mixtures. They differ in that the GDF apparatus uses a calculational method to determine the density of the test liquid, while NBS uses an absolute densimeter to determine density. The densimeter is absolute in that the density of the liquid is determined by the Archlmedes method, using a single crystal of silicon as the plummet. No calibration liquid is needed or used.

If densimeters are used in custody transfer measurement of density, calibration fac1l1t1es will be required to maintain measurement accuracy. A means of intercomparing such calibration systems will be required. A portable version of the DRS densimeter has been built for this purpose [13]. Th1s unit. the portable reference densimeter (PRD), has been used to intercompare the DRS and the Gaz de France facility. 
This intercomparison of the two test facilities provides an opportunity to compare direct density measurement and the calculational method of density determination. The estimates of the systematic uncertainty of the two methods should overlap.

\subsubsection{The Density Calibration Systems}

The Gaz de France System. The Gaz de France system (GDFS) [6] has been used to test a number of commercial densimeters. It is essentially composed of a 32 liter sample container, figure 2.4.5.1, placed inside a vacuum insulated dewar. The temperature of this sample container is regulated by circulation of cold nitrogen gas around it. The vapor pressure in the sample container is kept approximately constant by a back pressure regulator. The sample temperature corresponds to the liquid-vapor equilibrium temperature at the control pressure. The temperature of the circulating nitrogen gas is regulated to correspond to the sample temperature to minimize evaporation of the sample. The sample liquid level is maintained at a sufficient height to immerse the densimeters tested. The level of liquid is maintained constant by injections of sample liquid. These injections also mix the sample.

The following parameters are measured:

1) The sample temperature, $T_{G D F}$ is measured with the platinum resistance thermometer shown in figure 2.4.5.1. This thermometer is calibrated at a number of $\mathrm{f}$ ixed points, and its uncertainty is estimated to be $\pm 0.03^{\circ} \mathrm{C}$.

2) The pressure in the vapor phase above the sample is also measured by a capacitance manometer. The uncertainty of this measurement is \pm 0.25 percent of the full scale reading or \pm 12 mbar, whichever if larger.

3) A small quantity of the liquid sample is continuously vaporized and then analyzed by a gas chromatograph. The technique of sampling and chromatographic analysis has been discussed in references [3 to 6]. The uncertainty of the mole fractions of the constituents other than methane has been estimated as \pm 1 percent of each fraction.

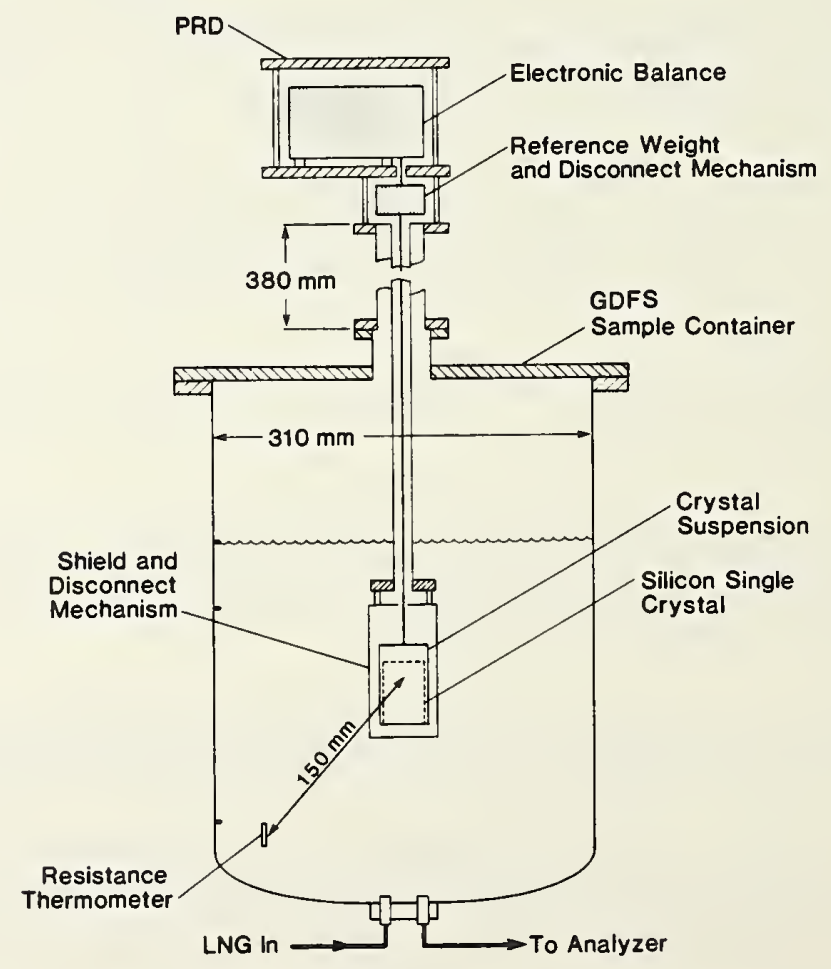

Figure 2.4.5.1. Schematic of the Gaz de France System (GDFs) sample container with the portable reference densimeter installed. 
When a satisfactory liquid-vapor equilibrium is reached in the apparatus, the sample temperature, the vapor pressure, and the composition in the case of mixtures, are recorded at approximately 10-minute intervals. Then, after changing the control pressure, possibly modifying the composition of the mixture, and injecting liquid until a new equilibrium is reached, a new set of data are recorded.

The sample density is calculated from the measurements of temperature, pressure, and composition of the sample. To determine the pure liquid methane density, only the temperature, T GDF of the liquid near the bottom of the sample container is needed.

The density $\rho$ GDF for pure saturated liquid methane as a function of temperature is given by a correlation developed by Haynes and Hiza [2]:

$$
\rho-\rho_{c}=a\left(1-\frac{T}{T_{C}}\right)^{0.35}+b_{1}\left(1-\frac{T}{T_{C}}+b_{2}\left(1-\frac{T}{T_{C}}\right)^{4 / 3}\right.
$$

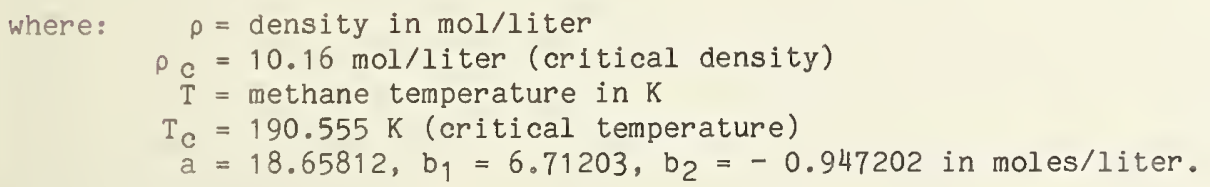

To obtain the mass density values given here, $\rho$ must be multiplied by the molecular weight, 16.04303 $\mathrm{g} / \mathrm{mol}$.

The experimental apparatus was designed to obtain a good liquid-vapor equilibrium, and, ideally, TGDF and the temperature $\mathrm{T}_{\mathrm{P}}$ calculated from the vapor pressure, using a parabolic interpolation of the pressure-temperature values in table 13 of reference [14], should agree. Since the sample chamber is not likely to be perfectly at equilibrium,

$$
\Delta T=T_{P}-T_{G D F}
$$

can be used as a measure of the quality of the equilibrium within it.

From equation (1) and the two temperatures $\mathrm{T}_{\mathrm{GDF}}$ and $\mathrm{T}_{\mathrm{P}}$, two values for the sample density can be calculated: $\rho \mathrm{GDF}=\rho\left(\mathrm{T}_{\mathrm{GDF}}\right)$ and $\rho_{\mathrm{P}}=\rho\left(\mathrm{T}_{\mathrm{P}}\right)$. The difference $\delta \rho=\rho \mathrm{GDF}-\rho \mathrm{P}$ provides an estimate of the measurement uncertainty when measuring the density of pure methane. This estimated uncertainty, $\delta \rho /$ $\rho$ was found to be \pm 0.15 percent assumes $\Delta T$ is entirely caused by a liquid temperature gradient.

The density of a mixture is calculated from measurements of $T_{G D F}, P$ and from measurements of the composition by the liquid sampling and calculation methods discussed earlier. The corresponding states method $[1,15]$, with an estimated uncertainty of \pm 0.1 percent, was used for this calculation. The vapor pressure and composition are used to calculate a sample temperature [16] as in the case of methane. This temperature is used along with the composition to calculate a second density value by the corresponding states method. This second density value again serves as an evaluation of the equilibrium. This apparatus has been used to test a number of commercially built densimeters.

The determination of LNG density from correlations of density with composition and temperature is subject to a number of sources of error. The estimated uncertainty of the mathematical models for predicting LNG density is 0.1 percent [1]. The composition determination is subject to uncertainty or the composition of the chromatograph calibration gas, the uncertainty of the chromatograph measurement and the uncertainty introduced by the sampling procedure. The uncertainty of the temperature measurement must also be included. The uncertainties associated with"the various parameters are as follows:

1) The uncertainty assigned to the sampling has been estimated to be 0.1 to 0.15 percent from tests of the sampling system [6].

2) The gas chromatograph used for the composition analysis is a state-or-the-art instrument. The uncertainty it introduces into a density determinat1on w111 be dependent on the composition of the sample and the accuracy of the callbration gas. 
The uncertainty that the chromatographic measurement contributes to the density determination has been estimated in one study to be 0.11 percent for LNG [5]. Parrish et al. [3] estimated an uncertainty contribution of 0.1 percent by the chromatograph to the heating value calculation. The uncertainty introduced into the density calculation is generally equal to or slightly less than the heating value uncertainty.

3) Errors introduced by the calibration gas uncertainty are based on estimates of the weighing uncertainties when the standard is prepared and is probably no more than \pm 0.03 percent [3]. An estimate for uncertainty introduced by deviations of the constituent gases was not available.

4) The density uncertainty introduced by the temperature measurement is about \pm 0.01 percent for a $\pm 30 \mathrm{mK}$ temperature uncertainty.

Parrish et al. [5] estimated that in the laboratory, the saturated density based on composition determinations from sampling and analysis combined with temperature and pressure measurement could be calculated to an uncertainty of 0.1 percent. This value seems optimistic since combining the uncertainties above in quadrature gives the uncertainty of a laboratory determination of density calculated from temperature and composition measurements of at least 0.2 percent. This includes no estimate of the uncertainty of the calibration mixture for the chromatograph other than that introduced by weighing the mixture.

The Density Reference System (NBS). The NBS densimeter test facility, the density reference system (DRS), has been described in the literature $[5,8,9,11,12]$ and in 2.4 .1 . It is a vacuum insulated sample container into which 16 liters of methane or LNG-like mixtures can be condensed. The system is completely closed. No gas vents at any time. Radiation shields cooled by liquid nitrogen reduce the heat leaks into the sample container, and cooling coils remove the remainder. The liquid can be stirred by a turbine pump. The thermal isolation of the sample maintains its isothermal state for minutes after the stirring has ceased. The density of the sample liquid is measured by an absolute densimeter. This densimeter uses the Archimedes principle, a silicon single crystal immersed in the liquid is weighed by an electronic balance. The density of single crystal silicon is known to a few $\mathrm{ppm}$, and if the vacuum weight of the crystal is measured, no calibrating fluid is necessary. The density of the liquid, $\rho_{1}$, is

$$
\rho_{1}=\rho_{S}\left(1-M / M_{S}\right)
$$

where $\rho_{S}$ is the crystal density, $M_{S}$ is its vacuum weight and $M$ is its apparent weight when completely immersed in the liquid.

When commercial densimeters were found to lack adequate calibrations, a transfer standards program was initiated by NBS to provide a better method of calibration to manufacturers and users (see 2.4.2). Densimeters of a manufacturer's or user's choice were calibrated in the DRS then returned to them. They would then use these calibrated instruments in their own sample container to calibrate densimeters sold. The calibration should be done in an LNG-like mixture for best results.

The transfer standard method of providing calibrations has disadvantages, however. The calibration of a standard could change with time or through shipping and handling. Repeated calibrations are necessary at least until a long term stability is established for the instrument. If the long term stability is adequate, there still remains the question of how well the calibration system in which the transfer standard is employed transfers the calibration from the standard to the densimeter under calibration. Testing calibrated densimeters in the DRS or GDFS could establish whether the calibration has been adequately transferred. Continual monitoring of the calibration operation is desirable to ensure the best calibration of the commercially available densimeters.

During the course of testing the commercial densimeters, both the DRS and the DRS densimeter were improved. The densimeter was improved by replacing the original electronic balance with a more compact and rugged one of greater range. The greater range permits the silicon crystal to be weighed directly instead of relative to a reference weight. The reference weight is retained in the new densimeter but now provides a means of adjusting and monitoring the balance calibration. This redesign suggested the possibility of making a portable standard densimeter of a design similar to the new DRS densimeter. This portable standard could be transported to other calibration facilities to measure their calibration uncertainty and to intercompare calibration facilities. A portable standard, the portable reference densimeter (PRD), has been built for this purpose. The PRD is shown schematically in figure 2.4.5.1 in place in the GDFS. This densimeter and comparison density measurements between it and the DRS are described in [12] and [13]. 
The densities of methane and methane-propane-nitrogen mixtures from simultaneous measurements by the PRD and the DRS densimeter were indistinguishable. Because of the larger crystal and the tungsten reference welght, the PRD is estimated to be slightly more accurate than the DRS densimeter. The density of pure methane liquid measured by the PRD is estimated to be within \pm 0.033 percent of the true density 99.7 percent of the time.

One densimeter user built a calibration system with a densimeter of a similar design for his standard. This unit, tested also in the DRS [9], gave densities that agreed with the DRS values to better than 0.005 percent \pm 0.01 percent, supporting the contention that the DRS densimeter is an accurate, wellcharacterized calibration instrument.

\subsubsection{Intercomparison of Calibration Systems}

The two test facilities were compared via the PRD. The PRD was air freighted from Boulder, Colorado, to the Centre de Recherches Gazieres of Gaz de France, located at St. Denis, near Paris, where it was inserted into the Gaz de France system. Densities of pure liquid methane samples and some mixtures were measured simultaneously with the PRD and the GDFS. These measurements are the first experimental comparisons between LNG-like mixture density from correlations and from direct density measurement with an absolute densimeter.

Upon arrival in Paris, the PRD balance was out of calibration by the amount expected because of the difference in the acceleration of gravity between Boulder and Paris. The balance was recalibrated with the reference weight, installed in the GDFS, and some initial data were taken with assistance from an NBS representative. The rest of the measurements and the calculations were done by Gaz de France personnel.

\subsubsection{Experimental Results}

Pure Methane. The pure methane data were taken at two separate periods of time. Measurements were made at seven different values of sample vapor pressure; hence, temperature. Table 2.4 .5 .1 gives a summary of these measurements. The values shown are averages of a series of measurements at the conditions. The pressure in the first column was nominally constant during the set of measurements at that pressure. The average measured temperature is shown in column two, and the average densities from GDFS and PDR are listed in the next two columns. The average error

$$
\Delta \rho / \rho=(\rho \mathrm{GDF}-\rho \mathrm{NBS}) / \rho \text { NBS }
$$

is given in percent in the fifth column and the standard deviation of $\Delta \rho / \rho$ is shown in the last column.

Table 2.4.5.1 Pure Methane Data

\begin{tabular}{|c|c|c|c|c|c|c|}
\hline $\begin{array}{c}\overline{\mathrm{P}} \\
(\mathrm{mbar})\end{array}$ & $\begin{array}{c}\overline{\mathrm{T}}_{\mathrm{GDF}} \\
(\mathrm{O} \mathrm{C})\end{array}$ & $\begin{array}{c}\bar{\rho}_{\mathrm{GDF}} \\
\mathrm{kg} / \mathrm{m} 3\end{array}$ & $\begin{array}{c}\bar{\rho}_{\mathrm{NBS}} \\
\mathrm{kg} / \mathrm{m} 3\end{array}$ & $\begin{array}{c}\overline{\Delta \rho / \rho} \\
(\%)\end{array}$ & $\begin{array}{c}\sigma(\Delta \rho / \rho) \\
(\%)\end{array}$ & $\begin{array}{c}\text { Number } \\
\text { of } \\
\text { observations }\end{array}$ \\
\hline \hline 1018 & -161.39 & 422.59 & 422.08 & 0.121 & 0.005 & 18 \\
1038 & -161.25 & 422.38 & 421.83 & 0.131 & 0.015 & 17 \\
1514 & -156.37 & 415.14 & 414.62 & 0.124 & 0.034 & 13 \\
2009 & -152.50 & 409.02 & 408.75 & 0.066 & 0.021 & 25 \\
2435 & -149.49 & 404.77 & 404.36 & 0.102 & 0.006 & 21 \\
2960 & -146.59 & 399.92 & 399.52 & 0.101 & 0.010 & 14 \\
3092 & -145.81 & 398.66 & 398.39 & 0.069 & 0.069 & 6 \\
\hline
\end{tabular}

The value of $\Delta \mathrm{T}$ from equation (2) varied during each set of measurements at a nominal temperature. In figure 2.4.5.2 $\Delta \rho$ is shown as a function of $\Delta \mathrm{T}$. Some density gradient in the sample is apparent irom these results, since $\Delta p$ increases with $\Delta T$. To remove this effect, the $\Delta T$ dependence shown was flt by the method of linear least squares to obtain

$$
\Delta \rho=0.51+1.093 \Delta T\left(\mathrm{~kg} / \mathrm{m}^{3}\right)
$$

The first term in equation (5) amounts to a constant of fset of $0.51 \mathrm{~kg} / \mathrm{m}^{3}$, which is about 0.12 percent at a density of $425 \mathrm{~kg} / \mathrm{m}^{3}$. A similar of set ( 0.10 percent) is observed at Boulder when the Dhs densimeter density value was compared to the density value calculated from the average temperature of a saturated liquid methane sample using equation (1). 


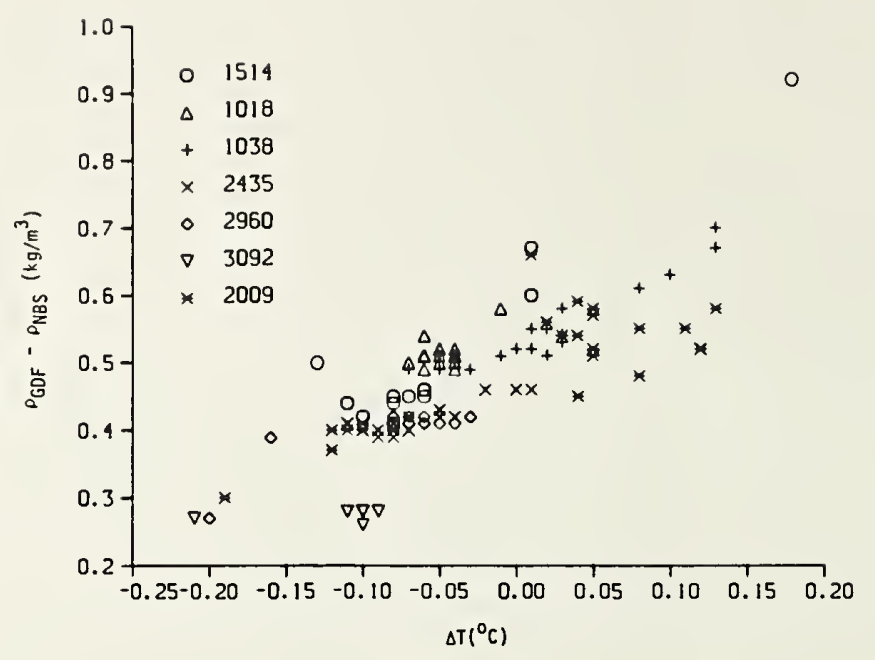

Figure 2.4.5.2. Difference in measured pure methane density by GDFs and NBS.

The close agreement of the magnitude of these two of sets supports the contention that this indirect method gives densities about 0.1 percent higher than the direct method as used in the DRS. The Boulder comparisons between the direct and indirect density values gives a 99 percent upper bound on the standard deviation of a single measurement to be $0.046 \mathrm{~kg} / \mathrm{m} 3$. This includes variation within a test on a sample and variation between samples. For the Gaz de France data on the seven different samples, the variation between tests is estimated to have a standard deviation of $0.044 \mathrm{~kg} / \mathrm{m}^{3} \mathrm{with}$ an upper 99 percent confidence limit of $0.115 \mathrm{~kg} / \mathrm{m}^{3}$ when the measured data is referenced to equation (5). The variation within tests has an estimated standard deviation of $0.041 \mathrm{~kg} / \mathrm{m} 3$ ( $114 \mathrm{observations}$ in seven tests) with an upper 99 percent confidence limit of $0.048 \mathrm{~kg} / \mathrm{m}^{3}$. The upper $99 \mathrm{percent}$ confidence limit for a single observation is then

$$
\left.\left[(0.115)^{2}+(0.048)^{2}\right)\right]^{1 / 2}= \pm 0.125 \mathrm{~kg} / \mathrm{m}^{3}
$$

Fluid Mixtures. Table 2.4.5.2 shows the averaged compositions of each of the mixtures used in these comparisons along with the averaged values of pressure, temperature (GDF), density (GDF), density (NBS), average density difference and the standard deviation of the average density difference. The compositions shown are the average over a set of determinations at a particular composition and vapor pressure setting. The mixtures in table 2.4.5.2 are listed in the order the data were taken. The individual values of $\Delta \rho$ are shown as a function of the same order in figure 2.4.5.3. The gap in the data shown was for some pure methane measurements. Two methane samples were also measured prior to the start of the mixture data acquisition.

The standard deviations about the mean of a set of measurements on a binary mixture are estimated to be $0.15 \mathrm{~kg} / \mathrm{m}^{3}$. This will be called the within test standard deviation, and it is judged the same for all ten tests of the binary mixtures. The means themselves vary much more than the within test variability allows. The means have a standard deviation of $0.35 \mathrm{~kg} / \mathrm{m}^{3}$. We infer from this that circumstances are changing significantly from test to test. Part of the change in circumstances is the change in composition, but a large shift does occur between two tests of the same mixture; i.e., $0.7 \mathrm{~kg} / \mathrm{m} 3$ between the first two tests shown in figure 2.4.5.3. These shifts in the means are larger than those observed for the pure methane data.

Equilibrium between the vapor phase and the liquid phase for mixtures is not easily obtained and probably accounts for the larger values for $\Delta T$ than observed for pure methane. The values of $\Delta T$ for the ten binary mixtures had ranges from 0.1 to 0.4 degrees Centigrade. Correlations between $\Delta \rho$ and $\Delta \mathrm{T}$ for any of these ten tests were of no practical significance in explaining the scatter of the data. The scatter is probably due to the sampling and analysis procedures. Approximately 98 percent of the data fall within \pm 0.18 percent of the overall mean. 
Table 2.4.5.2 Mixture Data

Averages of the data at each mixture and vapor pressure. The balance of the composition of each mixture is methane.

\begin{tabular}{|c|c|c|c|c|c|c|c|c|c|}
\hline \multirow[b]{2}{*}{$\|$} & \multirow[b]{2}{*}{$\begin{array}{c}\overline{\mathrm{P}} \\
\text { mbar }\end{array}$} & \multirow[b]{2}{*}{$\overline{\mathrm{T}}_{\mathrm{GDF}}$} & \multicolumn{2}{|c|}{$\begin{array}{l}\text { Composition } \\
\text { (balance } \mathrm{CH}_{4} \text { ) }\end{array}$} & \multicolumn{2}{|c|}{$\begin{array}{c}\text { Average } \\
\text { Densities }\end{array}$} & \multirow{2}{*}{\begin{tabular}{|c}
$\begin{array}{c}\text { Average } \\
\text { Difference }\end{array}$ \\
$\begin{array}{c}\overline{\Delta \rho / \rho} \\
\%\end{array}$
\end{tabular}} & \multirow{2}{*}{$\begin{array}{c}\text { S.D. of } \\
\text { Average } \\
\text { Difference }\end{array}$} & \multirow[b]{2}{*}{$\begin{array}{l}\text { \# of } \\
\text { Pt }\end{array}$} \\
\hline & & & $\begin{array}{l}\overline{\mathrm{X}} \mathrm{C}_{2} \mathrm{H}_{6} 6 \\
\text { mol } \%\end{array}$ & $\begin{array}{l}\overline{\mathrm{XC}}_{3} \mathrm{H}_{8} \\
\text { mol\% }\end{array}$ & $\begin{array}{l}\bar{\rho}_{\mathrm{GDF}} \\
\mathrm{kg} / \mathrm{m} 3\end{array}$ & $\begin{array}{l}\bar{\rho}_{\mathrm{NBS}} \\
\mathrm{kg} / \mathrm{m} 3\end{array}$ & & & \\
\hline 1 & 1460 & -156.40 & 4.13 & 0.00 & 427.81 & 427.61 & 0.044 & 0.044 & 10 \\
\hline 2 & 1378 & -157.22 & 3.87 & 0.00 & 427.92 & 427.04 & 0.207 & 0.030 & 23 \\
\hline 3 & 1446 & $-156 \cdot 30$ & 3.43 & 1.32 & 432.32 & 433.01 & -0.153 & 0.144 & 16 \\
\hline 4 & 1494 & -155.75 & 5.45 & 1.31 & 437.31 & 437.10 & 0.048 & 0.123 & 13 \\
\hline 5 & 1237 & -158.54 & 4.45 & 1.12 & 437.63 & 437.76 & -0.030 & 0.213 & 6 \\
\hline 6 & 1330 & -157.52 & 3.94 & 1.01 & 433.87 & 434.04 & -0.038 & 0.099 & 16 \\
\hline 7 & 1356 & -161.14 & 3.62 & 0.94 & 440.73 & 440.14 & 0.132 & 0.015 & 16 \\
\hline 8 & 1930 & -152.67 & 4.25 & 0.00 & 422.41 & 421.99 & 0.101 & 0.053 & 14 \\
\hline 9 & 2390 & -149.10 & 4.71 & 0.00 & 418.46 & 418.58 & 0.055 & 0.032 & 16 \\
\hline 10 & 3034 & $-145 \cdot 36$ & 4.55 & 0.00 & 412.15 & 412.06 & 0.018 & 0.024 & 12 \\
\hline 11 & 3026 & -144.99 & 6.98 & 0.00 & 418.85 & 418.83 & 0.003 & 0.028 & 12 \\
\hline 12 & 2992 & -145.01 & 8.00 & 0.00 & 421.91 & 421.83 & 0.020 & 0.047 & 4 \\
\hline 13 & 2970 & -145.12 & 8.29 & 0.00 & 423.00 & 422.94 & 0.002 & 0.099 & 18 \\
\hline 14 & 2454 & -148.24 & 7.60 & 0.00 & 425.66 & 425.60 & 0.014 & 0.032 & 10 \\
\hline 15 & 2040 & -150.17 & 12.63 & 0.03 & 442.99 & 443.39 & -0.090 & 0.120 & 12 \\
\hline 16 & 1980 & -150.72 & 13.00 & 0.03 & 444.83 & 445.18 & -0.078 & 0.027 & 11 \\
\hline 17 & 1491 & -156.55 & 0.00 & 2.85 & 430.61 & 430.54 & 0.025 & 0.131 & 16 \\
\hline 18 & 1958 & -153.06 & 0.00 & 2.91 & 425.83 & 426.14 & -0.071 & 0.023 & 12 \\
\hline
\end{tabular}

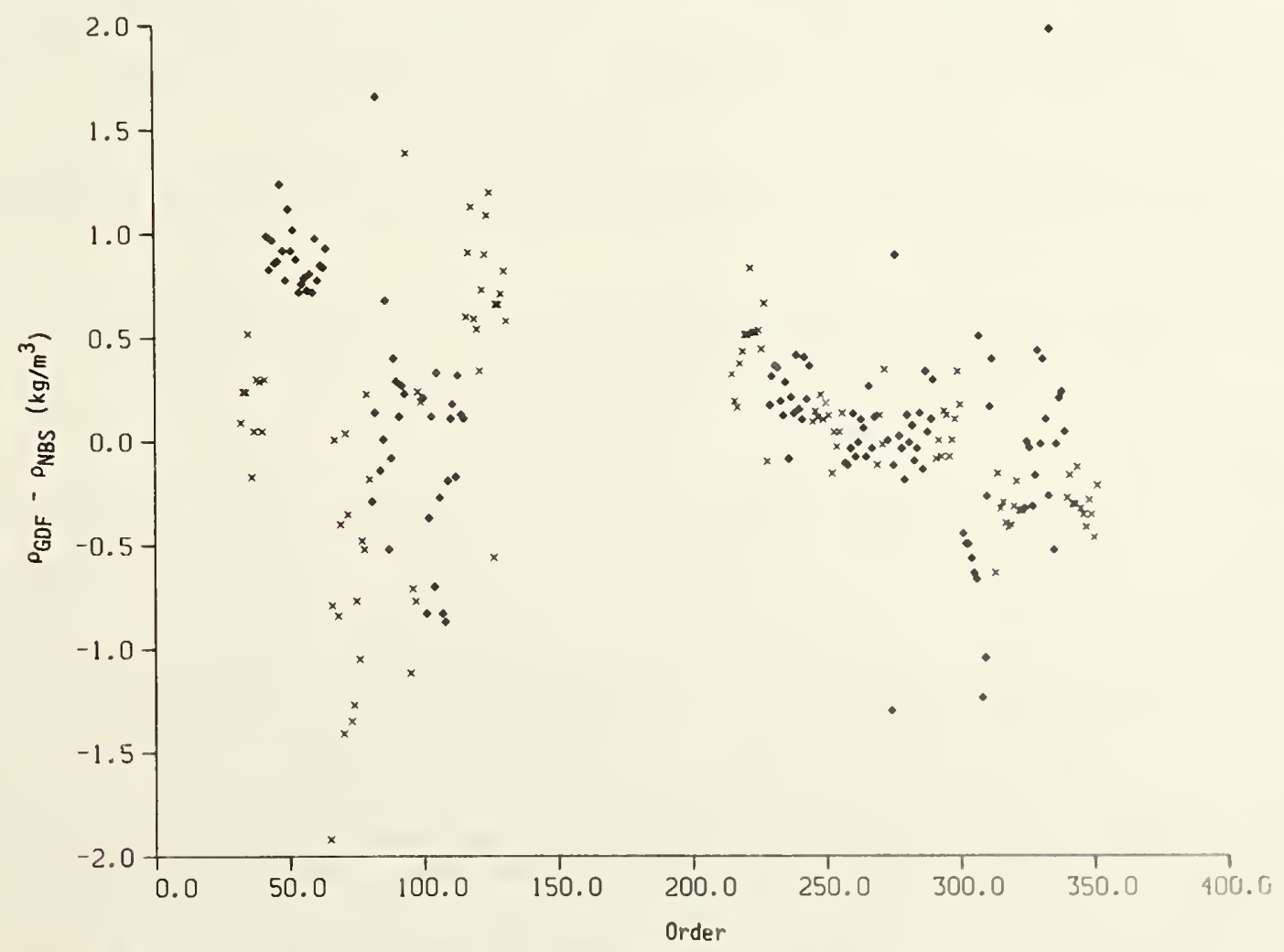

Figure 2.4.5.3. Differences in density measurements as a punction of data order for various mixtures. 
The estimated standard deviations about the mean of a set of measurements of a ternary mixture are mostly around $0.5 \mathrm{~kg} / \mathrm{m}^{3}$. One is as $10 \mathrm{w}$ as $0.1 \mathrm{~kg} / \mathrm{m} 3$ and one as high as $0.9 \mathrm{~kg} / \mathrm{m} 3$. The eight means themselves are varying with an estimated standard deviation of $0.3 \mathrm{~kg} / \mathrm{m}^{3}$. Approximately 95 percent of this data fall within \pm 0.25 percent of the overall mean.

\subsubsection{Conclusions}

Though the density determined from temperature and composition measurements using a correlation has been compared to the density measured by commercial densimeters [6], this work represents the first known comparison to an absolute densimeter. This comparison provides a direct experimental estimate of the uncertainty associated with the method of determining density from correlations using measured temperature and composition.

The density measured by the PRD in pure liquid methane was 0.12 percent smaller than the density determined by the GDFS by calculation from a sample temperature measurement, provided a correction is introduced for $\triangle \mathrm{T}$. Similar results were obtained in the DRS. When the directly measured liquid methane density is compared to the density calculated from the averaged readings of two thermometers, the directly measured density is 0.10 percent low. This means that the GDFS and the DRS agree to within 0.02 percent on density measurements in pure methane when a correction is made for the 0.1 percent offset between direct density measurement by the DRS or PRD and the Haynes-Hiza density from temperature correlation [2]. Thus, the two test systems show good agreement on 1 iquid methane density. The upper 99 percent 1 imit of a measurement of density in this comparison is $0.125 \mathrm{~kg} / \mathrm{m} 3$ compared to a similar limit of $0.046 \mathrm{~kg} / \mathrm{m}^{3}$ for the DRS [9].

The densities of the mixtures examined in this work measured by the GDFS and the PRD differed by no more than 0.25 percent in 95 percent of the measurements. This value does not conflict with the estimated errors for the two measurement methods but is larger than the 0.1 percent estimated from the heating value studies [5]. The uncertainty in heating value was estimated to be \pm 0.15 percent in the laboratory [5] and \pm 0.3 percent for measurements on shipboard [3]. Based on this, the uncertainty in field measurement of LNG density by sampling and analysis of the liquid could be larger than the \pm 0.25 percent obtained in the comparison of the DRS and GDFS described here. 
2.4.5.6 References

[1] McCarty, R. D., Four mathematical models for prediction of LNG densities, Nat. Bur. of Stand. (U.S.), Technlcal Note 1030 (December 1980). Also, McCarty, R. D., Mathematical models for the prediction of 11 quefled natural gas densities, J. Chem. Thermodynamics 14, 837, 1982.

[2] Haynes, W. M. and M. J. H1za, Measurements of orthobaric liquid densities of methane, ethane, propane 1sobutane and normal butane, J. Chem. Thermodynamics 9, 179 (1977).

[3] Parrish, W. R., J. M. Arvidson and J. F. LaBrecque, Development and evaluation of an LNG sampling measurement system, Nat. Bur. of Stand. (U.S.), NBSIR 78-887 (July 1978).

[4] Parrish, W. R., J. M. Arvidson and J. F. Labrecque, Evaluation of LNG sampling measurement systems for custody transfer, AGA Operating Section Proceedings, Proceedings of the Transmission Conference; Montreal, Quebec, T-236 (1978).

[5] Parrish, W. R., J. A. Brennan and J. D. Siegwarth, LNG custody transfer research at the National Bureau of Standards, AGA operating Section Proceedings; Proceedings of the Transmission Conference, Montreal, Quebec, Canada, T-243 (1978).

[6] Dewerdt, E. and P. Mousset, Contribution a l'amelioration du comptage du GNL - evaluation de denslmetres et echant1llonneur pour GNL, Sixth International Conference on Liquefied Natural Gas, Kyoto, Japan (1980), Appendix, Paper 8.

[7] The work was supported first by the American Gas Association, 1515 Wilson Blvd., Arlington, VA 22209 and later by the Gas Research Institute, 8600 West Bryn Mawr Avenue, Chicago, IL 60631.

[8] Siegwarth, J. D., B. A. Younglove and J. F. LaBrecque, Cryogenic fluids density reference system: provisional accuracy statement, Nat. Bur. of Stand. (U.S.), Technical Note 698 (November 1977).

[9] Siegwarth, J. D. and J. F. LaBrecque, Cryogenic fluids density reference system: provisional accuracy statement (1980), Nat. Bur. of Stand. (U.S.), Technical Note 1041 (1981).

[10] Siegwarth, J. D., B. A. Younglove and J. F. Labrecque, An evaluation of commercial densimeters for use in LNG, Nat. Bur. of Stand. (U.S.), Technical Note 697 (October 1977).

[11] Siegwarth, J. D., J. F. LaBrecque and 3. A. Younglove, Test of densimeters for use in custody transfer of LNG, Proceedings 53rd International School of Hydrocarbon Measurement, Norman, Oklahoma (1978) p 385 .

[12] Siegwarth, J. D. and J. A. Brennan, LNG instrumentation for custody transfer, AGA Operating Section Proceedings; Proceedings of the Transmission Conference, Atlanta, Georgia, T-119 (1981).

[13] Siegwarth, J. D. and J. F. LaBrecque, A portable calibration densimeter for use in cryogenic liquids, Nat. Bur. of Stand. (U.S.), Technical Note 1035 (1981).

[14] Goodwin, R. D., The thermophysical propert1es of methane from 90 to $500 \mathrm{k}$ at pressures to 700 bar, Nat. Bur. of Stand. (U.S.), Technical Note 653, (April 1974).

[15] McCarty, R. D., A comparison of mathematical models for the prediction of LNG dens1t1es, Nat. Bur. Of Stand. (U.S.), Interagency Report 77-867 (October 1977).

[16] The method is described in an unpublished note by Gaz de France. The method 1s based on the work by B. Lee, J. H. Erbar, and W. C. Edminster, Prediction of thermodynamic propertles for low temperature hydrocarbon process calculations, AIChE Journal 19, 349 (1973).

[17] Roncier, R. P., J. Saint-Just, J. E. Dewerdt, J. D. Siegwarth and J. F. LaBrecque, Est1mated uncertainty of calculated liquefied natural gas density from a comparison of WBS and Gaz de Erance densimeter test faclilities, Nat. Bur. Stand. (U.S.), J. Research, 88 No.3, 163-70 (May-June, 1983). 




\section{LNG MEASUREMENT}

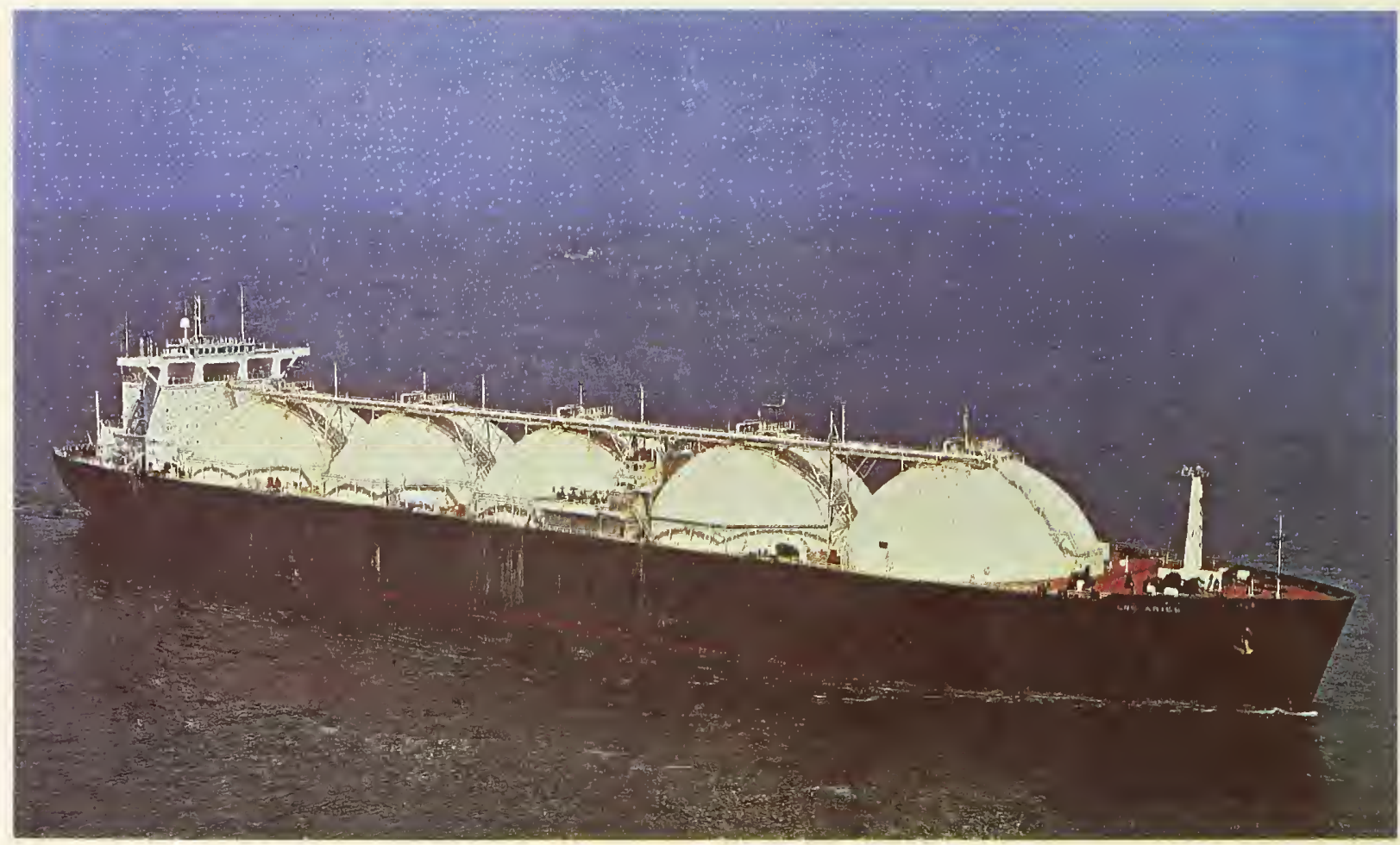

\section{LNG ARIES}

The LNG Aries is a 125,000 cubic meter capacity tanker constructed by General Dynamics Corporation (U.S.) and completed in 1977. The design was of the Moss-Rosenberg sphericai free standing type, and the owner was Wilmington Trust Company, Ltd. The ship is one of eight tankers of the same design constructed by Generai Dynamics during the period 1977-1979 and used to deliver LNG from Indonesia to Japan.

Measurements to establish the cargo value are described in the Measurement Manual. Of particuiar interest is the method of determining the original volume of the primary spherical tanks. A new technique is described, and the estimated error is presented. (Photograph by courtesy of The Japan Gas Association, Tokyo, Japan, on behalf of the 1981 Japan LNG Congress) 


\section{0 MEA SUREMENT ELEMENTS}

Vol ume

2.5

$2 \cdot 5 \cdot 1$

$2.5 \cdot 1 \cdot 1$

$2 \cdot 5 \cdot 1 \cdot 2$

$2 \cdot 5 \cdot 1 \cdot 3$

$2 \cdot 5 \cdot 1 \cdot 4$

$2 \cdot 5 \cdot 1 \cdot 5$

$2.5 \cdot 1 \cdot 6$

2.5 .2

$2.5 \cdot 2 \cdot 1$

$2.5 .2 \cdot 2$

$2 \cdot 5 \cdot 2 \cdot 3$

$2 \cdot 5 \cdot 2 \cdot 4$

$2 \cdot 5 \cdot 2 \cdot 5$

$2 \cdot 5 \cdot 2 \cdot 6$

$2 \cdot 5 \cdot 3$

$2.5 \cdot 3 \cdot 1$

$2 \cdot 5 \cdot 3 \cdot 2$

2.5 .3 .3

2.5 .3 .4

$2 \cdot 5 \cdot 3 \cdot 5$

2.5 .3 .6

2.5 .4

2.5 .4 .1

2.5 .4 .2

$2 \cdot 5 \cdot 4 \cdot 3$

$2 \cdot 5 \cdot 4 \cdot 4$

2.5 .4 .5

2.5 .4 .6

2.5 .4 .7

2.5 .5

$2.5 \cdot 5 \cdot 1$

2.5 .5 .2

2.5 .5 .3

2.5 .6 2.5 .6 .1 $2 \cdot 5 \cdot 6 \cdot 2$ 2.5 .6 .3 2.5 .6 .4 2.5 .6 .5 2.5 .6 .6 $2 \cdot 5 \cdot 6 \cdot 7$ $2 \cdot 5 \cdot 6 \cdot 8$
LNG Vol ume

Liquid Level Measurement

In troduction

Gage Types

Capacitance Type Level Gages

Cable Gage Tests

Bubbler Type Level Gage

References

Volume of Membrane-Type LNG Ship Tanks

Volume Measurement Methods

Analysis.

Integration Techniques

Results

LNG Ship Membrane Tank Calibrations

References

Volume of Freestanding Prismatic LNG Ship Tanks

In $\mathrm{t}$ roduction

Tank Description

The Phototgrammetric Calibration Method

Accuracy of the Photogrammetric Calibration Method

Accuracy of Tanks in Service

References

Volume of Spherical LNG Ship Tanks

Int roduction

Photogrammetric Survey

Analysis of Spherical Ship Tank

Calculation of Sounding Tables

e-Dimensional Triangulation Survey

Comparison of Survey Methods

References

Volume of Cylindrical LNG Shore Tanks

Photogrammetric Survey

Vol ume Computation

References

Vol ume From Totalized Flow Rate Metering

In troduction

The Measurement Station

Component Element Test ing

Flowmeter Scaling

Densimeter Tests

Conclusions.

Recommendations

References
Page

$2 \cdot 5 \cdot 1-1$

$2 \cdot 5 \cdot 1-1$

2. 5. 1- 1

$2 \cdot 5 \cdot 1-2$

2. 5 . 1-2

$2 \cdot 5 \cdot 1-5$

$2 \cdot 5 \cdot 1-19$

2. 5. 1-22

$2 \cdot 5 \cdot 2-1$

$2 \cdot 5 \cdot 2-1$

$2 \cdot 5 \cdot 2-5$

2. 5. 2-7

2. 5. 2-7

$2.5 .2-8$

2. $5.2-10$

2. 5. 3-1

2. 5. 3-1

2. 5. 3-2

2. 5. 3-7

2 . 5 . $3-8$

$2.5 \cdot 3-9$

2. 5. 3-12

$2.5 \cdot 4-1$

2. 5. 4-1

$2 \cdot 5 \cdot 4-1$

$2.5 \cdot 4-4$

$2.5 .4-8$

$2.5 .4-10$

$2.5 .4-14$

2. 5. 4-18

2. 5. 5-1

$2 \cdot 5 \cdot 5-1$

$2 \cdot 5 \cdot 5-4$

$2.5 .5-5$

2. 5.6-1

$2.5 \cdot 6-1$

2.5.6-1

$2.5 .6-3$

$2.5 \cdot 6-7$

2. 5.6-18

2. 5. 6-21

$2.5 \cdot 6-22$

$2.5 .6-23$ 



\subsection{LNG Volume}

\subsubsection{Liquid Level Measurement}

The level of liquid in a fixed volume container is a measure of quantity but is in itself of limited practical use. Combination of this measurement with that of volume-height calibrations (see 2.5 .2 and following sections) allow calculation of volume quantities as a function of liquid level above a fixed datum plane. A direct or calculated density (see 2.4), which must be representative of the entire volume, can then be combined with the volume calculation to give the mass of LNG associated with the liquid level. Liquid level is then only a portion of a rather complex process which is designed to provide a measure of quantity and quality of LNG. A critical assessment of the measurement uncertainties associated with the performance of several types of these devices has been made by Siegwarth [23]. This work, conducted at NBS-Boulder and sponsored by the Maritime Administration of the U.S. Department of Commerce, is presented in edited form in the following sections.

The measurement uncertainties of three types of gages commonly used in large liquefied natural gas storage and ship transport tanks were studied. The types are bubbler, capacitance, and cable gages. Measurement uncertainties for various types can be determined, but the test conditions must be carefully specified, because many of the largest errors result from parameters external to the gaging device. The gage installation, the tank design, and the liquid properties all influence gaging accuracy. A modification of cable gage installations that should significantly improve gaging accuracy is presented. Intrinsic accuracies of some representative cable gages are reported from tests done at ambient temperature under nearly isothermal conditions. The gages tested, if calibrated, can all measure level to about one millimeter under the test conditions. Capacitance gages should be tested in liquefied natural gas to establish their measurement uncertainty. Bubbler gages require knowledge of liquid density, the temperature distributions in and contents of the connecting tubes, pressure drops, and head corrections; otherwise, the errors can be several centimeters.

\subsubsection{Introduction}

Custody transfer of shipload quantities of liquefied natural gas (LNG) is currently accomplished by measurement of the volume, density and heating value per unit mass of the liquid. The volume in custody transfer is determined by measuring liquid level in calibrated ship transport tanks with liquid level gages. The National Bureau of Standards has had an active program in custody transfer measurement in which measurement methods have been examined and improved, and uncertainties for many of the measurements have been established (see References 1 through 11).

Extensive work has been completed and reported on all custody transfer measurements but liquid level measurement. The only cryogenic liquid level work at NBS to date has been a survey of level gaging methods $[12]$.

Level gage manufacturers usually supply gage accuracy estimates for their instruments. These estimates are based on design analysis, and perhaps ambient temperature tests of the gages, but generally not cryogenic tests except on a very limited scale. This is not surprising, since the great heights of tanks, up to 40 meters in some cases, preclude full scale cryogenic testing of gages by any one manufacturer because of the high cost of both the facility and the tests. A few papers in the published literature discuss some of the gaging methods and usually give some estimates of gage accuracy [13 through 19]. Few sub-ambient temperature tests have been done on any gages, and no independent tests of gages or tests of a number of gages in a single facility have been reported in the literature. The following should be considered an initial step in independently evaluating level gage accuracy.

The three types of level gages considered are the main choice for custody transfer measurement of LNG in shipload quantities. They are the capacitance type, the cable type and the bubbler type. These types are described in more detail in later sections. The capacitance type is generally used as the primary gage for custody transfer, while the cable gage or the bubbler usually serves as a back up gage. Gaging methods used for small tanks, such as truck tanks, are not included in this study.

The measurement uncertainties of some representative gages of the three types have been measured or estimated from available information. Level gaging for custody transfer is assumed to take place when the tank level is static, so the liquid surface is relatively quiet. Gage accuracy when the surface is moving is not estimated. Some of the gaging devices are too slow to even follow the liquid surface if the tank is rapidly filled. The uncertainty and hysteresis of four different types of cable gages have been measured at ambient conditions. 
Because the measurement of level by capacitance gages and bubbler gages depends on bulk parameters rather than the difference between liquid and vapor, as do the cable gages, adequate testing requires the use of a liquid, preferably LNG. No test facility presently exists that is capable of such measurements. Limited resources precluded such tests in this work. The measurement uncertainties for capacitance gages and bubbler gages have been estimated from existing information. The temperature within a tank and the external environment affect cable gage accuracies. The uncertainties introduced into cable gage measurements by the environment and ways of reducing these uncertainties are covered in Siegwarth [23].

\subsubsection{Gage Types}

Capacitance Gage Capacitance level gages are conceptually simple devices. One type has been described in the literature [18]. They are cylindrical capacitors consisting of two uniform coaxial tubes whose total capacitance is proportional to length if end effects are neglected. A vertical coaxial capacitor in vacuum has a capacitance of $C_{1}$. If this capacitor is filled with a dielectric liquid to a height $\mathrm{h}$, then

$$
C=2 \pi h\left(\varepsilon_{\ell}^{-1) l n} b / a+C_{1}=h C_{1 i q}+C_{1}\right.
$$

where $\varepsilon_{l}$ is the dielectric constant of the liquid, b and a are the outer tube ID and inner tube OD respectively, and $C_{l i q}$ is the capacitance over and above the vacuum value added by the 1 iquid dielectric. Actually, the empty portion of the gage contains a gas, so the term $\varepsilon_{\ell}-1$ is replaced by the term $\varepsilon_{\ell}-\varepsilon_{g}$ where $\varepsilon_{g}$ is the gas dielectric constant and $C_{1}$ is now the gas filled value of $C$. For $C_{1 i q}$ to remain proportional to $h, \varepsilon_{\ell}$ and $\varepsilon_{g}$ must remain independent of time and fill level.

Capacitance gages have limited application because the liquids in which they are used must leave no residue on the surfaces of the capacitor. Also, the liquids must have very low electrical conductivity. Liquefied natural gas appears to meet both these requirements.

Cable Gage Cable type level gages consist of a surface sensor suspended on a vertical wire, tape or cable $[14-17,19]$. As the liquid surface and the surface sensor move, the wire, tape or cable is wound on, or unwound from, a storage drum in the gage head to maintain the sensor at the liquid surface. The change in length of wire, etc., is measured either by the drum rotation or by a calibrated sprocket located in the gage head. The drum or sprocket drives a counter which gives the level of the liquid. This type of gage is widely used and can be used for almost any liquid.

A number of manufacturers build cable operated gages in a range of designs. Some gages, called automatic gages, are mechanical, while others are servo driven. In the latter the surface sensor transmits signals enabling the drive motor to maintain the sensor at the liquid surface.

Bubbler Gage Bubbler gages measure hydrostatic head. The pressure of a noncondensing gas required to displace all the liquid out the open bottom end of a drop tube that extends to the tank bottom is approximately the hydrostatic pressure. Usually, gas is bubbled slowly out the bottom of the tube to insure the liquid level has been forced to the bottom. From the hydrostatic head and the liquid density, the liquid level can be calculated.

\subsubsection{Capacitance Type Level Gages}

General Description A schematic diagram of a capacitance level gage is shown in figure 2.5.1.1. The gage consists of flanged sections of tube 1 with sections of smaller tube 2 mounted concentrically inside and supported by insulating posts 3. The inner tube sections are electrically isolated from each other by either a gap or a dielectric plug 4. The outer tube flanges 6 are bolted together so the outer tubes form a single conductor. This outer tube is electrically isolated by the insulator 5 from the tank 8. One lead from the outer tube and leads from each of the center tube sections are brought out of the tank through a seal 7. The center sections of a capacitance gage are often connected together electrically within the tank so that leads from only a top, bottom and center section need be brought out along with a lead from the outer tube.

A properly installed gage is supported vertically by the floor of the tank and is supported in the transverse directions but not constrained in the vertical direction at all other points. The segmented design insures that the gage can be handled and installed. It also permits selecting tubes of uniform dimension to minimize variation in the capacitance per unit length. The main purpose of segmenting the gages is to electrically isolate the center segments. Isolating sections allows the gage segments to be electrically switched so only the segment containing the liquid surface and a 


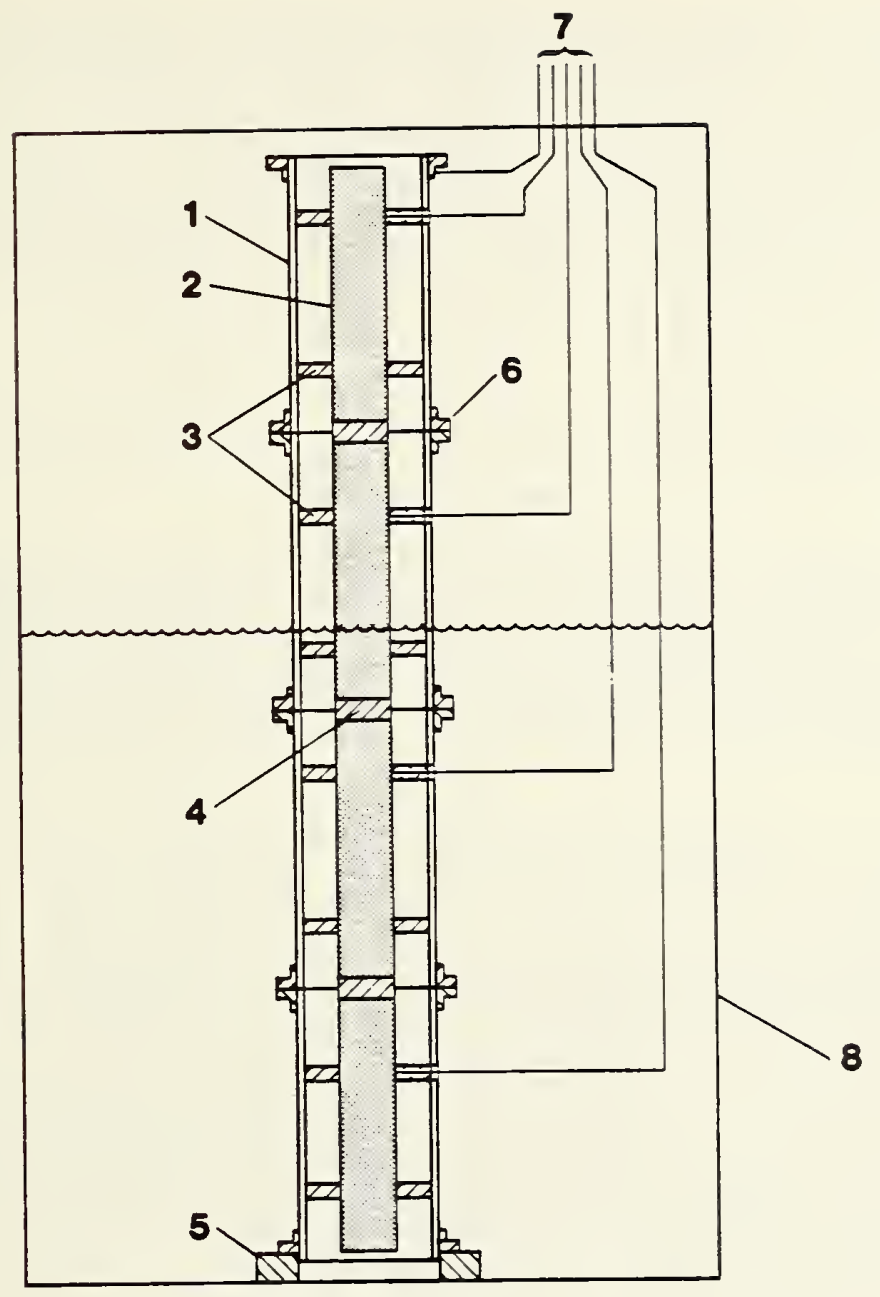

Figure 2.5.1.1. Schematic diagram of a capacitance level gage.

nearby completely filled segment used for reference are electrically active. Activating only the segment containing the liquid surface and ratioing that capacitance value to the nearby full section capacitance greatly reduces sensitivity of the measurement to changes in the liquid dielectric constant. The effect of uncertainties in the dielectric constant of the gas in the unfilled sections are reduced or eliminated. When a section is filled or emptied, the gage readout automatically switches to the next higher or next lower segment respectively.

The absence of moving parts is an attractive feature of this gage especially for shipboard applications and for tanks subjected to rapid filling and emptying rates. The measurement accuracies estimated are for the tank under static conditions and may not apply while the level is changing.

The two commercially available capacitance level gages are described briefly below.

First Capacitance Gage The gage described in this section [18] consists of two thin walled alumlnum tubes. The outer tube is about 6 centimeters ID, and the inner tube is about 4 centimeters OD. The inner tube is supported by the outer tube via two orthogonal insulated bolts along diameters. The various centering and supporting insulators are shielded to eliminate leakage currents to the center tube. Shims are placed at the insulator points to compensate for non-linearities introduced by the insulators, so that $\mathrm{dc} / \mathrm{dh}=$ constant where $\mathrm{C}$ is the capacitance and $\mathrm{h}$ is the helght. The $\mathrm{flanges}$ are fitted and riveted to the outer tube in a jig. The flange face separations are tested with a gage rod to $\pm 1 / 4$ millimeter. The sections can be 3.6 meter or 5 meter in length with one sectlon an odd length as required to bring the assembled gage to the desired height in the tank. Entrance holes for the LNG are provided at the flange collars but nowhere between flanges to avold weakening the tube. 
The gage and associated electronic circuitry is designed so that the full and empty conditions can be simulated at ambient conditions. This provides a method of calibrating the gage when the tank and gage are at ambient temperature. This calibration procedure cannot be used in an empty cold tank, because variations in the gas temperature result in sufficiently large variations in the capacitance of the gage sections. When the tanks are empty and cold, the zero values of each segment can be tested by manually switching in each segment and observing the level reading. If the level reading is not the known height to the bottom of the segment, the segment zero level value is adjusted to read the correct value. When the tank is filled with LNG, and the same segment is switched in, the gain or span of the electronics can be adjusted to give the correct full reading. This is done for each segment.

Measurement Uncertainties The lengths of the segments at the LNG boiling temperature of about - 160 degrees Centigrade, are known to

$$
\pm 1 / 4 \pm 0.03 \Delta l \text { millimeters }
$$

The second term in this uncertainty arises from the uncertainty of the thermal expansion coefficient of aluminum and $\Delta l$ is the change in length from ambient to the LNG boiling point. The gage sections above the liquid surface can be expected to be at some temperature other than the liquid temperature, but these sections do not enter the level measurement. The temperature uncertainty there contributes no error. The uncertainty of a level measurement when the level reaches a joint between two electrically separated segments $n$ and $n+1$ is

$$
\sigma=\left[0.25 \sqrt{n}+0.35 \sum_{1}^{n} \Delta \ell_{n}\right] \text { millimeters }
$$

Only the uncertainty of the section length and the uncertainty of the expansion coefficient [21] contribute to the measurement error when the liquid level is located at a joint. This equation assumes that no error is introduced by the readout circuitry.

As the level of the liquid moves above a joint between electrically separated segments, the linearity and resolution of the electrical components must be included in the gage accuracy. Some unpublished tests of gage sections using freon indicate a level measurement uncertainty no larger than \pm 3 millimeters [20]. This error should be maximum when the level is around the mid point of a segment. The non-linearity introduced by the gap between the ends of the center tube segments is small and included in the \pm 3 millimeters according to the manufacturer.

The manufacturer estimates the total uncertainty caused by calibration, capacitor dimensions and linearity, and the electronics as \pm 3.5 millimeters for a single section of the gage. The total uncertainty is placed at \pm 7.5 millimeters. The system measures level with a resolution of 0.1 millimeters and displays the measurement to 1 millimeter.

Second Capacitance Gage This gage consists of a 6.65 centimeter diameter ID 5 millimeter wall aluminum tube with a 1.3 centimeter diameter aluminum tube down the center. The 1.3 centimeter tube is centered by three plastic insulating rods inserted through holes placed 120 degrees apart around the tube in a plane perpendicular to the tube axis, and clamped against the inner tube by a band around the outside of the outer tube. These support assemblies are placed periodically along the tubes. No shielding of the insulators or compensation for them was found necessary.

The tubes are segmented usually in 5 meter lengths by flanges welded to the outer tube. The inner tube axial spacing is maintained by insulating spacers between adjacent ends. Holes are provided at intervals in the outer tube walls to permit entrance of the tank contents. The flanged outer tube is built to a length and measured. The variation in length is estimated to be \pm 1 millimeter.

The gage calibration is done when the tank is completely empty and completely full. With the tank empty, the segments are switched in one at a time and the zero reading of each segment is adjusted to the proper level reading. When the tank is filled, each segment is switched in and the span is adjusted to give the proper full reading. A point sensor on the top segment provides an intermediate point to calibrate the top segment.

Measurement Uncertainties The uncertainty $\sigma$ of the level at a joint for this gage is estimated to be

$$
\sigma=\left[\sqrt{n}+0.3 \sum_{1}^{n} \Delta \ell_{n}\right] \text { millimeters }
$$


where $\mathrm{n}$ is the number of segments submerged and $\Delta \mathrm{l}_{\mathrm{n}}$ is the thermal contraction of the cooled segments. The level measurement uncertainty increases as the liquid level moves up into a segment. Non-linearity of the gage results from variation in the tube dimensions $(-0.013$ millimeters on the inner tube $O D$ and 0.0025 millimeters on the outer tube ID). The height uncertainty from these variations is \pm 4.25 millimeters, but only a maximum of \pm 3 millimeters has been observed according to the manufacturer. The manufacturer estimates that temperature variations in the tank and variation of the electronics contribute errors of \pm 1.2 millimeters and \pm 5 millimeters respectively. The sum of these errors is 9.2 millimeters and the RMS value would be 6 millimeters. The systematic error from equation (3) for 5 meter sections with a 1 millimeter length uncertainty is \pm 5 millimeters at the top of a 25 meter high tank which raises the RMS uncertainty to about \pm 8 millimeters at the top of the tank.

Discussion The capacitance level gages of the two manufacturers differ in detail. Fundamentally, they are similar, and the experiences and needs of field use have resulted in modifications that have enhanced their similarities.

The uncertainty estimates cited in the manufacturers' 1 iterature are $\pm 7.5 \mathrm{millimeters}$ and \pm 10 millimeters for the first and second type gages respectively. If the error estimates by the manufacturers for linearity, gas temperature in the unfilled portion, electronics and thermal expansion coefficients of the aluminum tube are correct, then the error estimates by both manufacturers are conservative. Test data taken by one manufacturer, using freon at ambient temperature to simulate LNG, tends to confirm the conservative nature of the estimate, since the gage agreed with a ruled standard to \pm 3 millimeters [20].

Freon, however, will not behave the same as LNG. The latter can foam, the heavier hydrocarbons might preferentially adhere to the gage surfaces and cause larger level error readings than noted for freon. Variation in both gas and liquid density in the tank can introduce measurement error. Segmenting the gage eliminates any error that otherwise would have been introduced by the completely filled or completely empty segments. The gage accuracy cannot be tested or monitored readily. The full and empty calibration measurements are probably separated by 24 hours at least for transport tanks and perhaps by weeks or months in storage tanks. The top section-full calibration can only be tested by raising the liquid level above the gage either while filling or after filling by pumping liquid from tank to tank, unless an intermediate level sensor is provided.

Circulation of the liquid and gases during the filling and emptying of the tanks and ship motion while at sea can be expected to minimize liquid variations. However, special precautions are necessary to prevent density stratification at the very bottom of spherical tanks during filling. Stratification has been observed in spherical tanks especially when top filled. As the tank is cooled to the LNG cargo temperature, methane is flashed of while heavier components collect. Since a very small volume is incorporated in the bottom of a spherical tank, these heavy components can form a layer of sufficient depth to interfere with the automatic switching of the gage to higher segments as the liquid level rises. If the gage can be calibrated at ambient temperature, then in ship tanks it could presumably be calibrated once a year during the usual yearly inspection. Shore-based storage tank gages, since the tanks may never be warmed to ambient, do not present sufficient opportunities for ambient recalibration.

A possibility of overestimating capacitance gage accuracy exists. To establish accuracy of capacitance gages, the gages should be tested over a period of time in LNG service. This could be done in a special calibration facility or in a storage tank by comparing them to another gage of proven accuracy. Such a gage does not now exist, but a cable compensated gage, if tested ror accuracy, could be used.

\subsubsection{Cable Gage Tests}

Introduction Cable gages as presently installed can be subject to large level measurement errors. However, the compensation methods outlined in Siegwarth [23] could signiflcantly Improve gage accuracy. With compensation, cable gages could meet and probably exceed the estimated accuracy of capacitance level gages. The low cost of a cable gage and the relative ease with which it can be removed and serviced make the cable gage an attractive alternative for the capacitance gage.

The error estimates for cable gage measurements in Siegwarth [23] assume that the gage 1 ignlf contributes a negligible amount to the level measurement error when compared to those 1 ntroduced by temperature and tank movements. To ascertain that the intrinsic errors of the gage are lndeed small. 
the accuracy of four different types of cable gages representing most types commercially available, were tested in an ambient temperature test facility. The facility, the tests and the results are discussed in the remainder of this section.

Gage Accuracy Test Facility The accuracy of the gages, essentially the accuracy of the cable position measuring device, was tested in a facility installed in the air shaft of an existing building. Access to the shaft permitted testing gage accuracy at six points in a 31 meter height. A schematic of this installation is shown in figure 2.5.1.2. The horizontal cross section of the air shaft is about 1 meter by 3.5 meters. At alternate floor levels, a 0.8 meter wide by 2.8 meter high ventilation opening covered by a grill work penetrates the narrow side of the shaft. These openings occur on the even numbered floors on the side used for the tests. A 1.3 meter high frame was clamped in place across each opening supported by the floor. A right triangular plate with its plane vertical was attached to the top end of the frame as shown in figure 2.5.1.2. At the free end of this plate, a piece of aluminum angle was bolted with one flange horizontal and above the triangular plate. This horizontal or reference surface was milled flat. The arm could be rotated about two orthogonal horizontal axes, so the milled surface could be leveled.

The reference point at the shaft bottom consisted of two horizontal plates separated by a section of pipe, which had been machined square, on the ends and clamped between the plates. The top plate served as a reference surface and the lower plate, a base. The cantilevered end of the reference plate was leveled by adjusting leveling bolts at the corners of the triangular base plate.

The reference point on the 10 th floor frame was mounted approximately 0.5 meters above the floor to provide clearance between the gage heads and the reference surface to accommodate the surface sensors. The gage heads were mounted 1.3 meters of $f$ the floor on two horizontal aluminum channels supported on the 10th floor end by the frame supporting the reference point and on the opposite end by a frame hanging from the 11 th floor.

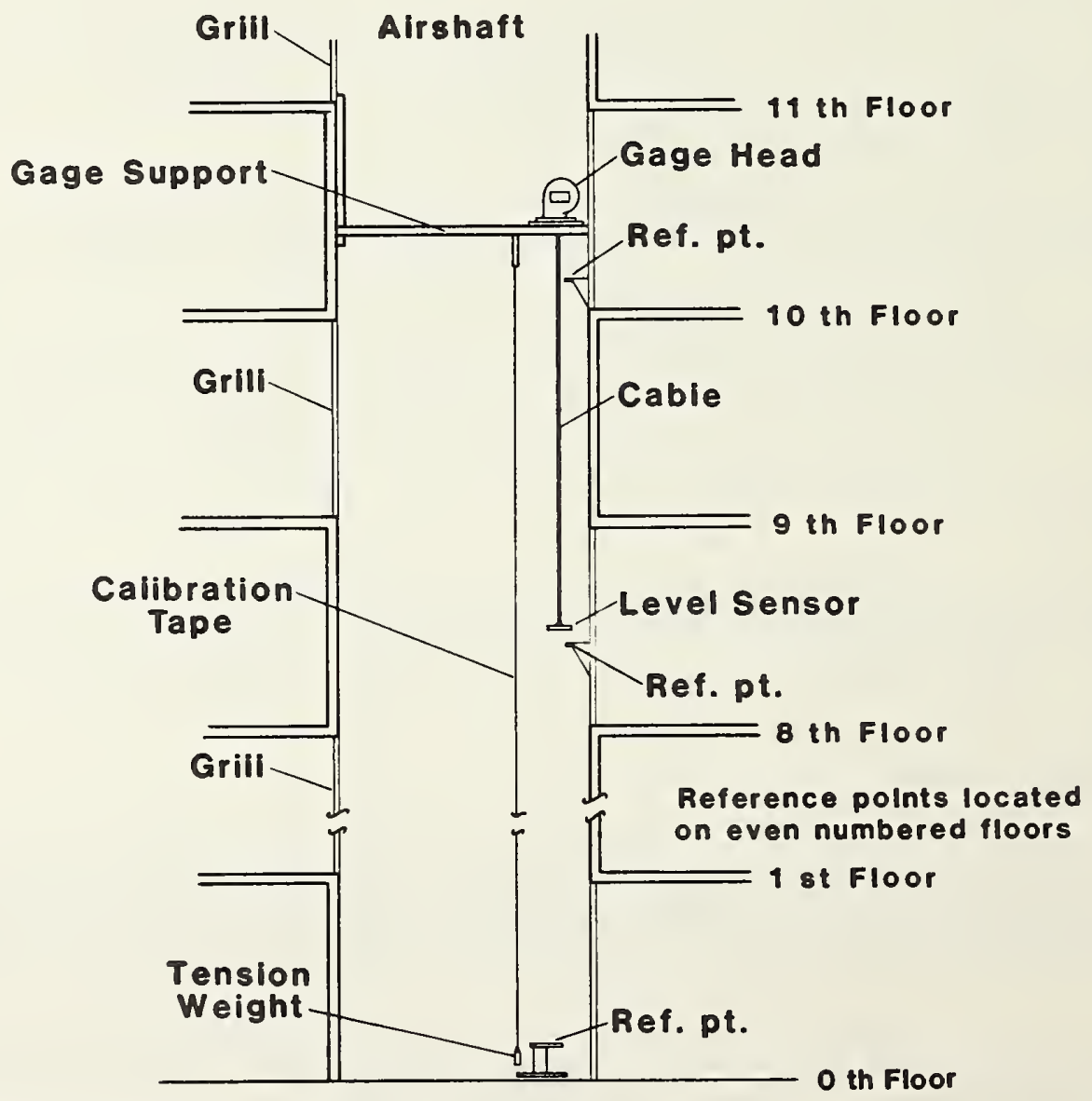

Figure 2.5.1.2. Ambient calibration facility for cable gages. 
These channels also supported an iron-36 percent nickel calibration tape used to measure the separation of the reference surfaces. This tape was suspended from a $U$ shaped frame that could be rolled forward to place the tape in the notches milled in each of the reference surfaces when measuring their separations. The notches were aligned so the tape hung straight through them. During gage tests, the tape was rolled back out of the way of the gage as shown in figure 2.5 .1 .2 .

With the calibration tape positioned in the notches and the 5.2 kilogram tape tensioning weight in place, the tape was scribed at a level approximately 16 millimeters above each reference point by using an gage block laid on each reference surface as a bottom guide for the scriber. The relative positions of the resulting six scribe marks were measured in the tape calibration facility of NBS at Gaithersburg, Maryland. The calibration certificate for this tape appears in Siegwarth [23].

This tape was used periodically during the tests to remeasure the relative positions of the six reference surfaces. The distance of each scribe line above the reference surface was measured as often as twice a day during tests. A steel scale was used to make these of fset measurements to an estimated precision of about 0.1 millimeter. The overall accuracy of the spacings between levels is estimated at \pm 0.3 millimeters. This estimate comes from some photogrammetric calibration accuracy tests in which this tape and similar tapes were used [22].

The air temperature in the shaft was monitored by liquid in glass thermometers at each level. The temperature varied by as much as $3.5^{\circ} \mathrm{C}$ from the top to the bottom of the shaft in early May when the outdoor temperature was generally lower than inside temperatures. The major part of the difference occurred between the 2nd floor and 0th floor (the shaft bottom) where the airshaft is not surrounded by offices. The air temperature at each floor changed less than $1^{\circ} \mathrm{C}$ over the period of a day or over the course of several days. During the summer months, the gradient up the airshaft was generally less than $2^{\circ} \mathrm{C}$, and the bottom level was often as warm as the top. Again, temperature changes over the course of a day or days were less than $1^{\circ} \mathrm{C}$. Mainly because the building is air conditioned from May to August, the average shaft air temperature only changed from about 22 to about $26^{\circ} \mathrm{C}$, and the total height change in the test area was less than 1.5 millimeters between the zero and 10 th floor. Because the shaft height and air temperature changed by such small amounts, reference point positions relative to the calibration tape were measured no more than twice a day during the actual tests.

Hysteresis Test Facility Tests using the fixed reference points in the air shaft only simulate a dropping liquid surface and thus did not provide a convenient means of examining gage hysteresis. Consequently, the servo gages were tested for hysteresis on a mill table. The servo gage hysteresls was assumed independent of the gage head elevation, as indeed it should be. The error of the fourth cable gage, an automatic gage, is not expected to be independent of position, hence the hysteresis of this gage was examined in the air shaft test facility.

To test the servo gages for hysteresis, they were mounted on a tower about 1.5 meters high clamped to the table of a large horizontal mill. The gageheads were offset from the tower on the ends of two approximately 15 centimeters channels as shown in figure 2.5.1.3. A horizontal aluminum plate was rigidly attached to the mill head to provide a movable reference surface in contrast to the air shaft test facility where the reference points were fixed. An electronic machine scale measured the vertical motion of the mill head to 0.1 millimeter resolution. The mill head hysteresis and scale accuracy was tested with a dial indicator calibrated in units of 0.0254 millimeters. The indicator was mounted on a vertical rod attached to the gage mounting plate. The dial indicator agreed with the machine scale to better than 0.02 millimeter and showed no evidence of hysteresis in the mill head. The dial indicator was compared to some gage blocks, with which it agreed to $\pm .0254 \mathrm{millimeter.}$

The hysteresis of the gages using buoyancy change to monitor liquid surface position was measured both against a solid surface and a liquid surface. The liquid surface was provided by water in a 30.5 centimeters diameter shallow pan. If the measured hysteresis differs between water and a solid surface, the hysteresis at an LNG surface can be inferred. A gage using thermal surface sensors required a liquid nitrogen surface to properly test it. The shallow pan was replaced by a 20 centimeters diameter by 90 centimeters deep nitrogen dewar.

The tests were done by raising and lowering the mill head, generally in millimeter steps, and recording the gage head and mill head height readings.

First Cable Gage The servo gage described in this section uses a displacer suspended on a wlre to detect the liquid surface. The wire winds in a spiral slot on a precisely machined drum [19]. The drum is connected through a magnetic coupling to a torque sensing device. When the liquld level rall 3 away from the displacer, the torque increases, and a switch contact closes in the torque sensor. motor, via a worm and gear, drives the displacer down until it contacts the 11quld. There the torque decreases which, opens the switch and stops the drive motor. Conversely, if the level rises, the 


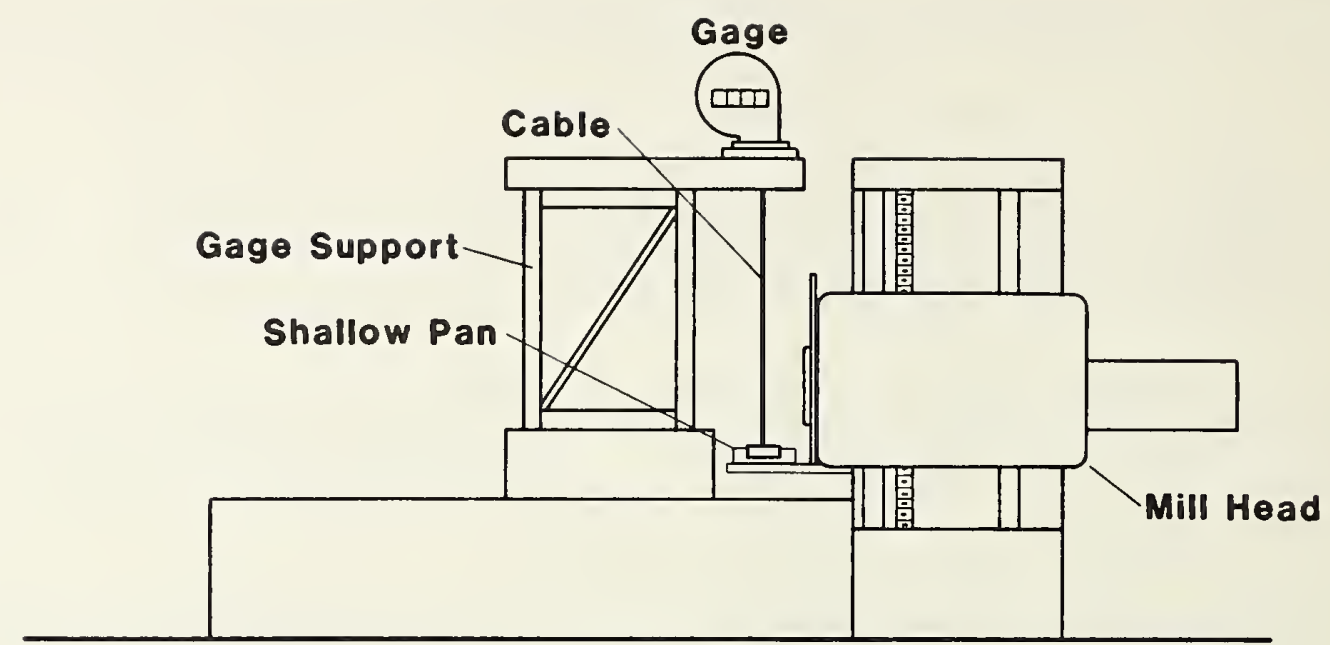

Figure 2.5.1.3. Hysteresis test facility for cable gages.

torque falls too low and the opposite switch closes in the torque sensor, causing the drive motor to lift the displacer back to the liquid surface. The torque sensor causes the drive motor to position the displacer so the torque is neither high nor low enough to close either switch. A counter is attached to the motor shaft and geared so the count is equal to the height in meters for the unit tested. The shaft also drives switches that control a stepping motor connected to a similar counter at a remote site. The counters have 0.5 millimeter divisions and were read to $0.1 \mathrm{millimeter}$.

For the tests performed, the displacer was sometimes replaced by a brass weight equal to the displacer weight of 223 grams, The bottom of the weight was machined flat so it would rest in a reproducible fashion on the reference surfaces. No compliance was introduced between the weight and reference point to simulate a displacer in liquid, since compliance already exists in the magnetic coupling within the gage.

Accuracy Tests The accuracy tests took place over a span of 6 days, with measurements made on the first two and last two days. The gage readings at the reference points on the first measurements down the shaft disagree with all succeeding values by about 3 millimeters. The later readings were smaller. The difference probably resulted from the wire crossing the ridge between channels on the drum somewhere between the 2nd and oth floor. This 3 millimeters decrease in reading was observed at one reference point during all the succeeding measurements, and that one was eliminated by dropping the level sensor (the brass weight on the end of the wire) to the next reference point down and raising it again. Probably, the wire crossed over a ridge again when it was pushed to one side to allow the weight to clear a reference arm. The data point was not used in the analysis.

During the tests the displacer weight was raised and allowed to settle again from 5 to 8 times on each reference surface before moving on the next. The average of each set of readings is shown in table 2.5.1.1a. The total scatter of these repeated readings was \pm 0.1 millimeter. The gage readings were not adjusted to zero at any reference point. The table is sectioned to show the day the data were taken, and the arrow shows whether the sensor was being raised or lowered.

During the period of time these tests were done, some of the reference level positions were measured by the calibration tape 7 times. Four of those times, all the reference level positions were measured. The measured offsets, after adjusting to the average value since the vertical position of the tape can vary, had a spread generally less than 0.1 millimeter with the largest being 0.15 millimeter. Since this spread is within the estimated resolution of a single reading of position relative to a tape scribe mark ( $t 0.2$ millimeter), no corrections for changes of reference point separations due to building temperature changes were necessary. 
Table 2.5.1.1 First Cable Gage Test Results

(a) Average readings in meters for the direct reading counter.

First column gives reading to 1 meter at each level.

\begin{tabular}{|c|c|c|c|c|c|c|c|c|c|c|c|}
\hline Floor & $5-7-81$ & & & $5-8-81$ & & & $5-11-81$ & & & & $5-12$ \\
\hline $\begin{array}{rr}10 \text { th } & 101 \\
8 \text { th } & 96 \\
6 \text { th } & 90 \\
4 \text { th } & 84 \\
\text { 2nd } & 78 \\
\text { 0th } & 70\end{array}$ & $\begin{array}{c}t \\
0.0498 \\
0.2450 \\
0.4598 \\
0.6875 \\
0.0904\end{array}$ & $\begin{array}{c}\stackrel{4}{4} \\
0.4632 \\
0.6845\end{array}$ & $\stackrel{+}{+}$ & $\begin{array}{c}0.1227 \\
0.0526 \\
0.2478 \\
0.4630 \\
0.6842 \\
\uparrow\end{array}$ & $\begin{array}{c}t \\
0.0526 \\
0.2477 \\
0.4630 \\
0.6842 \\
0.0902\end{array}$ & $\begin{array}{c}\uparrow \\
0.4631 \\
0.6844\end{array}$ & $\begin{array}{c}\uparrow \\
0.2447 \\
0.4629\end{array}$ & $\begin{array}{c}0.1229 \\
0.0527 \\
0.2479 \\
0.4631 \\
\uparrow\end{array}$ & $\begin{array}{c}0.1229 \\
0.0527 \\
0.2480 \\
0.4631 \\
0.6844 \\
0.0901 \\
+ \\
\end{array}$ & $\begin{array}{c}t \\
0.4630 \\
0.6843 \\
0.0902\end{array}$ & $\begin{array}{c}0.1226 \\
0.0525 \\
0.2477 \\
0.4629 \\
\uparrow\end{array}$ \\
\hline
\end{tabular}

(b) Comparison to calibrated table.

\begin{tabular}{|c|c|c|}
\hline $\begin{array}{l}\text { Tape (m) } \\
\ell_{\text {ref }}(m)\end{array}$ & $\begin{array}{l}\text { Cable Gage } 1 \\
\ell_{g}(\mathrm{~m}) \\
\text { Average }\end{array}$ & $\ell_{g}-\ell_{r e f}(\mathrm{~mm})$ \\
\hline $\begin{array}{l}5.0701 \\
10.8745 \\
16.65904 \\
22.4376 \\
31.0324\end{array}$ & $\begin{array}{r}5.0702 \\
10.8750 \\
16.6598 \\
22.4384 \\
31.0326\end{array}$ & $\begin{array}{l}0.1 \\
0.5 \\
0.8 \\
0.8 \\
0.2\end{array}$ \\
\hline
\end{tabular}

1 Remote readout gives precisely the same distances.

Since the spread of the averaged readings for each measurement at a reference level does not exceed 0.3 millimeter, the values for each measurement at a level were averaged and compared to the averaged calibration tape measurements of level position. In table 2.5.1.1b the measurements by the calibration tape and the level gage are converted to the distances from the 10 th floor reference surface to each of the others. The spacings given by the level gage are larger but only by 0.8 millimeter at the most. The errors suggest a small non-linearity, but only two of them exceed the scatter of the data. The results shown in table 2.5.1.1 are from the direct reading dial. The data from the remote readout gave precisely the same values for the reference level separations.

The manufacturer's specifications call for the gage error dL to be

$$
\begin{aligned}
& d L \leqq(0.5+0.035 L) \mathrm{mm} \quad \text { "fine precision" } \\
& \mathrm{dL} \leqq(0.5+0.13 L) \mathrm{mm} \quad \text { "commercial precision" }
\end{aligned}
$$

where $L=$ the level in meters, and for infinite density liquid which the reference surfaces slmulate. The gage tested was "commercial precision." At a level of 31 meters the allowable dL values are \pm 1.6 millimeters and \pm 4.6 millimeters for "fine" and "commercial precision," respectively. At i6.66 meters, where the measured difference was maximum, which was 0.8 millimeter, the error perm 1 ted $1 \mathrm{~s}$ 1.1 millimeters for the "fine precision" gage. Thus, this unit easily exceeds the requlrements ror "fine precision" at ambient conditions. The scatter of the data, only 0.3 millimeter total spread at each level, suggests that a non-linear correction could be applied to make the gage accuracy even greater.

The average air temperature in the shaft decreased by about $1{ }^{\circ} \mathrm{C}$ over the course of the tests. The temperatures in the air shaft decreased monotonically $3.5^{\circ} \mathrm{C}$ from the top to the bottom of the shaft. The approximate shrinkage of 31 meters of stainless steel wire for a $1^{\circ} \mathrm{C}$ temperature drop $1 \mathrm{~s} 0.5$ millimeter. A 0.5 millimeter change could be resolved. No change of that magnitude $1 \mathrm{~s}$ evident $1 \mathrm{n}$ the results. 
Hysteresis Tests The gage was tested for hysteresis on the horizontal mill first by using the brass weight against the flat table. The table was moved up and down between +1 , 0 , and -1 millimeter for five complete cycles. The five readings at each level generally showed a spread of 0.2 to 0.3 millimeter total. The averaged value of level reading for decreasing levels were 0.1 millimeter higher than the average values for increasing levels at the same mill head position and for both the local and remote readout. This indicated hysteresis is well within the scatter of the individual measurements.

When the reference surface was water, the direct readout suggested 0.1 to 0.2 millimeter hysteresis, while the remote readout suggested 0.2 to 0.3 millimeter. The displacer sensed a water surface that had a cross- sectional area about 8 times larger than the float, so the water height correction between the extremes of the displacer position relative to the surface is 0.04 millimeter. Hysteresis to a liquefied gas surface could be expected to double the hysteresis added by water, which could be the difference between the water and solid surface hysteresis, $0.2 \mathrm{millimeter} \mathrm{-} 0.1 \mathrm{millimeter}=0.1$ millimeter, doubled plus 0.1 millimeter or 0.3 millimeter for the direct readout. The above estimates of hysteresis are tenuous, because the magnitudes estimated are smaller than the scatter of the individual measurements at each level. Therefore, to within the measurement precision, hysteresis is not detectable. Some additional level measurement error results because the wire weight adds to the displacer weight as the level drops. The wire weighs 0.15 gram per meter which is 4.5 gram when extended 31 meters. The displacer is 14 centimeters diameter, so that in $\mathrm{LNG}$ at $500 \mathrm{~kg} / \mathrm{m}^{3}$, the displacer can be expected to ride about 0.6 millimeter lower at 30 meters depth than at 0 meters depth. This error is proportional to level, and if more precise measurements are desired, height corrections for the wire weight can easily be made.

Second Cable Gage The servo gage described in this section suspends the surface sensor on a stainless steel tape. The tape is actually two side by side strips of stainless steel electrically insulated from the environment and each other by a plastic coating. The level sensor is a 10 centimeters diameter foam float free to move over a range of about 1 centimeter with respect to the sensing head. A coil in the sensing head, driven by an AC voltage supplied from the gage head through the stainless steel tapes, senses the position of the same magnetic material attached to the float. A servo motor is controlied by the signal from the sensing head and adjusts the position of the head until a null position is achieved. The servo motor drives the tape storage drum. The tape passes over a sprocket wheel whose teeth engage in perforations in the wider of the two stainless steel strips then down to the sensing head. The sprocket wheel drives a mechanical counter and can also drive a transmitter to a remote readout. The least count on the counter drum corresponds to 1 millimeter. The 1 millimeter lines are about 1.5 millimeters apart, permitting the counter position to be estimated to 0.1 millimeter. Because the storage drum diameter increases as the tape is wound, the driving speed was greater than 0.6 meters/minute near full and about 0.4 meters/minute near empty. The control circuit drives at a rate proportional to of set near the null point, making overshoot almost undetectable as the sensing head approaches the rest position.

Accuracy Tests The gage accuracy was tested relative to the reference levels during three round trips up and down the air shaft. The gage head approaches the reference levels always from above so the float could rest on the reference surface. The bottom reference point was raised by a gage block approximately 10 centimeters because the tape was not long enough by a few centimeters to reach the bottom reference surface.

The averaged value for the level gage reading at each reference level for all the measurements at each level is shown in table 2.5.1.2a. The total spread of the data at each level is $0.5 \mathrm{mill}$ imeter or less. This precision approaches the resolution of the gage scale. The location of the dial reference causes some reduction of reading precision because of parallax.

Table 2.5.1.2b and figure 2.5.1.4 show the difference between the reference surface spacings determined by calibration tape and from the gage readings.

The bars on the averaged data points in figure 2.5.1.4 show the total spread of the data averaged at that point. The gage reads a value for the reference point readings 6 millimeters too small corresponding to a liquid level 6 millimeters high at the 31 meter level. The average differences for the intermediate levels fit a linear correction to \pm 0.2 millimeter, which is well within the 1 imits of the combined precision of the gage readability and the calibration tape measurement. A correction factor can readily be applied to the gage readings for high accuracy measurement. For this gage $1.00020 \mathrm{x}$ (gage reading) corrects the readings to the calibration tape scale. 
Table 2.5 .1 .2

Second Cable Gage Test Results

(a) Average reading and total spread?

\begin{tabular}{|c|c|c|}
\hline Floor & $\begin{array}{l}\text { Average Gage } \\
\text { Reading (m) }\end{array}$ & $\begin{array}{c}\text { Total } \\
\text { Spread (mm) }\end{array}$ \\
\hline $\begin{array}{l}10 \text { th } \\
8 \text { th } \\
6 \text { th } \\
4 \text { th } \\
2 n d \\
0 t h\end{array}$ & $\begin{array}{l}79.3940 \\
74.3250 \\
68.5210 \\
62.7376 \\
56.9602 \\
48.4678\end{array}$ & $\begin{array}{l} \pm 0.1 \\
+0.1 \\
-0.2 \\
+0.2 \\
-0.0 \\
+0.3 \\
-0.2 \\
+0.3 \\
-0.2 \\
+0.2 \\
-0.3\end{array}$ \\
\hline
\end{tabular}

1 Averages of 14 to 19 readings over 2 days. Each level approched three times each going up and going down.

(b) Comparison to calibration tape

\begin{tabular}{|c|c|c|c|}
\hline $\begin{array}{l}\text { Distance Below loth } \\
\text { Elocr Reference }\end{array}$ & $\begin{array}{c}\mathrm{lg}_{g} \\
\text { Gage Average } \\
\text { (II) }\end{array}$ & $\begin{array}{c}\text { lref } \\
\text { Calibration tape } \\
\text { (mm) }\end{array}$ & $\left.l_{g}-2_{\text {mef }}\right)$ \\
\hline $\begin{array}{l}10 \text { th } \\
8 \text { th } \\
6 \text { th } \\
4 \text { th } \\
\text { 2nd } \\
\text { oth }\end{array}$ & $\begin{array}{l}0 \\
5.9690 \\
10.8730 \\
16.6564 \\
22.4338 \\
30.9262\end{array}$ & $\begin{array}{l}0 \\
5.0701 \\
10.8748 \\
16.6594 \\
22.4382 \\
30.9322\end{array}$ & $\begin{array}{l}0 \\
1.1 \\
-1.8 \\
-3.0 \\
-4.4 \\
-6.0\end{array}$ \\
\hline
\end{tabular}

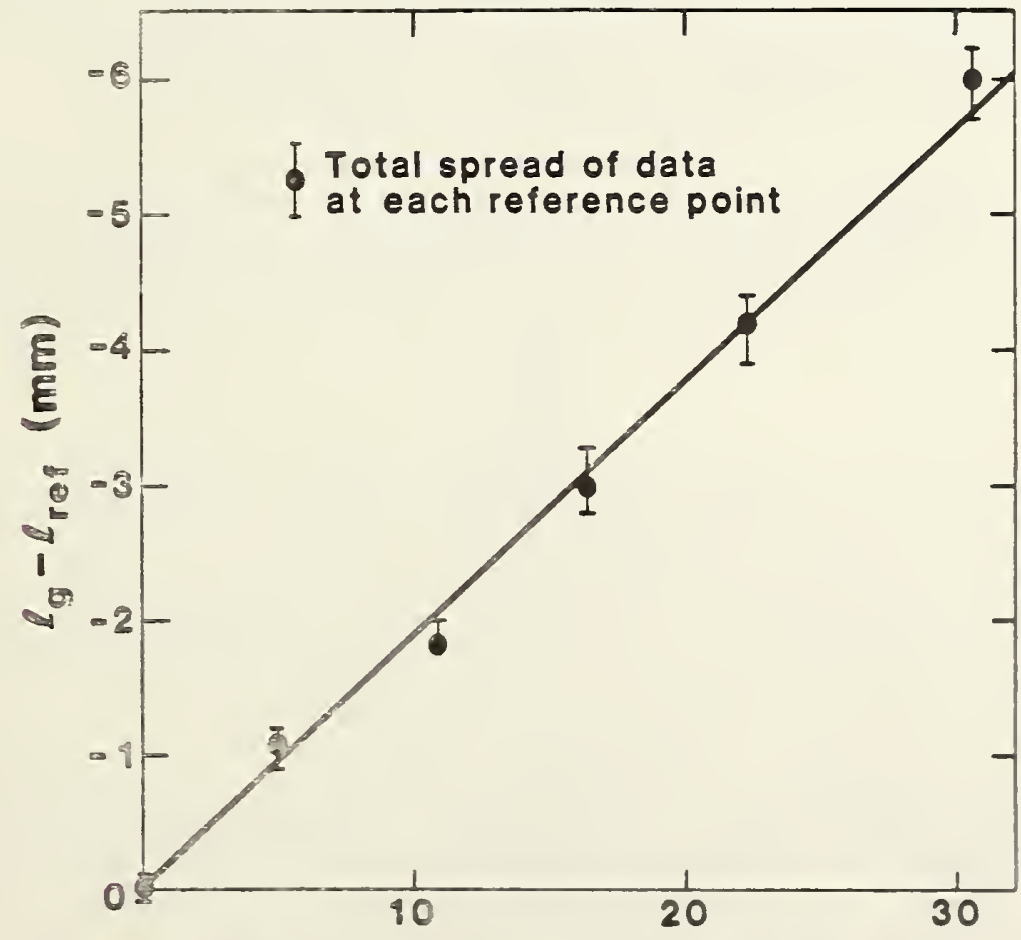

DISTANCE BELOW TOP REFERENCE (m)

Figure 2.5.1.4. Results of accuracy test of the second cable gage. 
The effect of cryogenic temperatures on the plastic coated tape was given cursory examination by cooling a section of the tape in liquid nitrogen. No reduction in the tape flexibility was evident in the section at liquid nitrogen temperature, nor was there any evident effects from differential contraction.

Hysteresis Tests The hysteresis tests on this gage were done first with the float in the sensor head resting on the shelf attached to the mill head. Three complete cycles (moving the table zero to +1 millimeter to zero to - 1 millimeter to zero is one cycle) and a cycle to \pm 0.2 millimeter resulted in no measurable difference between the gage and the mill head reading.

Next, the float was tested with a water surface in a 30.4 centimeters diameter pan. Over the course of three cycles through 1, 0 and - 1 millimeter, the maximum hysteresis was again less than 0.1 millimeter, i.e., no hysteresis was detected to the readability of the gage. During these water tests, the float settled 2 to 3 millimeters into the liquid. If the liquid were $500 \mathrm{~kg} / \mathrm{m} 3 \mathrm{natural}$ gas, the float would be expected to settle 4 to 6 millimeters into the liquid. If the LNG density changed by 10 percent, the gage would change by 0.6 millimeter at the same liquid level. If higher precision is desired, a correction can be introduced by measuring the liquid density and calculating the relative shift resulting from density change.

Third Cable Gage The cable gage described in this section uses a hollow cable to connect the surface sensor to the gage head [17]. A number of electrical leads pass through this hollow cable.

The leads connect a densimeter, a thermometer and the surface detectors in the surface sensor to their respective electronics in the gage head. The two surface detectors are thermal devices and cause the surface sensor to be driven to a position such that the lower detector is immersed, and the upper detector is above the liquid surface. The surface detectors are separated vertically by about 3 millimeters.

The cable consists of a flat wire wound in a tight coil around which one layer of 30 to 40 fine wires is twisted in a spiral of about one turn in 5.5 centimeters. Around the outside, a single much heavier wire is sprialled at a pitch of about one turn per 4 millimeters. A notched drive wheel meshes with this spiral wire and drives the sensor up and down. A stepping motor powers the drive wheel, and the sensor position is monitored by counting the pulses to the stepping motor. The instrument is provided with a calibration by setting an appropriate number into some switches. This number determines the advance of the counter per pulse to the stepping motor. The excess cable is stored on a spring-motor-driven drum in the drive head. The readout resolution of 1 millimeter cannot be interpolated, since this is the least count of an electronic counter.

This gage has a unique feature. Rather than using a point on the tank roof for a reference point for setting the gage zero, this gage can be rezeroed by allowing the surface sensor to come to rest on the tank bottom. The ensuing reduction of weight trips a switch in the gage head that resets the level readout to a zero value. Then the surface sensor is returned to the liquid surface. This method of determining the gage zero removes errors introduced by temperature distributions in the tank walls and ullage provided, the tank and environment are at steady state conditions (see Siegwarth [23] for details).

Accuracy Tests This device senses level by means of temperature probes and always hangs with full weight on the cable other than for a small buoyancy effect change. Thus, the level sensor could not be lowered against a reference surface to measure its position because the cable would slack. Instead the sensor was brought to a point where the counter read a fixed level, around 13 to 15 centimeters, above each reference surface, and the separation from the reference was measured with a dial indicator. The dial gage reading remained 15.57 millimeters throughout the test of this gage when placed between the two plates separated by $127.0 \mathrm{millimeters} \mathrm{by} \mathrm{a} \mathrm{gage} \mathrm{block.} \mathrm{The} \mathrm{dial} \mathrm{gage} \mathrm{read} \mathrm{so}$ that the spacing between the sensor bottom and the reference point was

$$
S=(5.000-r+0.613)(0.0254) \text { meters }
$$

where $r$ is the dial gage reading in inches. The spacing $S$ was subtracted from the gage reading $x$ to give the reference point position in the gage coordinates. The reference position $p$ is then

$$
p=x-(5-r+0.613)(0.0254) \text { meters }
$$


The spacing of the other reference points from the bottom reference point is

$$
p-p_{0}=x-x_{0}+\left(r-r_{0}\right)(0.0254)=\Delta p
$$

where $p_{0}, x_{0}$, and $r_{0}$ are the zero floor reference level values. The results have been examined by plotting $\Delta p_{\text {gage }}-\Delta p_{\text {actual }}$ as a function of $p-p_{0}$.

Inltially this gage was tested without the fixed guide wires, since the gage design did not require them if no currents were present that could deflect the level sensor and cable from vertical. However, during these initial tests, the level sensor rotated around the axis of the cable as much as 5 turns in the course of traversing the height of the shaft. The measurements often did not repeat by a few mllimeters when the sensor was returned to a reference point, and the gage reading was non-linear by about 1 centimeter over the height of the shaft.

A second set of tests was conducted with guide wires installed to constrain the sensor from rotating. In practice, most gages are installed with these guide wires.

The addition of the guide cables removed the non-repeatability observed in the first tests and significantly reduced the non-linearity. The results are shown in table 2.5.1.3 and figure 2.5.1.5.

Table 2.5.1.3

Third Cable Gage Test Results

(a) Differences from reference level positions.

\begin{tabular}{|c|c|c|c|c|c|c|}
\hline $\begin{array}{c}\text { Ref. Level } \\
\text { Floor }\end{array}$ & \multicolumn{6}{|c|}{ Deviation of the gage reading from the reference value } \\
$\ell_{\mathrm{g}}-\ell_{\text {ref }}$
\end{tabular}

(b) Comparisons of average gage separations to calibration tape values

\begin{tabular}{|c|c|c|}
\hline $\begin{array}{c}\text { lref } \\
\text { Calibration } \\
\text { Tape (m) }\end{array}$ & $\begin{array}{c}\ell_{\mathrm{g}} \\
\text { Gagerage } \\
(\mathrm{m})\end{array}$ & $\begin{array}{c}\ell_{\mathrm{g}}-\ell_{\mathrm{ref}} \\
(\mathrm{mm})\end{array}$ \\
\hline \hline 0 & -0.6 & -0.6 \\
8.5869 & 8.5803 & -6.6 \\
14.3653 & 14.3580 & -7.3 \\
20.1500 & 20.1424 & -7.6 \\
25.9544 & 25.9492 & -5.2 \\
31.0242 & 31.0209 & -3.3 \\
\hline
\end{tabular}

1 Changed $r_{0}$ because gage sensor stalled against top reference point.

As can be seen in figure 2.5.1.5, the scatter of the level readings are almost all with1n the resolution of the gage, estimated below to be \pm 1.0 millimeter. The scatter is shown by the brackets at each reference level. The estimated uncertainty of the dial 1ndicator measurement, \pm 0.2 millimeter, has not been included in the resolution. Only two repeat values of the zero reading at the shaft bottom were obtained, rather than three, because the sensor momentarily caught on the loth floor reference arm as it approached the top in the last measurement. This shlfts the zero, because the stepping motor stalls, but the counter continues counting steps. The going down zero was used ror the measurements back down the shaft. 


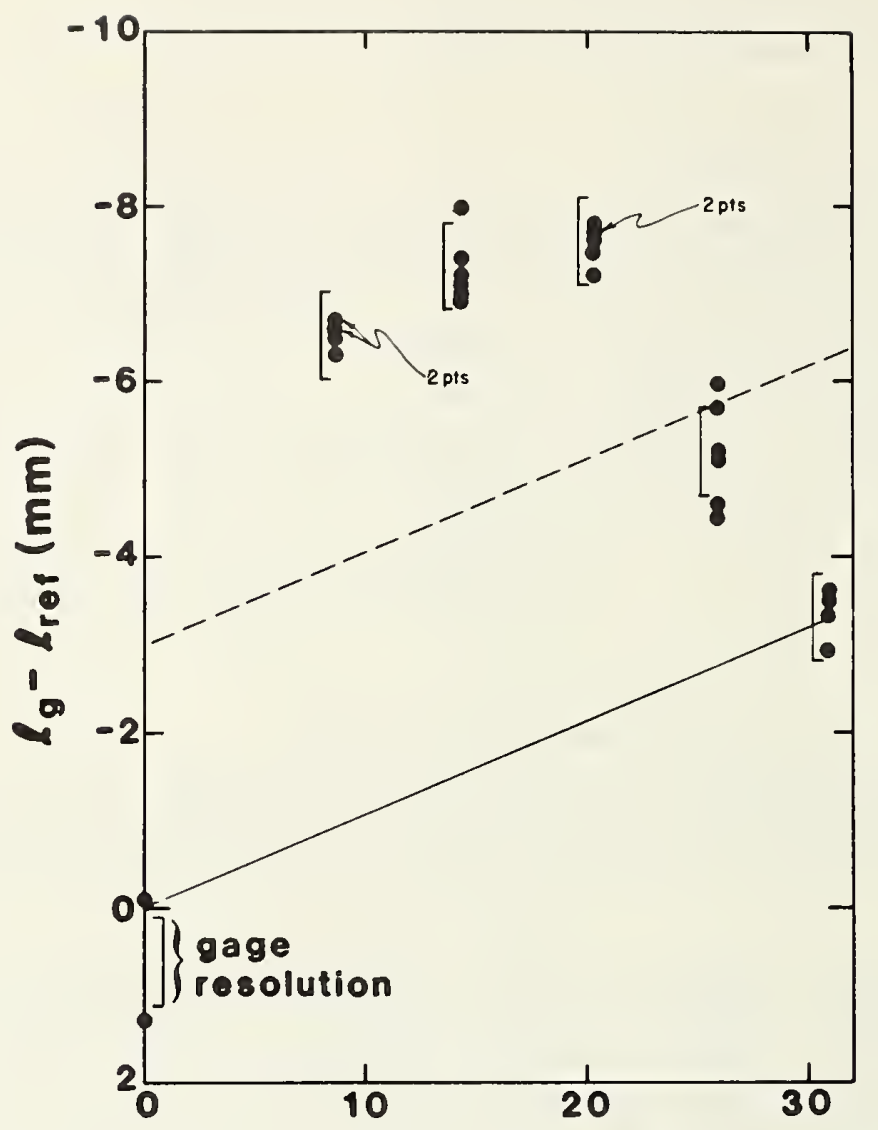

\section{DISTANCE FROM BOTTOM REFERENCE (m)}

\section{Figure 2.5.1.5. Results of accuracy tests of the third cable gage.}

As the dotted line in figure 2.5.1.5 shows, the gage calibration needs to be adjusted to count 3 millimeters higher over the 31 meters between top and bottom. With that adjustment and a shift of the zero reading to read 3 millimeters high, the gage accuracy would lie within \pm 3 millimeters of the true value over the range. If higher accuracy is needed, a curve may be fit through the data in figure 2.5.1.5 and a correction applied to the gage readings. This would yield levels accurate to about \pm 1 millimeter.

The non-linearity shown in figure 2.5.1.5 probably originates with the cable. Very slight differences in the spacing of spirals of the wrapping can accumulate to measurable errors. This variation could be introduced during the manufacture of the cable. Stresses in the cable introduced during the manufacture probably cause the center of the cable to twist relative to the ends. Because of the spiral wrap, this adds turns of the wrap between the center and one end while reducing by the same amount the number of wraps between the center and opposite end. The deviation from linearity shown in figure 2.5.1.6 would result if a 1.5 turn twist were present in the center of the cable, since the pitch of the wrap is about 4 millimeters per turn.

Before pulling the sensor up the last time, the cable was constrained from rotating and the center twisted counter clockwise 15 turns then released. This caused a slight change in the difference readings at the $8 \mathrm{th}$ and 10th floors. Any difference was less than 2 millimeters. This implies that the cable is quite stable with respect to twist at ambient temperature.

Because of the non-linearity of the instrument, the gage should be calibrated using several rather than two reference levels for best accuracy. 
The gage is driven by a stepping motor, and the calibration of 0.26229 millimeter per step is added to a reglster reading 0.00001 millimeter in the last place. The reading is displayed to the nearest mllimeter, so the readout uncertainty is \pm 1 millimeter. The uncertainty associated with the difference between two readings is also $\pm 1 \mathrm{millimeter}$.

The mechanism that zeros the counter when the sensor contacts the tank bottom is a weight-actuated switch that trips when some of the sensor load is taken up by the tank floor. During the tests without guide wires, the gage was allowed to zero several times on the bottom reference then raised to a helght reading of 130 millimeters above the bottom reference and the height measured with the dial indicator. The results are shown in table 2.5.1.4. The total spread of the data is less than 1 milimeter. This is within the resolution of the gage and suggests that the zeroing process does not add a significant error to the position measurement.

Table 2.5.1.4 Rezero Accuracy of The Third Cable Gage

\begin{tabular}{|c|c|}
\hline Rezero Date & Dial Gage* \\
\hline \hline $7-8-81$ & $130.5 \mathrm{~mm}$ \\
$7-14-81$ & $130.5 \mathrm{~mm}$ \\
$7-15-81$ & $131.1 \mathrm{~mm}$ \\
$7-24-81$ & $130.5 \mathrm{~mm}$ \\
" " " & $130.5 \mathrm{~mm}$ \\
" " & $130.3 \mathrm{~mm}$ \\
\hline
\end{tabular}

* Reading with sensing head raised to a reading of 13.0 centimeters above zero value.

Note: The average height measured by the dial indicator is 130.6 millimeters. The total spread of the data is 0.8 millimeter.

Hysteresis Tests This gage thermally senses the liquid surface. To do the hysteresis tests, a 90 centimeters deep by 20 centimeters diameter dewar containing liquid nitrogen was placed on the shelf on the milling machine head. A 16.5 centimeters diameter copper can was placed inside the dewar. A 0.5 centimeter diameter hole was drilled through the wall of this can so that when the level sensors were lowered to the height of the hole, the bottom of the sensing head was still about 8 centimeters above the bottom of the copper can.

The copper can was filled with liquid nitrogen to the level of the hole. The annular gap was also filled but to a lesser height. The level in the inner can could be maintained to the level of the hole by adding small amounts of liquid nitrogen and allowing the excess to run through the hole into the annulus. All of the boiling caused by heat leak takes place in the annular space. No boiling was noted in the inner can.

Hysteresis was first examined by varying the mill head position between +5 millimeters to -5 millimeters in 1 millimeter steps. The gage drive was set to automatic, the mode that causes the sensor to follow the liquid surface. The results of one such measurement is shown in figure 2.5 .1 .6$. The hysteresis is about \pm 3 millimeters. The gage would start to change between the millimeter steps. This is indicated by the curved dotted sections of the curve. If, instead, the table is stopped when the gage started to change, then the table position and new gage reading recorded, the hysteresis was similar in magnitude. The manufacturer's estimate of the hysteresis is 3 millimeters.

The gage was also tested in the manual mode by first driving the gage sensor up about 1 centlmeter then switching to automatic and allowing the gage to set itself at the liquid level. Next, the gage head was driven down and allowed to return to surface automatically again. This was done at other positive and negative table positions. The gage returns to the same position to within about millimeter regardless of whether the gage sensor was driven up or down. This was also true at points offset from the table zero position. Some change of the liquid nitrogen level and perhaps rotation of the sensor head, since no guide wires were used, caused shifts during the course of these measurements, but the results suggest that the level uncertainty resulting from hysteresis could be reduced to \pm 1 millimeter by following this procedure.

Fourth Cable Gage Historically, the earliest gages of the cable type were the automatic fapes. Automatic gages are completely mechanical devices, unless fitted with a remote display unit [15]. They are easy to make intrinsically safe, often a requirement for shipboard use. The gage tested hnr. is built especially for shipboard use on cryogenic liquids and is a roof mounted gage. A roam rloat 


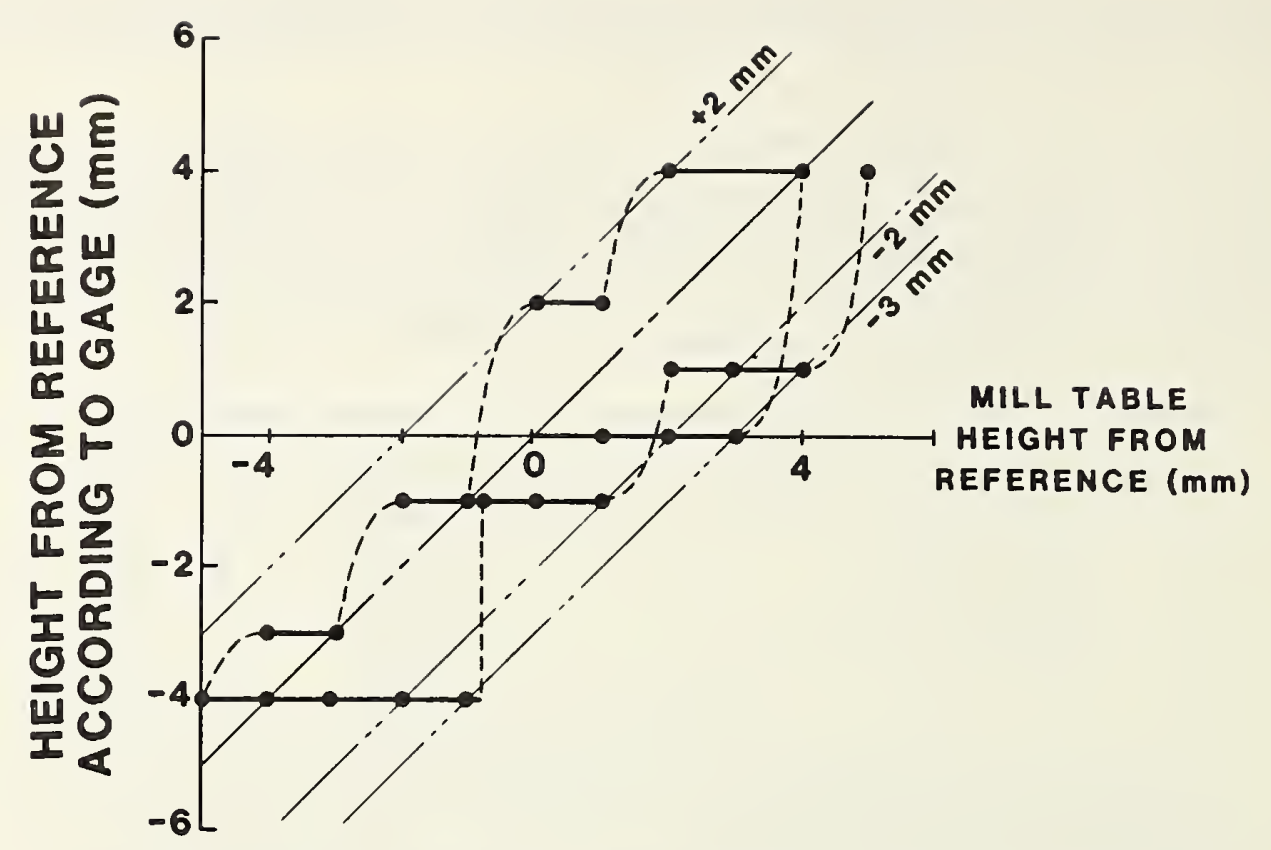

Figure 2.5.1.6. Results of hysteresis test on the third cable gage.

is connected to the gage head via a perforated stainless steel tape. In the gage head, the tape passes over a sprocket wheel, which drives a counter and is wound on a spring motor driven storage drum. The spring motor winds the tape on the drum as a rising liquid level raises the float but is sufficiently weak so that the weight of the float unwinds the tape as the level falls.

The gage tested is the automatic gage widely used in large LNG tanks. In marine service, the float is stored at the topmost position when the ship is in motion to prevent damage to the gage. A crank on the gage head engages the storage drum permitting the float to be raised. The float is held at the gage head by a latch mechanism.

The direct readout dial is in meters with graduations of 1 millimeter. The millimeter marks have about a 1 millimeter spacing, and the reading was estimated to 0.1 millimeter. This unit measures ullage height rather than liquid height. The zero of the gage was not changed from the zero setting when received.

Accuracy Tests to a Solid Surface The gage tests in the air shaft facility were done first by allowing the float to come to rest on each reference surface. An aluminum plate was attached to the bottom of the foam float to give it a more durable and reproducible surface. One set of gage readings at the reference levels were taken going down and back up the shaft. These results are shown in table 2.5.1.5. The gage and calibration tape distances agreed to \pm 0.8 millimeter.

Table 2.5.1.5

Comparison of Calibration Tape and Fourth Cable Gage Against a Solid Reference Surface

\begin{tabular}{|c|c|c|c|c|c|}
\hline \multirow[b]{2}{*}{ Floor } & \multirow{2}{*}{$\begin{array}{c}\text { Calibration } \\
\text { Tape }(\mathrm{m})\end{array}$} & \multicolumn{2}{|c|}{ Spacings $(m)$} & \multicolumn{2}{|c|}{$\ell g-\ell \operatorname{ref}(\mathrm{mm})$} \\
\hline & & Gage(down) & Gage (up) & (down) & (up) \\
\hline $10 t h$ & 0 & 0 & 0 & & \\
\hline 8 th & 5.0701 & 5.0700 & 6.0697 & -0.1 & -0.4 \\
\hline $6 \operatorname{th}$ & 10.8746 & 10.875 & 10.8745 & +0.4 & -0.1 \\
\hline 4 th & 16.6595 & 16.6590 & 16.6587 & -0.5 & -0.8 \\
\hline $2 \operatorname{th}$ & 22.4384 & 22.4380 & 22.4382 & -0.4 & +0.2 \\
\hline oth & 31.0338 & 31.0335 & 31.0332 & -0.3 & -0.6 \\
\hline
\end{tabular}


Accuracy to a Liquid Surface and Hysteresis Tests Servo-operated cable gages either reduce or eliminate of fset errors that can be introduced into automatic gage measurement by drag in the bearings or nonlinearities in the spring motors. Measuring hysteresis of a gage at closely spaced levels is adequate to characterize the measurement accuracy of a servo gage. However, hysteresis in an automatic gage should be tested over the entire height range of the gage. Some manufacturers use a single liquid level to test automatic gages over the measurement range by coiling and storing the tape at the float. Others use devices for measuring the effect of the spring motor tension over the length

of the tape.

The performance of this level gage when measuring a liquid surface was tested in the air shaft facility by placing a vessel of water on each reference surface large enough for the float. This vessel containing the float is shown in figure 2.5.1.7. The water level was maintained at a fixed height above the reference surface by a 9.5 millimeter 0.D. overflow pipe. The pipe passes vertically through the notch in the reference plate with the end open and about 7 centimeters above the vessel bottom. The pipe is soldered through a brass block which is clamped to the reference surfaces so the open end of the pipe is always the same distance above the reference surface. A 13 millimeter diameter hole up the center of the float permits the float to be placed over this tube. The bolt, nut and locknut used to attach the float to the tape was removed and replaced by an 8 millimeter diameter tube to provide a vent, so the water height in the hole would be the same as in the rest of the vessel. A spring, washer and split key kept the tube vertical on the float; a second split key attaches it to the link.

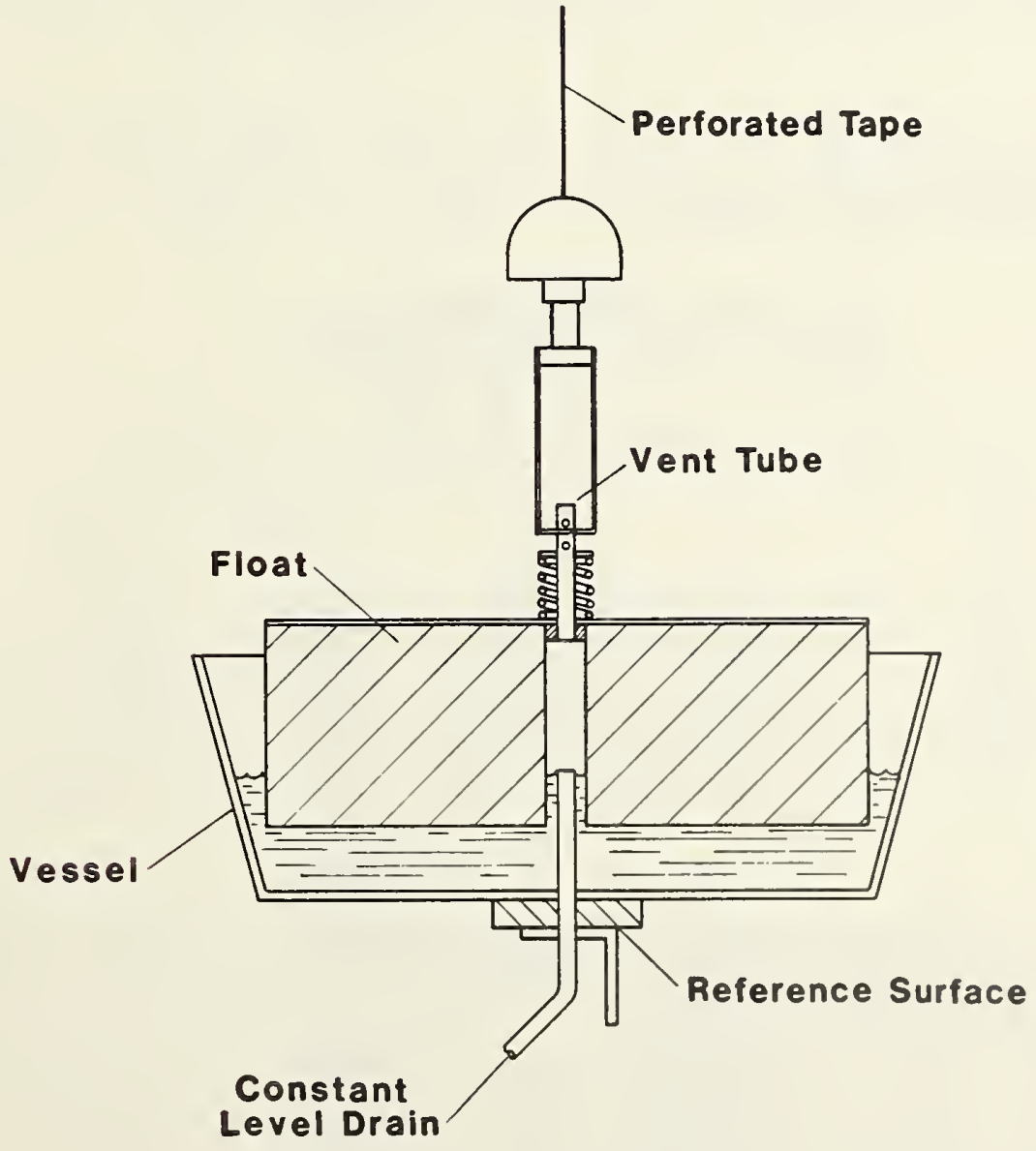

Figure 2.5.1.7. Schematic of the liquid reference surface devlce. 
The measurement process consisted of attaching the constant level pipe and water vessel to the reference level at which the gage was to be tested and pouring in sufficient water so that when the float was placed in the vessel, water overflowed. The water contained a small amount of detergent to reduce the surface tension. After the water drained down to a low flow rate and the gage reading had stopped changing, the gage was read. Then the float was raised about 1 centimeter and allowed to drop. When the gage reading was stable again, the reading was recorded, and 50 to 100 cubic centimeters of water was added to the vessel and allowed to drain away. When the draining had again nearly ceased, the gage reading was recorded. This raise and refill cycle was repeated at least once. After refilling, the gage was read when the flow dropped to the same rate rather than when the flow stopped completely. The gage reading had ceased changing well before a reading was recorded.

The data were recorded at each reference level for four trips, two down and two up the shaft. The differences measured between reference levels are shown in table 2.5.1.6a. Agreement is assumed between the gage and reference at the highest level. The latched position which is used in service as a calibration reference point is included. The reference surface separations are averaged for the measurements and compared to the tape determined reference level separations in table $2.5 .1 .6 \mathrm{~b}$ and figure 2.5.1.8. The bars on each data point in the figure show the full spread of the scatter in the data. The 3.2 millimeters bias can be expected to rise to 6.4 millimeters for a liquid of 500 $\mathrm{kg} / \mathrm{m}^{3}$ density.

The small scatter of the data relative to the bias suggests that a correction could be applied, therefore a measurement accuracy of \pm 1 millimeter is possible under laboratory conditions, well within the manufacturer's estimated uncertainty of \pm 1 centimeter. The \pm 0.5 millimeter scatter of the data has not been separated into the error due to the water level variation and the error due to the gage. The difference between the average float position when water was added and the position after the float was raised and dropped was usually less than 0.5 millimeter, and only occasionally slightly over. Some of the scatter could result from variations in the water level, the extent of which could not be easily determined. The hysteresis, then, in this gage is about 0.5 millimeter and possibly less.

These measurements suggest that a correction can be applied to the gage for the offset due to the spring motor tension, bringing the gage into the same accuracy $r$ ange as servo gages. However, the accuracy of this gage is probably more sensitive to wear and contamination than servo gages, so the accuracy could decrease with time in service.

Table 2.5.1.6

Fourth Cable Gage Test Results

(a) Gage readings

\begin{tabular}{|c|c|c|c|c|}
\hline Floor & \multicolumn{4}{|c|}{ Gage determined reference level spacing } \\
\hline latched & -0.4841 & -0.4872 & -0.4853 & -0.4840 \\
10th & 0 & 0 & 0 & 0 \\
8th & 5.0704 & 5.0711 & 5.0709 & 5.0711 \\
6th & 10.8764 & 10.8760 & 10.8767 & 10.8758 \\
4th & 16.6610 & 16.6614 & 16.6616 & 16.6620 \\
2nd & 22.4404 & 21.4410 & 22.4399 & 22.4408 \\
Oth & 31.0364 & 31.0367 & 31.0359 & 31.0371 \\
\hline
\end{tabular}

(b) Comparison of calibration tape and fourth cable gage

\begin{tabular}{|c|c|c|c|}
\hline Floor & $\begin{array}{c}\ell_{\mathrm{g}} \\
\text { Gage Average } \\
(\mathrm{m})\end{array}$ & $\begin{array}{c}\ell_{\text {ref }} \\
\text { Calibration tape } \\
(\mathrm{mm})\end{array}$ & $\begin{array}{c}\ell_{\mathrm{g}}-\ell_{\text {ref }} \\
(\mathrm{mm})\end{array}$ \\
\hline \hline latched & -0.4844 & & 0 \\
10th & 0 & 0 & 0.8 \\
6th & 5.0709 & 5.0701 & 1.6 \\
4th & 10.8762 & 10.8746 & 2.0 \\
2nd & 16.6615 & 16.6595 & 2.1 \\
Oth & 22.4405 & 22.4384 & 3.2 \\
\hline
\end{tabular}




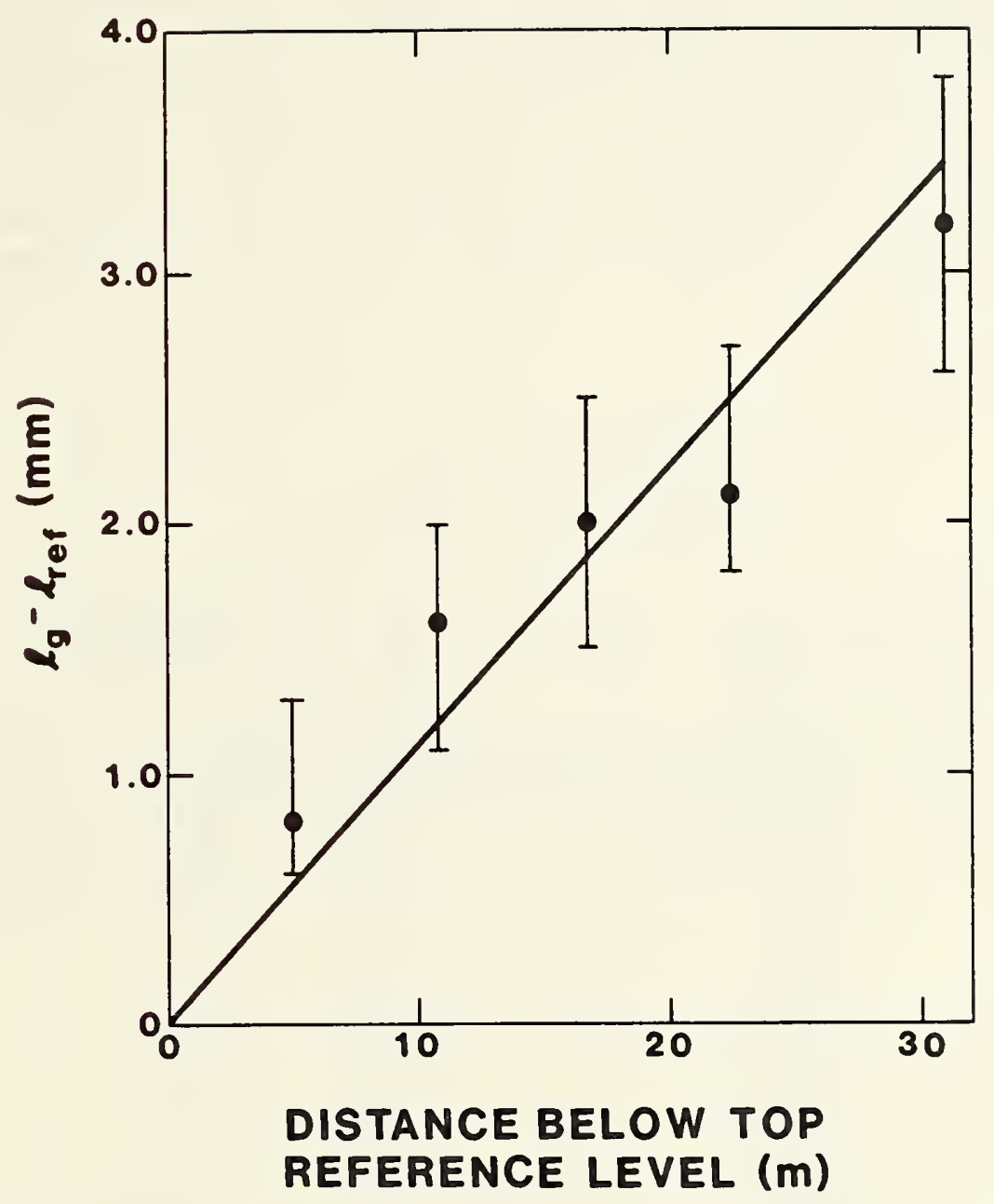

Figure 2.5.1.8. Results of the accuracy tests of the fourth cable gage.

\subsubsection{Bubbler Type Level Gage}

Bubbler level gages and differential pressure level gages have been described in the 1 iterature by $M$. P. Wilson, Jr. [13] Since these gages are usually assembled from commercially available components rather than manufactured as a package, no manufacturer's literature was found. The princlple of measurement is simple. The hydrostatic head of the liquid at the tank bottom is measured and converted to the liquid height in the tank by dividing by the liquid density. The denslty must be measured or calculated. The head pressure of a 25 meter depth tank filled with liquid methane at a density of $400 \mathrm{~kg} / \mathrm{m}^{3}$ is:

$$
P=\left(g_{0} \times 1000 \mathrm{~g} / \mathrm{cm}^{2}\right)
$$

If $g_{0}$, the acceleration of gravity, is in $\mathrm{cm} / \mathrm{sec}^{2}$ then the pressure is in Pascals. If a tank $1 \mathrm{~s}$ to be gaged to 1 millimeter, the pressure gage must resolve pressure to 1/25,000. Accurac 1 es of 1 part 1 n 10,000 of full scale are available with some of the commercially avallable pressure measuring instruments. However, this is a resolution of only 2.5 millimeters in a 25 meter deep tank. Both bubbler gage methods and static differential pressure measurement methods for Lic servlce are described by Wilson. The differential pressure method wherein a differential gage is connected acros the top and bottom of the tank is commonly used where penetrations Into the bottom of the tank are permitted. Since tank penetrations are often not allowed at the bottom of LNG tanks, espec $1 \mathrm{a} 1 \mathrm{ly}$ shlp tanks, and pressure gages are operated only with difficulty at low temperature, statlc differentlal gages often cannot be used in LNC service. The uncertainties of differential pressure gagos should be similar in magnitude to bubbler gages excluding the dynamic pressure drops in the latter. 
Two variations of the bubbler gage from the description by Wilson are shown in figure 2.5.1.9. The extra tube and differential pressure gage in the right side of figure 2.5 .1 .9 provide sufficient information so the liquid density in the region $\mathrm{h}$ can be calculated. The bubbler gage design on the left side illustrates a method of eliminating any pressure drop occurring in the bubbler 1 ine. When gas is bubbling through the lines shown in figure 2.5.1.9, the pressure measured by the differential pressure gage is the head pressure of the liquid above the bottom end of the bubbler tube.

An alternative method of measuring the liquid head is illustrated by Wilson but not shown here. Liquid gas interface detectors are located at the bottom of the tube, and when this interface is properly centered by adjusting the static pressure in the tube to the tank bottom, the pressure is recorded.

\section{Bubble Level Gages}

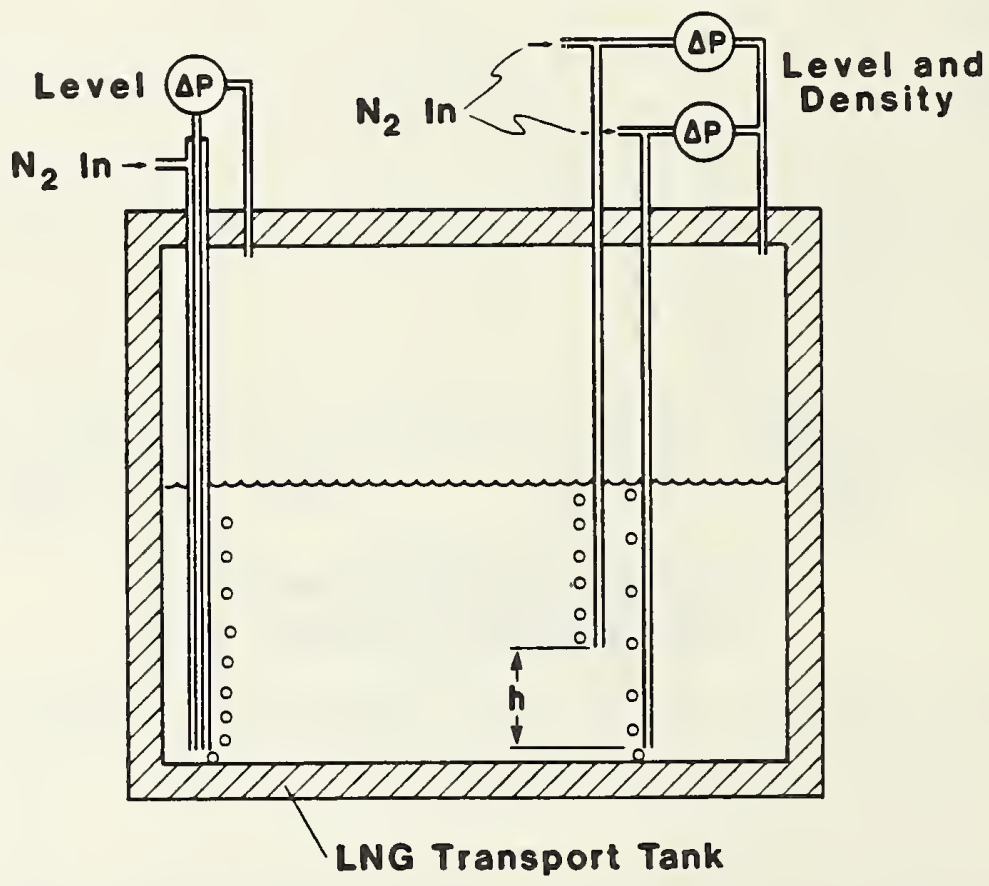

Figure 2.5.1.9. Schematic of some bubbler gages for LNG service.

Measurement Error The uncertainties discussed here are for the 25 meter deep tank discussed in a following section.

If the gas in the bubbler tubes were weightless, then the differential pressure observed would be the hydrostatic head. Since the gas has mass, a head pressure also exists for all the gas between the 1 iquid and the gage. The magnitude can be illustrated by the head correction for the bubbler line when the tank is full. The absolute pressure is then 2 bar in the bubbler line and the head correction is somewhere between ( $g_{0} \times 6 \mathrm{~g} / \mathrm{cm}^{2}$ ) Pa, for the nitrogen gas at ambient temperature and ( $g_{0} \mathrm{x}$ $\left.16.5 \mathrm{~g} / \mathrm{cm}^{2}\right) \mathrm{Pa}$ if the nitrogen gas is at the methane boiling temperature. Furthermore, since methane is a little over half as dense as nitrogen and probably fills the reference side of the differential pressure measurement, an additional correction of about $\left(g_{0} \times 11.7 \mathrm{~g} / \mathrm{cm}^{2}\right)$ Pa for each meter the gage is placed above the tank is required. This decreases as the tank level drops to about ( $g_{0} \times 0.5 \mathrm{~g} / \mathrm{cm}^{2}$ ) $\mathrm{Pa}$ as the tank approaches empty but now, the height from the liquid to the gage is much larger so the total correction required is still large. An uncertainty in the pressure correction of ( $g_{0} x$ $\left.1 \mathrm{~g} / \mathrm{cm}^{2}\right) \mathrm{Pa}$ is a 2.5 centimeters error in the liquid level measurement for a 1 iquid with a $400 \mathrm{~kg} / \mathrm{m}^{3}$ density. 
The density measurement should be made near the bottom of a tank to provide a density value at low filling levels. If $h$ in figure 2.5.1.9 represents 10 percent of the tank height, then the difference of the two gage readings from equation (4) will be ( $\left.g_{0} \times 100 \mathrm{~g} / \mathrm{cm}^{2}\right) \mathrm{Pa}$ for 1 iquid methane of $400 \mathrm{~kg} / \mathrm{m}^{3}$ density. The pressure gages can introduce a 1 percent error in the density measurement if they are 0.1 percent gages. Also, the uncertainty in the bubbler line nitrogen gas density contributes to the density uncertainty. The density measurement in a full tank may not represent the density at other levels in the tank especially in a shore storage tank where stratification can take place. The bubbling nitrogen also could alter the local density around the bubblers. A density measurement uncertainty of 1 percent does not seem unlikely. An error of 1 percent in density introduces an uncertainty of 1 percent in the level even before the gas column uncertainties are included.

If the gas is bubbling into the tank, probably the bubbler line contains nitrogen, but a pressure drop is present in the line. If the static method shown in figure 2.5.1.9, left side, is used or the interface detector method, the composition of the gas in the lines can be expected to be some mixture of methane and nitrogen. The uncertainty of the gas column density could easily be as large as any pressure drop that might be experienced in a line through which gas is flowing.

Bubbler gages have been relegated to backup use for capacitance gages on ship tanks. Gage accuracy has probably been a factor in this, but reliability has been a large consideration too. Very dry clean gas, usually nitrogen gas, must be used, or the bubbler nozzles rapidly plug with ice or other solids. Plugging has been a problem. Any leak in the lines from the pressure gage to the tank result in a false level reading, and errors introduced by small leaks can easily go undetected.

A bubbler gage can easily be in error by tenths of a meter in a 25 meter tank mainly because of uncertainty in the liquid density measurement and several centimeters additional because of uncertainty in gas head corrections. The poorer accuracy, along with failure due to leaks and plugging, limits the application of these gages to back up use for custody transfer measurement. If the gaging application does not require high accuracy, differential pressure and bubbler gages have advantages. They are least affected by surface turbulence, thus are useful for level monitoring during rapid filling of a tank. 


\section{5 .1 .6 References}

[1] Armstrong, G. T., Hydrocarbons for fuel -- 75 years of materials research at NBS, Nat. Bur. Stand. (U.S.), Spec. Publ. 434 (May 1976).

[2] Haynes, W. M., M. J. Hiza and R. D. MCCarty, Densities of LNG for custody transfer, LNG 5, International Conf. on Liquefied Natural Gas, Proc. 5th (Dusseldorf, Germany, Aug 29-Sep 1, 1977), Vol 2, Session III, Paper 11 (Institute of Gas Technology, Chicago, Ill., 1977).

[3] McCarty, R. D., Four mathematical models for the prediction of LNG densities, Nat. Bur. Stand. (U.S.), Tech. Note 1030 (Dec 1980).

[4] Brennan, J. A., Better LNG flow measurement sought, 0il Gas J. 76(5), 168+173-4+177 (Jan 1978).

[5] Parrish, W. R., J. M. Arvidson and J. F. LaBrecque, Development and evaluation of an LNG sampling system, Nat. Bur. Stand. (U.S.), NBSIR 78-887 (July 1978).

[6] Jackson, R. H. F., R. S. Collier, S. Haber and P. V. Tryon, Custody transfer systems for LNG ships: tank survey techniques and sounding tables, Nat. Bur. Stand. (U.S.), NBSIR $79-1751$ (1979).

[7] Haight, W. C., R. J. Hocken, B. R. Borchardt, C. L. Carroll, R. G. Hartsock, C. P. Reeve, F. E. Scire and R. C. Veale, Estimated accuracy of calibration of some membrane-type LNG transport tanks, Nat. Bur. Stand. (U.S.), NBSIR 80-2141 (1981).

[8] Hocken, R. J. and W. C. Haight, Multiple redundancy in the measurement of large structures, CIRP Ann.27, 357-60 (1978).

[9] Siegwarth, J. D., B. A. Younglove and J. F. LaBrecque, An evaluation of commercial densimeters for use in LNG, Nat. Bur. Stand. (U.S.), Tech. Note 697 (October 1977).

[10] Siegwarth, J. D. and J. F. LaBrecque, A portable calibration densimeter for use in cryogenic liquids, Nat. Bur. Stand. (U.S.), Tech. Note 1035 (March 1981).

[11] Siegwarth, J. D. and J. F. Labrecque, Cryogenic fluid density reference system: provisional accuracy statement (1980), Nat. Bur. Stand. (U.S.), Tech. Note 1041 (June 1981).

[12] Roder, H. M., ASRDI oxygen technology survey volume V: density and liquid level measurement instrumentation for cryogenic fluids oxygen, hydrogen, and nitrogen, NASA SP 3083 (1974).

[13] Wilson, Jr., M. P., LNG tank level measurement, AGA Operating Section Proceedings, Proceedings of the Transmission Conference, Las Vegas, Nevada, T-223 (1976).

[14] Jelffs, P. A. M., Calibration of containers and gauges, Journal of the Institute of Petroleum, 58, No. 561, pp117-25 (May 1972).

[15] Paterson, I.W.F., Standardization for installation of automatic gages, Journal of the Institute of Petroluem, 58, 209, (1972).

[16] Watson, P. B., Instrumentation for the Measurement of Refrigerated Hydrocarbon Liquids, Journal of the Institute of Petroleum, 58, No. 561, pp126-31 (May 1972).

[17] Halverson, G., Automatic continuous LNG level-gauging and temperature measuring systems, Instrumentation in Cryogenic Industry, 1, paper 602 Houston, Texas, (1976).

[18] Williams, R. A., High accuracy level gaging, Proceedings 55th International School of Hydrocarbon Measurement, Norman, Oklahoma (1980) pp 332. Similar papers appear in 1977 to 1982 schools. Also, see Moore, C. F. and Blanchard, R. L., Custody transfer instrumentation systems for LNG marine transport projects, Fourth International Conference on Liquefied Natural Gas, Algiers, Algeria (1974).

[19] Eilers, C., Modern automatic tankgauging systems, Proceedings 55th International School of Hydrocarbon Measurement, Norman, Oklahoma (1980) pg 345. 
[21] LNG Materials and Fluids - A user's manual of property data in graphic format, by D. B. Mann, Editor. Manual sponsored by American Gas Association, Maritime Administration, Office of Standard Reference Data and American Bureau of Shipping. Published by National Bureau of Standards, Thermophysical Properties Division 773, Boulder, Colo., 325 pages (1977).

[22] Siegwarth, J. D. and J. F. LaBrecque, Estimated uncertainty of calibrations of free standing prismatic Liquefied Natural Gas cargo tanks, Nat. Bur. Stand. (U.S.), NBSIR 81-1655, 294pp (January, 1982).

[23] Siegwarth, J. D., Measurement uncertainties of level gages for liquefied natural gas, NBSIR 82-1668, Nat. Bur. Stand. (U.S.), 52 pp (June, 1982). 


\subsubsection{Volume of Membrane-Type LNG Ship Tanks}

The following edited description of Hocken and Haight [1] will provide some insight into methods developed and used by NBS to determine or validate the large storage volumes required for economic LNG service. The work was sponsored by an industrial consortium, LNG Custody Transfer Measurements Committee. Although this particular work does not provide error values as such, the following report of Haight, et al. [4] does specify accuracy values for actual ship membrane tanks. The two papers together provide a consistent set of procedures and end-point values of uncertainty.

\subsubsection{Volume Measurement Methods [1]}

Traditionally, the dimensional metrologist has been involved with the mechanical measurements that insure interchangeability on the production line. The magnitude of this problem, both financial and technical, has led to a vast array of instrumentation and technique that allows measurement of objects of medium dimensions to an impressive degree of accuracy. These measurements seldom involved distances over a few meters or under one micrometer. In the past several years new technology has required increasingly accurate measurements outside of this range, both in the sub-micron range, which may be referred to as micrometrology and the range greater than 10 meters, which is referred to as macrometrology.

New instrumentation has been developed for measurements in micrometrology [2,3]. Such instrumentation is just being developed in the macrometrology area, which for years has been the province of the surveyor.

Techniques will be described which have recently been used to measure the volumes of large LNG tanks in an effort to improve on the accuracy of international custody transfers. The technique involves a combination of recently developed laser instruments and traditional surveyor's tools. Three types of ships have been considered, which are generally referred to by the type of tank they carry. The tank types are "spherical," "membrane," and "free-standing prismatic."

Although the National Bureau of Standards (NBS) has been involved in the measurement of all three types of tanks, only the algorithm for the so-called "membrane" tanks will be discussed here. A diagram of a typical tank of this type is shown in figure 2.5.2.1. Each LNG ship carries six of these tanks. Each tank is approximately 30 meters long, 25 meters high, and 28 meters wide, though these dimensions vary with location in the ship. As can be seen from the figure, these tanks are in the general shape of a ten-sided prismatic solid.

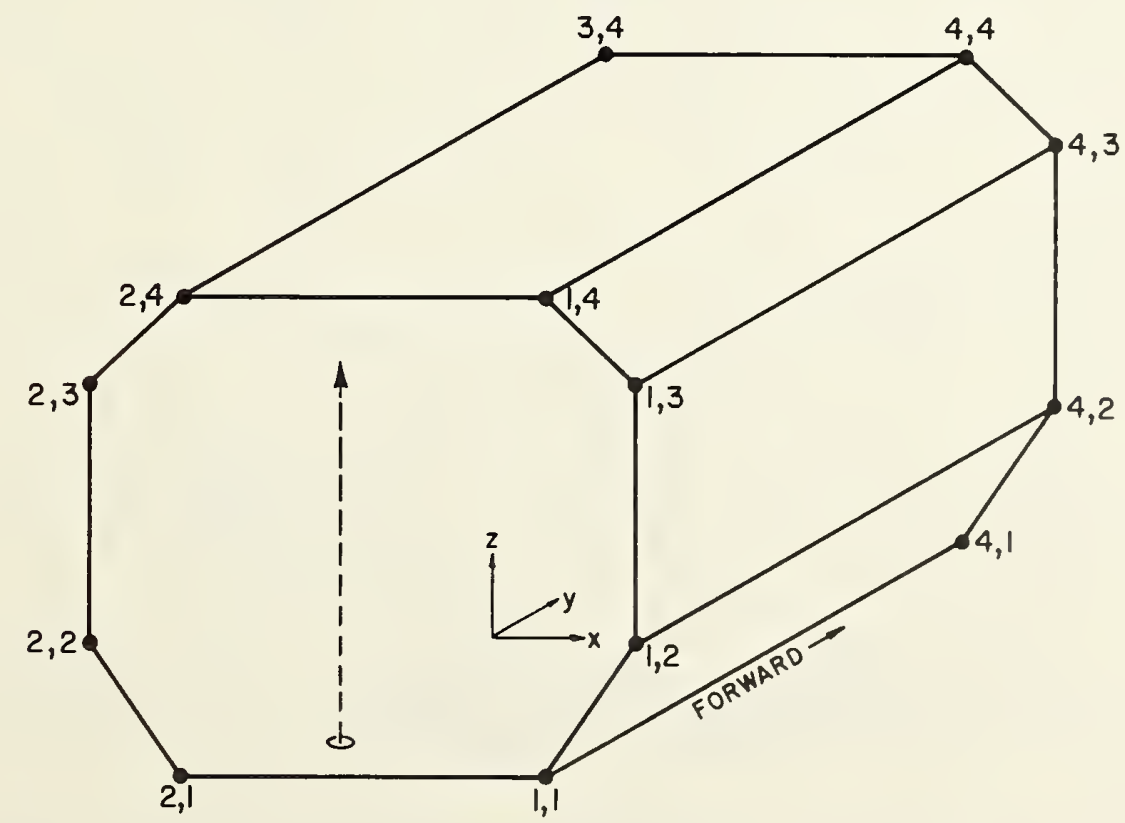

Figure 2.5.2.1. A typical LNG ship tank of the membrane type. 
These tarks are called membrane tanks because of the method of construction. They are made inside a large steel socket, the inner hull of the ship, by attaching layers of insulation and wood to the socket and then lining this insulation layer with a welded layer of thin, 1.2 millimeters, corrugated (or 0.7 millimeters, non-corrugated) stainless steel. This membrane merely serves to contain the liquid and serves little or no load bearing function. These tanks are cooled to about $-160^{\circ} \mathrm{C}$ and filled with LNG at the gas liquefaction site. The primary gaging system is a height gage located at the aft end of the tank. Measurements of the height of the liquid surface are made before and after filling (or emptying), and the price of the contents is determined from the known volume versus height tables for the tank. The sponsors of this project desired to know these tables to + 0.1 percent and to have some reasonable means of proving this accuracy. Unlike metrology for quality control, where suitability for end use is the primary criteria, in custody transfer situations "faith" in the accuracy of the measurements is of primary importance, particularly in such cases where custody transfers are international in character. This "faith" was the reason why NBS measurements were sought for these tanks, since there are private calibrators who also perform such measurements.

As a method, it seemed clear that the best approximation to such a tank would be a set of 10 planes which were "best fit" estimates of the actual tank surfaces. These planes would intersect at 16 corners to create a "best fit" tank. These corners would be in effect "gage points" for the solid. Once the set of 16 gage points was established in some orthogonal coordinate system, then the volume of the tank could be computed in any system, using the coordinate transformation techniques common in the field. It should be noted that the volume of the tank must be known in many orientations with respect to gravity, as when ships are loaded and unloaded, the level of the ship is constantly changing.

Once the model for the tank was chosen, the problem became one of trying to realize it in the field. There were several constraints which had to be considered. In the first place, the sheer physical labor involved in measuring such structures is quite significant. In order to minimize this labor, the experimental plan required the minimum number of measurements necessary to characterize the tank and still provide ourselves with enough redundancy to make a reasonable and defensible error estimate. The second constraint was simply one of speed. The period in which access to the tank was possible would be fairly short, and thus a measurement sequence was necessary which could be completed in two to three days. At the end of this time, the scaffolding which allows access to the tank walls is removed, so that remeasurement is out of the question. This third constraint led to the conclusion that on-line error checking was highly desirable.

Since the only access to the tanks was interior, the following measurement method was used. The corners of the tank were first labeled with subscripts, as shown in figure 2.5.2.1. Gage plates were then attached to the end walls just inside these corners. This method is nondestructive to the tank surfaces since the gage plates are attached to the membrane corrugations with teflon lined clamps. These gage plates serve to define a prismatic solid slightly smaller than the real tank and provide solid support for the necessary length measurements. An instrument called a laser level was then used to establish true planes between gage points. This device is shown schematically in figure 2.5.2.2. It consists conceptually of a collimated laser and a rotating pentaprism, which sweeps the laser beam in a plane. The gage plates were designed so that this "laser level" could be attached to the plates on a kinematic mount and sweep out planes just inside the tank surfaces. This laser instrument has the feature that, if so desired, it may also be use as a level. The laser is suspended in the device, and servo motors will level it to +0.10 seconds. This feature is used on the bottom survey in order to locate the tank with respect to true vertical. Each plane is thereby swept out in turn, and the line of passage of the laser beam is accurately marked as it crosses each of the four gage points which define that plane. At the same time, offsets from this plane to the real wall are measured with a laser level rod. These rods, which were refitted with higher accuracy scales and kinematic base plates, contain an active detector which searches for the beam, locks on it, and then automatically stops to allow reading of the measurement. Measurements of these offsets are made at a very large number of points for each plane in order to check for wall deformation and minimize cosine errors which result from operator misalignment of the rod with respect to the wall.

The laser surveys are shown schematically in figure 2.5.2.2, in two dimensions. The end result of the laser plane surveys is shown two dimensionally in figure 2.5.2.3, which is a view of the aft wall from outside. The distortions in the real walls are greatly exaggerated. (0ff sets from the plates to the end wall laser planes are recorded on special standoffs at each plate. These are not shown for reasons of clarity.)

Since the intersection points of the 10 laser planes are now marked on each plate and the offsets from these laser planes measured to all wall surfaces, the problem now reduces to measuring the coordinates of each of these intersection points in some orthogonal coordinate system. These measurements are made by laboratory type taping techniques which have, for these intermediate distances, higher 


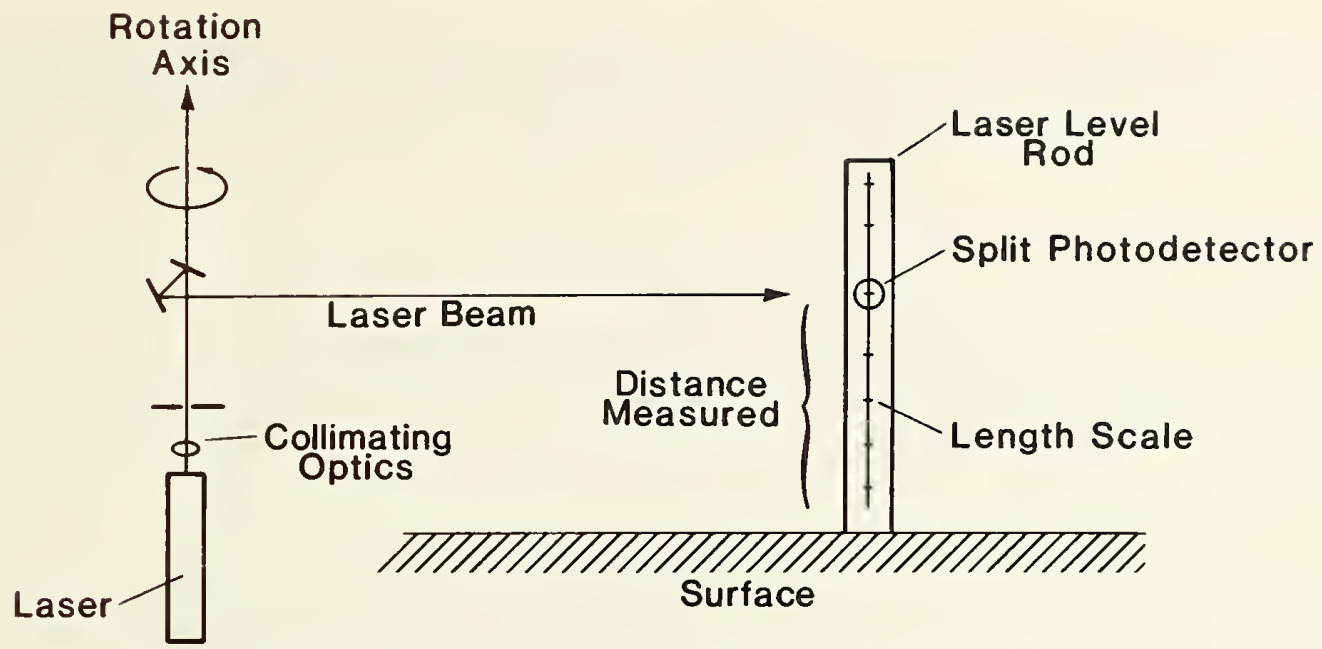

Figure 2.5.2.2. Schematic of laser level with beam seeking "laser" rod.

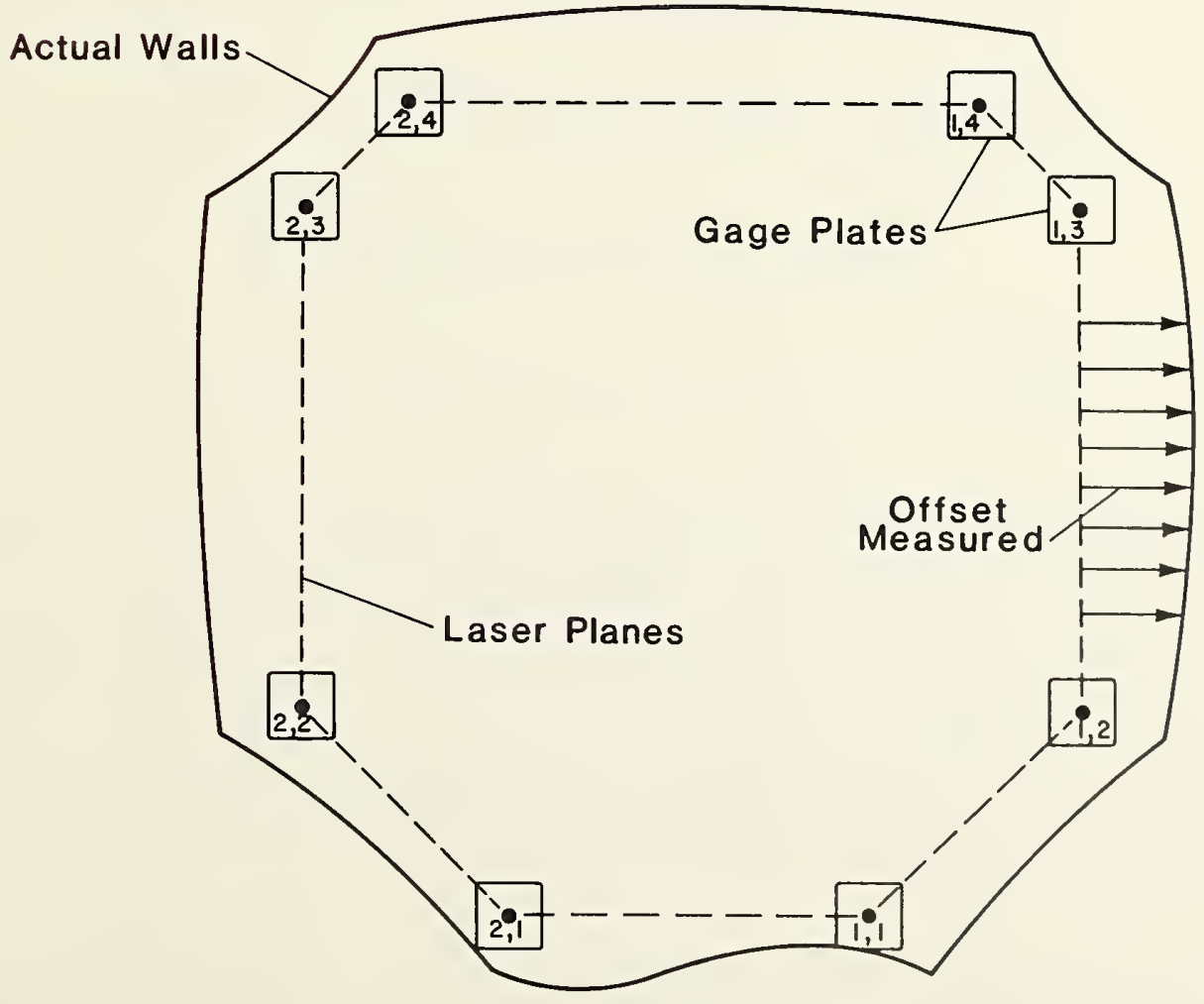

Figure 2.5.2.3. Schematic of tank aft wall (from outside) after complet lon of laser surveys. 
accuracy than more sophisticated laser ranging instrumentation. The arrangement for one end (which is duplicated at the other) is shown schematically in figure 2.5.2.4. Each end of the tape is supported over rollers, with one end clamped and the other end weighted, using calibrated brass weights.

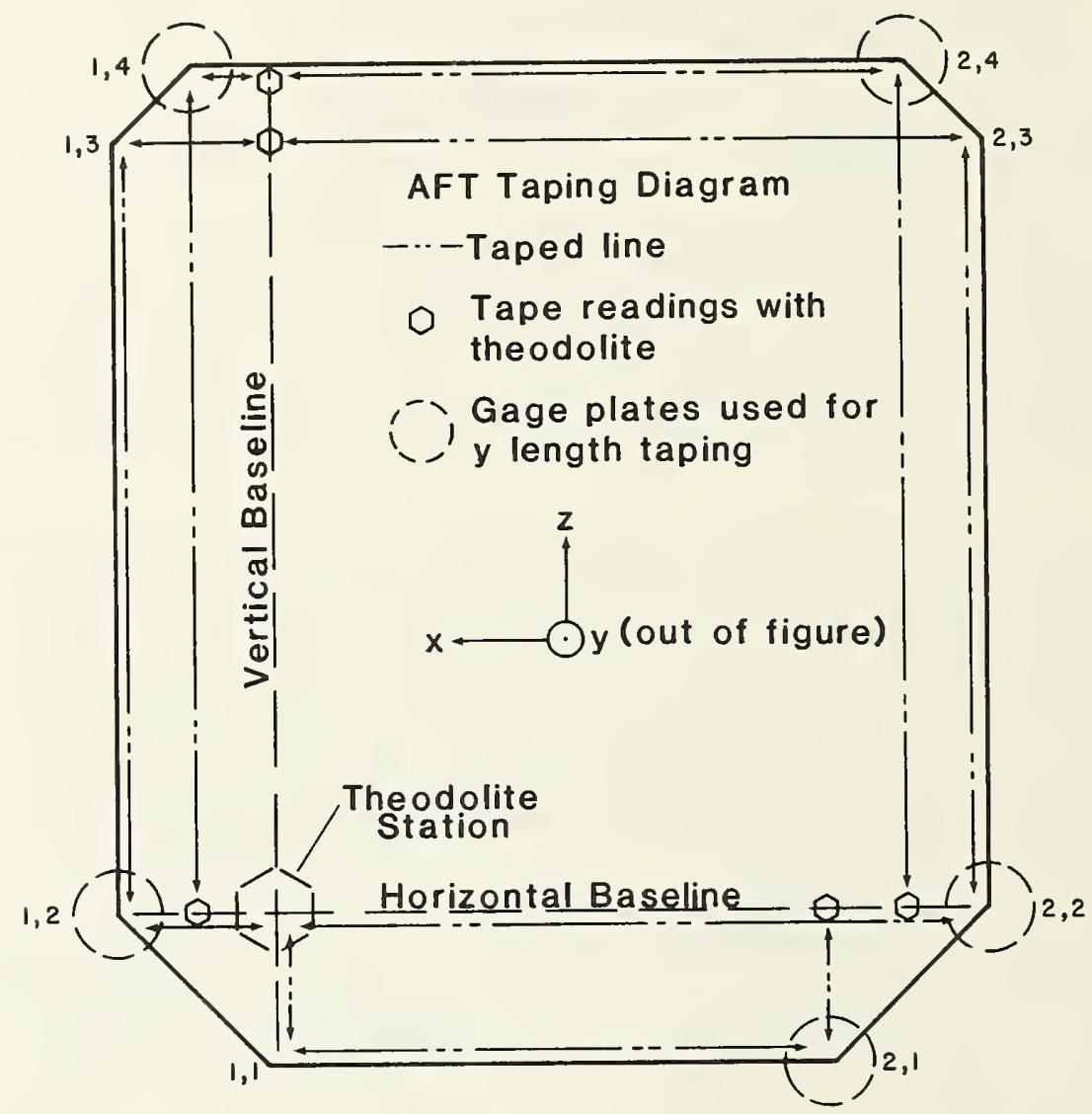

Figure 2.5.2.4. View of aft taping from inside.

In figure 2.5.2.4, the gage points are the marked intersections of the laser planes of the tank corners. At one position, an additional gage plate is attached to the tank wall. This plate is used to attach a theodolite, accurate to +1 arc seconds. This instrument has been modified by NBS to include a close up lens, so that it can be turned towards the wall for readings in that direction. This theodolite is used to establish an orthogonal, X,Y,Z system. Two axes of this system, the horizontal and vertical base lines, are shown on the figure. These two combine to generate the end wall plane. With the theodolite and a simple scale with a sliding cursor, visible to the theodolite operator, the following measurements are made:
a) Vertical distances ( $Z$ ) to gage points 1,2 and 2,2 from the horizontal base line.
b) Y distances (tank length) from the end base plane to the end laser plane at gage points 1,$2 ; 1,4 ; 2,1 ; 2,2 ; 2,4$.
c) Horizontal distance (X) to gage point 1,1 .
Next, the taping is done while the theodolite is still set up. 
Vertical tapings are made between 1,3 and 1,2; and 2,3 and 2,2, without using the theodolite. The tape is next suspended from 1,4 and read with the theodolite at the horizontal base 1 ine. This same procedure is followed for 2,4; 1,1, and 2,1. All vertical tapings are made with a 5 kg weight, and the temperature of the tape and wall are simultaneously monitored.

The horizontal $(x)$ tapings are made similarly with intermediate readings being made with the theodolite and special tape cursors. Tape tension for all horizontal measurements is $10 \mathrm{~kg}$. All intermediate readings of a tape using the theodolite are marked in the figure.

Before the theodolite is removed, it is used to make several readings of distances ( $Z$ ) on the forward wall between gage points there and the horizontal base plane. The theodolite is now moved to a conjugate setup on the forward wall, and the same procedure repeated. The cross measurements, aft to forward, are used to remove any small angles that will occur between coordinate systems generated by these two setups.

After the taping of both end walls, the $Y$ distances where the of sets between end wall theodolite plane and end wall laser plane were measured are taped down the length of the tank. These gage points are circled in the figure. As mentioned previously, temperature is closely monitored, and the tapes are always supported at enough points to render the catenary correction negligible. The initial alignment of gage points was made accurate enough to insure that cosine errors, which are neglected in the taping, are also negligible.

During the aft wall taping, several subsidiary measurements are made that are used to locate the height gage for future integration. These will be described later in the text.

\subsubsection{Analysis}

The end results of the measurement of a tank are:

1) A set of coordinates for the intersections of the 10 laser planes (16 intersections) in the aft coordinate system. (Three $Y$ coordinates have not been measured, and either the aft or forward coordinate system can be used at the convenience of the metrologist.)

2) A large number of deviations from each of the 10 planes to the corresponding wall.

3) The coordinates of several points along the height gage, which establish its location and orientation in the same coordinate system.

The data analysis may then proceed as follows. First, the known coordinates for five gage points on the fore and aft bulkheads are fit to planes and the $Y$ coordinate of the remaining three gage points calculated. (The coordinate system is shown in figure 2.5.2.1.) There is now a complete set of 16 coordinate triplets which specify the geometry of the laser generated solid. This set actually overconstrains the geometry, since only three points are required to specify a plane. Thus, for the 10 intersecting planes, 12 coordinate triplets are actually needed. This over-constraint allows an estimate of the closure of the algorithm and an estimate of how well the procedure has generated an ideal solid. This closure is estimated as follows. The four points for each plane are fit to a plane. Since there are only four such points, this is not done by least squares. Rather, coefficients of all possible combinations of three points are calculated and the resulting coefflclent set averaged to obtain the "best fit" plane (there are, by the way, four such combinatlons).

From these coefficients, it is possible to compute the intersection of the "best fit" planes taken three at a time. If the measurement technique were exact, these intersections would be at the measured gage points. Actually these intersections are at the "average" gage points and are not, in general, equal to the measured points. Deviations from these idealized points to the measured polnts are computed to estimate the closure. Typical results indicate an RMS closure of about. 2 millimeters.

The next step in the algorithm is to compute the "best fit" tank wall planes with respect to the 1 asn planes. Since there are often hundreds of points here, this is done by traditional least squares method. The results of one such fit are shown in figure 2.5.2.5. Plotted here are the deviations from a "best fit" plane as a function of position on that plane. The vertical axis $1 \mathrm{~s} 1 \mathrm{n}$ millimeterg. As can be seen, these tanks are remarkably well constructed, the RMS average deviation from a trun plane being only about 3 millimeters. 


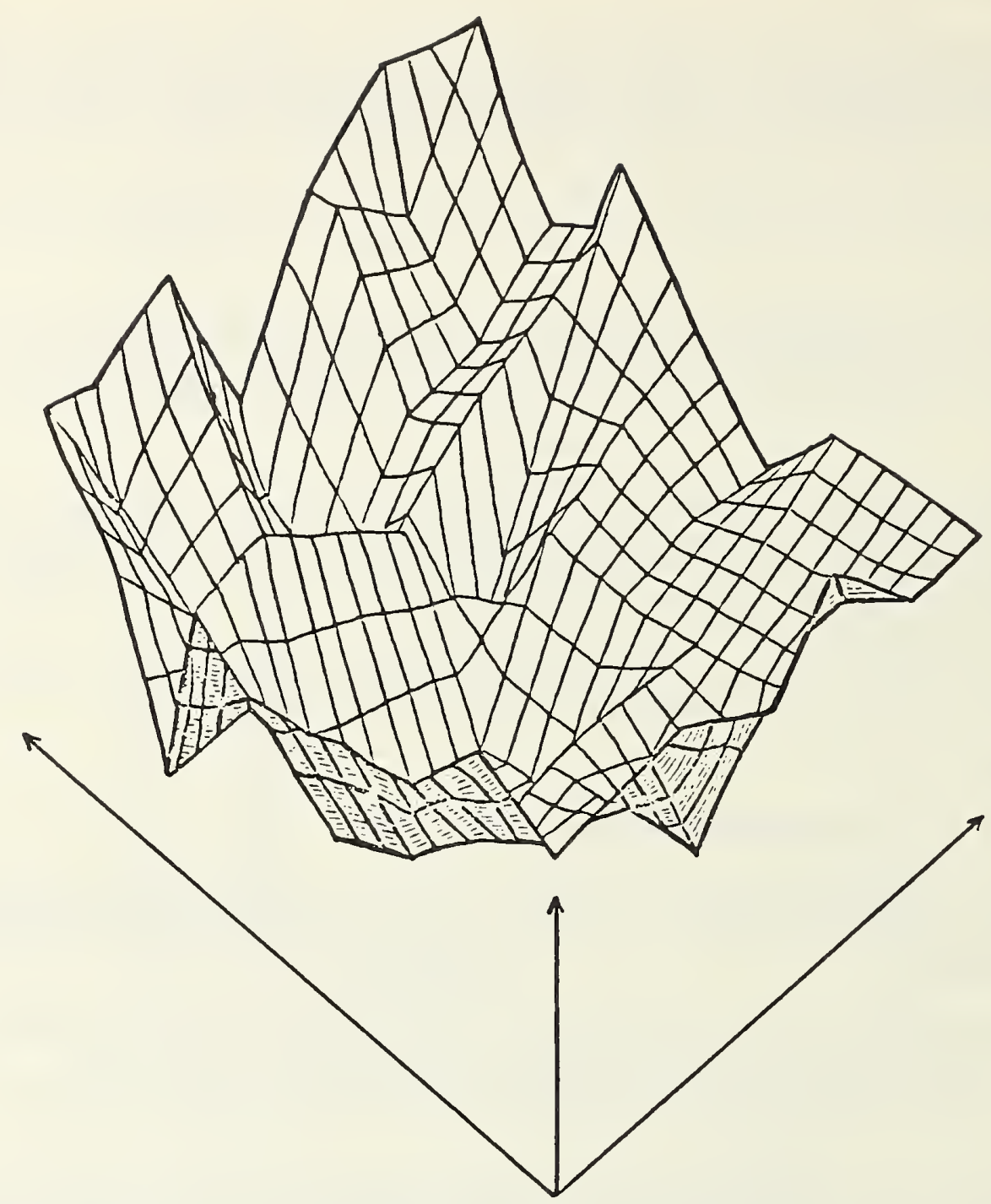

Figure 2.5.2.5. Contour of bottom of typical LNG ship tank.

In order to add these planes to the laser planes, a set of normals from the laser planes "average" gage points to the "best fit" wall planes are computed and the coefficients of these new planes calculated in the original reference system. It should be emphasized that this is not a statistical problem as the normals are from "best fit" plane to "best fit "plane. From these new coefficients the intersections of the "best fit" wall planes are calculated to obtain a set of 16 coordinate triplets, which fully specify the real tank geometry. It is during this process that a correction is made for the wall corrugations. Since the corrugations go inward, they effectively reduce the volume of the tank. Compensation for this fact is made by measuring a reduction per unit area in the lab and subtracting this fraction from the outward directed normals from the laser plane to the "best fit" wall plane.

At this point a further estimate of the survey accuracy is made, in particular, how these idealized gage points are affected by the density of laser plane survey points. This was done by reducing the number of survey points in the fit for the wall planes and examining how this reduction changes the intersection coordinates of the "best fit" plane. The minimum survey density is defined as that density where the computed intersection coordinates change by the error $( \pm 2$ millimeters) determined by the closure of the taping algorithm. 


\subsubsection{Integration Technique}

In the section on tank measurement, it was described how a set of 16 (or 12) tank coordinates are obtalned. These coordinates are in a system with its origin at the theodolite on the aft bulkhead. At the time of the actual tank measurement, several subsidiary measurements necessary for the integration were made. They were:

1) The location of base of the height gage in the theodolite coordinate system was established.

2) The orientation of the tank bottom plane with respect to the ship's list and trim marks was established. This was done using the laser's self leveling feature and nearly simultaneously reading the list and trim marks on the hull of the ship. (List and trim translated to roll and pitch, respectively.)

The integration algorithm now proceeds as follows. A translation is performed to put the tank coordinates into a system whose origin is at the origin of the height gage. These coordinates are in an orthogonal system, but in general this system is rotated with respect to the ship system (list and trim marks). From the level survey and the list and trim readings, two angles are calculated. These angles are used to rotate the tank system into the ship system. Thus, the coordinates of the tank in the ship system are now known except for some offset vector between the two origins (which is irrelevant anyway). It is assumed here that the tank has no yaw (rotation around the $Z$ axis in $f i g u r e$ 2.5.2.1) with respect to the ship. This is probably a non-trivial assumption, since the yaw of the tank is strongly coupled to the volume when the list and trim (roll and pitch) are non-zero. (This is easiest to see by imagining a yaw of 90 degrees). Nevertheless, since a method to accurately measure this angle was not available, it was assumed to be small.

The integration algorithm works entirely with this ship coordinate set, for each tank, and the pitch and roll angles. The following is a description of this algorithm for one orientation of pitch and roll.

1) The finite rotation matrix with pitch and roll is constructed and the coordinates are rotated to define the tank in this orientation. $Z$ is still the vertical axis, with respect to gravity.

2) The coordinate array is searched for the maximum and minimum $Z$ and the difference is divided into $\mathrm{N}$ steps; where $\mathrm{N}$ is approximately 10000 . The integration then proceeds by taking steps $\Delta Z$ and at each $Z$ height computing the intersections of the tank planes with the horizontal plane. These intersections define a $k$ sided figure, in the general case, with $k \leqq 8$. The area of each of these figures is calculated, and the integration proceeds by Simpson's rule. The number of steps $N$, was determined by integrating dummy tank data (with calculatable volumes, i.e., right angles, etc.) at arbitrary orientations and determining the number of steps necessary to reduce the numerical error to less than parts in one million. The height of the liquid at the gage is determined by calculating the $\mathrm{Z}$ intersection of the horizontal plane with a vector parallel to the height gage. (The origin is already at the gage zero.)

\subsubsection{Results}

Measurements have been completed on three tanks of this type. The algorithm described here was implemented in full on the last of these tanks. A crew of four metrologists took two days to complete the task, and data were entered to a remote computer using a telephone link at the end of the second day. This computer cross check showed an error which was removed by remeasurement the followlng morning, thus demonstrating the advantages of on-line checking. The closure of the algorlthm, after the error was corrected, was about \pm 2 millmeters RMS in three dimensions, which can be converted to a volume accuracy of parts in 10000 or better. The method has thus met the original criterla of belinf, fast, self checking, and highly accurate. The key elements of this system are; the generation of an ideal solid using laser techniques, measuring deviations from this solld in a properly dertned coordinate system and including enough redundancy for on-line error checking. The use of coordinates instead of lengths allows using the existing techniques of 3-D metrology, which greatly s1mpl 11 ed the software needed for the task. 
The original contract called for these measurements to be made on empty tanks at ambient temperature, and no attempt has been made to compute volume changes caused by liquid load or temperature effects, both of which may be considerable.

\subsubsection{LNG Ship Membrane Tank Calibrations [4]}

The National Bureau of Standards, supported by the Department of Commerce Maritime Administration, did some preliminary studies to establish an upper limit for the tank volume uncertainty of a spherical transport tank calibrated by photogrammetry [5]. Additional support from the Maritime Administration and the LNG Ship Custody Transfer Committee (an industrial consortium) has made possible a considerably expanded study of the calibration uncertainties of LNG transport tanks. The tank calibrations examined in this work are those of the three LNG Transports built by Newport News Shipbuilding and Drydock Company for El Paso Marine Company.

The cargo tanks on these nominal 125,000 cubic meter capacity ships are membrane types of the Technigaz design described in reference [6]. The tanks are ten-sided prismatic solids and rectangular in horizontal cross section. The walls are all plane surfaces. The end walls are vertical as are the side walls. The side walls are connected to the horizontal floor and ceiling by sloping chine planes. All eight of the longitudinal planes are parallel to the longitudinal axis of the ship. The supporting walls of the tanks are the inner hull of the ship. Layers of insulation are attached to these walls, and the internal surfaces of the insulation are covered with a liquid tight, 1.2 millmeters stainless steel membrane. This membrane has corrugations stamped into it forming a wafle pattern.

The measurements used to calculate the tank volume were generally made after the membrane installation was complete but before the removal of the construction scaffolding, since it is needed to provide access to the tank surface. Briefly, the calibration method used by the calibration contractor consisted of measuring horizontal cross section areas of the tank at intervals from the bottom to the top of the tank. The products of areas and the separation from the area immediately below summed to the level gives the volume to that level. This method is discussed in reference [7]. The cross sectional areas of these tanks were determined by measuring short distances to the wall from the sides of a horizontal rectangle of known dimension defined by laser beams.

A calibration method developed at NBS and described above and in reference [1] was used by NBS to assess the accuracy of the method used by the calibration contractor. The method used by NBS consists, in brief, of inscribing with laser planes a slightly smaller closed volume with the same shape as the tank. The dimensions of this laser-plane-defined volume are accurately measured. Perpendicular distances from the laser planes to the tank walls are measured at several hundred specified points distributed around the tank. The integration method used to determine volume as a function of height is also outlined in reference [1].

The calibration reports for each of the six tanks of the three ships, the El Paso Southern, The El Paso Arzew and the El Paso Howard Boyd, appear in the appendix of the original work [4]. Each individual tank report supplies the following information:

a. A calibration report or a report of test describing the measurement technique, measurement conditions, and error estimate.

b. A summary main gaging table showing tank volume as a function of gage height.

c. Summary list and trim correction tables to supplement the main table under expected conditions of ship list and trim.

d. A plot comparing the NBS main gaging tables at selected intervals to the gage books for each tank provided by the calibration contractor.

The staging was removed in four of the tanks before the NBS measurements were made; hence the calibration check was done only to the height accessible from the bottom of the tank.

The calibration accuracy required by the ship owner is \pm 0.2 percent of total tank volume. The NBS results range in accuracy from \pm 0.05 percent to \pm 0.1 percent as indicated in the Reports of Calibration [4]. In every case, the calibration contractor's results are well within \pm 0.2 percent of the NBS results. It therefore appears that the accuracy requirement has been achieved for the tanks at ambient temperature. The tank tables at ambient temperature are used by the transporter to determine LNG volume at operating temperature, because the tank dimensions have been assumed independent of cargo temperature. This assumption is based on the fact that the shape of the tank is 
determined by the external tank wall via the insulation. The corrugated design of the membrane allows the membrane to shrink on cooling without altering the internal volume.

In actual operation, the external tank wall might be as cold as $0^{\circ} \mathrm{C}$ depending on weather conditions. The temperature of the interior of the tanks was as high as $30^{\circ} \mathrm{C}$ during calibration of the tanks. Provided the external tank wall reached temperatures that high, The difference between the calibration and operating temperature could be as large as $30^{\circ} \mathrm{C}$.

The thermal expansion coefficient of iron as well as iron containing small quantities of nickel [8] is approximately $0.0000116 \mathrm{~m} / \mathrm{m}^{\circ} \mathrm{C}$. This contraction only affects the length and width of the tank with regard to calibration, since the liquid height is determined by the level gage rather than the tank height. The tank volume change between the calibration and operating temperature could be as much as -0.07 percent.

The shrinkage of the balsa wood insulation upon cooling (since the balsa is not a rigid body) as well as any bulging of the tank sides counteract the reduction in volume due to cooling the walls. The balsa shrinkage contributes a negligible change in tank volume. If this is also true with respect to tank wall bulging due to hydrostatic head, then the maximum systematic error introduced into the tank calibration due to cooling is estimated to be no more than -0.07 percent. 


\section{5 .2 .6 References}

[1] Hocken, R. J. and W. C. Haight, Multiple redundancy in the measurement of large structures, Annals of the CIRP, 27 (1978).

[2] Young, R. D., Length calibrations in the micrometer and sub-micrometer range, Annals of the CIRP, 25 (1977).

[3] R. Hocken, J. A. Simpson, B. Borchardt, J. Lazar, C. Reeve and P. Stein, Three-Dimensional Metrology, Annals of the CIRP, 26 (1977).

[4] Haight, W. C., R. J. Hocken, B. R. Borchardt, C. L. Carroll, R. G. Hartsock, C. P Reeve, F. E. Scire and R. C. Veale, Estimated accuracy of calibration of some membrane-type LNG transport tanks, Nat. Bur. Stand. (U.S.), NBSIR 80-2141, 79 pp (February, 1980).

[5] Jackson, R. H. F., R. S. Collier, S. Haber and P. V. Tryon, Custody transfer systems for LNG ships: tank survey techniques and sounding tables, Nat. Bur. Stand. (U.S.), NBSIR 79-1751, 84 pp (May, 1979).

[6] Jackson, R. G. and M. Kotcharian, Testing and technology of models of integrated tanks for LNG carriers, Paper 35, Session 6, First International Conference on LNG, Chicago, IL (April, 1968)

[7] Jelffs, P. A., Calibration of containers and gages, J. Inst. Pet., 58, No. 561, pp 117-25, (May, 1972).

[8] LNG Materials and Fluids User's Manual, D. B. Mann, Editor, Nat. Bur. Stand. (U.S.), Boulder, Co (1977). 


\subsubsection{Volume of Freestanding Prismatic LNG Ship Tanks}

The actual physical structure of the tank is composed of a set of plane surfaces attached at the edges and designed to conform to the internal structure of the ship. These tanks differ from the membrane tanks of 2.5 .2 in that the tank walls are self-supporting, or freestanding. This is accomplished by complex internal bracing and structure and allow tank construction to be performed separately from the ship yard.

A new technique, photogrammetry, was adapted to allow measurements of the volume enclosed by the outside walls of the tank, and then the wall material and internal structure volumes could be subtracted to give the required internal free volume and volume-height relationships for the tank tables. The following edited work of Siegwarth and LaBrecque [8] provides a description of the new measurement process and a detailed look at individual measurement techniques, estimated accuracies and precision.

The accuracy of the tank calibrated by the photogrammetric technique was examined during the calibration of fifteen freestanding prismatic LNG transport tanks. This examination indicated that the calibration accuracy of the tanks calibrated in the storage position was better than \pm 0.1 percent. Additional factors influencing the accuracy of the calibration of the tanks, such as the effects of installing the tanks into the ship and loading the ships with LNG, were examined in the course of this work and, the results are reported here. The various measurements used by NBS personnel to analyze the calibration accuracy are detailed in the eight appendices included in the original publication.

\subsubsection{Introduction}

The National Bureau of Standards has done preliminary studies [1] to establish an upper limit for the tank volume uncertainty of a spherical transport tank calibrated by photogrammetry [2,3]. The Maritime Administration of the Department of Commerce and the LNG Custody Transfer Measurements Supervisory Committee [4] have sponsored studies to test the accuracy of the present state of the art of tank calibration and to develop new methods of calibrating tanks that are suitable for industrial use. This additional support has made possible an expanded study of the calibration uncertainties of freestanding prismatic LNG transport tanks.

The objectives of this work were twofold. The first objective was to make more extensive measurements to provide an independent verification of the uncertainty of the photogrammetric method of determining spatial coordinates of points on the surface of the tanks. The second objective was to provide an upper limit for the uncertainty of the calibration of these particular tanks. This latter objective contains the first, but additional factors must be considered. As in the case of the spherical tanks, the photogrammetric survey may be completed before the tanks are transported to and installed in the ship. In addition, the photogrammetry is done with the tank empty. Effects of lifting, installing, cooling, and loading the tanks should be included in the calibration uncertainty.

The photogrammetric calibration method and effects on the tank volume of tank history following the tank calibration have been examined by independent measurements and calculations. The photogrammetric calibration has been analyzed for each of the tanks, using length standards. The NBS has tested the calibration of one tank by a completely independent volume determination method. The laser plane method developed by NBS for the conformation of the volume tables of membrane tanks [5] was modified and used on the exterior of the tank.

The method of volume calculations has also been tested for accuracy by independent calculations, using the photogrammetrically determined coordinate data for points on the tank surface. The dimensions of the aluminum in the tank walls and internal structure were measured for one tank to obtain an estimate of the error induced when using the blueprint dimensions in the correcting for volume taken by the tank walls and structure. The same tank dimensioned by laser planes was also dimensioned while filled with the maximum amount of water during the hydrostatic tests. A distortion study was performed In which the tank bottom was re-surveyed after the tanks were installed to determine whether the tank support blocks were properly shimmed so that the original bottom contour of the tank was retained. Finally, two of the early tanks were surveyed both before and after they were lifted into place aboard the ship, to investigate the effects the lifting might have on the tank volume calibration. The methods used and the results obtained from the various measurements by NBS are detalled in the reports contained in the original document [8]. An estimate of the uncertainty of the photogrammetric gurvey technique as applied to this particular calibration problem is also provided. 


\subsubsection{Tank Description}

Each ship contains five tanks similar to the tank shown in figure 2.5.3.1. Counting from the bow, the third and fourth tanks are identical; the second tank is nearly the same in capacity as tanks three and four but the sides taper slightly towards the bow. Tank five is shorter and tapered slightly toward the stern. Tank one is again shorter and heavily tapered towards the bow. The outer walls of the tanks are flat planes, eight in all. The bottoms and tops are horizontal planes; the ends are vertical planes. The sides are composed of two planes each. The topmost plane is vertical and connects to the bottom via a chine plane that slopes in toward the center line of the tank at the bottom. This narrowing of the bottom provides clearance for the curved corners between the sides and bottom of the hull. The larger of the tanks have widths and lengths of about 36 meters; all have heights of about 23 meters, and are constructed of rectangular plates of 5083 aluminum varying in thickness from approximately 0.5 to 3 centimeters. These plates are edge-welded to form panels. These panels are pre-assembled complete with the vertical stiffening channels and horizontal girders. The panels are lifted by crane, fitted into place and welded. The tanks are divided internally into four quadrants by vertical walls. The fore-to-aft wall is liquid tight. The port-to-starboard wall, or swash bulkhead, is not liquid tight. The external and internal walls of the tank are heavily braced internally by vertical 23 centimeters wide "L"-shaped ribs at roughly $2 / 3$ meter intervals. These ribs in turn are attached to seven wide flanged horizontal girders at intervals from the bottom to the top of the tank. Stiffening ribs are also welded to the top plates inside, and I-beam girders brace the floor panels at the same intervals as the ribs on the walls. These in turn are welded to heavy girders in both the floor and ceiling. Numerous triangular braces are fitted between the various stiffening girders. Figures 2.5.3.2 and 2.5.3.3 are photographs of this internal structure. The corners between the planes composing the tank surface are radiused. The radius is approximately 30.5 centimeters on the inside. The volume of the aluminum comprising the tank amounts to between one and two percent of the total volume of the tank. Figure 2.5.3.4 is a diagram of the tank showing the interior structure.

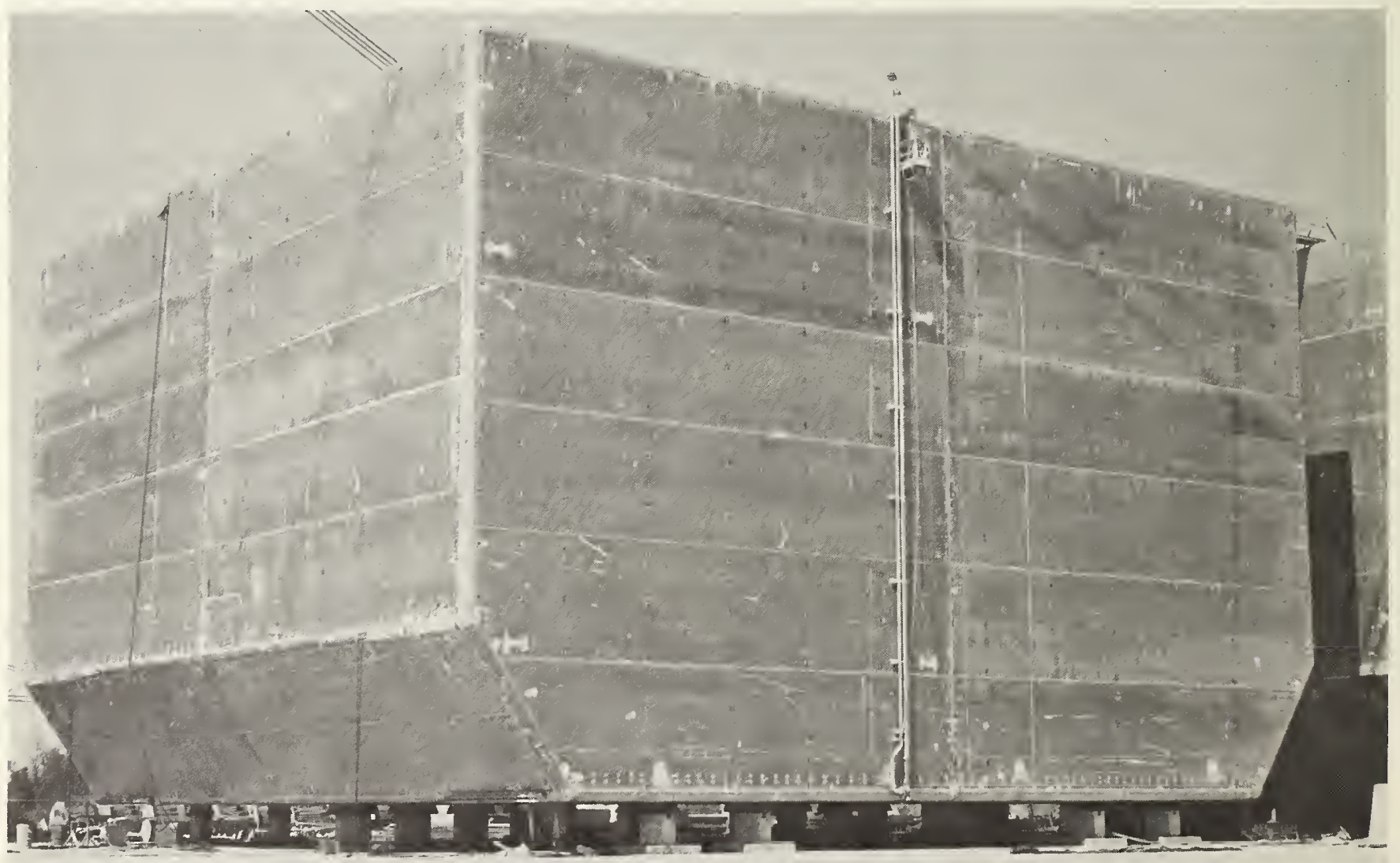

Figure 2.5.3.1. Exterior view of a completed tank waiting to be loaded aboard the ship. 


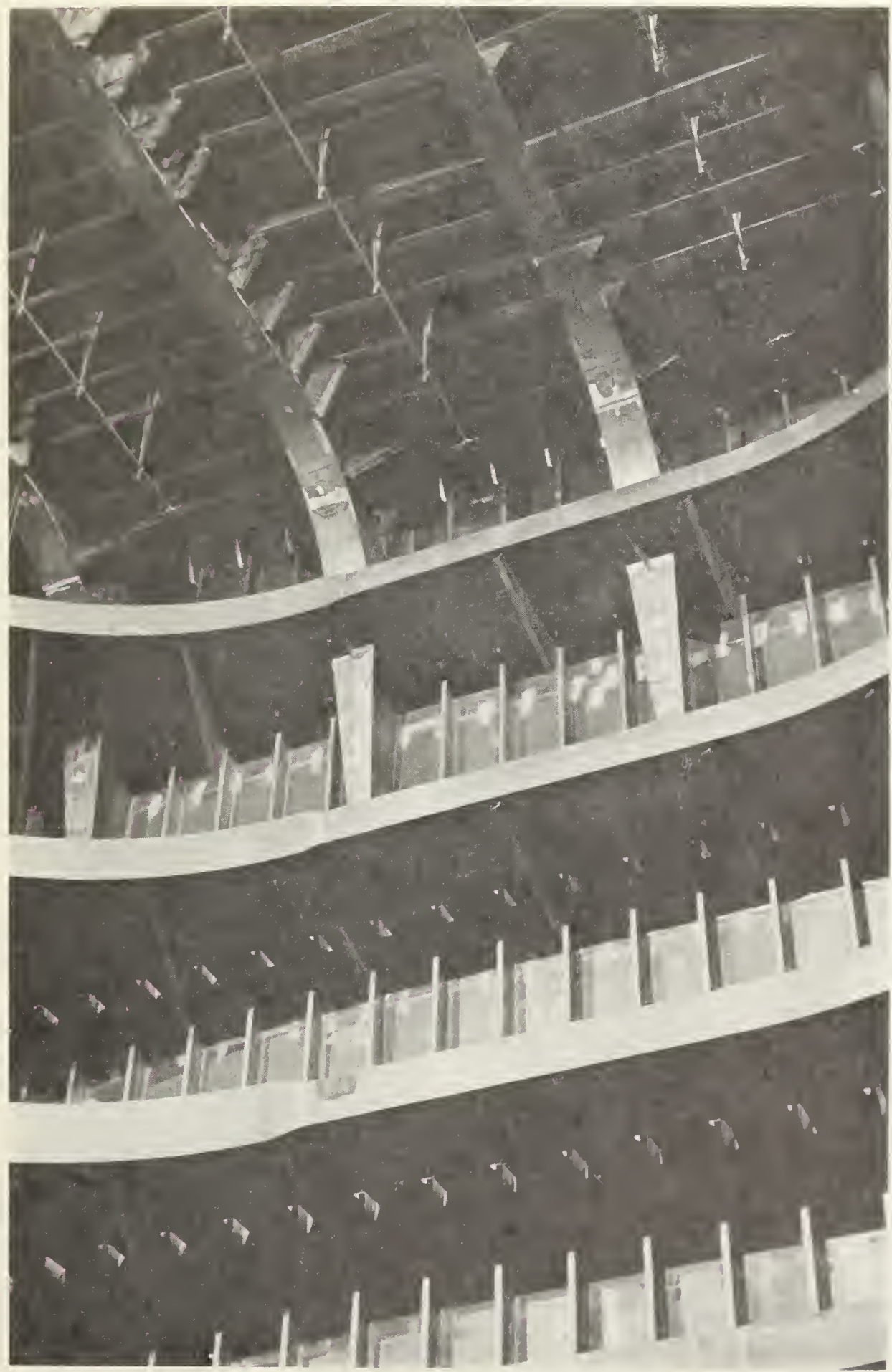

Figure 2.5.3.2. Interior view of tank showing ceiling and upper wall structure of tank. 


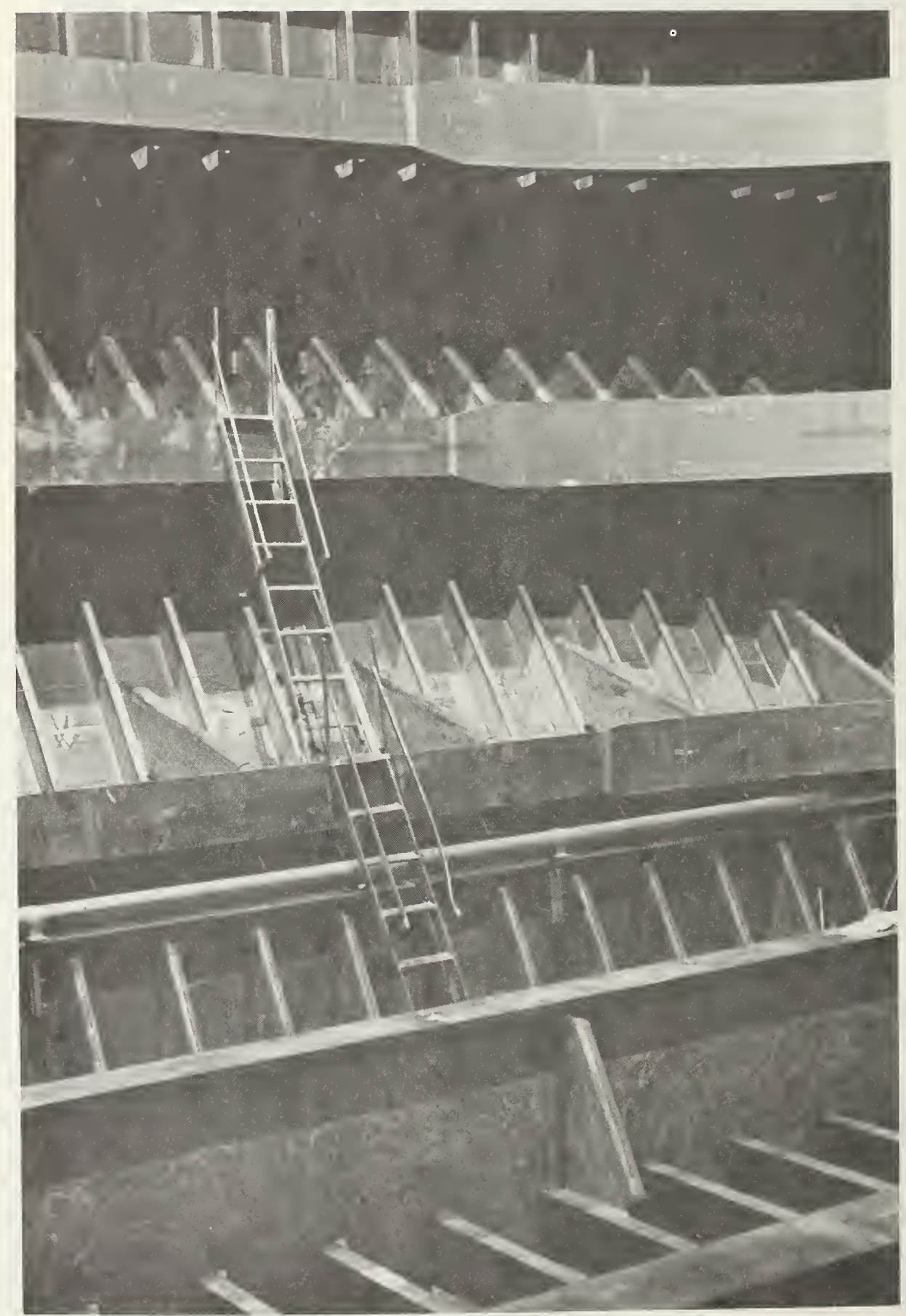

Figure 2.5.3.3. Interior view of tank showing floor and chine surfaces of tank. 


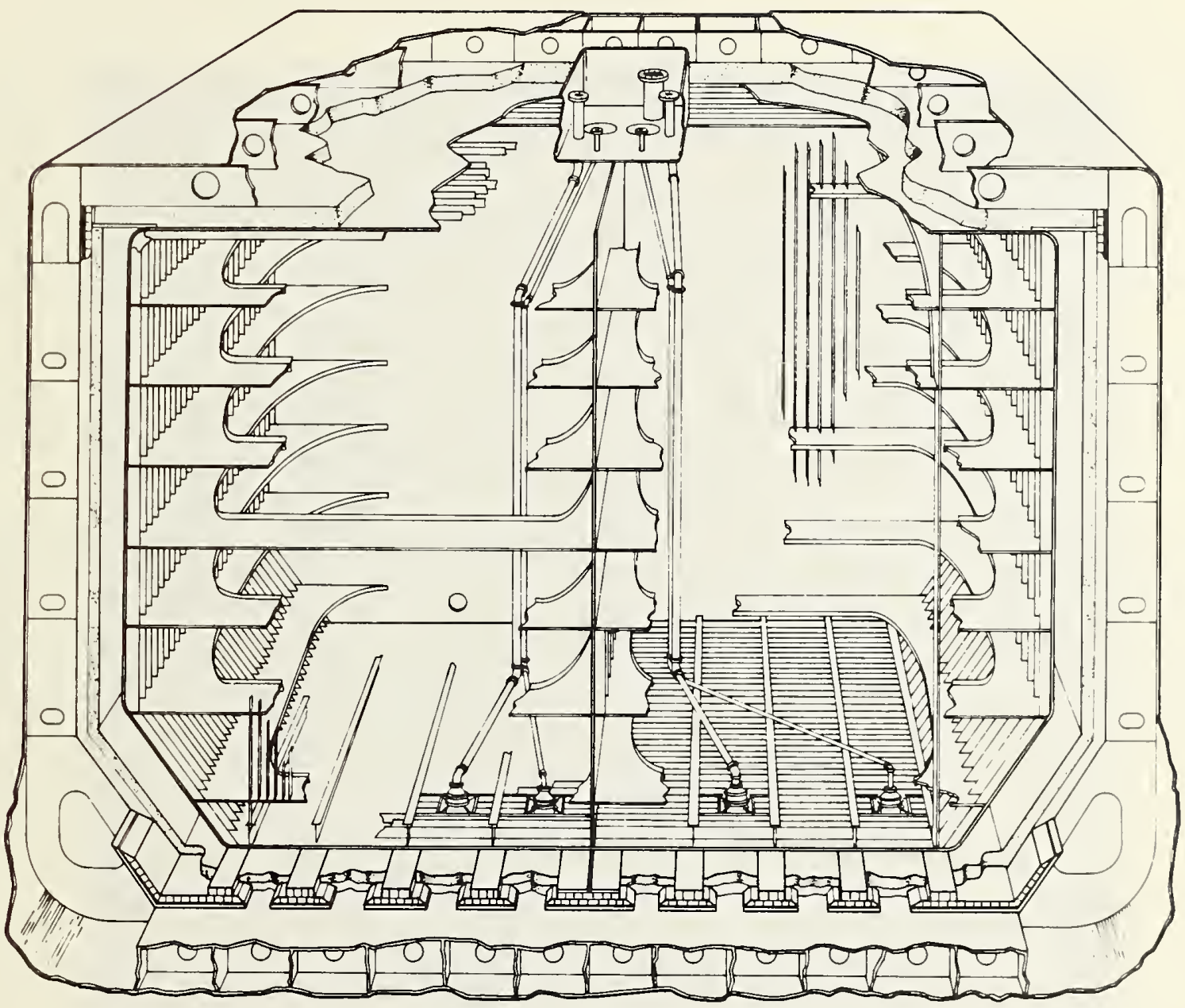

Figure 2.5.3.4. Cutaway drawing of two quadrants of a cargo tank show1ng the internal bracing configurations.

The tanks, after the assembly is complete or nearly complete, are transported from the assembly area to a hydrotest stand. The tanks are transported by four crawler tractors, one under each corner, lifting the tank by means of 1 ifting brackets. The tank is hydrotested by filling $1 \mathrm{t}$ a $11 \mathrm{ttle}$ more than half full with water. The tank is then pressurized with air to 0.14 bar gauge.

After hydrotesting, the tank is transported again by the crawlers to the storage area and placed on three rows of concrete pillars. The photogrammetric calibration usually takes place while the tanks are in this storage area. One tank of each ship set has been calibrated while on the hydrotest stand. Only the sides of the tanks are actually dimensioned photogrammetrically. The top and bottom of the tanks are profiled using conventional theodolite techniques. These surveys supply the top and bottom data used in the tank table calculation. This bottom survey is used to shim the surfaces of the support blocks on the ship located in the bottom of the tank socket so the tank $1 \mathrm{~s}$ unl formly supported. The tank and contents are supported completely on these blocks.

The ship is brought to the tank construction site to load the tanks. The tanks are moved up one at a time via the crawlers, then lifted, swung out over the ship and lowered 1nto the insulated tank socket by a $1.36 \times 10^{6} \mathrm{~kg}$ (1500 short tons) capacity crane. Figure 2.5 .3 .5 shows a tank being $11 \mathrm{rted}$ aboard the ship. The tank is connected to a horizontal lifting beam of the crane by a large number of cables supported by interconnected hydraulic cylinders so that the tank load 1 s equally supported on all 


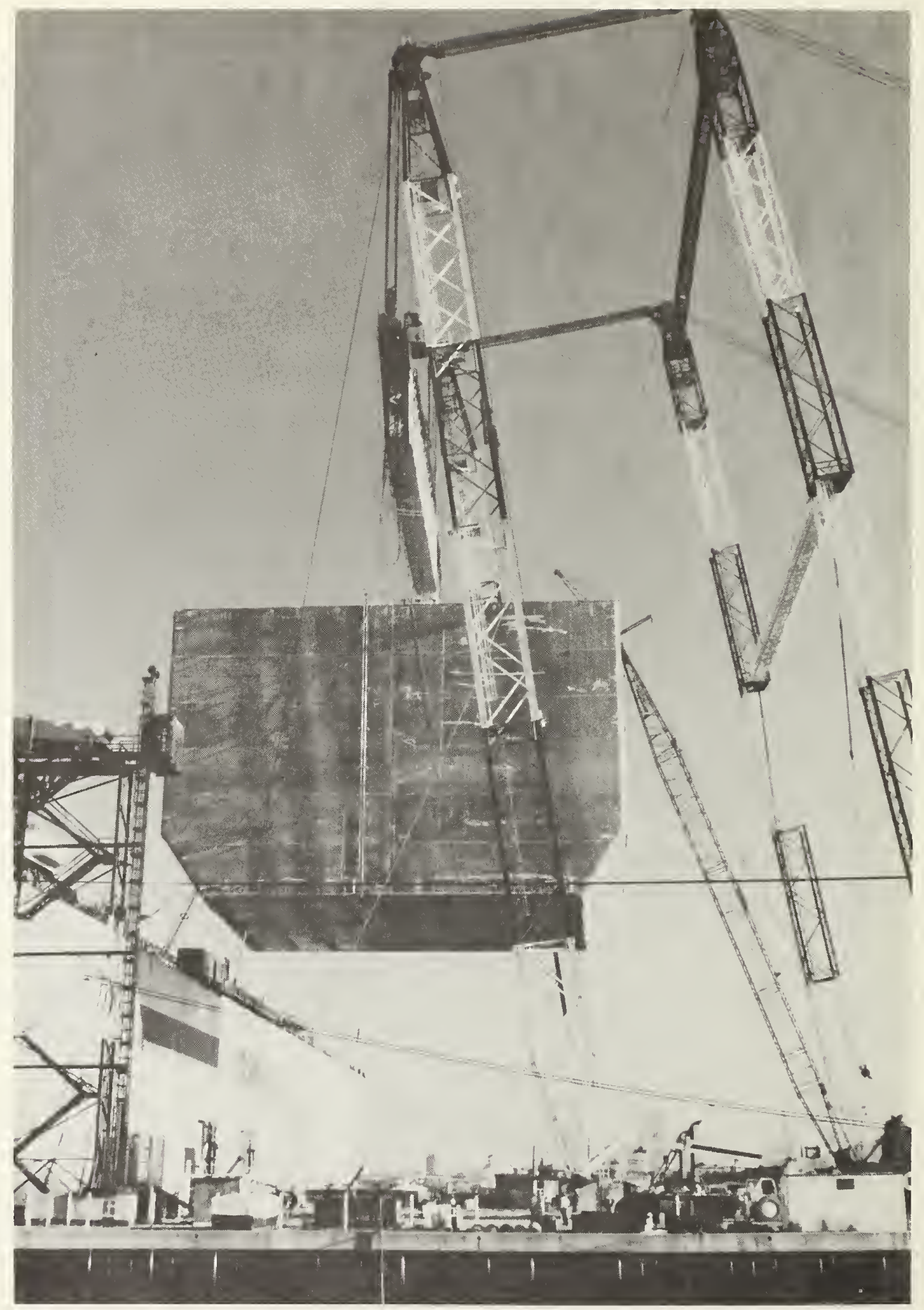

Figure 2.5.3.5. Tank \#5 being loaded on the Columbia. 
the cables. Insulation is placed over the top of the tank; then the deck section covering of the tank socket $1 \mathrm{~s}$ replaced. The walls of the tank are then accessible only from the inside of the tank.

\subsubsection{The Photogrammetric Calibration Method}

The photogrammetric process is employed to determine $x, y, z$ coordinates of a number of points distributed over the surface, in this case the outside surface, of the tank. These points are, physically, 19 millimeters diameter white dots placed on a black background or 19 millimeters diameter white balls placed on the tank prior to photography. The white dots and background are either painted on the tank wall or are printed on adhesive-backed paper and placed on the tank wall. The white balls are offset a known distance from the walls. The number and placement pattern are quite flexible and generally chosen to give sufficient detail of any wall variations with the minimum number of targets. The $x, y, z$ coordinates of the points on the tank can be in any arbitrary coordinate system. Figure 2.5.3.6 shows a schematic configuration of the photogrammetric method as used with the prismatic LNG tanks [9]. The number identifications indicate the individual camera positions used during the photographic portion of the procedure.

The coordinate system is later translated and rotated so that the $z$ axis is perpendicular to the gravitational plane when the tank is on the ship, and the $x$ axis is parallel to the center of the ship in the gravitational plane; hence, the y axis is transverse to the ship and in the gravitational plane.

The photogrammetric method of determining the coordinates of the tank surfaces is closely related to the method of using a theodolite to locate identifiable points on the surface. This is done with a theodolite by measuring vertical angles from the horizontal to the points on the surface and horizontal angles from each end of a baseline of known length. The axes of the theodolite determined coordinate system might be the horizontal baseline, the gravitational field direction and the axis perpendicular to both. One such theodolite set-up or station can be related to the next by repeating measurements of some of the targets located from the adjacent stations. The theodolite must be aimed twice at each point to be located, once from each end of the baseline, or one aimed from each end of the baseline if two theodolites are used.

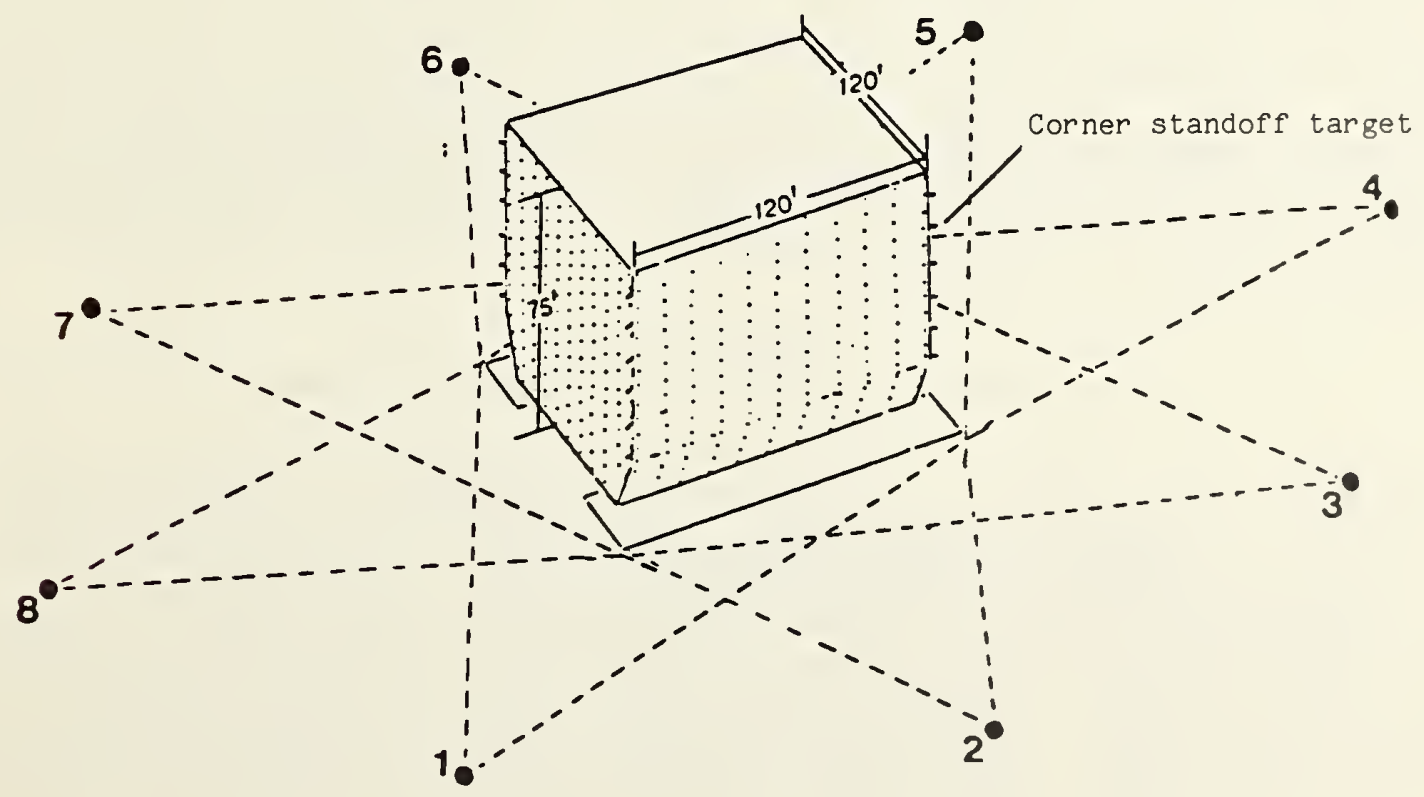

Figure 2.5.3.6. Typical geometric configuration employed ln the photogrammetric mensuration of prismatic LNG cargo tanks [9]. 
In the photogrammetric method, the eyepiece of the theodolite is replaced by a camera employing a very flat photographic plate. A photograph of the complete surface from each of the two ends of the baseline contains the same information as all the theodolite readings, since all the point images off the optical axis are recorded in each photograph. The angle data from a theodolite aimed at a point are contained in the position of the photographic image of the point. The $x, y$ coordinates of these photographic images can be measured to \pm 3 micrometers. Sufficient photographic image data are acquired so that not only $x, y, z$ coordinates of each target spot can be calculated but also the camera positions and orientations, a lens correction and an uncertainty for the coordinate position. In practice, the calibrated base line used with the theodolite can be replaced by a few known target spacings on the tank surfaces.

Although many of the details of the stereo-triangulation methods used by the photogrammetric consultants are proprietary, the general approach here is explained in references [2] and [3].

\subsubsection{Accuracy of the Photogrammetric Calibration Method}

The accuracy of calibration of the tanks has been independently estimated from a number of measurements made by NBS personnel. The various measurements and the results are described here in summary and in detail in appendices to the original document [8]. The tanks of the three ships Savannah, Cove Point and Columbia are examined.

1) The method used to verify the accuracy and precison of the photogrammetrically determined coordinates of designated points on the tank exterior walls is given and the results are presented for all fifteen tanks of the three ship sets.

2) Gaging tables, calculated at NBS using the photogrammetrically determined coordinate data, and gaging tables, calculated by the photogrammetrist from the same data, are compared in order to examine the computational method. The computational method and results for all fifteen tanks are also given in Siegwarth and LaBrecque [8].

3) The photogrammetric measurements were made on the tank under conditions assumed nearly isothermal and at ambient conditions. The tank volume can then be mathematically sized at the normal operating condition using the thermal expansion coefficient for 5083 Aluminum [7]. Possible errors introduced by lack of isothermal conditions, uncertainty of the expansion coefficient and incorrect determination of temperature are identified.

Tables $2.5 .3 .1,2.5 .3 .2$ and 2.5 .3 .3 give summaries of the estimated maximum errors of the volume determined by the photogrammetric method. The random errors are represented by 95 percent confidence limits for the random error source in question. While the errors due to volume calibration, scale factor, and target spacing are considered random, the thermal expansion coefficient error is systematic but unknown; the limits for this error are based on the bounds for the thermal expansion coefficient [6]. All errors are given in percent. In the tables a plus sign signifies an overestimate and a minus sign an underestimate.

Table 2.5.3.1

Error Summary (percent) for Savannah Tanks

\begin{tabular}{|c|c|c|c|c|c|c|c|}
\hline \multirow[b]{2}{*}{ Tank } & \multicolumn{4}{|c|}{ I-----Random Uncertainty ----- I } & \multirow{2}{*}{$\begin{array}{l}\text { Limits of } \\
\text { Systematic } \\
\text { Error for } \\
\text { Thermal } \\
\text { coef. }\end{array}$} & \multirow[b]{2}{*}{$\begin{array}{l}\text { Scale } \\
\text { bias }\end{array}$} & \multirow{2}{*}{$\begin{array}{c}\text { Scale Bias } \\
\pm \text { Systematic } \\
\text { and Random } \\
\text { error }\end{array}$} \\
\hline & $\begin{array}{c}\text { Volume } \\
\text { Calculation }\end{array}$ & $\begin{array}{l}\text { Target } \\
\text { spacing }\end{array}$ & $\begin{array}{l}\text { Scale } \\
\text { factor }\end{array}$ & $\begin{array}{l}\text { RMS } \\
\text { total }\end{array}$ & & & \\
\hline 1 & \pm 0.01 & \pm 0.014 & \pm 0.01 & \pm 0.020 & \pm 0.03 & -0.04 & $-0.04 \pm 0.05$ \\
\hline 2 & \pm 0.01 & \pm 0.014 & \pm 0.01 & \pm 0.020 & \pm 0.03 & -0.06 & $-0.06 \pm 0.05$ \\
\hline 3 & \pm 0.01 & 0.0 & \pm 0.01 & \pm 0.014 & \pm 0.03 & 0.00 & $0.00 \pm 0.044$ \\
\hline 4 & \pm 0.01 & 0.0 & \pm 0.01 & \pm 0.014 & \pm 0.03 & -0.03 & $-0.03 \pm 0.044$ \\
\hline 5 & \pm 0.01 & 0.0 & \pm 0.01 & \pm 0.014 & \pm 0.03 & -0.03 & $-0.03 \pm 0.044$ \\
\hline
\end{tabular}


Table 2.5.3.2

Error Summary (percent) for Cove Point Tanks

\begin{tabular}{|c|c|c|c|c|c|c|c|}
\hline \multirow[b]{2}{*}{ Tank } & \multicolumn{4}{|c|}{ I-------Random Uncertainty ------- I } & \multirow{2}{*}{\begin{tabular}{|l|} 
Limits of \\
Systematic \\
Error for \\
Thermal \\
coef.
\end{tabular}} & \multirow[b]{2}{*}{$\begin{array}{l}\text { Scale } \\
\text { bias }\end{array}$} & \multirow{2}{*}{$\begin{array}{c}\text { Scale Bias } \\
\pm \text { Systematic } \\
\text { and Random } \\
\text { error }\end{array}$} \\
\hline & $\begin{array}{c}\text { Volume } \\
\text { Calculation }\end{array}$ & $\begin{array}{l}\text { Target } \\
\text { spacing }\end{array}$ & $\begin{array}{l}\text { Scale } \\
\text { factor }\end{array}$ & $\begin{array}{l}\text { RMS } \\
\text { total }\end{array}$ & & & \\
\hline 1 & +0.01 & .014 & \pm 0.015 & \pm 0.023 & \pm 0.03 & 0.00 & $0.00 \pm 0.053$ \\
\hline 2 & \pm 0.01 & \pm 0.014 & \pm 0.009 & \pm 0.019 & \pm 0.03 & 0.00 & $0.00 \pm 0.049$ \\
\hline 3 & \pm 0.01 & +0.014 & \pm 0.005 & \pm 0.018 & \pm 0.03 & -0.02 & $-0.02 \pm 0.048$ \\
\hline 4 & \pm 0.01 & \pm 0.014 & \pm 0.011 & \pm 0.020 & \pm 0.03 & 0.01 & $0.01 \pm 0.050$ \\
\hline 5 & \pm 0.01 & \pm 0.014 & \pm 0.007 & \pm 0.019 & \pm 0.03 & 0.00 & $0.00 \pm 0.049$ \\
\hline
\end{tabular}

Table 2.5.3.3

Error Summary (percent) for Columbia Tanks

\begin{tabular}{|c|c|c|c|c|c|c|}
\hline \multirow[b]{2}{*}{ Tank } & \multicolumn{4}{|c|}{ I-------Random Uncertainty $------I$} & \multirow{2}{*}{$\begin{array}{l}\text { Limits of } \\
\text { Systematic } \\
\text { Error for } \\
\text { Thermal } \\
\text { coef. }\end{array}$} & \multirow{2}{*}{$\begin{array}{c}\text { Systematic } \\
\text { and Random } \\
\text { error }\end{array}$} \\
\hline & $\begin{array}{c}\text { Volume } \\
\text { Calculation }\end{array}$ & $\begin{array}{l}\text { Target } \\
\text { spacing }\end{array}$ & $\begin{array}{l}\text { Scale } \\
\text { factor }\end{array}$ & $\begin{array}{l}\text { RMS } \\
\text { total }\end{array}$ & & \\
\hline 1 & +0.01 & \pm 0.014 & \pm 0.025 & \pm 0.03 & \pm 0.03 & \pm 0.06 \\
\hline 2 & \pm 0.01 & \pm 0.014 & \pm 0.025 & \pm 0.03 & \pm 0.03 & \pm 0.06 \\
\hline 3 & \pm 0.01 & \pm 0.014 & \pm 0.025 & \pm 0.03 & \pm 0.03 & \pm 0.06 \\
\hline 4 & \pm 0.01 & \pm 0.014 & \pm 0.025 & \pm 0.03 & \pm 0.03 & \pm 0.06 \\
\hline 5 & \pm 0.01 & \pm 0.014 & \pm 0.025 & \pm 0.03 & \pm 0.03 & \pm 0.06 \\
\hline
\end{tabular}

The second column in the tables gives the estimate of the random uncertainty in the volume introduced by the computational method. The third column gives an estimate of the random uncertainty introduced when the number of identifiable points or targets on the tank used for the photogrammetric survey is reduced to half those used on tanks 3, 4, and 5 of the Savannah. The fourth column gives the random uncertainty associated with the magnitude of the scale factor required to convert coordinate number data to true lengths as determined from the NBS tape results. Column five gives the rms sum of these random errors.

A systematic error results from the uncertainty in the coefficient of thermal contraction for the aluminum tank cooled to operating temperature. The estimated limits of this error are given in column six. The seventh column of tables 2.5 .3 .1 and 2.5 .3 .2 give the systematic error in the tank volume resulting from systematic errors in the photogrammetric scale factor. Detectable scale factor systematic errors were observed only for some of the calibrations of the tanks of the Savannah and Cove Point. No scale factor systematic error was discernable for the tanks of the Columbia, so that column has been deleted. The scale factor systematic error can be eliminated by applying a multiplying factor to the gage table volumes.

The last column of the three tables shows a combined value of the uncertainty of the tank calibration for each tank. Some photogrammetrically determined numbers were used in this error estimate $1 \mathrm{n}$ combination with some independent measurements. A gaging table for tank 3 of the Columbla was calculated by NBS personnel using a completely independent measurement method. This method consisted of erecting a laser plane defined solid exterior to the tank wall, then measuring the offset from the laser-defined planes to the walls at a large number of points. The spatial relationship of the planes were measured using tapes and theodolites. The method was adapted from a tank volume measurement method used internally on membrane-type LNG tanks [5]. The results of the measurements of tank 3 or the Columbia are given in the original publication [8] Appendix D. These results agree w1th the photogrammetrically determined gaging tables and agree in total volume at 22.445 meters helght to 0.7 $\mathrm{m}^{3}$ or 0.003 percent. The largest difference is $3.0 \mathrm{~m}^{3}$ in the region of 15 meter helght.

\subsubsection{Accuracy of Tanks in Service}

The results of the last section are consistent with the tanks being calibrated within the accuricy claimed by the photogrammetrist. The tanks, however, are constructed and calibrated on shore and while empty. The procedures of loading the tanks aboard ship, or the hydrostatic loading, of the carfo 
could change the volume of the tank. Furthermore, the volumes discussed in the previous section were external, so that the volume of aluminum in the walls and internal framing must be subtracted from the external volume to obtain the actual liquid volume. This deadwood correction is 1.3 percent for the smallest tank (tank 1) and ranges down to 0.9 percent for the largest tank. The distortion of the tank caused by lifting it into the ship, the hydrostatic distortion and the deadwood have been studied by NBS personnel to determine what errors may be introduced into the gaging tables.

To test for lifting distortion and surface flatness, surveys as described in the original publication [8] Appendix E were performed on the top and bottom surfaces of tanks 4 and 5 of the Savannah. The tanks were surveyed in the storage yard and then immediately after being placed in the ship. The root mean square differences of the surveyed points from a fitted plane were calculated for each survey. The comparison showed that there was no significant change in the rms values of any of the before and after surveys; and, therefore, there could not be a significant change in tank volume as a result of tank moving process. Also, it was assumed that since the top and bottom of the tank, which would be most affected by the move, did not distort, then the walls did not distort either.

The hydrostatic pressure on the bottom of the tank when filled with LNG is about 1 bar. Since the tanks were hydrostatically tested by filling them half-full of water, it was possible for NBS personnel to measure hydrostatic deformation of the tank. Flatness measurements were made on the walls of tank 3 of the Columbia both with and without water in the tank. The results of these measurements are given in the original publication [8] Appendix F. The rms differences of the surveyed points from a fitted plane were calculated and compared for the survey done before the hydrostatic test and for the survey made during the test. As no significant differences were detected for the rms values, it was inferred that no significant volume change had occurred.

The tank volume comparisons are for the outside dimensions of the tank. To obtain the liquid volume, the volume of aluminum in the walls and internal structure must be calculated as a function of height. The deadwood volume at height $h$ must be subtracted from the external tank volume at height $h$ to get the liquid volume at this height. The photogrammetric contractor determined deadwood volume as a function of height from drawings of the tanks and the nominal dimensions of the various plates. In measurements made by NBS on tank 3 of the Columbia, it was found that the deadwood dimensions are generally slightly larger than nominal. For this tank, the larger dimensions decrease the total liquid volume by about $8 \mathrm{~m}^{3}$ or 0.03 percent. The actual measurements are reported in the original publication [8] Appendix G.

The survey effort and the results obtained for the tank bottom surveys of the 10 tanks of the first two ships are presented as Appendix $\mathrm{H}$ in the original publication [8]. The desired measurement accuracy was not achieved with these measurements, because the ship yard continued work during the measurements, which caused both constant movement and permanent changes of the list and trim. The flatness surveys of the four tank quadrants could not be satisfactorily correlated by the water tube measurements. The flatness surveys by quadrant did suggest, however, that little bottom distortion had resulted from placing the tanks. The motions of the ship during the surveys also precluded accurate measurement of the orientation of the tank with respect to the draft marks. The surveys of the third ship were not completed, because the gas trials of the Savannah showed the insulation system used was unsatisfactory for LNG service.

Because of the central location of the liquid level gages in these tanks, the volume error is not a strong function of list or trim. For example, a 5 centimeter list adds an error of $\pm 3 \mathrm{~m} 3$ to the volume of the largest tanks, the sign depending on whether the list is to starboard (positive) or to port. The attempt to measure the tank and gage orientations with respect to the draft marks was done when the ships were essentially complete. This measurement could be repeated anytime in the future when the ship tanks are brought up to air during the periodic inspections. This could best be done in a non-floating drydock. The measurements might also be done with the ship afloat in a quiet body of water when no other work is being done either on the ship or nearby.

Of the three sources of error considered in this section-lifting distortion, hydrostatic distortion, and errors in deadwood specification - only errors in the deadwood corrections are seen to be significant. For the lifting and hydrostatic distortions, no bounds have been calculated for how much change in volume could have occurred without having been detected by the methods of measurement used. The study by NBS of tank 3 of the Columbia showed the deadwood volume to be 2.9 percent 1 arger than the deadwood volume calculated from the tank's nominal dimensions. 
The uncertainty of this 2.9 percent value has been calculated to be appoximately \pm 0.2 percent. whlle 1 is not possible to predict what the error in the deadwood volume caused by oversized dimensions would be for the remaining fourteen tanks, the material irom which the other tanks were constructed is likely to be similar in sizing to the material for this tank, and it is not expected that this deadwood volume error would exceed 5 percent.

In conclusion, the NBS verification of the photogrametric method of determining the external tank volumes found the uncertainty of this method to be within \pm 0.1 percent of total volume. This includes the errors in the use of the temperature coefficient to calculate the volume at operating temperature and in the deadwood calculation, but not the errors in the bottom survey or the effects of liftlng, installing, and loading the tanks. The deadwood calculation error is expected to be within \pm 0.04 percent for the largest tank and within \pm 0.06 percent for the smallest. There exists no good estimate of the bottom survey error. There is no indication of any distortion due to hydrostatic loading or to moving the tanks, and no estimate of hom much volume change could have occurred without being detected by the techniques used in this study. 


\subsubsection{References}

[1] Jackson, R.H.F., R.S. Collier, S. Haber and R.V. Tryon, Custody transfer systems for LNG ships: tank survey techniques and sounding tables, Nat. Bur. Stand. (U.S.), NBSIR 79-1751(July 1, 1978).

[2] Brown, D.C., A solution to the general problem of multiple station analytical stereoangulation, Air Force Missile Test Center Report No. 58-8, Patrick AFB, Florida(1958).

[3] Kenefick, J.F., 1971. Ultra-precise analytics, Photogrammetric Engineering, Vol. 37, p. 1167.

[4] The members of the LNG Custody Transfer Measurements Supervisory Committee are Ivan W. Schmitt, Chairman, El Paso Marine Company; Bland Osborn, Columbia LNG Corporation; Howard S. Joiner, Consolidated Systems LNG Company; and Ed Crenshaw, Southern Energy Company.

[5] Hocken, R. J. and W. C. Haight, Multiple redundancy in the measurement of large structures, Annals of the International Institution of Production Engineering Research (CIRP), Volume 27, p. $1,(1978)$.

[6] Brown, D.C., Application of close-range photogrammetry to structures in orbit, Vol. 1 and 2, GSI Technical Report \#20-012. Available from Geodetic Services incorporated, 1511 South River View Drive, Melbourne, Florida 32901.

[7] Mann, D. B., Editor, LNG materials and fluids, Thermophysical Properties Division, National Bureau of Standards, Boulder, Colorado(1977).

[8] Siegwarth, J. D. and J. F. LaBrecque, Estimated uncertainty of calibrations of free standing prismatic liquefied natural gas cargo tanks, Nat. Bur. Stand. (U. S.), 294 pp (January 1982).

[9] Brown, D. C., LNG tank inventory measurement by photogrammetry, 1981 operating Section Proceedings, 81-T-55, ppT-127-37, Am. Gas Assoc., 1515 Wilson Blvd., Arlington, VA 22209 (1981). 


\subsubsection{Volume of Spherical LNG Ship Tanks}

\subsubsection{Introduction}

The 1 lquefled natural gas (LNG) program of the National Bureau of Standards (NBS) was designed primarlly to supply property data for the materials and fluids of interest and to aid in the establishment of measurement methods and instrumentation for custody transfer of this fluid in commerce. This section of the manual concerns work, supported in part by the Maritime Administration (MarAd) of the U.S. Department of Commerce, in the area of custody transfer: specifically, static measurements of the quantity of fluid contained in a storage tank before and after delivery, either into or out of the tank.

The analysis reported herein was performed by Jackson, et al [3] on spherical LNG tanks used in the LNG ship Aquarius. This edited account lacks some of the detailed mathematic and graphic development of the original publication but is designed to demonstrate the methods used.

The photogrammetric technique described in section 2.5 .4 .2 was a relatively new method, and MarAd requested the NBS to provide a third party review of the technique and verify where possible the conclusions. Subsequently, additional LNG ships were constructed by the same shipbuilder, and NBS was sponsored by the ship owners to conduct an independent measurement of several of the new ship tanks and to compare the resulting measurements with those found by the photogrammetric method, which was the method of choice by the ship owner. Section 2.5.4.5 of of the manual covers the NBS technique [4] and Section 2.5.4.6 the comparison with the photogrammetric method [6].

Previous methods for calibrating such tanks and calculating "list and trim" tables relied heavily on measurements taken with tapes, referred to as "strapping" [5]. Ship owners, and therefore ship builders, have a specific interest in these measurements and the resulting tables of volume versus height, since these tables are used by a ship's captain to determine the amount of cargo at any particular time. The resultant estimates of volume added or removed are then mathematically combined with fluid density measurements and calculations to determine mass transferred. Gas sampling and analysis provide average BTU content, and the combination of mass transferred and BTU content yields total heating value of the cargo.

In essence, "strapping" is a geometric survey of the inside of the ship tank, relating measured points on planes and surfaces to fixed reference points. The balance of the work concerns the mathematical treatment of the measured data to construct an accurate model of the tank geometry. (Error estimates can be made by relating the model to the measured and reference points.) The volume-height relationships are then derived from the mathematical model.

It should be pointed out that NBS had no specific contract authority to gather and analyze data. Only the extensive cooperation of the Maritime Administration, the ship builder, the ship owner, and the photogrammetric contractors made it possible to complete and publish this work.

\subsubsection{Photogrammetric Survey}

Rectangular and spherical tanks are usually dimensioned by conventional strapping techniques; sufficient lengths and circumferences are measured by taping so that the shape of the tank can be determined and used to calculate the volume as a function of height. Since the spherical tanks do not lend themselves well to conventional taping, a photogrammetric technique to measure tank volume has been developed (by a commercial firm).

Photogrammetric Surveying The wide-angle analytic photogrammetric technique used to determine the dimensions of the tanks offers potentially higher accuracy than strapping. The claimed preclsion for this technique approaches 1 part in 100,000 , or approximately \pm 0.4 millimeter for a 35 meter diameter tank.

The photogrammetric method, described in detail in [1], consists of determining the $x$, y, and $z$ coordinate values for targets on the inner tank surfaces by photographing the targets from nine different positions within a tank. Targets are required on the tank so that points on the surface can be identifiable from photograph to photograph (see figures 2.5.4.1 and 2.5.4.2 for tank target distributions). The 408 targets used in these tanks are 2.2 centimeters diameter white spots surrounded by a black background and are spray-painted on during construction of the tank. Special photographic plates consisting of thin emulsions of uniform thickness on very flat glass plates are used for the photography. 


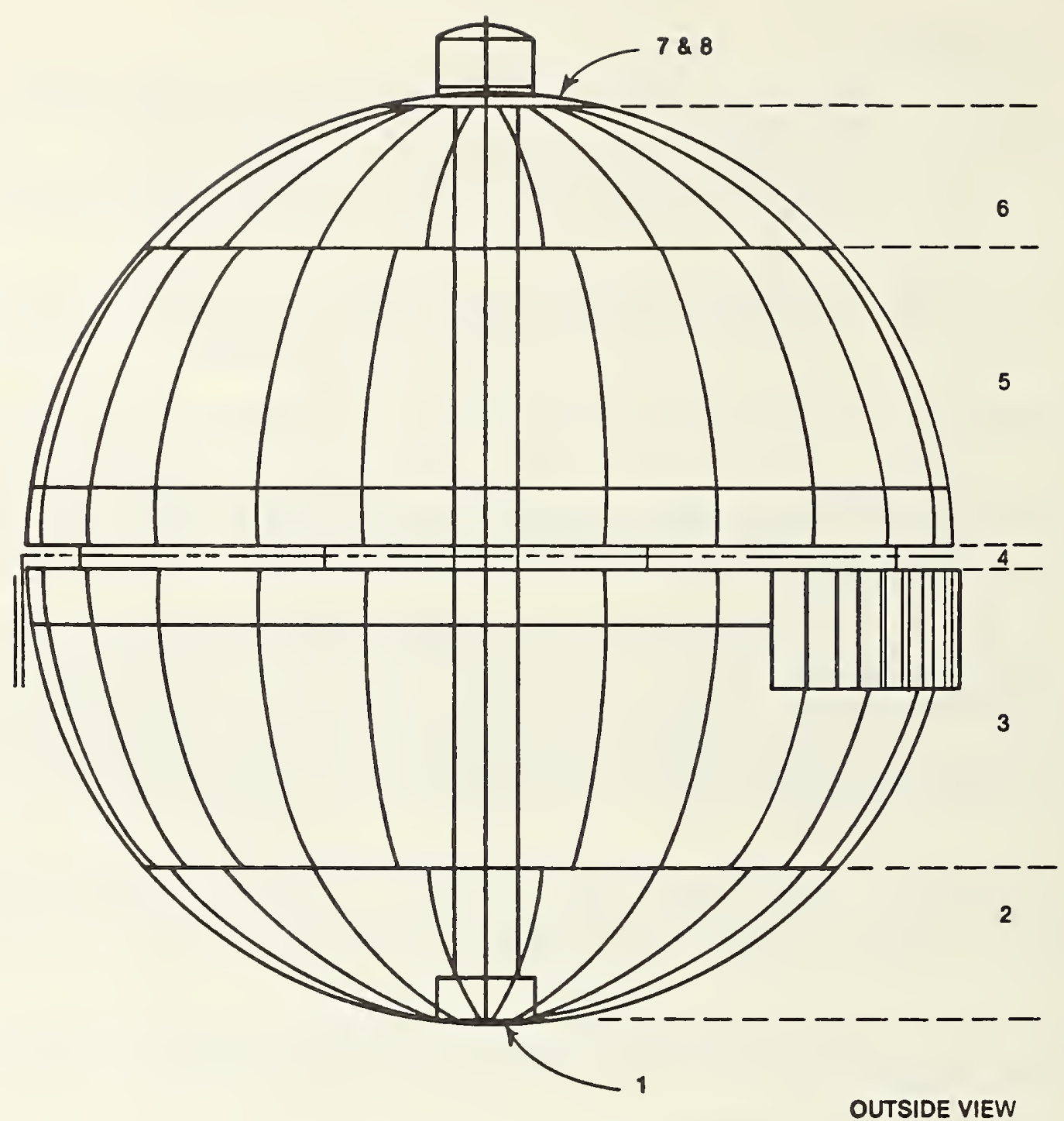

Figure 2.5.4.1. Tank schematic.

The two-dimensional positions of the target images on the plates are measured to within 3 micrometers with a monocomparator. These values are related to the $x, y$, and $z$ coordinates through a large set of mathematical equations that are solved by computer. The unknowns calculated are the $x$, $y$, and $z$ coordinates, the directions of the principal axis of the camera, the coefficients of the lens distortion correction equation, and the uncertainties for the coordinate values. Details of the technique, however, are proprietary. The resulting coordinates have an arbitrary scale; hence, calibration lengths must be known to correct to coordinates in real space. This information is provided by targets placed at known spacings on calibration tapes visible in the photographs. The targets should be photographed when the tank is isothermal, either after dark or after the tank has been insulated. 

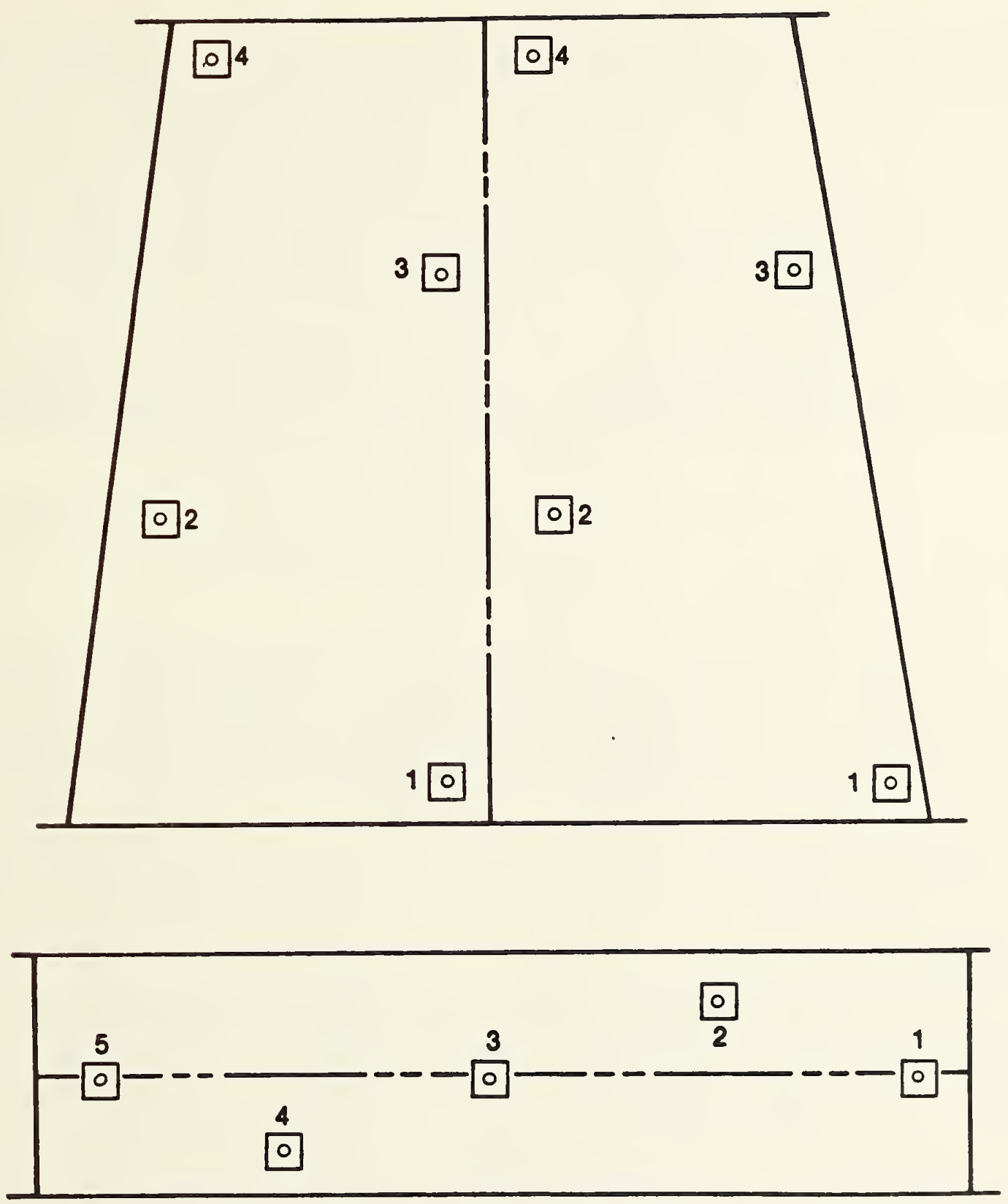

Figure 2.5.4.2. Distribution and numbering scheme for targets on inner plate and equatorial ring of spherical tank.

The Tank Model Construction of a mathematical model of the tank is essential to the calculation of tank capacity tables. The model is a mathematical description of the tank surface fitted to the target coordinates; its accuracy depends not only on the accuracy of the target coordinates but also on the density of targets on the tank surface and the regularity of the tank surface between target locations. It is clearly desirable to have as many targets as possible to reduce the uncertainty in interpolating between targets. However, there is a practical limit to the number of targets that can be measured because of the time and cost in reducing the data. With one exception (discussed 1n the next section), NBS had no control over the number or placement of targets painted on the tank surface.

Model construction usually begins with the calculation of some regular solid, e.g., a perfect sphere. prism, etc, which best fits the target coordinate data developed from the survey. Differences between actual target coordinates and the coordinates of the closest point on the surface of the model solld are then calculated. These "residuals" are then studied for patterns that would ind 1 cate systemat $1 \mathrm{c}$ distortions (bulges, bends, or twists) in the shape of the tank. 
On the basis of patterns discovered during this exploratory residual analysis, a special-purpose technique is developed to integrate numerically the composite tank model, which specifies a regular solid along with a table of deviations or residuals.

The final step in this analysis is the calculation of sounding tables giving tank volumes at regular intervals of liquid height in the tank. Error bounds on these tank values can then be developed.

Tank Table Modifications There are a number of modifications to the sounding tables that must be made before their construction is completed. List, trim, thermal contraction, and stress effects must be accounted for. List and trim corrections, however, are not addressed here, as NBS did not have access to the data or the procedures used in making these corrections.

It is standard practice to correct tank tables for thermal contraction by applying contraction coefficients as if the tank were mechanically unconstrained. The dimensions of the tank are decreased uniformly according to the thermal contraction of the material. Actually, tank mounting constraints and variations in the thermal expansions of the structural material can introduce strains in the tank that might affect cargo capacity. These thermal stress effects cannot presently be analyzed, because data on constraint effects and anisotropic thermal coefficients are lacking.

Another effect is the cargo load, which stresses both the tank and the ship hull. An analysis of the effects of these strains on the tank tables is beyond the scope of this work. Furthermore, the photogrammetric contractors do not include such corrections in their report.

\subsubsection{Analysis of Spherical Ship Tank}

As part of the Maritime Administration Custody Transfer Program mentioned earlier, NBS was asked to evaluate the methodology used to calibrate the spherical tanks. In addition, NBS was asked to comment on the accuracy of the sounding tables subsequently constructed. In this section, the NBS analysis is presented and described as to how it differs from the analysis performed by the photogrammetric consultants. Included also is a discussion of the accuracy of the sounding tables constructed and an assessment of the limitations, additional to those implicit in the last paragraph, to the applicability of those tables.

Each tank calibrated is spherical, with a diameter of approximately 36.5 meters and a capacity (volume) of 25,000 cubic meters. Constructed of four bands ("strakes") of aluminum plates in the shape of spherical trapezoids (see figure 2.5.4.1), with special cap plates at the top and bottom. The only internal structure is a cylindrical tower extending from cap, to cap containing piping, wiring, depth gauges, etc.

The first step in constructing the tanks discussed in this section is to weld together the equatorial ring by which the tank is supported. The ring is set up on jigs, and the tank plates making up the central strakes (previously welded into pairs) are welded in place above and below the equatorial ring. The top and bottom strakes are then welded in place. The bottom and top circular plates are welded in after the rest of the welding is completed, and the internal staging is removed.

After a tank is completed, it is loaded on a crawler, supported from beneath, and moved to the hydrostatic test stand. After the test, the tank is moved to the insulation building and insulated. Then it is moved to a barge for transporting to the shipyard, where it is lifted into place on the ship.

The procedure for constructing sounding tables consists of:

a) photogrammetrically surveying target coordinates on the inside surface of the tank;

b) constructing a mathematical model of the tank geometry;

c) developing a mathematical technique for computing volumes below specified heights in the tank; and

d) determining effects on tank shape (and therefore tank volume) of tank loading, cooling, liquid loading, sagging, sloshing, etc.

Each stage of calculation has associated with it an error bound on the accuracy of the result. These error bounds can be calculated using the appropriate statistical analysis; this can always be done and is straightforward. Difficulties arise only in regard to the "tightness" of those bounds, i.e., their balance between realism and conservatism. These error bounds will be discussed later in this section. 
The following is a result of an exploratory statistical analysis of the target coordinate data for Aquarlus tank number 4, obtained from the photogrammetric consultants. A best-fitting sphere is obtalned by using linear least squares to minimize the sum of the squares of the radial residuals. Analysis of these residuals then provides information on how the tank wall deviates from a true sphere, information of value to the numerical integration procedure described later.

In fitting a sphere, if the usual concept of a distance measured $d=\left(x^{2}+y^{2}+z^{2}\right)^{1 / 2}$ is used, the distance between a point and the surface of a sphere is a nonlinear function of the unknown parameters of the sphere. This complexity results in a nonlinear least squares problem for finding the best-fitting sphere. Witzgall [2] has developed a technique that avoids this complexity and reduces the problem to a linear one, allowing the use of linear least squares. This approach, is described as well as methods to remove its bias. The formulation is for an arbitrary n-dimensional space and thus applies equally well to circles in 2-space, spheres in 3-space, and hyperspheres in $n$-space ( $R^{n}$ ).

The detalled method used in calculating the best-fitting sphere is given in the original publication [3] and will not be repeated here. However, using the technique described, the radius of the best-fitting sphere for Aquarius tank number 4 was determined to be:

$r=18.2682$ meters,

with a standard error of

$S E=0.0071$ meters.

With respect to the coordinate system defined by the consultants, the coordinates of the center are:

$$
\begin{array}{ll}
a_{1}=x=.0005 \text { meters } & S E=.0013 \text { meters; } \\
a_{2}=y=-.0009 \text { meters } & S E=.0013 \text { meters; } \\
a_{3}=z=-.0137 \text { meters } & S E=.0011 \text { meters. }
\end{array}
$$

The standard errors given above are computed, using the usual least squares theory that assumes the radial residuals from a perfect sphere are random. As shown below, this assumption is incorrect. The purpose of the best-fitting sphere, however, is only to serve as a reference surface for further analysis.

Analysis of Residuals With the calculation of the best-fitting sphere, it is now possible to perform the exploratory analysis of residuals that provides specific information on tank shape. These "residuals" are the differences between actual target coordinates and the coordinates of the closest point on the surface of the model or best fitting sphere. A thorough residual analysis, such as was performed for the original publication [3], typically includes many types of graphical displays. In the interest of brevity, only a descriptive summary of the analyis will be presented here. If additional detail is required, the original publication should be consulted.

The targets are arranged in 21 rows around the sphere: 1 row on each of the top and bottom polar plates; 3 rows on the equatorial ring; and 4 rows on each of the four strakes. On each plate there are 4 targets arranged and numbered as indicated in figure 2.5.4.2.

The analysis of the residuals shows the nature of the tank distortion. The bottom polar plate and the bottom edge of the 非 1 strake are flattened (as if the tank were free-standing on a flat surface) and lie internal to the model sphere by as much as 6 centimeters. The 非 strake must have a "sharp" bend near the second row of targets, because the residuals for the upper three rows of targets on the 1 strake and all points on the 非 2 strake average 0.8 centimeter outside the sphere.

Table 2.5.4.1 summarizes the residuals by row. The term "Degrees of Freedom" used in the table $1 \mathrm{~s}$ equal to one less than the number of targets in a row. The most interesting fact from a study of this table is that in some regions the residuals are following some major distortion of the tank and are thus not random. In other areas (at joints between strakes), the residuals appear random bit have standard deviations similar to those in the nonrandom regions. In particular, the reglons of randomness are adjacent to the seams where 42 plates are joined. However, in only one case (bottco polar plate) do the "non-random" residuals appear to be such that the values could be approximated by a simple surface or polynomial. In fact the first strake, row 1 residuals can be 1 tted well by a sine curve, though not nearly as well as those for the bottom polar plate. 
Table 2.5.4.1

Summary of Target Residuals by Row

\begin{tabular}{|c|c|c|c|c|}
\hline Row Number & Average $(\mathrm{cm})$ & $\begin{array}{c}\text { Standard } \\
\text { Deviation }(\mathrm{cm})\end{array}$ & $\begin{array}{l}\text { Degrees of } \\
\text { Freedom }\end{array}$ & Random \\
\hline 1 Bottom plate & -5.75 & 0.70 & 7 & No \\
\hline 2 & -2.67 & 0.76 & 15 & No \\
\hline 3 First Strake & 0.72 & 0.31 & 17 & No \\
\hline 4 & 0.54 & 0.33 & 17 & No \\
\hline 5 & 0.66 & 0.53 & 17 & Yes \\
\hline 6 & 0.81 & 0.84 & 25 & Yes \\
\hline 7 Second Strake & 0.97 & 0.38 & 23 & No \\
\hline 8 & 0.72 & 0.70 & 23 & No \\
\hline 9 & 1.11 & 0.66 & 26 & No \\
\hline 10 & 0.14 & 1.05 & 8 & No \\
\hline 11 Equatorial & -1.45 & 0.90 & 26 & No \\
\hline 12 Ring & 0.06 & 0.82 & 8 & No \\
\hline 13 & 1.03 & 0.73 & 27 & No \\
\hline 14 Third Strake & 0.30 & 0.49 & 23 & No \\
\hline 15 & 0.33 & 0.38 & 23 & Yes \\
\hline 16 & 0.28 & 1.20 & 23 & Yes \\
\hline 17 & -0.62 & 0.89 & 21 & Yes \\
\hline 18 Fourth Strake & -0.94 & 0.70 & 17 & Yes \\
\hline 19 & 0.32 & 0.42 & 17 & No \\
\hline 20 & -0.43 & 0.69 & 17 & No \\
\hline 21 & -1.98 & 0.77 & 7 & No \\
\hline \multicolumn{2}{|c|}{ Pooled Standard Deviation } & 0.71 & 387 & \\
\hline
\end{tabular}

Target Measurement Errors Errors in the volume tables result in part from random and systematic errors in the target coordinate measurements. It is possible to next analyze the experimental evidence available in order to place bounds on the measurement errors. Later, the effects of the errors on the volume tables can be considered.

Two types of error are of concern: random and systematic (sometimes called bias). Systematic errors, e.g., scaling error, are the types that result in non-zero values for certain average errors: specifically, average error in target location and average error in length of a gauge rod. Random errors, e.g., photographic plate resolution error, cause errors in individual target coordinates that vary about the average error.

Systematic error thus refers to an average error for a given survey. Causes of systematic error may be the same for many surveys; for example, errors in photo equipment, computer programs, or survey techniques. On the other hand, the value of the systematic error in each survey may vary randomly from one survey to the next caused, for example, by errors in setting the scale. Since only one survey is available, it is not possible to study this effect; the limits to be set on systematic error apply to this survey alone and cannot be extended to other surveys.

The lack of a second independent survey also limits the analysis of random error, but there are two approaches. First, the residual standard deviation from a fitted model provides an upper bound to the measurement error standard deviation. However, it may not be a "sharp" bound because it includes the effects of model error (in this case, actual deviations in the tank surface from the circular cross section assumed by the model). The second approach involves using the internal estimates of the standard error of the estimated coordinates derived from the estimation algorithm. There may, however, be sources of random error that are not included in these internal estimates.

No definitive analysis is possible without two or more independent surveys. Random fluctuations caused by model error will be common to all surveys and could perhaps be separated from the measurement random error by variance-component analysis methods. 
Random Error An upper bound to the standard deviation of the random errors of the photogrammetric survey in the radial direction can be obtained from table 2.5.4.1. The standard deviation given for each horizontal row of targets is computed from the deviations of individual residuals from the average for that row. It is thus the standard deviation from the best-fitting circle at each height on the sphere. Each standard deviation includes both the target measurement error and the local deviations of the sphere from a circle at each level. These two error components cannot be separated without analyzing repeated independent surveys.

The standard deviations for each row appear to be reasonably consistent from top to bottom of the tank. The pooled value (rms weighted by the number of degrees of freedom) is $7.1 \mathrm{millimeters.} \mathrm{The}$ value of 7 millimeters will be used as the upper bound to the standard deviation of the target coordinates in the radial direction.

Systematic Error As a check for systematic error in the survey, seven gauge rods with targets at each end were placed in the tank during the survey. The rods were constructed from two-inch square 5093 aluminum tubing in two pieces, joined by a flange in the center.

Rods 1-5 were placed around the bottom of the tank, while rods 6 and 7 were placed near the top. The $x, y$, and $z$ coordinates locating the ends of each rod are given in table 2.5 .4 .2 . All rods were placed with the targets against the tank surface, so that length is in the tangential direction. The exact rod orientations can be determined from the above coordinates.

Table 2.5 .4 .2

Rod End-Point Coordinates (meters)

\begin{tabular}{|c|c|c|c|}
\hline Rod & $X$ & $Y$ & $Z$ \\
\hline \hline 1 & 2.1885 & -8.9500 & -15.7102 \\
& 1.3089 & -5.1156 & -17.4291 \\
2 & 4.6285 & -6.3103 & -16.4456 \\
3 & 7.1239 & -2.8493 & -16.5168 \\
& 7.5256 & 0.7120 & -16.5691 \\
4 & 5.8784 & 4.6593 & -16.5997 \\
& 3.0506 & 7.0377 & -16.5191 \\
5 & -1.1255 & 7.3089 & -16.6504 \\
& -4.0084 & 5.7746 & -16.8055 \\
6 & -6.6706 & 2.4913 & -16.7619 \\
& 0.9479 & -2.4609 & 17.8288 \\
7 & -2.6246 & -0.1752 & 17.8466 \\
& 2.6472 & 0.0806 & 17.8384 \\
& -0.8601 & 2.4881 & 17.8441 \\
\hline
\end{tabular}

The first six rods were measured by NBS-Washington (D.C.). Each rod was broken down, reassembled, and measured three times. The average length for each rod, corrected to $68^{\circ} \mathrm{F}$, is given in table 2.5 .4 .3$. The pooled standard deviation from the reassemblies of the rods was 0.024 millimeters ( 12 degrees of freedom), and a 95 percent tolerance interval for 95 percent of future reassembled lengths is 0.074 millimeter. It follows that the random error in the rod lengths (caused by reassembly, primarily) is a negligible component of the standard deviation of the differences between NBS measurements and the photo survey $(s=0.85 \mathrm{millimeter})$. The limit to systematic error in the NBS measurements $1 \mathrm{~s} \pm 0.001$ millimeter, which is a negligible fraction of the systematic difference observed.

Rod 7 is a special case, since it was measured only at NBS-Boulder (CO). The NBS-Boulder group also measured all 6 other rods. A bias between NBS-Boulder and NBS-Washington (caused in part by the measurement of the halves separately?) of 0.452 millimeter ( $S E=0.086$ millimeter) was observed. The length given for rod 7 is the NBS-Boulder length minus the estimated bias.

The lengths obtained by the photo survey and their differences from the NBS values are also given In table 2.5.4.3. The photo survey lengths have been adjusted from those supplied by the contractorg. The contractors originally corrected the lengths to $68^{\circ} \mathrm{F}$, assuming a common starting temperature. but NBS temperature measurements showed that the rods were actually at different temperatures (shown $1 \mathrm{n}$ table 2.5.4.3) when measured. The differences between the NBS and photo survey results or 1 . 1 nally showed a systematic error, which was removed by correcting the lengths for the actual temperature hi measurement. 
Table $2 \cdot 5 \cdot 4 \cdot 3$

Gage Rod Dimensional and Temperature Data

\begin{tabular}{|c|c|c|c|c|}
\hline $\begin{array}{l}\text { Rod } \\
\text { Number }\end{array}$ & $\begin{array}{c}\text { NBS Measurement } \\
\text { At } 68^{\circ} \mathrm{F}(\mathrm{m})\end{array}$ & $\begin{array}{l}\text { Consultants' } \\
\text { Measurement } \\
\text { Converted to } \\
68^{\circ} \mathrm{F}(\mathrm{m})\end{array}$ & $\begin{array}{c}\text { Consultant } \\
\text { NBS } \\
\text { Difference } \\
(\mathrm{mm})\end{array}$ & $\begin{array}{c}\text { Avg. Temp } \\
\text { During } \\
\text { Measurement } \\
\left({ }^{\circ} \mathrm{F}\right)\end{array}$ \\
\hline \hline 1 & 4.2927 & 4.2937 & 1.0 & 34.6 \\
2 & 4.2664 & 4.2680 & 1.6 & 35.0 \\
3 & 4.2783 & 4.2779 & -0.4 & 33.5 \\
4 & 4.1881 & 4.1876 & -0.5 & 34.2 \\
5 & 4.2278 & 4.2277 & -0.1 & 36.0 \\
6 & 4.2405 & 4.2416 & 1.1 & 38.0 \\
7 & 4.2534 & 4.2545 & 1.1 & 37.8 \\
\hline
\end{tabular}

The length differences range from -0.5 millimeter to +1.6 millimeters with an average of 0.54 millimeter $(\mathrm{SE}=0.32$ millimeter). A 99 percent confidence interval for bias in the rod lengths is thus $0.54 \pm 1.19$ millimeters, or the interval $(-0.65,1.73)$ millimeters.

This bound, however, applies only to the tangential direction. It is assumed that the appropriate factor for degrading this to the radial direction is the same factor 3 observed for between-measurement standard error in the radial and tangential directions. This leads to bounds on the bias of $(-2.0,5.2)$ millimeters in the radial direction.

While every effort has been made in the above analysis to make the bound on possible bias a conservative one, there are several factors which could cause it to be an underestimate.

1) Most of the rods were at the bottom of the tank where measurement errors (and perhaps bias) are smallest.

2) The rods were very short. Targets close together may have highly correlated measurement errors which cancel in the length computation. In addition, there may be other errors that accumulate in length measurement when the targets involved span several photographic plates.

3) The factors that resulted in a bias of 0.54 millimeter on this survey could vary randomly between independent surveys and have a standard deviation much larger than the standard deviation of the errors within a single survey.

The above bound on the bias applies uniquely to this survey and does not apply to the surveys of any other tanks.

These points suggest several concepts to consider in any future efforts to evaluate the precision and accuracy of photogrammetric surveys.

1) An additional survey, independent of the first, is essential.

2) Gauge rods should be distributed more uniformly throughout the tank.

3) Some longer rods should be used.

4) Some rods should be placed perpendicular to the tank surface, so that measurement errors in the radial direction can be evaluated directly.

5) The covariances of the coordinate estimates are a necessary part of the analysis and should be supplied by the contractor.

\subsubsection{Calculation of Sounding Tables}

The sounding tables constructed by Jackson, et al [3] are tables of volume versus elevation for the unloaded tank. The use of the term "elevation" requires comment. The term is intended to represent the depth of the LNG in the tank at a particular moment. That is, assuming that the tank is in its standard position (it has the same orientation with respect to the true vertical direction as it had 
while being measured), and assuming that the liquid surface is a horizontal plane, the elevation should be the distance from that horizontal plane to the (inside) bottom of the tank. However, that last point is apparently not accessible to measurement. It has been assumed that what is, in fact, measured is the distance from the liquid surface plane to an internal reference point called the pedestal probe point, and the elevation is then determined by adding to that measured distance the known vertical distance of the reference point above the bottom of the tank.

The elevation of the pedestal probe point, however, is not obtained through direct measurement. The photogrammetric consultants estimated the location of the tank bottom by using a mathematical model. After determining a "sphere of best fit", they determined a second, concentric sphere that best fit the bottom-most ring of targets on the bottom cap of the tank. They then took the bottom of this second sphere as the bottom of the tank.

In the Jackson, et al. analysis [3], the elevation of the pedestal probe point was also determined from a mathematical model of the bottom region of the tank. However, the model used here is more complicated and considered to be more reasonable. It is described below in the description of model 3.

Three different sounding tables were constructed and analysed in the original publication [3]. The tables were based on two different mathematical models and one system of volume corrections applied to the second model. The three tables are based on increasingly sophisticated analyses, but the final results differ only slightly. Only a general description of each model will be given here as an example of the extensive analysis, and reference is made to the original publication for details.

Tank Model 1 The first model developed was a simple mathematical sphere. The sphere that best fit the target location measurements (at ambient temperature) was found earlier in this section to have center $(.005,-.0009,-.0137)$ and radius 18.2682 . It is assumed that an isotropic contraction occurs as the tank is loaded with LNG. The sphere measurements were converted to the average temperature of LNG, $-160^{\circ} \mathrm{C}$, by use of the following linear thermal expansion coefficient obtained from the 1967 edition of the Alcoa Aluminum Handbook:

$$
\begin{aligned}
& L_{t}\left(0 \text { to }-320^{\circ} \mathrm{F}\right)=\mathrm{L}_{O}\left(1+C\left(11.74 t-0.00125 t^{2}-0.0000248 t^{3}\right) 10^{-6}\right), \\
& L_{t}\left(0 \text { to } 1000^{\circ} \mathrm{F}\right)=L_{O}\left(1+C\left(12.19 t+0.003115 t^{2}\right) 10^{-6}\right.
\end{aligned}
$$

where

$$
\begin{aligned}
\mathrm{L}_{0} & =\text { length at } 0^{\circ} \mathrm{F}, \\
\mathrm{L}_{\mathrm{t}} & =\text { length at } \mathrm{t}^{\circ} \mathrm{F} \text { within range indicated, } \\
\mathrm{C} & =\text { alloy constant }(1.020 \text { for } 5083 \text { aluminum })
\end{aligned}
$$

This resulted in a sphere with the same center but a radius of 18.20485 meters. However, 1 t was felt that for the purposes of building tank models a modification to this "contracted" best-fitting sphere was necessary. The modification considered was one that resulted in a sphere which would best fit the data if the points on the top and bottom caps of the tank and those on the equatorial ring were excluded. This resulted in a sphere with the same center but with a radius of 18.2113 meters. Tables for this model could then be calculated.

Tank Model 2 In this case, Model 1 is modified by replacing the top and bottom regions of the nom 1 nal sphere with segments (caps) of other, flatter spheres. The nominal sphere is now the sphere of Model 1. Each such flatter (higher radius) sphere was taken to have its center on the polar axis of the nominal sphere, but not to be concentric with the latter. If the spherical surfaces were concentric and touched somewhere--which they must for the model not to be discontinuous--the spherical surfaces would coincide.

Comparing the volume figures for Model 1 and 2 shows that the caps of Model 2 subtract 2.41 cubic meters from the volume of the tank and does not significantly affect the sounding table.

The calculation of the sounding tables involved accounting for the volume displaced by the tower structure inside the tank. This was called "tank internals" by the consultants, and the calculation was based on blueprint specifications rather than on direct measurement. This seems valid, as the total volume of the tower structure is about 7.6 cubic meters, so that high accuracy is not needed.

Tank Model 3 In this case the sounding table is constructed by a numer 1 cal integration of the discrepancy between the actual measurements and Model 2. The procedure is as follows: 
1) For each horizontal ring of targets, a mean z coordinate value is established, and the mean radial residual is determined. This is the same as integrating the radial residuals around each $r$ ing of targets by the trapezoid rule.

2) By linear interpolation between adjacent targets on a horizontal ring, a piecewise linear function of $\mathrm{z}$ is constructed, approximating the mean radial residual at all values of $z$; at the top and bottom of the model tank, the radial residuals are taken to be zero for this purpose.

3) The piecewise linear function is then integrated to give the volume corrections to Model 2 and results in the final sounding table of Model 3. The integration requires three parts; the bottom cap, the nominal sphere and the top cap.

A comparison of the tables generated from Tank Model 3 to those generated independently by the photogrammetric consultant show values of 0.05 percent to 0.07 percent higher than the consultant's figures. This is probably caused by the different methods used for the last numerical integration. The consultant used a polynomial of high degree.

The sources of other contributing sounding table errors were examined by Jackson, et al [3]. These included target coordinate error, model error and plate curvature error. After consideration of these error sources, Jackson, et al concludes that tables generated using Tank Model 3 are accurate to \pm 0.06 percent or \pm 2 cubic meters.

\subsubsection{Three-Dimensional Triangulation Survey}

In response to a request by a U.S. shipbuilder, NBS [4] developed a computer-based three-dimensional coordinate measurement system for determination of the spatial location of points on the interior surface of spherical LNG ship tank. This system utilizes three microprocessor-based digital theodolites in a precisely measured triangulation network for data acquisition, coupled to a dedicated central computer for real-time data processing. Also implemented is a mathematical model of the tank geometry under consideration, from which coordinate data on the tank surface is used to produce tables relating liquid cargo volume to liquid surface location.

The development goal of the NBS effort was to provide a precision determination of tank size and shape, unrelated to existing field measurement systems presently in use for such structures. This has been achieved through an application of state-of-the-art optical instrumentation and computer technology. The results reported here are intended to provide the basis for an independent verification of the accuracy of a photogrammetric measurement technique presently in use.

The following is an edited version of the final report of NBS to the sponsoring shipbuilder [4] and describes the NBS measurement system, the mathematical models used, and presents the results of a calibration conducted on Cargo Tank No. 3 of the LNG Tanker Lake Charles.

Measurement Method The three-dimensional coordinate measurement system used for this survey is based on the principle of simultaneous angular triangulation to a single target from three physically separate measurement stations. Three measurement stations are installed on the interior surface of the LNG tank at the vertices of a roughly equilateral triangle as shown in figure 2.5.4.3. Each station consists of an operator-support platform and a tripod-mounted digital theodolite. The operator platforms and tripods are physically separated from each other to ensure maximum instrument stability over the course of the survey. All support fixtures are bonded to the tank surface with a high strength epoxy to avoid damaging the tank shell and to assist in equipment removal at the end of the survey. Each theodolite incorporates a 30-power telescope with optically encoded metal film glass disks for measurement of horizontal and vertical telescope orientation. The angular encoder resolution is $1 / 4,096,000$, and readout is provided to 1 arc second. Additionally, a two axis gravitational level sensor is built in to correct horizontal and vertical angle data for instrument mislevel relative to the earth's gravitational field. Local data processing and scaling is performed in an internal microprocessor in the theodolite, and each unit is lined to a central desktop computer for on-line real-time data acquisition and analysis.

The target definition system is a laser projector consisting of a He-Ne laser illuminator, a cross hair target and a 600 millimeter zoom telephoto camera lens. The laser illuminated cross hair is projected through the camera lens and can be focused at any point on the tank surface. The lens zoom feature can be used to vary the cross hair size to meet measurement requirements. A $22-\mathrm{X}$ spotting telescope is attached to the system to aid in target centering over the white disks of the photogrammetric targets and for assistance in focusing. The centering overlap of this cross hair on the photogrammetric target is estimated to be \pm 5 millimeters, which is sufficlent for providing a 


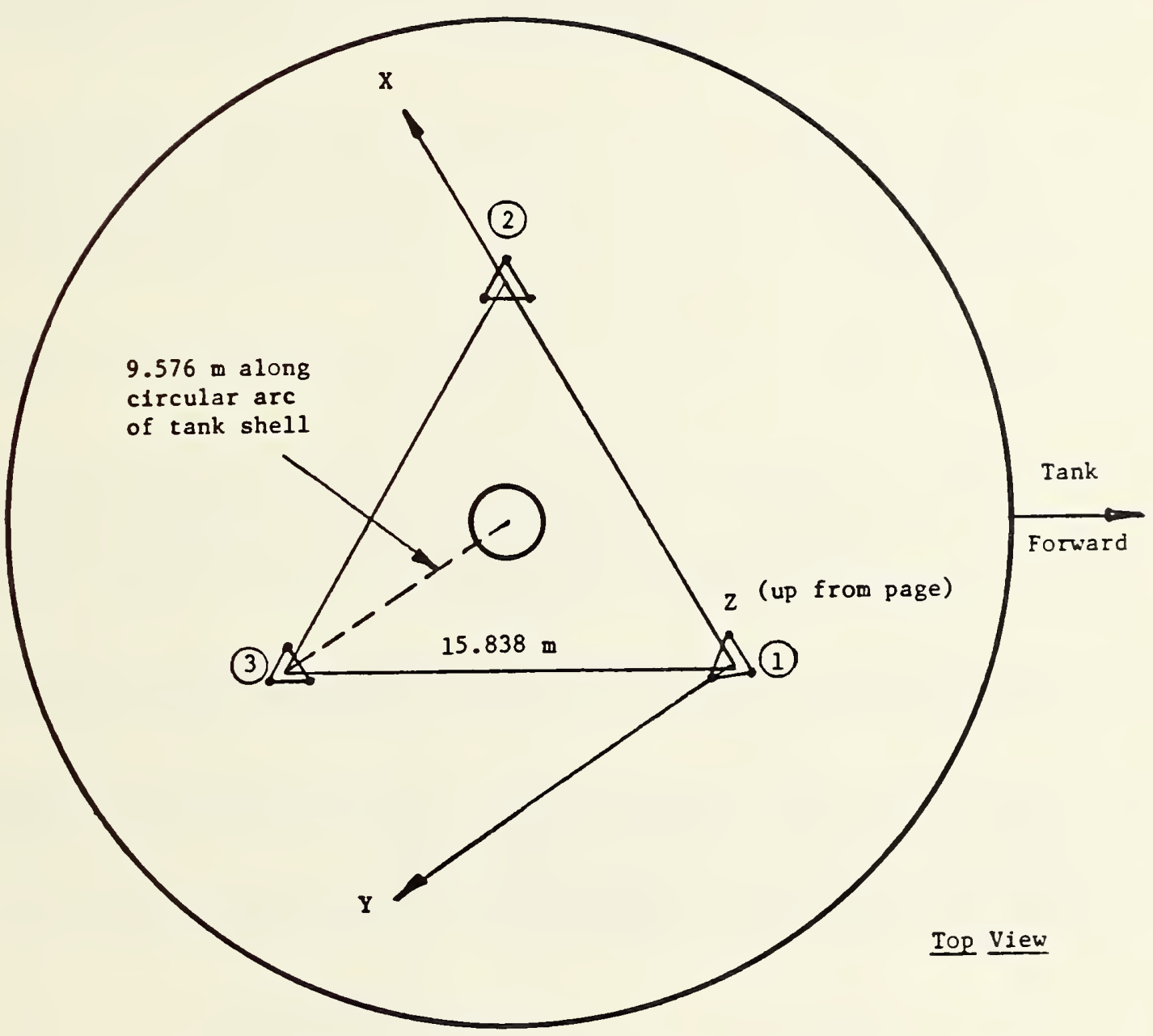

Figure 2.5.4.3. Stereotriangulation system base station locations. All dimensions represent typical nominals of an equilateral triangle.

basis for comparing the radial component of the target locations determined from photogrammetry with those reported here.

The digital theodolites have been calibrated at NBS to determine the angular accuracies of their encoders and to determine the orthogonality of their axes of rotation (height-of-standards). The results of these and other critical calibrations are provided in the final report [4].

The first step in the measurement procedure is to determine the (X,Y,Z) coordinates of each base station in a single coordinate system. To do this, the straight-line distances between the three theodolites are measured along the sides of the base station triangle. Speclal flxtures on the theodolite bases are used and an invar tape is suspended in catenary over rollers between statlons. Five repetitions of each measurement are made to allow assessment of random errors, and the tapes used were independently calibrated against a He-Ne laser interferometer system at NBS. The results of these tape calibrations were documented [4]. The tape temperature is also recorded at the t1me of base-line measurement using a temperature recorder whose calibration is included in the rinal report $[4]$. 
The second procedural step is to measure the horizontal and vertical angular orientation of the three base stations relative to each other. The technique used is to align two opposing units sighting on each others' cross hairs.

Throughout the course of base station triangulation, each theodolite operator monitors a fixed backsight on the central column. This backsight is an illuminated optical alignment target, and repeated observations are stored by the computer system for detection of any shift in theodolite angular orientation during the course of the target survey.

Upon completion of the base station triangulation, the target survey is started. Targets are identified one at a time by the laser projector, and each theodolite operator sights on the projected cross hair and transmits horizontal and vertical angle observations to the central computer. In the general case of all three theodolites being able to see the target, a total of 6 angular observations are acquired for determination of the $(X, Y, Z)$ coordinates of the point. This angle redundancy is used in a least squares solution of the measurement equations to compute point coordinates and angular residuals for each instrument. A statistical chi-squared test is used as a pass/fail criteria, and any target failing to close properly is immediately resurveyed.

For a small percentage of targets, only two of the three observers have clear lines of sight caused by obstruction by the central column and minumum focal distances of the theodolites. For these targets, 4 angular observations are acquired.

Supplemental measurements are made with the stereotriangulation system to determine the coordinate of the center of the pedestal upon which the capacitance gauge is mounted. In addition, a laser level is used to measure the vertical orientation of the gauge along the ships longitudinal and tranverse axes with respect to gravity. The laser level is also used to measure the list and trim of the ship from beneath the hull relative to the ship keel and draft marks. The results of these measurements are used to compute the list and trim correction tables reported [4].

All measurement data recorded during the course of the tank survey is stored on cassette tapes for later geometric modeling and table preparation. The on-line self-checking algorithms employed during the survey ensures the integrity of all measurement data before leaving the job site.

Mathematical Model of the Tank The output of the target survey discussed in the previous section is a set of $(X, Y, Z)$ coordinates for each point measured in the coordinate system shown in figure 2.5 .4 .1 . These data are reported at the tank ambient temperature at the time of determination of the measurement system length scale, which was $11.6^{\circ} \mathrm{C}$ for this tank.

The initial computation uses the method of least squares to fit the observed coordinate data for all surface points to a perfect sphere. The results of this computation are:

$\begin{array}{lc}\text { Tank Radius } & 18.27295 \text { meters } \\ \text { Tank Volume } & 25557.3 \text { cubic meters } \\ \text { Radial Standard Deviation } & 0.00085 \text { meters } \\ \text { Residual Standard Deviation } & 0.0166 \text { meters }\end{array}$

A detailed residual analysis of this data indicates that the major deviations from sphericity occur at the polar caps and at the equatorial ring. To quantify this, two additional data fits are performed.

First, the following targets are omitted:

- the 8 targets on the south polar cap,

-the 12 targets on the north polar cap,

-all targets on the rings adjacent to the polar cap weld seams,

-all equatorial ring targets.

The remaining targets were fitted to a sphere, yielding the following parameters:

Tank Radius

Radial Standard Deviation

Residual Standard Deviation

$$
\begin{array}{r}
18.27536 \text { meters } \\
0.00057 \text { meters } \\
0.00991 \text { meters }
\end{array}
$$

Next, the north polar cap points were fitted to a sphere, yielding the following parameters:

Tank Radius

Residual Standard Deviation 
This analysis indicates that a multi-parameter spherical model with an equatorial correction can be used for producing volume versus height tables. The geometry of this model is shown in figure 2.5.4.4, with equatorial ring details shown in figure 2.5.4.5. In these figures, the radius $R_{1}=$ 18.27536 meters is used from the data fit in that region of the tank. The radius $R_{2}=21.94682$ meters is used to model both the north and south polar cap regions. The extent of the polar caps is taken from the tank design dimensions, which were chord distances of 7.8486 meters and 4.9816 meters for the north and south caps respectively. The cross sectional shape of the equatorial ring segment was taken from the tank design drawings, and a volume correction was applied in this region.

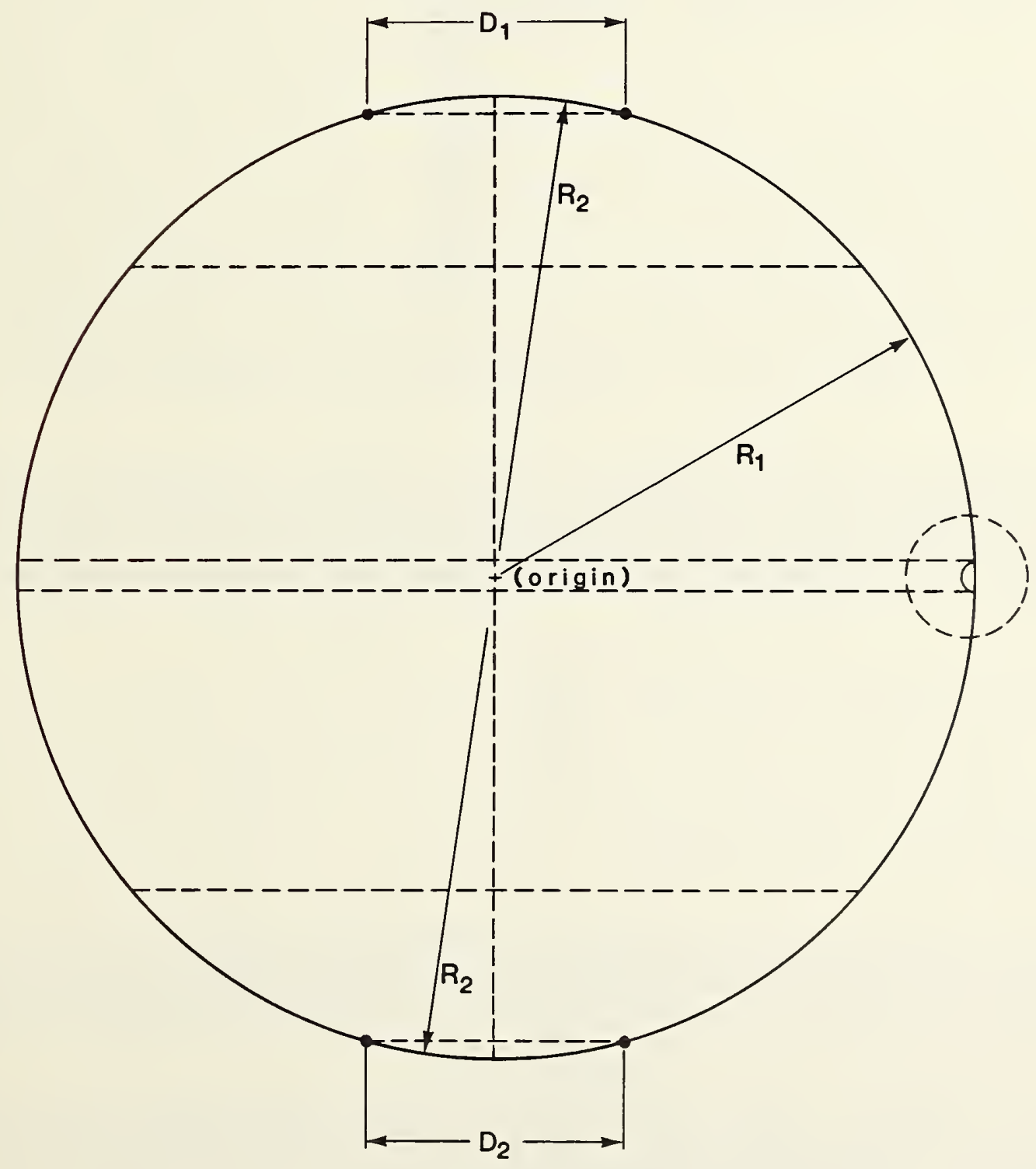

Figure 2.5.4.4. Mathematical mode depinition. 


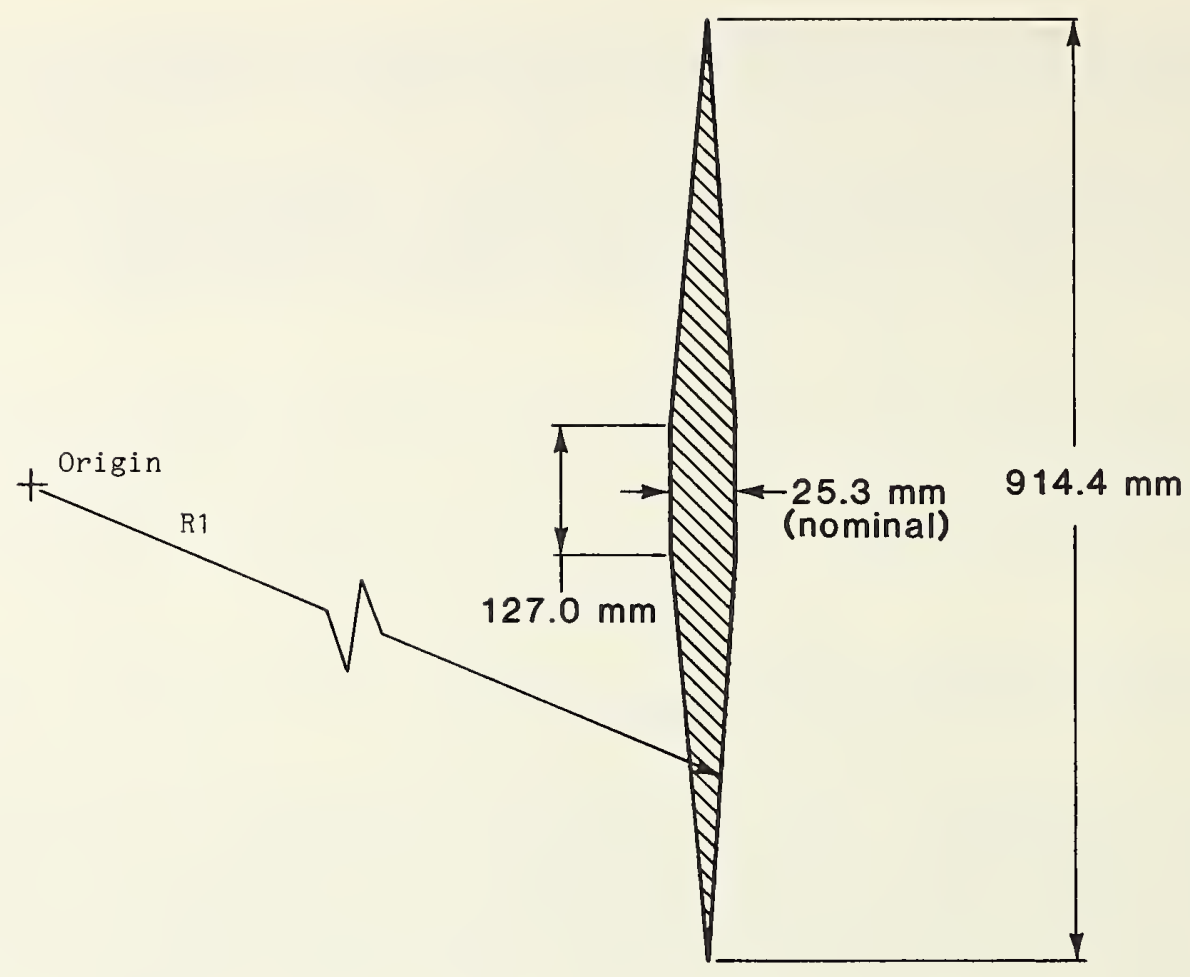

Figure 2.5.4.5. Tank equatorial ring geometery

Volume Error Estimate The volume-height tables and list-trim tables [4] are based on a multi-parameter model of a sphere with flattened polar caps (larger radius curvature than that for the tank body) and an inward bulge at the equatorial ring. For this model, the total tank volume is 25561.3 cubic meters with an uncertainty of 8.4 cubic meters $( \pm 0.05$ percent) at the three standard deviation limit. For any volume less than the total volume, the three standard deviation limit of uncertainty is:

$$
3 \sigma=[(\mathrm{V} /(\text { total volume })) 7.2+0.2] \text { cubic meters }
$$

for regions exclusive of the north polar cap, and

$3 \sigma=[(\mathrm{V} /($ total volume $)) 7.2+1.2]$ cubic meters

for regions including the north polar cap.

\subsubsection{Comparison of Survey Methods}

The preceding sections have described in detail the photogrammetric method of determining tank volume and methods of verifying the calculated volumes. The following discussion is included to show the type of analysis used when two independent measurement methods do not agree. In this case the photogrammetric company performing the survey was asked to comment on the differences found in the sounding tables generated by NBS (2.5.4.5) and those generated by the contractor. A highly edited version of the report [5] is provided for information only.

The differences between the sounding tables produced by NBS and those produced by GSI may be expected to arise from two sources, specifically differences in coordinate data and differences in the volumetric algorithm. The systematic differences in orientation may be attributed in part to the fact that the $z$-axis is defined by the direction of gravity.

The GSI survey was performed at Charleston, South Carolina, while the tank was located in the insulation shed, and the NBS survey was performed some months later at Quincy, Massachusetts, after the tank was installed in the ship's hull. The orientation of the tank with respect to the direction of 
gravity cannot be expected to coincide precisely for the two surveys. Some additional contribution to systematic differences for both orientation and origin arises from the fact that the GSI coordinates refer to the center of the painted targets, while NBS, for operational reasons, was obliged to survey coordinates of locations slightly displaced from the target centers. The systematic shift in origin is primarily attributable to the difference in definition of the origin. The GSI coordinates are referred to the center of a sphere which best-fits all targets on the spherical surface, whereas the NBS coordinates are referred to the center of a sphere which best-fits all targets except certain targets near the poles.

Direct comparison of the two sets of target coordinates does not reveal any significant differences which might account for the differences in the sounding tables. Therefore, it must be suspected that the differences are predominantly a result of the differences between the volumetric algorithms used by NBS and GSI. Accordingly, several additional volume computations were made. Each of these computatlons was made using the GSI volumetric algorithm with one modification. The original algorlthm utilizes an adaptive weighting scheme to determine the influence of each level row of targets to the polynomial used to represent departing volumes. Each level weight was a function of the computed sigmas of the individual targets in that level. Since these data are not supplied with the NBS data, it was decided to use unit weighting, i.e., weight equals one, for each level in place of the original adaptive weighting scheme.

Before describing the additional computations, it is necessary to review the manneir in which NBS elevation origin is defined. Specificaly, the terms "flattened" and "unflattened" bottom must be introduced, as they are basic to the discussion which follows. The NBS volumes are computed using reference spheres with one of two radii. The main portion, excluding the polar caps, is based on a reference sphere whose radjus has been designated $R_{\uparrow}$. The polar caps are computed from reference spheres having a radius which NBS has designated $R_{2}$. Contrary to the presentation shown in figure 2.5.4.4, these reference spheres do not share a common origin. Rather, they have origins which lie along a common z-axis. The vertical separation of the origins is very nearly equal to the difference in the two radii, $R_{1}$ and $R_{2}$. It can be shown that the vertical separation of the origins is exactly equal to the difference in the two radii plus the flattening of the pole. The actual value for the "flattening of the south pole" is given by the relation:

$$
F=R_{1}-R_{2}-\left[R_{1}-\left(D_{2} / 2\right)^{2}\right]^{1 / 2}+\left[R_{2}-\left(D_{2} / 2\right)^{2}\right]^{1 / 2}
$$

It now seems natural to adopt the term "NBS flattened bottom" for the horizontal plane which lies at a distance of $\left(R_{1}-F\right)$ below the origin of the sphere whose radius is R. Similarly, the term "NBS unflattened bottom" will be used when referring to the horizontal plane which lies at a distance of $R_{1}$ below the origin of the sphere whose radius is $R_{\uparrow}$. Thus the flattened bottom is situated above the unflattened bottom, at a distance equal to the flattening of the south pole.

The first computation was to process the NBS coordinates through the modified GSI algorithin. The elevation origin was adopted at the NBS flattened bottom, such that the elevation of the pedestal is the value given in the NBS report. The differences in volume from this computation with respect to the volume data in the NBS report are plotted in figure 2.5 .4 .6 as curve A. GSI asserted that a shift of the elevation origin of 2-3 centimeters could substantially account for differences of this natise Noting that the NBS report contained a value for the flattening of the south pole, and that this value was nearly 3 centimeters, another run was made using the NBS coordinates but with the elevati placed at the NBS unflattened bottom. The difference in volume with respect to the volume data in the NBS report is plotted on figure 2.5 .4 .6 as curve $B$.

With the objective of further evaluating the signiricance of coordinate differences on the volute computations, the GSI coordinates were run through the modified GSI algorithm first with the el vit 1 i origin at the NBS flattened bottom, and then with the elevation origin at the NBS unflattened bottom. The volume difference with respect to the respective NBS data computations using the modilled 2 l algorithm plotted in figure 2.5 .4 .6 as curves $C$ and $D$.

An examination of the plots suggests several conclusions. First, the NBS versug js 1 inlun differences caused by differences in target coordinate sets is almost negliglbie a shifts in elevation origin can account for significant volume differences which are shallest n in poles and maximal near the equator. Third, it appears likely that the elevaticn uri in for tn" ras data in the NBS report is their unflattened bottom, rather than the flattened bortom as reftrif. Finally, assuming for the sake of argument that this last conclusion is true, curve $B$ can oe $r$ raral 1 as a plot of volume differences caused by volumetric algorithm differences. 


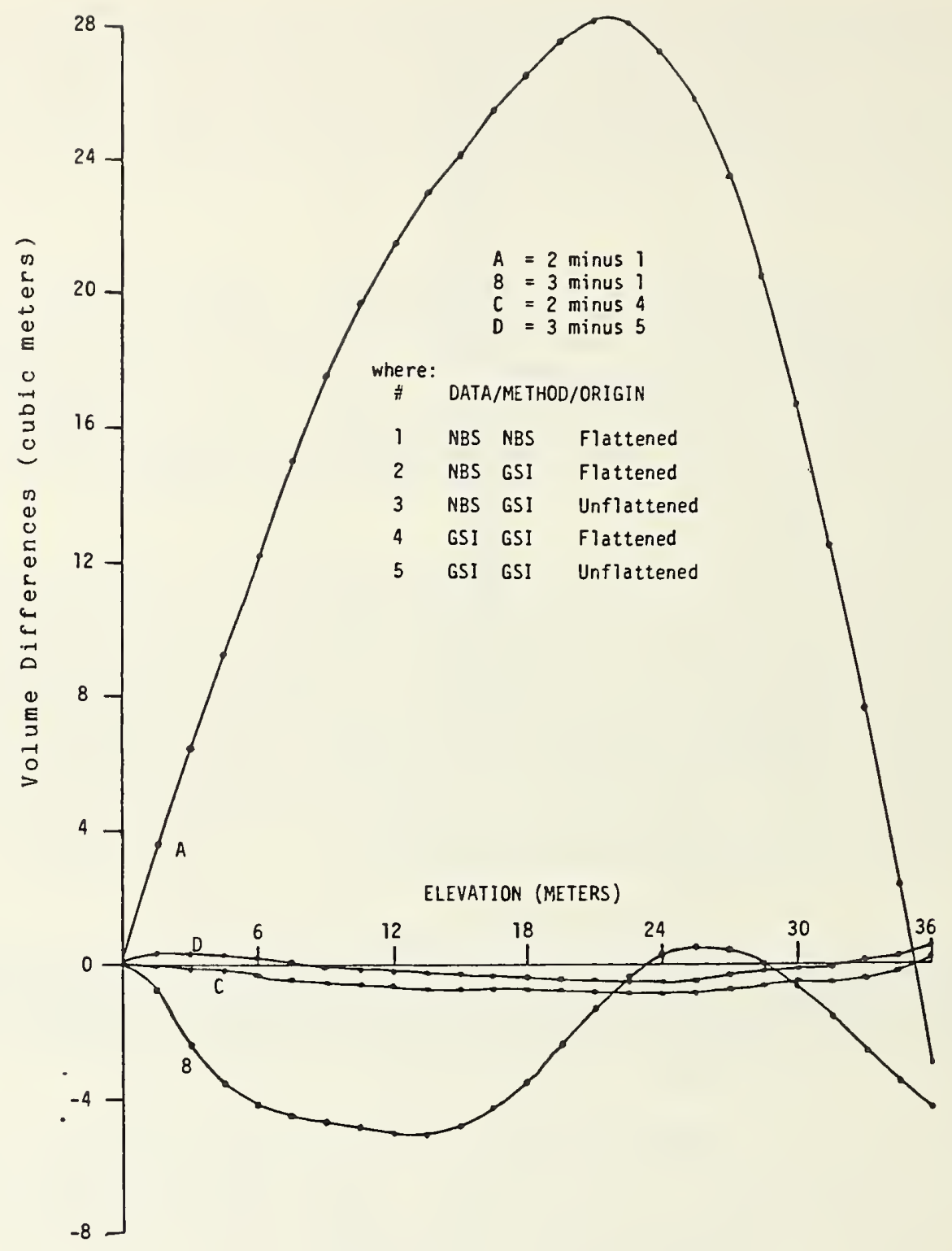

Figure 2.5.4.6. Volume differences of NBS and GSI for LNG ship cargo tank 41 .

Consider now an assessment of algorithm differences. One difference has already been addressed in part, namely the problem of elevation origin. The elevation origin, or "bottom", used by GSI is, like NBS, a mathematically deduced rather than a directly observed entity. Should the actual physical bottom differ from the mathematically deduced bottom by up to 4 or 5 centimeters, the volume error will be quite small, on the order of a fraction of a cubic meter, and will be a constant bias for all succeeding entries.

Thus, this error source is of no practical concern when using the volume tables to determine the amount of product transferred during a loading/unloading operation. An error of 3 centimeters in the elevation assigned to the pedestal with respect to the bottom (elevation origin error) will result in errors which vary as a function of height with almost no error near either pole, but errors of up to 30 cubic meters near the equator. In this case, the net error in determination of the quantity of product transferred will be a function of the gauge readings at the start and end of a loading/unloading operation. 
Another source of volume differences inherent in the two volume algorithms is the manner in which volume displaced by tank internal structures is handled. GSI accounts for the effects of all tank internal structures, as described in the individual tank reports. NBS accounts only for the volume displaced, caused by the intrusion of the equatorial ring structure, which represents approximately one-fourth of the total volume displaced by all internal structures. The remaining effects are more or less uniformly distributed with respect to elevation, with a total effect of approximately 6.8 cubic meters.

There are several differences in the modeling approach for volume computations. The first difference is that NBS utilized trapezoidal modeling to integrate volume; differences from a reference sphere, while GSI used a polynomial for this purpose. The volume differences caused by this modeling difference are estimated to be on the order of 3 cubic meters.

Another difference between the two algorithms is the manner in which horizontal departures are computed. The effects of this modeling error are dependent upon the actual departures of each level in any given tank, particularly the near-polar target levels. The actual value of this affect is estimated to be on the order of \pm 0.5 cubic meters, assuming that the actual departures from the reference sphere are near zero at the extreme near-polar target levels. If, however, the actual departures were significant, as would be the case if the reference sphere used for the integration was displaced vertically, then the resulting computed volumes would be adversely affected. A computation was made using NBS data through the modified GSI algorithm in which the reference sphere used to represent the middle (main) portion of the elevation range was displaced vertically downward by 2 centimeters. The resulting volumes were approximately 1.2 cubic meters smaller for all elevation entries greater than one meter. Thus, this error source seems to produce bias errors in the volumetric computations, which are of no practical concern when using the volume tables to measure product transfer.

Yet another difference between the volumetric algorithms is the difference in weighting schemes. The modified GSI algorithm (using unit weighting) is estimated to produce volumes that differ from those from the unmodified (adaptive weighting) by no more than 5 cubic meters. The actual differences will be largely a function of the size of the departing volumes for a given tank. The smaller the departing volumes the smaller the volume differences from these two weighting options. Experience suggests that volume differences caused by weighting choices will tend to average less than 3 cubic meters for these tanks.

A final note on volume differences is the effect of scaling. A scale change of one part in ten thousand is sufficient to produce volume changes of 7.5 cubic meters.

In summary, the coordinate sets determined by NBS and GSI agree very well, and the difference between NBS and GSI volume tables can be accounted for by the various algorithmic differences cited above. 


\subsubsection{References}

[1] Brown, D. C., Close-range camera calibration, Photogrammetric Engineering, 37, 855-66(1971).

[2] Witzgall, C., Best approximating sphere in $\mathrm{R}^{\mathrm{n}}$, unpublished working paper, Center for Applied Mathematics, Nat. Bur. Stand.(U.S.), Washington, D.C.

[3] Jackson, R. H. F., R. S. Collier, S. Haber and P. Tryon, Custody transfer systems for LNG ships: Tank survey techniques and sounding tables, Nat. Bur. Stand.(U.S.), NBSIR 79-1751, 84 pp (May 1979).

[4] Final report on three-dimensional triangulation survey of cargo tank no.3 on the LNG tanker Lake Charles, General Dynamics tank no. 41, prepared for General Dynamics Shipbuilding Division, Quincy, MA, by Nat. Bur. Stand.(U.S.), Automated Production Technology Division, Washington, D.C. $20234(1980)$.

[5] Jelffs, P. A., Calibration of containers and gages, J. Inst. Pet., 58, No. 561, pp 117-25, (May, 1972).

[6] Final report for General Dynamics cargo tank no. 41, tank no. 3 on hull no. 53, prepared for General Dynamics Shipbuilding Division, Quincy, MA, by Geodetic Services, Inc., P.0.Box 3669, Indialantic, FL 32903 (1981). 


\subsubsection{Volume of Cylindrical LNG Shore Tanks}

The shape and size of LNG land based storage tanks are more traditional since for more than thirty years, cryogenic tanks of this type have been constructed and installed for liquid oxygen, nitrogen, hydrogen and other atmospheric gases as well as LNG. The primary containment tank is generally cylindrical in shape and is surrounded by one or more meters in thickness of bulk insulation. A weather protective outside wall is constructed to protect and support the insulation. Tanks may be inground or above ground and have incorporated both metallic and non-metallic materials of construction.

Natural gas has been stored in liquid form for peak shaving purposes and in satellite storage to service customers remote from the main natural gas pipelines. The construction of large baseload importation terminals involved more than one large storage container to accommodate periodic LNG ship arrivals and to allow continuous high volume send out of natural gas. The measurement of quantity and quality became very important economically, and new instrumentation methods and procedures were adopted to ensure efficient operation of the import terminal. Many of these techniques are described in this part of the manual, in those sections dealing with volume measurements of LNG ship tanks.

The following edited section presents the results of a photogrammetric survey to determine the size, shape and volume of a 600,000 barrel (58 meters diameter by 37 meters in height) Liquid Natural Gas (LNG) Storage Tank, for Trunkline LNG Terminal at Lake Charles, LA. It is based on the final report of the photogrammetric contractor [7] and, as in the previous sections, it is designed to describe the process in detail and to provide an estimate of error for the measurement, which may then be integrated into the total custody transfer.

\subsubsection{Photogrammetric Survey [10]}

Preparatory to acquisition of the photographic data, which was obtained at Lake Charles, Louisiana, approximately 500 "bulls-eye" type targets were placed throughout the interior of the tank at pre-selected plate positions as shown by the following figures. Each target is a 1.27 centimeter diameter circle of a retro-reflective material mounted in the center of a black 2.22 centimeter diameter, 0.32 centimeter inch thick circular magnetic disc.

During the survey, the internal surface of the tank was photographed on special 0.64 centimeter thick micro-flat glass plates having a format of 24.13 by 24.13 centimeter by means of a custom-built metric camera, strategically positioned at six selected sites. Figure 2.5.5.1 shows the nominal positions of the exposure sites, which are located approximately 1.5 meters inside the inner surface of tank in a circular ring, at points approximately 7.6 meters above the tank floor, and distributed symmetrically about the vertical center line of the tank. At each of the six sites, three exposures were made for a total of eighteen exposures for the survey. The aim points of one of these exposures is diametrically opposite the exposure site, and ten degrees above the horizontal plane containing the exposure site. The aim points of the other two exposures at a site are approximately 35 degrees to the left and right of the center exposure, and also ten degrees above the horizontal plane. The combination of camera parameters, including location, angular field of view and direction of the optical axis, guarantees that each surface target will be imaged on no less than three photographic plates with an average or 4.5 images per target, and that no less than 25 percent of the internal surface of the tank will be imaged on each photographic plate.

In addition to the basic photographic equipment, auxiliary survey gear, including three calibrated survey tapes and two precision temperature sensors, were installed in the tank during the survey. The scale tapes provide an accurate scale reference for the final target coordinates, and the temperature sensors provide a means for determining the internal tank temperature at the time of the survey.

To ensure that the desired photographic results were obtained, the photographic plates were processed and carefully inspected on-site. Upon successful data acquisition, the plates were ready for subsequent data processing and reduction.

Every target on the photographic plates was identified and annotated with a unique 1 dent1rication number and measured on a two-axis monocomparator, calibrated to one micrometer along each axis. The plate coordinate data were then processed through a series of proprietary programs, whlch provide a simultaneous least squares adjustment of all measured data, ultimately yielding $X, Y, Z$ object spacc coordinates of the triangulated points. Detailed treatments on the mathematics of the least squarco adjustment, error propagation and lens calibration are available in references [1] through [5]. 

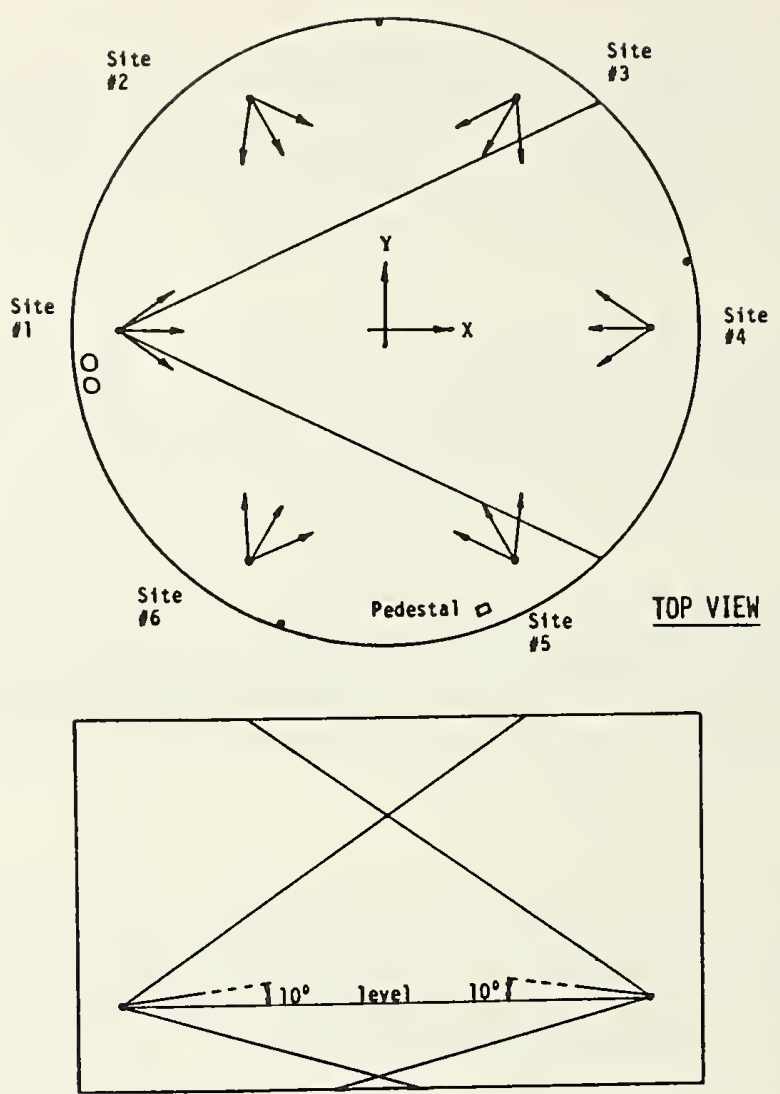

SIDE VIEW

Figure 2.5.5.1. Camera exposure sites within tank.

One of the unique features inherent in this computational process is self calibration of the camera, by which the internal geometry of the camera is simultaneously determined along with the desired target coordinates. Calibration of the camera includes recovery of the camera focal length, principal point and the coefficients of radial and decentering lens distortions. Application of these parameters significantly improves the projective relationship between targets and their image points, thereby greatly improving the resulting accuracy of the triangulated target coordinates.

During the stereotriangulation reduction, an arbitrary scale and coordinate orientation is enforced, and, as a final operation, it is necessary to perform a complete transformation (scale, translation and rotation) of the triangulated coordinates and their associated covariances into the desired survey coordinate system.

The coordinates are correctly scaled through application of an average scale factor, computed from comparison of thermally corrected calibrated distances and computed distances between targets on the scale tapes. Then orientation of the data is achieved by alignment of the $Z$ coordinate axis perpendicular to the horizontal plane as defined by leveled row of targets, as shown in figure 2.5.5.2. Lastly, the original of the final survey coordinate system is translated so that the $Z$ coordinate axis is located at the center of a cylinder which best fits the surveyed target coordinates and the $\mathrm{Z}$ coordinates of the level tie point matches the customer supplied value. The transformed survey coordinates represent the final survey results at the temperature at which the survey was performed $[7]$.

Figure 2.5.5.2 shows the fifteen levels starting with one at the bottom and ending with $\mathrm{f}$ ifteen at the top. The placement of the level 2 targets to within one millimeter of a constant height provides the basis for determination of the z-coordinate direction, which is defined to be perpendicular to the plane passing through the targets in level 2. Figure 2.5.5.3 shows, for each level, the individual plates which are numbered clockwise beginning with 1 near the downcomer. 


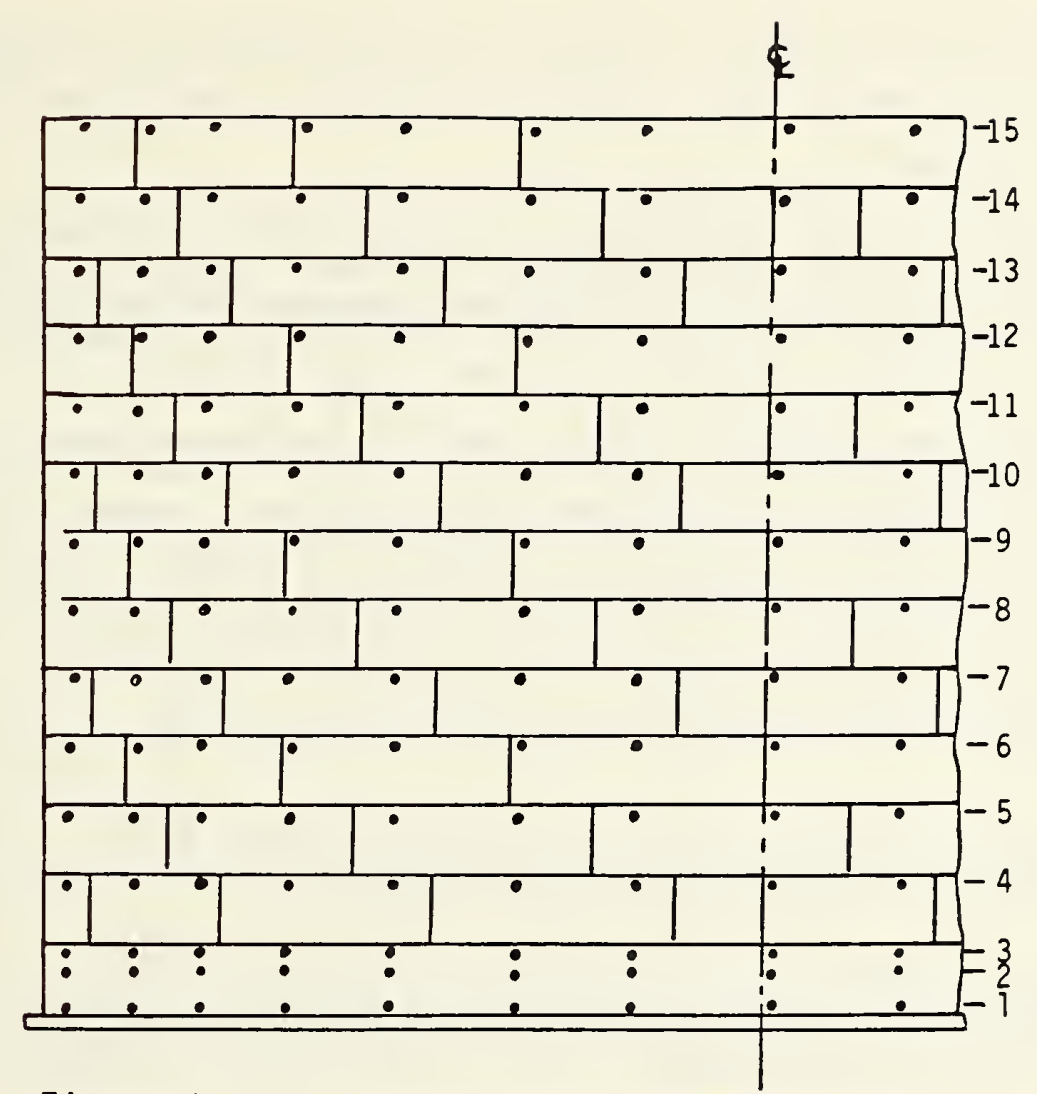

Figure 2.5.5.2. Tank level numbering scheme.

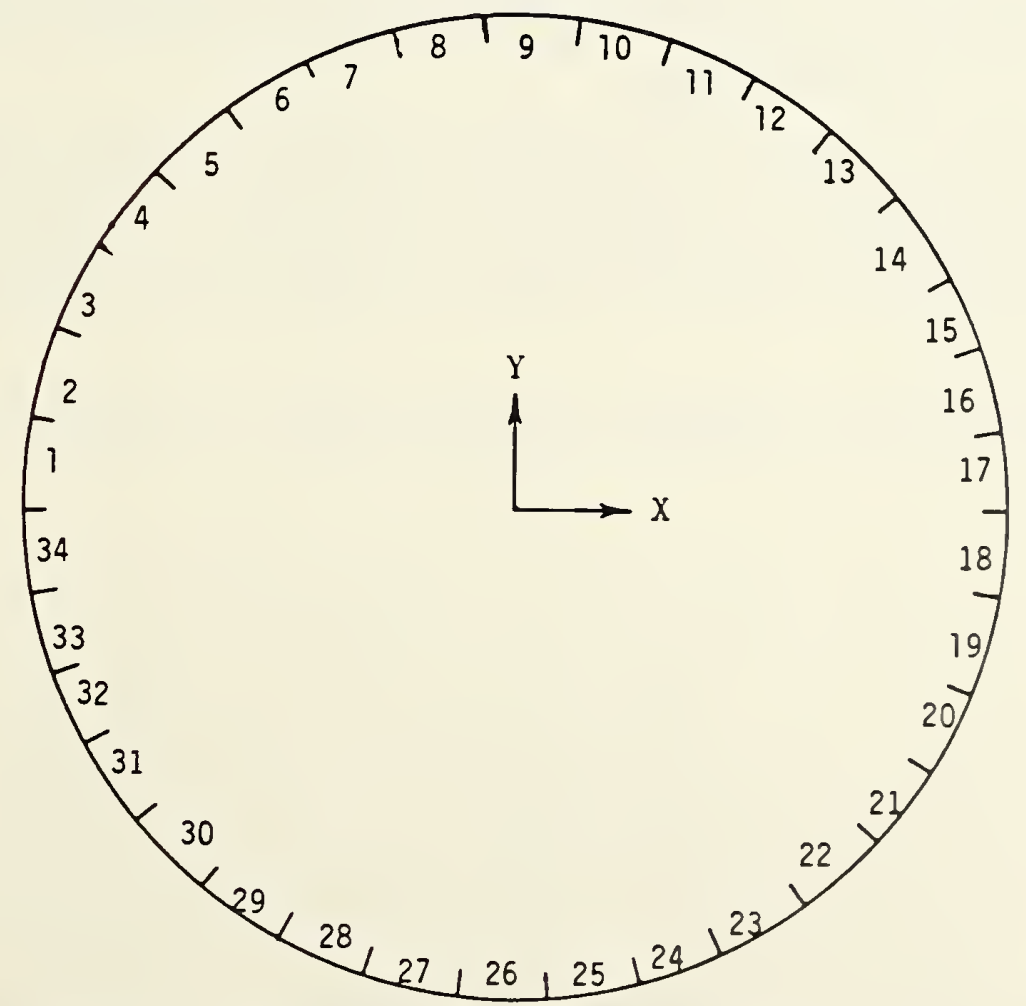

Figure 2.5.5.3. Tank column numbering scheme in plan view. 
The final photogrammetrically determined $X, Y, Z$ target coordinates for all targets within the tank are tabulated [7] at the survey temperature. These target coordinates have been adjusted to remove the thickness of the magnetic disc.

\subsubsection{Volume computation}

Since the storage tank very nearly approximates a cylindrical body, the total and partial volumes of the tank were derived from "cylindrical" computations. Basically, the final volume was determined by taking the computed volume of a reference cylinder and algebraically summing the computed differences in volume between the reference surface and the real surface plus the volume deviations caused by the tank internals and to the structural deformations ("barreling") which occur under loading.

A brief summary of the actual procedure follows. The X,Y,Z target coordinates were separated into fifteen layers or rings, so that all targets labeled "level number 1 " formed a ring, all targets labeled "level number 2 " formed another ring and likewise for the targets in the remaining levels. For each ring, the horizontal departures were modeled by Fourier polynomials as a function of longitude or angular distribution around the ring. Integration of the Fourier polynomial resulted in the departing cross-sectional area of the true targeted surface from the reference cylinder.

Through use of the principles of array algebra [6], the departing volume of the actual surface from the reference cylinder was determined by integration of the departing areas as a function of elevation.

The bottom of the tank is slightly coned, complicating rigorous volume computations for elevations between zero and the peak of the cone. However, the minimum product level will always be well above this elevation for the pumps to be able to function properly.

Before computing the final volume, it was necessary to remove the volumetric contribution of the permanent internal fixtures of the tank. The volumes of the tank internals were provided by the tank contractor at 10 centimeter increments of elevation. The tank was divided into 58 elevation ranges depending upon where significant changes in the concentration of the tank internals occured. The volumetric contribution with each volume range is obtained by linear interpolation with respect to elevation.

The final volume computation also required consideration of volume increases caused by barreling under loading conditions. The necessary data were supplied by the tank contractor, and the effects at 112.5 $\mathrm{K}$ are included in the final sounding tables.

The desired sounding table of partitional volumes as a function of vertical elevation, beginning with zero volume at the bottom of the tank and ending with the final total volume at the top of the tank, is included in the photogrammetric contractor final report $[7,8,9]$. The partial volumes of the tank (minus internals plus barreling) were then evaluated for every 1.52 centimeter of elevation range. The data in the table for each intermediate elevation included the total accumulated partial volume in both cubic meters and barrels.

The total single tank volume was calculated to be $96,493.91$ cubic meters at a tank temperature of $112.5 \mathrm{~K}$. The computed estimate of accuracy of the total volume at $112.5 \mathrm{~K}$ was 4.8 cubic meters or \pm 0.005 percent. 
2.5 .5 .3 References

[1] Brown, D., Advanced methods for calibration of metric cameras, DBA Systems, Inc., Final Report Part 1, U. S. Army Engineering Topographic Laboratories, Fort Belvoir, VA, (December 1968).

[2] Brown, D., Decentering distortion of lenses, Photogrammetric Eng., XXXII, No. 3 (May 1966).

[3] Brown, D., Precise calibration of surfaces of large radio reflectors by means of analytical photogrammetric triangulation, Research and Analysis Tech. Report No. 10, Instrument Corporation of Florida (November 1962).

[4] Brown, D., A solution to the general problem of multiple station analytical stereotriangulation, RCA Data Reduction Tech. Report No. 43, ASTIA Doc. No. 134278 (February, 1958 ).

[5] Brown, D., A treatment of analytical photogrammetry with emphasis on ballistic camera applications, RCA Data Reduction Tech. Report No. 39, (November 1956).

[6] Rauhala, U., A review of array algebra, Fotogrammetriska Meddelanden, 2:38, Division of Photogrammetry, Royal Inst. of Tech., S-100 44 Stockholm, Sweden (April 1976).

[7] Geodetic Services, Inc., Photogrammetric survey of Trunkline LNG Company 600,000 bbl LNG storage tank No. A at Lake Charles, LA, Final Report for Trunkline LNG Corp., 3000 Bissonnet Avenue, Houston, TX (April 1980).

[8] Geodetic Services, Inc., Photogrammetric survey of Trunkline LNG Company 600,000 bbl LNG storage tank No. B at Lake Charles, LA, Final Report for Trunkline LNG Corp., 3000 Bissonnet Avenue, Houston, TX (April 1980).

[9] Geodetic Services, Inc., Photogrammetric survey of Trunkline LNG Company 600,000 bbl LNG storage tank No. C at Lake Charles, LA, Final Report for Trunkline LNG Corp., 3000 Bissonnet Avenue, Houston, TX (April 1980).

[10] Brown, D. C., LNG tank inventory measurement by photogrammetry, 1981 operating Section Proceedings, 81-T-55, ppT-127-37, Am. Gas Assoc., 1515 Wilson Blvd., Arlington, VA 22209 (1981). 



\subsubsection{Volume from Totalized Flow Rate Metering}

\subsubsection{Introduction}

A general discussion of the measurement process as applied to LNG is included in this manual as section 2.1. Within that section, the component of volume measurement was divided into two separate methods. The first method was the traditional "strapping" or in-tank physical length measurement, starting generally at the lowest point in the container and resulting in a series of plane areas whlch, when combined with a incremental height, provided volume sums as a function of container height or depth. The technology of this method is covered in the first five sections of this part of the manual.

The second method is the subject of this section of the manual and provides a totalized volume measurement of the LNG flowing into or out of the storage tank. The measurement process involves a flowmeter installed in the LNG container supply piping which will indicate total flow per unit time. The actual flowmeter type is not important other than it may be an orifice meter, a turbine meter, a vortex device, a positive displacement meter or any other process device which develops an output indication which has a known relationship to a quantity of LNG passing through it during some period of time. Mass flowmeters have been adapted for cryogenic service [1], but are not available in the larger line sizes of interest in LNG service. Combination of the totalized volume measurement with LNG density and calorific value will give a total calorific value. All measurement components can be centralized in one location and considered a self-contained measurement station.

A recently completed study reported by Brennan [18] of the National Bureau of Standards (NBS) and sponsored by the Pipline Research Committee of the American Gas Association summarizes a long-term (1974-1983) program to develop and prove the concept of the measurement station. An edited version of this summary is given in the following sections. The description includes applications of a particular type of LNG in-line densimeter and calorific measurement technique in addition to the volume flowmeter. This was found necessary to provide continuity as the metering station capacity was scaled upward to LNG terminal size requirements.

\subsubsection{The Measurement Station}

The measurement station approach to LNG flow measurement is shown schematically in figure 2.5 .6 .1$. Each measurement station includes a flowmeter, a densimeter and a calorimeter with a gravitometer. Both the flowmeter and the densimeter are placed in the pipeline and measure the LNG directly. The densimeter does not extend across the pipeline but only measures the density of the LNG in a localized section of the pipe. In fully developed turbulent flow, this limitation does not lead to errors since there will not be a significant density gradient across the pipe cross section.

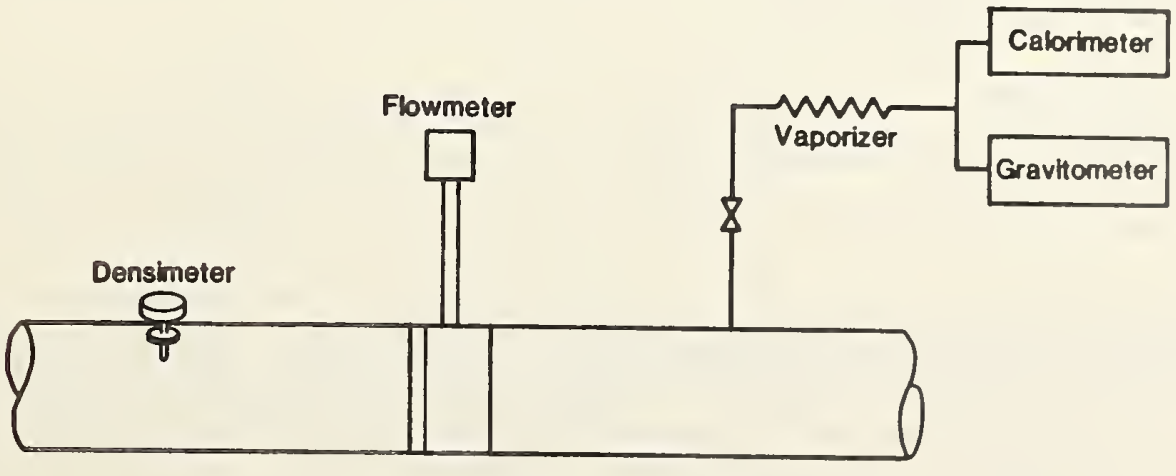

Figure 2.5.6.1. Typical measurement station schematic. 
The calorimeter and gravitometer must be supplied with a vaporized sample representative of the LNG flowing in the pipeline. This requires a well-designed and operated LNG sampling system. The sampling requirements are the same for any measurement technique, however, not just the measurement station method (see section 2.2).

LNG flow measurement with a measurement station where all measurement components are either in or attached to a pipeline has some very desirable characteristics. The measurement station can provide a continuous output of not only flow rates but also heating value on either an instantaneous or totalized basis. Individual elements are capable of independent calibration and, with the possible exception of the flowmeter, can be removed from the line for calibration or repair without taking the line out of service. Because of certain design features, it is usually not possible to remove the flowmeter from the line without removing a section of the pipeline.

To prove the feasibility of the measurement station approach, an extensive test program was started at the National Bureau of Standards in 1974. The purpose of the test program was to demonstrate that a measurement station could be used to accurately and reliably measure LNG flowing in a pipeline, including large diameter off-loading lines. Since only the flowmeter is line size dependent, the program logically divided into two parts. The first part was to show that individual measurements of flow rate, density, heating value and specific gravity could be integrated into a single measurement result. This work was completed on the NBS LNG flow facility. The second part of the program was to determine the factors for scaling the flowmeter to large diameter pipes.

Selection of components for a feasibility study of the measurement station approach was based on the following requirements:

A. Elowmeter

1. proven cryogenic performance

2. full line cross section measurement elements

3. no moving parts

4. ability to scale to large line sizes

5. at least 10:1 rangeability

B. Densimeter

1. proven cryogenic performance

2. ability to operate dynamically in LNG pipelines as opposed to a side stream or statically in a storage tank

C. Calorimeter

1. the standard industry calorimeter was specified because it already had the documented precision and accuracy

D. Gravitometer

1. continuous measurement

2. electrical output

3. proven precision and accuracy.

The type of components initially selected for the first tests were used in all subsequent tests throughout the program. All components were not included in all tests but, whenever tests were made, the same type elements were always used.

The flowmeter selected was a vortex shedding type with a thermal sensor for detecting the vortices. The selection was based on test results obtained in a previous flowmeter evaluation program at NBS in which all the different types of flowmeters used in custody transfer of cryogenic liquids were tested [1]. Of all the flowmeters tested in that extensive test program, the vortex shedding flowmeter was the only one that met all the selection criteria.

Selection of the densimeter was not based on a large body of test data, since none existed. Only one densimeter was found that had demonstrated cryogenic performance [2]. Therefore, that densimeter, which was a vibrating plate type, was selected for further study. During the later stages of the program, data on other densimeters operating in static cryogenic liquids became available [3]. Based on the data in [3], other choices are now available and include not only the vibrating plate type but also a vibrating cylinder type. A full description of the performance of these and other types of LNG densimeters are given in sections 2.4 .1 and 2.4 .2 of this manual. 
Installation requirements are somewhat different for the two vibrating element types of densimeters with the vibrating cylinder requiring larger pipeline cross sections. From that standpoint, the selected vibrating plate densimeter allow easier installation in the NBS flow reference facilities.

Calorimeter selection was based entirely on previous industrial experience. The calorimeter had been evaluated by NBS and the American Gas Association (AGA) [4] and is very widely accepted as the industry standard. Manufacturers data were collected on other types for comparative purposes, but none could be shown to have the potential for superior performance. Data made available since the selection of the calorimeter for this project may change this conclusion. other methods and techniques are presented and compared in section 2.3 of this manual.

Input information from several of the gas companies was used in selecting the gravitometer. Operating experience expressed by the company representatives combined with manufacturers data lead to the choice of a continuously sampling gravitometer which operates on an impulse ratio principle.

\subsubsection{Component Element Testing}

All components used in the measurement station were tested individually before integration into a system. Individual element tests were conducted so that a base line performance characteristic could be established. The base line was then used as the reference point for all later tests. Those tests also provided the first step in the flowmeter scaling work.

Flowmeter and densimeter liquid nitrogen tests The NBS liquid nitrogen flow facility was used for determining the flow characteristics of the flowmeter and densimeter. This flow facility has a flow range of 76-760 liters per minute over a range of temperatures and pressures. Facility accuracy on a mass basis is \pm 0.18 percent, which is composed of \pm 0.12 percent systematic error and \pm 0.06 percent random error (3 sigma) [5].

A two-inch flowmeter was chosen for those tests because it best matched the flow range of both the liquid nitrogen and the LNG flow facilities. When this work was started it was unknown how closely the manufacturer could predict the meter factor for the flowmeter. Therefore, the flowmeter was also calibrated in water at the NBS flow facility in Gaithersburg, Maryland. This was done because scaling factors could either be based on size alone or on both size and a meter factor in a surrogate fluid. It was anticipated that reliable data on larger diameter flowmeters to be tested later in the program might not exist. So all flowmeters tested in the project were calibrated in water to help establish a data base in a common liquid. Water was used as the calibration medium because no large capacity cryogenic flow facilities were in operation.

Figure 2.5.6.2 shows the results of the test on the two-inch flowmeter in liquid nitrogen and water. The change in meter factor between the two fluids was predicted by the manufacturer to be 0.9 percent. As can be seen in the figure, the change was nearly 1.6 percent. This fact illustrates the danger of relying on seemingly logical theoretical considerations that have not been verified.

Liquid nitrogen tests on the densimeter are shown in figure 2.5.6.3. These data covered almost a 13 percent density range with flow velocities of 0.3 to 3 meters per second. Densimeter output was a frequency that was related to density according to equation (1). The densimeter frequency was monitored and fit, by the method of least squares, to equation (1) to determine the equation. coefficients.

$$
\rho=A / f^{2}+B
$$

where

$$
\begin{aligned}
\rho & =\text { density } \\
\text { and } B & =\text { constants } \\
f & =\text { densimeter output frequency. }
\end{aligned}
$$

The deviation shown in figure 2.5.6.3 is the percentage difference between the densities calculated from equation (1) and from a PVT correlation by strobridge [6]. The residual standard deviation of the data from the calculated value was \pm 0.15 percent. 


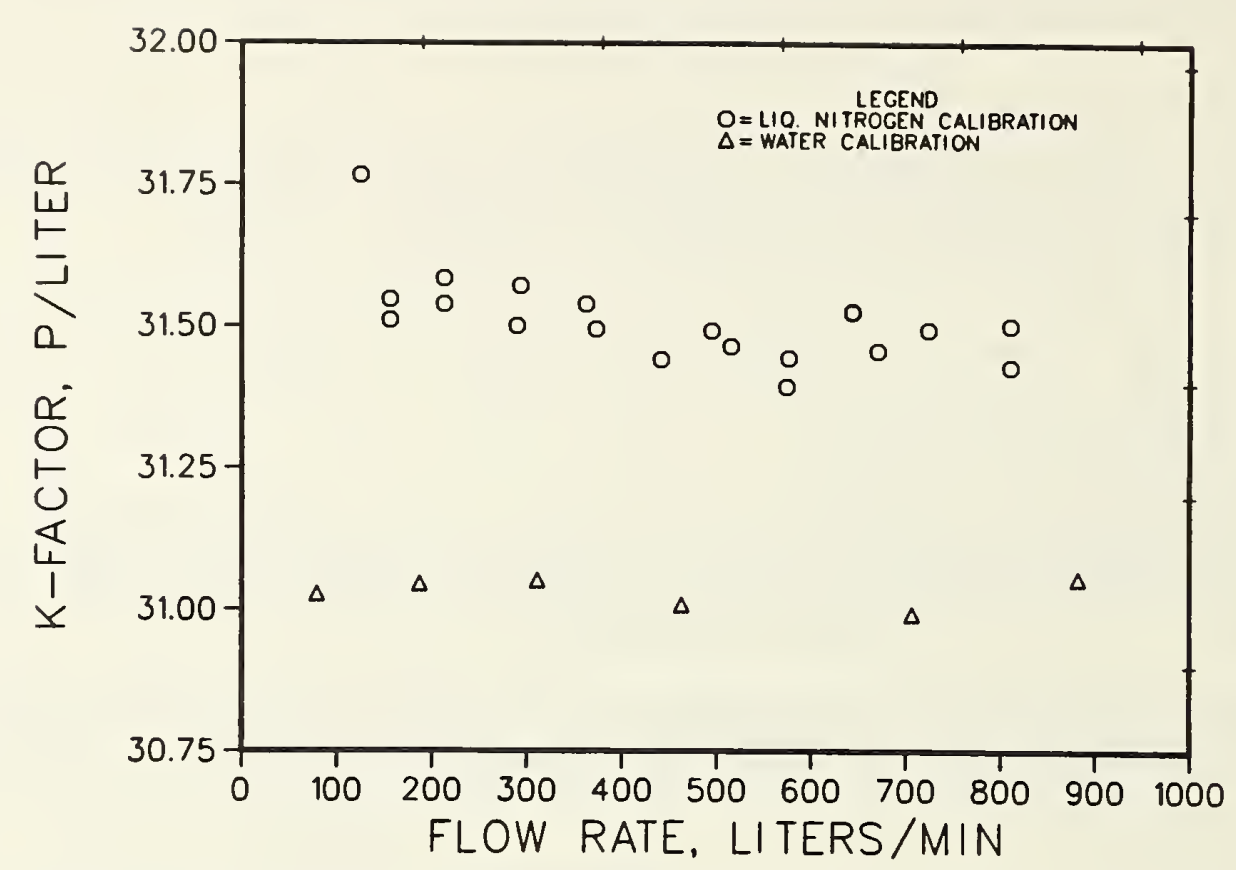

Figure 2.5.6.2. Two-inch vortex shedding flowmeter test results in liquid nitrogen and water.

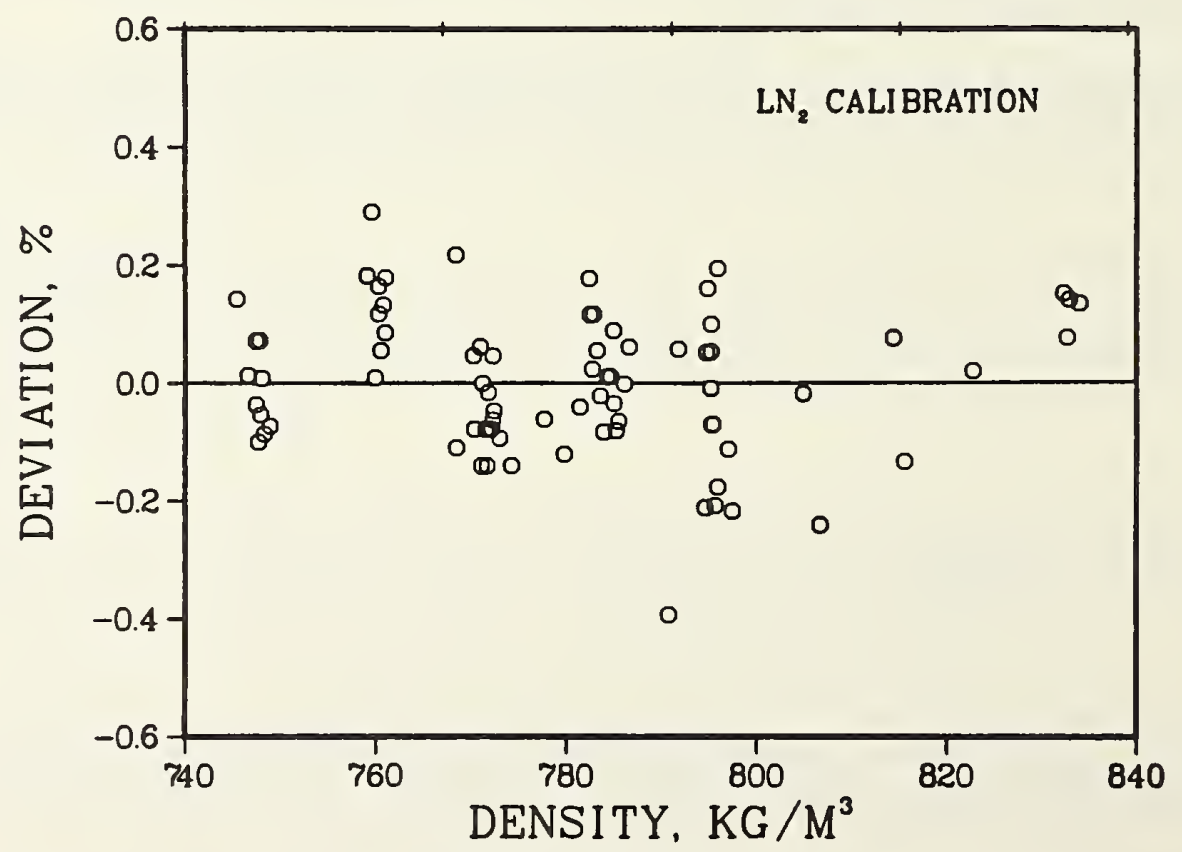

Figure 2.5.6.3. Densimeter test results in plowing liquid nitrogen. 
LNG flow Pacility. LNG tests on the measurement station components were performed on the LNG flow facility at NBS Boulder, Colorado. That facility contained all the measurement station components and a process chromatograph.

The process chromatograph was used for gas analysis of vaporized LNG samples. The chromatograph was insensitive to hydrocarbons above $C_{4}$, so it proved to be inadequate for most of the project requirements.

LNG samples could be taken from two locations within the test section or from the weigh tank. One sample tap was an adjustable probe using a dual pitot tube arrangement positioned so the tube axis was parallel to the flow stream with one inlet directed upstream and the other downstream. The vertical positioning of the pitot probe was adjustable and could traverse the flow stream so that a sample could be taken at any point across the inside pipe diameter. See section 2.2 , figures 2.2 .27 and 2.2.31 for general schematic of LNG flow facility at NBS Boulder, Colorado.

LNG from these sample taps was vaporized in an air or water heat exchanger and the pressure reduced to approximately $0.2 \mathrm{MPa}$ before entering the gas line supplying the chromatograph, calorimeter and gravi tometer.

Flow rate was variable from 150 to 760 liters per minute by means of a variable speed pump. Temperature control was provided with a liquid nitrogen heat exchanger. Pressure was dependent on temperature, flow rate and valve position up to maximum of 0.69 MPa. Helium gas was also used to pressurize the LNG. By maintaining constant pressure and temperature within the flow facility, it was possible to run with no venting. This condition ensured the constant LNG composition which was required to test the various elements.

The weigh tank was mounted on three load cells. Output from the three cells were summed to give a total reading of the change in weight in the tank. To reduce the flow disturbance on the load cells to a minimum, weight measurements were always made emptying rather than filling the tank.

LNG tests. Tests of each of the measurement station components were run on the LNG flow facility during 1975. These tests were designed to show that each of the separate components was functional and responsive to changes in flow rate, temperature, pressure, density and composition.

The vortex shedding flowmeter was unaffected by the wide variations in the LNG composition and continued to function properly throughout the test program. The vortex shedding flowmeter properly responded to all changes in flow rate.

Individual tests on the densimeter, calorimeter and gravitometer proved much more difficult than on the flowmeter. Densimeter output was being compared to calculated densities using the compositions obtained from vaporized LNG gas analysis. Those density calculations were performed using the extended corresponding states model as modified by McCarty [7]. See also sections 2.4 .3 and 2.4 .4 of this manual.

Calorimeter and gravitometer indications were also being compared to values calculated using the LNG composition. The calculations were performed following the procedures specified by Caldwell [8].

One of the main reasons that tests on the densimeter, calorimeter and gravitometer were difflcult was related to getting and analyzing the gas sample. While sampling is a critically important consideration in the application of the measurement station approach, it is not unique to that concept. In fact it is equally important to all measurement methods now in use. Since the samplinf problem was not unique to this project, an extensive investigation was not included within the scope of work. At the same time, however, it was not possible to fulfill the requirements of the project without spending considerable effort in the sampling area.

Fortunately another project was started within NBS specifically designed to develop the required sampling and gas analysis techniques. Facilities and personnel from the sampling project wre. utilized in some of the work on this project, and, when the sampling project was ready for rlalo testing the two projects were combined.

The results from the combined projects proved to be of great benefit to both projects. significant findings were: 
1. all LNG mixture fraction components must be shown, rather than assumed, to be absent when analyzing gas samples;

2. flow rate in the sampling tap must be above a minimum value;

3. laboratory sampling criteria are not directly transferable to field type measurements;

4. any one of several different vaporizer techniques can be used;

5. some method of time averaging the vaporized sample must be provided.

A complete description of the sampling project has been reported by Parrish [9], and an edited version is included as section 2.2 of this manual. The reader is referred to these sections for specific details. Ironically, the net result of the investigations demonstrated that the pitot tube sampling scheme originally installed in the LNG flow loop when used to supply gas to the calorimeter plus the gravitometer was an adequate sampling system. This may have been fortuitous, since the flow rate required to run both instruments was only about one half Parrish's [9] recommended 20 standard liters per minute minimum value. Therefore, it is recommended that Parrish's procedure be followed in any future design. The flow rate required by the calorimeter alone was not sufficient to provide good results, however. The measured heating value decreased from $41 \mathrm{MJ} / \mathrm{m} 3$ to that of methane $37.8 \mathrm{MJ} / \mathrm{m} 3$ when the flow rate was reduced to only that required by the calorimeter. Increasing the flow to higher values did not affect the measured heating value. The time response of the instruments also provided the time averaging required.

Parrish [9] found that a simple side tap flush with the inside wall of the flow line was somewhat better than a pitot tube tap. He found that there were fewer erroneous gas analyses with the side tap. In this project, no difference could be found between the two tap designs. The side tap is much simpler, so it would seem to be the logical choice for future work.

Integration of the individual elements of the measurement station was completed during 1976-1977. The method used for the integration was to bring all signals to a minicomputer, where the required data reduction was completed. Output from these tests was simply printed on an on-line printer. Alternative data handling schemes are numerous and include telemetering the data to a central location or storing the data on some mass storage device, such as punched paper tape, magnetic tape, discs, etc. for later computation. No attempt was made to include these devices. Each is dependent on user preferences and requirements, but each could be used with no adverse effect on the end result of the measurement.

Densimeter and flowmeter frequencies were calculated by bringing total counts into the computer and dividing by the elapsed time. Density and flow rate were calculated from equations derived from tests in liquid nitrogen. Nitrogen data were used for these calculations because they were obtained on the liquid nitrogen flow facility, which is much more accurate than the LNG flow facility. The two flow facilities operate on similar principles, but the nitrogen facility is of a different design, which minimizes the adverse affects of environmental conditions, tare weight and restraints. Consequently, it has higher accuracy capability. Also, it was necessary to know the LNG density in order to determine new values on the LNG facility. Since there was originally considerable uncertainty in the gas analysis and consequently the density calculation, it was decided to use the nitrogen values. This was changed in later tests completed after some sampling and analysis problems were resolved.

The gravitometer and calorimeter both had analog outputs which were entered into the computer, from which specific gravity and heating value were calculated. For these tests the following information was printed:

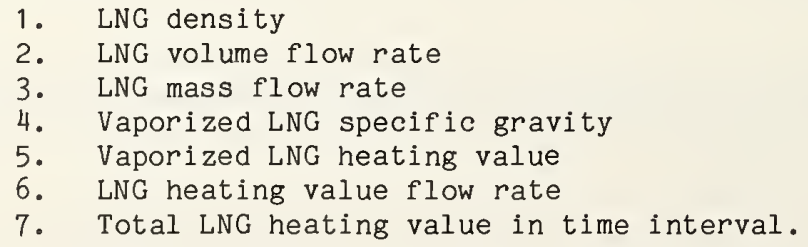

During the tests in which sampling and analysis problems were being investigated, the uncertainty in the integrated values was large, perhaps 3 to 4 percent. After these problems were resolved, the uncertainty was reduced to approximately \pm 1 percent. LNG flow facility uncertainty presently 
prohibits establishing measurement station uncertainties much better than this 1 percent value. Since It was not possible to accumulate large amounts of data, therefore, a value for the precision of the measurements was estimated from other tests using similar equipment. Those estimates were compared to the new measurements and found to be reasonable.

Figure 2.5.6.4 graphically shows the affect of improvement in the sampling and analysis that was made during the LNG tests. The deviation shown in the figure is between the calculated and the measured heating values with the measured value as the reference or zero line. As improvements were made, the deviation progressively approached zero.

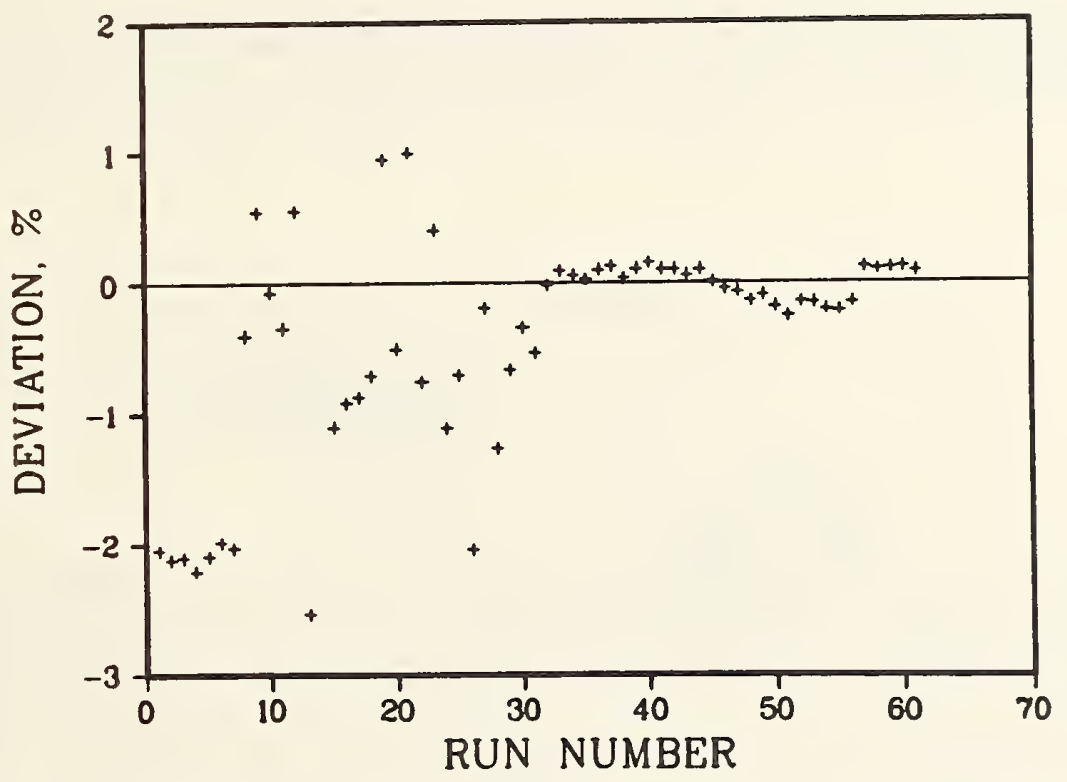

Figure 2.5.6.4. Effect of gas handling and analysis on calculated heating value showing progressive improvement in techniques.

\subsubsection{Flowmeter Scaling}

The two-inch flowmeter used in the component integration work also served as the starting point for the flowmeter scaling work. The two-inch size was the smallest flowmeter that was fabricated following the same design criteria as the larger flowmeters. Therefore, the scaling parameters would not have to include allowances for changes in design. That size also was a very convenient size to test on the NBS liquid nitrogen, liquefied natural gas and water flow facilities. All of those test data provided a good base line to start the scale-up procedure.

For the first two steps in the scale-up, the flowmeter size was doubled to four inch and then to elght inch. Flow range of even the four-inch flowmeter was much larger than the capabilities of the two NBS cryogenic flow facilities. Since no other operating cryogenic flow facilities large enough to test the flowmeters were available locally, alternative methods of testing had to be adopted.

In the absence of a conventional calibration facility, it was decided to use the capabllitles of existing LNG facilities for test purposes. That was done by installing the flowmeters elther in LiG feed lines to vaporizers or in LNG ship unloading lines. The liquid measurement was then comparrd with the gas measurement made after the LNG had been vaporized or to the rate determined by changes 1 n the tank levels. Using those methods of flowmeter testing allowed the flowmeter reading $1 \mathrm{n}$ the Lis to be directly compared to the customary industry methods of measurement.

Feasibility of the liquid-gas comparative test procedure was demonstrated with a four-inch flowmeter at a LNG peak shaving facility. The same facility was used to test the elght-1nch flowmeter. The same procedure was followed in the third scale up to a twelve-1nch flowmeter at a lic base 1 on facility. 
The final scale-up to a thirty-two inch flowmeter was tested at another LNG base load facility. In those tests, the flowmeter output was compared to the LNG volumes determined from storage tanks.

Four-and eight-inch flowmeter tests. The four-inch flowmeter was tested in the NBS water flow facility over the range of flow rates expected at the LNG peak shaving facility. The flowmeter was also tested in the NBS liquid nitrogen flow facility where the lower portion of the flow range could be evaluated. That flowneter test was critically important to the overall program since it was the only size that could be tested in both the peak shaving facility and the NBS cryogenic flow facility. Therefore, it was the only one that could be used to directly check the concept of comparing liquid and gas phase flow measurements.

Figure 2.5.6.5 shows the results of the water and liquid nitrogen tests on the four-inch flowmeter. The range of the liquid nitrogen test was limited by the capacity of the flow facility but was sufficient to determine a meter factor. There was more than normal scatter in the data from the liquid nitrogen test because of the combination of low flowmeter resolution and small flow facility capacity.

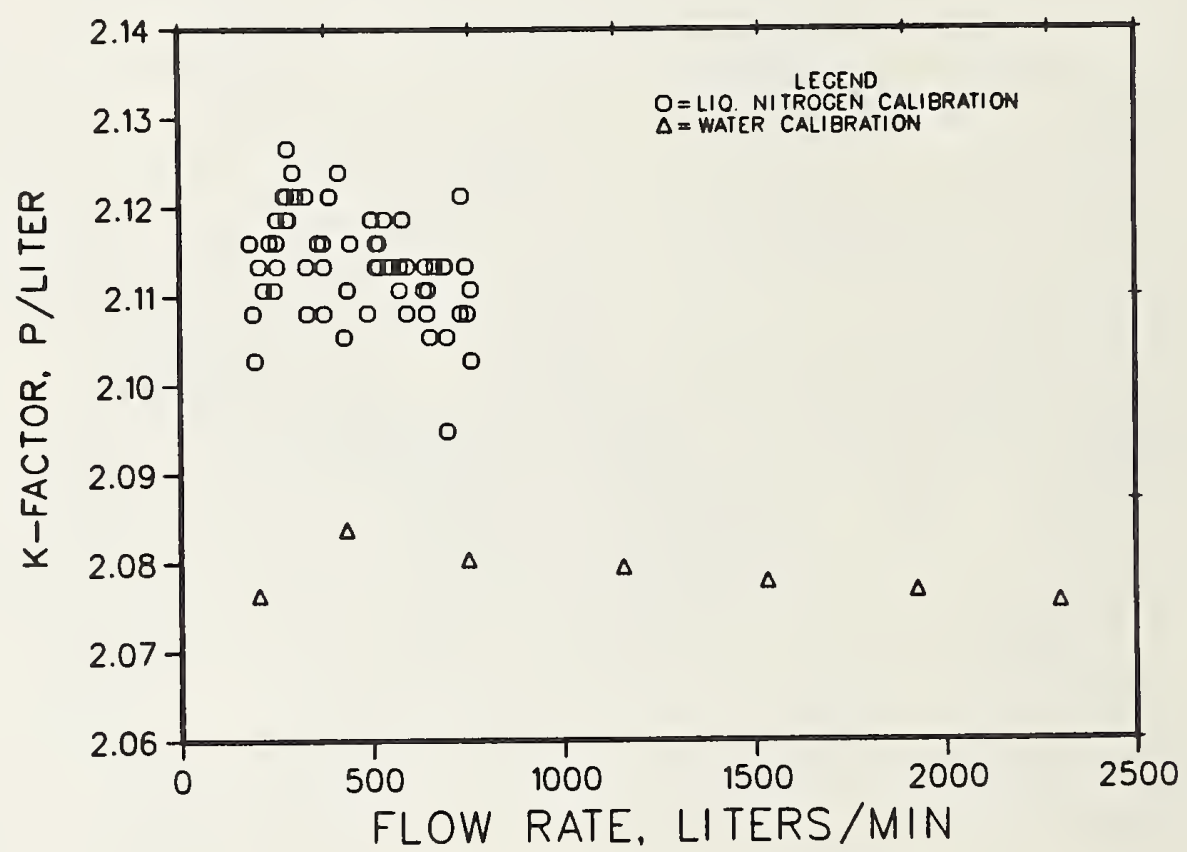

Figure 2.5.6.5. Four-inch vortex shedding flowmeter test results in liquid nitrogen and water.

In the peak shaving facility, LNG was pumped out of a storage tank through the liquid flowmeters, through the vaporizers, through the gas flowmeters and then into the gas transmission line. Just downstream of the gas flow measurement station provisions for taking a continuous gas sample were provided. The gas sample could be directed to a calorimeter and gravitometer. This was not always done, however, since the same instruments were also used to monitor the transmission line gas. Spot samples could also be taken upstream of the gas measurement station for later analysis, and this was done during each test.

The facility gas measurement consisted of two sixteen-inch and one twelve-inch orifice flowmeter runs. As part of this program, one of the sixteen-inch runs was water calibrated at the NBS facility in Gaithersburg, Maryland. Two nine-inch plates were tested in the one meter run. Those calibration data were used in the flow calculations for that meter run. 
An ultrasonic flowmeter had previously been installed in the peak shaving facility by its owner. That flowmeter was not part of this program, but data were collected during some of the tests for comparison with the vortex shedding flowmeter for informational purposes.

Flowmeter tests were made by recording the output of the vortex shedding flowmeter along with the LNG pressure and temperature on magnetic tape. The gas flow data consisting of line pressure, orifice pressure drop and temperature were all recorded on the peak shaving facilities circular chart recorders. Gas flow rates were calculated from those chart recordings following the procedures specified in AGA Report No. 3 [10]. If more than one orifice flowmeter was in use, a combined flow rate was calculated.

Gas equivalents of the LNG flow rates were calculated from the vortex shedding flowmeter using equation (3) and the meter factor previously determined. For the four inch flowmeter the meter factor determined in the Iiquid nitrogen test was used. For the eight-inch flowmeter a meter factor calculated from the water calibration was used.

$$
Q_{L}=(f / K)\left(\rho_{L}^{\prime} \rho_{G}\right)
$$

where

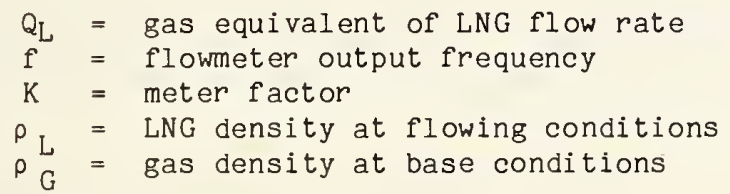

Recordings of the LNG temperature, pressure and flowmeter output were made every 10 seconds. Five individual temperature and pressure recordings were averaged and used in the density calculations. The LNG density was calculated from the gas analysis of the spot gas samples taken during each test. That analysis combined with the pressure and temperature data were used in the density calculation described by McCarty [7] as an extended corresponding states method. As one check on the correctness of the gas analysis, the heating value and specific gravity were also calculated following the procedures specified by Caldwell [8]. Whenever possible, the calculated values were compared to values measured on the peak shaving facilities calorimeter and gravitometer. As mentioned above, the measured values were not always available, but, whenever they were, they agreed with the calculated values to within the uncertainty limits specified by the instrument manufacturers.

Flowmeter frequency was determined by dividing the difference in the recorded flowmeter output by the elapsed time between selected recordings.

Comparisons between the $l$ iquid and gas flow measurements were made during periods of constant flow rate. Those periods were selected from the gas flow recordings. The comparisons were made by calculating the percentage deviation between them using equation (4).

$$
\text { Deviation (percent) }=100\left(Q_{L}-Q_{G}\right) / Q_{G}
$$

where

$$
\begin{aligned}
& \mathrm{Q}_{\mathrm{L}}=\text { gas equivalent of } \mathrm{LNG} \text { flow rate } \\
& \mathrm{Q}_{\mathrm{G}}=\text { gas flow rate from orifice flow measurement }
\end{aligned}
$$

As previously stated the four-inch flowmeter was the first to be tested in the peak shaving racil 1 ty. The facility piping was all eight inch, and the flowmeter test section had to be made to 11 between existing eight-inch flanges. After installation, the test section was wrapped with insulat1on and a plastic vapor barrier.

A very brief test of the four-inch flowmeter was finally run during a vaporizer test in December. 1975. The four-inch test section significantIy reduced the facility output, so the vaporizer test wis the only test permitted before the test section had to be removed and the eight-inch test section installed.

Since that test was the only opportunity available to test the four-inch flowmeter. an attmpt wi made to maximize the data obtained. Therefore, a test plan was adopted to give a range or rlow rates with some indication of reproducibility rather than collect all data at a single flow rate. This iy of test plan did not permit waiting long periods of time to ensure equilibrium was achleved, bul in 
almost all instances sufficient time was available for adequate stability. The biggest problem was achieving temperature stability at the gas phase orifice flowmeter. At the two lowest flow rates the temperature never stabilized as well as desired. Aside from that problem the test went very well, and some valuable data were obtained.

To aid in the data reduction of the pressure data from the gas orifice flowmeter, the recorder chart was driven at $1 / 2$ revolution per hour. The temperature recording chart was driven at the customary one revolution per day.

Results obtained in that test are shown in figure 2.5.6.6. One additional point at the lowest flow rate was attempted but is not included in the figure because the gas temperature at the orifice never stabilized. By taking an average temperature, the data point agreed with the other points, but much more uncertainty is associated with that point and hence is not included.

The results shown in figure 2.5.6.6 were calculated by comparing the flow rates indicated by each flowmeter. Since both meters are rate meters, this method of calculation eliminated the additional uncertainties associated with totalizing.

As can be seen in figure 2.5.6.6, there was a -1.03 percent bias between the two methods of measurement with a range of \pm 0.5 percent about the mean value. The data points lie within the uncertainty of the measurement method which was larger than normal because of the short test duration. The reproducibility of the data appeared reasonable, especially since the tests were of such short duration. After the LNG tests were completed, the four-inch flowmeter was retested on the NBS liquid nitrogen flow facility. The average meter factor determined in that test was within 0.12 percent of the meter factor determined before the LNG tests.

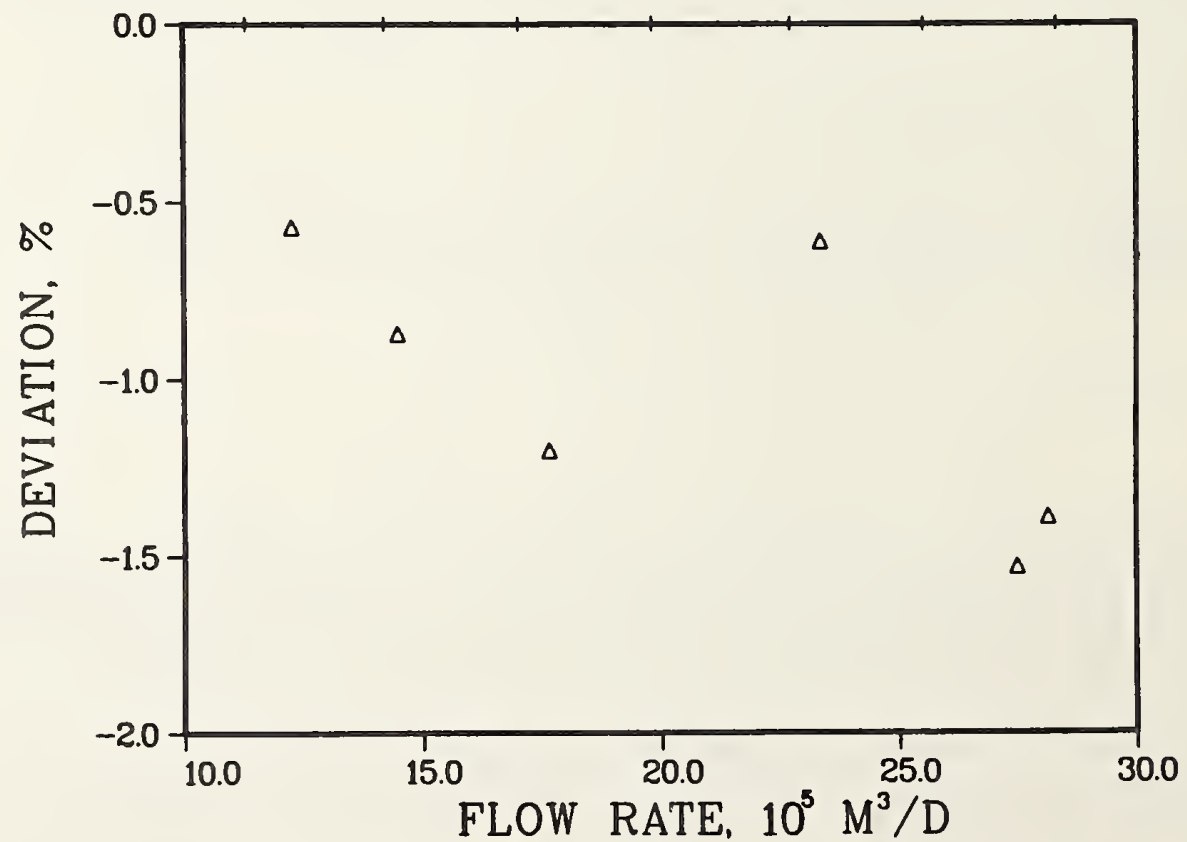

Figure 2.5.6.6. Four-inch vortex shedding flowmeter test results in LNG peak shaving facility. 
At the conclusion of the December 1975 test, the four-inch vortex shedding flowmeter was removed from the LNG line and an eight-inch vortex shedding flowmeter installed in the same location. Also, all the pressure and temperature instrumentation was transferred from the four-to the eight-inch test section. Since the eight-incb flowmeter matched the facility piping, it could be left installed without reducing the output capability. Therefore, it was fully foam insulated like the rest of the plping. In this flowmeter a redundant sensing technique was included so that if one sensor failed, a second one was already installed and ready. No sensor failure was experienced, but data from both sensors were collected throughout the test.

Like the four-inch flowmeter, the eight-inch vortex shedding flowmeter was calibrated in water before belng installed in the LNG 1 ine. The meter factor determined in the water test along with the knowledge of how the meter factor changes with temperature was used to calculate the factor used in LNG. The water calibration results are shown in figure 2.5.6.7.

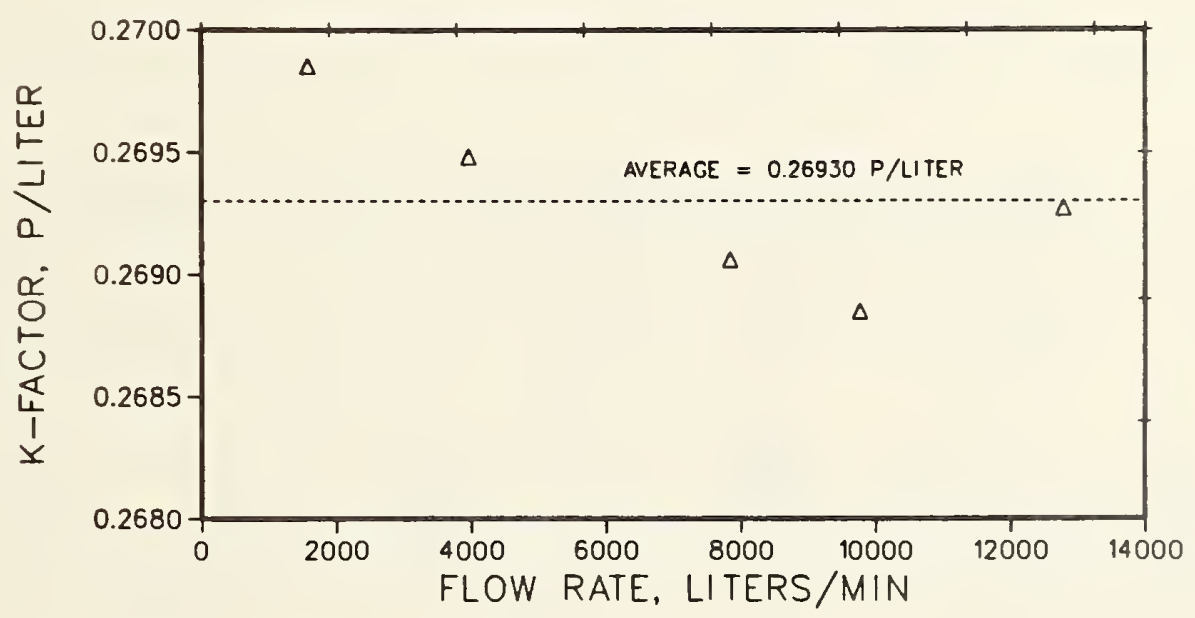

Figure 2.5.6.7. Eight-inch vortex shedding flowmeter test results in water.

LNG tests were run with the eight-inch flowmeter whenever LNG was vaporized during the remainder of the send-out season of 1975-1976 and 1976-1977. During the 1976-1977 send-out season, new instrumentation was installed on two of the gas orifice flowmeters. The third parallel orlflce flowmeter run was not instrumented, because it was not used in any of the previous tests. The new instrumentation consisted of pressure transducers for measuring orifice pressure drop and line pressure and a thermocouple for measuring the gas temperature. Another thermocouple was also installed to measure the ambient temperature in case the third orifice flowmeter was used. That thermocouple was required to make the manometer correction, which was only estimated in prevlous tests.

All of the gas-flow measurements were recorded on a second magnetic tape. Because of the long distance between the liquid-and gas-flow measurement locations, it was decided to record the data on separate recorders that were triggered to record data simultaneously. During those tests the ouiput from the ultrasonic flowmeter was also recorded on magnetic tape. The recorders were set to record all data every ten seconds throughout the test.

Both differential pressure transducers on the orifice pressure drop measurements were cal 1 brated us 1 in, water manometers provided by personnel at the peak-shaving facility. The clrcular charts wera calibrated simultaneously, both before and after the send-out season. 
Pressure transducers measuring line pressures were calibrated with a dead weight gauge at the facility either before or after the series of LNG tests.

Data were recorded during four send-outs with the fully instrumented meter runs. Two send-outs were during vaporizer tests normally run at the beginning of the heating season, and two were during demand send-outs.

LNG flow rates were calculated following the same procedures used for the four- inch flowmeter, except a different meter factor was used. Gas flow calculations were also done the same way, except the data recorded on magnetic tape rather than the circular charts were used. An exception to this procedure was necessary when the uninstrumented orifice flowmeter was in use. During those times the orifice pressure drop data were read from the chart recording, but the line pressure and temperature were taken from the tape recorded data on the adjacent meter run. It was necessary to use the chart recording on two of the tests.

Plots of the flow rates were used to select time intervals in which the flow rate was relatively constant. During those intervals an average for both the gas measurements and the liquid measurements was calculated. The deviations of the liquid measurements from the gas measurements were then calculated using those averages. Figure 2.5 .6 .8 shows all the results thus calculated for the tests run during the 1976-1977 send-out season.

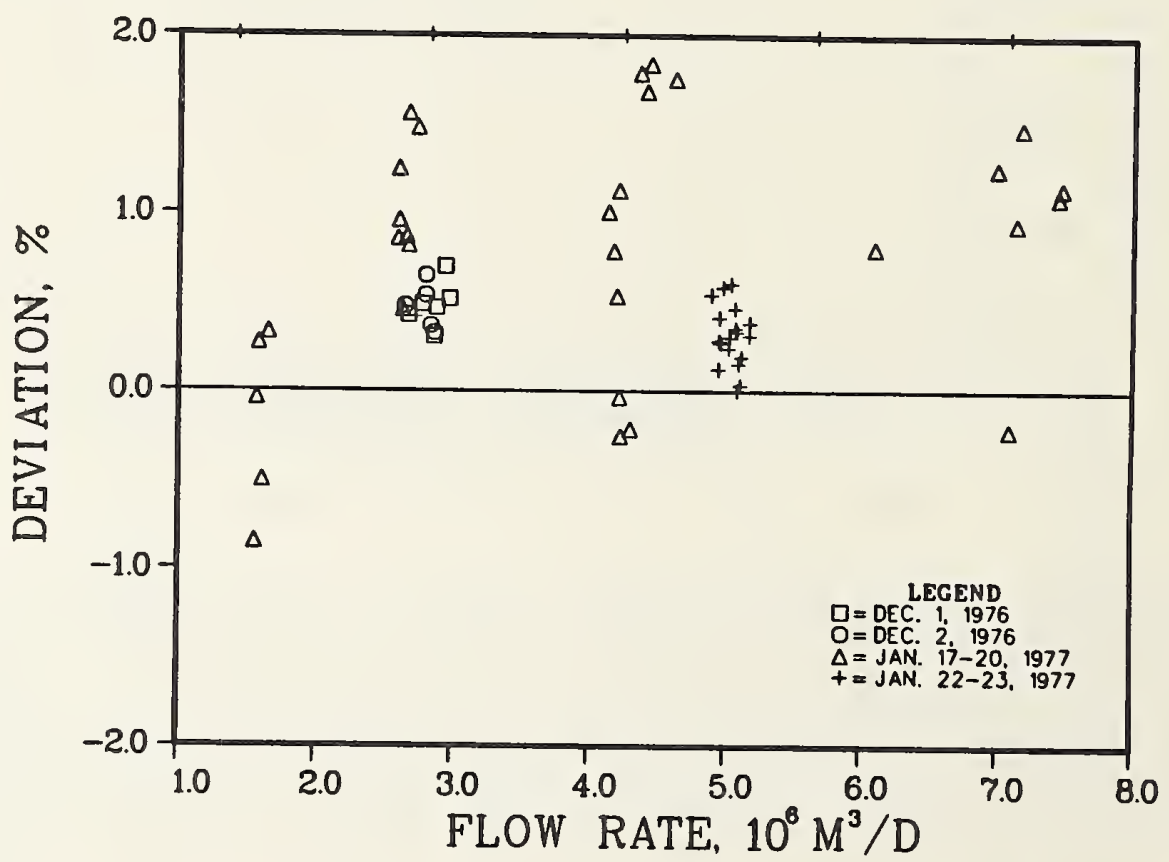

Figure 2.5.6.8. Eight-inch vortex shedding flowmeter test results in LNG peak shaving facility during second test series.

In general the data obtained during the vaporizer tests on December 1-2, 1976, and the demand send-out of January 22-23, 1977, show good agreement with each other and have relatively small scatter. During those tests, flow rates were held almost constant compared to the tests on January 17-20, 1977. The tests on January 22-23 did require the use of two orifice flowmeters, however, and one of them was the one that was not instrumented for recording on magnetic tape. The January 17-20, 1977, test required all three orifice flowmeters, including the one not recorded on magnetic tape.

Data on the ultrasonic flowmeter were also obtained during those tests, and the results indicated that with proper adjustment it may also be a viable choice for LNG flow measurement. 
Twelve-Inch Flowmeter Tests. In order to test larger size flowmeters, it was necessary to relocate the testing to a different facility. The largest line size available where comparisons could be made between liquid-and gas-flow measurements was at a new base-load import terminal. That facility was made avallable to the project for testing a twelve inch flowmeter. It was also possible to include a densimeter in the test section at that site. The test section was placed in the LNG feed line to the vaporizers just downstream of the secondary pumps. At that location, the LNG was at a pressure of approximately $9.65 \mathrm{MPa}$, which was more than twice the pressure of any prior tests.

In that installation, some of the gas was diverted for use as fuel within the facility after vaporization. Consequently the comparisons between the liquid-and gas-flow measurements had to be adjusted for the diverted gas. In all other respects the actual comparisons were performed using the same procedures used in the original four-and eight-inch flowmeter tests. That is, the gas flow measurements were calculated from facility circular charts rather than being recorded on magnetic tape. The charts were on six parallel twelve-inch orifice runs and were on $24-h o u r$ rotation.

Heating value and specific gravity measurements were made and recorded on facility instrumentation. Gas samples were taken from a tap on one of the orifice runs. These samples were analyzed, and the compositions used in density, heating value and specific gravity calculations were the same as was done for the previous tests.

In all previous flowmeter work completed on this project, a wafer-style flowmeter was used. The waferstyle flowmeter was installed in a pipeline by bolting between flanges. There was essentially no upstream or downstream piping attached to the flowmeter itself. Alignment within the pipe was assured by fitting the flowmeter into a recess machined into the flanges.

The twelve inch flowmeter was made directly in a piece of facility piping. The bluff body vortex generator was welded inside one end of a 6.1 meter length of pipe. That was the first time a flowmeter of that particular method of fabrication had been tested in any of the programs at NBS. Theoretically there should be no difference in the performance between the two methods of fabrication. The relationship between the bluff body and the pipe diameter determined the flow characteristics. The reasons the flowmeter fabricaton had to be changed were the larger size and the desire to eliminate the flanges in the high-pressure LNG pipeline.

The flowmeter was water calibrated at NBS-Gaithersburg. Figure 2.5.6.9 shows the results of that calibration. In figure 2.5.6.9 it can be seen that the shape of the calibration curve is very similar to that of the eight-inch flowmeter (figure 2.5.6.7), but the variation in meter factor with flow rate was more pronounced in the larger flowmeter. With previous flowmeters it was possible to use an average meter factor over the range of flow rates without introducing large errors. Although the potential for error is larger, the same procedure was also used for the twelve-inch flowmeter.

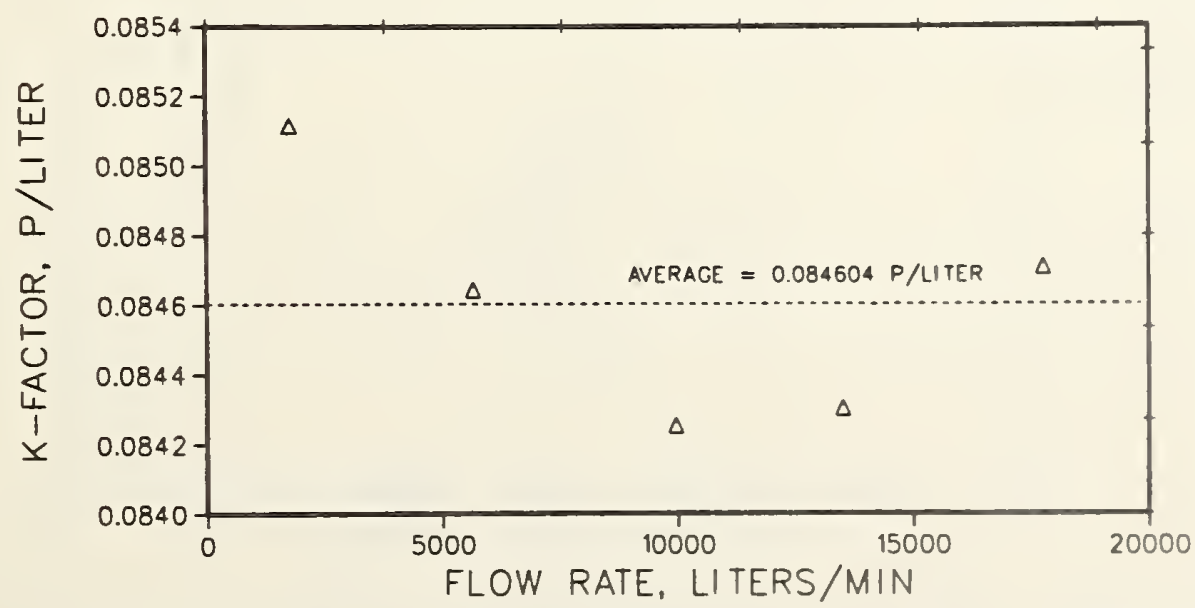

Figure 2.5.6.9. Twelve-inch vortex shedding flowmeter test results in water. 
Both the flowmeter and the densimeter were installed in one length of facility piping. The densimeter was installed within $30.5 \mathrm{~cm}$ of the inlet end and the flowmeter within $30.5 \mathrm{~cm}$ of the outlet end. A retractable densimeter was used in this installation, so the flowmeter water calibration was performed both with the densimeter inserted and retracted. No difference in flowmeter performance could be detected relative to the densimeter position.

Four flow tests were run on the flowmeter during 1979. During each test, data from the flowmeter, densimeter, thermometer and pressure transmitter were recorded on magnetic tape every 10 seconds. Those data were then averaged over five readings for comparison to the gas flow measurements. Periodically, gas samples were drawn from the vaporized gas stream and analyzed. From the composition thus determined, density, specific gravity and heating value were calculated. The fuel gas flow and temperature were calculated from facility strip-chart recordings. The flow rates were corrected for temperature, using the method described in [10] and added to the pipeline measurements.

Potential for errors in the fuel gas flow rate was much higher than other gas flow calculations, because the measurement was not intended by the terminal operator to be highly accurate. Consequently, the instrumentation was not as carefully documented as it might have been. Adding to the uncertainty of the measurement was the fact that during some of the tests, the recording was very noisy. During the periods of noisy chart recordings, an "eyeball" average value was used. The net effect of errors in that measurement is rather small, however, since the total fuel gas was about 2 percent of the total gas flow. So even if the fuel gas measurement was in error by 10 percent, the affect on the ultimate comparison between the gas and liquid flow measurements would only be about 0.2 percent.

Results of the tests are shown in figure 2.5.6.10. The data points in the figure are the deviations between the liquid measurement and the gas measurement, with the gas measurement being taken as the reference or zero line. Each point was determined during a period of stable flow, but the time interval over which the point was determined varied from a few minutes to several hours depending on the flow conditions.

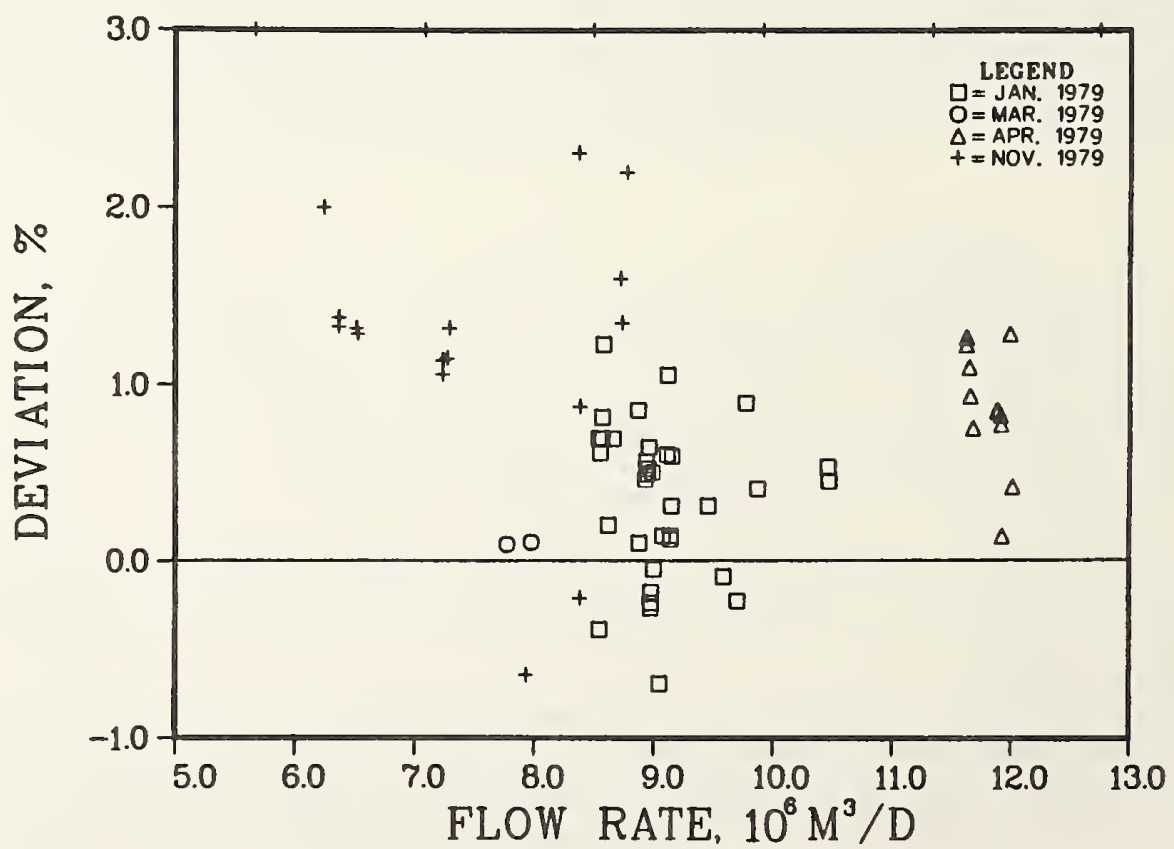

Figure 2.5.6.10. Twelve inch vortex shedding flowmeter test results in LNG at import terminal. 
With the exception of the November tests, the flow calculations were made following the same procedures used in the four-and eight-inch flowmeter tests. For the November tests, the liquid density was determined from the densimeter and the specific gravity read from facility recordings. In all previous tests those two values were calculated from gas compositions. The densimeter calibration constants used were determined during the March test. This change in calculational procedure appears to have worked reasonably well and is, of course, more in line with the objectives of the overall program.

The facility fuel gas measurement was not operating during most of the November test, so a complete comparison could not be made. Comparing the measurements without the fuel gas correction for that test with those from previous tests indicate the overall agreement should be the same.

The range of flow rates shown in figure 2.5 .6 .10 required from three to six orifice flowmeters in the gas phase measurement. The meter runs were used in such a way that no low orifice differential pressure data were obtained, which helped keep the potential errors to a minimum. In general, the range of the deviation in the data was very good. The total range was less than 2 percent, which is less than the uncertainty in the gas flow measurement alone. It may have been possible to reduce the amount of the scatter in the data by using different techniques in the data recordings, but since the total uncertainty was determined primarily by the uncertainty in the orifice flowmeters, no changes seemed justified. At the time the tests were run, there was very little that could be done to reduce the total uncertainty of the orifice flow measurement.

Uncertainty in the gas flow measurements is composed of \pm 0.75 percent per orifice flowmeter as stated in [10] plus on-site calibration and measurement errors. Table 2.5.6.1 lists the possible magnitude of the calibration and/or reading errors.

Table 2.5.6.1

Affect of Calibration and/or Chart Reading Errors for Errors of $1 / 2$ Smallest Chart Division

\begin{tabular}{|l|c|c|c|}
\hline \multirow{2}{*}{ Parameter } & Smallest Chart Division & $\begin{array}{c}\text { Affect on Flow Rate } \\
\text { Calculation } \\
\text { (Percent/1/2 Division) }\end{array}$ \\
\cline { 2 - 3 } & $\mathrm{Cm}$ & Magnitude & 0.79 \\
Orifice $\Delta \mathrm{P}$ & 0.18 & $0.50 \mathrm{kPa}$ & 0.80 \\
Orifice Pressure & 0.18 & $0.21 \mathrm{MPa}$ & 0.10 \\
Gas Temperature & 0.05 & $0.6^{\circ} \mathrm{C}$ & 0.10 \\
\hline
\end{tabular}

It is normally accepted practice to consider each orifice an independent measurement, therefore, the individual errors can be combined by the root mean square method to get an estimate of the total uncertainty of the measurement. Taking only the \pm 0.75 percent per orifice specified in [10] results in an uncertainty of \pm 1.30 percent for 3 orifices and \pm 1.84 percent when all six plates were being used. If the other errors mentioned above are also combined, the uncertainty increases to over 2 percent when all six plates were in use. A more detailed error analysis of the gas flow measurement would require a great deal of time and equipment and was beyond the scope of this project.

Errors in the liquid flow measurement were difficult to assess since the error in the predicted meter factor cannot be independently evaluated. Since the prediction was still based on values determined from a single two inch and a'single four inch flowmeter that were tested with liquid nitrogen and Lilg, a rather large uncertainty must be assigned to the meter factor. At this time, the uncertainty in that value is estimated to be approximately \pm 0.9 percent. The affect of pressure and temperature errors result in an additional \pm 0.3 percent uncertainty, and the density calculation adds another \pm 0.2 percent. Combining these errors in quadrature results in an uncertainty in the 11 quld measurement of \pm 1 percent.

As can be seen from the data in figure 2.5.6.10, the data generally lie within those error $11 \mathrm{mits}$.

Thirty-two Inch Flowmeter Tests. Full line size flowmeter tests were started at an import terminal $1 \mathrm{n}$ December 1982. The test instrumentation consisted of two flowmeters and a dens1meter. Al1 the instrumentation was located in the $81.3 \mathrm{~cm}$ ship off-loading line. The installation provided the rlnal objective in the scaling tests for the vortex shedding flowmeter. It also provided another tagt or a single path ultrasonic flowmeter and another test of a vibrating plate densimeter. 
There were three nominally 95, 400 cubic meter storage tanks which were used as volumetric references for the flowmeter comparisons. The volume of each of the tanks was determined photogrammetrically and sounding tables prepared with 1.5 centimeter increments $[11,12,13]$. Each tank was equipped with both a segmented capacitance type and a liquid-vapor interface tracking type liquid level gauge.

Before installation of the vortex shedding flowmeter in the LNG terminal, it was water calibrated. The complete calibration report is in reference [14]. Figure 2.5.6.11 shows the results of the water calibration. From the figure it is obvious that the maximum flow rate obtained during calibration was significantly lower than the 208,000 liters/minute LNG flow rates anticipated. The calibration rates were the maximum obtainable, however, and provided the necessary information for projecting the meter factor to LNG service.

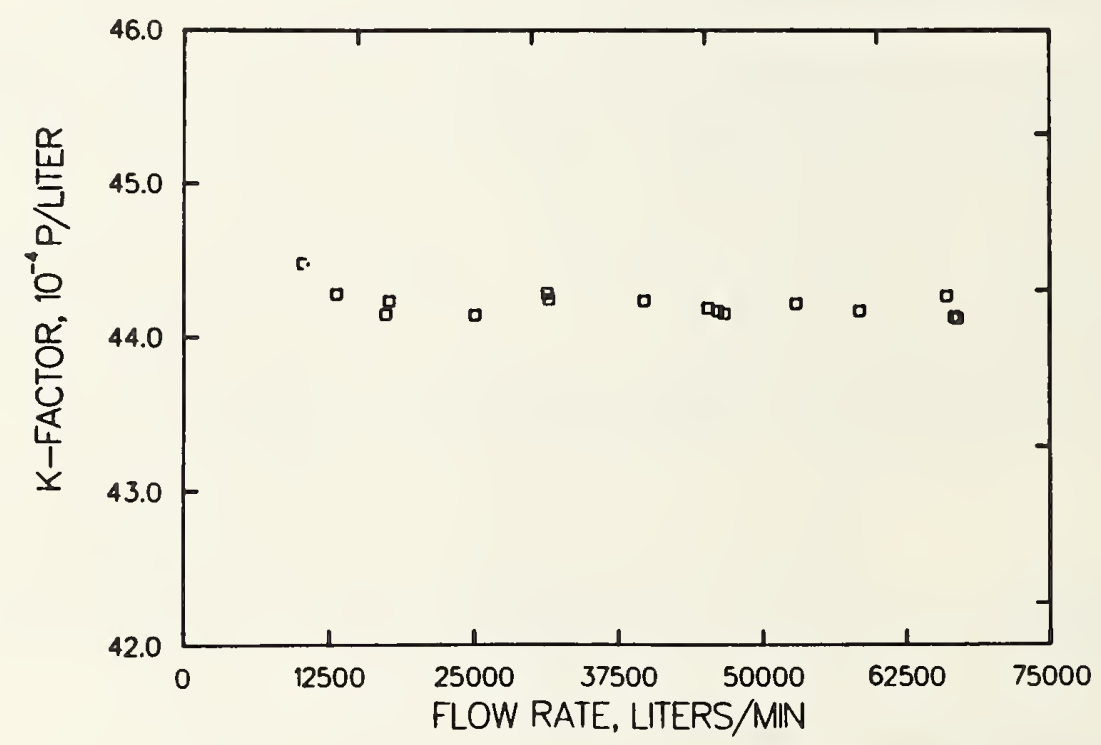

Figure 2.5.6.11. Thirty-two inch vortex shedding flowmeter test results in water.

There was no water calibration of the ultrasonic flowmeter. The transmitters were tested for cryogenic service in the NBS liquid nitrogen flow facility prior to installation in the $81.3 \mathrm{~cm}$ LNG line. Densimeter operation was also checked in the NBS liquid nitrogen flow facility before installation at the terminal.

Both the vortex shedding and the ultrasonic flowmeters have the capability of sensor replacement with the pipeline at operating conditions. That capability was used for replacing one of the two sensors in the vortex shedding flowmeter and for the initial installation of the ultrasonic flowmeter.

The densimeter was also removable under pipeline conditions and was retracted during terminal startup to prevent possible damage from foreign objects.

Some preliminary tests of the vortex shedding flowmeter were conducted at various times during 1982. The tests were run before all the other instrumentation at the LNG terminal was fully operational and were only used to verify that the flowmeter would work in LNG.

During 1983, a total of five tests were run. All of those tests included both vortex-shedding flowmeter data and storage tank liquid level data. Figure 2.5.6.12 shows a typical test with comparative data for the flowmeter and the flow rate calculated from the changing volume in the storage tanks. In each test the flowmeter output was computed using a calculated meter factor based on the water calibration corrected for cryogenic service. 


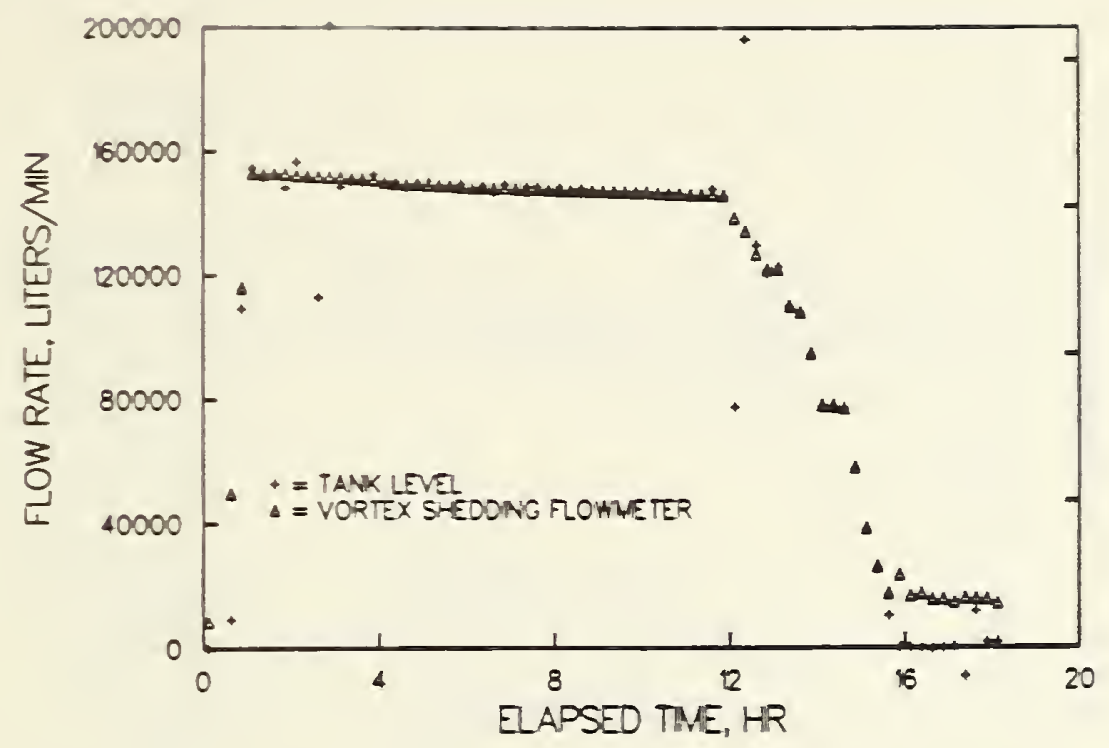

Figure 2.5.6.12, Septeaber 1983 test results showing average flow rates indicated by the rortex-shedding floweter and the tank level change.

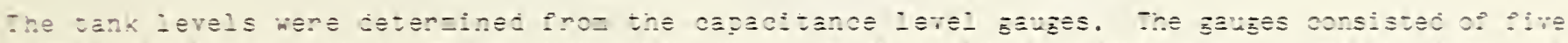

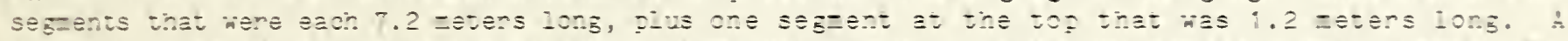

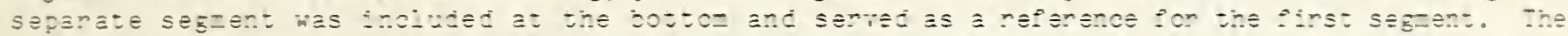

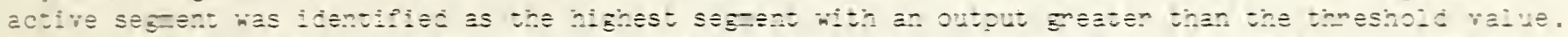

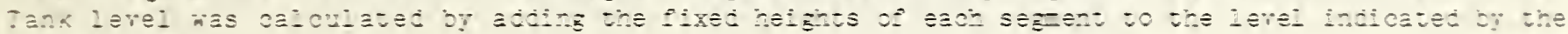

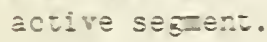

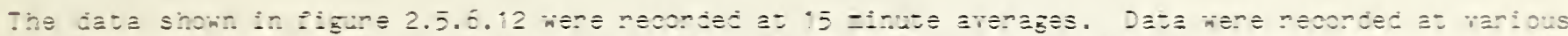

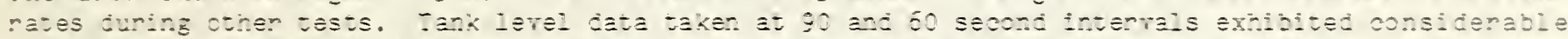

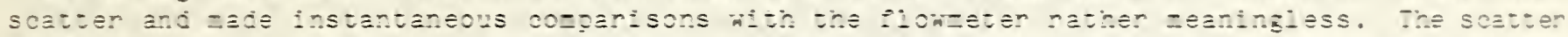

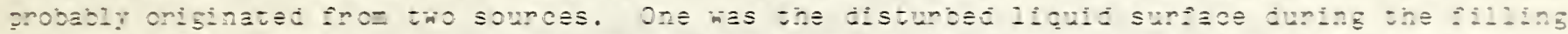

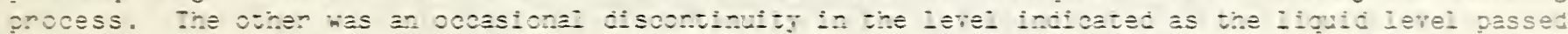

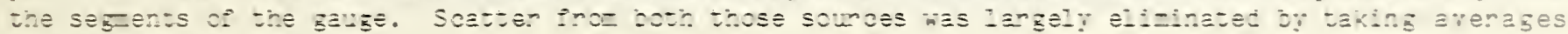

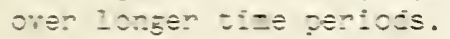

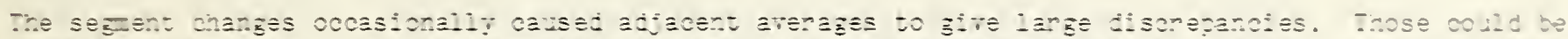

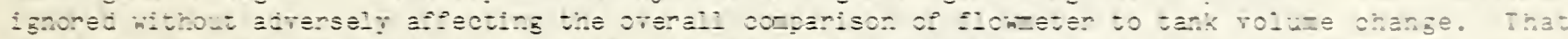

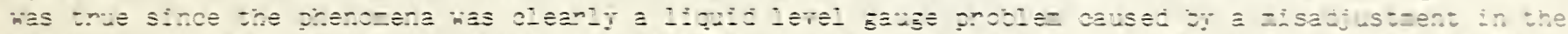

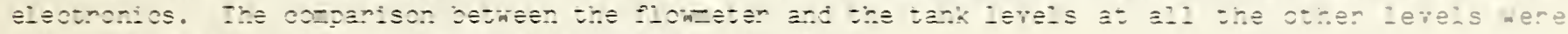
not couproised by those oocestonal enroneous indicaticns.

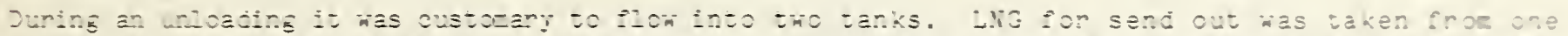

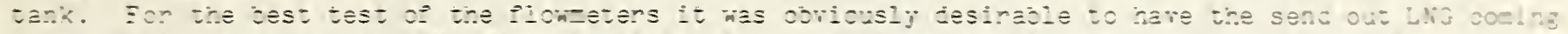

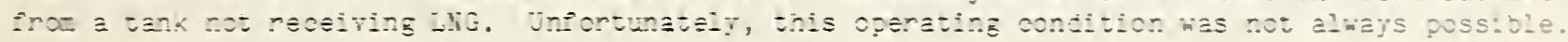

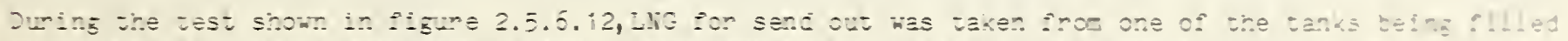

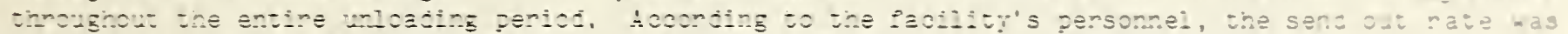

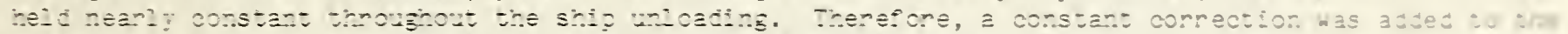

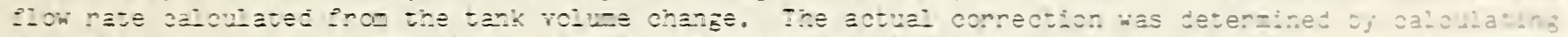

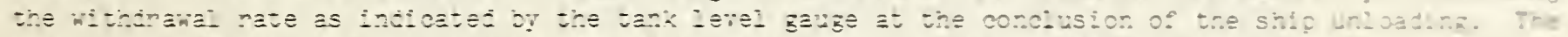
raะล

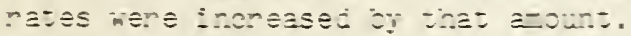

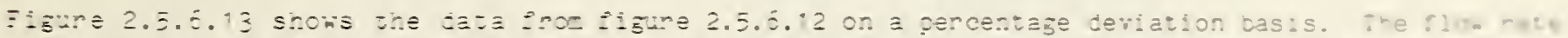

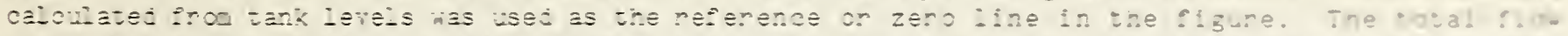

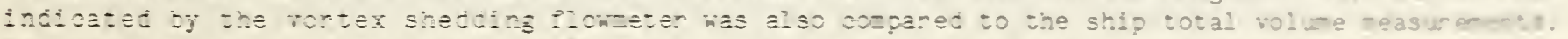

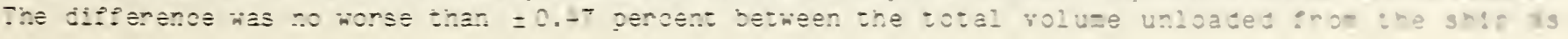




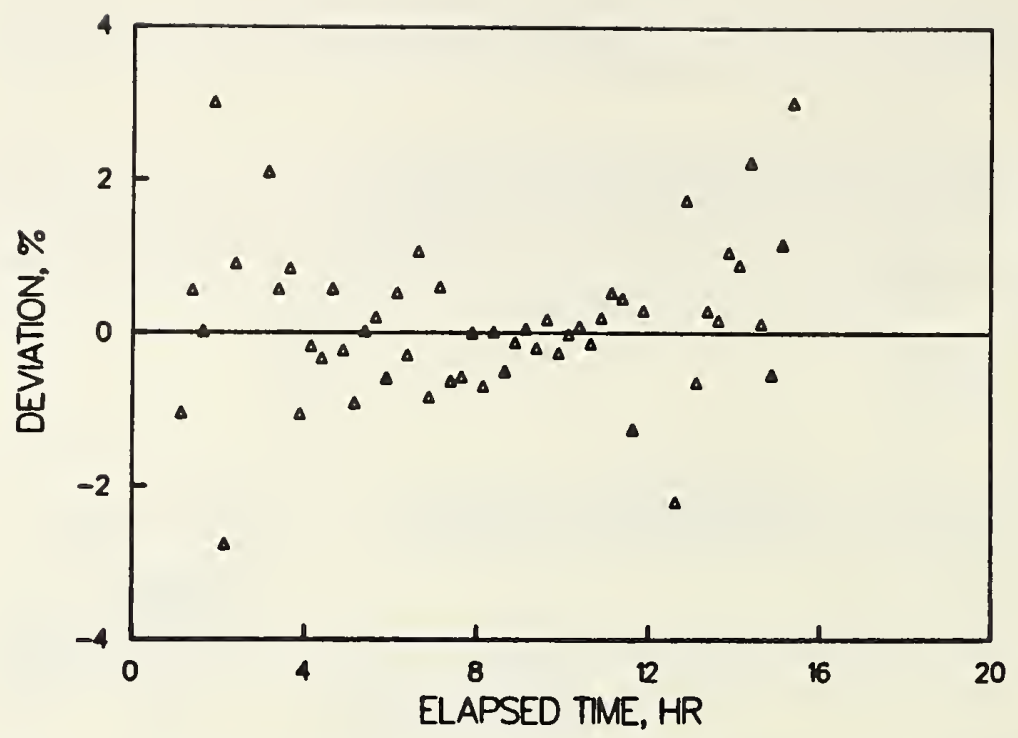

\section{Figure 2.5.6.13. September 1983 test results comparing flowmeter and tank indicated flow rates on a percentage basis.}

determined by ship level gauges and that indicated by the vortex-shedding flowmeter. The plus or minus uncertainty was caused by the low data recording rate, which prevented a determination of the actual start and stop times.

The ultrasonic flowmeter transducers were not installed in the LNG pipeline until August 1983. The first data were obtained during the September test. During three tests, the flowmeter appeared to work normally although with a higher variability in output than expected. This variability may have been caused by mounting the flowmeter too close to a tee section in the pipeline, or it may have been the result of the type of output signal. The output from the flowmeter was a $4-20$ ma current proportional to flow. That gave an instantaneous flow rate indication rather than an averaged rate over some specified time interval. The flowmeter had not been calibrated in water or any other fluid prior to being installed in the LNG terminal. Therefore, comparative data between the flowmeter and tank levels took on a different context than that for the vortex shedding flowmeter, which had been water calibrated.

Figure 2.5.6.14 shows the data from one test run on the ultrasonic flowmeter. The flowmeter signal was converted to flow rate by using data from the first test to determine a meter factor. The same meter factor was then used for the subsequent tests. The larger discrepancies shown after the 10-hour point were caused by incorrect tank readings.

\section{5 .6 .5 Densimeter Tests}

Densimeter operation during the LNG flow tests in the twelve inch diameter high pressure LNG pipeline is shown in figure 2.5.6.15. The density plotted in the figure was a calculated value from gas analysis. Density was calculated from the densimeter output frequency using equation (1) from section $2 \cdot 5 \cdot 6.4$.

$$
\rho=A / f^{2}+B
$$

where

$$
\begin{aligned}
\rho & =\text { density } \\
\mathrm{A} \text { and } \mathrm{B} & =\text { constants } \\
\mathrm{f} & =\text { densimeter output frequency }
\end{aligned}
$$

The data in figure 2.5.6.15 were fit to equation (1) by the method of least squares to determine the best values for the constants $A$ and $B$. The residual standard deviation after the fit was \pm 0.2 percent. 


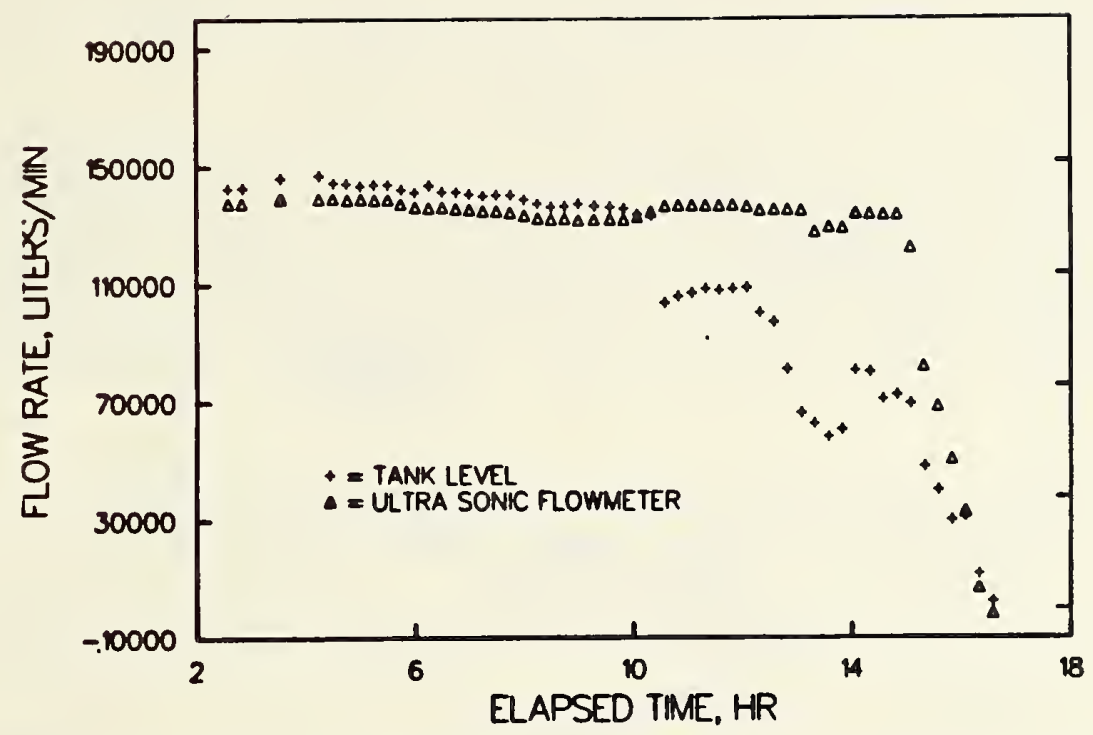

Figure 2.5.6.14. Ultrasonic flowmeter performance in LNG.

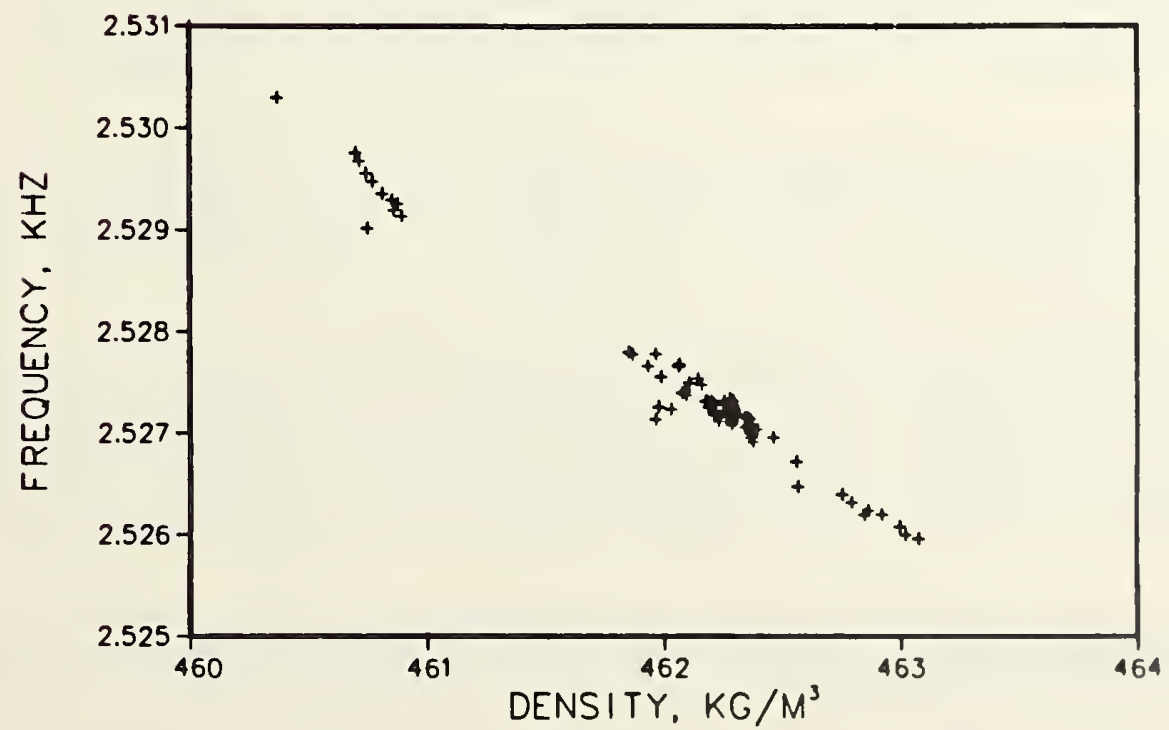

Figure 2.5.6.15. Densimeter test results in LNG at 1 mport terminal. 
The same type of densimeter that was tested in the-twelve inch diameter high pressure pipeline was installed in the thirty-two inch pipeline. Before installation in the LNG facility, it was tested in liquid nitrogen at NBS. The performance was monitored on four occasions as the instrument was cooled down in a bath of liquid nitrogen. The sensing head was left in the nitrogen for several hours each time it was cooled down, and then it was allowed to warm to room temperature. During that portion of the testing, the densimeter was in liquid nitrogen for approximately 43 hours. This test was performed to stabilize the densimeter before actual data-taking was attempted.

The densimeter was then installed in the NBS liquid nitrogen flow loop and tested over an 8 percent range of densities. The results were fit to equation (1) which gave a precision, based on three times the standard deviation, of \pm 0.09 percent with 26 degrees of freedom. Figure 2.5.6.16 shows the calibration data. Figure 2.5.6.17 shows the calibration data plus data obtained on three subsequent days. The densimeter was allowed to warm to room temperature between tests, and all tests were run with the liquid nitrogen flowing in the pipe. There were 47 additional data points taken during the three tests, and they had a bias of 0.06 percent from the calibration curve with a standard deviation of \pm 0.05 percent.

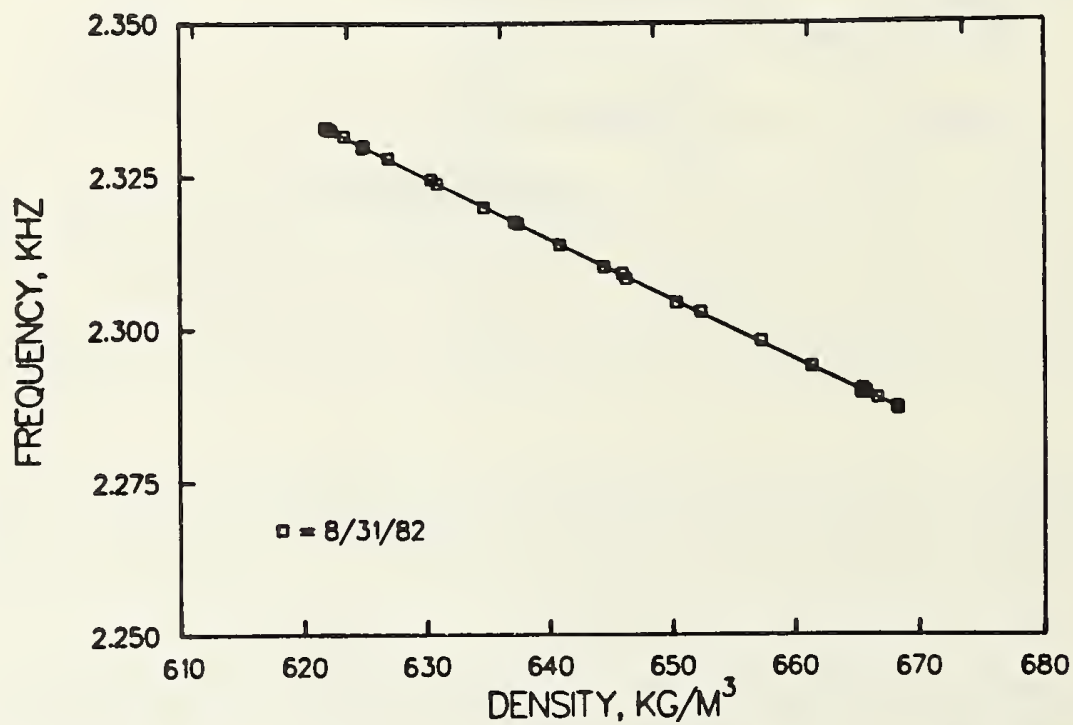

Figure 2.5.6.16 Liquid nitrogen calibration of densimeter.

The densimeter was installed in the LNG terminal, but the sensing head was left out of the pipeline during start up. The first data from the densitometer were obtained during a ship unloading in December 1982. Performance of the densimeter was checked by comparing the frequency output to the calculated density. Density was calculated from composition information using the revised Klosek and McKinley method [7] shown in section 2.4 .3 and 2.4 .4 of this manual.

Normally five LNG samples were taken at different times during the off loading by facility's personnel. The analyses from the various samples were then made available to this project and were used in the calculation of density. There was very little density change during any of the unloadings that were monitored. In fact there was only a very small density change from one unloading to another. The densimeter output also reflected that fact, since there was only a small change in frequency output.

During those tests there was not a large enough change in density to develop a new performance equation for LNG. Also, the density range was too far away from the nitrogen density that was used to develop the previous performance equation to permit accurate extrapolation.

To circumvent those problems some of the liquid nitrogen data were combined with the LNG data to develop a new equation over the whole range. That new equation then reproduced both sets of data to within 0.2 percent. More LNG data would be required to get an accurate representation of the standard deviation, but from the data available it can be stated that the densimeter responded correctly. 


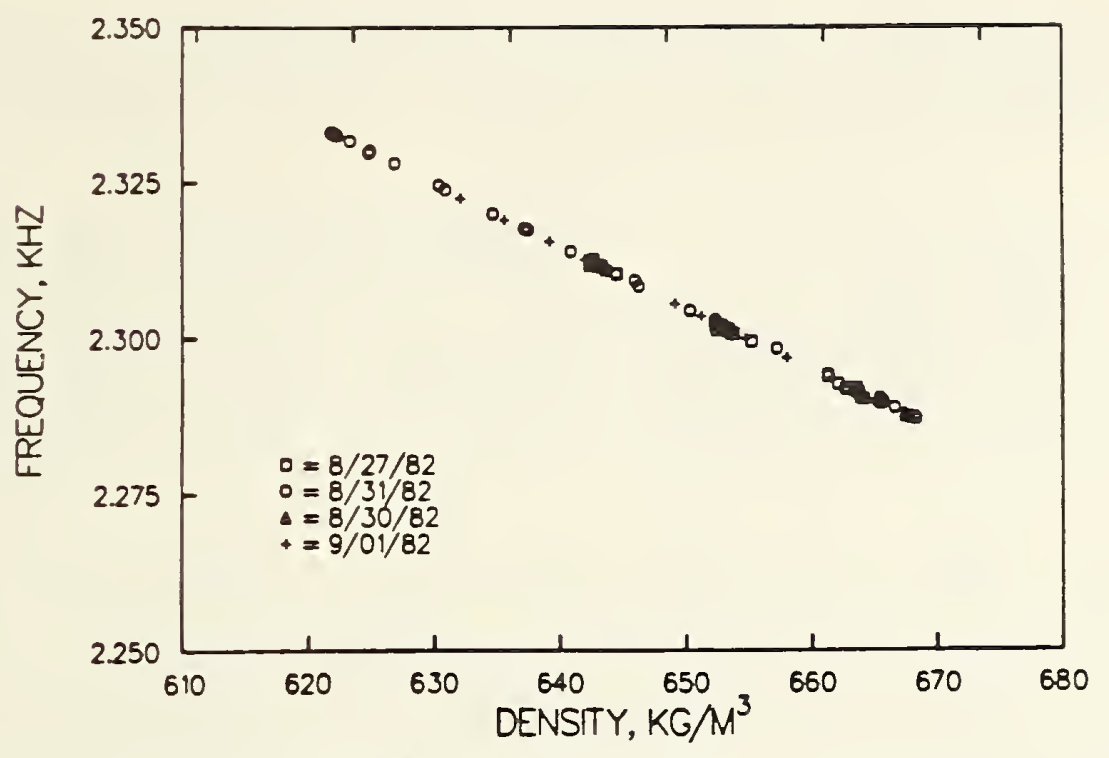

Figure 2.5.6.17. Densimeter performance in liquid nitrogen.

\subsubsection{Conclusions}

Although the flowmeter scaling work took longer than planned, no reason was found to discount the concept of a LNG measurement station. Indeed the measurements required are quite straightforward and have the advantage of being essentially continuous, whereas alternative methods rely on averaging spot samples in measurements.

With the possible exception of the flowmeter, all the components are capable of off-line independent calibration and can be removed for calibration and/or maintenance without shutting down the pipeline. The new flowmeter models also permit replacement of the sensors without shutting down the pipeline.

The overall concept of the measurement station has benefitted from the additional data that has become available during the time this project was in progress. There have been reports published on densimeter performance in LNG [3], LNG sampling [9], LNG density calculational methods [7] and density measurements on hydrocarbon mixtures $[15,16,17]$. All of those data sources have been used in the evaluation and/or cross checking of measurement station elements. Only this project and the sampling project [9] considered flowing systems, but all the data were used to help establish the fact that a measurement station is a viable means of measuring LNG flow.

The uncertainty in the measurement is no greater than the uncertainty in the gas flow measurements which were used for the initial comparisons. Those same gas flow measurements were also used for custody transfer of the vaporized LNG.

The largest uncertainty in the measurement station is in the flowmeter. Three methods of determining large diameter flowmeter performance in LNG were investigated. One method used conventional gas measurement techniques after vaporization to test a flowmeter measuring the LNG before vaporlzation. Another method predicted flowmeter performance in LNG based on a water calibration and the known change in performance between water and LNG of smaller flowmeters of the same type. The third method was to compare flowmeter output to volume change in storage tanks. To date, all methods have worked rather well, but available facilities to test floweters larger than twelve inches using the first method were not available.

The method of predicting performance based on smaller flowmeters has all been based on only two flowmeters; one two inch and one four inch. This is an extremely small sample on whlch to base an extrapolation method. In spite of this fact, the predictions have proven to be reasonably accurate.

Overall, the only lingering problem that might prove detrimental to the measurement stat 10 n $1 \mathrm{~s}$ 1: foreign material in the LNG interferes with the flowmeter or densimeter output. Such a conditlon was never definitely proved to exist, but it is a possibility. If this proves to be a problem $1 \mathrm{n}$ tha future, some provision will have to be made in the flowmeter and/or densitometer to allow $1 \mathrm{t}$ to be corrected while in operation. 
A calibration must be performed on the flowmeter before attempting to place it in service. Table 2.5.6.2 shows the relationship between the manufacturer's stated meter factors and those determined by calibration. From these data it can readily be seen that the meter factor stated by the manufacturer is inadequate for accurate measurement.

Table 2.5.6.2 Flowmeter Meter Factor Summary

\begin{tabular}{|c|c|c|c|c|c|}
\hline \multirow{3}{*}{$\begin{array}{c}\text { Size } \\
(i n)\end{array}$} & \multirow{3}{*}{$\begin{array}{l}\text { Manufacturer's } \\
\text { Stated Meter } \\
\text { Factor in water } \\
\text { (Pulses/gal) }\end{array}$} & \multicolumn{4}{|c|}{ Measured Meter Factor } \\
\hline & & \multicolumn{2}{|c|}{ Water } & \multicolumn{2}{|c|}{ Liquid Nitrogen } \\
\hline & & Pulses/liter & Pulses/gal & Pulses/liter & Pulses/gal \\
\hline 2 & 116 & 31.03 & 117.44 & 31.51 & 119.27 \\
\hline 4 & 7.910 & 2.078 & 7.867 & 2.114 & 8.00 \\
\hline 8 & 1.01 & 0.2693 & 1.0194 & $0.2737^{*}$ & $1.036^{*}$ \\
\hline 12 & 0.3 & 0.08461 & 0.32026 & $0.08600^{*}$ & $0.3255^{*}$ \\
\hline 32 & 0.016 & 0.004419 & 0.016729 & $0.004491 *$ & $0.017002^{*}$ \\
\hline
\end{tabular}

*Calculated value

\subsubsection{Recommendations}

To allow choices in instrument selection, different flowmeters and densimeters should be evaluated for LNG service. The particular choices used for the tests on this project are probably not the only ones that could be used, but it was beyond the scope of this work to evaluate other possible candidates.

Long-term tests on a complete measurement station need to be run. This will require an active import terminal and the assistance of facility personnel, but it is needed to establish the long term characteristics of the various elements of the measurement station. 


\section{5 .6 .8 References}

[1] Brennan, J.A., R. W. Stokes, C. H. Kneebone and D. B. Mann, An evaluation of selected angular momentum, vortex shedding and orifice cryogenic flowmeters, Nat. Bur. Stand (U.S.), Tech. Note $650,69 p p$ (March 1974).

[2] Brennan, J.A., R. W. Stokes, C. H. Kneebone and D. B. Mann, NBS-CGA cryogenic flow measurement program, ISA Transactions 14, 237-247 (1975).

[3] Siegwarth, J. D., B. A. Younglove and J. F. LaBrecque, An evaluation of commercial densimeters for use in LNG, Nat. Bur. Stand. (U.S.), NBSIR 77-867, (October 1977).

[4] Eiseman, J. H. and E. A. Potter, Accuracy of the Cutler-Hammer recording gas calorimeter when used with gases of high heating value, American Gas Association, 1515 Wilson Blvd., Arlington, VA (April 1957).

[5] Dean, J. W., J. A. Brennan, D. B. Mann and C. H. Kneebone, Cryogenic flow research facility provisional accuracy statement, Nat. Bur. Stand. (U.S.), Tech. Note 606, 40pp (July 1971).

[6] Strobridge, T. R., The thermodynamic properties of nitrogen from 64 to $300 \mathrm{~K}$ between 0.1 and 200 atmospheres, Nat. Bur. Stand. (U.S.), Tech. Note 129, 85pp (January 1962).

[7] McCarty, R. D., Four mathematical models for the prediction of LNG densities, Nat. Bur. Stand. (U.S.), Tech. Note 1030 (December 1980).

[8] Caldwell, B. J., Fuel gas energy metering, Transmission Measurement Committee Report No. 5 (Revised), American Gas Association, 1515 Wilson Blvd., Arlington, VA (1976).

[9] Parrish, W. R., J. M. Arvidson and J. F. LaBrecque, Development and evaluation of an LNG sampling measurement system, Nat. Bur. Stand. (U.S.), NBSIR 78-887 (July 1978).

[10] Orifice Metering of Natural Gas-Gas Measurement Committee Report No. 3, American Gas Association, 1515 Wilson Blvd., Arlington, VA (1969 Revision).

[11] Photogrammetric Survey of Trunkline LNG Company 600,000 bbl LNG Storage Tank No. A at Lake Charles, Louisiana, Geodetic Services, Inc. Indialantic, Florida (July 1982).

[12] Photogrammetric Survey of Trunkline LNG Company 600,000 bbl LNG Storage Tank No. B at Lake Charles, Louisiana, Geodetic Services, Inc. Indialantic, Florida (July 1981).

[13] Photogrammetric Survey of Trunkline LNG Company 600,000 bbl LNG Storage Tank No. C at Lake Charles, Louisiana," Geodetic Services, Inc., Indialantic, Florida (March 1981).

[14] Calibration of One 32 Vortex Meter Tag Number FEFT100, Alden Research Laboratory Worcester Polytechnic Institute, Holden, Massachusetts (October 1980).

[15] Hiza, M. J., W. M. Haynes and W. R. Parrish, Orthobaric liquid densities and excess volumes for binary mixtures of low molar-mass alkanes and nitrogen between 105 and $140 \mathrm{~K}$, J. Chem. Thermodynamics, 9 , 873-896 (1977).

[16] Haynes, W. M. and M. J. Hiza, Measurements of the orthobaric liquid densities of methane, ethane, propane, isobutane, and normal butane," J. Chem. Thermodynamics, 9, 179-187 (1977).

[17] Haynes, W. M., M. J. Hiza and R. D. McCarty, Densities of LNG for custody transfer, Proceedings of LNG-5 Conference, Dusseldorf, Germany, August 1977.

[18] Brennan, J. A., LNG flow measurement, Final Report to Pipeline Research Committee, American Gas Association on Project PR 50-104, (to be published by Pipeline Research Committee in 1985). 
6

6

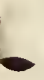

6

6

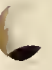

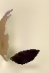






\subsection{MEASUREMENT APPLICATIONS}

Applications

Page

LNG Measurement Applications

3. 1- 1

3.1

$3 \cdot 1 \cdot 1$

3. 1.2

3.1 .3

3.2

3.2 .1

3. 2.2

3.2 .3

3.2 .4

3.2 .4 .1

3.2 .5

3.2 .6

3.2 .7

3.3

3. 3.1

3. 3.2

3.3 .3

3.3 .4

3. 3.4 .1

3.3 .5

3. 3.6

3.3 .7

3.4

3.4 .1

3.4 .2

3.4 .3

3.4 .4

3.4 .4 .1

3.4 .5

3.4 .6

3.4 .7
Measuremen $\mathrm{t}$ Uncer $\mathrm{ta}$ in $\mathrm{t}$ ies

Introduction

Estimates of Error for Individual Measurement Elements

S ummar y

Ship Loading/Unloading

In troduction

Examples of the Measurement Process.

Measurement Elements.

Sample Calculations for Case 1

Case 1 Error Summary.

Sample Calculations for Case 2

Sample Calculations for Case 3

Sample Calculations for Case 4

Pipeline Metering

lntroduction

Examples of the Measurement Process.

Measurement Elements.

Sample Calculations for Case 1

Case 1 Error Summary.

Sample Calculations for Case 2

Sample Calculations for Case 3

Sample Calculations for Case 4

Landbased Storage

In troduct ion

Examples of the Measurement Process.

Measurement Elements.

Sample Calculations for Case 1

Case 1 Error Summary.

Sample Calculations for Case 2

Sample Calculations for Case 3

Sample Calculations for Case 4
$3 \cdot 1-1$

3. $1-1$

3. $1-2$

$3.1-4$

$3 \cdot 2-1$

3. $2-1$

3. $2-2$

3. 2-3

3. 2- 6

3. 2- 12

3. 2-12

3. $2-13$

3. 2-14

3. 2- 1

3. $3-1$

3. $3-2$

3. $3-3$

3. $3-4$

3. $3-7$

3. $3-7$

3. $3-8$

3. $3-8$

3. 4- 1

3. 4- 1

3. 4-2

3. $4-3$

3. 4-5

$3.4-8$

3. 4-8

3. 4-9

$3.4-10$ 



\subsection{LNG Measurement Applications}

\section{1 Measurement Uncertainties}

The orlginal concept of this LNG Measurements Manual provided for extensive treatment of the measurement process as applied specifically to LNG baseload terminals and secondarily to LNG peak shaving and satellite operations. It was anticipated that extensive performance data would be available from measurement instrumentation for one or more of the U.S. LNG terminals. The results of the properties and instrumentation research at NBS and elsewhere were to be extensively documented in section 2 of this manual with estimates of error for each measurement element. The performance data of the field instruments could then be combined with basic physical property data of section 1 of this manual to give extensive examples of actual custody transfer measurements. Also included would be examples of cases where procedures could not be carried out because of equipment failure of one or more measurement elements and what could be done to complete the total measurement even though one or more elements were not available.

It has not been possible to complete the manual as planned. The importation of LNG to the U.S. at the date of this writing has been halted except for a relatively small quantity received at the Boston, Massachusetts, terminal. A limited amount of performance data has been taken at the Trunkline LNG Corporation terminal at Lake Charles, Louisiana, and this information has been included in section 2.5.6, but the data is not as extensive as anticipated.

This section of the manual has been revised to take into account the lack of field performance information and data. The approach is to be a set of example calculations based on assumed conditions at a typical LNG import terminal. The calculations will be based on the information provided and referenced to sections 1 and 2 of this manual, but the cases will, by necessity, be hypothetical and will not provide the practical sense of field applications.

However, the format of the manual is of loose-leaf design, and users may wish to add their own information to that included at this printing. If, in the near future, the LNG imports to the U.S. resume, then it may be possible to complete the manual as planned by replacing this section 3 with a revised section 3 based on actual LNG terminal measurement and instrumentation performance.

\subsubsection{Introduction}

The following sections describe three applications of the measurement process to establish the value of a quantity of LNG prior to sale. The first application is that of a LNG ship unloading (or loading) at a terminal. The second is where a flow measurement station is located in a LNG pipeline between the LNG ship and on-shore LNG tank storage; and the third application is where LNG is loaded into (or unloaded from) a shore LNG tank.

The first and third applications are considered static measurements, and the second is dynamic measurement. All three applications of the measurement process have a number of similarities.

1) All three allow calculation of the volume of LNG to be measured. The ship tanks are, in general, discrete volumes having individual measurement instrumentation such as liquid level gauges and densimeters. The same comments can be made for the shore tanks, which may be several times greater in volume but have similar instrumentation to that of the individual ship tank. Strapping and the calculation of tank tables are also similar operations for both ship tank and shore tank applications. Trim and list tables which may be used for correcting the measurement as a function of tank orientation are only required for the ship tanks.

Volume flowmeter measurements during loading and unloading operations are quite different. Strapping, tank tables and liquid level instrumentation are not required, but the volume passing through the meter must be totalized over the flow period.

2) Density measurements and instrumentation can be quite similar. However, not all of the commercially available densimeters can measure the density of a LNG flowing in a pipeline, but the calibration requirements are nearly the same. An "in-pipeline" densimeter will actually be exposed to all the contents of a tank during the loading or unloading operation where the "in-tank" densimeter may be exposed to only the limited volume of LNG in proximity to the primary measurement element. 
3) Sampling of LNG for calculation of density and/or calorific value is possible either by separate tank taps or by tapping main cargo piping at or near the flowmeter. Gas analysis is then made possible either on a continuous basis or by periodically filling sample bottles.

Based on the above, it is possible to assess the measurement process for all three applications. The assessment will take the form of estimating errors for the measurement processes involved in each of the applications and then performing sample calculations for each of the applications. The estimates of error are discussed in the following portions of this section, while sample calculations will be included with each of the applications sections.

\subsubsection{Estimates of Error for Individual Measurement Elements}

The following discussion is designed to provide estimates of error for each of the LNG measurement elements other than volume. The sources for the estimates of error are all within the text of LNG Manual and are referenced to the individual section, figure or table.

Volume This measurement is specific to each application and will be discussed with the sample calculations for each application (see sections 3.2, 3.3. and 3.4). There may be several values for the estimate of error depending on tank construction, tank size and liquid level devices used.

Gas Analysis This extremely important measurement process contributes to calculated density and calculated calorific value estimates. In addition, it is used to estimate the uncertainty in the calorific value found from the combustion calorimeter.

The estimate of total uncertainty is composed of an allocation for known sources of systematic error plus random error. Each component must include the value and a description of the source of error.

The estimate of random error for the gas analysis process comes from section 2.2.1. The random error of \pm 0.06 percent is three times the standard deviation of \pm 0.02 percent. This value represents the maximum value obtained from over 100 measurements of three or more repetitive analyses employing a properly operating gas chromatograph with programmable integrator system.

The known sources of systematic error are the uncertainty in the calibration gas composition of \pm 0.03 percent based on the uncertainty in the weighing process used to prepare the calibration mixtures. Great care must be taken to assure the purity of the components of the calibration gas in order to maintain this uncertainty value.

The total uncertainty in gas analysis is estimated to be \pm 0.09 percent, which is composed of an allocation of \pm 0.03 percent for known sources of systematic error plus a random error of \pm 0.06 percent. The random error is three times the standard deviation of \pm 0.02 percent found from over 100 measurements of three or more repetitive analyses employing a properly operating gas chromatograph with programmable integrator.

Calculated LNG Density The total uncertainty in calculated density is composed of two parts. The first is the estimate of error in calculation of density by means of the mathematical models which in turn are based on an extensive experimental data program described in section 2.4.3.1. "The total uncertainty of a single density measurement is approximately 0.1 percent at low temperatures ..." The most likely use of this data by the measurement engineer would be through the application of one or more of the mathematical models optimized to fit these experimental data. As described in section 2.4.3.7 "The result was that all four of the models originally chosen can be used to predict the density of LNG to within 0.1 percent of the true density, given the temperature, pressure (for these models) and composition." The resultant systematic error contribution would be these two errors combined in quadrature to give \pm 0.14 percent.

The second part of the total uncertainty in calculated density are known sources of random error which consist of errors in measurement of pressure, temperature and composition and are the required input variables for the above mathematical models. The error in composition is that found above from gas analysis and is assumed to be \pm 0.09 percent. 
Errors in pressure measurement are assumed not to exceed \pm 0.25 percent of reading at a pressure of $0.7 \mathrm{MPa}$. This error of about $\pm 1.8 \mathrm{kPa}$ in pressure must be converted to percent error in density. The isothermal compressibility of the fluid may be used which is:

$$
\text { Isothermal Compressibility }=\frac{\partial \rho}{\rho \partial \mathrm{P}} 1 / \mathrm{MPa}
$$

where $\rho$ is the density of the fluid and $\partial \rho / \partial P$ can be found from thermodynamic properties of the fluid. It is assumed for simplicity that pure methane is the fluid, and the work of Goodwin [24 of section 1.3] is used as a source for the thermodynamic data. The above value is calculated to be less than 0.001 percent change in density for a error of $\pm 1.8 \mathrm{kPa}$ and therefore can be neglected.

Temperature measurement errors have a greater influence on the calculated density error. Assume here that the error in temperature measurement does not exceed $\pm 0.1 \mathrm{~K}$.

The thermodynamic property of expansivity is:

$$
\text { Expansivity }=-\frac{\partial \rho}{\rho} \frac{\partial T}{T} 1 / K
$$

where $\rho$ is the density of the fluid and $\partial \rho / \partial T$ can be found for methane from Goodwin as above.

The error in density calculated from the expansivity value is \pm 0.016 percent for the $\pm 0.1 \mathrm{~K}$ error assumed for the temperature measurement.

Combining these sources of random error (pressure, temperature and composition) in quadrature gives a value of \pm 0.091 percent for these known sources of error.

The total uncertainty in calculated density is estimated to be \pm 0.23 percent, which is composed of \pm 0.14 percent for known sources of systematic error plus a random error of \pm 0.091 percent. The random error, estimated as a limit, is assumed to be at a 99.7 percent confidence level or three standard deviations.

Measured Density The uncertainty in direct density measurements is composed of the densimeter calibration error and the random error of the densimeter used. The value of the random error is found in section 2.4.2.7. Four commercial densimeters were evaluated, and it was found that performance depended on application, that for selected instruments at least, a calibration can be provided which is precise to better than 0.1 percent and stable to better than 0.1 percent. This is interpreted to be the limit of the random error of the densimeter and is consistent with the performance data included in the figures of section 2.4 .2 .

The allocation of known sources of systematic error is the estimated calibration error. This is found in section 2.4.1.7. The uncertainty of the density reference system for pure methane at the normal boiling point is \pm 0.055 percent. This value will vary somewhat with the density of the test fluid, but this value of \pm 0.055 percent is the maximum value and will be less for LNG mixtures of higher density.

The total calibration uncertainty in direct density measurement is estimated to be \pm 0.16 percent which is composed of \pm 0.06 percent for known sources of systematic error plus a random error of \pm 0.1 percent. The random error is the limit of calibration error for selected commercial denslmeters and is considered to be at a confidence level of 99.7 percent or three standard deviations.

In practice, an additional error must be added to this calibration error to reflect actual fleld measurements of the LNG density. It is estimated that this additional random error will not be less than the \pm 0.1 percent random error component found during calibration. This additional error $1 \mathrm{~s}$ applied by considering the above calculated total calibration error as the systematic component of the field density measurements and the additional random error as the random component. Total uncertalnty in direct density measurement is then \pm 0.26 percent, which is composed of \pm 0.16 percent from the calibration error plus a random error of \pm 0.1 percent. The random error, estimated as a $11 \mathrm{mlt}$, $1 \mathrm{~s}$ assumed to be at a confidence level of 99.7 percent or three standard deviations.

Calculated Calorific Value The uncertainty in calculated calorific value is composed of random and systematic components. The random component is taken from section 2.2.5.10. rLaboratory and iteld tests showed that it is feasible to obtain and analyze representative samples wlth a preclsion (based on three standard deviations) of better than \pm 0.3 percent in the computed heat 1 ing valug; th 13 includes the gas analysis precision of \pm 0.06 percent" (see above). 
The allocation of error for known sources of systematic error is composed of \pm 0.03 percent uncertainty in the calibration gas composition, which neglects the uncertainty of the individual components heating value and is based only on the uncertainty in the weighing process used to prepare the mixtures (see section 2.2.1). The estimate of error in the individual components' heating value is taken from table 1.3 .1 of section 1.3. There is a small variation in the estimated uncertainty in heating value depending on the composition. Referring to table 2.1 .1 which shows the mixture variations of LNG as a function of source, LNG may vary from 99.81 percent methane with small fractions of ethane and nitrogen to 71.9 percent methane and over 18 percent of the heavier hydrocarbons. Table 1.3 .1 shows that the greatest uncertainty in component heating value is that of methane which is $890.65 \pm 0.37 \mathrm{~kJ} / \mathrm{mol}$. This value of \pm 0.04 percent is assumed as the maximum value for the present calculations. The two values of systematic error, \pm 0.03 percent and \pm 0.04 percent are combined in quadrature to give \pm 0.05 percent.

The total uncertainty in measured calorific value is \pm 0.77 percent, which is composed of an allocation of \pm 0.06 percent for known sources of systematic error plus \pm 0.71 percent random error. The random error is assumed to be at a 99.7 percent confidence level.

Measured Calorific Value This uncertainty may have the largest value of all the measurement elements, because a greater number of assumptions have been made to establish the value. Additional research is required if this value is to be reduced. As discussed in section 2.3.2.1, a value of \pm 0.71 percent was calculated as the estimated error in calorific value on a mass basis as found by the recording combustion calorimeter. An additional estimate of error must be added to this value to account for the uncertainty in the calorific value of the calibration gas. This value, based on a 99.7 percent confidence level, has been found for methane to be \pm 0.06 percent (see reference [15], section 2.3 ).

The total uncertainty in measured calorific value is \pm 0.77 percent, which is composed an allocation of \pm 0.06 percent for known sources of systematic error plus \pm 0.71 percent random error. The random error is assumed to be at a 99.7 percent confidence level.

The unexpectedly large uncertainty in measured calorific value is probably a result of limited published research quality data. In Europe, where combustion calorimetry is, in many cases, the official accounting method, supporting data is believed to be adequate to show a lower error value. Actual performance data beyond that cited in the open literature are not available at this time.

\subsubsection{Summary}

The above calculations assume in all cases that the sampling process does in fact provide a representative sample of the LNG container under consideration. This may not be true in practice. This can also be said of the temperature and pressure measurements. Any variations in the above values caused by non-homogeneous fluid mixtures are unpredictable, and great care should be given to assuring uniform temperatures, pressures, densities and compositions. Cross checks using several combinations of the measurement elements are an excellent procedure to assure accurate and precise measurements. Table 3.1.1 contains a summary of the above developed estimates of error.

Table 3.1 .1

Estimates of Measurement Error LNG Measurement Process

\begin{tabular}{|l|c|c|}
\hline \multirow{2}{*}{$\begin{array}{l}\text { ENG Measurement } \\
\text { Element }\end{array}$} & Measured & \multicolumn{2}{|c|}{$\begin{array}{c}\text { Total } \\
\text { (pertainty }\end{array}$} \\
\cline { 2 - 3 } & \pm 0.26 & Calculated \\
\hline \hline Density & \pm 0.77 & \pm 0.23 \\
Calorific Value & \pm 0.35 \\
\hline
\end{tabular}




\section{LNG MEASUREMENT}

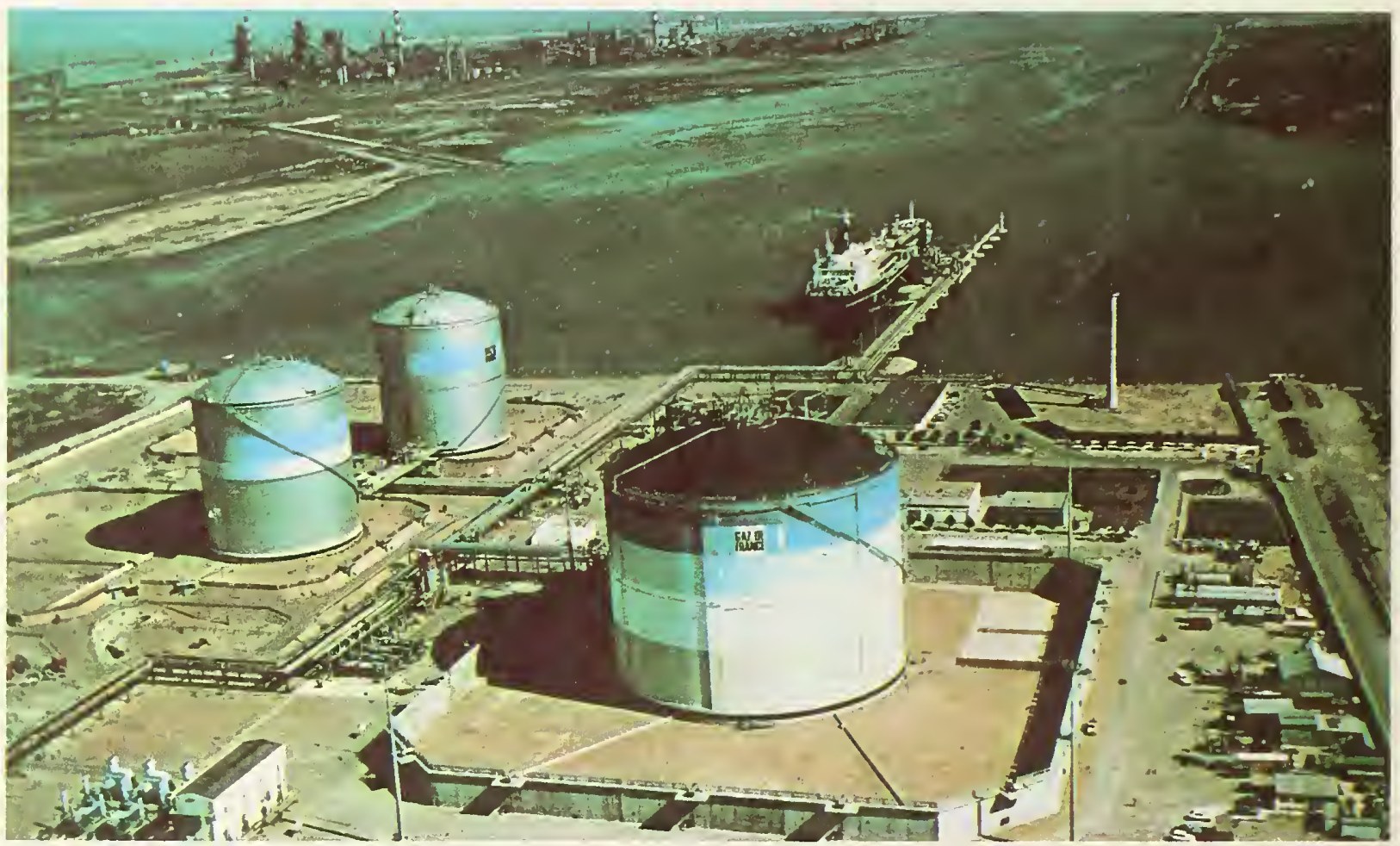

\section{FOS-SUR-MER TERMIMAL}

The Fos-sur-mer Terminal owned by Gaz de France (GDF is situated in an industrial zone west of Marseille, France, facing the Mediterranean Sea. The terminal began receiving LNG from Skikda, Algeria, in 1972. The receiving volume is of the order of 3 million tons per year.

The photograph shows two $35,000 \mathrm{~kL}$ and one $80,000 \mathrm{~kL}$ storage tanks, carrier berth and off-loading lines. Open rack type (550,000 m $\mathrm{m}^{3}$ ) and submerged combustion type vaporizer units are shown at the lower left. The LNG processing is integrated with a Le Air Liquid Company liquid air separation plant, providing refrigeration from the LNG in exchange for heated waste water from the air plant used in the rack type vaporizers. In addition, gaseous nitrogen is supplied from the air separation plant for use by the LNG terminal in natural gas calorific adjustments. (Photograph by courtesy of The Japan Gas Association, Tokyo, Japan, on behalf of the 1981 Japan LNG Congress) 


\subsection{Ship Loading/Unloading}

\subsubsection{Introduction}

The general case is assumed in which a LNG ship has been loaded with LNG of a known composition at a remote producer terminal, has then been in transit for some period of time and has arrived at the LNG importation terminal docking area. At the dock, the off-loading pipeline is connected to the LNG ship piping, and a quantity of LNG is removed from the ship tanks and deposited into a LNG shore tank. The ship unloading procedure is examined here in detail. The loading procedure would be similar.

In the following discussion no consideration is given to unexpected failure of instrumentation or other equipment which would require alternate measurements or measurement estimates of portions or all of the overall measurement process. Examples might be the failure of the capacitance liquid level device after the upper level had been read but before the lower level was read. A backup bubbler level gage (see section 2.5 .1 .5 ) might be used. The interruption of power to the LNG discharge pumps in one of the ship tanks may require a shift to a second tank and pump while the cause of the first interruption is repaired. These and other unanticipated changes in the expected measurement process are not unusual, particularly in the case of a new technology, and these types of unexpected problems will test the resources of the measurement engineer.

The measurement objective is to determine the quantity and composition of the LNG removed from the LNG ship. The most common measurement to be made is the total calorific value of the LNG removed from the ship. At the present time no single property measurement can accurately and precisely provide this value directly. It is necessary to use a multi-step procedure involving volume, density and specific calorific value.

Volume This is a measured quantity involving some predictive calculation. For the case of ship unloading, only static measurement states are considered. Each ship tank is assumed to be individually instrumented as to liquid level. Each tank is also considered as an individual measurement entity, because the liquid content may have been exposed to different heat leaks by reason of position in the ship, thickness or effectiveness of insulation, etc. These different quantities of heat energy absorbed by the LNG will result in slightly different tank liquid compositions at off-loading even though the same liquid composition was loaded into all the tanks.

Density A choice is given of either direct measurement using a calibrated densimeter or a density calculation based on sampling, analysis and the use of a LNG mixture state equation. Both methods can be used to provide a cross check.

As in the comments on volume above, each tank will be considered as a measurement entity in respect to an individual densimeter for each tank. However, in the case of sampling to provide a representative density or calorific value, the measurement process is complicated by the procedure of ship unloading. To limit unnecessary stresses on the ship structure, liquid is often removed from most or all of the tanks at the same time. It is therefore assumed that sampling is conducted by side tapping and monitoring (see section 2.2) the individual LNG tank off-loading line prior to the main liquid header. This should assure a representative sample of the contents from each individual ship tank.

Calorific Value This is the quantity of thermal energy released when the LNG mixture has been vaporized and burned in air to gaseous carbon dioxide and liquid water. The value may be determined directly by vaporizing a representative sample of LNG and burning the mixture in a combustion calorimeter which measures the thermal output compared to that of a calibration gas or by calculation from sampling, analysis and integrating the individual pure component calorific values. Both methods can be used as a cross check, but, because of the unloading procedure mentioned above, sampling procedures must be designed to assure a representative sample is taken for each separate tank.

The results of these procedures may be combined in the following manner:

\section{Total Calorific Value $=$ (Volume)(Density)(Specific Calorific Value)}

The term "total calorific value" means here the enthalpy of combustion of the contents of a tank or ship after these contents have been vaporized. In the testing literature, "total calor 1 f c value" for a gaseous fuel means something different, i.e. the enthalpy of combustion of a unit amount (mol, k $;$, cubic meter) when the products of combustion are gaseous carbon dioxide and $11 q u 1 d$ water. The term "specific calorific value" used here has that meaning for a kilogram sample. 


\subsubsection{Examples of the Measurement Process}

Variations of the above procedure are, of course, possible by combining one or more of the steps, but, as a descriptive matter, the above steps will be followed. Even with this restriction there are four possible individual procedures which may be followed:

Case 1. Total Calorific Value = LNG Volume $\mathrm{x}$ Density (calculated) $\mathrm{x}$ Specific Calorific Value (calculated)

Calculate the volume of LNG removed from each ship tank. Secure a representative LNG sample from each ship tank. Vaporize and analyze the sample to determine the fraction of individual components. Calculate the density of the LNG removed from each ship tank using the state equation for liquid density as a function of measured LNG temperature pressure and composition. Combine the density of each ship tank with the volume removed from each ship tank to give mass removed from each tank. Using previous sample analysis, calculate the calorific value of the LNG sample from calorific values of the individual components.

Calculate the density of the vaporized LNG sample and combine the calculated specific calorific values of each ship tank with the total mass removed from each tank to give the calorific value of LNG removed from each ship tank. Combine individual ship tank calorific values to give the total calorific value of LNG removed from ship.

Case 2. Total Calorific Value = LNG Volume $\mathrm{x}$ Density (measured) $\mathrm{x}$ Specific Calorific Value (calculated)

Calculate the volume of LNG removed from each ship tank. Measure the representative LNG density in each ship tank using a calibrated densimeter and calculate the mass of LNG removed from each ship tank. Secure a representative LNG sample from each ship tank. Vaporize and analyze the sample to determine the fraction of individual components. Calculate the calorific value of the LNG sample from calorific values of the individual components. Calculate the density of the vaporized LNG sample and combine the calculated specific calorific values of each ship tank with the total mass removed from each tank to give calorific value of LNG removed from each ship tank. Combine individual ship tank calorific values to give total calorific value of LNG removed from the ship.

Case 3. Total Calorific Value = LNG Volume $\mathrm{x}$ Density (calculated) $\mathrm{x}$ Specific Calorific Value (measured)

Calculate the volume of LNG removed from each ship tank. Secure a representative LNG sample from each ship tank. Vaporize and analyze the sample to determine the fraction of individual components. Calculate the density of the LNG removed from the ship tank using the state equation for liquid density as a function of measured LNG temperature, pressure and component fraction. Combine the density of each ship tank with volume removed from each ship tank to give mass removed for each tank. Secure a new representative tank sample or use the previous sample and burn the sample in a combustion calorimeter. Measure the density of the vaporized LNG sample and combine the specific calorific value determined from the combustion calorimeter with the mass removed from the ship tank to give the total calorific value of each ship tank. Combine the individual ship tank calorific values to give the total calorific value for the LNG removed from the ship.

Case 4. Total Calorific Value = LNG Volume $\mathrm{x}$ Density (measured) $\mathrm{x}$ Specific Calorific Value (measured)

Calculate the volume of LNG removed from each ship tank. Measure the representative LNG density in each ship tank using a calibrated densimeter and calculate the mass of LNG removed from each ship tank.

Secure a representative sample of LNG from each ship tank, vaporize the sample completely and burn the sample in a combustion calorimeter. Measure the density of the vaporized sample and combine the specific calorific value determined from the combustion calorimeter with the mass removed from each ship tank to give the calorific value of the LNG removed from each ship tank. Sum the individual calorific values to give the total calorific value for all the LNG removed from the ship. 


\subsubsection{Measurement Elements}

Volume. Each of the cases cited above require detailed examination of the individual measurement elements in order to determine the uncertainty of the measurement process. For example, each case clted requires a measurement of the volume of the LNG removed from the ship tank. The technique requires two measurements of the level of the LNG, one before unloading and the second after unloading. Table 3.2.1 lists the uncertainty in liquid level measurement using data from 2.5 .1 of this manual.

Table 3.2 .1

Liquid Level Measurement Uncertainty

\begin{tabular}{|l|c|}
\hline Level Device & $\begin{array}{r}\text { Height Uncertainty } \\
(\mathrm{mm})\end{array}$ \\
\hline \hline Capacitance I & \pm 7.5 \\
Capacitance II & \pm 10.0 \\
Cable & \\
Manufacturers Spec.(fine) & \pm 1.8 \\
Manufacturers Spec.(commercial) & \pm 5.3 \\
Cable (uncompensated) & \pm 80. \\
Cable (compensated- NBS estm.) & \pm 1.0 \\
\hline
\end{tabular}

The cable manufacturer's specification is a position specification at ambient temperatures. When installed in a cryogenic LNG tank, differential thermal expansion of the tank and level materials may cause significant error. Siegwarth (see reference [23] of section 2.5.1) has shown that the uncompensated error can amount to $80 \mathrm{~mm}$ in 41 meters, which accounts for the entry in table 3.2 .1 . Siegwarth in the same publication has shown that a compensated cable level device may be expected to show a precision of $\pm 1 \mathrm{~mm}$.

Three types of ship tanks were analyzed in section 2.5. These were the membrane type (2.5.2), the freestanding prismatic type (2.5.3) and the spherical type (2.5.4). One result of each analysis was an individual tank table which related a measured level to the volume of the tank at that level. After compensation for low temperature operation each of these tank tables could be used to measure the quantity of LNG removed during off-loading. It is assumed that the "list and trim" corrections for ship orientation have been made. Examples of calculations for each type of tank are given in the following tables. Tank LNG quantity removed was assumed to be from 95 percent tank capacity to 5 percent tank capacity. Other values may also be used. For simplicity, an error of \pm 7.5 mm is assumed in measurement of liquid level. of course, other values from table 3.2 .1 may also be used.

Table 3.2 .2

Ship Tank Volume Estimate of Error

Membrane-Type LNG Tanks

\begin{tabular}{|c|c|c|c|c|c|c|}
\hline Tank No & 1 & 2 & 3 & 4 & 5 & 6 \\
\hline
\end{tabular}


The first entry of table 3.2 .2 is the volume of each individual ship tank from the NBS Report of Calibration (reference 4 of section 2.5.2). The second and eighth entries are the volume error given as part of the Calibration Report. No estimate of incremental height-volume error was provided with this calibration so the total volume error for each tank was assessed at the measured Iiquid level.

The next four entries concern the estimate of error at the 95 percent volume level caused by the liquid level measurement uncertainty and are calculated using the actual tank table. The value $\Delta \mathrm{V} / \Delta \mathrm{H}$ is the change in volume per unit change in tank height at the 95 percent volume level. The liquid level ( $L L$ ) error when multiplied by this value gives the volume error attributed to the measurement of liquid level at the particular tank height.

This error estimate is repeated at the 5 percent volume level in entries seven, nine, ten and eleven. The total volume removed from each tank is given in entry twelve with the total error estimate of this volume calculated from the two volume and the two liquid level errors combined in quadrature.

The membrane tanks cited in table 3.2 .2 are designed so that cooling of the tank to operating temperatures would not result in a reduction in tank volume. The authors of the NBS Report of Calibration (see reference above) estimated that a possible additional bias could be estimated for thermal contraction and hydrostatic loading and deformation. However, this bias, if applied, was to be assessed uniformly over the volume and therefore would cancel when using the differences in volumes as shown in table 3.2.2.

Table 3.2 .3

Ship Tank Volume Estimate of Error Prismatic-Type LNG Tanks

\begin{tabular}{|l|c|c|c|c|c|}
\hline Tank No & 1 & 2 & 3 & 4 & 5 \\
\hline \hline Volume (m3) & 15618.5 & 29417.2 & 30290.3 & 30287.9 & 21614.3 \\
Volume-95\% (m3) & 14837.6 & 27946.3 & 28775.8 & 28773.5 & 20533.5 \\
Volume error (m3) & \pm 14.84 & \pm 27.95 & \pm 28.78 & \pm 28.77 & \pm 20.53 \\
H95\% (m) & 21.757 & 21.761 & 21.742 & 21.755 & 21.734 \\
$\Delta$ V/ H95\% (m3/m) & 700. & 1400. & 1400. & 1400. & 900. \\
LL Error $\pm 7.5 \mathrm{~mm}\left(\mathrm{~m}^{3}\right)$ & \pm 5.25 & \pm 10.50 & \pm 10.50 & \pm 10.50 & \pm 6.75 \\
Volume-5\% (m) & 780.9 & 1470.9 & 1514.5 & 1514.4 & 1080.7 \\
Volume error (m3) & \pm 0.78 & \pm 1.47 & \pm 1.51 & \pm 1.51 & \pm 1.08 \\
H5\% (m) & 1.446 & 1.361 & 1.362 & 1.365 & 1.361 \\
$\Delta$ V/ H5\% (m3)/m & 550. & 1200. & 1100. & 1100. & 800. \\
LL error $\pm 7.5 \mathrm{~mm}\left(\mathrm{~m}^{3}\right)$ & \pm 4.125 & \pm 9. & \pm 8.25 & \pm 8.25 & \pm 6. \\
Volume removed $(\mathrm{m} 3)$ & 14056.7 & 26475.4 & 27261.3 & 27259.1 & 19452.8 \\
Error estimate (m3) & \pm 16.29 & \pm 31.22 & \pm 31.76 & \pm 31.75 & \pm 22.45 \\
\hline
\end{tabular}

The first entry of table 3.2.3 is the volume of each individual ship tank from the cargo tank gauging tables. The third and eighth entries are the volume errors given with the tank gauging tables (reference [8], 2.5.3). This was stated as \pm 0.1 percent of height and was estimated for each tank at the measured liquid level.

The next four entries concern the estimate of error at the 95 percent volume level caused by the liquid level measurement uncertainty. The value $\Delta \mathrm{V} / \Delta \mathrm{H}$ is the change in volume per unit change in tank height at the 95 percent volume level. The Iiquid level (LL) error when multiplied by this value gives the volume error attributed to the measurement of liquid level at the particular tank height.

This error estimate is repeated at the 5 percent volume level in entries seven, nine, ten and eleven. The total volume removed from each tank is given in entry twelve with the total error estimate of this volume calculated from the two volume and liquid level errors combined in quadrature.

The volumes cited in table 3.2 .3 have been corrected for wall thickness and internal structures and further corrected to refer to cargo temperatures of $113.2 \mathrm{~K}$.

Table 3.2.4 provides information on only a single tank of the spherical type. However, since all the tanks are of nearly the same volume, the single tank information may be assumed to be the same for the other four tanks. The first entry of table 3.2.4 is the volume of an individual ship tank from the volume measurement analysis (references 9 and 10 of section 2.5.4). The third and eighth entries are the volume errors given with the tank gauging tables. This error estimate was calculated as a function of height from an equation included in the above references. 
Table 3.2 .4

Ship Tank Volume Estimate of Error Spherical-Type LNG Tank

\begin{tabular}{|l|c|}
\hline Volume $\left(\mathrm{m}^{3}\right)$ & 25558.07 \\
Volume-95\% (m3) & 24280.5 \\
Volume error (m3) & \pm 7.04 \\
H95\% (m) & 31.57 \\
$\Delta V / \Delta \mathrm{m}_{95 \%}\left(\mathrm{~m}^{3} / \mathrm{m}\right)$ & 491.7 \\
LL Error $\pm 7.5 \mathrm{Im}\left(\mathrm{m}^{3}\right)$ & \pm 3.69 \\
Volume-5\% $\left(\mathrm{m}^{3}\right)$ & 1277.6 \\
Volume error (m3) & \pm 0.56 \\
H5\% (m) & 4.94 \\
$\Delta V / \Delta \mathrm{m}_{5 \%}\left(\mathrm{~m}^{3}\right) / \mathrm{m}$ & 494. \\
LL error $\pm 7.5 \mathrm{~mm}\left(\mathrm{~m}^{3}\right)$ & \pm 3.71 \\
Volume removed $\left(\mathrm{m}^{3}\right)$ & 23002.9 \\
Error estimate (m3) & \pm 8.78 \\
\hline
\end{tabular}

The next four entries concern the estimate of error at the 95 percent volume level caused by the liquid level measurement uncertainty. The value $\Delta V / \Delta$ is the change in volume per unit change in tank height at the 95 percent volume level. The liquid level (LL) error when multiplied by this value gives the volume error attributed to the measurement of liquid level at the particular tank height.

This error estimate is repeated at the 5 percent volume level in entries seven, nine, ten and eleven. The total volume removed from the tank is given in entry twelve with the total error estimate of this volume calculated from the two volume and liquid level errors combined in quadrature.

The volumes cited in table 3.2 .4 have been corrected for internal structures and further corrected to refer to cargo temperatures of $113.2 \mathrm{~K}$.

Density. Definition of the values for LNG density within the individual ship tanks can be influenced greatly by actual field conditions. Direct density measurement utilizing an in-tank densimeter is not always possible, as many ships are not so equipped. An individual tank densimeter may malfunction or become inoperative at some critical point in the measurement process. The densimeter may actually be placed in a main piping header where several tank contents are pumped and mixed as the tanker is off-loaded, thus averaging and obscuring the true individual tank bulk density. Calculated LNG densities which depend on LNG sampling and gas analysis (see section 2.2) may not provide individual sampling lines to each tank or individual LNG pressure and temperature measurements for each tank, thus obscuring actual estimates of individual tank conditions. Few if any of these field conditions have been monitored in any detail prior to the writing of this manual. Documented comparisons of densities measured by densimeters and calculated under actual field operations are also not avallable at the present time. Therefore, a number of simplifying assumptions have been made in order to allow a description of the measurement process in the context of the four case examples cited above.

1) A representative value for LNG density will be calculated for each Liv ship tank. Gas sampling and analysis as described in section 2.2 is assumed to provide a representative composition of the LNG in each ship tank. Representative values o: tank liquid level (see tables 3.2.2, 3.2.3 and 3.2.4), liquid pressure and temperature are also assumed to be available. The process by which these values are established are assumed to be within the existing industrial practice.

2) In the absence of typical in-tank densimeter data, it is assumed that the measured density within the tank is numerically equal to the value calculated by means of sampling, gas analysis and use of the mathematical models. However, in assessing the estimated error, the total uncertainty of the densimeter used in the measurement w11 be that described in section 3.1 .2 of this manual. For example, 1: as a result of calculations to determine LNC density from measured pressure, temperature and assumed composition give a value of $480.23 \mathrm{~kg} / \mathrm{m}^{3}$, the uncertainty for this process is found from table 3.1 .1 as \pm 0.23 percent. To illustrate the sample calculations whlch follow, it is assumed that the density measured by densimeter is also $480.23 \mathrm{~kg} / \mathrm{m}^{3}$ but with an uncertainty from table 3.1 .1 of \pm 0.26 percent.

Calorific Value. Comments and restrictions to the process of measurement of L:iG sh!p calor $101 \mathrm{c}$ valum are similar to those of LNG density above. The acquisition of a representative sample from each sh1p tank which may be used in determining the calorific value directly from the combustlon calorlmetn or by calculation from gas analysis is necessary to provide a true representation of the total calor $1 \mathrm{I}_{\mathrm{c}}$ 
value of the LNG cargo. The field problems associated with sampling and analysis are referenced in section 2.2, and additional simplifying assumptions are made here to present the process in the context of the four case examples.

1) A representative calorific value will be calculated for each LNG ship tank as for density values above. It is known, of course, that each individual ship tank may be found to have slightly different values of bulk density and calorific value. These differences are certainly caused in part by differences in heat flux into each tank and the length of transit time. Since the following examples are to show method only, it is assumed that the densities and compositions for each tank are identical.

2) In the absence of typical field LNG calorific data, it is assumed that the calorific value for each LNG ship tank found from sampling and combustion calorimetry is numerically equal to the value calculated by means of sampling and gas analysis. However, in assessing the estimated error, the total uncertainty of the combustion calorimetry process used in the measurement will be that described in section 3.1 .2 of this manual. For example, if as a result of calculations to determine LNG calorific value from measured pressure, temperature and assumed composition give a value of $54.323 \mathrm{MJ} / \mathrm{kg}$, the uncertainty for this process is found from table 3.1 .1 as \pm 0.35 percent. To illustrate the sample calculations which follow, it is assumed that the calorific value measured by the combustion calorimeter is also $54.323 \mathrm{MJ} / \mathrm{kg}$ but with an uncertainty from table 3.1 .1 of \pm 0.77 percent.

3) Measurement of the calorific value of each ship tank is assumed to be an independent measurement process, and, therefore, the sum of the individual uncertainties may be made in quadrature.

\subsection{Sample Calculations for Case 1}

This example is composed of a measured volume of LNG removed from each ship tank (table 3.2.2), LNG sampling, gas analysis, LNG temperature and pressure measurement for calculation of LNG density and calorific values for each ship tank. These values are then summed to give the total calorific value (with uncertainty) of the LNG off-loaded. The source of the LNG is assumed to be Arzew, Algeria, and the composition has been confirmed by gas analysis to be as shown in table 2.1.1. It is further assumed that a LNG bulk liquid temperature of $108.00 \mathrm{~K}$ and a pressure of $138 \mathrm{kPa}$ have been measured for the LNG contents of each ship tank.

Calculation of the LNG density can be accomplished by using one or more of the mathematical models described in section 2.4 .3 and 2.4.4. One of the methods will be shown in detail and a comparison of density values from the other three methods will be shown for comparison purposes. The Revised Klosek and McKinley method (section 2.4.4.4) was selected, because this method does not require a computer for solution, although the method has been adapted to computer use.

The Klosek and McKinley (KM) method is a totally empirical recipe for calculating the density of the LNG-like mixture given the temperature and composition. Pressure is not taken into account. However, near atmospheric pressure LNG is nearly incompressible. Hence the density may be considered independent of pressure.

The procedure for calculating the LNG density is as follows:

$$
\mathrm{V}_{\operatorname{mix}}=\sum \mathrm{X}_{\mathrm{i}} \mathrm{V}_{\mathrm{i}}-\mathrm{kX}_{\mathrm{CH}_{4}}
$$

where $V_{\operatorname{mix}}$ is the volume of the mixture, $X_{i}$ and $V_{i}$ are the mole fraction and volume of the $i$ th component, $\mathrm{X}_{\mathrm{CH}_{4}}$ is the mole fraction of methane and $\mathrm{k}$ is a correction factor obtained from a table or graph. The $V_{i}$ and $k$ are obviously temperature dependent and in addition $k$ is dependent upon the molecular mass of the mixture. The original form of equation (1) was modified by adding a term to take into account the nitrogen when it is present. See section 2.4.4.4 for details.

$$
\mathrm{V}_{\mathrm{mix}}=\sum \mathrm{x}_{\mathrm{i}} \mathrm{V}_{\mathrm{i}}-\left[\mathrm{k}_{1}+\left(\mathrm{k}_{2}-\mathrm{k}_{1}\right) \mathrm{x}_{\mathrm{N}_{2}} / 0.0425\right] \mathrm{x}_{\mathrm{CH}_{4}}
$$


where $k_{1}$ refers to mixtures without nitrogen and $k_{2}$ refers to mixtures with nitrogen. All other terms are the same as in equation (1). Tabular values for solving the equation are given in section 1 , in the references of section 2.4 .4 and are reproduced here for convenience.

Table 3.2.5 provides initial data for the solution. Values for molecular mass are from tables 1.2 .1 and 1.2.2. Volumes of saturated liquid of the pure components are found from table 3.2.6. The slight differences in the molecular masses between tables 3.2 .5 and 3.2 .6 are caused by a revision of the values used in table 3.2 .6 in accord with section 1.2 of this manual. This revision is too small to affect the mass and volume fractions in table 3.2 .5 .

Table 3.2 .5

Molecular Mass and Volume of Algerian LNG Mixture

Temperature $=108 \mathrm{~K}$, Pressure $=138 \mathrm{kPa}$

\begin{tabular}{|l|l|l|l|l|l|}
\hline Constituent & $\mathrm{X}_{\mathrm{i}}$ & \multicolumn{1}{|c|}{$\begin{array}{c}\mathrm{M}_{\mathrm{W}} \\
\mathrm{g} / \mathrm{mol}\end{array}$} & $\begin{array}{c}\mathrm{X}_{\mathrm{i}} \mathrm{M}_{\mathrm{W}} \\
\mathrm{g} / \mathrm{mol}\end{array}$ & $\begin{array}{c}\mathrm{V}_{\mathrm{i}} \\
\mathrm{L} / \mathrm{mol}\end{array}$ & $\begin{array}{c}\mathrm{XV}_{\mathrm{i}} \\
\mathrm{L} / \mathrm{mol}\end{array}$ \\
\hline \hline methane & 0.8700 & 16.043 & 13.9574 & 0.037481 & 0.032608 \\
ethane & 0.0814 & 30.070 & 2.4477 & 0.047512 & 0.003867 \\
propane & 0.0230 & 44.097 & 1.0142 & 0.062033 & 0.001427 \\
n-butane & 0.0068 & 58.123 & 0.3952 & 0.076384 & 0.000519 \\
i-butane & 0.0046 & 58.123 & 0.2674 & 0.077836 & 0.000358 \\
n-pentane & 0.0002 & 72.150 & 0.0144 & 0.091042 & 0.000018 \\
nitrogen & 0.0140 & 28.0134 & $\frac{0.3922}{8.4885}$ & 0.043963 & $\underline{0.000615}$ \\
\hline
\end{tabular}

Values for the correction factors of equation (2) are found by linear interpolation of temperature and volume in table 3.2 .7 for correction factor $k_{1}$ and table 3.2 .8 for correction factor $k_{2}$. For the temperature and composition selection for the example (table 3.2.5), the values for the correction factors were found to be $k_{1}=0.434$ and $k_{2}=0.61$.

Substituting in equation (2) gives:

$$
\begin{aligned}
& V_{m}=0.0394124-\left\{0.434\left(10^{-3}\right)+\left[0.61\left(10^{-3}\right)-0.434\left(10^{-3}\right)\right][0.014] / 0.425\right\} 0.8700 \\
& V_{m}=0.038985 \text { liters } / \mathrm{mol} \\
& \rho(\text { density })=1 / V_{m}=25.6509 \mathrm{~mol} / \text { liter. }
\end{aligned}
$$

In terms of the actual mixture, the molar density is multiplied by the molecular mass of the mixture from table 3.2 .5 to give:

$$
\rho(\text { mixture density })=(25.6509) /(18.4885)=474.246 \mathrm{~kg} / \mathrm{m}^{3}
$$

Computer programs which provide a calculated value for the required density of the LNG mixture are discussed in detail in section 2.4.4 and include a simple computer program for solving equation (2) above and more extensive programs for the other models. A comparison of the mixture dens1ties predicted by the four mathematical models are shown in table 3.2.9. The four models are the klosew and McKinley (KM), the Corresponding States (CS), the Cell (C) and the Hard Sphere (HS).

The maximum error of the five calculation methods from the mean mixture density example as shown 1 n table 3.2 .9 is $\pm 0.122 \mathrm{kilograms/cubic}$ meter or \pm 0.026 percent. This is within the est $1 \mathrm{mate}$ or error for calculation of LNG density from the four mathematical models ( 0.1 percent) given in sect 10 ns 2 . 4.4 and 3.1.2. The value from equation (6) will be used in all the following example calculatlons. 
Table 3.2 .6

Volumes of Saturated Liquid of the Pure Components (Liters/mole)

\begin{tabular}{|c|c|c|c|c|c|c|c|c|}
\hline$T(K)$ & $\mathrm{CH} 4$ & $\mathrm{C}_{2} \mathrm{H}_{6}$ & $\mathrm{C}_{3} \mathrm{H}_{8}$ & $\mathrm{nC}_{4} \mathrm{H}_{10}$ & $\mathrm{iC}_{4} \mathrm{H}_{10}$ & $\mathrm{~N}_{2}$ & $\mathrm{nC}_{5} \mathrm{H}_{12}$ & $\mathrm{iC}_{5} \mathrm{H}_{12}$ \\
\hline 90. & 0.035441 & 0.046081 & 0.060461 & 0.074708 & 0.076084 & 0.037543 & 0.089173 & 0.089243 \\
\hline 92. & 0.035649 & 0.046235 & 0.060632 & 0.074891 & 0.076274 & 0.038081 & 0.089379 & 0.089454 \\
\hline 94. & 0.035861 & 0.046390 & 0.060804 & 0.075075 & 0.076466 & 0.038650 & 0.089586 & 0.089666 \\
\hline 96. & 0.036077 & 0.046547 & 0.060977 & 0.075259 & 0.076659 & 0.039254 & 0.089793 & 0.089878 \\
\hline 98. & 0.036298 & 0.046704 & 0.061151 & 0.075445 & 0.076853 & 0.039897 & 0.090000 & 0.090091 \\
\hline 100 & 0.036524 & 0.046863 & 0.061325 & 0.075631 & 0.077047 & 0.040586 & 0.090208 & 0.090304 \\
\hline 102. & 0.036755 & 0.047023 & 0.061501 & 0.075818 & 0.077243 & 0.041327 & 0.090416 & 0.090518 \\
\hline 104 & 0.036992 & 0.047185 & 0.061677 & 0.076006 & 0.077440 & 0.042128 & 0.090624 & 0.090733 \\
\hline 106. & 0.037234 & 0.047348 & 0.061855 & 0.076194 & 0.077637 & 0.043002 & 0.090833 & 0.090948 \\
\hline 108 & 0.037481 & 0.047512 & 0.062033 & 0.076384 & 0.077836 & 0.043963 & 0.091042 & 0.091163 \\
\hline 110. & 0.037735 & 0.047678 & 0.062212 & 0.076574 & 0.078035 & 0.045031 & 0.091252 & 0.091379 \\
\hline 112. & 0.037995 & 0.047845 & 0.062392 & 0.076765 & 0.078236 & 0.046231 & 0.091462 & 0.091596 \\
\hline 114. & 0.038262 & 0.048014 & 0.062574 & 0.076957 & 0.078438 & 0.047602 & 0.091673 & 0.091814 \\
\hline 116. & 0.038536 & 0.048184 & 0.062756 & 0.077150 & 0.078640 & 0.049179 & 0.091884 & 0.092032 \\
\hline 118. & 0.038817 & 0.048356 & 0.062939 & 0.077344 & 0.078844 & 0.050885 & 0.092095 & 0.092251 \\
\hline 120. & 0.039106 & 0.048529 & 0.063124 & 0.077539 & 0.079049 & 0.052714 & 0.092307 & 0.092470 \\
\hline 122. & 0.039404 & 0.048704 & 0.063309 & 0.077734 & 0.079255 & 0.054679 & 0.092520 & 0.092690 \\
\hline 124. & 0.039710 & 0.048881 & 0.063496 & 0.077931 & 0.079462 & 0.056797 & 0.092733 & 0.092911 \\
\hline 126. & 0.040025 & 0.049059 & 0.063684 & 0.078128 & 0.079671 & 0.059085 & 0.092947 & 0.093133 \\
\hline 128 & 0.040350 & 0.049239 & 0.063873 & 0.078327 & 0.079880 & 0.061565 & 0.093161 & 0.093355 \\
\hline 130. & 0.040685 & 0.049421 & 0.064063 & 0.078526 & 0.080091 & 0.064263 & 0.093376 & 0.093578 \\
\hline $\mathrm{MW}^{*}$ & 16.04303 & 30.07012 & 44.09721 & 58.1243 & 58.1243 & 28.0134 & 72.15139 & 72.15139 \\
\hline
\end{tabular}

* See 2.4.4.6 for an explanation of values of molecular weight.

Table 3.2 .7

Correction Factor $k_{1} \times 103$

\begin{tabular}{|r|c|c|c|c|c|c|c|c|c|c|}
\hline $\mathrm{T} / \mathrm{W}$ & 16 & 17 & 18 & 19 & 20 & 21 & 22 & 23 & 24 & 25 \\
\hline \hline 90 & -0.005 & 0.120 & 0.220 & 0.340 & 0.430 & 0.515 & 0.595 & 0.660 & 0.725 & 0.795 \\
95 & -0.006 & 0.135 & 0.260 & 0.380 & 0.500 & 0.590 & 0.665 & 0.740 & 0.810 & 0.885 \\
100 & -0.007 & 0.150 & 0.300 & 0.425 & 0.575 & 0.675 & 0.755 & 0.830 & 0.910 & 0.990 \\
105 & -0.007 & 0.165 & 0.340 & 0.475 & 0.635 & 0.735 & 0.840 & 0.920 & 1.045 & 1.120 \\
110 & -0.008 & 0.180 & 0.375 & 0.535 & 0.725 & 0.835 & 0.950 & 1.055 & 1.155 & 1.245 \\
115 & -0.009 & 0.220 & 0.440 & 0.610 & 0.810 & 0.945 & 1.065 & 1.180 & 1.280 & 1.380 \\
120 & -0.01 & 0.250 & 0.500 & 0.695 & 0.920 & 1.055 & 1.205 & 1.330 & 1.450 & 1.550 \\
125 & -0.013 & 0.295 & 0.590 & 0.795 & 1.035 & 1.210 & 1.385 & 1.525 & 1.640 & 1.750 \\
130 & -0.015 & 0.345 & 0.700 & 0.920 & 1.200 & 1.370 & 1.555 & 1.715 & 1.860 & 1.990 \\
135 & -0.017 & 0.400 & 0.825 & 1.060 & 1.390 & 1.590 & 1.800 & 1.950 & 2.105 & 2.272 \\
\hline
\end{tabular}

Table 3.2 .8

Correction Factor $k_{2} \times 10^{3}$

\begin{tabular}{|c|c|c|c|c|c|c|c|c|c|c|}
\hline$T / W$ & 16 & 17 & 18 & 19 & 20 & 21 & 22 & 23 & 24 & 25 \\
\hline \hline 90 & -0.004 & 0.10 & 0.22 & 0.35 & 0.50 & 0.60 & 0.69 & 0.78 & 0.86 & 0.95 \\
95 & -0.005 & 0.12 & 0.28 & 0.43 & 0.59 & 0.71 & 0.83 & 0.94 & 1.05 & 1.14 \\
100 & -0.007 & 0.16 & 0.34 & 0.49 & 0.64 & 0.79 & 0.94 & 1.08 & 1.17 & 1.27 \\
105 & -0.01 & 0.24 & 0.42 & 0.61 & 0.75 & 0.91 & 1.05 & 1.19 & 1.33 & 1.45 \\
110 & -0.015 & 0.32 & 0.59 & 0.77 & 0.92 & 1.07 & 1.22 & 1.37 & 1.52 & 1.71 \\
115 & -0.024 & 0.41 & 0.72 & 0.95 & 1.15 & 1.22 & 1.3 & 1.45 & 1.65 & 2.00 \\
120 & -0.032 & 0.60 & 0.91 & 1.23 & 1.43 & 1.63 & 1.85 & 2.08 & 2.30 & 2.45 \\
125 & -0.043 & 0.71 & 1.13 & 1.48 & 1.73 & 1.98 & 2.23 & 2.48 & 2.75 & 2.90 \\
130 & -0.058 & 0.95 & 1.46 & 1.92 & 2.20 & 2.42 & 2.68 & 3.00 & 3.32 & 3.52 \\
135 & -0.075 & 1.30 & 2.00 & 2.40 & 2.60 & 3.00 & 3.40 & 3.77 & 3.99 & 4.23 \\
\hline
\end{tabular}


Table 3.2 .9

LNG Mixture Density Values Using

Several Calculation Methods

\begin{tabular}{|l|l|}
\multicolumn{1}{c|}{} & $\begin{array}{c}\text { Density } \\
\mathrm{kg} / \mathrm{m}^{3}\end{array}$ \\
\hline (from equation (6) and tables) & 474.246 \\
(from computer program- KM) & 474.242 \\
(from computer program- CS) & 474.446 \\
(from computer program- C) & 474.276 \\
(from computer program- HS) & 474.409 \\
(mean value) & 474.324 \\
(maximum error) & \pm 0.122 \\
\hline
\end{tabular}

Calorific Value The calorific value of the selected LNG mixture may be found for the example of Case 1 by calculation from the gas analysis. The following method is suggested as one which includes the most recent analysis of the heats of combustion and atomic and molecular mass. The source of these values and suggested methods is the NBS study sponsored by Groupe International des Importateurs de Gaz Naturel Liquefie (GIIGNL). The results of the study have been published by NBS as NBSIR $82-2401$ "Heating Values of Natural Gas and Its Components" by G. T. Armstrong and T. L. Jobe (1982). As explained in section 1 of this manual, some of the values reported in the reference have since been revised (references $12 \mathrm{a}, \mathrm{b}$ of section 1). The publication and revision are extensive in scope and detail, and only portions are cited in this section and section 1 . However, the content of the study is the basis of all calculations involving combustion enthalpies used in this manual.

The virial equation $P V / R T=1+B(T) / V$ where $P$ is absolute pressure, $V$ is molar volume, $R$ is the gas constant, $T$ is the absolute temperature and $B(T)$ the second virial coefficient is used by Armstrong and Jobe in the development of the combustion enthalpies of LNG mixtures. The equation is simple to use, has a basis in theory and allows an understanding of the application of the developed combustion enthalpies to ideal and real gas states for both pure fluids and mixtures.

The composition of the gas mixture in terms of the mole fractions X(i) of the individual substances present, or in terms of the mass fractions $W(i)$ of the individual substances present, is presumed to be known (see table 3.2 .5 ) and $\sum X(i)$ or $\sum W(i)$ is unity.

The calculation of the heating value involves five steps: (1) the calculation of the ideal gas molar heating value of the mixture; (2) the calculation of the virial coefficient of the gas mixture and its temperature derivative; (3) the calculation of the molar volume of the real gas mixture based upon the virial coefficient of the gas mixture from step (2); (4) the adjustment of the molar heating value for the term $\mathrm{H}-\mathrm{H}^{\circ}$ which is the difference in enthalpy of the real and ideal gas based on the virial coefficient of the gas mixture and its derivative; (5) calculation of the heating value on a mass basis and the volumetric heating value of the real gas mixture. Molar heating value $\Delta_{\mathrm{c}} \mathrm{H}_{\mathrm{m}}$ (mixture) can be converted to the heating value on a mass basis $\Delta_{\mathrm{C}} \mathrm{H}_{\mathrm{W}}$ (mixture) by use of the mass fractions of the substances in the gas. From the molar volume calculated in step ( 3 ), the molar enthalpy of combustion can be converted to the volumetric enthalpy of combustion, $\Delta_{C} H_{V}(m i x t u r e)$. According to Armstrong and Jobe (see above), step (4) and the calculation of the temperature derivative of the virial coefficient can be omitted with errors not exceeding an estimated 50 J/mol.

The calculation procedure is considered to be the the most accurate of those consldered. It $1 \mathrm{~s}$ applicable to both dry and humid gases, including the water-saturated gas. The dry gas conditions are assumed here. Notation and symbols are those of Armstrong and Jobe.

Step 1 Calculation of the heating value of the ideal gas mixture. The composition of the example gas mixture (see table 3.2.5) in terms of mole fractions $x(1)$ of the individual substances present is presumed to be given, and the sum of the $x(1)$ fractions is unity.

The enthalpy of combustion of the ideal gas mixture is calculated by adding the molar enthalpies of combustion of the individual components weighted according to the molar fractions using the equation:

$\Delta_{c^{H}}{ }_{m}=X(1) \Delta_{c} H_{m}^{\circ}(1)+X(2) \Delta_{c^{H}}{ }^{\circ}(2)+\ldots+X(n) \Delta_{c} H_{m}^{\circ}(n)$

where $\Delta \mathrm{c}^{\circ} \mathrm{m}$ is the enthalpy of combustion per mole, of the 1 deal gas. 
Standard enthalpies of combustion of the hydrocarbons at ideal gas conditions and specified temperatures are taken from table 1.3.2. The results of the calculation from equation (7) are shown in table 3.2.10. The value of molar volume Iisted in the table and used to calculate standard enthalpies of combustion on a volume basis is found from $V_{m}=R T / P$ where $R$ is the gas constant $8.31441 \mathrm{~J} / \mathrm{mol}-\mathrm{K}$ (or 8.31441 $\mathrm{m}^{3}$-pascal/mole-K). (See equation (1) of section 1.2).

Table 3.2 .10

Sample Calculation of the Standard Enthalpy of Combustion of Algerian Gas Mixture Assuming Ideal Gas

$P=101.325 \mathrm{kPa}, \mathrm{T}=288.15 \mathrm{~K}$ $v_{m}^{i d}=0.0236447 \mathrm{~m}^{3} / \mathrm{mol}$

\begin{tabular}{|c|c|c|c|c|c|c|}
\hline Component & $X(i)$ & $M(i)$ & $\begin{array}{c}-\Delta_{\mathrm{c}}^{\mathrm{H}^{\circ}}{ }_{\mathrm{m}}(\mathrm{i}) \\
\mathrm{kJ} / \mathrm{mol}\end{array}$ & $\begin{array}{c}-\mathrm{X} \text { (i ) } \Delta_{\mathrm{c}^{\mathrm{H}}}{ }_{\mathrm{m}}(\mathrm{i}) \\
\mathrm{kJ} / \mathrm{mol}\end{array}$ & $\begin{array}{c}-\Delta_{\mathrm{C}^{\mathrm{O}} \mathrm{O}_{\mathrm{V}}(i)} \\
\mathrm{MJ} / \mathrm{m} 3\end{array}$ & $\begin{array}{c}-\mathrm{X}(\mathrm{i}) \Delta_{\mathrm{MJ} / \mathrm{m} 3} \mathrm{H}^{\circ}{ }_{\mathrm{v}}(\mathrm{i}) \\
\end{array}$ \\
\hline methane & 0.8700 & 16.043 & 891.58 & 775.674 & 37.707 & 32.8051 \\
\hline ethane & 0.0814 & 30.070 & 1562.14 & 127.158 & 66.067 & 5.3779 \\
\hline propane & 0.0230 & 44.097 & $2221 \cdot 10$ & 51.083 & 93.976 & 2.1614 \\
\hline n-butane & 0.0068 & 58.123 & 2879.76 & 19.582 & 121.793 & 0.8282 \\
\hline i-butane & 0.0046 & 58.123 & 2870.58 & 13.205 & 121.405 & 0.5585 \\
\hline n-pentane & 0.0002 & 72.150 & 3538.60 & 0.708 & 149.657 & 0.0299 \\
\hline ni trogen & 0.0140 & 28.0134 & 0.0 & $\frac{0.0}{987.410}$ & 0.0 & $\frac{0.0}{41.7610}$ \\
\hline
\end{tabular}

The molar combustion enthalpy of the Algerian gas mixture example (ideal gas basis) is calculated to be $-\Delta_{c} \mathrm{H}^{\circ} \mathrm{m}=987.410 \mathrm{~kJ} / \mathrm{mol}$. The volumetric combustion enthalpy of the gas mixture (ideal gas basis) is calculated to be $-\Delta_{\mathrm{c}^{\circ}}{ }_{\mathrm{v}}=41.7610 \mathrm{MJ} / \mathrm{m}^{3}$.

Step 2 Calculation of the second virial coefficient B(mixture) of the real gas mixture. The second virial coefficient $B$ (mixture) is a function of $T$ and of the virial coefficients of the components and of the virial coefficients of the binary mixtures of the components. The equation used is taken from Armstrong and Jobe.

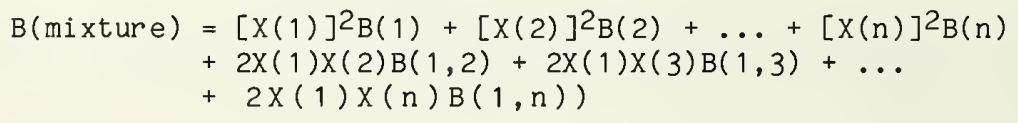

Table 3.2 .11

Correlated Second Virial Coefficients, $B_{12}(T)$, for Binary Mixtures From Equation 11, Section 1.3 Por Use in Calculations on Natural Gas Mixtures

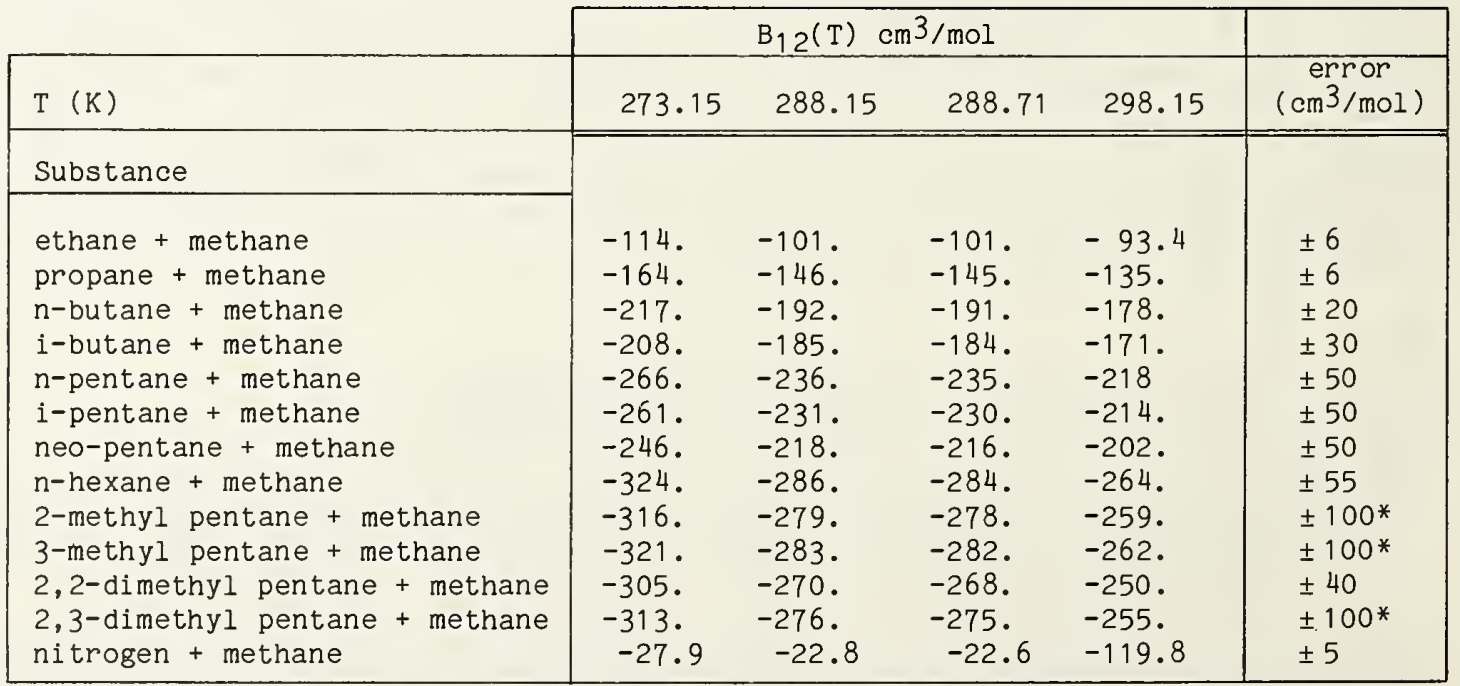

* estimated- no data. 
Component 1 is taken to be methane in every case. This equation is applicable only to natural gas containing methane as the major component. The values for second virial coefficients are from table 1.3.4 (section 1.3). The binary mixtures data are from table 3.2 .11 and the correlation given in section 1.3 .

Entering the composition fractions and virial coefficients into equation ( 8 ) for the selected temperature of $288.15 \mathrm{~K}$ and pressure of $101.325 \mathrm{kPa}$. gives:

$$
\begin{aligned}
\mathrm{B}(\text { mixture })= & {\left[(0.8700)^{2}(-47.0)+(0.0814)^{2}(-203 .)+(0.0230)^{2}(-428 .)\right.} \\
& +(0.0068)^{2}(-805 .)+(0.0046)^{2}(-716 .)+(0.0002)^{2}(-1334 .) \\
& \left.+(0.0140)^{2}(-7.1)\right]+[2(0.8700)(0.0814)(-101 .) \\
& +2(0.8700)(0.0230)(-146 .)+2(0.8700)(0.0068)(-192 .) \\
& +2(0.8700)(0.0046)(-185 .)+2(0.8700)(0.0002)(-236 .) \\
& +2(0.8700)(0.0140)(-22.8)] \\
B(\text { mixture })= & -61.738 \mathrm{~cm} 3 / \mathrm{mol}
\end{aligned}
$$

Step 3 Calculate the real gas molar volume of the mixture using the equation:

$$
v_{m}(\text { mixture }) / v_{m}^{i d}=1 / 2+1 / 2\left[1+4 B(m i x t u r e) / v_{m}^{i d}\right]^{1 / 2}
$$

where $V i \underset{m}{d}$ may be calculated as $V_{m}^{i d}=R T / P=23644.7 \mathrm{~cm} 3 / \mathrm{mol}$ (see table 3.2 .9 and preceding text) and $\mathrm{B}$ (mixture) is obtained from equation (9).

Then:

$$
\begin{aligned}
& \mathrm{V}_{\mathrm{m}}(\mathrm{mixture}) / \mathrm{V}_{\mathrm{m}}^{\mathrm{id}}=1 / 2+1 / 2[1+(4)(-61.738) / 23644.7]^{1 / 2} \\
& \left.\mathrm{~V}_{\mathrm{m}} \text { (mixture }\right) / \mathrm{V}_{\mathrm{m}}^{i d}=0.99738
\end{aligned}
$$

and:

$$
V_{m}(\text { mixture })=(0.0236447)(0.99738)=0.023583 \mathrm{~m}^{3} / \mathrm{mol}
$$

Step 4 Correct to real gas by calculating the enthalpy difference between real and ideal gas $\left(\mathrm{H}-\mathrm{H}^{\circ}\right)$ for the gas mixture using:

$$
\mathrm{H}-\mathrm{H}^{\circ}=\left(\mathrm{RT} / \mathrm{V}_{\mathrm{m}}\right)[\mathrm{B}-\mathrm{T}(\mathrm{dB} / \mathrm{dT})]
$$

According to Armstrong, this calculation can be omitted with errors that do not exceed $50 \mathrm{~J} / \mathrm{mol}$. Since from step 1 above the ideal combustion enthalpy for the mixture is $987410 \mathrm{~J} / \mathrm{mol}$, a correction of $50 \mathrm{~J} / \mathrm{mol}$ would amount to 0.005 percent and is considered negligible. Therefore, step 4 is not considered here but should not be neglected for all cases and must be considered for all mixtures which may vary extensively from the example.

Step 5 Calculate $\Delta_{c} H_{m}$ (mixture), and $\Delta_{c} H_{v}$ (mixture) from the following:

$$
\Delta_{c} H_{m} \text { (mixture) }=\Delta_{c} H_{m}^{O} \text { (mixture) }+\left(\Delta_{c} H_{m}-\Delta_{c} H_{m}\right) \text { (mixture) }
$$

where the second term is the result of step 4 and is considered zero. Therefore, from step 1 ,

$$
-\Delta_{c} H_{m}(m i x t u r e)=987.409 \mathrm{~kJ} / \mathrm{mole}
$$

Using the molecular mass of the mixture calculated in table 3.2 .5 ,

$$
-\Delta_{c H_{m}}(\text { mixture })=(987.410) /(18.4885)=53.407 \mathrm{~kJ} / \mathrm{g}=53.407 \mathrm{MJ} / \mathrm{kg}
$$

The volumetric combustion enthalpy comes from equation (15) and step 3.

$$
\begin{aligned}
& -\Delta_{\mathrm{c}^{H}} \mathrm{H}_{\mathrm{V}} \text { (mixture) }=-\Delta_{\mathrm{C}} \mathrm{H}_{\mathrm{m}} \text { (mixture) } / \mathrm{V}_{\mathrm{m}} \text { (mixture) } \\
& -\Delta_{\mathrm{C}} \mathrm{H}_{\mathrm{V}}(\text { mixture })=(987.410) /(0.023583)=41.870 \mathrm{MJ} / \mathrm{m}^{3}
\end{aligned}
$$




\subsubsection{Case 1 Error Summary}

From the above calculations of volume, density and calorific value, it is possible to assemble the total calorific value and estimate of error of the LNG removed from the LNG ship.

From table 3.2 .2 the volume removed from tank 1 is $11,298.9 \mathrm{~m} 3$. Equation (6) gives a density of $474.246 \mathrm{~kg} / \mathrm{m}^{3}$ for the LNG within the tank, and, therefore, the mass removed is the volume removed multiplied by the density or $5,358.46 \mathrm{Mg}$. The calorific value of the LNG removed is the mass removed multiplied by the specific calorific value on a mass basis from equation (16). This value of 53.407

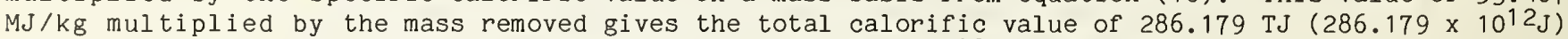
removed from tank 1. ( $T$ is the SI prefix symbol for Tera or $10^{12}$, see section 1.1 )

From table 3.2 .2 the volume error is $\pm 18.33 \mathrm{~m}^{3}$ or \pm 0.16 percent of the tank 1 volume removed. From table 3.1.1 the estimated error in calculated density is \pm 0.23 percent, and the estimated error in calculated calorific value is \pm 0.35 percent. These individual estimates of error are independent variables and may be combined in quadrature. The combination of volume, density and specific calorific value is a simple multiplication, and, therefore, the error in total calorific value of the LNG removed is:

$$
\text { Estimated Tank Error Case } \begin{aligned}
1 & =\left[e_{\mathrm{V}}^{2}+e_{d^{2}}+e_{c}^{2}\right]^{1 / 2} \\
& = \pm 0.45 \text { percent }
\end{aligned}
$$

where $e_{\mathrm{v}}$ is the estimated volume error, $e_{\mathrm{d}}$ is the estimated density error, and $e_{c}$ is the estimated error in calorific value. The total error in LNG removed from tank 1 is $(286.179 \mathrm{TJ})( \pm 0.45) / 100)=$ $1.288 \mathrm{TJ}$.

In like manner, total calorific values and estimated error may be calculated for ship tanks 2, 3 , 4, 5 and 6. The results are summarized in table 3.2.11.

\subsubsection{Sample Calculations for Case 2}

The example is composed of a measured volume of LNG removed from each ship tank of the LNG membrane-type ship (table 3.2.2). A representative LNG density is measured for each tank using a calibrated LNG densimeter. A representative sample of LNG is removed from each ship tank, vaporized and analyzed to determine the fraction of each of the LNG components. Calculations are then made to determine the calorific value of the representative LNG sample on a mass basis.

As has been stressed in earlier sections of this manual, actual field data taken and assessed under controlled conditions are not available at the present time. The case in point would normally require individual measurements of the in-tank densimeters of the LNG ship prior to, during and after off-loading. Since these data are not available, the representative LNG density calculated for Case 1 above (Algerian LNG of table 3.2.5) will be used for this example. In addition, the required sampling, analysis and calculation of calorific value described under Case 1 will also be assumed to be valid for this Case 2. This procedure will allow simple calculations and comparisons of the estimates of error for each of the combinations of measurement elements. For the membrane-ship of the example, the individual tank quantities and calorific values will remain the same as in Case 1. The total calorific values will also be the same for all the cases. Only the estimated errors will change, and these will be a function of the measurement elements selected for each case. The values of estimated error for each element are given in table 3.1.1.

In a manner similar to that described under Case 1, it is possible to assemble the total calorific value and estimate of error of the LNG removed from the LNG ship for the measurement assumptions of Case 2.

From table 3.2 .2 the volume removed from tank 1 is $11,298.9 \mathrm{~m}^{3}$. Equation (6) gives a density of $474.246 \mathrm{~kg} / \mathrm{m}^{3}$ for the LNG within the tank, and, therefore, the mass removed is the volume removed multiplied by the density or $5,358.46 \mathrm{Mg}$. The calorific value of the LNG removed is the mass removed multiplied by the specific calorific value on a mass basis from equation (16). This value of 53.407 $\mathrm{MJ} / \mathrm{kg}$ multiplied by the mass removed gives the total calorific value of $286.179 \mathrm{TJ}\left(286.179 \mathrm{x} 10^{12 \mathrm{~J}}\right.$ ) removed from tank 1 .

From table 3.2 .2 the volume error is $\pm 18.33 \mathrm{~m} 3$ or \pm 0.16 percent of the tank 1 volume. From table 3.1 .1 the estimated error in measured density is \pm 0.26 percent, and the estimated error in calculated calorific value is \pm 0.35 percent. These individual estimates of error are independent variables and may be combined in quadrature. The combination of volume, density and specific calorific value is a 
slmple multiplication, and, therefore, the error in total calorific value of the LNG removed is:

$$
\begin{aligned}
\text { Estimated Tank Error Case } 2 & =\left[e_{v}^{2}+e_{d}^{2}+e_{c}^{2}\right]^{1 / 2} \\
& = \pm 0.46 \text { percent }
\end{aligned}
$$

where $e_{\mathrm{y}}$ is the estimated volume error, $e_{\mathrm{d}}$ is the estimated density error and $e_{\mathrm{c}}$ is the estimate error in calorific value. The total error in LNG removed from tank 1 is $(286.179 \mathrm{TJ})( \pm 0.46) / 100)=1.316$ TJ.

In 11 ke manner, total calorific values and estimated error may be calculated for ship tanks 2 , 3 , 4, 5 and 6 . The results are summarized in table 3.2.11.

\subsubsection{Sample Calculations for Case 3}

This third example is composed of a measured volume of LNG removed from each ship tank of the LNG membrane-type ship (table 3.2.2). A representative sample of LNG is removed from each ship tank, vaporized and analyzed to determine the fraction of each of the LNG components. The density of the sample is calculated using the mathematical models as shown in the example of Case 1 . From the previous sample or a newly drawn one, the sample is burned in the combustion calorimeter to determine the calorific value. A measured sample gas density will allow calculation of the calorific value on a mass basis.

The assumptions for Case 2 are made also for Case 3. For the membrane-type ship of the example, the individual tank quantities and calorific values will remain the same as in Case 1 . The total calorific values will also be the same for all the cases. Only the estimated errors will change, and these will be a function of the measurement elements selected for each case. The values of estimated error for each element is given in table 3.1 .1 .

In a manner similar to that described under Case $1_{1}$ it is possible to assemble the total calorific value and estimate of error of the LNG removed from the LNG ship for the measurement assumptions of Case 2.

From table 3.2 .2 the volume removed from tank 1 is $11,298.9 \mathrm{~m}^{3}$. Equation (6) gives a density of $474.246 \mathrm{~kg} / \mathrm{m}^{3}$ for the LNG within the tank, and, therefore, the mass removed is the volume removed multiplied by the density or $5,358.46 \mathrm{Mg}$. The calorific value of the LNG removed is the mass removed multiplied by the specific calorific value on a mass basis from equation (16). This value of 53.407 $\mathrm{MJ} / \mathrm{kg}$ multiplied by the mass removed gives the total calorific value of $286.179 \mathrm{TJ}$ (286.179 $\mathrm{x} 10^{12} \mathrm{~J}$ ) removed from tank 1 .

From table 3.2 .2 the volume error is $\pm 18.33 \mathrm{~m}^{3}$ or \pm 0.16 percent of the tank 1 volume. From table 3.1 .1 the estimated error in calculated density is \pm 0.23 percent, and the estimated error in measured calorific value is \pm 0.77 percent. These individual estimates of error are independent variables and may be combined in quadrature.

The combination of volume, density and specific calorific value is a simple multiplicat1on, and, therefore, the error in total calorific value of the LNG removed is:

$$
\text { Estimated Tank Error Case } \begin{aligned}
3 & =\left[e_{v}^{2}+e_{d}^{2}+e_{c}^{2}\right]^{1 / 2} \\
& = \pm 0.81 \text { percent }
\end{aligned}
$$

where $e_{\mathrm{v}}$ is the estimated volume error, $e_{\mathrm{d}}$ is the estimated density error and e $1 \mathrm{~s}$ the estlmated error in calorific value. The total error in LNG removed from tank 1 is $(286.179 \mathrm{TJ})( \pm 0.81) / 100)$ $2.318 \mathrm{TJ}$.

In like manner, total calorific values and estimated error may be calculated for shlp tanks 2 , 3 , 4,5 and 6 . The results are summarized in table 3.2 .11 . 


\subsubsection{Sample Calculations for Case 4}

This final example is composed of a measured volume of LNG removed from each ship tank of the LNG membrane-type ship (table 3.2.2). A representative LNG density is measured for each tank using a calibrated LNG densimeter. A representative sample is taken from each ship tank and is burned in the combustion calorimeter to determine the calorific value. A measured sample gas density will allow calculation of the calorific value on a mass basis.

The assumptions for Case 2 are made also for Case 4. For the membrane-type ship of the example, the individual tank quantities and calorific values will remain the same as in Case 1 . The total calorific values will also be the same for all the cases. Only the estimated errors will change, and these will be a function of the measurement elements selected for each case. The values of estimated error for each element are given in table 3.1.1.

In a manner similar to that described under Case 1, it is possible to assemble the total calorific value and estimate of error of the LNG removed from the LNG ship for the measurement assumptions of Case 2 .

From table 3.2 .2 the volume removed from tank 1 is $11,298.9 \mathrm{~m}^{3}$. Equation (6) gives a density of $474.246 \mathrm{~kg} / \mathrm{m} 3$ for the LNG within the tank, and, therefore, the mass removed is the volume removed multiplied by the density or $5358.46 \mathrm{Mg}$. The calorific value of the LNG removed is the mass removed multiplied by the specific calorific value on a mass basis from equation (16). This value of 53.407 $\mathrm{MJ} / \mathrm{kg}$ multiplied by the mass removed gives the total calorific value of $286.179 \mathrm{TJ}$ (286.179 $\mathrm{x} 10^{12 \mathrm{~J}}$ ) removed from tank 1 .

From table 3.2 .2 the volume error is $\pm 18.33 \mathrm{~m}^{3}$ or \pm 0.16 percent of the tank 1 volume. From table 3.1 .1 the estimated error in calculated density is \pm 0.26 percent and the estimated error in measured calorific value is \pm 0.77 percent. These individual estimates of error are independent variables and may be combined in quadrature. The combination of volume, density and specific calorific value is a simple multiplication and therefore the error in total calorific value of the LNG removed is:

Estimated Tank Error Case $4=\left[e_{v}^{2}+e_{d}^{2}+e_{c}^{2}\right]^{1 / 2}$

where $e_{\mathrm{V}}$ is the estimated volume error, $e_{\mathrm{d}}$ is the estimated density error and $e_{\mathrm{c}}$ is the estimate error in calorific value. The total error in LNG removed from tank 1 is $(286.179 \mathrm{TJ})( \pm 0.83) / 100)=2.375$ TJ.

In like manner, total calorific values and estimated error may be calculated for ship tanks 2 , 3 , 4, 5 and 6 . The results are summarized in table 3.2.11.

Table 3.2 .11

Summary Results for Case 1, 2, 3, and 4

Calorific Value and Estimated Error for

LNG Removed From Membrane-Type Ship Tanks

\begin{tabular}{|c|c|c|c|c|c|c|}
\hline \multirow{2}{*}{ Tank } & \multirow{2}{*}{$\begin{array}{c}\text { Volume } \\
\text { Removed } \\
(\mathrm{m} 3)\end{array}$} & \multirow{2}{*}{$\begin{array}{c}\text { Calorific } \\
\text { Value } \\
(\mathrm{TJ})\end{array}$} & & \multicolumn{4}{|c|}{ Estimated Error (TJ) } \\
\cline { 6 - 8 } & & Case 1 & Case 2 & Case 3 & Case 4 \\
\hline \hline & $11,298.9$ & 286.179 & \pm 1.288 & \pm 1.316 & \pm 2.318 & \pm 2.375 \\
2 & $22,360.0$ & 566.334 & \pm 2.549 & \pm 2.605 & \pm 4.587 & \pm 4.701 \\
3 & $24,854.0$ & 629.502 & \pm 2.833 & \pm 2.896 & \pm 5.099 & \pm 5.225 \\
4 & $5,624.3$ & 142.452 & \pm 0.641 & \pm 0.655 & \pm 1.154 & \pm 1.182 \\
5 & $24,873.6$ & 630.000 & \pm 2.835 & \pm 2.898 & \pm 5.103 & \pm 5.229 \\
6 & $24,868.0$ & 629.857 & \pm 2.834 & \pm 2.897 & \pm 5.102 & \pm 5.228 \\
Total & $113,878.8$ & $2,884.324$ & \pm 5.715 & \pm 5.842 & \pm 10.288 & \pm 10.541 \\
& & & $( \pm 12.980)$ & $( \pm 13.267)$ & $( \pm 23.363)$ & $( \pm 23.940)$ \\
\hline
\end{tabular}


It should be noted that the error for each case shown in Table 12 has been found by combining the errors from each tank in quadrature. It was assumed that measurements for each tank are independent of each other. This may not be true in practice and a simple sum is included in parenthesis. It is one objective of this manual to provide enough of the basic data and a description of the method so that if other procedures are preferred then these procedures may be followed using the original data.

The estimates of error for the total ship off-loading vary as to the measurement elements used and the level of detail and control of the measurement process. For example; the total error (independent tank measurements) varies from \pm 0.20 percent to \pm 0.37 percent, a rather small number and range considering the magnitude of the individual error estimates of the measurement elements. To hold the total uncertainty to a minimum, it pays to plan carefully for the entire measurement process. 



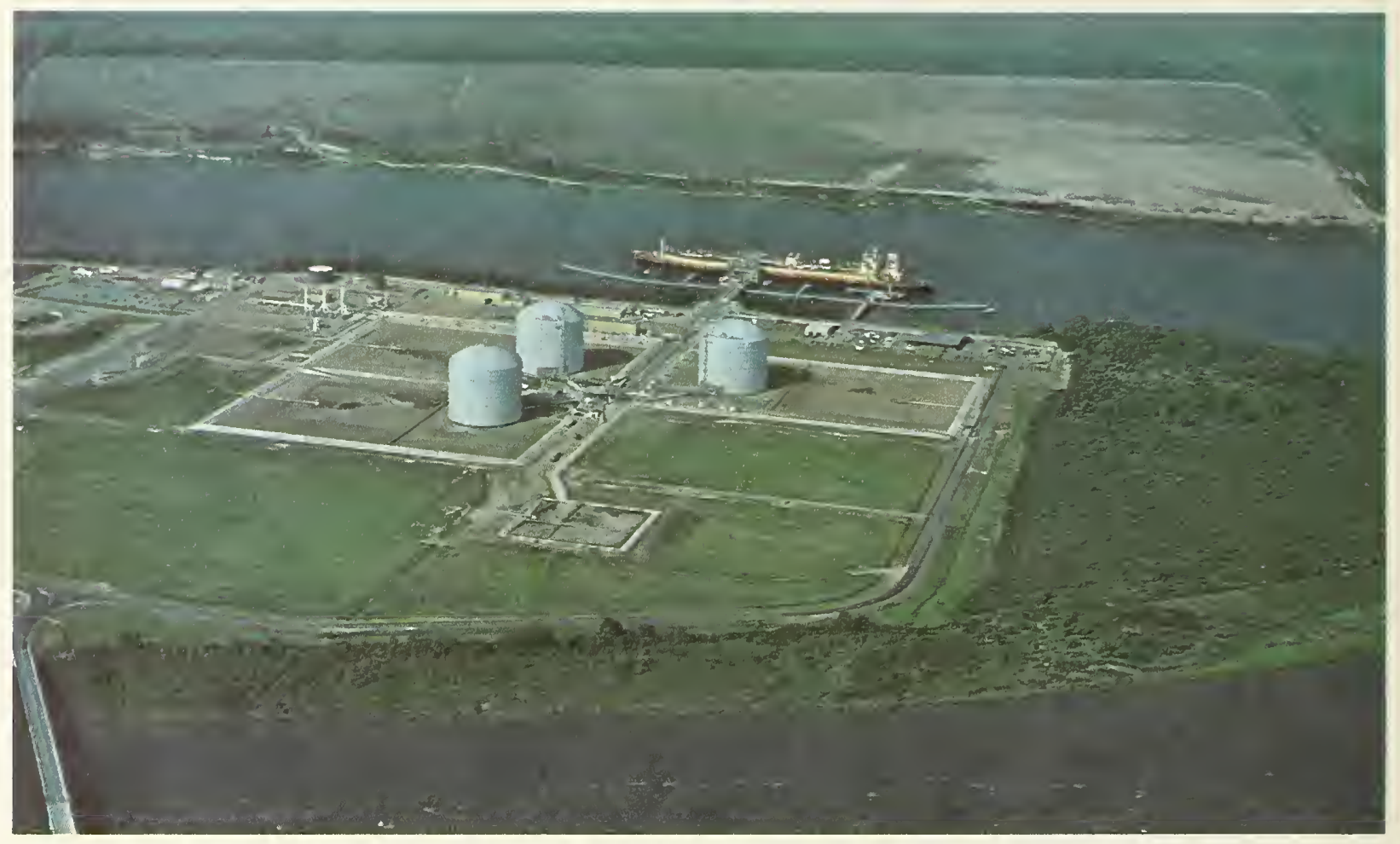

\section{ELBA ISLAND TERMINAL}

The terminal is located on Elba Island near Savannah, Georgia, at a point 16 $\mathrm{km}$ upstream of the mouth of the Savannah River, which flows along the border between South Carolina and Georgia. Construction of the terminal, which is owned by Southern Energy, a wholly owned subsidiary of Southern Natural Gas, was started in 1973. The first shipment of LNG was received in June 1978 under a sales contract between El Paso and Algeria's Sonatrach. The terminal has received LNG in an amount equivalent to 9.7 million cubic meters per day.

The photograph shows three storage tanks of 64,000 kL capacity and five submerged combustion type vaporizers (100 t/day capacity each) located to the left of the storage tanks. The LNG is vaporized and carried by pipeline about $20 \mathrm{~km}$ to a regulator station where it can be fed into existing pipelines owned by Southern Natural Gas. Currently no LNG is being imported at this terminal. (Photograph by courtesy of Southern Energy Company, Elba Island, Georgia) 


\subsection{Pipeline Metering}

\subsubsection{Introduction}

The general case is assumed in which an LNG ship has been loaded with LNG of a known composition at a remote producer terminal, has then been in transit for some period of time and has arrived at the LNG Importation terminal docking area. At the dock, the off-loading pipeline is connected to the LNG ship piping and a quantity of LNG is removed from the ship tanks and deposited into a LNG shore storage tank. The off-loading pipeline contains a measurement station composed of instrumentation for the dynamic measurements of LNG volume flow, liquid density and calorific value (see section 2.5.6.2). For this example, it is not necessary to define either the ship or shore tank instrumentation or capacities, as the only consideration is the quantity and composition of the LNG passing through the measurement station. However, in order to allow comparisons between the methods, accuracy and precision of the measurement station with that of the ship loading/unloading ( 3.2 ) and 1andbased storage (3.4) measurements, it will be assumed that a quantity of $114,000 \mathrm{~m}^{3}$ of LNG (approximately net from one $130,000 \mathrm{~m}^{3}$ ship load) is metered through the station, and the composition of the LNG was that of the Algerian LNG mixture shown in table 2.1.1.

It should be noted that in practice the quantity and composition of the LNG measured in the pipeline will not be precisely the same as that measured in the LNG ship. Pipeline heat leak and cooldown losses will result in changes in the quantity and composition of the liquid. The same caution is made here as to the expected changes of quantity and composition of the LNG delivered to the individual shore tank. Shore tank liquid content and composition prior to loading will additionally complicate the measurement process. In general, the following process will be concerned with the quantity and composition of the liquid passing through the metering station. Other considerations must be made in comparing the actual individual measurements of LNG removed from the ship, LNG metered in the off-loading line and LNG loaded into the shore tanks.

As in the preceding examples, no consideration is given to unexpected failure of instrumentation or other equipment which would require alternate measurements or measurement estimates of portions or all of the overall measurement process. Examples might be the interruption of power to the LNG discharge pumps in one of the ship tanks which may delay LNG flow through the off-loading line. Since the measurement station is an online dynamic system designed to integrate in time a series of short term observations, any departure from steady state flow conditions will certainly have some effect on the total measurement precision and accuracy. If the measurement station is chosen as the primary measurement, every effort should be made to minimize variations in flow rate, pressure and temperature.

The measurement objective is to determine the quantity and composition of the LNG passing through the off-loading line. The most common measurement to be made is the total caloriflc value. At the present time, no single property measurement can accurately and precisely provide this value directly. It is necessary to use a multi-step procedure involving volume, density and specific calorific value.

Volume. Dynamic quantitative totalized flow measurement of cryogenic fluids applied specifically to LNG is discussed in detail in section 2.5.6. Both mass and volume metering could have been used successfully with LNG. As the quantities of LNG and plpeline size requirements increased, volumetric metering became the meter of choice because of ease of adaptation and scaling cost when compared to the direct reading mass flowmeter. The generic vortex type flowmeter was selected as an example of volume metering only because extensive experimental data was avallable (see section 2.5 .6 ). 0 ther metering may certainly be used if demonstrated accuracy, precision and scaling laws have been established. Continuous observation of the meter output and an accurate measurement of elapsed time to give totalized flow is necessary to achieve maximum measurement accuracy and precision.

Density. A choice is available of either direct measurement using a calibrated inline flow dens1meter or a density calculation based on sampling, analysis and a mathematical model LilG mixture gtate equation. Both methods can be used to provide a cross check. Continuous monitoring of the $\delta 2$ w is necessary to achieve greatest accuracy and precision of measurement.

Calorific Value. This is the quantity of thermal energy released when the LNG mixture has been vaporized and burned in air to gaseous carbon dioxide and liquid water. The value may be determined directly by vaporizing a representative sample of LNG drawn from the flowing fluid in the plpellne and burning the mixture in a combustion calorimeter which measures the thermal output compared to that of a calibration gas or by calculation from sampling, analysis and integrating the ind!v!dual pure component calorific values. Both methods can be used as a cross check, but continuous monlicor!nf: 13 necessary to achieve greatest accuracy and precision of measurement. 
The results of these procedures may be combined in the following manner:

Total Calorific Value = (Totalized Volume)(Density)(Specific Calorific Value)

The term "total calorific value" means here the enthalpy of combustion of the contents of a tank or ship after these contents have been vaporized. In the testing literature, "total calorific value" for a gaseous fuel means something different, i.e. the enthalpy of combustion of a unit amount (mol, kg, cubic meter) when the products of combustion are gaseous carbon dioxide and liquid water. The term "specific calorific value" used here has that meaning for a kilogram sample.

\subsubsection{Examples of the Measurement Process}

Variations of the above procedure are, of course, possible by combining one or more of the steps, but as a descriptive matter, the above steps will be followed. Even with this restriction there are four possible individual procedures which may be followed:

Case 1 Total Calorific Value = LNG Volume $\mathrm{x}$ Density (calculated) $\mathrm{x}$ Specific Calorific Value (calculated)

Calculate the total volume of LNG passing through the measurement station by integrating the flow rate over the total time of metering. Secure a continuous representative LNG sample from the off-loading pipeline. Vaporize and analyze the sample to determine the fraction of individual components. Calculate the density of the flowing LNG using the state equation for liquid density as a function of measured LNG temperature, pressure and composition. Combine the density found from the equation with the volume passing through the measurement station to give the total mass metered by the station. This may be done by using an average volume flow rate during the sampling period and then integrating over the full off-loading time period or by averaging the calculated density from the individual samplings and multiplying this value by the totalized flow.

Using the previous sample analysis and averaging technique calculate the average specific calorific value of the LNG sample from calorific values of the individual components. Calculate the average density of the vaporized LNG sample and combine the calculated specific calorific values with the total mass measured at the measurement station.

Case 2 Total Calorific Value = LNG Volume $\mathrm{x}$ Density (measured) $\mathrm{x}$ Specific Calorific Value (calculated)

Calculate the total volume of LNG passing through the measurement station by integrating the flow rate over the total time of metering. Continuously measure the density of the LNG passing through the measurement station using a calibrated "in-line" densimeter and calculate the total mass of LNG passing through the measurement station. Secure a continuous representative LNG sample from the off-loading pipeline. Vaporize and analyze the sample to determine the fraction of individual components. Calculate the specific calorific value of the LNG sample from calorific values of the individual components. Calculate the density of the vaporized LNG sample and combine the calculated specific calorific values with the total mass passing through the measurement station to give the total calorific value of LNG.

Case 3 Total Calorific Value = LNG Volume $\mathrm{x}$ Density (calculated) $\mathrm{x}$ Specific Calorific Value (measured)

Calculate the total volume of LNG passing through the measurement station by integrating the flow rate over the total time of metering. Secure a continuous representative LNG sample from the off-loading pipeline. Vaporize and analyze the sample to determine the fraction of individual components. Calculate the density of the LNG using a state equation for liquid density as a function of measured LNG temperature, pressure and component fraction. Combine the density found from the equation with the volume passing through the measurement station to give the total mass metered by the station. This may be done by using an average volume flow rate during the sampling period and then integrating over the full off-loading time period or by averaging the calculated density from the individual samplings and multiplying this value by the totalized flow. 
Secure a representative pipeline LNG sample and burn the sample in a combustion calorimeter to give a specific calorific value. Measure the density of the vaporized LNG sample and combine the specific calorific value determined from the combustion calorimeter with the measured mass passing through the off-loading pipeline at the measurement station.

Case 4 Total Calorific Value = LNG Volume $\mathrm{x}$ Density (measured) $\mathrm{x}$ Specific Calorific Value (measured)

Calculate the total volume of LNG passing through the measurement station by integrating the flow rate over the total time of metering. Continuously measure the density of the LNG passing through the measurement station using a calibrated "in-line" densimeter and calculate the total mass of LNG passing through the measurement station. Secure a continuous representative sample of LNG the pipeline, completely vaporize the sample and burn the sample in a combustion calorimeter to give the specific calorific value. Measure the density of the vaporized sample and combine the specific calorific value determined from the combustion calorimeter with the total mass passed through the measurement station to give the total calorific value of the LNG passing through the measurement station.

\subsubsection{Measurement Elements}

Volume. The basis for analysis of volume error measurement is taken from section 2.5 .6 of this manual. Admittedly, the direct experimental evidence which may be used to establish the total uncertainty in the total LNG volume measured at the measurement station is minimal. However, the estimates of error given in 2.5.6 are those available and are believed to be representative of what will be found in the future as additional studies are completed. The selection of error values will be defined so that if new information becomes available or the reader wishes to use other data, this may be done.

At this time it is estimated that the uncertainty in measurement of the total volume of LNG passed through a typical measurement station is \pm 0.5 percent. This value is the sum in quadrature of two error estimates. The first estimate is that from section 2.5.6.4 which describes field testing of a thirty-two inch vortex shedding flowmeter. The total volume flow measured by the vortex shedding flowmeter was compared to the total volume removed from the LNG ship referenced to the ship level gauges. The difference was found not to exceed \pm 0.47 percent between the two measurements.

The second value used to provide flowmeter total volume uncertainty is found from section 3.2 .3 of this manual which provides an estimate of error for LNG ship tank volume measurement which includes liquid level and tank volume error estimates. From table 3.2.2, the error estimate from tank 1 is listed as \pm 0.16 percent of the tank volume. This value when added in quadrature to the above flowmetering error is found to be \pm 0.5 percent as stated.

Density. Definition of the values for LNG density as the fluid passes through the off-loading pipeline measurement station can be influenced greatly by actual field conditions. Direct density measurement utilizing an in-line densimeter is not always possible, as many pipelines are not so equipped. An individual densimeter may malfunction or become inoperative at some critical point in the measurement process. To realize maximum accuracy and precision, the densimeter primary element must be located in the main pipeline flow and must not be sensitive to fluid velocities. Calculated LNG densities which depend on LNG sampling and gas analysis (see section 2.2) must be located at or near the measurement station and should continuously monitor the fluid density.

Few if any of these field conditions have been monitored in any detail prior to the writing of this manual. Documented comparisons of densities measured by densimeters and calculated under actual ${ }^{1}$ leld operations are also not available at the present time. The summary of LNG flowmeter testing of section 2.5 .6 contains some data on in-line densimeters, but the small variation in density observed during the tests was not adequate to assess process measurement error. Therefore a number of simplifying assumptions have been made in order to allow a description of the measurement process in the context of the four case examples cited above.

1) A representative value for LNG density will be calculated for the LNG of $-10 a d i n g$ pipeline flowmetering process. Gas sampling and analysis as described in section 2.2 is assumed to provide a representative composition of the LNG in the plpelino. Accurate measurement of pipeline liquid pressure and temperature are also assumed to be available. The process by which these values are established are assumed to be within the existing industrial practice. 
2) In the absence of typical in-line densimeter data, it is assumed that the measured LNG density within the pipeline is numerically equal to the value calculated by means of sampling, gas analysis and use of the mathematical models. However, in assessing the estimated error, the total uncertainty of the densimeter used in the measurement will be that described in section 3.1 .2 of this manual. For example, if as a result of calculations to determine LNG density from measured pressure, temperature and assumed composition give a value of $480.23 \mathrm{~kg} / \mathrm{m}^{3}$, the uncertainty for this process is found from table 3.1 .1 as \pm 0.23 percent. To illustrate the sample calculations which follow, it is assumed that the density measured by the in-line densimeter is also found to be $480.23 \mathrm{~kg} / \mathrm{m}^{3}$ but with an uncertainty from table 3.1 .1 of \pm 0.26 percent.

Calorific Value. Comments and restrictions to the process of measurement of LNG calorific value are similar to those of LNG density above. The continuous acquisition of a representative sample from the pipeline which may be used in determining the calorific value directly from the combustion calorimeter or by calculation from gas analysis is necessary to provide a true representation of the total calorific value of the LNG cargo. The field problems associated with sampling and analysis are referenced in section 2.2, and additional simplifying assumptions are made here to present the process in the context of the four case examples.

1) A representative calorific value will be calculated as for density values above. It is known, of course, that the continuous observation will show variations in composition and density. These differences may be caused in part by differences in compositions of liquid in the individual ship tanks and in practice must be considered and averaged. Since the following examples are to show method only, it is assumed that the densities and compositions for each tank are constant values.

2) In the absence of typical field LNG calorific data, it is assumed that the calorific value of the pipeline fluid found from sampling and combustion calorimetry is numerically equal to the value calculated by means of sampling and gas analysis. However, in assessing the estimated error, the total uncertainty of the combustion calorimetry process used in the measurement will be that described in section 3.1 .2 of this manual.

For example, if as a result of calculations to determine LNG calorific value from measured pressure, temperature and assumed composition give a value of $54.323 \mathrm{MJ} / \mathrm{kg}$, the uncertainty for this process is found from table 3.1 .1 as 0.35 percent. To illustrate the sample calculations which follow, it is assumed that the calorific value measured by the combustion calorimeter is also found to be $54.323 \mathrm{MJ} / \mathrm{kg} \mathrm{but}$ with an uncertainty from table 3.1 .1 of \pm 0.77 percent.

\subsection{Sample Calculations for Case 1}

This example is composed of a volume of LNG passed through the off-loading pipeline measurement station. It is assumed that the measurement station provides instrumentation for LNG sampling, gas analysis, LNG temperature and pressure measurement used in the calculation of LNG density and calorific values. These values are then combined to give the total calorific value (with uncertainty) of the LNG passed through the pipeline. The source of the LNG is assumed to be Arzew, Algeria, and the composition has been confirmed by gas analysis to be as shown in table 2.1.1. It is further assumed that an LNG bulk liquid temperature of $108.00 \mathrm{~K}$ and a pressure of $138 \mathrm{kPa}$ are the results of measured averages of the pipeline liquid over the off-loading time period. These liquid conditions are the same as those cited in sections 3.2 and 3.4 .

Calculation of the LNG density can be accomplished by using one or more of the mathematical models described in section 2.4.3 and 2.4.4. One of the methods will be shown in detail, and a comparison of density values from the other three methods will be shown for comparison purposes. The Revised Klosek and McKinley method (section 2.4.4.4) was selected, because this method does not require a computer for solution; although the method has been adapted to computer use.

The Klosek and McKinley (KM) method is a totally empirical recipe for calculating the density of the LNG-like mixture given the temperature and composition. Pressure is not taken into account. However, at near atmospheric pressure LNG is nearly incompressible, hence the density may be considered independent of pressure. 
The procedure for calculating the LNG density is as follows:

$$
\mathrm{V}_{\text {mix }}=\sum \mathrm{x}_{\mathrm{i}} \mathrm{V}_{\mathrm{i}}-\mathrm{kX}_{\mathrm{CH}_{4}}
$$

where $V_{m i x}$ is the volume of the mixture, $x_{i}$ and $V_{i}$ are the mole fraction and volume of the $i$ th component, $\mathrm{X}_{\mathrm{CH}_{4}}$ is the mole fraction of methane and $\mathrm{k}$ is a correction factor obtained from a table or graph.

The $V_{1}$ and $k$ are obviously temperature dependent, and in addition $k$ is dependent upon the molecular mass of the mixture. The original form of equation (1) was modified by adding a term to take into account the nitrogen when it is present. See section 2.4.4.4 for details.

$$
\mathrm{V}_{\operatorname{mix}}=\left[\mathrm{x}_{\mathrm{i}} \mathrm{v}_{\mathrm{i}}-\left[\mathrm{k}_{1}+\left(\mathrm{k}_{2}-\mathrm{k}_{1}\right) \mathrm{x}_{\mathrm{N}_{2}} / 0.0425\right] \mathrm{x}_{\mathrm{CH}_{4}}\right.
$$

where $k_{1}$ refers to mixtures without nitrogen and $k_{2}$ refers to mixtures with nitrogen. All other terms are the same as in equation (1). Tabular values for solving the equation are given in section 1 , 2.4. 4 and 3.2.

Table 3.3. 4 provides initial data for the solution. Values for molecular mass are from tables 1.2 .1 and 1.2.2. Volumes of saturated liquid of the pure components are found from table 3.2 .6 . The slight differences in the molecular masses are a result of the revision cited in section 1.2 and do not change the values of mixture molecular mass shown in table 3.3 .4 .

Table 3.3 .4

Molecular Mass and Volume of Algerian LNG Mixture Temperature $=108 \mathrm{~K}$, Pressure $=138 \mathrm{kPa}$

\begin{tabular}{|l|l|l|l|l|l|}
\hline Constituent & $X_{i}$ & $\begin{array}{c}\mathrm{M}_{\mathrm{W}} \\
\mathrm{g} / \mathrm{mol}\end{array}$ & $\begin{array}{c}\mathrm{X}_{\mathrm{i}} \mathrm{M}_{\mathrm{W}} \\
\mathrm{g} / \mathrm{mol}\end{array}$ & $\begin{array}{c}\mathrm{V}_{\mathrm{I}} \\
\mathrm{L} / \mathrm{mol}\end{array}$ & $\begin{array}{c}\mathrm{XV}_{\mathrm{I}} \\
\mathrm{L} / \mathrm{mol}\end{array}$ \\
\hline \hline methane & 0.8700 & 16.043 & 13.9574 & 0.037481 & 0.032608 \\
ethane & 0.0814 & 30.070 & 2.4477 & 0.047512 & 0.003867 \\
propane & 0.0230 & 44.097 & 1.0142 & 0.062033 & 0.001427 \\
n-butane & 0.0068 & 58.123 & 0.3952 & 0.076384 & 0.000519 \\
i-butane & 0.0046 & 58.123 & 0.2674 & 0.077836 & 0.000358 \\
n-pentane & 0.0002 & 72.150 & 0.0144 & 0.091042 & 0.000018 \\
nitrogen & 0.0140 & 28.0134 & $\frac{0.3922}{8.4885}$ & 0.043963 & $\frac{0.000615}{0.0394124}$ \\
\hline
\end{tabular}

Values for the correction factors of equation (2) are found by linear interpolation of temperature and volume in table 3.2.7 for correction factor $k_{1}$ and table 3.2 .8 for correction factor $k_{2}$. For the temperature and composition selection for the example (table 3.3.4), the values for the correction factors were found to be $k_{1}=0.434$ and $k_{2}=0.61$.

Substituting in equation (2) gives:

$$
\begin{aligned}
& V_{m}= 0.0394124 \\
&-\left\{0.434\left(10^{-3}\right)+\left[0.61\left(10^{-3}\right)-0.434\left(10^{-3}\right)\right][0.014] / 0.0425\right\} 0.8700 \\
& V_{m}= 0.038985 \text { liters } / \mathrm{mol} \\
& \rho(\text { density })=1 / V_{m}=25.6509 \mathrm{~mol} / 1 \text { iter }
\end{aligned}
$$

In terms of the actual mixture, the molar density is multiplied by the molecular mass of the mixture from table 3.3 .4 to give:

$$
\rho(\text { mixture density })=(25.6509)(18.4885)=474.246 \mathrm{~kg} / \mathrm{m}^{3}
$$

Computer programs which provide a calculated value for the required density of the Lil mlxture are discussed in detail in section 2.4.4. A comparison of the mixture densities predicted by the sour mathematical models are shown in table 3.3.5. The four models are the Klosek and Mck $1 \mathrm{nley}$ (KM). $\mathrm{kh}$ Corresponding States (CS), the Cell (C) and the Hard Sphere (HS). 
Table $3 \cdot 3 \cdot 5$

LNG Mixture Density Values Using Several Calculation Methods

\begin{tabular}{|l|c|}
\cline { 2 - 2 } \multicolumn{1}{c|}{} & $\begin{array}{c}\text { Density } \\
\mathrm{kg} / \mathrm{m} 3\end{array}$ \\
\hline (from equation (2) and tables) & 474.246 \\
(from computer program- KM) & 474.242 \\
(from computer program- CS) & 474.446 \\
(from computer program- C) & 474.276 \\
(from computer program- HS) & 474.409 \\
(mean value) & 474.324 \\
(maximum error) & \pm 0.122 \\
\hline
\end{tabular}

The maximum error of the five calculation methods from the mean mixture density example as shown in table 3.3 .5 is \pm 0.026 percent. This is within the estimate of error for calculation of LNG density from the four mathematical models ( 0.1 percent) given in sections 2.4 .4 and 3.1 .2 . The value from equation (6) will be used in all the following example calculations.

Calorific Value. The calorific value of the selected LNG mixture may be found for the example of Case 1 by calculation from the gas analysis. The following method is suggested as one which includes the most recent analysis of the heats of combustion and atomic and molecular masses. The source of these values and suggested methods was an NBS study sponsored by Groupe International des Importateurs de Gaz Naturel Liquefie (GIIGNL). The results of the study have been published by NBS as NBSIR $82-2401$ "Heating Values of Natural Gas and Its Components" by G. T. Armstrong and T. L. Jobe (1982). As explained in section 1 of this manual, some of the values reported in the reference have since been revised (references $12 \mathrm{a}$ and $\mathrm{b}$ of section 1 ). The publication and revision are extensive in scope and detail and only portions are cited in this section and section 1 . However, the content of the study is the basis of all calculations involving combustion enthalpies used in this manual.

The virial equation $\mathrm{PV} / \mathrm{RT}=1+\mathrm{B}(\mathrm{T}) / \mathrm{V}$ where $\mathrm{P}$ is absolute pressure, $\mathrm{V}$ is molar volume, $\mathrm{R}$ is the gas constant, $T$ is the absolute temperature and $B(T)$ the second virial coefficient is used by Armstrong and Jobe in the development of the combustion enthalpies of LNG mixtures. The equation is simple to use, has a basis in theory and allows an understanding of the application of the developed combustion enthalpies to ideal and real gas states for both pure fluids and mixtures.

The composition of the gas mixture in terms of the molar fractions $x(i)$ of the individual substances present, or in terms of the mass fractions $W(i)$ of the individual substances present, is presumed to be known (see table 3.3 .4 ) and $\left[X(i)\right.$ or $\sum W(i)$ is unity.

The calculation of the heating value involves five steps: (1) the calculation of the ideal gas molar heating value of the mixture; (2) the calculation of the virial coefficient of the gas mixture and its temperature derivative; (3) the calculation of the molar volume of the real gas mixture based upon the virial coefficient of the gas mixture from step (2); (4) the adjustment of the molar heating value for the term $\mathrm{H}-\mathrm{H}^{\circ}$ which is the difference in enthalpy of the real and ideal gas based on the virial coefficient of the gas mixture and its derivative; (5) the calculation of the heating value on a mass basis and the volumetric heating value of the real gas mixture.

Molar heating value $\Delta_{c} H_{m}$ (mixture) can be converted to the heating value on a mass basis $\Delta{ }_{\mathrm{C}} \mathrm{H}_{\mathrm{W}}$ (mixture) by use of the mass fractions of the substances in the gas. From the molar volume calculated in step (3), the molar enthalpy of combustion can be converted to the volumetric enthalpy of combustion, $\Delta_{c} H_{v}$ (mixture). According to Armstrong and Jobe (see above), step (4) and the calculation of the temperature derivative of the virial coefficient can be omitted with errors not exceeding an estimated $50 \mathrm{~J} / \mathrm{mol}$.

The calculation procedure is considered to be the the most accurate of those considered. It is applicable to both dry and humid gases, including the water-saturated gas. The dry gas conditions are assumed here. Notation and symbols are those of Armstrong and Jobe. The detailed description of the procedure is given in section 3.2 and the results of that procedure are used in this section. 
From step 5 of section 3.2:

$$
-\Delta_{c^{H}} H_{m}(\text { mixture })=987.410 \mathrm{~kJ} / \mathrm{mole}
$$

Using the molecular mass of the mixture calculated in table 3.3 .4 ,

$$
-\Delta_{c} H_{m}(\text { mixture })=(987.410) /(18.4885)=53.407 \mathrm{MJ} / \mathrm{kg}
$$

The volumetric combustion enthalpy comes from equation (15) and step 3 of section 3.2 .

$$
\begin{aligned}
-\Delta_{c^{H}} H_{V}(\text { mixture }) & =\Delta_{c} H_{m}(\text { mixture }) / V_{m}(\text { mixture }) \\
-\Delta_{c} H_{V}(\text { mixture }) & =(987.410) /(0.023583) \\
& =41.870 \mathrm{MJ} / \mathrm{m}^{3}
\end{aligned}
$$

\subsubsection{Case 1 Error Summary}

From the above calculations of volume, density and calorific value, it is possible to assemble the total calorific value and estimate of error of the LNG passed through the off-loading line and measurement station.

From section 3.3 .1 the volume of LNG measured at the measurement station was $114,000 \mathrm{~m} 3$. Equation (6) gives an average density of $474.246 \mathrm{~kg} / \mathrm{m}^{3}$ for the off-loading process and therefore, the mass of LNG measured at the measurement station is the volume measured multiplied by the density or 54,064.0 $\mathrm{Mg}$. The calorific value of the LNG added is the mass added multiplied by the specific calorific value on a mass basis from equation ( 8 ). This value of $53.407 \mathrm{MJ} / \mathrm{kg}$ multiplied by the mass added gives the total calorific value of $2.887 \mathrm{PJ}\left(2.887 \times 10^{15} \mathrm{~J}\right)$ at the measurement station. ( $P$ is the SI prefix symbol for Peca or $10^{15}$, see section 1.1 ).

From section 3.3 .1 the totalized volume error is \pm 0.50 percent. From table 3.1 .1 the estimated error in calculated density is \pm 0.23 percent, and the estimated error in calculated calorific value is \pm 0.35 percent. These individual estimates of error are independent variables and may be combined in quadrature. The combination of volume, density and specific calorific value is a simple multiplication, and therefore the error in total calorific value passed through the measurement station is:

$$
\text { Estimated Error Case } \begin{aligned}
1 & =\left[e_{v}^{2}+e_{d}^{2}+e_{c}^{2}\right]^{1 / 2} \\
& = \pm 0.65 \text { percent }
\end{aligned}
$$

where $e_{\mathrm{v}}$ is the estimated volume error, $e_{d}$ is the estimated density error and $e_{c}$ is the estimated error in calorific value. The total error in calorific value of the LNG at the measurement station $1 \mathrm{~s}$ $(2,887$. TJ $)( \pm 0.65) / 100)= \pm 19.76 \mathrm{TJ}$.

\subsubsection{Sample Calculations for Case 2}

The example is composed of a measured volume of LNG passed through the off-loading plpeline measurement station. An average LNG density is determined for the entire off-loading process from readings of the calibrated LNG in-line densimeter. A continuous sampling of the pipeline LNG is vaporized and analyzed to determine the fraction of each of the LNG components. Calculat1ons are then made to determine the calorific value of the representative LNG sample on a mass basls.

In a manner similar to that described under Case 1, it is possible to assemble the total calorlr $1 \mathrm{c}$ value and estimate of error of the LNG measured at the measurement stat1on for the measurement assumptions of Case 2 .

From Case 1, the total calorific value of the LNG passed through the measurement stat1on $1 \mathrm{~s} 2.887$ PJ. From section 3.3 .1 the volume error is \pm 0.50 percent. From table 3.1 .1 the est $1 \mathrm{mated}$ error $1 \mathrm{n}$ measured density is \pm 0.26 percent, and the estimated error in calculated calor $1 \mathrm{f} 1 \mathrm{c}$ value $1 \mathrm{~s} \pm 0.35$ percent. These individual estimates of error are independent variables and may be comblned $1 \mathrm{n}$ quadrature. The combination of volume, density and specific caloriflc value $1 \mathrm{~s}$ a simple multiplication, and therefore the error in total calorific value of the LNG passed through tha measurement station is: 
Estimated Error Case $2=\left[e_{\mathrm{v}}^{2}+e_{d^{2}}+e_{c}^{2}\right]^{1 / 2}$

$$
= \pm 0.66 \text { percent }
$$

where $e_{V}$ is the estimated volume error, $e_{d}$ is the estimated density error and $e_{c}$ is the estimated error in calorific value. The total error in calorific value of the metered LNG is ( 2,887 . PJ)( $\pm 0.66 / 100)= \pm 19.05 \mathrm{TJ}$.

\subsubsection{Sample Calculations for Case 3}

This third example is composed of a measured volume of LNG passed through the off-loading pipeline measurement station. A representative sample of LNG is continuously removed from the pipeline, vaporized and analyzed to determine the fraction of each of the LNG components. The density of the sample is calculated using the mathematical models as shown in the example of Case 1 . From the previous sample or a newly drawn one the sample is burned in the combustion calorimeter to determine the calorific value. A measured sample gas density will allow calculation of the calorific value on a mass basis.

Erom Case 1 the total calorific value of the LNG passed through the measurement station is 2.8864 PJ. From section 3.3.1 the volume error is \pm 0.50 percent. From table 3.1 .1 the estimated error in calculated density is \pm 0.23 percent, and the estimated error in measured calorific value is \pm 0.77 percent. These individual estimates of error are independent variables and may be combined in quadrature. The combination of volume, density and specific calorific value is a simple multiplication and therefore the error in total calorific value of the LNG added is:

Estimated Error Case $3=\left[e_{v}^{2}+e_{d}^{2}+e_{c}^{2}\right]^{1 / 2}$

$$
= \pm 0.95 \text { percent }
$$

where $e_{v}$ is the estimated volume error, $e_{d}$ is the estimated density error and $e_{c}$ is the estimated error in calorific value. The total error in calorific value of the LNG at the measurement station is $(2,887$. PJ $)( \pm .95) / 100)= \pm 27.43 \mathrm{TJ}$.

\subsubsection{Sample Calculations for Case 4}

This final example is composed again of a measured volume of LNG passed through the off-loading pipeline measurement station. An average LNG density is determined for the entire off-loading process using a calibrated LNG densimeter. A representative sample is taken continuously from the pipeline and is burned in the combustion calorimeter to determine the calorific value. A measured sample gas density will allow calculation of the calorific value on a mass basis.

From Case 1, the total calorific value of the LNG passed through the off-loading pipeline was 2.8864 PJ. From section 3.3 .1 the volume error \pm 0.50 percent. From table 3.1 .1 the estimated error in measured density is \pm 0.26 percent, and the estimated error in measured calorific value is \pm 0.77 percent. These individual estimates of error are independent variables and may be combined in quadrature. The combination of volume, density and specific calorific value is a simple multiplication, and therefore the error in total calorific value of the LNG added is:

Estimated Error Case $4=\left[e_{v}^{2}+e_{d}^{2}+e_{c}^{2}\right]^{1 / 2}$

$$
= \pm 0.95 \text { percent }
$$

where $e_{v}$ is the estimated volume error, $e_{d}$ is the estimated density error and $e_{c}$ is the estimated error in calorific value. The total error in calorific value of the LNG at the measurement station is $(2,887$. TJ $)( \pm .95) / 100)= \pm 27.43 \mathrm{TJ}$. 
Table 3.3 .6

Summary Results for Case 1, 2, 3, and 4

Calorific Value and Estimated Error for

LNG Passed through Measurement Station

\begin{tabular}{|l|c|c|c|c|c|}
\hline $\begin{array}{l}\text { Volume } \\
\text { Measured } \\
\left(\mathrm{m}^{3}\right)\end{array}$ & $\begin{array}{c}\text { Calorific } \\
\text { Value } \\
(\mathrm{TJ})\end{array}$ & \multicolumn{4}{|c|}{ Estimated Error (TJ) } \\
\cline { 3 - 6 } & Case 1 & Case 2 & Case 3 & Case 4 \\
\hline \hline $114,000.0$ & $2,887$. & \pm 18.76 & \pm 19.05 & \pm 27.43 & \pm 27.43 \\
\hline
\end{tabular}

The estimates of error for the total measurement station off-loading will vary as to the measurement elements used and the level of detail and control of the measurement process. For example, the total error (independent measurements) varies from \pm 0.65 percent to \pm 0.95 percent, a rather small number and change considering the magnitude of the individual error estimates of the measurement elements. To hold the total uncertainty to a minimum, it pays to plan carefully for the entire measurement process. 



\section{LMG MEASUREMEMT}

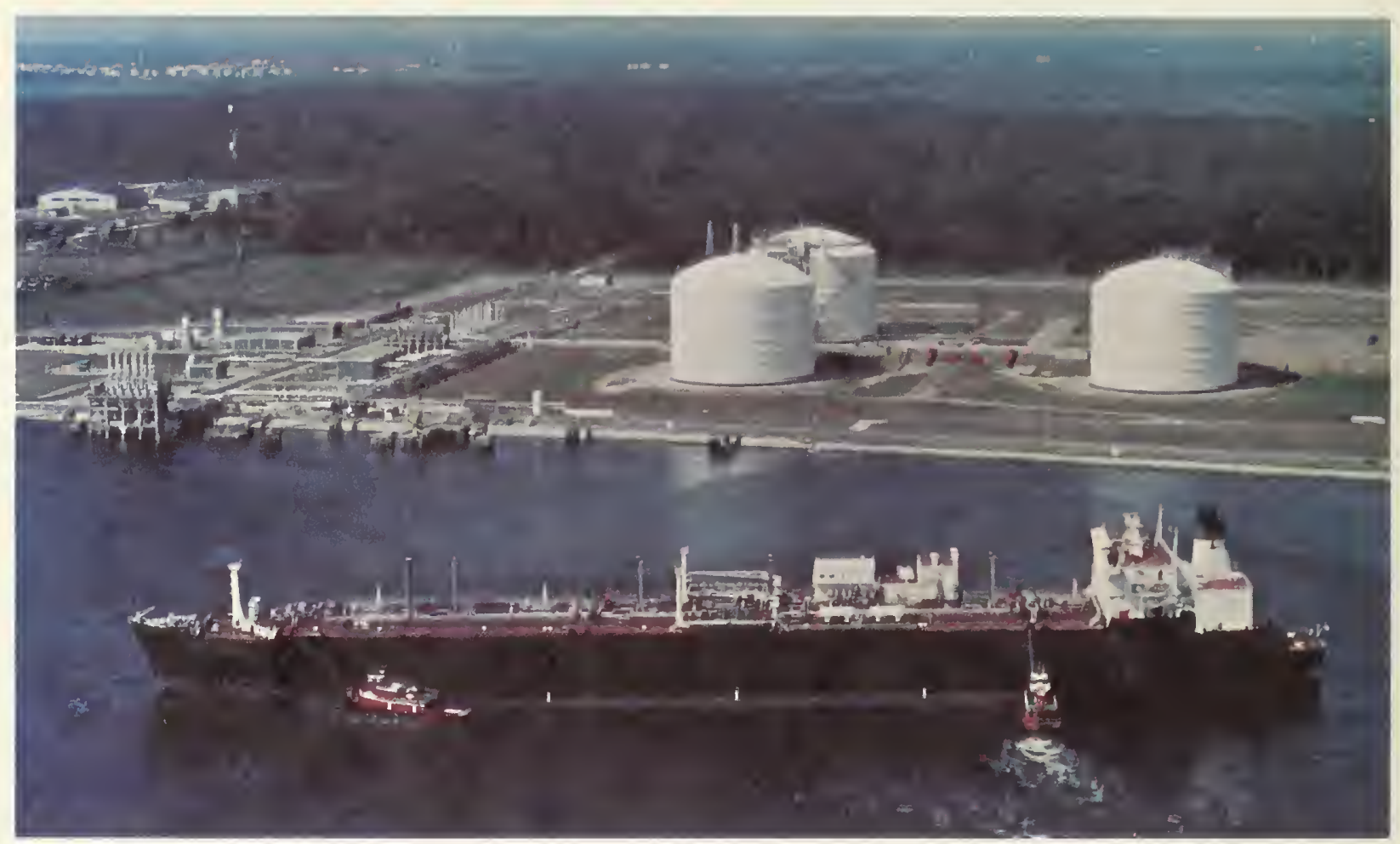

\section{LAKE CHARLES TERMIHAL}

The Lake Charles LNG Terminal is located on an industrial canal near Lake Charles, Louisiana, and at a distance of about $40 \mathrm{~km}$ from the Gulf of Mexico. Construction of the terminal was begun in 1978 by Trunkline LNG, a wholly owned subsidiary of Panhandle Eastern. Plans called for receiving LNG from Algeria at a rate of about 3.7 milijon tons annually. The major terminal facilities are shown in the photograph. They include the berth, three 95,000 KL above ground double-walled metali ic storage tanks and seven units of submerged combustion type vaporizers (to the left of the tanks) each having a capacity of $90 \mathrm{t} / \mathrm{h}$.

Extensive calibration and measurement studies were initiated with this facility and are described in the Measurement Manual sections on shore tank volume measurements and totalized volume flowmeter measurements. Currently no LNG is imported through this terminal. (Photograph by courtesy of Trunkine LNG Company, Lake Charles, Louisiana) 


\section{4 Landbased Storage}

\subsubsection{Introduction}

The general case is assumed in which an LNG ship has been loaded with LNG of a known composition at a remote producer terminal, has then been in transit for some period of time and has arrived at the LNG importation terminal docking area. At the dock, the off-loading pipeline is connected to the LNG ship plping, and a quantity of LNG is removed from the ship tanks and deposited into a LNG shore tank. There are, of course, large differences in LNG ship capacities and shore storage facilities. For this example, it is assumed that the volume of LNG off-loaded to the shore facility is nearly that of the membrane-type LNG ship having tank capacities and characteristics as shown in table 3.2.2. It is also assumed that more than one shore tank will be required to contain and store the contents of the LNG ship. This section of the manual considers the off-loading of LNG to the shore tanks.

It was noted in the previous section on pipline flowmetering that the quantity and composition of the LNG measured in the pipeline will not be precisely the same as that measured in the LNG ship tanks. Plpeline heat leak and cooldown losses will result in changes in composition of the liquid. The same caution is made here as to the expected changes of quantity and composition quality of the LNG delivered to the individual shore tank. Shore tank liquid content and composition prior to loading will additionally complicate the measurement process. In general, the following process will be concerned with the quantity and composition of the liquid content of the shore tank. 0 ther considerations must be made in comparing the individual measurements of LNG removed from the ship, LNG metered in the off-loading line and LNG loaded into the shore tanks.

As in the preceding examples, no consideration is given to unexpected failure of instrumentation or other equipment which would require alternate measurements or measurement estimates of portions or all of the overall measurement process. Examples might be the failure of the capacitance liquid level device after the upper level had been read but before the lower level was read. A backup bubbler level gage or other liquid level measurement device (see section 2.5.1) might be used. The interruption of power to the LNG discharge pumps in one of the ship tanks may delay LNG flow into the shore tank, and the off-loading may be halted for a period of time. These and other unanticlpated changes in the expected measurement process are not unusual, particularly in the case of a new technology; and these types of unexpected problems will test the resources of the measurement engineer.

The measurement objective is to determine the quantity and composition of the LNG delivered and stored in the shore tanks. The most common measurement to be made is the total calorific value of the LNG stored. At the present time, no single property measurement can accurately and precisely provide this value directly. It is necessary to use a multistep procedure involving volume, density and specific calorific value.

Volume. This is a measured quantity involving some predictive calculation. For the case of shore tank storage (see section 2.5.5), only static measurement states are considered. Each shore tank is assumed to be individually instrumented as to liquid level. Each tank is also considered as an individual measurement entity, because the temperature, pressure and composition of the liquid may vary during the loading operation.

Density. A choice may be made of either direct density measurement using a calibrated densimeter or a density calculation based on sampling, analysis and a LNG mixture state equation. Both methods can be used to provide a cross check. As in the comments on volume above, each tank will be considered as a measurement entity in respect to an individual densimeter for each tank. Sampling and analysis of the liquid from each individual tank is also assumed.

Calorific Value. This is the quantity of thermal energy released when the LNG mixture has been vaporized and burned in air to gaseous carbon dioxide and liquid water. The value may be determlned directly by vaporizing a representative sample of LNG and burning the mixture in a combustlon calorimeter which measures the thermal output compared to that of a calibration gas or by calculat 1 on from sampling, analysis and integration of the individual pure component calorific values. Both methods can be used as a cross check, but because of the loading procedure mentioned above, sampling procedures must be designed to assure that a representative sample is taken for each separate tank.

The results of these procedures may be combined in the following manner: 
The term "total calorific value" means here the enthalpy of combustion of the contents of a tank or ship after these contents have been vaporized. In the testing literature, "total calorific value" for a gaseous fuel means something different, i.e. the enthalpy of combustion of a unit amount (mol, kg, cubic meter) when the products of combustion are gaseous carbon dioxide and liquid water. The term "specific calorific value" used here has that meaning for a kilogram sample.

\subsubsection{Examples of the Measurement Process}

Variations of the above procedure are, of course, possible by combining one or more of the steps, but, as a descriptive matter, the above steps will be followed. Even with this restriction there are four possible individual procedures which may be followed:

Case 1 Total Calorific Value = LNG Volume $\mathrm{x}$ Density (calculated) $\mathrm{x}$ Specific Calorific Value (calculated)

Calculate the volume of LNG added to each shore tank. Secure a representative LNG sample from each shore tank. Vaporize and analyze the sample to determine the fraction of individual components. Calculate the density of the LNG added to each shore tank using the state equation for liquid density as a function of measured LNG temperature, pressure and composition. Combine the density of each shore tank with the volume added to give mass added to each tank. Using the previous sample analysis calculate the specific calorific value of the LNG sample from calorific values of the individual components. Calculate the density of the vaporized LNG sample and combine the calculated specific calorific values of each shore tank to give the total calorific value of LNG delivered.

Case 2 Total Calorific Value = LNG Volume $x$ Density (measured) $x$ Specific Calorific Value (calculated)

Calculate the volume of LNG added to each shore tank. Measure the representative LNG density in each shore tank using a calibrated densimeter and calculate the mass of LNG added to each tank. Secure a representative LNG sample from each shore tank. Vaporize and analyze the sample to determine the fraction of individual components. Calculate the specific calorific value of the LNG sample from calorific values of the individual components. Calculate the density of the vaporized LNG sample and combine the calculated specific calorific values of each shore tank with the total mass added to each tank to give calorific value of LNG removed. Combine individual tank calorific values to give the total calorific value of LNG delivered.

Case 3 Total Calorific Value = LNG Volume $\times$ Density (calculated) $\times$ Specific Calorific Value (measured)

Calculate the volume of LNG added to each shore tank. Secure a representative LNG sample from each tank. Vaporize and analyze the sample to determine the fraction of individual components. Calculate the density of the LNG added to the shore tank using the state equation for liquid density as a function of measured LNG temperature, pressure and component fraction. Combine the density of each shore tank with volume added to each tank to give mass added to each tank. Secure a new representative tank sample or use the previous sample and burn the sample in a combustion calorimeter. Measure the density of the vaporized LNG sample and combine the specific calorific value determined from the combustion calorimeter with the mass added to each shore tank to give the total calorific value of each tank. Combine the individual shore tank calorific values to give the total calorific value for the LNG delivered.

Case 4 Total Calorific Value = LNG Volume $\mathrm{x}$ Density (measured) $\mathrm{x}$ Specific Calorific Value (measured)

Calculate the volume of LNG added to each shore tank. Measure the representative LNG density in each tank using a calibrated densimeter and calculate the mass of LNG added to each shore tank. Secure a representative sample of LNG from each shore tank, vaporize the sample completely and burn the sample in a combustion calorimeter. Measure the density of the vaporized sample and combine the specific calorific value determined from the combustion calorimeter with the mass added to each shore tank to give the calorific value of the LNG added to tank. Sum the individual calorific values to give the total calorific value for all the LNG delivered. 


\subsubsection{Measurement Elements}

Volume. Each of the cases cited above requires detailed examination of the individual measurement elements in order to determine the uncertainty of the measurement process. For example; each case clted requires a measurement of the volume of the LNG added to the shore tanks. The technique requires two measurements of the level of the LNG, one before loading and the second after loading. Table 3.4.1 lists the uncertainty in liquid level measurement using data from 2.5 .1 of this manual.

Table 3.4 .1

Liquid Level Measurement Uncertainty

\begin{tabular}{|l|c|}
\hline Level Device & $\begin{array}{r}\text { Height Uncertainty } \\
\text { (mm) }\end{array}$ \\
\hline \hline Capacitance I & $\begin{array}{l} \pm .5 \\
\pm 10.0\end{array}$ \\
Capacitance II & \\
Cable & \pm 1.8 \\
Manufacturers Spec.(fine) & \pm 5.3 \\
Manufacturers Spec.(commercial) & \pm \\
Cable (uncompensated) & \pm 80. \\
Cable (compensated- NBS estm.) & \pm 1.0 \\
\hline
\end{tabular}

The cable manufacturers specification is a position specification at ambient temperatures. When installed in a cryogenic LNG tank, differential thermal expansion of the tank and level materials may cause significant error. Siegwarth (see reference [23] of section 2.5.1) has shown that the uncompensated error can amount to $80 \mathrm{~mm}$ in 41 meters which, accounts for the entry in table 3.4 .1 . Siegwarth in the same publication has shown that a compensated cable level device may be expected to show a precision of $\pm 1 \mathrm{~mm}$.

A single type of LNG shore tank was analyzed in section 2.5.5. One result of the analysis was an individual tank table which related a measured level to the volume of the tank at that level. After compensation for low temperature operation each tank table could be used to measure the quantity of LNG added or removed. Examples of calculations for the cylindrical type of tank are given in the following tables. It is assumed that the tanks are cold and of uniform temperature. Tank Liv quantity added was assumed to be from 5 percent tank capacity to 95 percent tank capacity. When the first tank reaches 95 percent capacity (table 3.4.2), flow is diverted to the second tank(table 3.4.3). Other values may also be used. For simplicity, an error of $\pm 7.5 \mathrm{~mm}$ is assumed in measurement of liquid level in both tanks. Of course, other values from table $3.4 .1 \mathrm{may}$ also be used. The total volume off-loaded from the LNG ship is assumed for this example to be $114,000 \mathrm{~m}^{3}$ exclusive of transfer losses.

The first entry of table 3.4 .2 is the volume of shore tank 1 from the photogrammetric survey (reference 7,8 and 9 of section 2.5.5). The second and eighth entries are the volume error given as part of the survey. No estimate of incremental height-volume error was provided with this calibration, so the total volume error for each tank was assessed at the measured liquld level.

The next four entries concern the estimate of error at the 5 percentile volume level caused by the liquid level measurement uncertainty and calculated using the actual tank table. The value $\Delta \mathrm{V} / \Delta \mathrm{H}$. $\mathrm{s}$ the change in volume per unit change in tank height at the 5 percent volume level. The $11 q u 1 d$ level (LL) error when multiplied by this value gives the volume error attributed to the measurement of liquid level at the particular tank height.

This error estimate is repeated at the 95 percent volume level in entries seven, nine, ten and eleven. The total volume added to the tank is given in entry twelve with the total error estimate of this volume calculated from the two volume, and the two liquid level errors combined in quadrature. 
Table 3.4 .2

Shore Tank Estimate of Error Cylindrical Shore Tank Number 1

\begin{tabular}{|c|c|}
\hline $\begin{array}{l}\text { Volume }\left(\mathrm{m}^{3}\right) \\
\text { Volume error }\left(\mathrm{m}^{3}\right) \\
\text { Volume @ } 5 \%\left(\mathrm{~m}^{3}\right) \\
\mathrm{H}_{5 \%}(\mathrm{~m}) \\
\Delta \mathrm{V} / \Delta \mathrm{H}\left(\mathrm{m}^{3} / \mathrm{m}\right) \\
\text { LL error } \pm 7.5 \mathrm{~mm}\left(\mathrm{~m}^{3}\right) \\
\text { Volume @ } 95 \%\left(\mathrm{~m}^{3}\right) \\
\text { Volume error (m3) } \\
\text { H95\% (m) } \\
\Delta \text { V/ } \Delta \mathrm{H}\left(\mathrm{m}^{3} / \mathrm{m}\right) \\
\text { LL error } \pm 7.5 \mathrm{~mm}\left(\mathrm{~m}^{3}\right) \\
\text { Volume added (m3) } \\
\text { Error estimate (m3) }\end{array}$ & $\begin{array}{c}96493.91 \\
\pm 4.8 \\
4824.7 \\
1.81 \\
2623.4 \\
\pm 19.7 \\
91669.2 \\
\pm 4.8 \\
34.91 \\
2627.3 \\
\pm 19.7 \\
86844.5 \\
\pm 28.68\end{array}$ \\
\hline
\end{tabular}

Table 3.4 .3

Shore Tank Estimate of Error Cylindrical Shore Tank Number 2

\begin{tabular}{|l|c|}
\hline Volume (m3) & 96628.32 \\
Volume @ 5\% (m3) & 4831.4 \\
Volume error (m3) & \pm 7.1 \\
H5\% (m) & 1.81 \\
$\Delta V / \Delta$ (m3/m) & 2625.3 \\
LL error $\pm 7.5 \mathrm{~mm}\left(\mathrm{~m}^{3}\right)$ & \pm 19.7 \\
Volume e end (m3) & 31986.9 \\
Volume error (m3) & \pm 7.1 \\
Hend (m) & 12.15 \\
$\Delta V / \Delta H\left(\mathrm{~m}^{3} / \mathrm{m}\right)$ & 2627.3 \\
LL error $\pm 7.5 \mathrm{~mm}\left(\mathrm{~m}^{3}\right)$ & \pm 19.7 \\
Volume added (m3) & 27155.5 \\
Error estimate (m3) & \pm 29.61 \\
\hline
\end{tabular}

The entries of table 3.4.3 have the same explanation as those of table 3.4.2. The difference is the total quantity of liquid added. The volumes cited in the tables have been corrected for internal structures and corrected to refer to cargo temperatures of $112.5 \mathrm{~K}$.

Density. Definition of the values for LNG density within the individual shore tanks can be influenced greatly by actual field conditions. Direct density measurement utilizing an in-tank densimeter is not always possible as many shore tanks are not so equipped. An individual tank densimeter may malfunction or become inoperative at some critical point in the measurement process. Calculated LNG densities which depend on LNG sampling and gas analysis (see section 2.2) may not provide individual sampling lines to each tank or individual LNG pressure and temperature measurements for each tank thus may obscure actual estimates of individual tank conditions. Few if any of these field conditions have been monitored in any detail prior to the writing of this manual. Documented comparisons of densities measured by densimeters and calculated under actual field operations are also not available at the present time. Therefore a number of simplifying assumptions have been made in order to allow a description of the measurement process in the context of the four case examples cited above.

1) A representative value for LNG density will be calculated for each LNG shore tank. Gas sampling and analysis as described in section 2.2 is assumed to provide a representative composition of the LNG in each tank. Representative values of tank liquid level (see tables 3.4.2, 3.4.3), liquid pressure and temperature are also assumed to be available. The process by which these values are established are assumed to be within the existing industrial practice.

2) In the absence of typical in-tank densimeter data, it is assumed that the measured density within the tank is numerically equal to the value calculated by means of sampling, gas analysis and use of the mathematical models. However, in assessing the estimated error, the total uncertainty of the densimeter used in the measurement will be that described in section 3.1 .2 of this manual. For example, if, as a result of 
calculations to determine LNG density from measured pressure, temperature and assumed composition give a value of $480.23 \mathrm{~kg} / \mathrm{m}^{3}$, the uncertainty for this process is found from table 3.1 .1 as \pm 0.23 percent. To illustrate the sample calculations which follow, it is assumed that the density measured by densimeter is also found to be $480.23 \mathrm{~kg} / \mathrm{m} 3$ but with an uncertainty from table 3.1 .1 of \pm 0.26 percent.

Calorific Value. Comments and restrictions to the process of measurement of LNG calorific value are similar to those of LNG density above. The acquisition of a representative sample from each shore tank which may be used in determining the calorific value directly from the combustion calorimeter or by calculation from gas analysis is necessary to provide a true representation of the total calorific value of the LNG cargo. The field problems associated with sampling and analysis are referenced in section 2.2 and additional simplifying assumptions are made here to present the process in the context of the four case examples.

1) A representative calorific value will be calculated for each LNG shore tank as for density values above. It is known, of course, that each individual tank may be found to have slightly different values of bulk density and calorific value. These differences are certainly caused in part by differences in compositions of liquid originally in the tank and that added into each tank. Since the following examples are to show method only, it is assumed that the densities and compositions for each tank are identical.

2) In the absence of typical field LNG calorific data, it is assumed that the calorific value for each LNG shore tank found from sampling and combustion calorimetry is numerically equal to the value calculated by means of sampling and gas analysis. However, in assessing the estimated error, the total uncertainty of the combustion calorimetry process used in the measurement will be that described in section 3.1 .2 of this manual.

For example, if as a result of calculations to determine LNG calorific value from measured pressure, temperature and assumed composition give a value of $54.323 \mathrm{MJ} / \mathrm{kg}$, the uncertainty for this process is found from table 3.1.1 as \pm 0.35 percent. To illustrate the sample calculations which follow, it is assumed that the calorific value measured by the combustion calorimeter is also found to be $54.323 \mathrm{MJ} / \mathrm{kg}$ but with an uncertainty from table 3.1 .1 of \pm 0.77 percent.

3) Measurement of the calorific value of each shore tank is assumed to be an independent measurement process, and therefore the sum of the individual uncertainties may be made in quadrature.

\subsubsection{Sample Calculations for Case 1}

This example is composed of a measured volume of LNG added to each shore tank (table 3.4 .2 and 3.4 .3 ), LNG sampling, gas analysis, LNG temperature and pressure measurement for calculation of LNG density and calorific values for each tank. These values are then summed to give the total caloriflc value (with uncertainty) of the LNG loaded. The source of the LNG is assumed to be Arzew, Algeria and the composition has been confirmed by gas analysis to be as shown in table 2.1.1. It is further assumed that an LNG bulk liquid temperature of $108.00 \mathrm{~K}$ and a pressure of $138 \mathrm{kPa}$ have been measured for the LNG contents of each ship tank. These liquid conditions are the same as those cited 1 n sections 3.2 and 3.3 .

Calculation of the LNG density can be accomplished by using one or more of the mathematical models described in section 2.4.3 and 2.4.4. One of the methods will be shown in detall, and a comparison or density values from the other three methods will be shown for comparison purposes. The Rev1sed Klosek and McKinley method (section 2.4.4.4) was selected because this method does not require a computer ror solution, although the method has been adapted to computer use.

The Klosek and McKinley (KM) method is a totally empirical recipe for calculating the density of the LNG-like mixture given the temperature and composition. Pressure is not taken 1 nto account. However. near atmospheric pressure LNG is nearly incompressible, hence the denslty may be cons 1 dernd independent of pressure. 
The procedure for calculating the LNG density is as follows:

$$
\mathrm{V}_{\mathrm{mix}}=\sum \mathrm{X}_{\mathrm{i}} \mathrm{V}_{\mathrm{i}}-\mathrm{kX}_{\mathrm{CH}_{4}}
$$

where $V_{m i x}$ is the volume of the mixture, $X_{i}$ and $V_{i}$ are the mole fraction and volume of the $i$ th component, $\mathrm{x}_{\mathrm{CH}_{4}}$ is the mole fraction of methane and $\mathrm{k}$ is a correction factor obtained from a table or graph.

The $V_{i}$ and $k$ are obviously temperature dependent, and in addition $k$ is dependent upon the molecular mass of the mixture. The original form of equation (1) was modified by adding a term to take into account the nitrogen when it is present. See section 2.4.4.4 for details.

$$
\mathrm{V}_{\operatorname{mix}}=\sum \mathrm{X}_{\mathrm{i}} \mathrm{V}_{\mathrm{i}}-\left[\mathrm{k}_{1}+\left(\mathrm{k}_{2}-\mathrm{k}_{1}\right) \mathrm{x}_{\mathrm{N}_{2}} / 0.0425\right] \mathrm{x}_{\mathrm{CH}_{4}}
$$

where $k_{1}$ refers to mixtures without nitrogen, and $k_{2}$ refers to mixtures with nitrogen. All other terms are the same as in equation (1). Tabular values for solving the equation are given in section $1,2.4 .4$ and 3.2 .

Table 3.4.4 provides initial data for the solution. Values for molecular mass are from tables 1.2 .1 and 1.2.2. Volumes of saturated liquid of the pure components are found from table 3.2.6. The slight differences in the molecular masses are a result of the revision cited in section 1.2 and do not change the values of mixture molecular mass shown in table 3.4.4.

Table 3.4 .4

Molecular Mass and Volume of Algerian LNG Mixture Temperature $=108 \mathrm{~K}$, Pressure $=138 \mathrm{kPa}$

\begin{tabular}{|l|l|l|l|l|l|}
\hline Constituent & $\mathrm{X}_{\mathbf{i}}$ & $\begin{array}{c}\mathrm{M}_{\mathrm{W}} \\
\mathrm{g} / \mathrm{mol}\end{array}$ & $\begin{array}{c}\mathrm{X}_{\mathrm{i}} \mathrm{M}_{\mathrm{W}} \\
\mathrm{g} / \mathrm{mol}\end{array}$ & $\begin{array}{c}\mathrm{V}_{\mathrm{i}} \\
\mathrm{L} / \mathrm{mol}\end{array}$ & $\begin{array}{c}\mathrm{XV}_{\mathrm{i}} \\
\mathrm{L} / \mathrm{mol}\end{array}$ \\
\hline \hline methane & 0.8700 & 16.043 & 13.9574 & 0.037481 & 0.032608 \\
ethane & 0.0814 & 30.070 & 2.4477 & 0.047512 & 0.003867 \\
propane & 0.0230 & 44.097 & 1.0142 & 0.062033 & 0.001427 \\
n-butane & 0.0068 & 58.123 & 0.3952 & 0.076384 & 0.000519 \\
i-butane & 0.0046 & 58.123 & 0.2674 & 0.077836 & 0.000358 \\
n-pentane & 0.0002 & 72.150 & 0.0144 & 0.091042 & 0.000018 \\
nitrogen & 0.0140 & 28.0134 & $\frac{0.3922}{8.4885}$ & 0.043963 & $\frac{0.000615}{0.0394124}$ \\
\hline
\end{tabular}

Values for the correction factors of equation (2) are found by linear interpolation of temperature and volume in table 3.2.7 for correction factor $k_{1}$ and table 3.2 .8 for correction factor $k_{2}$. For the temperature and composition selection for the example (table 3.4.4), the values for the correction factors were found to be $k_{1}=0.434$ and $k_{2}=0.61$.

Substituting in equation (2) gives:

$$
\begin{aligned}
& V_{m}= 0.0394124 \\
&-\left\{0.434\left(10^{-3}\right)+\left[0.61\left(10^{-3}\right)-0.434\left(10^{-3}\right)\right][0.014] / 0.0425\right\} 0.8700 \\
& V_{m}= 0.038985 \text { liters } / \mathrm{mol} \\
& \rho(\text { density })=1 / V_{m}=25.6509 \mathrm{~mol} / 1 \text { iter. }
\end{aligned}
$$

In terms of the actual mixture, the molar density is multiplied by the molecular mass of the mixture from table 3.4 .4 to give:

$$
\rho(\text { mixture density })=(25.6509) /(18.4885)=474.246 \mathrm{~kg} / \mathrm{m}^{3}
$$

Computer programs which provide a calculated value for the required density of the LNG mixture are discussed in detail in section 2.4.4. A comparison of the mixture densities predicted by the four mathematical models are shown in table 3.4.5. The four models are the revised Klosek and McKinley $(\mathrm{KM})$, the Corresponding States (CS), the Cell (C) and the Hard Sphere (HS). 


\section{Table 3.4 .5}

LNG Mixture Density Values Using

Several Calculation Methods

\begin{tabular}{|l|c|}
\cline { 2 - 2 } \multicolumn{1}{c|}{} & $\begin{array}{c}\text { Density } \\
\mathrm{kg} / \mathrm{m}^{3}\end{array}$ \\
\hline (from equation (2) and tables) & 474.246 \\
(from computer program- KM) & 474.242 \\
(from computer program- CS) & 474.446 \\
(from computer program- C) & 474.276 \\
(from computer program- HS) & 474.409 \\
(mean value) & 474.324 \\
(maximum error) & \pm 0.122 \\
\hline
\end{tabular}

The maximum error of the five calculation methods from the mean mixture density example as shown in table 3.4 .5 is \pm 0.026 percent. This is within the estimate of error for calculation of LNG density from the four mathematical models ( 0.1 percent) given in sections 2.4 .4 and 3.1 .2 . The value from equation (6) will be used in all the following example calculations.

Calorific Value. The calorific value of the selected LNG mixture may be found for the example of Case 1 by calculation from the gas analysis. The following method is suggested as one which includes the most recent analysis of the heats of combustion and atomic and molecular masses. The source of these values and suggested methods was an NBS study sponsored by Groupe International des Importateurs de Gaz Naturel Liquefie (GIIGNL). The results of the study have been published by NBS as NBSIR $82-2401$ "Heating Values of Natural Gas and Its Components" by G. T. Armstrong and T. L. Jobe (1982). As explained in section 1 of this manual, some of the values reported in the reference have since been revised (references $12 \mathrm{a}$ and b of section 1). The publication and revision are extensive in scope and detail, and only portions are cited in this section and section 1 . However, the content of the study is the basis of all calculations involving combustion enthalpies used in this manual.

The virial equation $P V / R T=1+B(T) / V$ where $P$ is absolute pressure, $V$ is molar volume, $R$ is the gas constant, $T$ is the absolute temperature and $B(T)$ the second virial coefficient is used by Armstrong and Jobe in the development of the combustion enthalpies of LNG mixtures. The equation is simple to use, has a basis in theory and allows an understanding of the application of the developed combustion enthalpies to ideal and real gas states for both pure fluids and mixtures.

The composition of the gas mixture in terms of the molar fractions $x(i)$ of the individual substances present, or in terms of the mass fractions $W(i)$ of the individual substances present, is presumed to be known (see table 3.4 .4 ) and $\sum X(i)$ or $\sum W(i)$ is unity.

The calculation of the heating value involves five steps: (1) the calculation of the ideal gas molar heating value of the mixture; (2) the calculation of the virial coefficient of the gas mixture and its temperature derivative; (3) the calculation of the molar volume of the real gas mixture based upon the virial coefficient of the gas mixture from step (2); (4) the adjustment of the molar heating value for the term $\mathrm{H}^{\circ} \mathrm{H}^{\circ}$ which is the difference in enthalpy of the real and ideal gas based on the virial coefficient of the gas mixture and its derivative; (5) the calculation of the heating value on a mass basis and the volumetric heating value of the real gas mixture.

Molar heating value $\Delta_{c} H_{m}$ (mixture) can be converted to the heating value on a mass basls $\Delta \mathrm{cH}_{\mathrm{W}}$ (mixture) by use of the mass fractions of the substances in the gas. From the molar volume calculated in step (3), the molar enthalpy of combustion can be converted to the volumetrlc enthalpy of combustion, $\Delta_{c} H_{v}$ (mixture). According to Armstrong and Jobe (see above), step ( 4 ) and the calculation of the temperature derivative of the virial coefficient can be omltted wlth errors not exceeding an estimated $50 \mathrm{~J} / \mathrm{mol}$.

The calculation procedure is considered to be the the most accurate of those consldered. It 13 applicable to both dry and humid gases, including the water-saturated gas. The dry gas cond1tions are assumed here. Notation and symbols are those of Armstrong and Jobe. The detalled descrlptlon or tha procedure is given in section 3.2, and the results of that procedure are used in thls sectlon. 
From step 5 of section 3.2 :

$$
-\Delta_{\mathrm{c}} \mathrm{H}_{\mathrm{m}}(\mathrm{mixture})=987.410 \mathrm{~kJ} / \mathrm{mole}
$$

Using the molecular mass of the mixture calculated in table 3.4.4,

$$
-\Delta_{c^{H}}(m i x t u r e)=(987.410) /(18.4885)=53.407 \mathrm{MJ} / \mathrm{kg}
$$

The volumetric combustion enthalpy comes from equation (15) and step 3 of section 3.2 .

$$
\begin{aligned}
-\Delta_{C^{H}} H_{V}(m i x t u r e) & =\Delta_{c} H_{m}(\text { mixture }) / V_{m}(m i x t u r e) \\
-\Delta_{C} H_{v}(\text { mixture }) & =(987.410) /(0.023583) \\
& =41.870 \mathrm{MJ} / \mathrm{m}^{3}
\end{aligned}
$$

\subsubsection{Case 1 Error Summary}

From the above calculations of volume, density and calorific value it is possible to assemble the total calorific value and estimate of error of the LNG added to the LNG shore tank.

From table 3.4 .2 the volume added to tank 1 is $86,844.5 \mathrm{~m}^{3}$. Equation (6) gives a density of 474.246 $\mathrm{kg} / \mathrm{m}^{3}$ for the LNG within the tank and therefore the mass added is the volume added multiplied by the density or $41,185.7 \mathrm{Mg}$. The calorific value of the LNG added is the mass added multiplied by the specific calorific value on a mass basis from equation (8). This value of $53.388 \mathrm{MJ} / \mathrm{kg} \mathrm{multiplied} \mathrm{by}$ the mass added gives the total calorific value of 2.200 PJ $\left(2.200 \times 10^{15} \mathrm{~J}\right.$ ) added to tank 1 . ( $P$ is the SI prefix symbol for peta or $10^{15}$, see section 1.1 ).

From table 3.4 .2 the volume error is $\pm 28.68 \mathrm{~m}^{3}$ or \pm 0.033 percent of the tank 1 volume added. From table 3.1.1 the estimated error in calculated density is \pm 0.23 percent, and the estimated error in calculated calorific value is \pm 0.35 percent. These individual estimates of error are independent variables and may be combined in quadrature. The combination of volume, density and specific calorific value is a simple multiplication, and therefore the error in total calorific value of the LNG added to the shore tank is:

Estimated Tank Error Case $1=\left[e_{v}^{2}+e_{d}^{2}+e_{c}^{2}\right]^{1 / 2}$

$$
= \pm 0.42 \text { percent }
$$

where $e_{\mathrm{v}}$ is the estimated volume error, $e_{d}$ is the estimated density error and $e_{c}$ is the estimated error in calorific value. The total error in calorific value of the LNG removed from tank 1 is $(2,188.6 \mathrm{TJ})( \pm 0.42) / 100)=9.24 \mathrm{TJ}$.

In like manner, total calorific values and estimated error, may be calculated for the shore tank 2. The results are summarized in table 3.4.6.

\subsubsection{Sample Calculations Por Case 2}

The example is composed of a measured volume of LNG added to each shore tank (table 3.4.2 and 3.4.3). A representative LNG density is measured for each tank using a calibrated LNG in-tank densimeter. A representative sample of LNG is removed from each tank, vaporized and analyzed to determine the fraction of each of the LNG components. Calculations are then made to determine the calorific value of the representative LNG sample on a mass basis.

As has been stressed in earlier sections of this manual, actual field data taken and assessed under controlled conditions are not available at the present time. The case in point would normally require individual measurements of the in-tank densimeters prior to, during and after loading. Since this information is not available, the representative LNG density calculated for Case 1 above (Algerian LNG of table 3.4.4) will be used for this example. In addition, the required sampling, analysis and calculation of calorific value described under Case 1 will also be assumed to be valid for Case 2 . This procedure will allow simple calculations and comparisons of the estimates of error for each of the combinations of measurement elements. For the shore tank of the example, the individual tank quantities and calorific values will remain the same as in Case 1 . The total calorific values will also be the same for all the cases. Only the estimated errors will change, and these will be a function of the measurement elements selected for each case. The values of estimated error for each element are given in table 3.1.1. 
In a manner similar to that described under Case 1 it is possible to assemble the total calorific value and estimate of error of the LNG added to the shore tank for the measurement assumptions of Case 2 .

From table 3.2 .2 the volume added to tank 1 is $86,844.5 \mathrm{~m}^{3}$. Equation (6) gives a density of 474.246 $\mathrm{kg} / \mathrm{m}^{3}$ for the LNG within the tank, and therefore the mass added is the volume added multiplied by the density or $41,185.7 \mathrm{Mg}$. The calorific value of the LNG added is the mass added multiplied by the specific calorific value on a mass basis from equation ( 8 ). This value of $53.407 \mathrm{MJ} / \mathrm{kg} \mathrm{multiplied} \mathrm{by}$ the mass added gives the total calorific value of $2.200 \mathrm{PJ}\left(2.200 \times 10^{15} \mathrm{~J}\right)$ added to tank 1 .

From table 3.4 .2 the volume error is $\pm 28.68 \mathrm{~m}^{3}$ or \pm 0.033 percent of the tank 1 volume. From table 3.1.1 the estimated error in measured density is \pm 0.26 percent, and the estimated error in calculated calorific value is \pm 0.35 percent. These individual estimates of error are independent variables and may be combined in quadrature. The combination of volume, density and specific calorific value is a simple multiplication, and therefore the error in total calorific value of the LNG added to the shore tank is:

Estimated Tank Error Case $2=\left[e_{\mathrm{v}}^{2}+e_{\mathrm{d}}^{2}+e_{\mathrm{c}}^{2}\right]^{1 / 2}$

$$
= \pm 0.44 \text { percent }
$$

where $e_{\mathrm{y}}$ is the estimated volume error, $e_{\mathrm{d}}$ is the estimated density error and $e_{\mathrm{c}}$ is the estimated error in calorific value. The total error in calorific value of the LNG added to tank 1 is ( $2,199.6$ $\mathrm{TJ})( \pm 0.44) / 100)=9.67 \mathrm{TJ}$.

In like manner, total calorific values and estimated error may be calculated for shore tank 2 . The results are summarized in table 3.4 .6 .

\subsubsection{Sample Calculations for Case 3}

This third example is composed of a measured volume of LNG added to each shore tank ( $t a b l e 3.4 .2$ and 3.4.3). A representative sample of LNG is removed from each shore tank, vaporized and analyzed to determine the fraction of each of the LNG components. The density of the sample is calculated using the mathematical models as shown in the example of Case 1. From the previous sample or a newly drawn one the sample is burned in the combustion calorimeter to determine the calorific value. A measured sample gas density will allow calculation of the calorific value on a mass basis.

The assumptions for Case 2 are made also for Case 3. For the shore tanks of the example, the individual tank quantities and calorific values will remain the same as in Case 1 . The total calorific values will also be the same for all the cases. Only the estimated errors will change, and these will be a function of the measurement elements selected for each case. The values of est 1 mated error for each element are given in table 3.1 .1 .

In a manner similar to that described under Case 1 it is possible to assemble the total calorifle value and estimate of error of the LNG added to the LNG shore tank for the measurement assumptions of Case 2 .

From table 3.4 .2 the volume added to tank 1 is $86,844.5 \mathrm{~m}^{3}$. Equation ( 6 ) gives a dens1ty of 474.246 $\mathrm{kg} / \mathrm{m}^{3}$ for the LNG within the tank and therefore the mass added is the volume added mult1pl1ed by the density or $41,185.7 \mathrm{Mg}$. The calorific value of the LNG added is the mass added mult1plied by the specific calorific value on a mass basis from equation (8). This value of $53.407 \mathrm{MJ} / \mathrm{kg} \mathrm{mult1} / \mathrm{pl} 1 \mathrm{ed} \mathrm{by}$ the mass added gives the total calorific value of $2.200 \mathrm{PJ}(2.200 \times 1015 \mathrm{~J})$ added to tank 1 .

From table 3.4 .2 the volume error is $\pm 28.68 \mathrm{~m}^{3}$ or \pm 0.033 percent of the tank 1 volume. From table 3.1 .1 the estimated error in calculated density is \pm 0.23 percent, and the estimated error $1 \mathrm{n}$ measured calorific value is \pm 0.77 percent. These individual estimates of error are independent varlables and may be combined in quadrature. The combination of volume, density and specific calor $1 \mathrm{f} 1 \mathrm{c}$ value 13 a simple multiplication, and therefore the error in total calorific value of the LNG added $1 \mathrm{~s}$ :

Estimated Tank Error Case $3=\left[e_{v}^{2}+e_{d}^{2}+e_{c}^{2}\right]^{1 / 2}$

$$
= \pm 0.80 \text { percent }
$$

where $e_{\mathrm{v}}$ is the estimated volume error, $e_{d}$ is the estimated density error, and $e_{c} 1 \mathrm{~s}$ the estlmated error in calorific value. The total error in calorific value of the LNG added to tank 113 ( 2.139 .6 $\mathrm{TJ})( \pm 0.80) / 100)=17.6 \mathrm{TJ}$. 
In like manner, total calorific values and estimated error may be calculated for shore tank 2 . The results are summarized in table 3.4 .6 .

\subsubsection{Sample Calculations for Case 4}

This final example is composed of a measured volume of LNG added to each shore tank (3.4.2 and 3.4.3). A representative LNG density is measured for each tank using a calibrated LNG densimeter. A representative sample is taken from each tank and is burned in the combustion calorimeter to determine the calorific value. A measured sample gas density will allow calculation of the calorific value on a mass basis.

The assumptions for Case 2 are made also for Case 4 . For the shore tanks of the example, the individual tank quantities, and calorific values will remain the same as in Case 1. The total calorific values will also be the same for all the cases. Only the estimated errors will change, and these will be a function of the measurement elements selected for each case. The values of estimated error for each element are given in table 3.1 .1 .

In a manner similar to that described under Case 1 it is possible to assemble the total calorific value and estimate of error of the LNG added to the LNG shore tanks for the measurement assumptions of Case 2 .

From table 3.4 .2 the volume added to tank 1 is $86,844.5 \mathrm{~m}^{3}$. Equation (6) gives a density of 474.246 $\mathrm{kg} / \mathrm{m} 3$ for the LNG within the tank, and therefore the mass added is the volume added multiplied by the density or $41,185.7 \mathrm{Mg}$. The calorific value of the LNG added is the mass added multiplied by the specific calorific value on a mass basis from equation (8). This value of $53.407 \mathrm{MJ} / \mathrm{kg} \mathrm{multiplied} \mathrm{by}$ the mass removed gives the total calorific value of $2.200 \mathrm{PJ}(2.200 \times 1015 \mathrm{~J})$ added to tank 1 .

From table 3.4 .2 the volume error is $\pm 28.68 \mathrm{~m}^{3}$ or \pm 0.033 percent of the tank 1 volume. From table 3.1 .1 the estimated error in measured density is \pm 0.26 percent, and the estimated error in measured calorific value is \pm 0.77 percent. These individual estimates of error are independent variables and may be combined in quadrature. The combination of volume, density and specific calorific value is a simple multiplication, and therefore the error in total calorific value of the LNG added is:

Estimated Tank Error Case $4=\left[e_{v}^{2}+e_{d}^{2}+e_{c}^{2}\right]^{1 / 2}$

$$
= \pm 0.81 \text { percent }
$$

where $e_{\mathrm{v}}$ is the estimated volume error, $e_{d}$ is the estimated density error, and $e_{c}$ is the estimated error in calorific value. The total error in calorific value of the LNG added to tank 1 is ( 2.199 .6 $\mathrm{TJ})( \pm 0.81) / 100)=17.80 \mathrm{TJ}$.

In like manner, total calorific values and estimated error may be calculated for shore tank 2 . The results are summarized in table 3.4.6.

Table 3.4 .6

Summary Results for Case 1, 2, 3, and 4

Calorific Value and Estimated Error for LNG Added to Shore Tanks

\begin{tabular}{|c|c|c|c|c|c|c|}
\hline \multirow[b]{2}{*}{ Tank } & \multirow{2}{*}{$\begin{array}{l}\text { Volume } \\
\text { Added } \\
\left(\mathrm{m}^{3}\right)\end{array}$} & \multirow{2}{*}{$\begin{array}{c}\text { Calorific } \\
\text { Value } \\
\text { (TJ) }\end{array}$} & \multicolumn{4}{|c|}{ Estimated Error $(\mathrm{TJ})^{*}$} \\
\hline & & & Case 1 & Case 2 & Case 3 & Case 4 \\
\hline $\begin{array}{l}1 \\
\frac{2}{\text { Total }}\end{array}$ & $\begin{array}{r}86,844.5 \\
27,155.5 \\
114,000.0\end{array}$ & $\begin{array}{r}2,199.6 \\
687.8 \\
2,887.4\end{array}$ & $\begin{array}{r} \pm 9.24 \\
\pm 2.89 \\
\pm 9.681 \\
( \pm 12.13)\end{array}$ & $\begin{array}{r} \pm 9.67 \\
\pm 3.02 \\
\pm 10.13 \\
( \pm 12.69)\end{array}$ & $\begin{array}{l} \pm 17.60 \\
\pm 5.50 \\
\pm 18.40 \\
( \pm 23.10)\end{array}$ & $\begin{array}{l} \pm 17.80 \\
\pm 5.57 \\
\pm 18.65 \\
( \pm 23.37)\end{array}$ \\
\hline
\end{tabular}


It should be noted that the total error for each case has been found by combining the errors from each tank in quadrature. It was assumed that measurements for each tank are independent of each other. This may not be true in practice, and a simple sum is included in parenthesis. It is one objective of this manual to provide enough of the basic data and a description of the method so that if other procedures are preferred, then these procedures may be followed using the original data.

The estimates of error for the total shore loading will vary as to the measurement elements used, and the level of detail and control of the measurement process. For example, the total error (independent tank measurements) varies from \pm 0.34 percent to \pm 0.65 percent, a rather small number and change considering the magnitude of the individual error estimates of the measurement elements. To hold the total uncertainty to a minimum, it pays to plan carefully for the entire measurement process. 



\begin{tabular}{|c|c|c|c|}
\hline $\begin{array}{l}\text { U.S. =EFT EF COMM. } \\
\text { BIBLIOGRAPHIC DATA } \\
\text { SHEET, See ins!'UCLIONSI }\end{array}$ & $\begin{array}{l}\text { 3. PUBLICATION OR } \\
\text { FEPORT NO. } \\
\text { NBSIR-85/3028 }\end{array}$ & 2. Performing Organ. Report Na. & $\begin{array}{l}\text { 3. Publication Date } \\
\text { June } 1986\end{array}$ \\
\hline \multicolumn{4}{|c|}{$\begin{array}{l}\text { A'TITLE AND SUBTITLE } \\
\text { LNG MEASUREMENT } \\
\text { A Users' Manua } 7 \text { for Custody Transfer }\end{array}$} \\
\hline \multicolumn{4}{|c|}{$\begin{array}{l}\text { 5. AUTHOR(S) } \\
\text { Douglas B. Mann, General Editor }\end{array}$} \\
\hline \multicolumn{3}{|c|}{$\begin{array}{l}\text { 6. PERFORMING ORGANIZA.TION (If joint or other than NBS, see instructions) } \\
\text { National Bureau of Standards } \\
\text { Department of Commerce } \\
\text { Gaithersburg, Md. } 20899\end{array}$} & $\begin{array}{l}\text { 8. Type of Report \& Period Covered } \\
\text { Final }\end{array}$ \\
\hline
\end{tabular}

9. SPONSORING ORGANIZATION NAME AND COMPLETE ADDRESS (Street. City. Stote, ZIP)

Document describes a computer program: SF-185, FIPS Software Summary, is attached.

11. ABSTRACT /A 200-word or less factual summory of most significont informotion. If document includes o significont titliogrophy or literoture survey. mention it here)

The LNG Measurement Manual will provide measurement engineers and others with a source of critically evaluated basic physical property data, a description of recent relevant measurement research and detailed examples of several methods of establishing the quantity and quality of liquefied natural gas (LNG) as a commercial commodity at the custody transfer point of sale. The contents of the manual are edited condensations of published research on properties and measurement processes. Explanations of the several methods of cargo valuation are considered as examples only and are not intended as recommended practice. Other methods of determining LNG cargo value may certainly be used. The procedures examined here utilize a consistent set of basic physical and thermophysical properties data and conversion factors which must be considered when comparing other measurement methods with those considered in this study. The concept of measurement as a process operating on a system is used both to describe the individual measurement elements and to show the integration of these elements into a total measurement process for LNG.

12. KEY VOORDS (Six to iwelve entries; alphobeticol order; copitalize only proper names: ond separate key words ty semicolons, analysis: calorific values: density: LNG: instrumentation: measurement: sampling: volume

13. AVAILABILITY

X Unlimited

W For Official Distribution. Do Not Release to NTIS

$\square$ Order From Superintendent of Documents, U.S. Government Printing Office, Washinglon, D.C. 20402.

14. NO. OF PRINTED PAGES

326

13. Price

X Order Fom National Tect.nical Information Service (NTIS), Springlield, VA. 22IEI 


